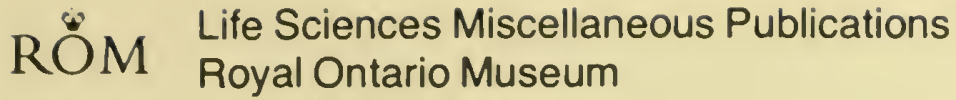

\title{
Catalogue of Type Invertebrate, Plant, and Trace Fossils in the Royal Ontario Museum
}

Janet Waddington Peter $\mathrm{H}$. von Bitter Desmond Collins

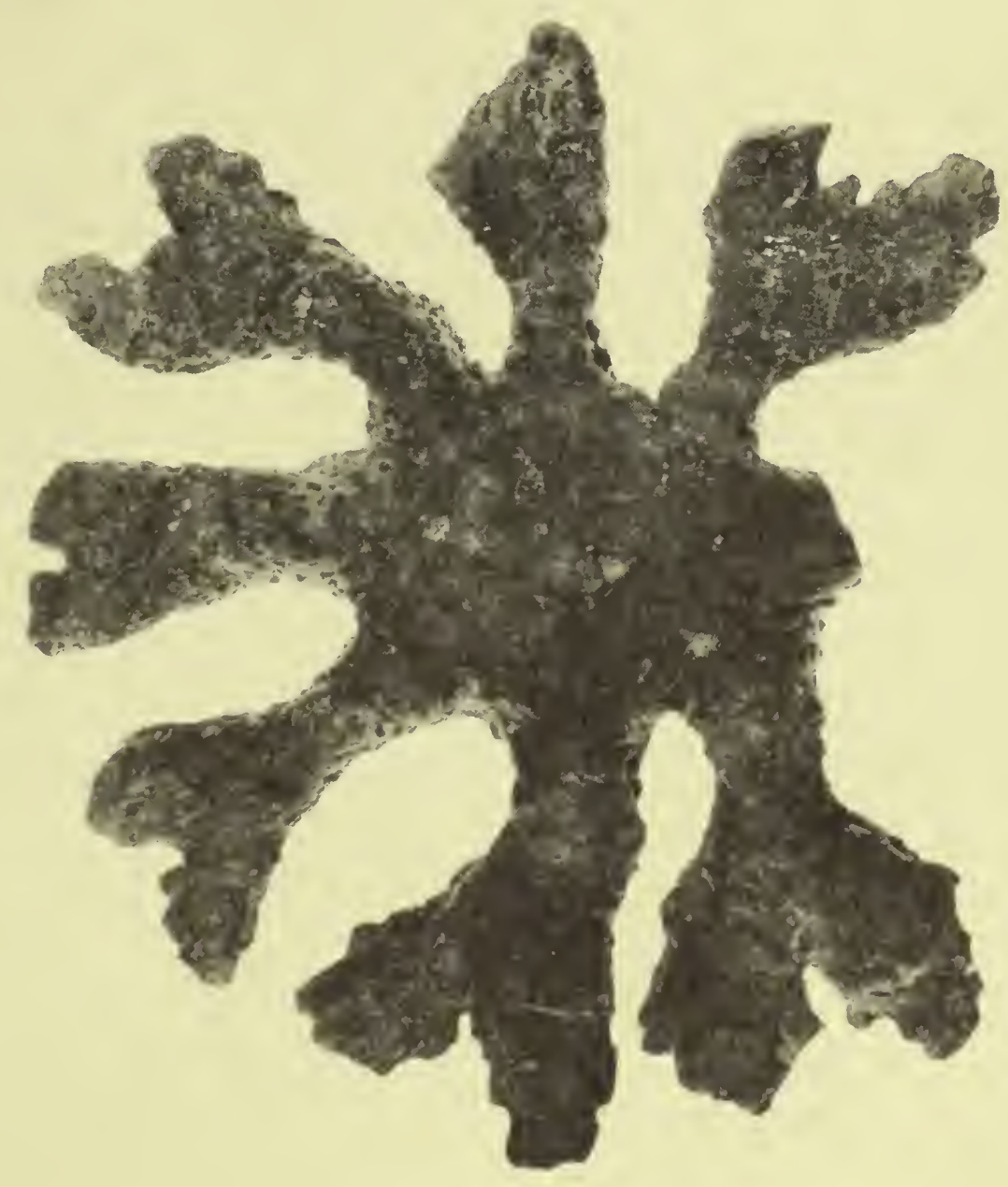




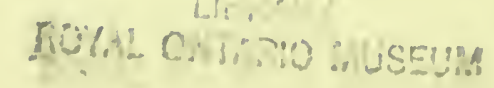


JANET WADDINGTON PETER H. VON BITTER DESMOND COLLINS

\section{Catalogue of Type Invertebrate, Plant, and Trace Fossils in the Royal Ontario Museum}


The Royal Ontario Museum publishes three series in the Life Sciences: LIFE SCIENCES CONTRIBUTIONS, a numbered series of original scientific publications including monographic works.

LIFE SCIENCES OCCASIONAL PAPERS, a numbered series of original scientific publications, primarily short and usually of taxonomic significance.

LIFE SCIENCES MISCELLANEOUS PUBLICATIONS, an unnumbered series of publications of varied subject matter and format.

All manuscripts considered for publication are subject to the scrutiny and editorial policies of the Life Sciences Editorial Board, and to review by persons outside the museum staff who are authorities in the particular field involved.

\section{LIFE SCIENCES EDITORIAL BOARD}

Chairman: A. R. EMERY

Senior Editor: A. G. EDMUND

Editor: A. J. BAKER

Editor: J. H. MCANDREWS

JANET WADDINGTon is a Curatorial Assistant in the Department of Invertebrate Palaeontology, Royal Ontario Museum.

PETER H. von BITTER is Associate Curator-in-charge in the Department of Invertebrate Palaeontology, Royal Ontario Museum, and Associate Professor in the Department of Geology, University of Toronto.

DESmond Collins is Curator in the Department of Invertebrate Palaeontology, Royal Ontario Museum, and Associate Professor in the Department of Zoology, University of Toronto.

\section{Cover photo:}

Brachiospongia tuberculata James

28299 Ref Spec-F

Trenton Gp, Bobcaygeon Fm

Kirkfield, Ont $(\times .6)$ 


\section{Contents}

Introduction 5
Characteristics of the Catalogue 5
Acknowledgenents 7
Catalogue of Types 9
Protozoa 11
Archaeocyatha 11
Porifera 11
Coelenterata, Conulariida 11
Coelenterata, Stromatoporoidea 12
Coelenterata, Anthozoa 21
Bryozoa 26
Brachiopoda 35
Mollusca, Monoplacophora 86
Mollusca, Gastropoda 86
Mollusca, Bivalvia 90
Mollusca, Cephalopoda 97
Mollusca, Miscellaneous 102
Arthropoda, Trilobita 104
Arthropoda, Ostracoda 118
Arthropoda, lnsecta 124
Arthropoda, Miscellaneous 130
Echinodermata, Crinozoa 130
Echinodermata, Asterozoa 132
Echinodermata, Echinozoa 132
Annelida 133
Conodontophorida 135
Graptolithina 140
Miscellaneous and Plants 143
Literature Cited 147


Digitized by the Internet Archive in 2012 with funding from Royal Ontario Museum 


\section{Catalogue of Type Invertebrate, Plant, and Trace Fossils in the Royal Ontario Museum}

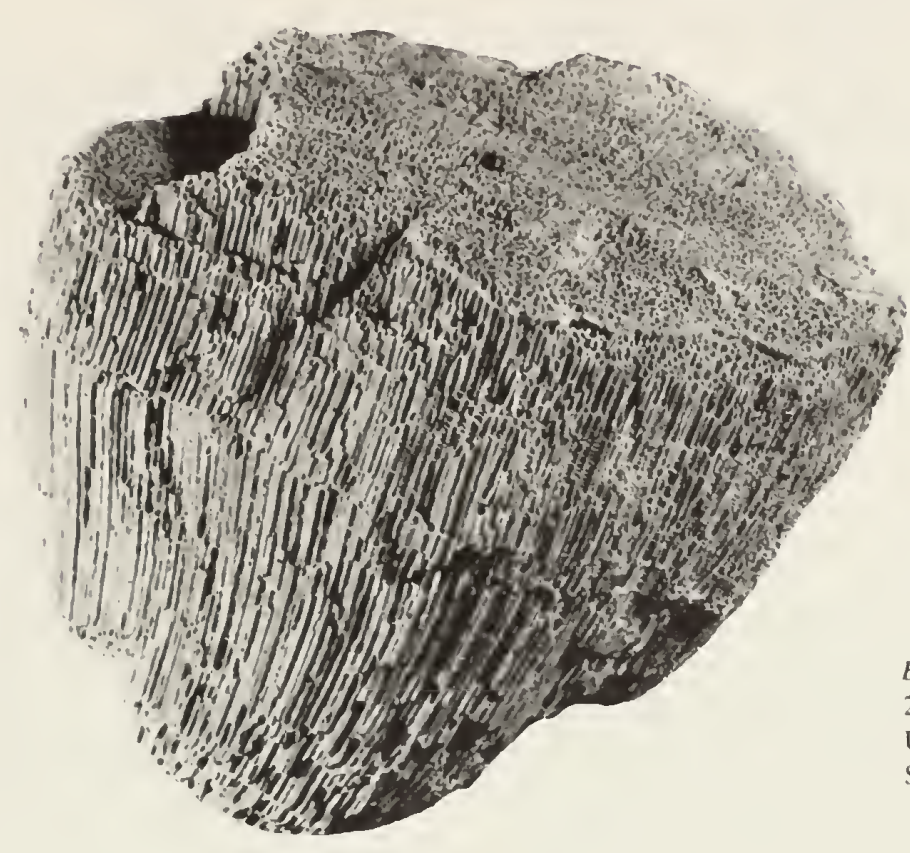

Emmonsia tuberosa (Rominger) 27075 Ref Spec-F

U. Abilibi River Fm

Sextant Rapids. Abitibi R, Ont $(\times .34)$

\section{Introduction}

Between 1941 and 1946 Madeleine A. Fritz produced a Catalogue of Types in the Royal Ontario Museum of Palaeontology, published in six short volumes. 1t included fossil invertebrates, fossil plants, and trace fossils. Since then, many more types have been added to the collections, so that publication of a new catalogue has become necessary.

The new catalogue includes all types, figured and referred specimens of fossil invertebrates, fossil plants, and trace fossils at present deposited in the Department of Invertebrate Palaeontology of the Royal Ontario Museum. It differs considerably from the earlier one in the addition of types that have either been located or designated since 1946, and in the deletion of manuscript types and fragments of type specimens housed at other institutions. The latter have been returned to those institutions.

Production of the new type catalogue is part of a much more extensive project to enter all data on the collections in the Department of Invertebrate Palacontology onto a computer-based storage and retrieval system. The task of standardizing and transferring the specimen records was begun in 1970 by von Bitter and was carried out from 1971 to the present by Waddington. Decisions on standards and projected characteristics of the catalogue were arrived at by consensus of all three authors.

\section{Characteristics of the Catalogue}

The computer used was a PDP8/1 with memory limitations requiring fixed-length fields in the record format. This has necessitated a certain amount of abbreviation and coding. Species and author are combined in a single field, making it necessary to abbreviate or omit the author in cases of a very long species name. Time rock units are coded (Table 1).

Entries are ordered alphabetically by genus and species within groupings chosen for curatorial and taxonomic convenience. Because of the nature of the computer programme used to sort the records taxonomically, specimen records of the same species are ordered randomly, with the result that the holotype of a species in some instances is listed after paratypes or other referred specimens.

Each specimen appears under the name to which the specimen was most recently referred in print, except for cases in which a prinary type has subsequently been referred to a different species. All specimen synonymy is mentioned in the main entry. In addition, all tix il to which a specimen has been referred occur as short entries crossreferenced to the main entry. No attempt has been made to cover species synonymy except for individual specimens that have been cited in print.

The Royal Ontario Museum houses types of many species which were described at a time when types were not always designated. Specimens recorded as types in Rom 


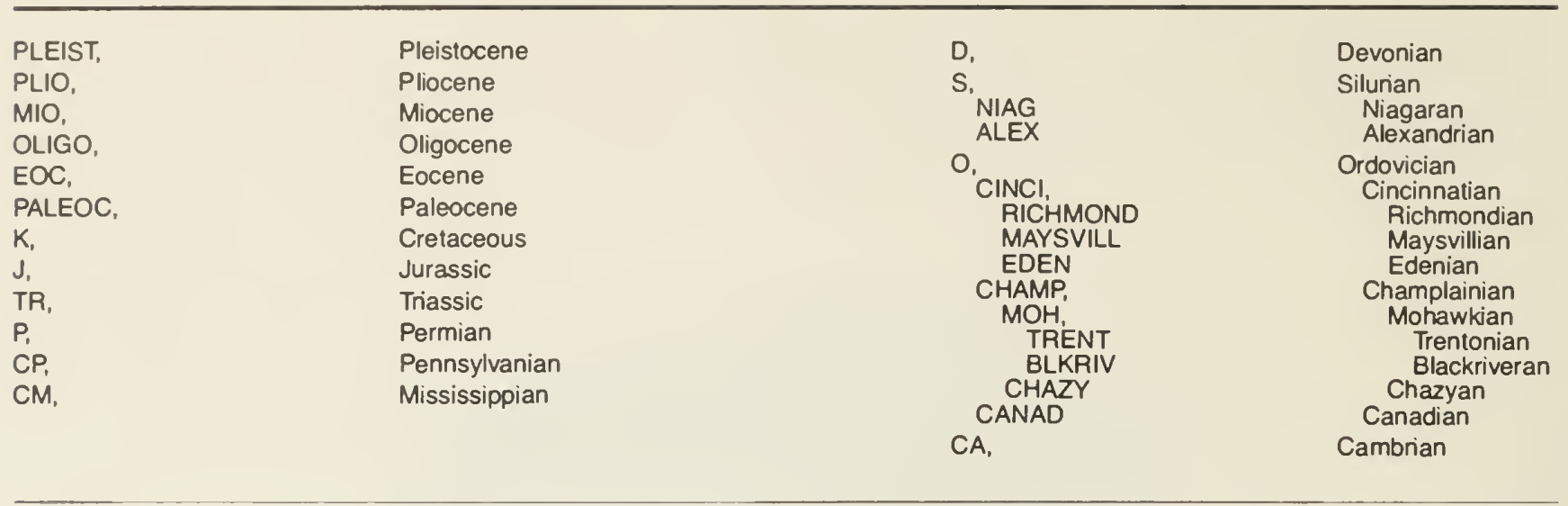

$$
\mathbf{U}=\text { Upper } \quad \mathbf{M}=\text { Middle } \quad \mathbf{L}=\text { Lower }
$$

files have been checked against original figures and descriptions where possible. If the authenticity of a type specimen is in doubt, a note has been made in the record. Some specimens listed in the earlier catalogues as holotypes or paratypes have been redesignated syntypes or lectotypes after investigation of the literature. A few specimens are recorded in the ROM files as "types" with no reference given. These records have been included in this catalogue.

Primary types are recorded as follows:

P-HOLO Holotype - the single specimen taken as the "type" by the original author of a species.

P-PARA Paratype - a specimen (or specimens), supplementary to the holotype, used by the original author as the basis of a new species. Specimens need only be mentioned in the original description.

P-SYN Syntype - one of several specimens of equal rank upon which a species is based, with no one specimen being designated as holotype.

P-LECTO Lectotype - a single specimen, selected from a syntypic series subsequent to the original description, to serve as the holotype.

P.PARAL Paralectotype - any other specimen of a syntypic series from which a lectotype has been designated.

P-NEO Neotype - a specimen selected to replace the holotype when the primary type material of a species has been lost or destroyed.

(Definitions after Schenk \& McMasters, 1956, and International Commission on Zoological Nomenclature, 1964.)
All secondary type specimens are listed in the catalogue as referred specimens:

REF SPEC-F Figured specimen.

REF SPEC-M Specimen mentioned in a publication but not figured.

REF SPEC ? Material that has been recorded as seconREF SPEC dary types but for which no reference or only a questionable reference could be found at the time of compilation of this catalogue.

Listed as referred specimens are many specimens formerly designated hypotypes, plesiotypes, figured specimens, heautotypes, onomotypes, or morphotypes.

"Types" not recognized by the ICZN (1964), such as topotype, metatype, homeotype, ideotype, and tophomeotype, are omitted from this catalogue. Taxa published as "variety", "mutation", or "form" have been treated as subspecies to the extent that this catalogue recognizes them as taxa, and their type specimens are treated as primary types.

Specimen records have been updated as far as possible from the original hand-written file cards. Unless otherwise noted, rock stratigraphic units are standardized according to the Geology and Economic Minerals of Canada (Douglas 1970) and the Lexicon of United States Geologic Names (Keroher et al. 1966). The standard for suprageneric classification is the Treatise of Invertebrate Paleontology and it has been adhered to for consistency even when it may be outdated. Bibliographic information is of necessity abbreviated. The first entry is usually a number with a letter prefix which gives the page reference for the genus in the Treatise of Invertebrate Paleontology or, when the appropriate volume of the Treatise has not been published, in 
Zittel (1927) using prefix $Z$. This is followed by a complete bibliographic history of the specimen including at least author, year of publication, page, and illustrations for all known references to the specimen. Abbreviations throughout the records have been kept to a minimun, and familiar usages have been followed whenever possible.

Records are printed in the following format, with each category being the total contents of one field in the computer file:

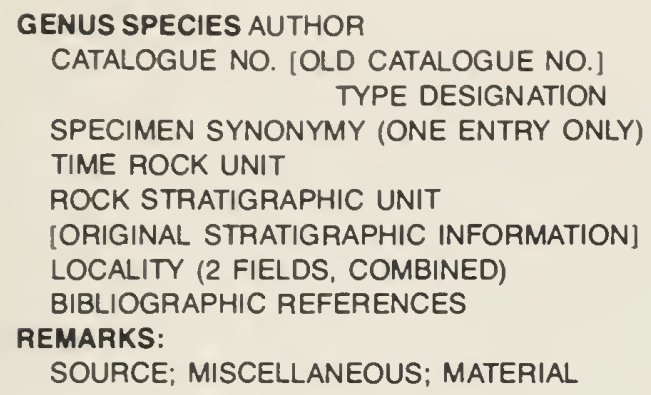

The catalogue number follows a purely numeric sequential system. The old catalogue number, when present, is part of an earlier system using a letter suffix, and has been retained because specimens are usually cited by this number. In some cases, for example, syntypic suites, more than one specimen has the same number. Whenever possible, specimens within these suites have been differentiated.

Source information is abbreviated as follows:

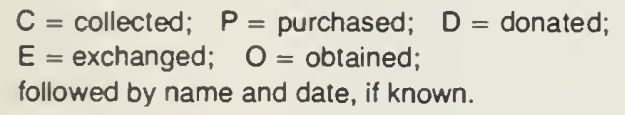

Locality abbreviations correspond to Blackadar (1972).

\section{Acknowledgements}

The initial impetus for this project was provided by the installation of a PDP8/1 computer in the Royil Ontario Museum in July 1970, as part of a Negotiated Development Grant from the National Research Council to the Department of Zoology of the University of Toronto for the development of a programme in systenatic and evolutionary zoology. The catalogue could not have been produced without the aid of the computer and operator funded by this grant.

Most of the data in the catalogue was entered onto computer tape with the support of a $\$ 10,000$ Research Agreement (\#1135-D13-4-47/72) from the Department of Energy, Mines and Resources to Collins and von Bitter in 1972/73 for the "Development and Testing of a Computer-Based System for the Storage and Retrieval of Palaeontological Data". Assistance in inputting and updating data from 1973 to 1976 was provided by a Cataloguing Assistance Grant from the National Museums Corporation.

Mrs. E. P. McGann ably handled both the programming and the practical aspects of inputting the data. Programmes for alphabetic and taxonomic sorting and other manipulation of data were developed by J. Kornatowski of the Department of Zoology, University of Toronto.

Although production of the catalogue was begun in 1970 , the assembly of the types, which were scattered throughout the collection, was carried out by John Monteith well before this time, and is acknowledged here.

Thomas E. Bolton, Geological Survey of Canada, and Frederick J. Collier, Smithsonian Institution, read the manuscript and offered helpful suggestions for its improvement. 


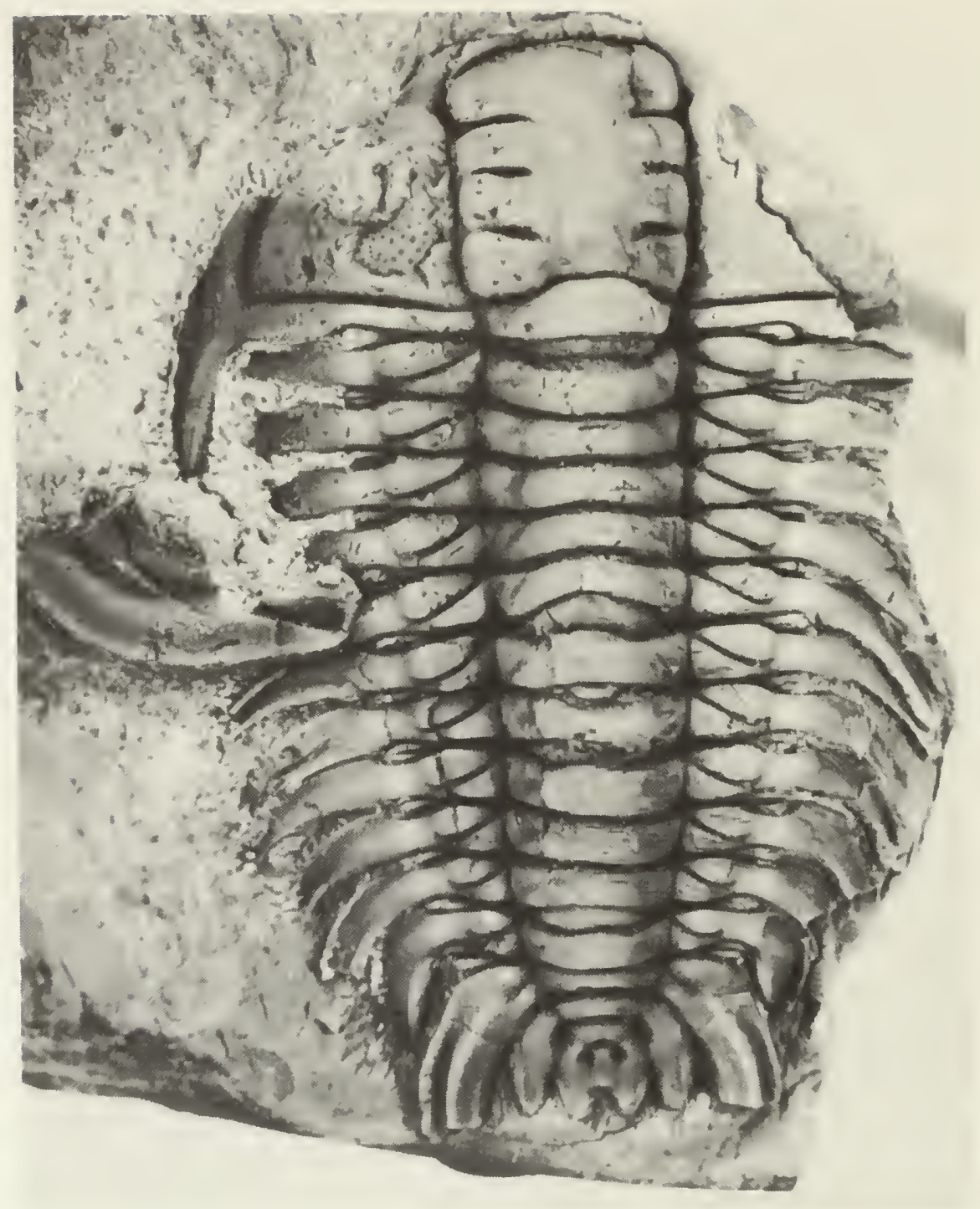


Catalogue of Types 



\section{PROTOZOA}

GL OBIGERINA CAMBRICA MATTHEW

$8291[661 \mathrm{CM}]$ P-SYN

$\mathrm{MCA}$.

ST JOHN GP

[ST JOHN.DIV 1C2]

HANFORD BK ST MARTINS,ST JOHN CONB

C669;MATTHEW GF, TRANS NY ACAD SCI,VOL

14,1895,P111,PL1,FIG 5A-C

REMARKS: D-W MACKENZIE ; NO TYPES DESIGNATED IN PUBL'N:SPEC LABELLED COTYPE; STRAT GIVEN AS BAND 1B IN PUBL'N,BAND 1C2 IN ROM RECORDS ; 1 SPEC

GLOBIGERINA GRANDIS MATTHEW

$8290[660 \mathrm{CM}] \mathrm{P}-\mathrm{HOLO}$

$\mathrm{MCA}$.

ST JOHN GP

[ST JOHN,DIV 1B2]

HANFORD BK, ST MARTINS,ST JOHN CO,NB

C669:MATTHEW GF. TRANS NY ACAD SCI, VOL

14, 1895, P111, PL1, FIG 6

REMARKS: D-W MACKENZIE;NO TYPES DESIGNATEDIN

PUBL' $N$; 1 SPEC

ORBULINA INGENS MATTHEW

8145 [515CM ] P-SYN

$\mathrm{MCA}$

ST JOHN GP

[ST JOHN,DIV 1B2]

HANFORD BK, ST MARTINS,ST JOHN CO,NB

C675; MATTHEW GF, TRANS NY ACAD SCI, VOL

14, 1895,P110,PL1,FIG 4

REMARKS: D-W MACKENZIE ;LABELLED COTYPES ; 3 SPEC

ORBULINA INTERMEDIA MATTHEW

$8148[518 \mathrm{CM}]$ P-SYN

$\mathrm{MCA}$

ST JOHN GP

[ST JOHN,DIV 1B2]

HANFORD BK, ST MARTINS,ST JOHN CO,NB

C675;MATTHEW GF,TRANS NY ACAD SCI,VOL

14, 1895,P110,PL 1,FIG 3

REMARKS: D-W MACKENZIE ; LABELLED COTYPES ; 3 SPEC

\section{ORBULINA CF UNIVERSA LAMARCK}

8293 [663CM] REF SPEC-F

$\mathrm{MCA}$.

ST JOHN GP

[ST JOHN,DIV 1B2]

HANFORD BK, ST MARTINS,ST JOHN CO,NB

C675:MATTHEW GF,TRANS NY ACAD SCI,VOL

14,1895,P109-110,PL 1,FIG 1

REMARKS: D-W MACKENZIE ;RECORDS STATE 2 SPEC;

LABELLED MORPHOTYPE; 1 SPEC

ORBULINA ? OVALIS MATTHEW

$8292[662 C M]$ P-SYN

$\mathrm{MCA}$

ST JOHN GP

[ST JOHN, DIV 1B2

HANFORD BK, ST MARTINS,ST JOHN CO,NB

C675;MATTHEW GF, TRANS NY ACAD SCI, VOL

14.1895.P110,PL1,FIG 2

REMARKS: D-W MACKENZIE ; LABELLED HOLOTYPE ; 1 SPEC

\section{ARCHAEOCYATHA}

ARCHAEOCYATHUS ? PAVONOIDES MATTHEW

REMARKS: SEE MATTHEWCYATHUS PAVONOIDES

(MATTHEW)
ARCHAEOSYCON VESICULOSUM OKULITCH

25640 P-HOLO

LCA.

FORTEAU FM

POINT AMOUR,LABRADOR

E(R) 123;OKULITCH VJ, 1943,GSA SPEC PAP,N48,P82-

83,PL $15, \mathrm{FIG} 1,2$

REMARKS: C,D-CO DUNBAR:SLIDES NO 65A,B;CUT FROM PEABODY MUS SPEC NO $17046 ; 2$ SLIDES

MATTHEWCYATHUS PAVONOIDES (MATTHEW)

7872 [242CM] P-HOLO

ARCHEOCYATHUS ? PAVONOIDES MATTHEW

$M C A$,

ST JOHN GP

[ST JOHN,DIV 1C]

HANFORD BK, ST MARTINS,ST JOHN CO,NB

E20:MATTHEW GF, TRANS ROY SOC CAN, 1886,SECT

4,P29,PL5,FIG 1A-D;OKULITCH VJ,1B 1D, 1940, VOL

34,SECT 4,P83,PL3,FIG 4,5

REMARKS: D-W MACKENZIE ;OKULITCH VJ,GSA SPEC PAP 48,1943,P85,PL14,FIG 6,PL15,FIG 5; 1 SPEC

\section{PORIFERA}

AULOCOPIUM GIGANTEA PARKS

$22661[417$ S] P-HOLO

AULOCOPIUM (AULOCOPELLA ?) GIGANTEA PARKS

O,

LOWER RAPIDS, GODS R MAN

E52; PARKS WA, TRANS ROY CAN INST, VOL 11,1915,P8,PL5,FIG 7

REMARKS: D-WH HEARST, 1915 ;C-JB TYRRELL, 1912;LOC GIVEN AS SHAMATTAWA R,NOW CALLED GODS RTRIBUTARY OF HAYES R ; 1 SPEC

BRACHIOSPONGIA TUBERCULATA JAMES

28299 REF SPEC-F

MO,CHAMP, MOH, TRENT

TRENTON GP,BOBCAYGEON CR LRMOST VERULAM FM

S SIDE ABANDONED QUARRY, $1.5 \mathrm{MIN}$ OF KIRKFIELD, KIRKFIELD,ONT

E76;RIGBY JK,J PALEO,V44,N6, 1970,P1139,TEXT FIG 2 REMARKS: C,D-N VOUTT ;FRAG AND 2 SLIDES DONATED TO ROM;SPEC PLACED ON PERMANENT LOAN TO ROM, 1973,FROM NEIL VOUTT ; 1 SPEC, 1 FRAG,SLIDES

\section{EOCORYNE GEMINUM MATTHEW}

8239 [609CM] P-SYN

$M C A$

ST JOHN GP

[ST JOHN,DIV 1C2]

PORTER'S BK, ST MARTINS, ST JOHN CO,NB

E 103;MATTHEW GF, TRANS ROY SOC CAN, 1886,SECT 4,P31,PL5, FIG 4,4A, 4B

REMARKS: D-W MACKENZIE ; LOC AND STRAT GIVEN IN PUBL'N AS DIV 1C AT HANFORD BK AND DIV ID AT PORTERS BK:LABELLED COTYPES : 3 SPEC

\section{COELENTERATA,CONULARIIDA}

\section{ARCHAEOCONULARIA ATTENUATA SINCLAIR} 23280 P.HOLO

MS,NIAG

CLINTON GP,ROCHESTER FM

[ROCHESTER]

GRIMSBY,ONT

F61:SINCLAIR GW,TRANS ROY SOC CAN, VOL 38 ,SECT 4,1944,P88-89,PL1,FIG 1,2

REMARK S: D-BE WALKER, 1905 ;MISTAKENLY PUBL'D AS ROM NO $1020 \mathrm{~N}$; 1 SPEC 
CONULARIA FORMOSA MILLER\&DYER

17454 [1215HR] REF SPEC-F

U O.CINCI,MAYSVILL

DUNDAS FM

DON VALLEY QUARRY,TORONTO,ONT

F60;PARKS \& FRITZ,ONT DEPT MINES,VOL

31,PT9, 1923,P13-14,PL2,FIG 4

REMARKS:5 SPEC

\section{CONULARINA NARRAWAYISINCLAIR}

18905 P-HOLO

MO,CHAMP,MOH,BLKRIV

BLACK RIVER GP

[BLACK RIVER

OTTAWA R, VAL TETREAU,QUE

F65; SINCLAIR GW,ANN CARNEGIE MUS, VOL 29,ART 10, 1942.P224-225,PL2 FIG 2

REMARKS: C-JE NARRAWAY;P-1938; 1 SPEC

EOCONULARIA AMOENA SINCLAIR

23279 P-HOLO

MO.CHAMP,MOH,BLKRIV

BLACK RIVER GP

TETREAUVILLE,QUE

F65:SINCLAIR GW, TRANS ROY SOC CAN,VOL 38,SECT 4,1944,P92,PL 1,FIG 7,8

REMARKS: P-JE NARRAWAY COLL, 1938 ;LOCALITY

UNCERTAIN;PUBL'N STATES TETREAUVILLE,OLD

RECORDS STATE VAL TETREAU;MISTAKENLY PUBL'D

AS ROM NO $18905 ; 1$ SPEC

\section{METACONULARIA GIBRALTARENSIS SINCLAIR}

$7537[829 \mathrm{CL}] \mathrm{P}-\mathrm{HOLO}$

S,ALEX

CATARACT GP, MANITOULIN FM

GIBRALTAR CLIFF,SE OF MANITOWANING, MANITOULIN

IS,ONT

F6 1:SINCLAIR GW, TRANS ROY SOC CAN, VOL 34,SECT

4,1940,P111-112,PL2,FIG 2

REMARKS: C-HG WAY, 1932 ;SPECIES PROPOSED BY WAY

IN U OF T THESIS MSS,PUBLISHED BY SINCLAIR; 1 SPEC

\section{METACONULARIA ? DUBIA SINCLAIR}

$18889 \mathrm{P}-\mathrm{HOLO}$

MO,CHAMP,MOH,BLKRIV

BLACK RIVER GP, LERAY FM

[OTTAWA LS,LERAY HORIZ]

VAL TETREAU,QUE

F61:SINCLAIR GW,TRANS ROY SOC CAN,VOL 34,SECT

4,1940,P107-108,PL3,FIG 1-2

REMARKS: C-JE NARRAWAY:D-1938; 1SPEC

\section{COELENTERATA,STROMATOPOROIDEA}

ACTINODICTYON VAGANS PARKS

9375[1572CN] P-SYN

$M D$

COLUMBUS FM

[ONONDAGA,COLUMBUS LS ]

S SIDE OF QUARRY, KELLEY'S IS,OHIO,USA

F130:PARKS WA,UNIV TOR STUDIES,GEOL

SER,N39, 1936,P 113-116,PL 18,FIG 1,2

REMARKS: C-J TOWNSEND :SLIDES 2214,2215,AND ONE

FROM 'PART OF SPEC MARKED X':ORIG 3 PCES OF

SAME SPEC-ONE SENT TO USNM WASHINGTON,MARCH $1942 ; 2$ SPEC, 3 SLIDES

\section{ACTINODICTYON VAGANS PARKS}

$9376[1573 C N]$ P-SYN

MD

COLUMBUSFM

[ONONDAGA,COLUMBUS LS

S SIDE OF QUARRY, KELLEY'S IS,OHIO,USA

F130;PARKS WA,UNIV TOR STUDIES,GEOL

SER,N39, 1936,P113-116,PL 18,FIG 5

REMARKS: C-J TOWNSEND :SLIDES 2213A,B;A FIG'D;2

PCES FROM SAME SPEC ; 2 PCES, 2 SLIDES
ACTINODICTYON VAGANS PARKS

$9377[1574 C N]$ P-SYN

MD.

COLUMBUS FM

[ONONDAGA,COLUMBUS LS]

S SIDE OF QUARRY, KELLEY'S IS, OHIO, USA

F130;PARKS WA, UNIV TOR STUDIES, GEOL

SER,N39, 1936,P113-116,PL 18,FIG 3,4

REMARKS: C-J TOWNSEND ; 3 PCES FROM ONE SPEC;

SLIDES 2211,$2212 ; 3$ PCES, 2 SLIDES

ACTINODICTYON VAGANS PARKS

$9378[1575 \mathrm{CN}]$ P-SYN

$M D$,

COLUMBUS FM

[ONONDAGA,COLUMBUS LS]

S SIDE OF QUARRY, KELLEY'S IS,OHIO, USA

F130;PARKS WA,UNIV TOR STUDIES,GEOL

SER,N39, 1936,P113-116,PL18,FIG 6,7

REMARKS: C-J TOWNSEND ;SLIDES 2229A,B AND ONE

FROM 'PART OF SPEC MARKED X';ORIG 3 PCES FROM ONE SPEC; 1 FRAG SENT TO M LECOMPTE,BRUSSELS, MARCH $1949 ; 3$ PCES, 3 SLIDES

ACTINOSTROMA CF EXPANSUM (HALL\&WHITFIELD)

$16554[2258 \mathrm{H}]$ REF SPEC-F

ACTINOSTROMA EXPANSUM VAR NOV PARKS

UD,

SHUEYVILLE,IOWA,USA

F127;PARKS WA,UNIV TOR STUDIES, GEOL

SER,N39, 1936,P120,PL13,FIG 3,4

REMARKS: P- ;5 PCES FROM 1 SPEC;SLIDES 2137,2138, 2139;2137 NOT LOCATED;HOLOTYPE IN FRITZ'S ROM TYPE CAT ; 5 PCES, 3 SLIDES

ACTINOSTROMA EXPANSUM (HALL\&WHITFIELD)

13211 [2622D ] REF SPEC-F

UD

S OF CHARLES CITY,IOWA,USA

F127:PARKS WA,UNIV TOR STUDIES, GEOL

SER,N39, 1936,P118-121,PL 19,FIG 3-6

REMARKS: P-CL FENTON;2 PCES OF ONE SPEC;SLIDES 2115A-D; 2 PCES, 4 SLIDES

\section{ACTINOSTROMA PARKSI FRITZ\&WAINES}

27358 P-HOLO

$M D$,

U ABITIBI RIVER FM

ABITIBI R, CORAL RAPIDS, ONT

F127;FRITZ \& WAINES,GAC PROC,VOL8, 1956,P9697,PL2,FIG 2

REMARKS: C-MA FRITZ,J MONTEITH ;C-1950;SLIDES 2358A,B ; 4 PCES,2 SLIDES

ACTINOSTROMA TYRRELLI NICHOLSON 13200 [2611D ] REF SPEC-F

$M D$

PRESQU'ILEFM

[PRESQU'ILEDOL]

PRESQU'ILE PT , GREAT SLAVE L,NWT

F127:PARKS WA,UNIV TOR STUDIES,GEOL

SER,N39, 1936,P121-124,PL 19,FIG 1,2

REMARKS: D-PS WARREN, U OF A, 1934 : SLIDES 1913 A,B

PART OF U OF ALTA NO DV674:C-WJ DEAN 1927:4 FRAG,2 SLIDES

\section{ACTINOSTROMA TYRRELLI NICHOLSON}

13201 [2612D ] REF SPEC-M

$M D$

PRESQU'ILEFM

[PRESQU'ILE DOL

PRESQU'ILE PT , GREAT SLAVE L,NWT

F127:PARKS WA,UNIV TOR STUDIES,GEOL

SER, N39, 1936,P121-124

REMARKS: D-PS WARREN,U OF A, 1934 ;C-AE CAMERON 1920:SLIDES 1914A,B:PART OF U OF ALTA NO DV673; NOT FIG'D; 5 FRAG, 2 SLIDES 
ACTINOSTROMA VULCANA PARKS

15088 [478G] P-SYN

MS,NIAG

GUELPH FM

DURHAM,ONT

F127:PARKS WA, UNIV TOR STUDIES, GEOL

SER, N4, 1907,P 10-11,PL 1,FIG 1,2,\&?

REMARKS: D-BE WALKER ;SLIDES 22,23;ONE FRAG SENT

TO USNM,MAY $1942 ; 5$ FRAGS, 2 SLIDES

ACTINOSTROMA WHITEAVESII VAR NIAGARENSIS PARKS

20994 [684N] P-HOLO

MS,NIAG

LOCKPORT GP

THOROLD.ONT

F127; PARKS WA,UNIV TOR STUDIES, GEOL

SER,N5, 1908,P11-13,PL7,FIG 4,5

REMARKS: D-BE WALKER ;SLIDES 156-166; 1 SPEC, 11 SLIDES

? ANOSTYLOSTROMA HAMILTONENSE PARKS

$16529[2233 \mathrm{H}]$ REF SPEC-F

M D.ERIAN

HAMILTON GP

ROCKY PT,ALPENA, MICH, USA

F---;PARKS WA.UNIV TOR STUDIES,GEOL

SER, N39, 1936,P49-50,PL7,FIG 6,7

REMARKS: C-WA PARKS ;SLIDES 1931A,B;SPEC LISTED BY PARKS AS BEING COMPARABLE TO ANOSTYLOSTROMA HAMILTONENSE AND CLATHRODICTYON UNDULATUM; IDENT UNCERTAIN ; 1 SPEC,3 FRAG,SLIDES

ANOSTYLOSTROMA HAMILTONENSE PARKS

$16537[2241 \mathrm{H}]$ P-PARA?

MD,ERIAN

HAMILTONGP

LONG L,ALPENA,MICH, USA

F---:PARKS WA,UNIV TOR STUDIES,GEOL SER, N39, 1936,P46-50

REMARKS: C-WA PARKS :LABELLED PARATYPE;NOT FIG'D OR MENTIONED SPECIFICALLY IN PUBL'N:SLIDES 2095A, $B ; 2$ SPEC,2 SLIDES

ANOSTYLOSTROMA HAMILTONENSE PARKS

$16536[2240 \mathrm{H}] \mathrm{P}-\mathrm{HOLO}$

$M D$ DRIAN

HAMILTON GP

LONG L,ALPENA,MICH,USA

F-.-;PARKS WA,UNIV TOR STUDIES,GEOL

SER, N39, 1936, P46-50,PL8, FIG 1,3,4

REMARKS: C-WA PARKS ;SLIDES 2097-2099;ORIG 3 PCES OF 1 SPEC; 1 FRAG SENT TO USNM,MARCH 1942;GENUS NOT IN TREATISE ; 2 PCES, 3 SLIDES

ANOSTYLOSTROMA HAMILTONENSE PARKS

5657 P.PARA

$M D, E R I A N$

HAMILTONGP

LONG L,ALPENA,MICH,USA

F---;PARKS WA, UNIV TOR STUDIES, GEOL

SER, N39, 1936, P46-50,PL8,FIG 2

REMARKS: C-WA PARKS ;GENUS NOT IN TREATISE ; 1 SPEC,SLIDE 2096

\section{ANOSTYLOSTROMA HAMILTONENSE PARKS}

$16555[2259 \mathrm{H}]$ P-PARA?

M D.ERIAN

HAMILTON GP

LONG L,ALPENA,MICH,USA

F---:PARKS WA, UNIV TOR STUDIES, GEOL

SER, N39, 1936, P48, PL 13,FIG 5,6

REMARKS: C-WA PARKS :SPEC LABELLED PARATYPE BUT NOT MENTIONEDIN PUBL' $N$ : 1 SPEC, 2 SLIDES

ANOSTYLOSTROMA HAMILTONENSE VAR PAPILLATUM

$16548[2252 \mathrm{H}]$ P-SYN

MD.ERIAN

HAMILTON GP

LONG L,ALPENA,MICH,USA

F---:PARKS WA, UNIV TOR STUDIES,GEOL
SER,N39, 1936,P50-52,PL9,FIG 1.4

REMARKS: C-WA PARKS ; SLIDES 2153-2156;RECORDS STATE 13 PCES; 1 FRAG SENT TO USNM MARCH 1942; GENUS NOT IN TREATISE ; 11 FRAG, 4 SLIDES

AULOCERIUM SAVAGEI PARKS

21447 [ $1267 \mathrm{~N}]$ P-HOLO

MS,NIAG

WILMINGTON,ILL,USA

F140;PARKS WA, UNIV TOR STUDIES, GEOL

SER,N6, 1909,P44-46, PL 18,FIG 13,15

REMARKS: C,D-TE SAVAGE, 1908 ;PART OF HOLOTYPE; REMAINDER DEPOSITED AT GEOL SURV ILL, URBANA, ILL;SLIDES 448,449; 1 FRAG, 2 SLIDES

BEATRICEA UNDULATA BILLINGS

17073 [819HR] REF SPEC ?

U O,CINCI,RICHMOND

RICHMOND GP

[RICHMOND]

ANTICOSTIIS, QUE

F142:PARKS WA, UNIV TOR STUDIES, GEOL

SER, N7, 1910,P336

REMARKS: D-HAMILTON SCI ASS, 1910 ;SPEC IS PART OFA LONG COENOSTEUM-NOT THE FIG'D PART OF SPEC

UNCERTAIN IF THIS IS REALLY PART OF THE FIG'D SPEC; SLIDE NO $360 ; 1$ SPEC, 1 SLIDE

\section{CLATHRODICTYON ARVENSE PARKS}

$9347[1438 \mathrm{CN}] \mathrm{P}-\mathrm{HOLO}$

$M D, E R I A N$

ONONDAGAGP

TOWNSEND TP,NR SIMCOE,ONT

F128:PARKS WA, UNIV TOR STUDIES, GEOL

SER,N39, 1936,P23-24,PL3,FIG 1,2

REMARKS: C-J TOWNSEND :SLIDES 1861A,B:NO SPEC : 2 SLIDES

\section{CLATHRODICTYON ARVENSE PARKS}

$9348[1439 \mathrm{CN}]$ P-PARA

$M D, E R I A N$

ONONDAGA GP

W ONT - LOC UNCERTAIN

F128:PARKS WA UNIV TOR STUDIES GEOL

SER,N39, 1936, P23-24,PL3,FIG 3,4

REMARKS: C-J TOWNSEND;SLIDES $1873 \mathrm{~A}, \mathrm{~B}$; RECORDS STATE 2 FRAGS; 1 FRAG SENT TO USNM, MARCH 1942; 1 FRAG,2 SLIDES

CLATHRODICTYONINSULARE PARKS

13187 [2598D]P-HOLO

COLUMBUS FM

[COLUMBUS LS, ONONDAGA]

S SIDE OF QUARRY, KELLEY'S IS, OHIO, USA

F128:PARKS WA UNIV TOR STUDIES, GEOL

SER,N39, 1936,P29-30,PL3,FIG 7,8

REMARKS: C-J TOWNSEND : SLIDES 1872A,B:RECORDS STATE 4 PCES OF ONE SPEC:ONE FRAG SENT TO USNM, MARCH $1942 ; 3$ PCES, 2 SLIDES

CLATHRODICTYONLATUM PARKS

$16526[2230 \mathrm{H}] \mathrm{P}-\mathrm{HOLO}$

$M D, E R I A N$

HAMILTON GP

LONG L, ALPENA, MICH,USA

F128:PARKS WA,UNIV TOR STUDIES, GEOL

SER,N39, 1936,P33-34,PL6,FIG 4,5

REMARKS: C-WA PARKS ;SLIDES 1897A,B;SPEC MISSING ; 2 SLIDES

CLATHRODICTYON LAXUM NICHOLSON

13171 [2582D ] REF SPEC-F

$M D$

COLUMBUS FM

[COLUMBUS LS,ONONDAGA]

KELLEY'S IS, OHIO,USA

F128:PARKS WA,UNIV TOR STUDIES, GEOL

SER,N39, 1936,P 13-16, PL 1, FIG 2

REMARKS: C-J TOWNSEND :SLIDE 1833;SPEC MISSING: 
SEE SPEC NO 25316 WHICH MAY BE THIS SPEC:LOC AND STRAT GIVEN IN PUBL'N AS ONONDAGA LS, ONTARIO ; 1 SLIDE

\section{CLATHRODICTYON LAXUM NICHOLSON}

13172 [2583D] REF SPEC-F

$\mathrm{MO}$.

COLUMBUS FM

[COLUMBUS LS,ONONDAGA]

KELLEY'S IS,OHIO,USA

F128;PARKS WA,UNIV TOR STUDIES,GEOL

SER, N39, 1936,P13-16,PL 1,FIG 1,4

REMARKS: C-J TOWNSEND;SLIDES 1834A,B;LOC GIVEN IN PUBL'N AS ONTARIO ; 6 FRAG, 2 SLIDES

\section{CLATHRODICTYON LAXUM NICHOLSON}

13173 [2584D] REF SPEC-F

$M$ D,ERIAN

ONONDAGA GP

WATERFORD,ONT

F128:PARKS WA,UNIV TOR STUDIES,GEOL

SER,N39, 1936,P 13-16,PL 1,FIG 3

REMARKS: SLIDES 1835A,B; 1 PCE, 2 SLIDES

\section{CLATHRODICTYON CF LAXUM NICHOLSON}

13174 [2585D] REF SPEC-F

MDERIAN

ONONDAGA GP

HAGERSVILLE,ONT

F128:PARKS WA,UNIV TOR STUDIES, GEOL

SER, N39, 1936,P 13-16, PL 1,FIG 5,6

REMARKS: C-J TOWNSEND :SLIDES 1836A,B;5 PCES FROM ONE SPEC ; 5 PCES, 2 SLIDES

\section{CLATHRODICTYON LAXUM NICHOLSON}

$13175[25860]$ REF SPEC-F

MD

[M DEVONIAN LS]

KWATABOAHEGAN R,ONT

F128;PARKS WA,UNIV TOR STUDIES,GEOL

SER,N39, 1936,P13-16,PL 1,FIG 8

REMARKS: C-WA PARKS ;SLIDES 1837 A-E; 1837 A FIG'D ; 5 PCES, 5 SLIDES

\section{CLATHRODICTYON LAXUM NICHOLSON}

13205 [26 16D ] REF SPEC-M

MD,

[MIDDLE DEVONIAN]

KWATABOAHEGAN R,ONT

F128;PARKS WA, 1936, UNIV TORONTO STUDIES,GEOL SER,N39,P 15

REMARKS: C-WA PARKS ;2 PCES OF 1 SPEC;SLIDE NO $2100 ; 2$ PCES, 1 SLIDE

\section{CLATHRODICTYON LAXUM VAR COLUMNARE PARKS}

$9374[1571 \mathrm{CN}] \mathrm{P}-\mathrm{HOLO}$

$M D, E R I A N$

ONONDAGA GP

MARBLEHEAD,OHIO,USA

F128;PARKS WA,UNIV TOR STUDIES,GEOL

SER,N39, 1936,P 16-18,PL9,FIG 5,6

REMARKS: C-J TOWNSEND ;SLIDES $2143,2144,2145 A, B$

$2143+2144$ FIG'D;FRAG SENT TO USNM.MARCH 1942;1

FRAG MISSING ; 1 SPEC, 4 SLIDES

\section{CLATHRODICTYON CF LAXUM,CF SUBSTRIATELLUM}

13176 [2587D ] REF SPEC-F

$\mathrm{MD}$.

COLUMBUS FM

[COLUMBUS LS,ONONDAGA]

MARBLEHEAD,OHIO,USA

F128:PARKS WA, UNIV TOR STUDIES,GEOL SER,N39, 1936,P 15,PL 1, FIG 7,PL2,FIG 4

REMARKS: C-J TOWNSEND -SLIDES 1838A-D A\&B FIG'D - 3 PCES, 4 SLIDES
CLATHRODICTYON MOOSENSE (PARKS)

13208 [2619D] P-HOLO

ACTINOSTROMA MOOSENSIS PARKS

$M D$

KWATABOAHEGAN R,ONT

F132:PARKS WA,ONT BUR

MINES,N13,PT 1, 1904,P183, 184,PL3,FIG 3,4:PARKS

WA, 1936,P34-35,PL8,FIG 5-7

REMARKS: C-WA PARKS :SLIDES 2113A-D;SPECIES

DESCRIBED 1904,REDESCRIBED 1936; 1 SPEC, 4 SLIDES

CLATHRODICTYON MOOSENSE VAR PROXIMALE PARKS

$13210[26210]$ P-HOLO

$M D$

KWATABOAHEGAN R,ONT

F128:PARKS WA,UNIV TOR STUDIES, GEOL

SER,N39, 1936,P35-36,PL 19,FIG 7,8

REMARKS: C-WA PARKS ;SLIDES $2111 \mathrm{~A}-\mathrm{C}$; 10 PCES FROM ONE SPEC ; 10 FRAG, 3 SLIDES

CLATHRODICTYON OHIOENSE PARKS

13184 [2595D] P-HOLO

MD.

COLUMBUS FM

[COLUMBUS LS,ONONDAGA]

KELLEY'S IS, OHIO USA

F128;PARKS WA,UNIV TOR STUDIES,GEOL SER,N39, 1936,P24-26,PL3,FIG 5,6

REMARKS: C-J TOWNSEND :SLIDES 1860A,B;RECORDS

STATE 5 PCES FROM ONE SPEC;ONE FRAG SENT TO

USNM, MARCH $1940 ; 4$ PCES, 2 SLIDES

\section{CLATHRODICTYON OHIOENSE PARKS}

13185 [2596D ] P-PARA

$M D$,

COLUMBUS FM

[COLUMBUS LS,ONONDAGA]

KELLEY'S IS,OHIO,USA

F128:PARKS WA, UNIV TOR STUDIES,GEOL

SER,N39, 1936,P24-26

REMARKS: C-J TOWNSEND;NOT FIG'D; 1 SPEC

\section{CLATHRODICTYON OHIOENSE PARKS}

13186 [2597D]P-PARA

$M D$.

COLUMBUS FM

[COLUMBUS LS,ONONDAGA]

KELLEY'S IS,OHIO,USA

F128:PARKS WA,UNIV TOR STUDIES, GEOL

SER,N39, 1936,P24-26

REMARKS: C-J TOWNSEND ;NOT FIG'D ; 4 PCES

\section{CLATHRODICTYON PONDEROSUM (NICHOLSON)}

13193 [2604D ] REF SPEC-F

$M D$,

COLUMBUS FM

[COLUMBUS LS,ONONDAGA]

MARBLEHEAD,OHIO,USA

F 128:PARKS WA UNIV TOR STUDIES, GEOL SER,N39, 1936,P42-44,PL5,FIG 5,6

REMARKS: C-J TOWNSEND ;SLIDES 1878A,B;1 SPEC AND 2 SMALL FRAGS ; 3 PCES, 2 SLIDES

CLATHRODICTYON PROBLEMATICUM PARKS

REMARK S: SEE STICTOSTROMA PROBLEMATICUM (PARKS)

\section{CLATHRODICTYON RECTUM PARKS}

$21438[1258 \mathrm{~N}]$ P-SYN?

MS,NIAG

LOUISVILLE,KY,USA

F128:PARKS WA,UNIV TOR STUDIES,GEOL SER,N5, 1908,P28-29,PL 14,FIG 5-7

REMARK S: C-GK GREENE? : SLIDES 107-109;1 SPEC AND ONE PCE,NOT FROM SAME SPEC; UNCERTAIN WHICH

SLIDES WERE MADE FROM;PARKS DOES NOT

DESIGNATE HOLOTYPE:UNCERTAIN IF SLIDES ARE FIG'D ; 2 PCES, 3 SLIDES 
CLATHRODICTYON REGULARE (VON ROSEN)

27354 REF SPEC-F

$M D$,

U ABITIBI RIVER FM

ABITIBI R, CORAL RAPIDS,ONT

F128:FRITZ\&WAINES,GAC PROC, VOL 8, 1956,P8889, PL 1,FIG 1-3

REMARKS: C-MA FRITZ,J MONTEITH ;C-1950;SLIDES $2354 A, B ; 8$ PCES.2 SLIDES

CLATHRODICTYON RETIFORME NICHOLSON \& MURIE $16523[2227 \mathrm{H}]$ REF SPEC-M

MD,ERIAN

HAMILTON GP

CHARLESTON,IND,USA

F128;PARKS WA.UNIV TOR STUDIES,GEOL SER, N39, 1936,P33

REMARKS: P- ;SLIDES NO 1901;NOT FIG'D ; 2 PCES,2 SLIDES

CLATHRODICTYON RETIFORME NICHOLSON \& MURIE $16520[2224 \mathrm{H}]$ REF SPEC-F

MD,ERIAN

HAMILTONGP

? PROBABLY THEDFORD OR ARKONA, ONT

F128:PARKS WA,UNIV TOR STUDIES,GEOL

SER,N39, 1936,P32,PL6,FIG 6,7

REMARKS: C-WA PARKS ;SLIDES 1902A,B;4 PCES FROM ONE SPEC ; 4 PCES, 2 SLIDES

\section{CLATHRODICTYON RETIFORME NICHOLSON\&MURIE}

$16522[2226 \mathrm{H}]$ REF SPEC-F

MD.ERIAN

HAMILTON GP

LONG L,ALPENA,MICH

F128; PARKS WA,UNIV TOR STUDIES,GEOL

SER,N39, 1936, P32, PL6,FIG 8

REMARKS: C-WA PARKS ;SLIDES 1903A,B;A IS FIG'D;3 PCES,2 SLIDES

CLATHRODICTYON STRIATELLUM (D'ORBIGNY)

15123 [515G ] REF SPEC?

MS,NIAG

GUELPHFM

DURHAM,ONT

F128;PARKS WA,UNIV TOR STUDIES,GEOL

SER,N4,1907,P 14-18,PL1,FIG 3?

REMARKS: C-J TOWNSEND;SLIDES NO 4,5;UNCERTAIN WHETHER THIS MATERIAL WAS FIG'D:ROM RECORDS STATE 'SEC FIG'D UNIV TOR STUDIES,GEOL SER NO 4'; NOT CITED IN PUBL'N ; 10 PCES.2 SLIDES

\section{CLATHRODICTYON STRIATELLUM (D'ORBIGNY)}

15056 [445G] REF SPEC?

MS.NIAG

GUELPHFM

ONT

F128;PARKS WA,UNIV TOR STUDIES,GEOL

SER,N4, 1907, P 14-18,PL1,FIG 4

REMARKS: C-J TOWNSEND :SLIDES NO 8.9:UNCERTAIN WHETHER THIS IS PUBLISHED MATERIAL;ROM RECORDS STATE 'SEC FIG'D UNIV TOR STUD,NO 5,GEOL SER'; GEOL SER NO 4,PL 1,FIG 4-WRITTEN ON SPEC ; 2 PCES, 2 SLIDES

\section{CLATHRODICTYON SUBSTRIATELLUM (NICHOLSON)}

13179 [2590D] REF SPEC-F

$M D$

COLUMBUS FM

[COLUMBUS LS,ONONDAGA]

MARBLEHEAD,OHIO,USA

F128:PARKS WA, UNIV TOR STUDIES,GEOL

SER, N39, 1936,P 18-23,PL2,FIG 1-3

REMARKS: C-J TOWNSEND;SLIDES 1867 A-C; 4 PCES

FROM ONE SPEC : 4 PCES. 3 SLIDES
CLATHRODICTYON SUBSTRIATELLUM (NICHOLSON)

13180 [2591D] REF SPEC-F

$M D$

COLUMBUS FM

[COLUMBUS LS.ONONDAGA]

MARBLEHEAD,OHIO,USA

F128:PARKS WA,UNIV TOR STUDIES, GEOL

SER N39, 1936,P22 PL2,FIG 7

REMARKS: C-J TOWNSEND :SLIDES 1868A-D;D FIG'D;3

PCES FROM ONE SPEC ; 3 PCES, 4 SLIDES

CLATHRODICTYON SUBSTRIATELLUM (NICHOLSON)

$13181[2592 D$ ] REF SPEC-F

$M D$,

COLUMBUSFM

[COLUMBUS LS.ONONDAGA]

MARBLEHEAD,OHIO,USA

F128;PARKS WA,UNIV TOR STUDIES,GEOL

SER,N39, 1936, P22,PL2,FIG 8

REMARKS: C-J TOWNSEND :SLIDES 1869A,B;B FIG'D;3

PCES FROM ONE SPEC ; 3 PCES, 2 SLIDES

\section{CLATHRODICTYON TOWNSENDI PARKS}

13189 [2600D] P-HOLO

$M D$,

COLUMBUS FM

[COLUMBUS LS,ONONDAGA

S SIDE OF QUARRY, KELLEY'S IS,OHIO,USA

F128;PARKS WA,UNIV TOR STUDIES,GEOL

SER N39, 1936, P26-29 PL4 FIG 1-5

REMARKS: C-J TOWNSEND :SLIDES 1898A,B;3 PCES FROM ONE SPEC : 3 PCES. 2 SLIDES

\section{CLATHRODICTYON UNDULATUM PARKS}

$16528[2232 \mathrm{H}]$ P-HOLO

$M D, E R I A N$

HAMILTON GP

LOCALITY UNCERTAIN

F128:PARKS WA,UNIV TOR STUDIES,GEOL SER,N39, 1936,P40-42,PL7,FIG 4,5

REMARKS: C-WA PARKS :SLIDES 1932 A B:3 PCES FROM ONE SPEC; 1 PCE SENT TO USNM,MARCH $1942 ; 3$ PCES.2 SLIDES

CLATHRODICTYON VARIOLARE VON ROSEN

$21419[1239 N]$ REF SPEC-F

MS,NIAG

LOCKPORT GP

ASSIGINACK TP, MANITOULIN IS,ONT

F128:PARKS WA UNIV TOR STUDIES, GEOL

SER,N5, 1908,P 19-21,PL7,FIG 2,PL8,FIG 9

REMARKS:D-BE WALKER; 1SPEC,SLIDE 111

CLATHRODICTYON VESICULOSUM MINUTUM (ROMINGER)

$21423[1243 N]$ REF SPEC-F

$M S, N I A G$

LOCKPORT GP

[LOCKPORT]

L TEMISKAMING,ONT

F128:PARKS WA,UNIV TORONTO STUDIES,GEOL

SER,N5, 1908,P 18-19,PL8,FIG 5

REMARKS: D-BE WALKER; UNCERTAIN WHICH SLIDE

FIG'D;SLIDES NO 141,$142 ; 3$ PCES, 2 SLIDES

CLATHRODICTYON VULGARE PARKS

$16550[2254 \mathrm{H}]$ P-HOLO

MD,ERIAN

HAMILTON GP

STONEY CK ALPENA MICH, USA

F128:PARKS WA,UNIV TOR STUDIES,GEOL SER,N39, 1936,P38-40,PL9,FIG 7.8

REMARKS: C-WA PARKS : SLIDES $2140,2141.9$ PCES FROM ONE SPEC;ONE FRAG SENT TO USNM,MARCH $1942 ; 9$ PCES, 2 SLIDES

CYSTOSTROMA FRITZAE GALLOWAY\&ST JEAN

27285 P-HOLO

U O, CINCI,RICHMOND

LISKEARD FM

FARR QUARRY, HAILEYBURY ONT 
F---;GALLOWAY \& ST JEAN,BULL AM PALEO,VOL

43, N 194, 1961,P16-17

REMARKS: C-MA FRITZ,J MONTEITH ;SLIDES 2327 A-D; SPEC NOT LOCATED;C-1949;ROM MATERIAL NOT FIG' D BUT PL2,FIG 1A,B ARE UNIV IND SLIDES 309-21,301-89, MADE FROM FRAG OF HOLOTYPE;FRAG STORED AT UNIV INDIANA; 1 SPEC,4 SLIDES

\section{DERMATOSTROMA CORRUGATUM PARKS}

$17071[817 \mathrm{HR}]$ P-HOLO

U O.CINCI,RICHMOND

TOP OF WHITEWATER BED

DUTCH CK, NR WILMINGTON,OHIO,USA

F140:PARKS WA,UNIV TOR STUDIES,GEOL

SER,N7, 1910,P34-35,PL24,FIG 7,10,11,14

REMARKS: C-GM AUSTIN, 1907 ;D-AF FOERSTE;SLIDES 355,$356 ; 1$ FRAG, 2 SLIDES

DERMATOSTROMA GLYPTUM PARKS

17070 [816HA] P-HOLO

U O,CINCI,RICHMOND

TOP OF WHITEWATER BED

DUTCH CK, NR WILMINGTON,OHIO,USA

F140;PARKS WA,UNIV TOR STUDIES, GEOL

SER,N7, 1910,P33-34,PL24,FIG 4-6

REMARKS: C-GM AUSTIN, 1907 ;D-AF FOERSTE;SLIDES 352-354; 5 FRAGS, 3 SLIDES

\section{DERMATOSTROMA PAPILLATUM (JAMES)}

17069 [815HR] REF SPEC-M

U O.CINCI,RICHMOND

RICHMOND GP,WAYNESVILLE FM

CLARKSVILLE,OHIO,USA

F 140;PARKS WA,UNIV TOR STUDIES, GEOL

SER, N7, 1910,P30-31

REMARKS: C-GM AUSTIN;D-AF FOERSTE ;SLIDE 351; SPEC AND SMALL FRAGS;NOT FIG'D; 1 SPEC, 1 SLIDE

\section{DERMATOSTROMA SCABRUM (JAMES)}

17075 [821HR] REF SPEC-F

U O,CINCI,MAYSVILL

DUNDAS FM

HUMBER R? TORONTO, ONT

F 140; FRITZ MA,ONT DEPT MINES, VOL 32,PT7, 1925,P2,PL 1,FIG 1

REMARKS: C-J TOWNSEND :SLIDES 761,$762 ; 1$ SPEC, 2 SLIDES

\section{HERMATOSTROMA GUELPHICA PARKS}

15089 [479G] P-SYN

MS,NIAG

GUELPH FM

ELORA,ONT

F 131:PARKS WA,UNIV TOR STUDIES,GEOL

SER,N4, 1907,P34-36,PL4,FIG 1,2,PL6,FIG 3,4

REMARKS: C-J TOWNSEND;SLIDES 72,73;ROM RECORDS STATE 3 PCES:ONE FRAG SENT TO USNM, MAY $1942 ; 2$ PCES,2 SLIDES

\section{LABECHIA DURHAMENSIS PARKS}

15060 [449G ] P-SYN

MS,NIAG

GUELPH FM

ELORA ONT

F140:PARKS WA,UNIV TOR STUDIES,GEOL SER,N4,1907,P20-22,PL2,FIG 4,5

REMARKS: C-J TOWNSEND ;SLIDES 76,77;ROM RECORDS STATE 3 PCES FROM 1 SPEC;ONE FRAG SENT TO USNM, MAY $1942 ; 2$ PCES,2 SLIDES

\section{LABECHIA DURHAMENSIS PARKS}

15082 [471G] P-SYN

MS NIAG

GUELPHFM

DURHAMONT

F 140;PARKS WA,UNIV TOR STUDIES,GEOL SER, N4, 1907,P20-22PL6,FIG 2?

REMARKS: C-J TOWNSEND :SLIDES 80,81 ; 1 SPEC.2 SLIDES
LABECHIA DURHAMENSIS PARKS

15092 [482G] P-SYN

MS,NIAG

GUELPHFM

DURHAM,ONT

F140;PARKS WA,UNIV TOR STUDIES,GEOL

SER, N4, 1907,P20-22,PL6,FIG 1

REMARKS: C-J TOWNSEND ; 1 SPEC

LABECHIA MINORA PARKS

15077 [466G ] P-SYN

MS,NIAG

GUELPHFM

ELORA,ONT

F 140;PARKS WA,UNIV TOR STUDIES,GEOL

SER, N4, 1907,P22-23,PL3,FIG 2,5,6

REMARKS: D-J TOWNSEND;SLIDES 84,85;3 PCES FROM SPEC;UNCERTAIN WHETHER THIS IS ALL THE FIG'D MATERIAL;LISTED AS HOLOTYPE IN FRITZ'S ROM TYPE CAT;NO TYPES DESIGNATED BY PARKS ; 3 PCES, 2 SLIDES

ROSENELLA GLENELGENSIS PARKS

15090 [480G] P-SYN

MS,NIAG

GUELPHFM

ELORA,ONT

F141;PARKS WA,UNIV TOR STUDIES,GEOL

SER,N4, 1907,P23-25,PL2,FIG 3,PL3,FIG 4,PL6,FIG 5

REMARKS: C-J TOWNSEND ;SLIDES 88,89,90;UNCERTAIN WHETHER THIS IS ALL THE FIG'D MATERIAL;NOTYPES DESIGNATED BY PARKS ; 17 FRAGS, 3 SLIDES

\section{ROSENELLA? MANITOULINENSIS PARKS}

$21443[1263 N]$ P-SYN

MS,NIAG

LOCKPORT GP

TEHKUMMAH TP, MANITOULIN IS,ONT

F 141; PARKS WA,UNIV TOR STUDIES,GEOL

SER,N5, 1908,P42-44,PL12,FIG 3,PL14,FIG 8 ?

REMARKS: C-J TOWNSEND, 1907 ;SLIDES 268,269;

UNCERTAIN WHETHER THIS IS THE FIG'D MATERIAL;

PARKS STATES 'HAVE MADE SPECS FROM MANITOULIN IS THE TYPE',BUT DOES NOT MENTION ROM ; 4 PCES,2 SLIDES

STICTOSTROMA ALPENENSE PARKS

16547 [2251H] P-HOLO

MD,ERIAN

HAMILTONGP

ROCKY PT, ALPENA, MICH,USA

F---:PARKS WA, UNIV TOR STUDIES, GEOL

SER,N39, 1936,P85-86,PL 13,FIG 1,2

REMARKS: C-WA PARKS :SLIDES 2191,$2192 ; 1$ PCE, 2 SLIDES

STICTOSTROMA BOREALIS FRITZ\&WAINES

27376 P-HOLO

MD.

U ABITIBI RIVER FM

ABITIBIR,CORAL RAPIDS, ONT

F---;FRITZ \& WAINES,GAC PROC,VOL8, 1956,P113

114,PL2,FIG 10-12

REMARKS: C-MA FRITZ,J MONTEITH :C-1950;SLIDES

2365A,B;FRAGS OF ONE SPEC ; 7 PCES, 2 SLIDES

STICTOSTROMA ERIENSE PARKS

13192 [2603D ] P-PARA

$\mathrm{MD}$

COLUMBUS FM

[COLUMBUS LS,ONONDAGA]

MARBLEHEAD,OHIO,USA

F-..;PARKS WA,UNIV TOR STUDIES,GEOL SER, N39, 1936,P81-83,PL5, FIG 4

REMARKS: $\mathrm{C}-\mathrm{J}$ TOWNSEND :SLIDES 1881A,B;EXPL'N OF PL 5 STATES FIG 4 FROM SAME SPEC AS FIG 3;TEXT STATES FIG 3 \& 4 ARE DIFFERENT SPECS ; 3 PCES, 2 SLIDES 
STICTOSTROMA ERIENSE PARKS

$13190[26010]$ P-HOLO

$M D$,

COLUMBUS FM

[COLUMBUS LS,ONONDAGA]

MARBLEHEAD,OHIO,USA

F---:PARKS WA,UNIV TOR STUDIES,GEOL

SER,N39, 1936,P81-83,PL5,FIG 1,2

REMARKS: C-J TOWNSEND :SLIDES 1882A,B;4 PCES FROM ONE SPEC ; 4 PCES, 2 SLIDES

STICTOSTROMA ERIENSE PARKS

$13191[2602 D]$ P.PARA

$M D$,

COLUMBUS FM

[COLUMBUS LS,ONONDAGA]

MARBLEHEAD,OHIO,USA

F---:PARKS WA,UNIV TOR STUDIES,GEOL

SER,N39, 1936,P81-83,PL5,FIG 3

REMARKS: C-J TOWNSEND;ROM RECORDS STATE 5 PCES

FROM 1 SPEC; 1 FRAG SENT TO USNM, MARCH 1942;

SLIDE $1880 ; 4$ PCES, 1 SLIDE

STICTOSTROMA HURONENSE PARKS

$16525[2229 \mathrm{H}]$ P.HOLO

M D,ERIAN

HAMILTONGP

LONG L,ALPENA,MICH,USA

F-.-.PARKS WA,UNIV TOR STUDIES,GEOL

SER,N39, 1936,P83-84,PL7,FIG 1-3

REMARKS: C-WA PARKS :SLIDES 1911A,B;ONE FRAG SENT

TO USNM,MARCH 1942; 3 PCES, 2 SLIDES

STICTOSTROMA INSOLITUM PARKS

$13207[2618 \mathrm{D}]$ P-HOLO

$M D$,

COLUMBUS FM

[COLUMBUS LS,ONONDAGA]

MARBLEHEAD,OHIO,USA

F---:PARKS WA,UNIV TOR STUDIES, GEOL

SER, N39, 1936,P86-88,PL15, FIG 4,5

REMARKS: C-J TOWNSEND :SLIDES 2112A,B;ROM

RECORDS STATE 5 PCES FROM ONE SPEC;ONE PCE

SENT TO USNM,MARCH 1942; 4 PCES, 2 SLIDES

STICTOSTROMA MAMMILLATUM (NICHOLSON)

$9361[1552$ CN ] REF SPEC-M

$M D$ ERIAN

ONONDAGA FM

ASHTON'S OUARRY, GORRIE TP, ONT

F---:PARKS WA, UNIV TOR STUDIES, GEOL

SER,N39, 1936,P78-81

REMARKS: NOT FIG'D; 1 SPEC

STICTOSTROMA MAMMILLATUM (NICHOLSON)

$9360[1551$ CN ] REF SPEC-F

$M D, E R I A N$

ONONDAGA FM

ASHTON'S QUARRY, GORRIE TP.ONT

F-.-:PARKS WA,UNIV TOR STUDIES,GEOL

SER,N39, 1936,P78-81,PL14,FIG 3-6

REMARKS: C-J TOWNSEND :SLIDES 2149-2152;ROM

RECORDS STATE 10 PCES FROM ONE SPEC ; 9 PCES

STICTOSTROMA PROBLEMATICUM (PARKS)

13196 [2607D]P-HOLO

CLATHRODICTYON PROBLEMATICUM PARKS

$M D$.

KWATABOAHEGAN R,ONT

F128:PARKS WA,ONT BUR

MINES,N 13,PT 1, 1904,P 184,PL4,FIG 5,6:PARKS

WA 1936,P88-90,PL5,FIG 7-8, PL6, FIG 1-3

REMARKS: C-WA PARKS :RECORDS STATE 3 PCES OF 1

SPEC:ONE FRAG SENT TO USNM, MARCH 1942:SLIDES

1883A-C : 2 PCES, 3 SLIDES
STICTOSTROMA PROBLEMATICUM (PARKS)

27375 REF SPEC-F

$M D$.

U ABITIBI RIVER FM

ABITIBI R, CORAL RAPIDS, ONT

F---;FRITZ \& WAINES,GAC PROC,VOL 8, 1956,P111113,PL2,FIG 7-9

REMARKS: C-MA FRITZ,J MONTEITH ;C-1950;SLIDES $2364 A, B ; 10$ PCES, 2 SLIDES

STICTOSTROMELLA BIFIDAPILA FRITZ\&WAINES

27356 P-HOLO

$M D$,

U ABITIBI RIVER FM

ABITIBI R,CORAL RAPIDS,ONT

F131:FRITZ \& WAINES, GAC PROC VOL 8,1956, P9294,PL1,FIG 6-9

REMARKS: C-MA FRITZ,J MONTEITH :C-1950:SLIDES

1356A-C:STICTOSTROMELLA = STROMATOPORELLA

SEE ADDENDUM TO FRITZ + WAINES, 1956,P126;2

PCES, 3 SLIDES

STICTOSTROMELLA CREBRAPILA FRITZ\&WAINES

27357 P-HOLO

$M D$,

U ABITIBI RIVER FM

ABITIBI R CORAL RAPIDS, ONT

F131;FRITZ \& WAINES,GAC PROC,VOL 8, 1956,P9495,PL1,FIG 10-12

REMARKS: C-MA FRITZ,J MONTEITH ;C-1950;SLIDES 2357A,B,:STICTOSTROMELLA = STROMATOPORELLA SEE ADDENDUM TO FRITZ + WAINES, 1956,P $126 ; 5$ PCES, 2 SLIDES

STROMATOCERIUM CANADENSE NICHOLSON\&MURIE

23503 [ 182T] REF SPEC-F

MO,CHAMP.MOH,BLKRIV

BLACK RIVER GP

[BLACK RIVER]

PAOUETTE'S RAPIDS, RIDEAUR,ONT

F141;PARKS WA, UNIV TOR STUDIES, GEOL

SER N7 1910 P 15-20,PL21,FIG 8

REMARKS: C-J TOWNSEND, $1909 ; 3$ PCES FROM ONE SPEC: SLIDES 388,$389 ; 389$ FIG'D ; 3 PCES, 2 SLIDES

STROMATOCERIUM CANADENSE NICHOLSON\&MURIE 23500 [179T] REF SPEC-F MO.CHAMP,MOH,BLKRIV

BLACK RIVER GP

[BIRDSEYE LS ]

LOTS 3\&4,CONC GLOUCESTER TP CARLETON CO.ONT

F141:PARKS WA, UNIV TOR STUDIES,GEOL SER,N7, 1910,P15-20,PL22,FIG 1,2

REMARKS: D-WR BILLINGS, 1909 ;SLIDES 382-384;2 PCES FROM ONE SPEC : 2 PCES, 3 SLIDES

STROMATOCERIUM HURONENSE BILLINGS

17072 [818HR] REF SPEC-F

U O, CINCI,RICHMOND

RICHMOND GP,WAYNESVILLE FM

WILMINGTON,OHIO,USA

F141;PARKS WA,UNIV TOR STUDIES,GEOL SER,N7, 19 10,P20-24,PL22,FIG 8,9

REMARKS: C.GM AUSTIN:D-AJ FOERSTE;D-1907:SLIDES 357-359;8 PCES FROM ONE SPEC ; 8 PCES, 3 SLIDES

STROMATOCERIUM RUGOSUM HALL

23498 [177T] REF SPEC-F

MO,CHAMP,MOH,BLKRIV

BLACK RIVER GP

PAOUETTE'S RAPIDS, RIDEAU R,ONT

F141:PARKS WA,UNIV TOR STUDIES,GEOL SER,N7, 1910,P11-15,PL21,FIG 5

REMARKS: C-J TOWNSEND, 1909;1 SPEC AND 1 FRAG SLIDES $376-379 ; 2$ PCES, 4 SLIDES 
STROMATOCERIUM RUGOSUM HALL

23499 [178T ] REF SPEC-F

MO,CHAMP,MOH,BLKRIV

BLACK RIVER GP

PAQUETTE'S RAPIDS, RIDEAU R,ONT

F141;PARKS WA, UNIV TOR STUDIES, GEOL

SER,N7, 1910,P11-15,PL21,FIG 6

REMARKS: C-J TOWNSEND, 1909 ;SLIDES $380,381,400 ; 2$ PCES,3 SLIDES

STROMATOCERIUM RUGOSUM HALL

23504 [183T ] REF SPEC-F

MO,CHAMP,MOH,TRENT

TRENTON GP

KIRKFIELD,ONT

F141:PARKS WA,UNIV TOR STUDIES,GEOL

SER,N7, 1910,P11-15,PL21,FIG 4

REMARK S: C-J TOWNSEND, $1909 ; 1$ SPEC AND 8 FRAGS:

SLIDES $390-392 ; 9$ PCES,3 SLIDES

\section{STROMATOCERIUM RUGOSUM HALL}

23509 [188T] REF SPEC-F

MO,CHAMP,MOH,BLKRIV

BLACK RIVER GP

PAQUETTE'S RAPIDS, RIDEAU R,ONT

F141;PARKS WA, UNIV TOR STUDIES,GEOL

SER,N7,1910,P11-15,PL21,FIG 7

REMARKS: C-J TOWNSEND, 1909 ;SLIDE 397;2 PCES FROM 1 SPEC;SMALLER PCE FIG'D ; 2 PCES, 1 SLIDE

STROMATOPORA GALLOWAYI FRITZ\&WAINES

27359 P-SYN

$M D$.

U ABITIBI RIVER FM

ABITIBI R,CORAL RAPIDS,ONT

F133;FRITZ \& WAINES,GAC PROC,VOL 8, 1956,P98100, PL2,FIG 3,4

REMARKS: C-MA FRITZ,J MONTEITH ;C-1950;SLIDES 2359A-C ; 4 PCES, 3 SLIDES

\section{STROMATOPORA GALLOWAYI FRITZ\&WAINES}

27360 P-SYN

$M D$,

U ABITIBI RIVER FM

ABITIBI R,CORAL RAPIDS,ONT

F133;FRITZ \& WAINES, GAC PROC, VOL 8, 1956,P98-100

REMARKS: C-MA FRITZ,J MONTEITH;C-1950; 4 PCES

\section{STROMATOPORA GALLOWAYI FRITZ\&WAINES}

27361 P-SYN

$M D$,

U ABITIBI RIVER FM

ABITIBI R, CORAL RAPIDS,ONT

F133;FRITZ \& WAINES,GAC PROC, VOL 8,1956,P98-100 REMARKS: C-MA FRITZ,J MONTEITH :C-1950; 10 PCES

STROMATOPORA GALTENSIS DAWSON

15063 [452G] REF SPEC?

MS,NIAG

GUELPHFM

DURHAM,ONT

F133:PARKS WA,UNIV TOR STUDIES, GEOL

SER,N4, 1907,P25-28,PL4,FIG 3,4

REMARKS: C-J TOWNSEND :SLIDES 32,33;ROM RECORDS

STATE 6 PCES;ONE FRAG SENT TO USNM,MAY 1942;

UNCERTAIN WHETHER SLIDES ARE THE FIG'D SLIDES

AS NO RECORD IN PUBL' $N$; 5 PCES,2 SLIDES

STROMATOPORA SAINTJEANI FRITZ\&WAINES

27363 P-SYN

$M D$

U ABITIBI RIVER FM

ABITIBI R, CORAL RAPIDS, ONT

F 133:FRITZ \& WAINES, GAC PROC, VOL 8, 1956,P 100-102

REMARKS: C-MA FRITZ,J MONTEITH :C-1950; 11 PCES
STROMATOPORA SAINTJEANI FRITZ\&WAINES

27362 P-SYN

$M D$,

U ABITIBI RIVER FM

ABITIBI R,CORAL RAPIDS,ONT

F 133;FRITZ \& WAINES,GAC PROC,VOL 8, 1956,P 100 102,PL2,FIG 5,6

REMARKS: C-MA FRITZ,J MONTEITH ;C-1950;SLIDES 2360A,B ; 4 PCES, 2 SLIDES

STROMATOPORELLA CELLULOSA (NICHOLSON\&MURIE) $9349[1440 \mathrm{CN}]$ REF SPEC-F

$M$ D,ERIAN

ONONDAGA GP (DRIFT)

NR SIMCOE,ONT

F131;PARKS WA,UNIV TOR STUDIES, GEOL SER,N39, 1936,P 108-110,PL4,FIG 6,7

REMARKS: D-BE WALKER;SLIDES 1884A-C ; 2 PCES, 3 SLIDES

STROMATOPORELLA ELEVATA PARKS

$16544[2248 \mathrm{H}]$ P-HOLO

MD,ERIAN

HAMILTON GP

ARKONA,ONT

F131;PARKS WA,UNIV TOR STUDIES, GEOL

SER, N39, 1936,P110-111,PL 15,FIG 1-3

REMARKS: C-WA PARKS ;SLIDES $2146,2147,2148 ; 1$ PCE, 3 SLIDES

STROMATOPORELLA ELORA PARKS

15069 [458G] P-SYN

$M S, N I A G$

GUELPH FM

DURHAM,ONT

F131;PARKS WA, UNIV TOR STUDIES, GEOL SER,N4, 1907,P29-31,PL6,FIG 6

REMARKS: C-J TOWNSEND ;UNCERTAIN WHETHER FIG REPRESENTS THIS SPEC;NO PARTICULAR SPECS MENTIONED IN PUBL' $N$; 2 PCES (1SPEC)

STROMATOPORELLA ELORA PARKS

15067 [456G ] P-SYN

MS,NIAG

GUELPH FM

DURHAM,ONT

F131:PARKS WA.UNIV TOR STUDIES,GEOL SER,N4,1907,P29-31,PL3,FIG 3

REMARKS: D-BE WALKER ;3 PCES FROM 1 SPEC;SLIDES 49-52; UNCERTAIN WHETHER FIG REPRESENTS THIS SPEC;NO PARTICULAR SPECS MENTIONED IN PUBL' $N$; 3 PCES,4 SLIDES

STROMATOPORELLA ELORA PARKS

15068 [457G] P-SYN

MS,NIAG

GUELPH FM

DURHAM ONT

F131; PARKS WA,UNIV TOR STUDIES, GEOL SER,N4, 1907,P29-31,PL5,FIG 1,3,4

REMARKS: C-J TOWNSEND ; 3 PCES FROM 1 SPEC;SLIDES 53-56;UNCERTAIN WHETHER FIGS REPRESENT THIS SPEC;NO PARTICULAR SPECS MENTIONED IN PUBL'N; ROM RECORDS STATE 4 PCES ; 3 PCES, 4 SLIDES

STROMATOPORELLA ELORA VAR MINUTA PARKS

15070 [459G] P-SYN

M S,NIAG

GUELPH FM

DURHAM,ONT

F131:PARKS WA UNIV TOR STUDIES, GEOL SER, N4, 1907,P31-34,PL5,FIG 2,5,6,PL6, FIG 7

REMARKS: C-J TOWNSEND :SLIDES 58-63;UNCERTAIN WHICH SLIDES ARE FIG'D; 1 PCE SENT TO USNM,MAY $1942 ; 8$ PCES, 6 SLIDES 
STROMATOPORELLA CF GRANULATA NICHOLSON 9366 [1563CN ] REF SPEC-F

$M D, E R I A N$

ONONDAGAGP

TEITZ QUARRY, SPRINGVALE,ONT

F131:PARKS WA.UNIV TOR STUDIES, GEOL

SER,N39, 1936, P 100,PL 15,FIG 6,7

REMARKS:C-WA PARKS :SLIDES 2193,$2194 ; 3$ PCES, 2 SLIDES

STROMATOPORELLA GRANULATA NICHOLSON

$16540[2244 \mathrm{H}]$ REF SPEC-F

$M D, E R I A N$

HAMILTONGP

ARKONA.ONT

F 131:PARKS WA,UNIV TOR STUDIES,GEOL

SER,N39, 1936, P95-100,PL16,FIG 3

REMARKS: C-J TOWNSEND :SLIDES 2103A,B;A IS FIG'D;6 PCES FROM 1 SPEC; 6 PCES,2 SLIDES

STROMATOPORELLA GRANULATA NICHOLSON

$16541[2245 \mathrm{H}]$ REF SPEC-F

MD,ERIAN

HAMILTONGP

ARKONA,ONT

F131:PARKS WA.UNIV TOR STUDIES, GEOL

SER,N39, 1936,P95-100,PL16,FIG 1,2

REMARKS: C-J TOWNSEND ;SLIDES 2104 A,B-FIG'D ; 1 SPEC,2 SLIDES

STROMATOPORELLA CF GRANULATA NICHOLSON $9365[1562 \mathrm{CN}]$ REF SPEC-M

$M D$ DERIAN

ONONDAGA GP ?

ASHTON'S QUARRY, GORRIE TP,ONT

F131:PARKS WA,UNIV TOR STUDIES,GEOL

SER,N39, 1936,P 100

REMARKS: C-J TOWNSEND ;NOT FIG'D ; 4 PCES

STROMATOPORELLA GRANULATA VAR DISTANS PARKS

$16546[2250 \mathrm{H}]$ P-PARA

MD.ERIAN

HAMILTON GP

MIDDLE L, ALPENA CO,MICH,USA

F131:PARKS WA,UNIV TOR STUDIES,GEOL

SER N39, 1936,P101

REMARKS: C-WA PARKS ;SLIDES $2120 A$, B; NOT FIG'D CARDS STATE 4 PCES FROM 1 SPEC; 1 FRAG SENT TO USNM, MARCH $1942 ; 3$ PCES, 2 SLIDES

STROMATOPORELLA GRANULATA VAR DISTANS PARKS $16542[2246 \mathrm{H}]$ P-HOLO

MD.ERIAN

HAMILTONGP

ARKONA ONT

F131;PARKS WA,UNIV TOR STUDIES,GEOL

SER,N39, 1936,P 100-101,PL14,FIG 7,8,PL15,FIG 8

REMARKS: C-J TOWNSEND :SLIDE $2190 ; 1$ SPEC, 1 SLIDE

STROMATOPORELLA KAYI PARKS

$13216[2627 D(B)]$ P-HOLO

UD,

HACKBERRYFM

[HACKBERRY SH]

HACKBERRY GROVE,IOWA,USA

F131:PARKS WA UNIV TOR STUDIES, GEOL

SER,N39, 1936,P111-112,PL13,FIG 7,8

REMARKS: P-WARD, 1921 ;SLIDES 2205,2206;PUBL' $N$

GIVES NO. AS 2627D(A)-SHOULD BE (B); 1 SPEC,2

SLIDES

STROMATOPORELLA SELWYNII NICHOLSON

9357 [1448CN] REF SPEC-M

$M D$ ERIAN

ONONDAGAGP

[ONONDAGA,DEVONIAN]

BEACHVILLE,ONT

F 131:PARKS WA, 1936,UNIV TORONTO STUDIES,GEOL SER,N39,P 104

REMARKS: C-J TOWNSEND : LOC SAID TO BE BEACHVILLE SPEC MENTIONED BY LOC ONLY IN PUBL'N : 1 SPEC
STROMATOPORELLA SELWYNII NICHOLSON $9358[1449 C N]$ REF SPEC-F

$M D, E R I A N$

COLUMBUS FM

[ONONDAGA]

S SIDE OF QUARRY, KELLY'S IS,OHIO,USA

F 131:PARKS WA.UNIV TOR STUDIES, GEOI

SER,N39, 1936,P101-104,PL17,FIG 5, 6

REMARKS: C-J TOWNSEND ;SLIDES $2106 \mathrm{~A}, \mathrm{~B} ; 1$ SPEC AND SEVERAL SMALL FRAGS ; 1 SPEC, 2 SLIDES

STROMATOPORELLA SELWYNII NICHOLSON

9355 [ $1446 \mathrm{CN}$ ] REF SPEC-F

$M D, E R I A N$

ONONDAGA GP

SELKIRK,ONT

F131;PARKS WA,UNIV TOR STUDIES,GEOL

SER,N39, 1936,P101-104,PL17,FIG 1,2

REMARKS: C-J TOWNSEND, $1889 ; 2$ PCES FROM ONE SPEC SLIDES 2105 A, B $; 2$ PCES, 2 SLIDES

STROMATOPORELLA TUBERCULATA NICHOLSON 9359 [1450CN] REF SPEC-F

$M D, E R I A N$

ONONDAGAGP

HAGERSVILLE,ONT

F131:PARKS WA,UNIV TOR STUDIES,GEOL SER,N39, 1936,P 104-107,PL 14,FIG 1,2

REMARKS: C-BE WALKER ;SLIDES 2108 A-D;A,B FIG'D ; 4 PCES, 4 SLIDES

STROMATOPORELLA TUBERCULATA NICHOLSON $9356[1447 \mathrm{CN}]$ REF SPEC-F

$M D, E R I A N$

ONONDAGA GP

ROCKFORD,ONT

F131:PARKS WA,UNIV TOR STUDIES, GEOL SER,N39, 1936,P 104-107,PL17,FIG 3,4

REMARKS: C-J TOWNSEND ;SLIDES $2107 \mathrm{~A}, \mathrm{~B} ; 1$ SPEC, 2 SLIDES

STYLODICTYON ROBUSTUM FRITZ\&WAINES

27355 P-HOLO

$M D$,

U ABITIBI RIVERFM

ABITIBI R,CORAL RAPIDS,ONT

F 131; FRITZ \& WAINES, GAC PROC, VOL 8, 1956,P9092,PL1,FIG 4,5

REMARKS: C-MA FRITZ,J MONTEITH ;C-1950:SLIDES 2355A,B;2 PCES OF 1 SPEC ; 2 PCES, 2 SLIDES

SYRINGOSTROMA AURORELLA FRITZ\&WAINES

27364 P-HOLO

$M D$

U ABITIBI RIVER FM

ABITIBI R,CORAL RAPIDS,ONT

F131;FRITZ \& WAINES,GAC PROC,VOL 8, 1956,P 103104.PL3,FIG 1.4

REMARKS: C-MA FRITZ,J MONTEITH ;C-1950;SLIDES $2361 \mathrm{~A}, \mathrm{~B} ; 4$ PCES, 4 SLIDES

SYRINGOSTROMA CREBRICOLUMNAE FRITZ\&WAINES 27365 P-HOLO

$M D$,

U ABITIBI RIVER FM

ABITIBI R.CORAL RAPIDS,ONT

F131;FRITZ \& WAINES, GAC PROC, VOL 8, 1956,P 104 106,PL3,FIG 7,10

REMARKS: C-MA FRITZ,J MONTEITH :C-1950:SLIDES

2362A,B; 1 LGE SPEC AND 11 PCES; 12 PCES,2 SLIDES

SYRINGOSTROMA DISTINCTICOLUMNAE FRITZ\&WAINES 27379 P-PARA

MD.

U ABITIBI RIVER FM

ABITIBI R. CORAL RAPIDS ONT

F 131;FRITZ \& WAINES, GAC PROC, VOL 8, 1956,P 108-109 REMARKS: C-MA FRITZ,J MONTEITH ;C-1950; NOT FIG'D ; 6 PCES 
SYRINGOSTROMA DISTINCTICOLUMNAE FRITZ\&WAINES

27367 P.HOLO

$M D$.

U ABITIBI RIVER FM

ABITIBI R, CORAL RAPIDS,ONT

F131;FRITZ \& WAINES,GAC PROC,VOL 8, 1956,P 108 109,PL3,FIG 9.12

REMARKS: C-MA FRITZ,J MONTEITH;C-1950;SLIDES 2367A,B;6 PCES OF 1 SPEC ; 6 PCES, 2 SLIDES

SYRINGOSTROMA DISTINCTICOLUMNAE FRITZ\&WAINES 27378 P-PARA

$M D$,

U ABITIBI RIVER FM

ABITIBI R, CORAL RAPIDS, ONT

F131;FRITZ \& WAINES,GAC PROC, VOL 8, 1956,P 108-109 REMARKS: C-MA FRITZ,J MONTEITH ;C-1950;NOT FIG'D ; 5 PCES

SYRINGOSTROMA PROPINQUICOLUMNAE FRITZ\&WAINES 27366 P-HOLO

$M D$.

U ABITIBI RIVER FM

ABITIBI R,CORAL RAPIDS,ONT

F131;FRITZ \& WAINES,GAC PROC,VOL 8, 1956,P 106107. PL3,FIG 8,11

REMARKS: C-MAFRITZ,J MONTEITH ;C-1950;SLIDES 2363A,B; 1 SPEC AND 15 FRAGS ; 16 PCES, 2 SLIDES

\section{SYRINGOSTROMA RECTICOLUMNAE FRITZ\&WAINES}

27377 P-PARA

$M D$,

U ABITIBI RIVER FM

ABITIBI R,CORAL RAPIDS,ONT

F13 1;FRITZ \& WAINES,GAC PROC,VOL 8, 1956,P 109-111 REMARKS: C-MA FRITZ,J MONTEITH ;C- 1950; NOT FIG'D ; 1 SPEC (3 PCES)

\section{SYRINGOSTROMA RECTICOLUMNAE FRITZ\&WAINES}

27380 P-HOLO

$M D$,

U ABITIBI RIVER FM

ABITIBI R,CORAL RAPIDS,ONT

F131; FRITZ + WAINES,GAC PROC,VOL 8,1956,P109-

111, PL3,FIG $2,3,5,6$

REMARKS: C-MA FRITZ,J MONTEITH ;C- 1950;SLIDES

2366 A-C; UNCERTAIN WHICH OF A AND B FIG'D,C IS FIG'D; 19 PCES, 3 SLIDES

\section{SYRINGOSTROMA RECTICOLUMNAE FRITZ\&WAINES}

27381 P-PARA

$M D$

U ABITIBI RIVER FM

ABITIBI R,CORAL RAPIDS,ONT

F131; FRITZ \& WAINES, GAC PROC, VOL 8, 1956,P 109-111 REMARKS: C-MA FRITZ,J MONTEITH ;C-1950;NOT FIG'D ; 1 SPEC

SYRINGOSTROMA RECTICOLUMNAE FRITZ\&WAINES

27373 P-PARA

$M D$

U ABITIBI RIVER FM

ABITIBI R.CORAL RAPIDS,ONT

F131; FRITZ \& WAINES,GAC PROC VOL 8, 1956,P 109-11 REMARKS: C-MA FRITZ,J MONTEITH ;C-1950;NOT FIG'D ; 1 SPEC

SYRINGOSTROMA RECTICOLUMNAE FRITZ\&WAINES 27374 P-PARA

$M D$

U ABITIBI RIVER FM

ABITIBI R, CORAL RAPIDS, ONT

F 131:FRITZ \& WAINES GAC PROC VOL 8, 1956,P 109-111 REMARKS: C-MA FRITZ,J MONTEITH ;C-1950;NOT FIG'D ; 1 LGE SPEC
TRISTYLOTUS HEXAGONUS PARKS

12978 [2368D ] P-SYN

$M D$.

KWATABOAHEGAN R,ONT

REMARKS: C-WA PARKS, 1903 -SLIDES 1433 A B:SPECS MARKED COTYPES-NO TYPES DESIGNATED:STORED WITH STROMATOPOROID TYPES ; 13 PCES, 2 SLIDES

TRUPETOSTROMA CORALVILLENSE (THOMAS)

13212 [2623D] P-SYN

UD,

CORALVILLE-CEDAR VALLEY FM

HAND'S QUARRY,RIVER PRODUCTS CO.

CORALVILLE,OHIO,USA

F132;PARKS WA,UNIV TOR STUDIES, GEOL SER,N39, 1936,PL 10, FIG 7,8

REMARKS: C-AO THOMAS:D-G KAY:SLIDES $2133 A-C \cdot A+$ B FIG'D;FRAGS OF NO 2600,STATE UNIV OF IOWA, WHICH IS A SYNTYPE OF THE SPECIES ; 4 PCES, 3 SLIDES

TRUPETOSTROMA IOWENSE PARKS

$13148[2559 \mathrm{D}] \mathrm{P}-\mathrm{HOLO}$

UD,

CEDAR VALLEY FM

MASON CITY,IOWA,USA

F132; PARKS WA,UNIV TOR STUDIES,GEOL

SER, N39, 1936,P57-60,PL 10,FIG 3-6

REMARKS: P :SLIDES $1912 \mathrm{~A}-\mathrm{E} ; \mathrm{B}, \mathrm{D}, \mathrm{E}$ FIG'D; 1 FRAG SENT

TO USNM, MARCH $1942 ; 1$ SPEC, 5 SLIDES

TRUPETOSTROMA MACULOSUM PARKS

$13215[2626 \mathrm{D}(\mathrm{A})]$ P-HOLO

UD

CEDAR VALLEYFM

CHARLES CITY,IOWA,USA

F132;PARKS WA,UNIV TOR STUDIES, GEOL

SER,N39, 1936, P67-70,PL11,FIG 4-6

REMARKS: P-CL FENTON :SLIDES 2195-2200;2 195,2 196

FIG'D;2 PCES FROM 1 SPEC ; 2 PCES, 6 SLIDES

TRUPETOSTROMA PLANULATUM (HALL\&WHITFIELD) 12549 [1935D ] REF SPEC-F

U D

HACKBERRY FM

[HACKBERRY SH]

HACKBERRY GROVE,IOWA USA

F132; PARKS WA, UNIV TOR STUDIES, GEOL SER, N39, 1936,P62-64,PL 11,FIG 2,3

REMARKS: P-CLFENTON :SLIDES 2 187-2 189;2 187,2188 FIG'D; 1 SPEC, 3 SLIDES

TRUPETOSTROMA SOLIDULUM (HALL\&WHITFIELD)

11905 [1899D ] REF SPEC-F

UD,

HACKBERRYFM

[HACKBERRY SH]

ROCKFORD, IOWA,USA

F132:PARKS WA,UNIV TOR STUDIES, GEOL

SER,N39, 1936,P64-66,PL 12,FIG 3,4

REMARKS: P-CL FENTON :SLIDES 2 180-2 186;2 182,2 183

FIG'D : 5 PCES, 7 SLIDES

TRUPETOSTROMA SOLIDULUM (HALL\&WHITFIELD)

11906 [1900D ] REF SPEC-M

U D,

HACKBERRYFM

[HACKBERRY SH]

HACKBERRY CK, IOWA, USA

F132:PARKS WA,UNIV TOR STUDIES,GEOL

SER,N39, 1936,P64-66

REM ARKS: P-CL FENTON ; SLIDES 2170-2 175; NOT FIG'D ; 9 PCES,6 SLIDES

TRUPETOSTROMA SOLIDULUM (HALL\&WHITFIELD) 13213 [2624D] REF SPEC-F

UD

IOWA,USA

F 132.PARKS WA, UNIV TOR STUDIES, GEOL

SER, N39, 1936,P64-66,PL 12,FIG 5

REMARKS: P-CL FENTON :SLIDES $2121 \mathrm{~A}, \mathrm{~B} ; \mathrm{A}$ IS FIG 'D ; 7 PCES, 2 SLIDES 
TRUPETOSTROMA WARRENI PARKS

$13179[2608 \mathrm{D}] \mathrm{P}-\mathrm{HOLO}$

MD,

PRESQU'ILE PT , GREAT SLAVE L,NWT

F132;PARKS WA, UNIV TOR STUDIES,GEOL SER,N39, 1936,P55-57,PL 10,FIG 1,2

REMARKS: C,D-AE CAMERON :C-1920;SLIDES 1885A-C;A AND B FIG'D;FRAGS OF NO DV 677, U OF ALTA ; 5 FRAGS, 3 SLIDES

TRUPETOSTROMA ? ERRATICUM (HALL\&WHITFIELD)

13215 [? $626 \mathrm{D}(\mathrm{B})]$ REF SPEC-F

UD,

CEDAR VALLEY FM

[CEDAR VALLEY LS ]

CHARLES CITY,IOWA, USA

F132; PARKS WA,UNIV TOR STUDIES,GEOL

SER,N39, 1936,P74-77,PL 12,FIG 7.8

REMARKS: P-CL FENTON ; SLIDES 2201,2202; ; 1 SPEC,2 SLIDES

TRUPETOSTROMA ? INCRUSTANS (HALL\&WHITFIELD)

12547 [1932D] REF SPEC-F

UD.

HACKBERRY FM

[HACKBERRY SH]

HACKBERRY GROVE, IOWA.USA

F132; PARKS WA,UNIV TOR STUDIES, GEOL

SER,N39, 1936,P7 1-74,PL11,FIG 8,PL12,FIG 1

REMARKS: P-WARD'S ;SLIDES 2176-2179;2176 AND 2177

FIG'D; 1 SPEC 4 SLIDES

\section{COELENTERATA,ANTHOZOA}

ACROPHYLLUM ONEIDAENSE (BILLINGS)

27055 REF SPEC-F

$M D$

U ABITIBI RIVER FM

[U ABITIBI RIVER LS ]

W BANK OF R, ABITIBI R, CORAL RAPIDS, ONT

F272:CRANSWICK \& FRITZ, GAC PROC, VOL 10,1958, P33-

34,PL1,FIG 4-7

REMARKS: C-MA FRITZ,J MONTEITH ; 3 SPEC

ALVEOLITES SUBORBICULARIS LAMARCK

27076 REF SPEC-F

$M D$

U ABITIBI RIVER FM

[U ABITIBI RIVER LS ]

W BANK OF R, ABITIBI R, CORAL RAPIDS, ONT

F466;CRANSWICK \& FRITZ, GAC PROC,VOL 10,1958,P5758,PL5,FIG 5,PL6,FIG 1-3

REMARKS: C-MA FRITZ,J MONTEITH :C- $1950 ; 8$ PCES

AMPLEXUS SEVERNENSIS (PARKS)

22548 [300S ] P-HOLO

TYRRELLIA SEVERNENSIS PARKS

$S$,

LIMESTONE RAPIDS, SEVERN R,DIST OF PATRICIA,ONT

F264:PARKS WA, IN TYRRELL, ONT BUR MINES 22ND

REPT, 1913,P 193;PARKS WA, TRANS ROY CAN INST, VOL 11.PT 1,1915,P36,PL3,FIG 12

REMARKS: D-WH HEARST, 1914 ;C-JB TYRRELL, $1912 ; 1$ SPEC

AULOPORA CONFERTA WINCHELL

27335 REF SPEC-F

MD.ERIAN

WILLIAMS ISLAND FM

WILLIAMS IS, ABITIBI R,ONT

F472;FRITZ,LEMON,NORRIS, GAC PROC, VOL9, 1957,P31.

32, PL3,FIG 7

REMARKS: C-W NORRIS, R LEMON, 1953; 1 SPEC
AULOPORA MICHIGANENSIS FENTON

27338 REF SPEC-F

MD,ERIAN

WILLIAMS ISLAND FM

WILLIAMS IS,ABITIBI R,ONT

F472;FRITZ,LEMON,NORRIS, GAC PROC, VOL9, 1957,P3233,PL4.FIG 7-10

REMARKS: C-W NORRIS,R LEMON, $1953 ; 1$ SPEC, 1 SLIDE 2346

BILLINGSASTRAEA BILLINGSI (CALVIN) 27063 REF SPEC-F

$M D$,

UABITIBI RIVER FM

[U ABITIBI RIVER LS ]

W BANK OF R, ABITIBIR,CORAL RAPIDS, ONT

F280; CRANSWICK \& FRITZ,GAC PROC,VOL 10,1958,P4344,PL2,FIG 3

REMARKS: C-MA FRITZ,J MONTEITH ;C-1950:SLIDES 2307 PLUS 1 OTHER;ONE THICK SECTION; 1 SPEC,3 FRAG, SLIDES

BILLINGSASTRAEA VERNEUILI (EDWARDS\&HAIME) 27062 REF SPEC-F

$M D$,

U ABITIBI RIVER FM

W BANK OF R, ABITIBI R, CORAL RAPIDS, ONT

F280;CRANSWICK \& FRITZ,GAC PROC,VOL 10,1958,P4243,PL2,FIG $2,4,7$

REMARKS: C-MA FRITZ,J MONTEITH :C-1950:SLIDES 2308A,B PLUS 1 OTHER; 3 PCES,3 SLIDES

CLADOPORA RIMOSA ROMINGER

27077 REF SPEC-F

$M D$,

U ABITIBI RIVER FM

[U ABITIBI RIVER LS ]

W BANK OF R, ABITIBI R, CORAL RAPIDS, ONT

F466:CRANSWICK \& FRITZ, GAC

PROC, VOL 10, 1958,P59,PL5,FIG 6

REMARKS: C-MA FRITZ,J MONTEITH ;C- $1950 ; 8$ FRAGS

\section{CLADOPORA ROBUSTA ROMINGER}

27078 REF SPEC-F

$M D$,

U ABITIBI RIVER FM

[U ABITIBI RIVER LS ]

W BANK OF R, ABITIBI R,CORAL RAPIDS, ONT

F466; CRANSWICK \& FRITZ, GAC PROC, VOL 10, 1958,P5960,PL6, FIG 4-5

REMARKS: C-MA FRITZ,J MONTEITH ;C-1950; 6 SPEC

CLADOPORA ROEMERI (BILLINGS)

27079 REF SPEC-F

$M D$

U ABITIBI RIVER FM

[U ABITIBIRIVER LS]

W BANK OF R, ABITIBI R, CORAL RAPIDS, ONT

F466:CRANSWICK \& FRITZ, GAC PROC, VOL 10, 1958,P6061,PL6,FIG 6

REMARKS: C-MA FRITZ,J MONTEITH ;C- 1950 ; 1 SPEC, SLIDE 2310

COLUMNARIA ALVEOLATA DISCRETA FOERSTE REMARKS: SEE COLUMNARIA DISCRETA FOERSTE

COLUMNARIA DISCRETA FOERSTE

11241 REF SPEC-F

COLUMNARIA ALVEOLATA DISCRETA FOERSTE

MO,CHAMP,MOH,BLKRIV

BLACK RIVER GP

PAQUETTE'S RAPIDS,OTTAWA R,ONT

F298;FOERSTE AF,J CINCINNATI SOC NAT HIST,V

$21,1914, P 124$ :OKULITCH VJ,TRANS ROY SOC CAN V

32, SEC 4, 1938,P 107, PL 1, FIG 1,2

REMARKS: C-J TOWNSEND, $1906 ; 1$ SPEC 
COLUMNARIA MAGNIFICA OKULITCH

REMARKS: SEE FOERSTEPHYLLUM MAGNIFICUM (OKULITCH)

CORAL-INCERTAE SEDIS

22553 [305S ] REF SPEC-M

CF PYCNOSTYLUS SP OR CF AMPLEXUS SP

S,

LIMESTONE RAPIDS, SEVERN R,DIST OF PATRICIA ONT

-.-;PARKS WA, TRANS ROY CAN

INST, VOL 11,PT 1, 1915,P45-46

REMARKS: C-JB TYRRELL, 1912 :D-WH HEARST, 1914; 1 SPEC

CORAL-INCERTAE SEDIS

22586 [339S ] REF SPEC-M

CF PYCNOSTYLUS SP

S,

LIMESTONE RAPIDS, SEVERN R,DIST OF PATRICIA,ONT -...:PARKS WA, TRANS ROY CAN INST, VOL 11,PT 1, 1915,P46

REMARKS: C-JB TYRRELL, 1912 ;D-WH HEARST, 1914; 1 SPEC (3 PCES)

CYSTIPHYLLOIDES AGGREGATUM (BILLINGS)

25417 [895UH] REF SPEC-F

L D.ULSTER

?BOIS BLANCFM

[ONONDAGA]

HAGERSVILLE,ONT

F312; MCLEAN RA, 1976,.GEOL SURV CAN,BULL 274,P78,PL1,FIG 2,PL2,FIG 6

REMARKS: D-BE WALKER ; 23 PCES, 14 SLIDES

CYSTIPHYLLOIDES VESICULOSUM (GOLDFUSS)

27065 REF SPEC-F

$M D$,

U ABITIBI RIVER FM

[U ABITIBI RIVER LS]

W BANK OF R, ABITIBI R, CORAL RAPIDS,ONT

F319;CRANSWICK \& FRITZ,GAC PROC, VOL 10,1958,P4647,PL3,FIG 6-7

REMARKS: C-MA FRITZ,J MONTEITH ;C- $1950 ; 3$ SPEC

DISPHYLLUM DYER CRANSWICK\&FRITZ

27059 P-HOLO

$\mathrm{MD}$,

U ABITIBI RIVER FM

[U ABITIBI RIVER LS]

W BANK OF R, ABITIBI R, CORAL RAPIDS,ONT

F280;CRANSWICK \& FRITZ,GAC PROC, VOL 10,1958,P3839, PL 1,FIG 8,9

REMARKS: C-MA FRITZ,J MONTEITH :C-1950;SLIDE NO

2304 ; 1 SPEC, 13 FRAG,SLIDE

DRYMOPORA CYSTIFORMIS FRITZ,NORRIS, LEMON

27330 P-SYN

MDERIAN

WILLIAMS ISLAND FM

WILLIAMS IS,ABITIBI R,ONT

...-;FRITZ,LEMON,NORRIS, GAC PROC, VOL9, 1957,P3335.PL3,FIG 1-3

REMARKS: C-W NORRIS,R LEMON, 1953 ;SLIDE NO 2341; 1 SPEC + 1 SLIDE

DRYMOPORA CYSTIFORMIS FRITZ, NORRIS, LEMON

27331 P-SYN

M D.ERIAN

WILLIAMS ISLAND FM

WILLIAMS IS,ABITIBI R.ONT

---;FRITZ,LEMON,NORRIS,GAC PROC,VOL9, 1957,P3335,PL3,FIG 4-6

REMARKS: C-W NORRIS,R LEMON, 1953 :SLIDE NO $2342 ; 1$ SPEC,2 FRAG, SLIDE

EMMONSIA EMMONSI (HALL)

27073 REF SPEC-F

$M D$

U ABITIBI RIVER FM

[U ABITIBI RIVER LS]
W BANK OF R, ABITIBI R, CORAL RAPIDS,ONT

F464;CRANSWICK \& FRITZ, GAC

PROC.VOL 10,1958,P54,PL4,FIG 10

REMARKS: C-MA FRITZ,J MONTEITH ;C- 1950; 4 PCES

\section{EMMONSIA EPIDERMATA (ROMINGER)}

27074 REF SPEC-F

$M D$,

UABITIBI RIVER FM

[U ABITIBI RIVER LS]

W BANK OF R, ABITIBI R CORAL RAPIDS, ONT

F464:CRANSWICK \& FRITZ,GAC

PROC,VOL 10, 1958,P55,PL4,FIG 7-9,PL5, FIG 7

REMARKS: C-MA FRITZ,J MONTEITH ;C-1950;6 PCES

EMMONSIA TUBEROSA (ROMINGER)

27075 REF SPEC-F

$M D$,

U ABITIBI RIVER FM

[U ABITIBI RIVER LS]

E BANK OF R, ABITIBI R, SEXTANT RAPIDS,ONT

F464;CRANSWICK \& FRITZ,GAC PROC, VOL 10,1958,P5556.PL5, FIG 1-4

REMARKS: C-MA FRITZ,J MONTEITH ;C-1950;5 FRAGS FROM CORAL RAPIDS ALSO HAD THIS NUMBER-GIVEN NEW NO 28159; 1 LGE SPEC

FAVOSITES ABITIBIENSIS FRITZ,LEMON,NORRIS 27321 P-SYN

MD,ERIAN

WILLIAMS ISLAND FM

WILLIAMS IS,ABITIBI R,ONT

F461;FRITZ,LEMON,NORRIS,GAC PROC, VOL9, 1957,P3637,PL2,FIG $1-3$

REMARKS: C-W NORRIS,R LEMON, 1953 ; SLIDES 2339 A,B,C 6 PCES, 3 SLIDES

FAVOSITES ABITIBIENSIS FRITZ,LEMON,NORRIS 27322 P-SYN

$M D, E R I A N$

WILLIAMS ISLAND FM

WILLIAMS IS,ABITIBIR,ONT

F461;FRITZ,LEMON,NORRIS,GAC PROC, VOL9,1957,P36 37.PL2,FIG 4

REMARKS: C-W NORRIS, R LEMON, $1953 ; 1$ SPEC

FAVOSITES ABITIBIENSIS FRITZ,LEMON,NORRIS

27323 P-SYN

$M D, E R I A N$

WILLIAMS ISLAND FM

WILLIAMS IS,ABITIBI R,ONT

F461.FRITZ IEMON NORRIS GAC PROC VOL9, 1957,P36.

37,PL2,FIG 5

REMARKS: C-W NORRIS,R LEMON, $1953 ; 1$ SPEC

FAVOSITES ABITIBIENSIS FRITZ, LEMON, NORRIS 27324 P-SYN

$M D, E R I A N$

WILLIAMS ISLAND FM

WILLIAMS IS,ABITIBIR,ONT

F461;FRITZ,LEMON,NORRIS, GAC PROC, VOL9, 1957,P36

37,PL2,FIG 6

REMARKS: C-W NORRIS, R LEMON, $1953 ; 1$ SPEC

FAVOSITES ABITIBIENSIS FRITZ,LEMON,NORRIS

27325 P-SYN

$M D$ ERIAN

WILLIAMS ISLAND FM

WILLIAMS IS,ABITIBI R,ONT

F461; FRITZ,LEMON.NORRIS, GAC PROC, VOL9, 1957,P36-

37,PL2, FIG 7

REMARKS: C-W NORRIS,RLEMON, 1953 ; 1 SPEC

FAVOSITES ABITIBIENSIS FRITZ,LEMON,NORRIS

27326 P-SYN

$M D, E R I A N$

WILLIAMS ISLAND FM

WILLIAMS IS, ABITIBI R,ONT

F461; FRITZ,LEMON,NORRIS,GAC PROC, VOL9, 1957,P3637,PL2, FIG 8

REMARKS: C-W NORRIS, R LEMON, 1953 ; 1 SPEC 
FAVOSITES ABITIBIENSIS FRITZ,LEMON, NORRIS 27327 P-SYN

$M$ D.ERIAN

WILLIAMS ISLAND FM

WILLIAMS IS,ABITIBIR,ONT

F461; FRITZ, LEMON,NORRIS,GAC PROC.VOL9, 1957,P3637,PL2, FIG 9

REMARKS: C-W NORRIS.R LEMON ; 1 SPEC

FAVOSITES CF ALPENENSIS ALPENENSIS WINCHELL

27066 REF SPEC-F

$\mathrm{MD}$.

U ABITIBI RIVER FM

[ABITIBI RIVER LS ]

W BANK OF R, ABITIBI R,CORAL RAPIDS, ONT

F461:CRANSWICK \& FRITZ,GAC PROC, VOL 10, 1958,P47-

48,PL3,FIG 3

REMARKS: C-MAFRITZ,J MONTEITH ;C-1950; 4 PCES

\section{CF FAVOSITES ASPERA D'ORBIGNY}

22536 [288S ] REF SPEC-M

0.

LOWER RAPIDS, GODS R,MAN

F461:PARKS WA, 1915, TRANS ROY CAN INST, V11,PT1,P9.

11

REMARKS: C-JB TYRRELL, 1912 ;D-WH HEARST, 1914;SPEC RECORDED AS CF FAVOSITES ASPERA D'ORBIGNY,CF COLUMNARIA FRANKLINI SALTER; LOC GIVEN AS SHAMATTAWA R-NOW CALLED GODS R,TRIBUTARY OF HAYES R ; 1 SPEC (3 PCES)

\section{CF FAVOSITES ASPERA D'ORBIGNY}

22535 [287S ] REF SPEC-M

0 .

LOWER RAPIDS GODS R.MAN

F461; PARKS WA, 1915, TRANS ROY CAN INST, V11,PT1,P911

REMARKS: C-JB TYRRELL, 1912 :D-WH HEARST, 1914:SPEC RECORDED AS CF FAVOSITES ASPERA D'ORBIGNY,CF COLUMNARIA FRANKLINI SALTER;LOC GIVEN AS SHAMATTAWA R-NOW CALLED GODS R,TRIBUTARY OF HAYES R; 1 SPEC (4 PCES)

\section{FAVOSITES CLAUSUS ROMINGER}

27067 REF SPEC-F

MD.

U ABITIBI RIVER FM

[U ABITIBI RIVER LS ]

W BANK OF R, ABITIBI R, CORAL RAPIDS, ONT

F461;CRANSWICK \& FRITZ,GAC PROC, VOL 10,1958,P48 49.PL3,FIG 4,5

REMARKS: C-MA FRITZ,J MONTEITH :C-1950; 1 SPEC,5 FRAGS

\section{FAVOSITES CONCAVA GRABAU}

27068 REF SPEC-F

$M D$

U ABITIBI RIVER FM

[U ABITIBI RIVER LS ]

W BANK OF R, ABITIBIR, CORAL RAPIDS, ONT

F461; CRANSWICK \& FRITZ,GAC PROC, VOL 10,1958,P49 50,PL3,FIG 8,9

REMARKS: C-MA FRITZ,J MONTEITH ;C- $1950 ; 3$ SPEC

\section{FAVOSITES DUMOSA WINCHELL}

27069 REF SPEC-F

$\mathrm{MD}$,

U ABITIBI RIVER FM

[U ABITIBI RIVER LS ]

W BANK OF R, ABITIBI R,CORAL RAPIDS,ONT

F461:CRANSWICK \& FRITZ,GAC PROC,VOL 10, 1958,P50-

51.PL4,FIG 1-3

REMARKS: C-MA FRITZ,J MONTEITH ;C- 1950 ; 3 PCES

FAVOSITES DUMOSA VAR PARVA CRANSWICK\&FRITZ 27070 P-HOLO

$M D$

UABITIBI RIVER FM

[U ABITIBI RIVER LS

W BANK OF R, ABITIBI R,CORAL RAPIDS,ONT
F461:CRANSWICK \& FRITZ,GAC

PROC,VOL 10, 1958,P51.PL4,FIG 4

REMARKS: C-MA FRITZ,J MONTEITH ; 1 SPEC

\section{FAVOSITES FORBESI DYERENSIS}

7562 [855CL) ]P-PARA

$M S, N I A G$

CLINTON GP,DYER BAY FM

BIDWELL TP,UPPER WEST BAY CLIFF, MANITOULIN

IS,ONT

F461;BOLTON TE,GSC MEM 289,1957,P65,PL5,FIG 3.PL11,FIG 2

REMARKS: C-HG WAY, $1932 ; 1$ SLIDE NO 1761;AUTHOR WAY IN BOLTON; 1 SPEC, 5 FRAGS,SLIDE

\section{FAVOSITES FORBESI DYERENSIS}

7538 [830CL] P-HOLO

MS.NIAG

CLINTONGP,DYER BAY FM

BIDWELL TP,UPPER WEST BAY CLIFF, MANITOULIN IS.ONT

F461;BOLTON TE,GSC MEM 289,1957,P65,PL5,FIG 1,2

REMARKS: C-HG WAY, 1932 ;AUTHOR WAY IN BOLTON; 1 SPEC

FAVOSITES GIBSONI PARKS

25459 [942UH ] P-HOLO

MD,

KWATABOAHEGAN R, JAMES BAY,ONT

F461; PARKS WA,ONT BUR OF

MINES,V13,1904,P181,PL1,FIG 1-5,PL2,FIG5,PL7,FIG 1

REMARKS: D-WA PARKS ; 6 SLIDES NO 1698A-F ; 1 SPEC(3PCES),SLIDES

FAVOSITES INTERTEXTUS ROMINGER

27071 REF SPEC-F

$M D$,

U ABITIBI RIVER FM

[U ABITIBI RIVER LS

W BANK OF R, ABITIBI R,CORAL RAPIDS, ONT

F461:CRANSWICK \& FRITZ, GAC

PROC,VOL 10, 1958,P52,PL4,FIG 11,12

REMARKS: C-MA FRITZ.J MONTEITH :SLIDES 2309 A,B;C$1950 ; 3$ SPEC, 2 SLIDES

FAVOSITES TUBEROIDES GRABAU

22514 [266S ] P-SYN

$M D, E R I A N$

DETROIT RIVER GP,AMHERSTBURG FM

[U MONROE]

LIVINGSTONE CUT,DETROIT R, OPP AMHERSTBURG,ONT

F461:GRABAU AW, MICHIGAN GEOL \& BIOL SURV,PUBL2,GEOL SER 1,1909,P112

REMARKS: D-T NATTRESS, 1914 ; NO MENTION MADE OF

PARTICULAR SPECIMENS IN PUBL'N ; 1 SPEC

\section{FAVOSITES TUBEROIDES GRABAU}

22513 [265S ] P-SYN?

$M D, E R I A N$

DETROIT RIVER GP,AMHERSTBURG FM

[UMONROE]

LIVINGSTONE CUT,DETROIT R, OPP AMHERSTBURG, ONT

F461; GRABAU AW, MICHIGAN GEOL \& BIOL SURV,PUBL2,GEOL SER 1, 1909,P112,PL 14,FIG 2

REMARKS: D-T NATTRESS, 1914 ; THIS IS PROBABLY THE FIGURED SPEC;NO MENTION OF PARTICULAR SPECIMENS IN PUBL'N ; 1 SPEC

FAVOSITES TURBINATUS BILLINGS

27072 REF SPEC-F

M D

U ABITIBI RIVER FM

[U ABITIBI RIVER LS ]

W BANK OF R, ABITIBI R,CORAL RAPIDS,ONT

F461:CRANSWICK \& FRITZ,GAC PROC, VOL 10, 1958,P5253,PL4,FIG 5,6

REMARKS: C-MA FRITZ,J MONTEITH :C-1950; 1 SPEC (2 PCES) 
FLETCHERIA SINCLAIRI OKULITCH

6871 P-HOLO

MO,CHAZY

U CHAZY GP ?

NR PEMBROKE,ONT

F298;OKULITCH VJ,TRANS ROY CAN

INST, VOL21,PT2, 1937,P3 15,PL1,FIG 5

REMARKS: D-VJ OKULITCH, 1937 ;C-W SINCLAIR; 1 SPEC

FLETCHERIA SINCLAIRI OKULITCH

6870 P-PARA

$M O, C H A Z Y$

UCHAZYGP?

NR PEMBROKE,ONT

F298;OKULITCH VJ,TRANS ROY CAN

INST VOL21,PT2, 1937,P315,PL1,FIG 6.7

REMARKS: D-VJ OKULITCH, 1937 ;C-W SINCLAIR; 1 SPEC

FOERSTEPHYLLUM MAGNIFICA (OKULITCH)

11245 P-HOLO

COLUMNARIA MAGNIFICA OKULITCH

MO,CHAMP,MOH,BLKRIV

BLACK RIVER GP,CHAUMONT OR ROCKLAND FM

[BLACK RIVER GP]

PAQUETTE'S RAPIDS, OTTAWA R,ONT

F458;OKULITCH VJ,TRANS ROY SOC CAN,V32,SEC

4, 1938,P 107,108,PL2,FIG 7;JULL RK, 1976,J PALEO,VOL

50,P387-388 PL 1.FIG 4 A B

REMARKS: C-J TOWNSEND, $1906 ; 1$ SPEC

HALLIA ABITIBIENSIS CRANSWICK\&FRITZ

27057 P-SYN

$M D$

U ABITIBI RIVER FM

[U ABITIBI RIVER LS ]

W BANK OF R, ABITIBI R,CORAL RAPIDS, ONT

F273;CRANSWICK \& FRITZ,GAC PROC, VOL 10,1958,P3536.PL1,FIG 3,6

REMARKS: C-MA FRITZ,J MONTEITH ;C-1950;POLISHED

SECTION,FIG 6,PARTLY CUT;SLIDES 2307 A,B,C ; 3 SPEC, 3 SLIDES

HELIOPHRENTIS ALTERNATUM MUT MAGNA GRABAU

22486 [238S ] P-HOLO

$M D, E R I A N$

DETROIT RIVER GP,AMHERSTBURG FM

U MONROE FM

R OPP AMHERSTBURG, DETROIT R,AMHERSTBURG,ONT

F278:GRABAU AW,MICHIGAN GEOL \& BIOL

SURV PUBL2,GEOL SER 1,1909,P101,PL13,FIG 6

REMARKS: D-T NATTRESS, $1914 ; 1$ SPEC

HELIOPHYLLUM HALLI VAR DILATISEPTATUM C\&F

27058 P-SYN

$M D$

U ABITIBI RIVER FM

[U ABITIBI RIVER LS ]

ABITIBI R,CORAL RAPIDS,ONT

F278;CRANSWICK \& FRITZ,GAC PROC,VOL 10,1958,P37-

38,PL1,FIG 5

REMARKS: C-MA FRITZ,J MONTEITH :C-1950;2 SPEC

HEXAGONARIA MARTISONI CRANSWICK\&FRITZ

27061 P-SYN

$M D$

U ABITIBI RIVER FM

[U ABITIBI RIVER LS ]

W BANK OF R, ABITIBI R, CORAL RAPIDS, ONT

F280:CRANSWICK \& FRITZ,GAC PROC, VOL 10, 1958,P40$41, \mathrm{PL} 2, \mathrm{FIG} 5,6$

REMARKS: C-MA FRITZ,J MONTEITH :C-1950;RECORDS

STATE 2 SPEC \& 4 FRAG;SLIDES 2306 A,B,C:COULD NOT

IDENTIFY ACTUAL FIG'D SECTION FOR PL2,FIG $5 ; 1$

SPEC, 4 FRAG,SLIDES

HEXAGONARIA PRISMA (LANG\&SMITH)

27060 REF SPEC-F

$M D$

U ABITIBI RIVER FM

[U ABITIBI RIVER LS ]
W BANK OF R, ABITIBI R, CORAL RAPIDS,ONT

F280:CRANSWICK \& FRITZ,GAC PROC,VOL 10,1958,P39

$40, P L 2, F I G 1$

REMARKS: C-MA FRITZ,J MONTEITH ;C-1950;SLIDES

2304A,B PLUS 1 OTHER ; 6 PCES,3 SLIDES

LICHENARIA COBOCONKENSIS OKULITCH

16560 P-HOLO

MO,CHAMP,MOH,BLKRIV

BLACK RIVER GP,COBOCONK FM

COBOCONK,ONT

F454;OKULITCH VJ,J PALEO,VOL 13,N5, 1939,P514,PL59,FIG

REMARKS: C-VJ OKULITCH, $1938 ; 1$ SPEC

LICHENARIA COBOCONKENSIS OKULITCH

16561 P-PARA

MO,CHAMP,MOH,BLKRIV

BLACK RIVER GP, COBOCONK FM

COBOCONK,ONT

F454;OKULITCH VJ,J PALEO,VOL 13,N5, 1939,P514,PL59,FIG

REMARKS: C-VJ OKULITCH, 1938; 1 SPEC

\section{LYELLIA THEBESENSIS PAUCIVESICULOSA}

$7491[783 \mathrm{CL}] \mathrm{P}-\mathrm{HOLO}$

L S,ALEX

CATARACT GP,MANITOULIN FM,U BIOHERMAL BEDS

[BIDWELL MBR,CATARACT FM]

BIDWELL TP,LOT 24,CON 11, MANITOULIN IS,ONT

F460;BOLTON TE,GSC MEM 289,1957,P64-65,PL6,FIG $3,5,6,7$

REMARKS: C-HG WAY :VAR AUTHOR WAY IN BOLTON: SLIDES NO 1760A-F; 1 SPEC,6 SLIDES

\section{LYELLIA THEBESENSIS PAUCIVESICULOSA}

$7490[782 \mathrm{CL}]$ P-PARA

L S,ALEX

CATARACT GP,MANITOULIN FM,U BIOHERMAL BEDS

[BIDWELL MBR,CATARACTFM ]

BIDWELL TP,LOT 24,CON 11, MANITOULIN IS,ONT

F460;BOLTON TE,GSC MEM 289, 1957,P64-65,PL6,FIG 4

REMARKS: C-HG WAY;VAR AUTHOR WAY IN BOLTON ; 1 SPEC

MULTISOLENIA TORTUOSA FRITZ

1154 P-HOLO

$M S, N I A G$

LOCKPORT GP

MANN IS, L TIMISKAMING, ONT

F464;FRITZ MA,J PALEO, VOL11,NO3,1937,P233,TEXT FIG 1 4

REMARKS: C-J TOWNSEND ; 3 SLIDES NO 1753-1755:MANN IS = BURNT IS; 1 FRAG OF HOLOTYPE SENT TO BMNH, 1939;1 FRAG SENT TO USNM, 1941; 1 SPEC,3 FRAGS SLIDE

PALEOALVEOLITES PAQUETTENSIS OKULITCH 11242 P-HOLO

M O,CHAMP,MOH,BLKRIV

BLACK RIVER GP

PAQUETTE'S RAPIDS, OTTAWA R,ONT

F454; OKULITCH VJ,TRANS ROY SOC CAN, VOL32,SEC 4, 1938, P96-97, PL1,FIG 3

REMARKS: C-J TOWNSEND, $1906 ; 1$ SPEC

\section{PLEURODICTYUM CONVEXUM (D'ORBIGNY)}

27080 REF SPEC-F

$M D$,

U ABITIBI RIVER FM

[U ABITIBI RIVER LS ]

W BANK OF R, ABITIBI R,CORAL RAPIDS,ONT

F466;CRANSWICK \& FRITZ,GAC PROC,VOL 10, 1958,P6263, PL2,FIG 7,8

REMARKS: C-MA FRITZ,J MONTEITH :C-1950; 1 SPEC 
PRISMATOPHYLLUM WILLIAMSI FRITZ,LEMON,NORRIS 27334 P-SYN

$M D, E R I A N$

WILLIAMS ISLAND FM

WILLIAMS IS,ABITIBI R,ONT

F280;FRITZ,LEMON,NORRIS, GAC PROC, VOL9, 1957,P30. 31.PL4,FIG 3

REMARKS: C-W NORRIS,R LEMON, 1953 ; 1 SPEC

PRISMATOPHYLLUM WILLIAMSI FRITZ, LEMON,NORRIS

27332 P.SYN

$M D, E R I A N$

WILLIAMSISLAND FM

[U ABITIBI RIVER LS ]

WILLIAMS IS,ABITIBI R,ONT

F280;FRITZ,LEMON,NORRIS,GAC PROC, VOL9, 1957,P3031,PL4,FIG 1

REMARKS: C-W NORRIS,R LEMON, 1953 ;SLIDE 2343; FRAG, 1 SLIDE

PRISMATOPHYLLUM WILLIAMSI FRITZ,LEMON,NORRIS 27333 P.SYN

MD,ERIAN

WILLIAMS ISLAND FM

WILLIAMS IS, ABITIBIR,ONT

F280;FRITZ, LEMON,NORRIS, GAC PROC, VOL9, 1957,P3031,PL4,FIG 2

REMARKS: C-W NORRIS,R LEMON, 1953 ;SLIDE $2344 ; 2$ FRAGS, 1 SLIDE

PROTARAEA WALKERI (SPENCER)

$21427[1247 N]$ REF SPEC-F

MS,NIAG

LOCKPORT GP

[NIAGARA LS]

HAMILTON,ONT

F458;PARKS WA, UNIV TOR STUDIES, GEOL

SER,N5, 1908,P60-63,PL 15,FIG 1,2

REMARKS: D-BE WALKER: 1 SPEC, 2 SLIDES

PYCNOSTÝLUS ELEGANS WHITEAVES

22550 [302S ] REF SPEC-F

$\mathrm{S}$,

LIMESTONE RAPIDS, SEVERN R,DIST OF PATRICIA, ONT F298:PARKS WA TRANS ROY CAN

INST, VOL 11,PT1, 1915,P40,PL1,FIG 3

REMARKS: C-JB TYRRELL, 1912 ;D-WH HEARST, $1914 ; 2$ SPEC

PYCNOSTYLUS MAXIMUS PARKS

22551 [303S ] P-SYN

S.

LIMESTONE RAPIDS, SEVERN R,DIST OF PATRICIA,ONT F298; PARKS WA, TRANS ROY CAN

INST, VOL 11,PT1, 1915,P40-41,PL1,FIG 2

REMARKS: C-JB TYRRELL, 1912 ;D-WH HEARST, $1914 ; 2$ SPEC

ROMINGERIA UMBILLIFERA (BILLINGS)

22497 [249S ] REF SPEC-F

MD,ERIAN

DETROIT RIVER GP,AMHERSTBURG FM

[U MONROE FM]

DETROIT R BED, OPP AMHERSTBURG,ONT

F470;GRABAU AW,MICHIGAN GEOL \& BIOL SURV,PUBL 2.GEOL SER 1,1909,P 108,PL 14,FIG 7

REMARKS:D-T NATTRESS, $1914 ; 1$ SPEC

\section{SCENOPHYLLUM CONIGERUM (ROMINGER)}

27056 REF SPEC-F

$M D$.

U ABITIBI RIVER FM

[U ABITIBI RIVER LS ]

W BANK OF R, ABITIBI R.CORAL RAPIDS, ONT

F272; CRANSWICK \& FRITZ, GAC PROC, VOL 10, 1958,P3435.PL1.FIG 1,2

REMARKS: C-MA FRITZ,J MONTEITH ;C- 1950 : 2 SPEC (5 PCES)
STREPTELASMA INTEGRISEPTATUM PARKS $22534[286$ S ] P-SYN

0 .

LOWER RAPIDS, SHAMATTAWA R,ONT

F268;PARKS WA, TRANS ROY CAN

INST.VOL 11,PT1, 1915,P13-15,PL5,FIG 1-3

REMARKS: C-JB TYRRELL;D-WH HEARST ;C-1912;D- 1914 ; 3 SPEC

STREPTELASMA CF RUSTICUM (BILLINGS)

28533 [285S ] REF SPEC-F

O.

LOWER RAPIDS , SHAMATTAWA R,ONT

F268;PARKS WA, TRANS ROY CAN

INST, VOL 11,PT1, 19 15,P 12-13,FIG 4,5

REMARKS: C-JB TYRRELL, 1912 ;D-WH HEARST, $1914 ; 2$ SPEC (SEV PCES)

SYNAPTOPHYLLUM MULTICAULE (HALL)

22459 [210S] REF SPEC-F

$M D, E R I A N$

DETROIT RIVER GP.AMHERSTBURG FM

[U MONROE FM]

LIVINGSTONE CUT,DETROIT R OPP AMHERSTBURG,ONT

F280;GRABAU AW.MICHIGAN GEOL + BIOL SURV,PUBL

2,GEOL SER 1,1909,P105-106,PL 12,FIG 6

REMARKS: D-REV T NATTRESS, $1910 ; 1$ SPEC \& 1 CAST

SYNAPTOPHYLLUM SIMCOENSE (BILLINGS)

27064 REF SPEC-F

$M D$,

U ABITIBI RIVER FM

[U ABITIBI RIVER LS ]

E BANK OF RIVER, ABITIBI R,CORAL RAPIDS,ONT

F280;CRANSWICK \& FRITZ, GAC PROC, VOL 10, 1958,P45 46.PL3,FIG 1,2

REMARKS: C-MA FRITZ,J MONTEITH ;C- 1950 ; 6 FRAGS, 3 SLIDES

SYRINGOPORA CF TABULATA EDWARDS\&HAIME 27081 REF SPEC-F

$M D$.

U ABITIBI RIVER FM

[U ABITIBI RIVER LS ]

W BANK OF RIVER, ABITIBI R,CORAL RAPIDS,ONT

F472; CRANSWICK \& FRITZ,GAC PROC,VOL 10, 1958,P6364, PL6,FIG 9

REMARKS: C-MA FRITZ,J MONTEITH :C-1950; 1 SPEC

THAMNOPHYLLUM DYERI FRITZ,LEMON,NORRIS

27687 P-SYN

$M D$,ERIAN

WILLIAMS ISLAND FM

WILLIAMS IS,ABITIBI R,ONT

F281:FRITZ,LEMON,NORRIS, GAC PROC, VOL9, 1957,P26-

28,PL3,FIG 8

REMARKS: C-W NORRIS,R LEMON, $1953 ; 1$ SPEC

THAMNOPHYLLUM DYERI FRITZ,LEMON, NORRIS

27336 P-SYN

MD,ERIAN

WILLIAMS ISLAND FM

WILLIAMS IS,ABITIBI R,ONT

F480;FRITZ,LEMON,NORRIS,GAC PROC,VOL9,1957,P26 28,PL3,FIG 9

REMARKS: C-W NORRIS.R LEMON ;RECORDS STATE 5 PCES,FIG'D SECTION PRESENT ; 4 PCES

THAMNOPORA MARTISONI FRITZ, LEMON, NORRIS

27328 P-HOLO

MD.ERIAN

WILLIAMS ISLAND FM

WILLIAMS IS,ABITIBI R,ONT

F464;FRITZ, LEMON,NORRIS, GAC PROC, VOL9, 1957,P3738,PL2,FIG 10-12

REMARKS: C-W NORRIS,R LEMON. 1953 ; SLIDES NO 2340 A, B: 1 SPEC,5 FRAG,SLIDES 
TORTOPHYLLUM CYSTICUM WINCHELL

27688 REF SPEC-M

MD.ERIAN

WILLIAMS ISLAND FM

WILLIAMS IS, ABITIBI R,ONT

F278-FRITZ,LEMON NORRIS GAC PROC VOL9, 1957.P28-29

REMARKS: C-W NORRIS, R LEMON, 1953 ;SLIDES $2370 A, B$,

C:NOT FIG'D; 1 SPEC,7 PCES,SLIDES

TORTOPHYLLUM CYSTICUM WINCHELL

27337 REF SPEC-F

MDERIAN

WILLIAMS ISLAND FM

WILLIAMS IS,ABITIBI R,ONT

F278;FRITZ,LEMON,NORRIS,GAC PROC, VOL9, 1957,P28-

29,PL4,FIG 4-6

REMARKS: C-W NORRIS, R LEMON, 1953 ;SLIDES $2345 A, B$ : 1 SPEC, 4 FRAG,SLIDES

TYRRELLIA SEVERNENSIS PARKS

REMARKS: SEE AMPLEXUS SEVERNENSIS (PARKS)

\section{BRYOZOA}

AMPLEXOPORA PERVERSA CALEY

17751 [1532HR] P-HOLO

U O.CINCI,MAYSVILL

WEKWEMIKONGSING FM

CLAY CLIFF, MANITOULIN IS, ONT

G108;CALEY JF,GSC MEM 202,1936,P75-76,PL2,FIG 7,8

PL3,FIG 5

REMARKS: C-JF CALEY, 1932 ;SLIDE $1937 ; 1$ SPEC, 1 SLIDE

\section{AMPLEXOPORA SOLITARIA DYER}

12147 P-HOLO

U O,CINCI,MAYSVILL

MEAFORD FM

[STREETSVILLE MBR (RICHMOND) MAYSVILLE]

CREDIT R, STREETSVILLE,ONT

G108;DYER WS,ONT DEPT MINES, VOL 32,PT 7, 1925,P6162.PL 1,FIG 1,3,PL7,FIG 2

REMARKS: C-WS DYER, 1923 ;SLIDES 803,804 (803 FIG'D): SPEC FIG'D ON PL $7 ; 2$ PCES, 2 SLIDES

ARTHROPORA SHAFFERI (MEEK)

17343 [1102HR] REF SPEC-F

U O,CINCI,MAYSVILL

DUNDAS FM

[DUNDAS ]

DON VALLEY QUARRY, TORONTO ONT

G 136;PARKS \& DYER, ONT DEPT MINES,VOL

30,PT7, 1922,P23-24,PL5, FIG 5

REMARKS: C-J TOWNSEND ; 1 SPEC

\section{ASCOPORA ALANI FRITZ}

27762 P-SYN

$\mathrm{CP}$

ROCKY MOUNTAIN GP, KANANASKIS FM

MCGUGAN SAMPLE 1OF , NR BANFF, ALTA

G 131; FRITZ MA,BULL CAN PET GEOL, VOL 11,N1,1963,P5458,PL1,FIG 1,2,PL2,FIG 2

REMARKS: C-A MCGUGAN,GSC ;SLIDES A-C FIG'D ; 4 FRAGS, 6 SLIDES

\section{ASPIDOPORA CF AREOLATA ULRICH}

17323 [1082HR] REF SPEC-F

U O,CINCI,MAYSVILL

DUNDASFM

[DUNDAS ]

5 FT LEVEL, OLD SHALE PIT, LAMBTON, ONT

G57:PARKS \& DYER ONT DEPT MINES VOL 30 PT7, 1922,P4-

5,PL1,FIG 8,PL2,FIG 8,PL6,FIG 7,8

REMARKS: SLIDE $754 ; 1$ SPEC, 1 SLIDE
ATACTOPORA MACULATA ULRICH

16591 [250HR] REF SPEC-F

U O,CINCI,MAYSVILL

DUNDASFM

DON VALLEY QUARRY,TORONTO,ONT

G97;PARKS + DYER,ONT DEPT MINES, VOL

30,PT7, 1922,P8-9,PL1,FIG 10,PL2,FIG 9, 10,PL5,FIG 1

REMARKS: C-J TOWNSEND ;SLIDES 753A-D;A \& B FIG'D ; 3 PCES, 4 SLIDES

ATACTOPORELLA DENSA DYER

16595 [254HR] REF SPEC-M

U O,MAYS ORRICHMOND

MEAFORD FM

[MEAFORD,RICHMOND]

STREETSVILLE,ONT

G95;FRITZ MA, 1977, LIFE SCI CONTR, ROM,N111,P2-4

REMARKS: C-J TOWNSEND, 1923 ;FOUND IN ASSOCIATION WITH HOLOTYPE OF HOMOTRYPELLA EXPANSA DYER; STORED UNDER H.EXPANSA; 1 SLIDE

\section{ATACTOPORELLA DENSA DYER}

12148 P-HOLO

U O.MAYS OR RICHMOND

MEAFORD FM

[STREETSVILLE MBR (RICHMOND) MEAFORD]

CREDIT R , STREETSVILLE ONT

G95:DYER WS, 1925, ONT DEPT MINES, VOL

32,PT7, P62,PL1,FIG 2,4;FRITZMA, 1977,P2-4,FIG 1A,B

REMARKS: C-WS DYER, 1923 : SLIDE 811;ROM RECORDS

STATE 3 FRAGS BELONG TO 1 SPEC ; 3 PCES, 1 SLIDE

ATACTOPORELLA PECULIARIS (DYER)

12275 REF SPEC-F

UO,MAYS OR RICHMOND

MEAFORD FM

[STREETSVILLE MBR, RICHMOND, MEAFORD]

CREDIT R AT STREETSVILLE,ONT

G96;FRITZ MA, 1977, ROM,LIFE SCI CONTR,N111,P4-9,FIG 2A,B

REMARKS: C-WS DYER, 1922 ;SLIDES 1011 + 1012;1011 FIG'D; 1 SPEC,FRAGS,SLIDES

\section{ATACTOPORELLA PECULIARIS (DYER)}

12166 P-HOLO

HOMOTRYPELLA HOSPITALIS VAR PECULIARIS DYER U O.MAYS OR RICHMOND

MEAFORD FM

SECT 13,INT 5, STREETSVILLE,ONT

G96;DYER WS, 1925,ONT DEPT MINES, VOL 32,PT7,P69-

70,PL4,FIG 1;FRITZMA, 1977, P4-9,FIG 1C,D

REMARKS: C-WS DYER, 1923 ;SLIDES 796,797; 1 SPEC,2 SLIDES

BATOSTOMA ELONGATA CALEY

17747 [1528HR]P-HOLO

U O,CINCI,MAYSVILL

WEKWEMIKONGSING FM

CLAY CLIFF MANITOULIN IS ONT

G113:CALEY JF,GSC MEM 202,1936,P75-76,PL2,FIG 3,4.PL3,FIG 2

REMARKS: C-JF CALEY 1933 :SLIDES 1934A,B;A + SPEC ARE FIG'D ; 1 SPEC, 2 SLIDES

\section{BATOSTOMA SP}

24346 [1188T ] REF SPEC-F

MO.CHAMP MOH,TRENT

TRENTON GP

[TRENTON FM ]

RLWY CUT, LITTLE CURRENT MANITOULIN IS, ONT

G 113:CALEY JF,GSC MEM 202,1936,P74-75,PL4,FIG 5,6,PL3,FIG 11

REMARKS: C-JF CALEY, 1932 ;SLIDE $1928 ; 1$ SPEC, 3 FRAGS,SLIDE

\section{BATOSTOMA SP}

24347 [1189T ] REF SPEC-F

MO.CHAMP,MOH,TRENT

TRENTON GP

[TRENTON FM ] 
RLWY CUT, LITTLE CURRENT, MANITOULINIS,ONT G113:CALEY JF,GSC MEM 202, 1936,P74-75,PL3,FIG 10 REMARKS: C-JF CALEY, 1932; 1 SPEC,SLIDE 1929

\section{BATOSTOMA SP}

24348[1190T] REF SPEC-F

MO.CHAMP,MOH,TRENT

TRENTON GP

[TRENTON FM]

RLWY CUT, LITTLE CURRENT, MANITOULIN IS, ONT

G113:CALEY JF,GSC MEM 202, 1936,P74-75,PL6,FIG 1,2

REMARKS: C-JF CALEY, 1932 ;SLIDES 1930A,B : 1 SPEC, 2 SLIDES

BYTHOPORA ARCTIPORA (NICHOLSON)

17336 [1095HR] REF SPEC-F

U O,CINCI,MAYSVILL

DUNDASFM

[DUNDAS ]

DON VALLEY QUARRY, TORONTO.ONT

G99:PARKS \& DYER,ONT DEPT MINES, VOL

30,PT7, 1922, P18,PL5,FIG 11

REMARKS: C-J TOWNSEND; 1 SPEC

BYTHOPORA ARCTIPORA (NICHOLSON)

17335 [1094HR] REF SPEC-F

U O.CINCI,MAYSVILL

DUNDAS FM

[DUNDAS]

DON VALLEY QUARRY, TORONTO ONT

G99:PARKS + DYER,ONT DEPT MINES,VOL

30.PT7, 1922,P18,PL6,FIG 3

REMARKS: C-J TOWNSEND ; 2 SPEC

BYTHOPORA CF DELICATULATA (NICHOLSON)

17337 [1096HR] REF SPEC-F

U O,CINCI,MAYSVILL

DUNDAS FM

[DUNDAS ]

WESTON.ONT

G99:PARKS \& DYER,ONT DEPT MINES,VOL

30,PT7, 1922, P18-19, PL6,FIG 4

REMARKS: C-JTOWNSEND; 1 SPEC

\section{BYTHOPORA MEEKI (JAMES)}

12254 REF SPEC-F

U O,CINCI,MAYSVILL

MEAFORD FM

[STREETSVILLE MBR (RICHMOND) MEAFORD ]

CREDIT R,STREETSVILLE,ONT

G99:DYER WS.ONT DEPT MINES,VOL

32, PT7, 1925, P51, PL5, FIG 4

REMARKS: C-WS DYER, 1922 :SLIDE 961 ;SECT 14,13 FT

LEVEL, OF DYER:PL5,FIG 4 IS SURFACE OF SPEC,

ENLARGED : 1 SPEC, 1 SLIDE

\section{BYTHOPORA SIMILIS FRITZ}

12316 P.HOLO

U O.CINCI,MAYSVILL

MEAFORD FM

[VINCENT MBR,MEAFORD FM]

WORKMAN'S CK,ONT

G99;FRITZ MA, TRANS ROY SOC CAN,VOL 20,PT4,1926,P9697,PL2,FIG 8, PL4,FIG 1

REMARKS: C-MAFRITZ, 1925 :SLIDES 1171,$1172 ; 1172$ NOT

FIG'D : 1 SPEC, 2 SLIDES

\section{BYTHOPORA VACUAFRITZ}

12315 P-HOLO

U O,CINCI,MAYSVILL

MEAFORD FM

[VINCENT MBR,MEAFORD FM.RICHMOND]

WORKMAN'S CK.ONT

G99;FRITZ MA,TRANS ROY SOC CAN,VOL 20,PT4,1926,P9596, PL 1,FIG 1,2,PL4,FIG 3

REMARKS: C-MA FRITZ, 1925 ;SLIDES 1111,$1112 ; 1$ SPEC,2 SLIDES
CALLOPORELLA VACUA DYER

12149 P-HOLO

UO,CINCI,MAYSVILL

MEAFORD FM

[ERINDALE MBR(RICHMOND) MEAFORD

SECT NO 9,10 FT BELOW BRYOZOAN REEF, COOKSVILLE

CK,ONT

G112;DYER WS,ONT DEPT MINES, VOL

32. PT 7, 1925,P63, PL 1,FIG 5 , 6

REMARKS: C-WS DYER, 1923; 1 SPEC,SLIDE 814

CHILOPORELLA FLABELLATA ULRICH

17321 [1080HR] REF SPEC-F

U O.CINCI,MAYSVILL

DUNDASFM

DON VALLEY QUARRY,TORONTO,ONT

G82:PARKS + DYER,ONT DEPT MINES, VOL

30,PT7, 1922,P2,PL1,FIG 1,2,PL2,FIG 1,2,PL5,FIG

12,PL6,FIG 11

REMARKS: C-J TOWNSEND :SLIDES 755A-C:A + B FIG'D:

NAME MISSPELLED IN CAPTION PL6 ; 4 SPEC. 3 SLIDES

\section{CONOPEUM BICYSTOSUM SANDERSON}

10856 [5337CT]P-SYN

UK,SENON,MAAST

EDMONTON FM,DRUMHELLER MARINE TONGUE

SE $1 / 4$ SEC 33,TP 28,RGE 21, HORSESHOE CANYON , $8 \mathrm{MI} W$

DRUMHELLER,ALTA

G 156:ALLEN SANDERSON,RES COUNCIL ALTA, REPT

13,1945,P89,PL7

REMARKS: C-JOG SANDERSON. $1926 ; 2$ SPEC

CYCLOTRYPA BOREALIS PARKS

$12972[2362 D]$ P.HOLO

MD.ERIAN

KWATABOAHEGAN R ONT

G83;PARKS WA,ONT BUR MINES, VOL

13,PT 1, 1904,P185,PL2,FIG 1,2

REMARKS: C-WA PARKS, 1903 :SLIDES 1434A,B; 1 SPEC, 2 SLIDES

DEKAYELLA PRAENUNTIA ECHINATA ULRICH

24358 [1200T] REF SPEC.F

MO.CHAMP, MOH, TRENT

TRENTON GP

[TRENTON ]

LIGHTHOUSE,LITTLE CURRENT, MANITOULIN IS, ONT

G97:CALEY JF GSC MEM 202, 1936,P73,PL5,FIG

4,5,6,PL3,FIG 9

REMARKS: C-JF CALEY, 1932 :LARGER PCE FIG'D ; 2 PCES, SLIDE 1933

DEKAYIA APPRESSA VAR DISTINCTA FRITZ

17543 [1308HR] P-HOLO

U O.CINCI,MAYSVILL

DUNDASFM

[CHRISTIE MBR,DUNDAS ]

WORKMAN'S CK.ONT

G98;FRITZ MA,TRANS ROY SOC CAN,VOL 20,PT4, 1926,P9899.PL1,FIG 3.4

REMARKS: C-MA FRITZ, 1925 :SLIDES 1102-1104;1102, 1103 + SPEC ARE FIG'D; 1 SPEC, 3 SLIDES

DEKAYIA GRANULOSA FRITZ

17544 [1309HR]P-HOLO

UO.CINCI,MAYSVILL

DUNDASFM

[CHRISTIE MBR,DUNDAS ]

WORKMAN'S CK.ONT

G98;FRITZMA, TRANS ROY SOC CAN, VOL

20,PT4,1926, P99, PL 1,FIG 5,6

REMARKS: C-MA FRITZ, 1925; 1 SPEC.SLIDE $\} 105$

DEKAYIA MEAFORDENSIS FRITZ

17560 [1327HR]P-SYN

U O.CINCI,MAYSVILL

DUNDASFM

[CHRISTIE MBR,DUNDAS]

WORKMAN'S CK.ONT

G98;FRITZMA,TRANS ROY SOC CAN,VOL

20.PT4.1926.P 100.PL4.FIG 11

REMARKS: C-MA FRITZ, 1925, 1 SPEC 
DEKAYIA MEAFORDENSIS FRITZ

$17545[1310 \mathrm{HR}]$ P-SYN

U O,CINCI,MAYSVILL

DUNDAS FM

[CHRISTIE MBR,DUNDAS]

WORKMAN'S CK,ONT

G98;FRITZ MA, TRANS ROY SOC CAN, VOL

20,PT4, 1926, P100,PL1,FIG 7,8.PL4,FIG 4

REMARKS: C-MA FRITZ, 1925 ;SLIDES 1106,1107; 1106 + SPEC ARE FIG'D ; 1 SPEC,2 SLIDES

\section{ERIDOTRYPA PELLICULATA FRITZ}

17542 [1307HR]P-HOLO

U O,CINCI,MAYSVILL

DUNDAS FM

[HUMBER MBR,DUNDAS ]

WORKMAN'S CK ONT

G99;FRITZ MA, TRANS ROY SOC CAN, VOL 20,PT4,1926,P9798,PL2,FIG1,2,PL4,FIG 7

REMARKS: C-MA FRITZ, 1925 ;SLIDES 943-945;943 FIG'D ; SPEC, 3 SLIDES

\section{ERIDOTRYPA SIMILIS BASSLER}

$20921[611 \mathrm{~N}]$ P-SYN

MS,NIAG

CLINTON GP,ROCHESTER FM

[ROCHESTER SHALE]

GRIMSBYONT

G99:BASSLER RS, USGS BULL 292, 1906,P31-32,PL26,FIG 2 REMARKS: D-BE WALKER, $1906 ; 1$ SPEC

\section{FENESTRELLINA FORTINENSIS FRITZ}

20373 P-SYN

D.

GRANDE GREVE FM

RGE VII, 2 MIW OF FORTIN-MALBAY TP LINE, FORTIN

TP,GASPE CO,QUE

G122;FRITZ MA,CONTR ROMP,NO 4, 1940,P7-8,PL1,FIG 1,5

REMARKS: C-HW MCGERRIGLE;D- 1940 ;LOC-CLOSE TO

RVII-VIII LINE;D-QDM;QDM LOC 13 M 39 ; 3 SPEC,SLIDE 254

\section{FENESTRELLINA MULTISTRIATA FRITZ}

20973 P.SYN

D.

GRANDE GREVE FM

CAP DE ROSIERS TP,GASPE CO,QUE

G 122;FRITZ MA,J PALEONT, VOL 15,N1, 1941,P94-96,FIG 1-3 REMARKS: C-HW MCGERRIGLE.D-QDM - 1 SPEC 2 FRAGS

\section{FISTULIPHRAGMA JONESI FRITZ}

20374 P-HOLO

D.

GRANDE GREVEFM

FORTIN TP,GASPE CO, QUE

G85;FRITZMA,CONTR ROMP,NO 4, 1940,P5-6,PL1,FIG $2,3,4,7$

REMARKS: C-HW MCGERRIGLE;D- 1940 ;P-QDM ; 1 SPEC, SLIDE 255

\section{HALLOPORA AEQUALIS DYER}

12150 P-HOLO

UO. CINCIMAYSVILL

MEAFORD FM

[ERINDALE MBR,MEAFORD]

SECT 12 STREETSVILLE ONT

G112;DYER WS, 1925,P63-64,PL1,FIG 7,PL2,FIG 1,2,PL7,FIG 15:FRITZ MA, GAC PROC, VOL 21, 1970,P 17-18,PL3,FIG $1,3,4,6$

REMARKS: C-WS DYER, 1923 ;SLIDES 818,$819 ; 1$ SPEC, 2 SLIDES

\section{HALLOPORA CALEYI WAY IN BOLTON}

$7508[800 \mathrm{CL}]$ P-SYN

$M$ S,NIAG

CATARACT GP

[BIDWELL MBR, CATARACT FM]

BIDWELL TP,LOT 23,CON 10 , MANITOULIN IS, ONT

G112·BOLTON TE GSC MEM 289, 1957,P67,PL8,FIG

1,2,PL9,FIG 1,2
REMARKS: C-HG WAY, 1932 ;SLIDES 1705, 1706A,B,1707A B; 1705 NOT FIG'D;SPECS NUMBERED $800 \mathrm{CL}, 800 \mathrm{CLA}$, 800CLB;800CL FIG'D ; 3 SPEC, 5 SLIDES

HALLOPORA DALEI VAR SUBALTA PARKS + DYER

REMARKS: SEE HALLOPORA SUBALTA PARKS + DYER

HALLOPORA DANFORTHENSIS PARKS

$17340[1099 \mathrm{HR}]$ P-PARAL

HALLOPORA CF SUBPLANA (ULRICH) IN PARKS + DYER

U O.CINCI,MAYSVILL

DUNDAS FM

DON VALLEY QUARRY,TORONTO,ONT

G 112:PARKS \& DYER, 1922,P 19-21.PL6.FIG 9:PARKS

WA, 1925,P35;FRITZ MA, GAC PROC, VOL 21, 1970,P1516, PL $1, F I G 1,6$

REMARKS: C-J TOWNSEND ;SLIDE 747:PUBLISHED 1925

AS HALLOPORA ONEALLI VAR DANFORTHENSIS,

SYNTYPE;CHANGED TO SPECIES STATUS +

LECTOTYPE DESIGNATED BY FRITZ; 1 SPEC, 1 SLIDE

HALLOPORA DANFORTHENSIS PARKS

$17341[1100 \mathrm{HR}]$ P-LECTO

HALLOPORA CF SUBPLANA (ULRICH) IN PARKS + DYER U O.CINCI,MAYSVILL

DUNDAS FM

DON VALLEY QUARRY TORONTO ONT

G112;PARKS \& DYER, 1922,P 19-21,PL4,FIG 13,14;PARKS WA 1925, P35;FRITZ MA GAC PROC VOL 21, 1971,P1718.PL 1,FIG 2

REMARKS: C-J TOWNSEND ;SLIDE 746;PUBLISHED 1925 AS HALLOPORA ONEALLIVAR DANFORTHENSIS; SYNTYPE:CHANGED TO SPECIES STATUS + DESIGNATED LECTOTYPE BY FRITZ;SEE NO $17339+$ $17339 \mathrm{H}$. ONEALLI DANFORTHENSIS ; 2 SPEC, 1 SLIDE

HALLOPORA ENCRUSTANS CALEY

17748 [1529HR]P-HOLO

UO,CINCI,MAYSVILL

WEKWEMIKONGSING FM

CLAY CLIFF, MANITOULIN IS, ONT

G 122; CALEY JF,GSC MEM 202,1936,P75,PL3,FIG 8,PL4,FIG 7,8

REMARKS: C-JF CALEY, 1932 ; SLIDE 1940

\section{HALLOPORA MACULOSA DYER}

12151 P-HOLO

U O,CINCI,MAYSVILL

MEAFORD FM

[STREETSVILLE MBR (RICHMOND) MEAFORD]

SECT 13.CREDIT R STREETSVILLE,ONT

G 122;DYER WS, 1925,P64,PL2,FIG 3,4;FRITZMA,GAC PROC VOL 21,1970,P 18-19,PL2,FIG 1,2,4,5, PL3,FIG 5

REMARK S: C-WS DYER, 1923 :SLIDES 821,821A,821B,821C: SPEC RESECTIONED BY FRITZ - ONLY FRAGMENTS REMAIN ; 4 SLIDES, FRAGS

HALLOPORA OBLIQUIPORA JOHNSON IN BOLTON 7414 [649CL] P-HOLO

S,MED

CATARACT GP,MANITOULINFM

[U MANITOULIN DOL]

UPPER CONTACT MANITOULIN DOL WITH CABOT HEAD SHALES, CATARACT, ONT

G122:BOLTON TE, GSC MEM 289, 1957,P67-68,PL9,FIG 4,5

REMARKS: C-H JOHNSON, 1930 :SLIDE $1510 ; 1$ SPEC, 1 SLIDE

\section{HALLOPORA ONEALLI DANFORTHENSIS PARKS}

17338 [1097HR] P-PARAL

HALLOPORA CF SUBPLANA (ULRICH) PARKS\&DYER

UO.CINCI,MAYSVILL

DUNDAS FM

DON VALLEY QUARRY TORONTO, ONT

G 112:PARKS \& DYER, 1922,P19-21,PL5,FIG 4:PARKS WA, 1925, P35

REMARKS: C-J TOWNSEND :PARKS MENTIONED NO TYPES, BUT REFFERRED TO PARKS + DYER, IMPLYING

ALL MENTIONED SPECS FOR H. SUBPLANA WERE PARKS' SYNTYPES; FRITZ, 1970,DESIGNATED A LECTOTYPE FOR H. DANFORTHENSIS;SEE H. DANFORTHENSIS, NO $17340+17341 ; 1$ SPEC 
HALLOPORA ONEALLI DANFORTHENSIS PARKS

REMARKS: SEE ALSO HALLOPORA DANFORTHENSIS

PARKS

HALLOPORA ONEALLI DANFORTHENSIS PARKS

17339 [1098HR] P-PARAL

HALLOPORA CF SUBPLANA (ULRICH) PARKS\&DYER

U O.CINCI,MAYSVILL

DUNDAS FM

DON VALLEY QUARRY, TORONTO,ONT

G 122:PARKS \& DYER, 1922,P 19-21,PL6,FIG 9;PARKS

WA 1925.P35

REMARKS: C-J TOWNSEND :PARKS MENTIONED NO TYPES, BUT REFFERRED TO PARKS + DYER,IMPLYING ALL MENTIONED SPECS FOR H. DANFORTHENSIS;SEE H. DANFORTHENSIS,NO $17340+17341 ; 2$ SPEC

HALLOPORA ONEALLI VAR CREDITENSIS DYER

12152 P-LECTO

U O,CINCI,MAYSVILL

MEAFORD FM

[MEAFORD,RICHMOND]

STREETSVILLE,ONT

G112:DYER WS, 1925,P64-65;FRITZ MA,GAC PROC,VOL

21, 1970,P 18,PL1,FIG 3-5

REMARKS: C-WS DYER, 1923 ; NUMEROUS FRAGS,SLIDE

HALLOPORA ONEALLI VAR CREDITENSIS DYER

12153 P-PARAL

UO.CINCI,MAYSVILL

MEAFORD FM

[MEAFORD,RICHMOND]

STREETSVILLE,ONT

G 112;DYER WS.ONT DEPT MINES,VOL 32,PT7, 1925,P6465,PL1,FIG 8,PL5,FIG 6

REMARKS: C-WS DYER, 1923 ;SLIDE 820;LECTOTYPE NO 12152 DESIGNATED BY FRITZ, 1971 ; 1 SPEC, 6 FRAG, SLIDE

HALLOPORA PECULIARIS JOHNSON IN BOLTON

$7413[648 \mathrm{CL}]$ P-HOLO

S.MED

CABOT HEAD FM, LR LIMEY BEDS

CREDIT FORKS, 1 FT ABOVE CONTACT , CREDIT R, ONT

G122;BOLTON TE,GSC MEM 289, 1957,P68,PL 10,FIG 1,2

REMARKS: C-H JOHNSON, 1930 ;SLIDES 1509A,B;B NOT

FIG'D ; 1 SPEC,2 SLIDES

HALLOPORA SUBALTA PARKS\&DYER

16588 [247HR] REF SPEC-F

UO.CINCI,MAYSVILL

DUNDAS FM

DON VALLEY QUARAY,TORONTO,ONT

G 122;FRITZ MA, GAC PROC,VOL 21, 1970,P 16-17,PL2,FIG 6

REMARKS: C-J TOWNSEND ; SLIDE 664B FIG'D; SLIDES 661

664A AND SPECS STORED WITH REGULAR COLL'NS;

DESIGNATED PARALECTOTYPE BY FRITZ, 1971; NOT A

SYNTYPE OF PARKS \& DYER 1921, THEREFORE NOT A

PARALECTOTYPE; SEVERAL SPECS,SLIDES

HALLOPORA SUBALTA PARKS\&DYER

$17342[1101 \mathrm{HR}]$ P-LECTO

HALLOPORA DALEI VAR SUBALTA PARKS\&DYER

U O.CINCI,MAYSVILL

DUNDAS FM

DON VALLEY QUARRY,TORONTO.ONT

G 122:PARKS DYER, 1922,P21-22:FRITZ MA,GAC PROC,VOL 21, 1970,P 16-17,PL2,FIG 3,PL3,FIG 2

REMARKS: C-J TOWNSEND;SLIDES 740A-E;D \& E NOT FIG'D;DESIGNATED SYNTYPES BY PARKS \& DYER; REDESIGNATED LECTOTYPE BY FRITZ;UNCERTAIN WHICH SPEC IS TO BE CONSIDERED THE TYPE ; 2 SPEC, FRAGS,SLIDES

HALLOPORA CF SUBPLANA (ULRICH)

REMARKS: SEE HALLOPORA DANFORTHENSIS PARKS
HALLOPORA TOLLI BASSLER

23355 REF SPEC-F

MO.CHAMP,MOH,BLKRIV

BLACK RIVER GP

[BLACK RIVER]

JONCAS TP.GASPE CO, QUE

G122;FRITZ MA,J PALEONT,VOL 15.N5, 1941,P564,FIG

REMARKS: C-W MCGERRIGLE;D-1941,QDM; 1 SPEC

SLIDES 26A,B

\section{HETEROTRYPA DEFINITA DYER}

12154 P-LECTO

U O,CINCI,MAYSVILL

MEAFORD FM,ERINDALE MBR

[ERINDALE MBR,MEAFORD,RICHMOND]

MULLET CK,ERINDALE, ONT

G97:DYER WS,ONT DEPT MINES, VOL

32,PT7, 1925,P65,PL6,FIG 11:FRITZ MA 1975,P4-8,FIG 1A

REMARKS: C-WS DYER, 1923 ; SPEC FIG'D BY DYER:

TANGENITAL SECT POSSIBLY FIG'D BYFRITZ

DESIGNATED LECTOTYPE BY FRITZ; 1 SPEC, 2 SLIDES

HETEROTRYPA DEFINITA DYER

12155 P-PARAL

U O,CINCI,MAYSVILL

MEAFORD FM, ERINDALE MBR

[ERINDALE MBR,MEAFORD,RICHMOND]

CREDIT R,ONT

G97:DYER WS, ONT DEPT MINES, VOL

32, PT7, 1925,P65,PL2,FIG 5,6;FRITZ MA , 1975,P4-8,FIG 1B. C, $5 \mathrm{E}-\mathrm{F}$

REMARKS: C-WS DYER, 1923 :SLIDES $777-779+2$ OTHERS

SLIDE 779 IS FIG 1C,INCORRECTLY CITED AS NO 12154

BY FRITZ;779A IS FIG $1 B ; 6$ SPEC, 5 SLIDES

HETEROTRYPA MACULOSA DYER

12160 P.SYN

HETEROTRYPA SIMPLEX VAR MACULOSA DYER

U O.CINCI,MAYSVILL

MEAFORD FM

[MEAFORD, RICHMOND]

STREETSVILLE, ONT

G97:DYER WS, ONT DEPT MINES, VOL

32,PT7, 1925, P67,PL3,FIG 2;FRITZ MA, 1975,P $18-19$

REMARKS: C-WS DYER, 1923 ; SLIDES 785-787;785 FIG'D BY DYER; 1 SPEC.3 SLIDES

HETEROTRYPA MEAFORDENSIS FRITZ

REMARKS: SEE HETEROTRYPA SUBPULCHELLA VAR PARVULIPORA DYER

HETEROTRYPA PROLIFICA ULRICH

12226 REF SPEC-F

U O,CINCI,MAYSVILL

MEAFORD FM,ERINDALE MBR

SECT 9,35 FT LEVEL , COOKSVILLE CK,ONT

G97;DYER WS,ONT DEPT MINES, VOL

32,PT7, 1925,P53,PL5, FIG 3;FRITZ MA, 1975,P8-12,FIG 2 A. B.5C

REMARKS: C-WS DYER, 1922 ;SLIDES 917-919;SPEC FIG'D BY DYER;SLIDES 917A,918A FIG'D BY FRITZ; CONSIDERED H. SUBRAMOSA BY FRITZ; 2 SPEC, 5 SLIDES

HETEROTRYPA ROBUSTA DYER

12158 P-SYN

UO CINCIMAYSVILL

MEAFORD FM,STREETSVILLE MBR

[STREETSVILLE MBR,MEAFORD,RICHMOND]

SECT 14,INT 3 , STREETSVILLE.ONT

G97;DYER WS,ONT DEPT MINES,VOL

32,PT7, 1925,P66,PL2,FIG 7;FRITZ MA, 1975,P12-15

REMARKS: C-WS DYER, 1923 :SLIDE 784 . FIG'D BY DYER ; 2 PCES, 1 SLIDE

HETEROTRYPA ROBUSTA DYER

12156 P-SYN

UO.CINCI.MAYSVILL

MEAFORD FM

[MEAFORD, RICHMOND] 
STREETSVILLE,ONT

G97:DYER WS,ONT DEPT MINES,VOL

32,PT7, 1925,P66,PL6,FIG 2;FRITZ MA, 1975,P12-15,FIG 3A-B, 4 A-B

REMARKS: C-J TOWNSEND, 1923 :SLIDES 770-773:SPEC

FIG'D BY DYER; SLIDES 770,771 FIG'D BY FRITZ; 1 SPEC FRAGS,SLIDES

\section{HETEROTRYPAROBUSTA DYER}

12157 P-SYN

UO.CINCI,MAYSVILL

MEAFORD FM.STREETSVILLE MBR

[STREETSVILLE MBR,MEAFORD,RICHMOND]

SECT 14,INT 3 , STREETSVILLE,ONT

G97:DYER WS, ONT DEPT MINES, VOL

32,PT7, 1925,P66,PL2,FIG 8;FRITZ MA, 1975,P12-15,FIG $3 \mathrm{C}, 4 \mathrm{C}$

REMARKS: C-WS DYER, 1923 ;SLIDES 788,$789 ; 788$ FIG'D BY FRITZ;SECT 14,INT 3 OF DYER ; 3 SPEC, 2 SLIDES

\section{HETEROTRYPA SIMPLEX DYER}

12159 P-SYN

U O,CINCI,MAYSVILL

MEAFORD FM,STREETSVILLE MBR

[STREETSVILLE MBR,MEAFORD,RICHMOND ]

SECT 14, STREETSVILLE, ONT

G97; DYER WS,ONT DEPT MINES, VOL 32,PT7, 1925,P66-

67.PL3,FIG 1:FRITZ MA, 1975,P15-18,FIG 2C-D 5D

REMARKS: C-WS DYER, $1923 ; 2$ SLIDES,NO 781,BOTH FIG'D BY FRITZ;SECT 14 OF DYER; 1 SPEC, 2 SLIDES

HETEROTRYPA SIMPLEX VAR MACULOSA DYER

REMARKS: SEE HETEROTRYPA MACULOSA DYER

\section{HETEROTRYPA SIMPLEX VAR MACULOSA DYER}

12175 P-SYN

U O,CINCI,MAYSVILL

MEAFORD FM,STREETSVILLE MBR

STREETSVILLE,ONT

DYER WS,ONT DEPT MINES, VOL 32,PT7, 1925,P67,PL7,FIG 9:FRITZ MA, 1975,P 18-19, FIG 5A-B,6A-B

REMARKS: C-WS DYER, 1923 :SLIDES 774-776:775 + 776

FIG'D BY FRITZ; CONSIDERED BY FRITZ TO BE THE

SPECIES H. MACULOSA DYER; 3 SPEC, 3 SLIDES

\section{HETEROTRYPA SUBPULCHELLA VAR PARVULIPORA DYER}

12161 P-HOLO

U O,CINCI,MAYSVILL

MEAFORD FM

[MEAFORD, RICHMOND FM]

STREETSVILLE,ONT

G97:DYER WS, ONT DEPT MINES, VOL 32 PT7 1925.P6768,PL6,FIG 9;FRITZ MA, 1975,P20-24,FIG 6C-D

REMARKS: C-j TOWNSEND, 1923 ;SLIDES $782,783+2$ OTHERS:SPEC FIG'D BY DYER:SLIDES 782B + ONE NEW SLIDE FIG'D BY FRITZ;RENAMED H. MEAFORDENSIS BY FRITZ; 1 SPEC, 4 SLIDES

\section{HOMOTRYPA CINCINNATIENSIS VAR MEAFORDENSIS}

12317 P-HOLO

U O,CINCI,MAYSVILL

MEAFORD FM ERINDALE MBR

[ERINDALE,MEAFORD, RICHMOND]

WORKMAN'S CK,ONT

G96:FRITZ MA 1926. TRANS ROY SOC CAN, VOL 20,SECT 4,P100-101,PL2,FIG 3,4,PL4,FIG 12

REMARKS: C-MA FRITZ, 1925:SLIDES 1113,$1114 ; 1113+$ SPEC FIG'D : 1 SPEC, 2 SLIDES

HOMOTRYPA CREDITENSIS DYER

12294 REF SPEC-M

UOMAYS OR RICHMOND

MEAFORD FM

IMEAFORD, RICHMOND

CREDIT R,STREETSVILLE,ONT

G96;FRITZ MA, 1977.LIFE SCI CONTR,ROM N111.P 10- 15

REMARK S: C-J TOWNSEND :SLIDE NO 1047; NOT'FIG'D; 2 SPEC, 1 SLIDE
HOMOTRYPA CREDITENSIS DYER

12263 REF SPEC-M

UO,MAYS OR RICHMOND

MEAFORDFM

[STREETSVILLE MBR, RICHMOND,MEAFORD]

CREDIT R,STREETSVILLE,ONT

G96;FRITZ MA, 1977,LIFE SCI CONTR,ROM,N111,P10-15

REMARKS: C-WS DYER, 1922 ;SLIDE NO 980;NOT FIG'D ; 2 SPEC, 1 SLIDE

\section{HOMOTRYPA CREDITENSIS DYER}

12162 P-HOLO

UO,CINCI,MAYSVILL

MEAFORD FM

[MEAFORD, RICHMOND]

STREETSVILLE,ONT

G96;DYER WS, 1925, ONT DEPT MINES, VOL

32,PT7,P68,PL3,FIG 3,4,PL7,FIG 5;FRITZ MA, 1977,P10-

15, FIG $3 A, B$

REMARKS: C-J TOWNSEND, 1923 ;SLIDES 790-791; 1 SPEC 2 SLIDES

HOMOTRYPA CRENULATA CALEY

24355 [1197T ] P-HOLO

M O,CHAMP, MOH,TRENT

TRENTON GP

[TRENTON]

RLWY CUT, LITTLE CURRENT, MANITOULIN IS,ONT

G96;CALEY JF,GSC MEM 202,1936,P70,PL2,FIG 1,2,PL3,FIG

6

REMARK S: C-JF CALEY, 1933 ;SLIDES 1948A-C;SPEC + SLIDES FIG'D; 1 SPEC,FRAGS,SLIDES

\section{HOMOTRYPA LEWISI WAY IN BOLTON}

7513 [805CL ] P-SYN

L S,ALEX

CATARACT GP,MANITOULIN FM

[MANITOULIN FM,BASE OF U BIOHERMAL BED]

LOT 7,CON 9 , ALLEN TP,MANITOULIN IS,ONT

G96;BOLTON TE,GSC MEM 289,1957,P66,PL10,FIG 4,5,6

REMARK S: C-HG WAY, 1932 ;SLIDES 1712,$1713 ; 1713$ FIG'D 2 SPEC, 2 SLIDES

\section{HOMOTRYPA STREETSVILLENSIS DYER}

12164 P-PARAL

UO,MAYS OR RICHMOND

MEAFORD FM

[STREETSVILLE MBR,MEAFORD]

STREETSVILLE,ONT

G96:DYER WS, 1925, ONT DEPT MINES,VOL 32,PT7,P68-

69,PL3,FIG 6;FRITZMA, 1977,P 15-19

REMARKS: C-WS DYER, 1923 :SLIDES 793-795;793 FIG'D BY

DYER;CONSIDERED BY FRITZ TO BE HOMOTRYPA SP

INDET ; 11 PCES, 3 SLIDES

HOMOTRYPA STREETSVILLENSIS DYER

12165 P-LECTO

UO,MAYS OR RICHMOND

MEAFORD FM

STREETSVILLE,ONT

G96:DYER WS, 1925,ONT DEPT MINES,VOL 32,PT7,P6869,PL3,FIG 5,PL7,FIG 4;FRITZ MA, 1977,P 15-19, FIG 4A-C

REMARKS: C-J TOWNSEND, 1923 :SLIDES 829,830;829 FIG'D;DESIGNATED LECTOTYPE BY FRITZ ; 1 SPEC, 2 SLIDES

HOMOTRYPA STREETSVILLENSIS DYER

12163 P-PARAL

UO,MAYS OR RICHMOND

MEAFORD FM

STREETSVILLE,ONT

G96;DYER WS, 1925,ONT DEPT MINES,VOL 32,PT7,P6869,FIG 5A-C;FRITZ MA, 1977,P 15-19

REMARKS: C-J TOWNSEND, 1923 ;SLIDE $792 ; 2$ SPEC, 1 SLIDE 
HOMOTRYPA STREETSVILLENSIS VAR SIMILIS C.

12447 P-HOLO

UO,CINCI,MAYSVILL

MEAFORD FM

KAGAWONG FALLS, MANITOULIN IS,ONT

G96:CALEY JF, GSC MEM 202, 1936,P70-71,PL5,FIG 1-3

REMARKS: C-JF CALEY, 1932 ;VAR AUTHOR CALEY:SLIDE 1936 ; 1 SPEC, 1 SLIDE

HOMOTRYPELLA DUBIA (CUMINGS\&GALLOWAY)

12270 REF SPEC-F

U O.CINCI,MAYSVILL

MEAFORD FM,STREETSVILLE MBR

SECT 14,13 FT LEVEL, CREDIT R,STREETSVILLE,ONT

G96:DYER WS,ONT DEPT MINES,VOL

32,PT7, 1925,P54,PL7,FIG 10

REMARKS: C-WS DYER, 1922 ;SLIDES 1002,$1003 ; 4$ SPEC, 2 SLIDES

\section{HOMOTRYPELLA EXPANSA DYER}

16595 [254HR] P-HOLO

U OMAYS OR RICHMOND

MEAFORD FM

[MEAFORD,RICHMOND]

STREETSVILLE,ONT

G96:DYER WS, 1925, ONT DEPT MINES, VOL

32,PT7,P69,PL5,FIG 11;FRITZ MA, 1977, ROM LIFE SCI

CONTR,N111,P19-22,FIG 5A,B

REMARKS: C-J TOWNSEND, 1923 :SLIDES 798-800 + 2

OTHERS; CONSIDERED BY FRITZ TO BE SYNONYM OF

HOMOTRYPELLA HOSPITALIS (NICHOLSON); 1 SPEC, 5

SLIDES

HOMOTRYPELLA HOSPITALIS (NICHOLSON)

REMARKS: SEE ALSO HOMOTRYPELLA EXPANSA DYER

HOMOTRYPELLA HOSPITALIS (NICHOLSON)

12295 REF SPEC-F

U O.CINCI,MAYSVILL

MEAFORD FM

[MEAFORD,RICHMOND]

CREDIT R.STREETSVILLE.ONT

G96;DYER WS,ONT DEPT MINES, VOL

32, PT 7, 1925,P54,PL5,FIG 1

REMARK S: C-J TOWNSEND :SLIDES 1048-1050,NOT FIG'D 1 SPEC,3 SLIDES

\section{HOMOTRYPELLA HOSPITALIS (NICHOLSON)}

12248 REF SPEC-F

U O.CINCI,MAYSVILL

MEAFORD FM

[MEAFORD, RICHMOND]

CREDIT R,STREETSVILLE,ONT

G96;DYER WS,ONT DEPT MINES, VOL

32,PT7, 1925,P54,PL5, FIG 2

REMARKS: C-WS DYER, 1922 ;SLIDE $949 ; 1$ SPEC, 1 SLIDE

HOMOTRYPELLA HOSPITALIS VAR PECULIARIS DYER REMARKS: SEE ATACTOPORELLA DENSA (DYER)

HOMOTRYPELLA PECULIARIS DYER REMARKS: SEE ATACTOPORELLA PECULIARIS (DYER)

\section{LEPTOTRYPA EXPANSA PARKS\&DYER}

$17326[1085 \mathrm{HR}]$ P-HOLO

U O.CINCI,MAYSVILL

DUNDAS FM

4 FT LEVEL,OLD SHALE PIT, LAMBTON, ONT

G98;PARKS \& DYER,ONT DEPT MINES, VOL

30,PT7, 1922, P 10-11,PL1, FIG 12,PL2, FIG 3

REMARKS: C-H STEWART, 1919 ;SLIDE $758 ; 1$ SPEC,1SLIDE

LEPTOTRYPELLA SPINULIFERA FRITZ

$16407[2108 \mathrm{H}]$ P-SYN

MD.ERIAN

HAMILTON GP

[HAMILTON]

THEDFORD.ONT
G98:FRITZ MA TRANS ROY CAN INST VOL

17,PT2, 1930,P224-225,PL 12, FIG 3-6

REMARK S: SLIDE 1479;ROM RECORDS STATE 2 SPECS: SPECIN FIG 4 NOT LOCATED; 1 SPEC, 1 SLIDE

LIOCLEMA MCGERRIGLEI FRITZ

20375 P-HOLO

LD, ?

MALBAY TP,GASPE CO,QUE

G 103;FRITZ MA,ROMP CONTRIB,NO 4, 1940,P6-7,PL 1,FIG 6,9

REMARKS: D-QDM, 1940 ;SLIDES 256A,B:QDM LOC F25-M$39 ; 1$ SPEC, 2 SLIDES

LOCULIPORA ULRICHI BASSLER 20922[612N] P-SYN

MS,NIAG

CLINTON GP,ROCHESTER FM

[ROCHESTER SHALE]

GRIMSBY ONT

G124;BASSLER RS,USGS BULL 292, 1906,P53,PL25,FIG 23 REMARKS: D-BE WALKER, $1906 ; 1$ SPEC

MESOTRYPA DISTINCTA PARKS

30726 P-PARAL

MO,CINCI,MAYSVILL

DUNDAS FM

[DUNDAS ]

HUMBER R, TORONTO,ONT

G96;PARKS WA, 1925, ONT DEPT MINES, VOL

32,PT7, P36, PL 4,FIG 4, 5; FRITZ MA, 1976, LIFE SCI

CONTR,ROM,N 107,P7-10,FIG 2 A

REMARKS: C-J TOWNSEND :CAPTION FOR FIG 2 A GIVES SPEC INCORRECTLY AS ROM 1200HR,LECTOTYPE : 5 SPEC, 13 SLIDES

MESOTRYPA DISTINCTA PARKS

17440 [1200HR] P-LECTO

U O,CINCI,MAYSVILL

DUNDASFM

[DUNDAS ]

HUMBER R, TORONTO ONT

G96;PARKS WA,ONT DEPT MINES, VOL

32,PT7, 1925,P36,PL4,FIG 4,5;FRITZ MA, 1976, LIFE SCI CONTR ROM N107.P7-10.FIG 2B

REMARKS: C-J TOWNSEND :CAPTION FOR FIG 2 A SHOULD READ PARALECTOTYPE ROM $30726 ; 1$ SPEC + 1 SLIDE

MESOTRYPA DIVERSA (PARKS\&DYER)

17330 [1089HR ] P-HOLO

STIGMATELLA CATENULATA VAR DIVERSA PARKS\&DYER

UO,CINCI,MAYSVILL

DUNDAS FM

[DUNDAS]

LR 4 FT OF EXPOSURE, E SIDE HUMBER R, BELOW

WESTON,ONT

G98:PARKS + DYER ONT DEPT MINES, VOL

30,PT7, 1922,P 14,PL3,FIG 8,8A;FRITZ MA, 1971,ROM LIFE SCI OCC PAP 18,P 1-8,FIG 1-2

REMARKS: C-WA PARKS, 1920 :SLIDES 713 A,B PLUS ONE

OTHER; 1 SPEC, 3 SLIDES

MESOTRYPA MACULOSA CALEY

24349 [1191T] P-HOLO

MO.CHAMP.MOH,TRENT

TRENTON GP

[TRENTON] 
RLWY CUT, LITTLE CURRENT, MANITOULIN IS,ONT G96;CALEY JF,GSC MEM 202,1936,P7 1-72,PL3,FIG

1.PL4,FIG 3,4

REMARKS: C-JF CALEY;SLIDE 1950; 1 SPEC, 1 SLIDE

\section{MESOTRYPA PATELLA (ULRICH)}

12283 REF SPEC-F

U O.CINCI,MAYSVILL

MEAFORD FM,STREETSVILLE MBR

[STREETSVILLE MBR,MEAFORD, RICHMOND]

8 FT 6 IN LEVEL, CREDIT R,STREETSVILLE,ONT

G96;DYER WS, ONT DEPT MINES, VOL

32,PT7, 1925,P54,PL5, FIG 9

REMARKS: C-WS DYER, 1922 ;SLIDE 1024,NOT FIG'D;SECT

14 OF DYER ; 1 SPEC( 4 PCES),SLIDE

\section{MESOTRYPA PROLIFICA FRITZ}

25157 [747U] P-HOLO

U O,CINCI,EDEN

LCOLLINGWOOD FM

[L COLLINGWOOD]

BTWN COLLINGWOOD AND CRAIGLEITH, ONT

G96;FRITZ MA,IN PARKS WA,TRANS ROY SOC CAN,VOL

22.SECT 4, 1928,P76-78,FIG 22,23,PL 1,FIG 28

REMARKS: C-WA PARKS, 1927 ;SLIDES 1294,$1295 ; 4$ PCES, 2 SLIDES

\section{MESOTRYPA? SIMPLEX FRITZ}

$17546[1312 \mathrm{HR}]$ P-HOLO

U O,CINCI,MAYSVILL

DUNDAS FM,CHRISTIE MBR

WORKMAN'S CK,ONT

G96;FRITZ MA,TRANS ROY SOC CAN,VOL 20,SECT

4,1926,P 101-102,PL2,FIG 5,6,7

REMARKS: C-MA FRITZ, 1925;SLIDES 1098-1101;1100 AND 1101 NOT FIG'D; 1 SPEC, 4 SLIDES

MONOTRYPELLA CF AEQUALIS ULRICH

17766 [1548HR] REF SPEC-F

UO,CINCI,MAYSVILL

WEKWEMIKONGSING FM

CLAY CLIFF, MANITOULIN IS, ONT

G109;CALEY JF,GSC MEM 202,1936,P76,PL3,FIG 4,PL4,FIG 7-9

REMARKS: C-JF CALEY 1932 :LARGER PCE FIG'D

UNCERTAIN WHICH THIN SECTIONS FIG'D ; 2 PCES,

SLIDE 1983

\section{MONOTRYPELLA CURVATA DYER}

12167 P-HOLO

U O,CINCI,MAYSVILL

MEAFORD FM

[MEAFORD,RICHMOND]

STREETSVILLE,ONT

G 109;DYER WS,ONT DEPT MINES, VOL

32,PT7, 1925, P70,PL 4,FIG 3,4,PL6,FIG 3

REMARKS: C-J TOWNSEND, 1923 ;SLIDES 807,$808 ; 1$ SPEC\&FRAG,2 SLIDES

\section{MONOTRYPELLA ? DUBIA CALEY}

24354 [1196T ] P-HOLO

MO,CHAMP,MOH,TRENT

TRENTON GP

[TRENTON ]

RLWY CUT, LITTLE CURRENT, MANITOULIN IS, ONT

G 109;CALEY JF, GSC MEM 202, 1936,P77,PL6,FIG 1012.PL3,FIG 13

REMARKS: C-JF CALEY ; SLIDES 1949A,B;SPEC IN 3 PCES : 1 SPEC, 2 SLIDES

\section{MONTICULIPORA MANITOULINENSIS CALEY}

$24343[1184 \mathrm{~T}] \mathrm{P}-\mathrm{HOLO}$

MO,CHAMP,MOH,TRENT

TRENTON GP

[TRENTON]

LIGHTHOUSE, LITTLE CURRENT, MANITOULIN IS, ONT

G94:CALEY JF,GSC MEM 202, 1936,P72,PL5,FIG 7,8,9

REMARKS: C-JF CALEY, 1932 :SLIDES 1924A, B ; 1 SPEC, 2 SLIDES
MONTICULIPORA MULTIPORA DYER

12168 P-LECTO

MONTICULIPORA PARASITICA VAR MULTIPORA DYER

U O,CINCI,MAYSVILL

MEAFORD FM.STREETSVILLE MBR

[STREETSVILLE MBR,MEAFORD, RICHMOND]

SECT 14,INT 3 , STREETSVILLE,ONT

G94;DYER WS, ONT DEPT MINES, VOL

32,PT7, 1925,P7 1,PL4,FIG 2;FRITZ MA, 1976, LIFE SCI

CONTR,ROM,N 107,P3-6,FIG 1A,B

REMARKS: C-WS DYER ;SLIDE 842;2 PCES FROM 1 SPEC ; 2 PCES, 1 SLIDE

\section{MONTICULIPORA MULTIPORA DYER}

12169 P-PARAL

MONTICULIPORA PARASITICA VAR MULTIPORA DYER

UO, CINCI,MAYSVILL

MEAFORD FM,STREETSVILLE MBR

[STREETSVILLE MBR,MEAFORD, RICHMOND]

SECT 13,INT 4 , STREETSVILLE, ONT

G96;DYER WS, 1925,ONT DEPT MINES,VOL

32,PT7,P71,PL6,FIG 8;FRITZ MA, 1976,LIFE SCI

CONTR,ROM,N107,P3-6

REMARK S: C-WS DYER, 1923 ;THIS SPEC ALSO HOLOTYPE OF PRASOPORA RICHMONDENSIS FRITZ;STORED UNDER PRASOPORA RICHMONDENSIS ; 1 SPEC, 1 SLIDE

MONTICULIPORA PARASITICA VAR MULTIPORA DYER REMARKS: SEE MONTICULIPORA MULTIPORA DYER

PALESCHARA BEANI (JAMES)

17344 [1103HR] REF SPEC-F

U O,CINCI,MAYSVILL

DUNDAS FM

DON VALLEY QUARRY TORONTO,ONT

G 147;PARKS \& DYER,ONT DEPT MINES, VOL

30,PT 7, 1922,P24-25,PL5,FIG 7

REMARKS: C-J TOWNSEND ; 1 SPEC

PERONOPORA VERA NICKLES

17324 [1083HR] REF SPEC-F

PERONOPORA VERA ULRICH

U O,CINCI,MAYSVILL

DUNDAS FM

DON VALLEY QUARRY,TORONTO,ONT

G96;PARKS + DYER,ONT DEPT MINES, VOL

30,PT 7, 1922,P7,PL 1,FIG 4,5,PL2,FIG 4,5,PL5,FIG 3;FRITZ MA, 1976,P 10-13,FIG 3A-D

REMARKS: C-J TOWNSEND ;SLIDES 757A,B;SPEC MARKED 'A' IS FIG'D BY PARKS + DYER; 5 PCES, 2 SLIDES

PRASOPORA DONENSIS PARKS + DYER

32317 P-PARAL

U O,CINCI,MAYSVILL

DUNDAS FM

DON VALLEY QUARRY,TORONTO, ONT

G96;PARKS + DYER, 1922.ONT DEPT MINES,VOL 30,PT7,P7:FRITZMA, 1976, LIFE SCI

CONTR,ROM,N 107,P 14-17

REMARKS: C-J TOWNSEND;ORIG WITH NO 1084HR,PARKS + DYER'S SYNTYPES:LECTOTYPE DESIGNATED BY

FRITZ; 1 SPEC + 2 SLIDES 
PRASOPORA DONENSIS PARKS + DYER

32318 P-PARAL

U O.CINCI,MAYSVILL

DUNDAS FM

DON VALLEY QUARRY,TORONTO,ONT

G96:PARKS + DYER, 1922, ONT DEPT MINES, VOL

30,PT7,P7:FRITZ MA, 1976, LIFE SCI

CONTR.ROM,N 107,P 14-17

REMARKS: C-J TOWNSEND ; ORIG WITH NO 1084HR,PARKS + DYER'S SYNTYPES:CONSIDERED 8Y FRITZ TO BE PRASOPORA CF DONENSIS:SPEC MARKED 'B' IS A BRACHIOPOD; 4 SPEC + 6 SLIDES

PRASOPORA DONENSIS PARKS\&DYER

17325 [1084HR] P-LECTO

UO.CINCI,MAYSVILL

DUNDAS FM

DON VALLEY QUARRY,TORONTO,ONT

G96:PARKS + DYER, ONT DEPT MINES, VOL

$30, P T 7,1922$, P 7-8,PL1,FIG 6,7,PL2,FIG 6,7,PL5,FIG 6;

FRITZ MA, 1976,P 14-17,FIG 4A-C

REMARKS: C-J TOWNSEND: 1 OF 6 SYNTYPES

DESIGNATED LECTOTYPE BY FRITZ; 1 SPEC + 3 SLIDES

PRASOPORA ISOLATA CALEY

$17750[1531 \mathrm{HR}]$ P-HOLO

U O.CINCI,MAYSVILL

WEKWEMIKONGSING FM

CLAY CLIFF, MANITOULIN IS, ONT

G96:CALEY JF,GSC MEM 202,1936,P69-70,PL2,FIG $5,6, P L 3, F I G 3$

REMARK S: C-JF CALEY, 1933 ;SLIDES 1942A-D;A,B + SPEC FIG'D; 1 SPEC, 4 SLIDES

PRASOPORA RICHMONDENSIS FRITZ

12169 P-HOLO

MONTICULIPORA MULTIPORA DYER

UO,MAYS OR RICHMOND

MEAFORD FM

[STREETSVILLE MBR,MEAFORD,RICHMOND]

E SIDE OF CREDIT R, STREETSVILLE, ONT

G96;DYER WS, 1925,P71,PL6,FIG 8;FRITZ MA, 1976,LIFE SCI

CONTR, ROM,N107,P18-21,FIG 5A-C

REMARKS: C-WS DYER, 1923 ;THIS SPEC ALSO

PARALECTOTYPE OF MONTICULIPORA MULTIPORA

DYER;STORED UNDER PRASOPORA RICHMONDENSIS

FRITZ; 1 SPEC 2 SLIDES

PRASOPORA RICHMONDENSIS FRITZ

12169

MONTICULIPORA MULTIPORA DYER

UO.MAYS OR RICHMOND

MEAFORDFM

[STREETSVILLE MBR,MEAFORD,RICHMOND]

E SIDE OF CREDIT R, STREETSVILLE,ONT

G96;DYER WS, 1925,P71,PL6,FIG 8;FRITZ MA, 1976, LIFE SC CONTR.ROM N 107,P18-21,FIG 5A-C

REMARKS: C-WS DYER, 1923 ; THIS SPEC ALSO

PARALECTOTYPE OF MONTICULIPORA MULTIPORA

DYER:STORED UNDER PRASOPORA DONENSIS FRITZ : 1

SPEC +2 SLIDES

RHOMBOTRYPA QUADRATA (ROMINGER)

12296 REF SPEC-F

UO.CINCI,MAYSVILL

MEAFORD FM

[MEAFORD,RICHMOND]

CREDIT R.STREETSVILLE,ONT

G109;DYER WS,ONT DEPT MINES, VOL
30,PT7, 1925,P54,PL4,FIG 5.PL6,FIG 4

REMARKS: C-J TOWNSEND ; SLIDE 1051;SPEC MARKED 'A' IS FIG'D ; 3 SPEC, 1 SLIDE

RHOPALONARIA LAMBTONENSIS FRITZ

27119 P-HOLO

MD,ERIAN

HAMILTON GP

THEDFORD,LAMBTON CO ONT

G35;FRITZ MA,J PALEONT, VOL 28,N1, 1954,P118,FIG 1 REMARKS: D-BE WALKER ; 1 SPEC

SPATIOPORA DELICATULA FRITZ

25158 [748U] P-HOLO

U O. CINCI,EDEN

BLUE MOUNTAIN FM

[BLUE MOUNTAIN (UP GLOUCESTER)]

CK $1 / 2$ MI W OF CRAIGLEITH,ONT

G83;FRITZ MA,IN PARKS WA, TRANS ROY SOC CAN, VOL

22,SECT 4,1928,P78-79,PL1,FIG 17

REMARKS: C-WA PARKS, $1927 ; 1$ SPEC

SPATIOPORA VARIANS PARKS\&DYER

17322 [1081HR] P-HOLO

U O,CINCI,MAYSVILL

DUNDAS FM

4 FT LEVEL, OLD SHALE PITS, LAMBTON,ONT

G83;PARKS \& DYER,ONT DEPT MINES,VOL

30,PT7, 1922,P3,PL1,FIG 3,PL5,FIG 10

REMARKS: C-H STEWART, 1919; 1 SPEC,SLIDE 760

STIGMATELLA ALCICORNIS CUMINGS\&GALLOWAY

$17421[1180 \mathrm{HR}]$ REF SPEC-F

U O,CINCI,MAYSVILL

DUNDAS FM

16 FT LEVEL, HUMBERVALE QUARRY, ONT

G98;ARMSTRONG HS,J PALEONT,VOL 19,1945,P 150,FIG $3,4,10$

REMARKS: C-H STEWART, 1919 ;SLIDE $722 ; 1$ SPEC, 1 SLIDE

STIGMATELLA CATENULATA VAR (A)

17328 [1087HR] REF SPEC-F

U O.CINCI,MAYSVILL

DUNDAS FM

SHALE PIT, 5 FT LEVEL, LAMBTON,ONT

G98;PARKS \& DYER, 1921,P13,PL3,FIG 1,2,PL4,FIG 1,2

FRITZ MA, LIFE SCI CONTR,ROM,N87, 1973,P4-7,FIG 1B-C REMARKS: C-H STEWART, 1919 ;SLIDES 718A,B PLUS 2

OTHERS;SPECIES AUTHORS CUMMINGS\&GALLOWAY : SPEC, 4 SLIDES

STIGMATELLA CATENULATA VAR (B) PARKS + DYER

REMARKS: SEE STIGMATELLA HALYSA ARMSTRONG

STIGMATELLA CATENULATA DIVERSA PARKS + DYER REMARKS: SEE MESOTRYPA DIVERSA (PARKS + DYER)

STIGMATELLA CRENULATA ULRICH + BASSLER

12220 REF SPEC-F

U O.CINCI,MAYSVILL

MEAFORD FM

[ERINDALE MBR,RICHMOND,MEAFORD]

CREDIT R,STREETSVILLE,ONT

G98;DYER,WS,ONT DEPT MINES, VOL

32,PT7, 1923,P54,PL7,FIG 8:ARMSTRONG HS,J

PALEONT, VOL 19,1945.P151

REMARKS: C-WS DYER, 1922 :SLIDE NO 933:SPEC MARKED 'A' FIG'D BY DYER; 4 SPEC + 1SLIDE

STIGMATELLA CRENULATA ULRICH + BASSLER REMARK S: SEE ALSO STIGMATELLA VULGARIS PARKS + DYER 
STIGMATELLA CRENULATA ULRICH\&BASSLER

12297 REF SPEC-F

U O,CINCI,MAYSVILL

MEAFORD FM

[MEAFORD, RICHMOND]

CREDIT R,STREETSVILLE,ONT

G98:DYER WS,ONT DEPT MINES, VOL

32,PT7, 1925,P54,PL4,FIG 6:ARMSTRONG

HS, 1945,P 151,FIG 1,2;FRITZMA, 1973,P 13-14,FIG 4A,C

REMARKS: C-J TOWNSEND :SLIDES 1052, 1053,FIG'D ; 1 SPEC, 2 SLIDES

STIGMATELLA CRENULATA VAR MEAFORDENSIS FRITZ 17547 [ 1313 HR ] P-HOLO

U O.CINCI,MAYSVILL

DUNDAS FM, HUMBER MBR

WORKMAN'S CK, ONT

G98;FRITZ MA,TRANS ROY SOC CAN, VOL 20,1926,P $102-$ 103,PL3,FIG 1,2,PL4,FIG 13

REMARKS: C-MA FRITZ, 1925 ;SLIDES 1094,$1095 ; 1$ SPEC. 2 SLIDES

STIGMATELLA HALYSA ARMSTRONG

17329 [1088HR] P-HOLO

STIGMATELLA CATENULATA VAR (B) PARKS\&DYER

U O.CINCI,MAYSVILL

DUNDAS FM

SHALE PITS, 7 FT LEVEL, LAMBTON,ONT

G98;ARMSTRONG HS,J PAL, VOL 19, 1945,P 153-154,FIG 5, 12:PARKS + DYER, 1922,P12,PL3,FIG 3;FRITZ, 1973,P78,FIG $2 A-D$

REMARK S: C-H STEWART, 1919 ;SLIDES 717 A-D PLUS 1 OTHER ; 4 PCES, 5 SLIDES

STIGMATELLA HALYSA CRASSA (DYER)

17554 [ 1321 HR ] REF SPEC-M

U O.CINCI,MAYSVILL

DUNDAS FM,CREDIT MBR

SEC 6,3' LEVEL, ERINDALE,CREDIT R,ONT

G98:FRITZ MA.ROM LIFE SCI CONTR,N87, 1973,P11

REMARKS: C-WS DYER, 1922 ;SLIDE 929;TOPOTYPE ; 5 PCES, 1 SLIDE

\section{STIGMATELLA HAL YSA CRASSA (DYER)}

17555 [1322HR ] REF SPEC-M

U O,CINCI,MAYSVILL

DUNDAS FM,CREDIT MBR

ERINDALE,CREDIT R,ONT

G98:FRITZ MA,ROM LIFE SCI CONTR,N87, 1973,P11

REMARKS: C-WS DYER, 1922 ;TOPOTYPE;SLIDES $930-931$ 9 PCES \& 2 SLIDES

STIGMATELLA HALYSA CRASSA (DYER)

17556 [ 1323HR ] REF SPEC-M

U O,CINCI,MAYSVILL

DUNDAS FM CREDIT MBR

OPP MOUTH OF MULLET CK,SECT 7,5' TO 10' LEVEL,

CREDIT R,ONT

G98;FRITZ MA,ROM LIFE SCI CONTR,N87,1973,P11

REMARKS: C-WS DYER, 1922 ;SLIDE 932;CITED AS

TOPOTYPE BY FRITZ; NOT FROM TYPE LOCALITY; 1 SLIDE

STIGMATELLA HALYSA VAR CRASSA (DYER)

$17530[1292 \mathrm{HR}]$ P-HOLO

STIGMATELLA SESSILIS VAR CRASSA DYER

U O,CINCI,MAYSVILL

DUNDAS FM.CREDIT MBR

$1 / 2$ MI S OF ERINDALE,ONT

G98;DYER WS, 1925,P72,PL6,FIG 10;ARMSTRONG HS, 1945,P 154,FIG 6,7:FRITZ MA, 1973,P 10-11, FIG 1A,3D

REMARKS: C-WS DYER ; SLIDE 809 AND 4 OTHERS; 1 SPEC 5 SLIDES

\section{STIGMATELLA HALYSA VAR ERINDALENSIS A.}

12244 P-HOLO

STIGMATELLA LAMBTONENSIS PARKS\&DYER

U O,CINCI,MAYSVILL

MEAFORD FM,ERINDALEMBR

[ERINDALE MBR,MEAFORD,RICHMOND]
SECT 12 CREDIT R STREETSVILLE ONT

G98;ARMSTRONG HS,J PALEONT, VOL 19, 1945,P154155,FIG 8,9;DYER WS, 1925,P55,PL5,FIG 8;FRITZ MA, 1973,P 11-13,FIG 3A-C

REMARKS: C-WS DYER, 1922 ;PUBL'D BY DYER AS HYPOTYPE OF S. LAMBTONENSIS:SLIDE NO $937 ; 1$ SPEC, 1 SLIDE

STIGMATELLA HYBRIDA DYER

12170 P-HOLO

U O CINCIMAYSVILL

MEAFORD FM,ERINDALE MBR

BRYOZOAN REEF, COOKSVILLE CK, ONT

G98;DYER WS,ONT DEPT MINES,VOL 32,PT7,1923,P7172,PL4,FIG 7,8,PL7;FRITZ MA, 1976,P18-25

REMARKS: C-WS DYER, 1923 ;SLIDE 763 + 3 OTHERS;SPEC IN 2 PCES ; 1 SPEC, 4 SLIDES

STIGMATELLA INCRUSTANS CUMINGS\&GALLOWAY

12298 REF SPEC-F

$O, C I N C I, M E A F O R D$

MEAFORD FM

[MEAFORD,RICHMOND]

CREDIT R,STREETSVILLE, ONT

G98;DYER WS,ONT DEPT MINES,VOL

32,PT7,1925,P55,PL6,FIG 7

REMARKS: C-J TOWNSEND ;SLIDE $1054 ; 1$ SPEC, 1 SLIDE

\section{STIGMATELLA INTERMEDIA FRITZ}

17515 [1276HR] P-HOLO

U O,CINCI, MAYSVILL

DUNDAS FM, HUMBER MBR

S OF BRIDGE OVER CK, WORKMAN'S CK,ONT

G98;FRITZ MA, 1925,P36.PL4,FIG 1;FRITZ

MA, 1926,P 103,PL3,FIG 3,4

REMARKS: C-MA FRITZ, 1925 ;SLIDE 1093:1276HR + 1314 HR ARE THE SAME SPEC ; 1 SPEC, 1 SLIDE

STIGMATELLA LAMBTONENSIS PARKS + DYER

REMARKS: SEE ALSO STIGMATELLA HALYSA VAR ERINDALENSIS ARMSTRONG

STIGMATELLA LAMBTONENSIS PARKS\&DYER

17331 [1090HR] P-HOLO

U O,CINCI,MAYSVILL

DUNDAS FM

5 FT LEVEL, OLD SHALE PIT, LAMBTON, ONT

G98:PARKS + DYER, 1922,P 14-15,PL2,FIG 13;ARMSTRONG HS, 1945, J PALEO,VOL 19,P153

REMARKS: C-H STEWART;MENTIONED BY ARMSTRONG'NOT STIGMATELLA'; 1 SPEC, 3 SLIDES

STIGMATELLA PECULIARIS FRITZ

12318 P-HOLO

U O,CINCI,MAYSVILL

MEAFORD FM, VINCENT MBR

WORKMAN'S CK, ONT

G98;FRITZ MA,TRANS ROY SOC CAN,VOL

20,PT4, 1926,P 104.PL3,FIG 5,6,PL4,FIG 8

REMARKS: C-MA FRITZ, 1925 ;SLIDE $1108 ; 1$ SPEC, 1 SLIDE

STIGMATELLA PECULIARIS SIMILIS CALEY

17749 [1530HR ] P-HOLO

UO,CINCI,MAYSVILL

WEKWEMIKONGSING FM

CLAY CLIFF, MANITOULIN IS,ONT

G98;CALEY JF,GSC MEM 202,1936,P73,PL3,FIG 7,PL4,FIG 12

REMARKS: C-JF CALEY ;SLIDE $1941 ; 1$ SPEC, 1 SLIDE

STIGMATELLA CF PERSONATA ULRICH + BASSLER REMARKS: SEE STIGMATELLA VULGARIS VAR MONTICULATA ARMSTRONG 
STIGMATELLA PERSONATA LOBATA DYER

$12171 \mathrm{P} \cdot \mathrm{HOLO}$

STIGMATELLA PERSONATA VAR LOBATA DYER

U O.CINCI.MAYSVILL

MEAFORD FM

[MEAFORD, RICHMOND]

STREETSVILLE.ONT

G98:DYER WS, ONT DEPT MINES, VOL

32,PT7, 1923,P72,PL6,FIG 1;FRITZ MA, 1973,P 17-18,FIG $5 A-C$

REMARKS: C-WS DYER, 1923 :SLIDES $746+3$ OTHERS; SPEC FIG'D BY DYER; 1 SPEC, 4 SLIDES

STIGMATELLA SESSILIS VAR DELICATULA FRITZ

17549 [1316HR]P-SYN

UO.CINCI,MAYSVILL

DUNDAS FM

[DUNDAS, HUMBER MBR]

WORKMAN'S CK.ONT

G98;FRITZ MA.TRANS ROY SOC CAN,VOL 20,SECT

4, 1926,P 104-105,PL3,FIG 7,8,PL4,FIG 9,10

REMARKS: C-MAFRITZ, 1925 ; SLIDES 1096, 1097;1097 NOT FIG'D ; 2 SPEC,2 SLIDES

STIGMATELLA SESSILIS VAR CRASSA DYER

REMARKS: SEE STIGMATELLA HALYSA VAR CRASSA (DYER)

STIGMATELLA VULGARIS PARKS\&DYER

17332 [1091HR] P-HOLO

U O.CINCI,MAYSVILL

DUNDAS FM

DON VALLEY QUARRY.TORONTO,ONT

G98:PARKS + DYER, ONT DEPT MINES, VOL

30,PT7, 1922,P15,PL1,FIG 13,14,PL6,FIG 12; FRITZ

MA, 1973,P20-21, FIG 6A-B

REMARK S: C-J TOWNSEND:SLIDES 715A,B, PLUS 4

OTHERS ; 1 SPEC,6 SLIDES

STIGMATELLA VULGARIS PARKS\&DYER

17327 [1086HR] REF SPEC-F

STIGMATELLA CRENULATA ULRICH\&BASSLER

U O,CINCI,MAYSVILL

DUNDASFM

HUMBER R TORONTO ONT

G98:PARKS \& DYER, 1922,P 12,PL3,FIG 4,5,7,PL4,FIG 4,5; ARMSTRONG HS, 1945,P153

REMARKS: C-HSTEWART : 1 SPEC, 2 SLIDES

STIGMATELLA VULGARIS VAR RAMOSA FRITZ

17550 [1317HR]P-HOLO

U O.CINCI,MAYSVILL

DUNDAS FM HUMBER MBR

WORKMAN'S CK, ONT

G98:FRITZ MA, TRANS ROY SOC CAN,VOL

20.PT4, 1926,P 105-106.PL3,FIG 9, 10 PL4,FIG 2

REMARKS: C-MA FRITZ, 1925; 1 SPEC,2 SLIDES

\section{STIGMATELLA VULGARIS VAR MONTICULATA}

17735 [1516HR]P-HOLO

U O. CINCI,MAYSVILL

DUNDAS FM

HUMBER R, TORONTO,ONT

G98:ARMSTRONG HS,J PALEONT,VOL 19, 1945,P155156,FIG 11; FRITZ MA, 1973,P21,FIG 6C

REMARK S: C-H STEWART -SLIDES 1703A B.PLUS 1 OTHER: CONSIDERED BY FRITZ TO BE SYNONYMOUS WITHS VULGARIS ; 1 SPEC, 3 SLIDES

\section{STIGMATELLA VULGARIS VAR MONTICULATA}

17333[1092HR] P.PARA

STIGMATELLA CF PERSONATA ULRICH\&BASSLER

UO,CINCI,MAYSVILL

DUNDASFM

25 FT LEVEL, HUMBER R CUT BELOW RLWY BRIDGES,

LAMBTON.ONT

G98:PARKS \& DYER, 1922,P 16:ARMSTRONG HS, 1945, P 155156

REMARKS: SLIDE $714 ; 9$ SPEC, 1 SLIDE

\section{BRACHIOPODA}

ACROTHELE ABAVIA MATTHEW

$7804[174 C M] P-S Y N$

$\mathrm{MCA}$

BOURINOT GP,DUGALD FM

[UETCHEMINIAN,DIV E3A-E]

DUGALD BK \& INDIAN BK, ESCASONIE,CAPE BRETON.NS

H280; WALCOTT CD, 1912,P635-636;MATTHEW

GF 1902,NB.P398-400,PL15.FIG 3A-D 4A-B

REMARKS: C-GF MATTHEW:D-MACKENZIE ; LABELLED COTYPES; UNCERTAIN WHICH SPECS FIG'D;STRAT REV AS IN HUTCHINSON, 1952, GSC MEM 263; 14 SPEC

ACROTHELE AVIA MATTHEW

7776 [146CM] P-PARAL

MCA,

BOURINOT GP,DUGALDFM

[U ETCHEMINIAN, DIV E3D]

DUGALD BK, ESCASONIE, CAPE BRETON, NS

H280; WALCOTT CD, 1912,P634-636,PL61,FIG 7,7AB,PL62,FIG 1D, 1T;MATTHEW GF, 1899, NB,P202. 203,PL3,FIG 1A-H

REMARKS: C-GF MATTHEW:D-MACKENZIE ;SPEC NO 8360(B) DESIGNATED LECTOTYPE BY WALCOTT:STRAT REV AS IN HUTCHINSON, 1952, GSC MEM 263;STUDIED BY AJ ROWELL, $1972 ; 2$ SPEC

\section{ACROTHELE AVIA MATTHEW}

$7805[175 \mathrm{CM}]$ P-PARAL

$\mathrm{MCA}$

BOURINOT GP,DUGALD FM

[U ETCHEMINIAN,DIV E3D]

DUGALD BK, ESCASONIE,CAPE BRETON,NS

H280; WALCOTT CD, 1912,P634-636,PL61,FIG 7,7B,PL62,FIG 10,11:MATTHEW GF, 1899,NB,P202-203,PL3,FIG 1A-H

REMARKS: C-GF MATTHEW:D-MACKENZIE ;SPEC NO 8360(B) DESIGNATED LECTOTYPE BY WALCOTT:STRAT REV AS IN HUTCHINSON, 1952, GSC MEM 263;STUDIED BY AJ ROWELL, $1972 ; 11$ SPEC

\section{ACROTHELE AVIA MATTHEW}

8360(A) [730CM(A)] P-PARAL

$\mathrm{MCA}$,

BOURINOT GP,DUGALD FM

[U ETCHEMINIAN,DIV E3B]

DUGALD BK, ESCASONIE,CAPE BRETON,NS

H280; WALCOTT CD, 1912,P634-636,PL61,FIG

7,7A, 7B,PL62, FIG 1D, 11:MATTHEW GF, 1899, NB,P202 203,PL3,FIG 1A-H

REMARKS: C-GF MATTHEW:D-MACKENZIE :SPEC NO 8360(B) DESIGNATED LECTOTYPE BY WALCOTT:STRAT REV AS IN HUTCHINSON, 1952, GSC MEM 263;STUDIED BY AJ ROWELL, $1972 ; 4$ SPEC (OF 5)

ACROTHELE AVIA MATTHEW

8360(B) [730CM(B)] P-LECTO

$\mathrm{MCA}$.

BOURINOT GP,DUGALD FM

[U ETCHEMINIAN,DIV E3D]

DUGALD BK, ESCASONIE,CAPE BRETON,NS

H280:MATTHEW GF, 1899,P202-203,PL3,FIG 1A-H;

WALCOTT CD, 1912,P634-636,PL61,FIG 7A

REMARK S: C-GF MATTHEW:D-MACKENZIE :SPEC 'B' OF MATTHEW'S SYNTYPES:DESIGNATED LECTOTYPE BY WALCOTT; STRAT REV AS IN HUTCHINSON, 1952, GSC MEM 263:STUDIED BY AJ ROWELL, 1972; 1 SPEC (OF 5 )

ACROTHELE AVIA MUT PUTEIS MATTHEW

$7798[168 \mathrm{CM}]$ P-SYN

$M C A$,

BOURINOT GP.DUGALDFM

[UETCHEMINIAN,DIVE3]

GREGWA BK, ESCASONIE,CAPE BRETON,NS

H280:WALCOTT CD, 19 12.P636:MATTHEW GF, 1902,NB,P398,PL 16,FIG 5A-8

REMARKS: C.GF MATTHEW:D-MACKENZIE :STRAT REV AS IN HUTCHINSON 1952 GSC MEM 263: UNCERTAIN WHICH SPECS FIG'D ; 5 SPEC 
ACROTHELE AVIA MUT PUTEIS MATTHEW

$7801[171 \mathrm{CM}]$ P-SYN

$M C A$,

BOURINOT GP,DUGALD FM

[U ETCHEMINIAN,DIV E3]

GREGWA BK, ESCASONIE,CAPE BRETON,NS

H280; WALCOTT CD, 1912,P636; MATTHEW

GF, 1902,NB,P398,PL 16,FIG 5A-B

REMARKS: C-GF MATTHEW;D-MACKENZIE ;STRAT REV AS IN HUTCHINSON, 1952, GCS MEM 263; UNCERTAIN WHICH SPECS FIG'D ; 5 SPEC

\section{ACROTHELE AVIA MUT PUTEIS MATTHEW}

8793 [1163CM] P-SYN

$\mathrm{MCA}$,

BOURINOT GP.DUGALD FM

[U ETCHEMINIAN,DIV E3D]

GREGWA BK, ESCASONIE,CAPE BRETON,NS

H280; WALCOTT CD, 19 12,P636; MATTHEW

GF, 1902,NB,P398,PL 16,FIG 5A-B

REMARKS: C-GF MATTHEW;D-MACKENZIE ;STRAT REV AS IN HUTCHINSON, 1952,GSC MEM 263; UNCERTAIN WHICH SPECS FIG'D ; 3 SPEC

\section{ACROTHELE MATTHEWI (HARTT)}

$8143[513 \mathrm{CM}]$ REF SPEC-F

$M C A$,

ST JOHN GP

[ST JOHN,ACADIAN,DIV 1C]

SEELYST ST JOHN NB

H280;MATTHEW GF, TRANS ROY SOC CAN,VOL 3,SECT 4,1886,P39-41,PL5,FIG 15, 15A

REMARK S: C-GF MATTHEW:D-MACKENZIE :LABELLED PLESIOTYPES; UNCERTAIN WHICH SPEC IS FIG'D; ROM RECORDS STATE 8 SPEC-SPECS MARKED F + $G$ MISSING;NO MATERIAL FROM ST JOHN MENTIONED IN PUBL' $N$; 6 SPEC

\section{ACROTHELE MATTHEWI VAR LATA MATTHEW}

8144 [5 14CM] P-SYN

$M C A$.

ST JOHN GP

[ST JOHN,ACADIAN,DIV $1 C$ ]

PORTERS BK, ST MARTINS, ST JOHN CO,NB

H280;MATTHEW GF,TRANS ROY SOC CAN.VOL 3,SECT

4,1886,P41,PL5,FIG 17,17A

REMARKS: C-GF MATTHEW;D-MACKENZIE :UNCERTAIN WHICH SPEC IS FIG'D ; 12 SPEC

\section{ACROTHELE MATTHEWI MUT MULTICOSTATA MATT.}

7787 [157CM] P-SYN?

$\mathrm{MCA}$

ST JOHN GP

[ST JOHN,ACADIAN]

PORTERS BK, ST MARTINS,ST JOHN CO,NB

H280:MATTHEW GF, TRANS ROY SOC CAN, SER 2, VOL

3,SECT 4,1897,P 168

REMARKS: C-GF MATTHEW;D-MACKENZIE;LABEL STATES PORTERS BK,ST MARTINS AND HASTINGS COVE,KINGS CO:LABELLED COTYPES;SPECIES NOT FIG'D;NO TYPES MENTIONED ; 3 SPEC

ACROTHELE MATTHEWI VAR COSTATA MATTHEW

7810 [180CM ] P-SYN

$\mathrm{LCA}$

ST JOHNGP

[ST JOHN.ACADIAN.DIV 1B2-5]

HANFORD BK, ST MARTINS, ST JOHN CO,NB H280; MATTHEW GF, TRANS NY ACAD SCI, VOL 14,1895,P 128,PL5,FIG 9

REMARKS: C-GF MATTHEW;D-MACKENZIE ;UNCERTAIN WHICH SPEC IS FIG'D:LABELLED COTYPES ; 6 SPEC

ACROTHELE MATTHEWI VAR PRIMA MATTHEW REMARKS: SEE ACROTHELE PRIMA MATTHEW
ACROTHELE PRIMA MATTHEW

7786 (A) [156CM(A)] P-LECTO

ACROTHELE MATTHEWI VAR PRIMA MATTHEW

LCA,

ST JOHN GP

[ST JOHN GP,ACADIAN,DIV 1B1]

HANFORD BK. ST MARTINS.ST JOHN CO,NB

H280:MATTHEW GF, TRANS ROY SOC CAN, VOL 3,SECT 4.1886,P41,PL5,FIG 16,16A;WALCOTT CD, 19 12,PL60,FIG

REMARK S: C-GF MATTHEW:D-MACKENZIE :SPEC 'C' OF MATTHEW'S SYNTYPES;DESIGNATED LECTOTYPE BY WALCOTT:STUDIED BY AJ ROWELL, 1972: 1 SPEC

ACROTHELE PRIMA MATTHEW

7786(B) [ 156CM(B)] P.PARAL

ACROTHELE MATTHEWI VAR PRIMA MATTHEW

LCA

ST JOHN GP

[ST JOHN,ACADIAN,C 1B 1]

HANFORD BK, ST MARTINS,ST JOHN CO,NB

H280;MATTHEW GF, 1886,P41,PL5,FIG 16, 16A;WALCOTT CD.USGS MON 51,1912,P653

REMARKS: C-GF MATTHEW;D-MACKENZIE ;SPEC 7786(A) DESIGNATED LECTOTYPE BY WALCOTT:STUDIED BY AJ ROWELL, $1972 ; 2$ SPEC

ACROTHELE PROLES MATTHEW

$7808(B)[178 \mathrm{CM}(\mathrm{B})]$ P-PARAL

$M C A$,

BOURINOT GP.DUGALD FM

[UETCHEMINIAN,DIV E3F]

DUGALD BK \& INDIAN BK, ESCASONIE, CAPE BRETON, NS H280;WALCOTT CD, 1912,P654-655,PL62,FIG 4:MATTHEW GF, 1902,NB,P 400-401,PL 17,FIG 3B-E

REMARKS: C-GF MATTHEW:D-MACKENZIE :SPEC 7808(A) DESIGNATED LECTOTYPE BY WALCOTT;UNCERTAIN WHICH OTHER SPECS FIG'D; STRAT REV AS IN HUTCHINSON, 1952,GSC MEM 263:STUDIED BY AJ ROWELL, $1972 ; 6$ SPEC

\section{ACROTHELE PROLES MATTHEW}

7784 [154CM ] P-PARAL

$M C A$.

BOURINOT GP,DUGALD FM

[UETCHEMINIAN,DIV E3F]

DUGALD BK \& INDIAN BK, ESCASONIE, CAPE BRETON, NS H280;WALCOTT CD, 1912,P654-655,PL62,FIG 4;MATTHEW GF, 1902,NB,P400-401,PL17.FIG 3B-E

REMARKS: C-GF MATTHEW;D-MACKENZIE ;SPEC 7808(A) DESIGNATED LECTOTYPE BY WALCOTT;UNCERTAIN WHICH OTHER SPECS FIG'D:STRAT REV AS IN HUTCHINSON, 1952, GSC MEM 263;STUDIED BY AJ ROWELL, $1972 ; 2$ SPEC

\section{ACROTHELE PROLES MATTHEW}

7808 (A) $[178 \mathrm{CM}(\mathrm{A})]$ P-LECTO

$M C A$,

BOURINOT GP.DUGALD FM

[UETCHEMINIAN,DIV E3F]

DUGALD BK \& INDIAN BK, ESCASONIE, CAPE BRETON, NS H280:MATTHEW GF, 1902,P400-401,PL17,FIG 3A:WALCOTT CD,USGS MON 51, 1912,P654-655,PL62,FIG 4

REMARKS: C-GF MATTHEW:D-MACKENZIE;SPEC 'F' OF MATTHEW'S SYNTYPES:DESIGNATED LECTOTYPE BY WALCOTT;STRAT REV AS IN HUTCHINSON, 1952,GSC MEM 263;STUDIED BY AJ ROWELL, $1972 ; 1$ SPEC

\section{ACROTHYRA PROAVIA MATTHEW}

7807 [177CM ] P-SYN

$M C A$.

BOURINOT GP,DUGALD FM

[UETCHEMINIAN DIV E3E]

DUGALD BK, ESCASONIE,CAPE BRETON,NS

H277:WALCOTT CD, 19 12,P7 17-7 18,PL76,FIG 3C,3G MATTHEW GF, 1899,NB,P203,PL3,FIG 2A-F; 1902 ,P386

REMARKS: C-GF MATTHEW;D-MACKENZIE ; LABELLED COTYPES IN OLD RECORDS:STRAT REV AS IN HUTCHINSON, 1952,GSC MEM 263;STUDIED BY AJ ROWELL, $1972 ; 20$ SPEC 
ACROTHYRA PROAVIA MATTHEW

8643 [ $1013 \mathrm{CM}]$ P-SYN?

$\mathrm{MCA}$.

BOURINOT GP.DUGALDFM

[UETCHEMINIAN,DIVE3E]

DUGALD BK. ESCASONIE,CAPE BRETON,NS

H277;MATTHEW GF, 1899,P203,PL3,FIG 2A-F; 1902,P386;

WALCOTT CD, 1912,P7 17-7 18,PL76,FIG 3C,3G

REMARKS:C-GF MATTHEW, $1900 ?$ ? NOT LABELLED TYPES

IN OLD RECORDS:STUDIED 19728 Y AJ ROWELL-MAY BE

PART OF ORIG SYNTYPIC SUITE:STRAT REV AS IN

HUTCHINSON, 1952,GSC MEM 263; 1 SLAB

\section{ACROTHYRA PROAVIA MATTHEW}

$8810[1180 \mathrm{CM}]$ P-SYN?

$\mathrm{MCA}$.

BOURINOT GP.DUGALDFM

[UETCHEMINIAN,DIV E3E ]

DUGALD BK, ESCASONIE,CAPE BRETON,NS

H277:MATTHEW GF, 1899,P203,PL3,FIG 2A-F; 1902,P386;

WALCOTT CD, 1912,P717-718,PL76,FIG 3C,3G

REMARKS: C-GF MATTHEW, 1900 ? :LABELLED

TOPOTYPES IN OLD RECORDS:STUDIED 1972 BY AJ

ROWELL-MAY BE PART OF ORIG SYNTYPIC SUITE;

STRAT REV AS IN HUTCHINSON, 1952,GSC MEM 263; 1 SPEC

\section{ACROTHYRA PROAVIA MATTHEW}

$8812[1182 \mathrm{CM}]$ P-SYN?

$\mathrm{MCA}$

BOURINOT GP.OUGALDFM

[UETCHEMINIAN,DIV E3E

DUGALD BK, ESCASONIE,CAPE BRETON,NS

H277;MATTHEW GF, 1899,P203,PL3,FIG 2A-F;1902,P386;

WALCOTT CD, 1912,P717-718,PL76,FIG 3C.3G

REMARK S: C-GF MATTHEW :LABELLED TOPOTYPES IN

OLD RECORDS;STUDIED 1972 BY AJ ROWELL-MAY BE

PART OF ORIG SYNTYPIC SUITE;REV AS IN

HUTCHINSON, 1952,GSC MEM 263; 1 SLAB

\section{ACROTHYRA PROAVIA MUT CRASSA MATTHEW}

$8361[731 \mathrm{CM}]$ P-HOLO

$M C A$,

BOURINOT GP.DUGALDFM

[UETCHEMINIAN.DIVE3E]

DUGALD BK, ESCASONIE, CAPE BRETON NS

H277; WALCOTT CD, 19 12,P7 17,PL76,FIG 3D;MATTHEW GF, 1902,N8,P389-390,PL 14,FIG 5A-C

REMARKS: C-GF MATTHEW:D-MACKENZIE ; STRAT REV AS IN HUTCHINSON, 1952, GSC MEM 263; STUDIED BY AJ ROWELL, $1972 ; 1$ SPEC \& ROCK FRAG

\section{ACROTHYRA PROAVIA MUT PRIMA MATTHEW}

25648 P-SYN?

$M C A$.

BOURINOT GP,DUGALD FM

[UETCHEMINIAN, DIVE3A]

DUGALD BK, ESCASONIE,CAPE BRETON,NS

H277;MATTHEW GF, 1902,P389,PL14,FIG 4A-E; WALCOTT CD. 1912,P717,PL76,FIG 3

REMARKS: C-GF MATTHEW:D-MACKENZIE :LABELLED TOPOTYPES IN OLD RECORDS;STUDIED 1972 BY AJ ROWELL-MAY BE PART OF ORIG SYNTYPIC SUITE:REV AS IN HUTCHINSON, 1952, GSC MEM $263 ; 3 \operatorname{SLABS}(A, 8, D)$

\section{ACROTHYRA PROAVIA MUT PRIMA MATTHEW}

\section{$8818[1188 \mathrm{CM}]$ P-SYN ?}

LCA

BOURINOT GP.DUGALDFM

[U ETCHEMINIAN,DIV E3A]

DUGALD BK, ESCASONIE,CAPE 8RETON,NS

H277:MATTHEW GF, 1902,P389,PL14.FIG 4A-E;WALCOTT

CD. 1912,P7 17,PL76,FIG 3

REMARKS:C-GF MATTHEW:D-MACKENZIE, NOT

LABELLED TYPES IN OLD RECORDS:STUDIED 1972 BY AJ ROWELL-MAY BE PART OF ORIG SYNTYPIC SUITE, REV AS IN HUTCHINSON 1952, GSC MEM 263;OLD RECORDS STATE 3 SLABS,FOR ORIENTATION : 2 SLABS
ACROTHYRA PROAVIA MUT PRIMA MATTHEW $7740[110 \mathrm{CM}]$ P-SYN

$\mathrm{MCA}$,

BOURINOT GP.DUGALD FM

[U ETCHEMINIAN DIV E3A]

DUGALD BK. ESCASONIE.CAPE BRETON,NS

H277; WALCOTT CD, 1912,P717,PL76, FIG 3:MATTHEW GF, 1902,NB,P389,PL.14,FIG 4A-E

REMARKS: C-GF MATTHEW:D-MACKENZIE :LABELLED COTYPES IN OLD RECORDS:STRAT REV AS IN HUTCHINSON, 1952, GSC MEM 263:STUDIED 8Y AJ ROWELL, $1972 ; 1$ SLAB

ACROTHYRA PROAVIA MUT PRIMA MATTHEW 7777 [147CM ] P-SYN?

MCA

BOURINOT GP,DUGALD FM

[UETCHEMINIAN]

DUGALD 8K, ESCASONIE CAPE BRETON NS

H277:MATTHEW GF, 1902,P389,PL14,FIG 4A-E;WALCOTT CD, 1912,P717,PL76,FIG 3

REMARKS: C-GF MATTHEW:D-MACKENZIE :LABELLED TOPOTYPES IN OLD RECORDS:STUDIED 1972 BY AJ ROWELL-MAY BE PART OF ORIG SYNTYPIC SUITE; STRAT REV AS IN HUTCHINSON, 1952, GSC MEM 263 ; 5 SLABS

ACROTHYRA SERA MATTHEW

7711 [81CM] P-SYN?

ACROTHYRA (SIGNATA) SERA MATTHEW

$M C A$

BOURINOT GP.DUGALDFM

[LETCHEMINIAN]

DUGALD BK, ESCASONIE, CAPE BRETON,NS

H277; MATTHEW GF, 1902,P383-384,PL 13,FIG 3A-F

WALCOTT CD 1912,P718-719,PL80,FIG 48-C

REMARKS: C-GF MATTHEW;D-MACKENZIE :LABELLED TOPOTYPE IN OLD RECORDS;STUDIED 1972 BY AJ ROWELL-MAY BE PART OF ORIG SYNTYPIC SUITE: STRAT REV AS IN HUTCHINSON, 1952, GSC MEM 263; 6 SPEC

\section{ACROTHYRA SERA MATTHEW}

$7716[86 \mathrm{CM}]$ P-SYN?

ACROTHYRA (SIGNATA) SERA MATTHEW

$M C A$,

BOURINOT GP,DUGALD FM

[LETCHEMINIAN]

DUGALD BK, ESCASONIE,CAPE BRETON,NS

H277 MATTHEW GF 1902,P383-384.PL 13,FIG 3A-F:

WALCOTT CD, 1912,P7 18-719,PL80,FIG 4B-C

REMARKS: C-GF MATTHEW;D-MACKENZIE;LABELLED TOPOTYPE IN OLD RECORDS:STUDIED 1972 BY AJ ROWELL-MAY BE PART OF ORIG SYNTYPIC SUITE: STRAT REV AS IN HUTCHINSON, 1952, GSC MEM 263 ; 3 SPEC

ACROTHYRA SERA MATTHEW

7891 [261CM] P-SYN?

ACROTHYRA (SIGNATA) SERA MATTHEW

MCA

BOURINOT GP.DUGALD FM

[LETCHEMINIAN ]

DUGALDBK, ESCASONIE, CAPE BRETON,NS

H277: MATTHEW GF 1902.P383-384,PL 13,FIG 3A-F WALCOTT CD. 1912,P7 18-7 19.PL80,FIG 4 B-C

REMARKS: C-GF MATTHEW:D-MACKENZIE :LABELLED TOPOTYPES IN OLD RECORDS:STUDIED 1972 BY AJ ROWELL-MAY BE PART OF ORIG SYNTYPIC SUITE;REV AS IN HUTCHINSON, 1952.GSC MEM $263 ; 6$ SPEC

ACROTHYRA SERA MATTHEW

7699 [69CM] P-SYN?

ACROTHYRA (SIGNATA) SERA MATTHEW

$M C A$,

BOURINOT GP.DUGALD FM

[LETCHEMINIAN ]

DUGALD BK, ESCASONIE,CAPE BRETON.NS

H277:MATTHEW GF, 1902,P383-384.PL 13.FIG 3A-F;

WALCOTT CD, 19 12,P7 18-719,PL80,FIG 4B-C

REMARKS: C-GF MATTHEW:D-MACKENZIE :LABELLED

TOPOTYPE IN OLD RECORDS:STUDIED 1972 BY AJ 
ROWELL-MAY BE PART OF ORIG SYNTYPIC SUITE: STRAT REV AS IN HUTCHINSON, 1952, GSC MEM 263; 1 SPEC

\section{ACROTHYRA SERA MATTHEW}

7721[91CM]P-SYN

ACROTHYRA (SIGNATA) SERA MATTHEW

$M C A$

BOURINOT GP,DUGALDFM

[LETCHEMINIAN,OIV E1C]

DUGALD BK, ESCASONIE, CAPE BRETON,NS

H277; WALCOTT CD, 1912,P7 18-719,PL80, FIG 4B-C;

MATTHEW GF, 1902,P383-384,PL 13,FIG 3A-F

REMARKS: C-GF MATTHEW:D-MACKENZIE :STRAT REV AS IN HUTCHINSON, 1952, GSC MEM 263;STUDIED BY AJ ROWELL, $1972 ; 1$ SLAB

\section{ACROTHYRA SERA MATTHEW}

8637 [1007CM ]P-SYN?

ACROTHYRA (SIGNATA) SERA MATTHEW

$M C A$.

BOURINOT GP DUGALD FM

[L ETCHEMINIAN DIV E1C

DUGALD BK, ESCASONIE,CAPE BRETON,NS

H277:MATTHEW GF, 1902,P383-384,PL 13,FIG 3A-F WALCOTT CD 1912,P718-719,PL80, FIG 4B-C

REMARKS: C-GF MATTHEW ; NOT LABELLED TYPES IN OLD RECORDS;STUDIED 1972 BY AJ ROWELL-MAY BE PART OF ORIG SYNTYPIC SUITE.STRAT REV AS IN HUTCHINSON, 1952,GSC MEM 263; 3 SPEC

\section{ACROTHYRA SIGNATA MATTHEW}

$7710(B)[80 \mathrm{CM}(\mathrm{B})]$ P-PARAL

$M C A$

BOURINOT GP DUGALDFM

[LETCHEMINIAN,DIVE1B]

DUGALD BK, ESCASONIE, CAPE BRETON,NS

H277:WALCOTT CD 1912.P719 PL80,FIG 1D:MATTHEW GF, 1902,NB,P381-382,PL13,FIG 2A-E

REMARKS: C-GF MATTHEW;D-MACKENZIE ;6 VENTRAL, 3

DORSAL VALVES;SPEC 7710 (A) DESIGNATED

LECTOTYPE BY WALCOTT:STRAT REV AS IN

HUTCHINSON, 1972,GSC MEM 263;STUDIED 1972 BY AJ ROWELL : 4 SMALL SLABS

\section{ACROTHYRA SIGNATA MATTHEW}

$7710(A)[80 \mathrm{CM}(A)]$ P-LECTO

$M C A$.

BOURINOT GP,DUGALDFM

[LETCHEMINIAN DIV E1B]

DUGALD BK, ESCASONIE, CAPE BRETON,NS

H277; WALCOTT CD 19 12,P7 19,PL80,FIG 1; MATTHEW GF, 1902,P381-382,PL13,FIG 2B

REMARKS: C-GF MATTHEW:D-MACKENZIE :SPEC ' $C$ ' OF MATTHEW'S SYNTYPES;DESIGNATED LECTOTYPE BY WALCOTT:STRAT REV AS IN HUTCHINSON, 1952, GSC MEM 263;STUDIED 1972 BY AJ ROWELL; 1 SPEC

ACROTHYRA SIGNATA ORTA MATTHEW

7694(B) [64CM(B)] P-PARAL

$M C A$,

BOURINOT GP DUGALD FM

[L ETCHEMINIAN DIV E2C

DUGALD BK, ESCASONIE,CAPE BRETON,NS

H277:WALCOTT CD, 1912,P7 19-720,PL80,FIG 3B;MATTHEW GF 1902.P385-386. PL 13,FIG 4A-F

REMARKS: C-GFMATTHEW;D-MACKENZIE ;SPEC 7694(A) DESIGNATED LECTOTYPE BY WALCOTT: STRAT REV AS IN HUTCHINSON, 1952,GSC MEM 263:STUDIED 1972 BY AJ ROWELL ; 2 SPEC

ACROTHYRA SIGNATA ORTA MATTHEW

7694(A) [64CM(A)]P-LECTO

$M C A$

BOURINOT GP DUGALDFM

[LETCHEMINIAN,DIVE2C]

DUGALD BK, ESCASONIE, CAPE BRETON,NS

H277:MATTHEW GF, 1902,P385-386,PL13,FIG 4A-F WALCOTT CD, 1912,P7 19-720,PL80,FIG 3A

REMARKS: C-GF MATTHEW;D-MACKENZIE ;SPEC 'C' OF MATTHEW'S SYNTYPES:DESIGNATED LECTOTYPE BY WALCOTT; STRAT REV AS IN HUTCHINSON, 1952, GSC MEM 263;STUDIED 1972 BY AJ ROWELL; 1 SPEC
ACROTHYRA SIGNATA PRIMA MATTHEW

7734 [104CM] P.PARAL

$M C A$

BOURINOT GP,ESKASONI FM

[COLDBROOK TERRANE,FINE GREY SHALES]

DUGALD BK, ESCASONIE, CAPE BRETON,NS

H277; WALCOTT CD, 1912,P720,PL80,FIG 2,2B;MATTHEW GF, 1902,P382-383,PL 13,FIG 1A-G

REMARKS: C-GF MATTHEW, 1900 :LECTOTYPE

DESIGNATED BY WALCOTT,SEE NO 8636(A);STUDIED

1972 BY AJ ROWELL;STRAT REV AS IN HUTCHINSON,

1952, GSC MEM $263 ; 4$ SPECS

\section{ACROTHYRA SIGNATA PRIMA MATTHEW}

8636(A) [1006CM-A] P-LECTO

$M C A$

BOURINOT GP,ESKASONI FM

[COLDBROOK GP]

DUGALD BK, ESCASONIE,CAPE BRETON, NS

H277;MATTHEW GF, 1902,P382-383,PL 13,FIG 1A-G;

WALCOTT CD, 1912,P720,PL80,FIG 2

REMARKS: C-GF MATTHEW, 1900 ;LABELLED TOPOTYPE IN

OLD RECORDS;STUDIED 1972 BY AJ-ROWELL-MAY BE

THE SPEC FIG'D + DESIGNATED LECTOTYPE BY

WALCOTT;STRAT REV AS IN HUTCHINSON, 1952,GSC MEM 263: 1 SPEC

\section{ACROTHYRA SIGNATA PRIMA MATTHEW}

8636(B) [ 1006CM-B] P-PARAL

$M C A$

BOURINOT GP,ESKASONI FM

[COLDBROOK GP ]

DUGALD BK, ESCASONIE,CAPE BRETON,NS

H277:MATTHEW GF, 1902,P382-383,PL13,FIG 1A-G:

WALCOTT CD, 1912,P720,PL80,FIG 2,2B

REMARK S: C-GF MATTHEW, 1900 ;LABELLED TOPOTYPE IN OLD RECORDS;STUDIED 1972 BY AJ ROWELL-MAY BE PART OF ORIG SYNTYPIC SUITE;LECTOTYPE DESIGNATED BY WALCOTT,SEE NO 8636(A):STRAT REV AS IN HUTCHINSON, 1952, GSC MEM 263; 1 SPEC

ACROTHYRA SIGNATA PRIMA MATTHEW

7730 [100CM ] P-PARAL

$\mathrm{MCA}$,

BOURINOT GP.ESKASONI FM

[COLDBROOK GP]

DUGALD BK, ESCASONIE, CAPE BRETON,NS

H277:MATTHEW GF, 1902,P382-383,PL 13,FIG 1A-G;

WALCOTT CD, 19 12,P720,PL80,FIG 2B

REMARKS: C-GF MATTHEW.D-MACKENZIE :LABELLED

TOPOTYPES IN OLD RECORDS:STUDIED 1972 BY AJ ROWELL-MAY BE PART OF ORIG SYNYTPIC SUITE: LECTOTYPE DESIGNATED BY WALCOTT, SEE NO 8636(A): STRAT REV AS IN HUTCHINSON, 1952, GSC MEM 263; 13 ROCK FRAGS

\section{ACROTHYRA SIGNATA TARDA MATTHEW}

$7691[61 \mathrm{CM}]$ P-SYN

$M C A$,

BOURINOT GP DUGALD FM

[L ETCHEMINIAN, RED \& GREY SHALES,E 1C-D]

DUGALD BK, ESCASONIE, CAPE BRETON,NS

H277:WALCOTT CD, 1912,P718:MATTHEW

GF, 1902, NB,P384-385,PL14,FIG 1A

REMARKS: C-GF MATTHEW:D-MACKENZIE ;STRAT REV AS IN HUTCHINSON, 1952,GSC MEM 263:STUDIED BY AJ ROWELL, $1972 ; 3$ SPEC

\section{ACROTRETA BAILEYIMATTHEW}

$7831(\mathrm{~A})[201 \mathrm{CM}(\mathrm{A})]$ P-LECTO

$M C A$

ST JOHN GP

[ST JOHN,ACADIAN,DIV C1D?]

LONG REACH,KINGS CO,NB

H277; MATTHEW GF, 1886,P36-37,PL5,FIG 13, 13A,C,D;

WALCOTT CD 1912 P676-677,PL77,FIG 2,2, 2"

REMARKS: C-GF MATTHEW:D-MACKENZIE ; SPEC ' $K$ ' OF MATTHEW'S SYNTYPES;THIS SPEC PROBABLY THE ONE DESIGNATED LECTOTYPE BY WALCOTT, ACCORDING TO AJ ROWELL, $1972 ; 1$ SPEC 
ACROTRETA BAILEYIMATTHEW

$7831(B)[201 \mathrm{CM}(\mathrm{B})]$ P-PARAL

$M C A$.

ST JOHN GP

[ST JOHN, ACADIAN,DIV 1D?]

LONG REACH, KINGS CO,NB

H276; WALCOTT CD, 1912,P676-677,PL77,FIG 2A-D;

MATTHEW GF, 1886,P36-37,PL5,FIG 13, 13A,C,D

REMARKS: C-GF MATTHEW;D-MACKENZIE;CARD GIVES

LOC AS BELYEA'S LNDG + LONG REACH;ONLY LONG

REACH MENTIONED IN PUBL' N; LECTOTYPE

DESIGNATED BY WALCOTT,SEE NO $7831($ A); SPEC

' $J$ ' = WALCOTT'S FIG $2 A$; 8 SPEC

ACROTRETA GEMMULA MATTHEW

$7756[126 \mathrm{CM}]$ P-PARAL

LCA.

ST JOHN GP

[ST JOHN,ACADIAN,DIV 183 ]

HANFORD BK, ST MARTINS.ST JOHN CO,NB

H276;WALCOTT CD. 19 12,P686,PL66,FIG 3,3A-B;MATTHEW GF, 1894.P87-88,PL 16,FIG 2A-D

REMARKS: C-GF MATTHEW;D-MACKENZIE;LECTOTYPE DESIGNATED BY WALCOTT:STUDIED 1972 BY AJ ROWELL-BELIEVES SPEC WALCOTT FIG'D IS MISSING ; 5 SPEC

ACROTRETA INFLATA (MATTHEW)

$7760(B)[130 C M(B)]$ P-PARAL

LINGULELLA? INFLATA MATTHEW

LCA,

ST JOHN GP

[ST JOHN,ACADIAN,C1B1]

HANFORD BK, ST MARTINS,ST JOHN CO,NB

H276:WALCOTT CD, 1912,P691-692,PL76,FIG 1D, 1F-G;

MATTHEW GF, 1886,P33,PL5,FIG 7,7A

REMARKS: C-GF MATTHEW;D-MACKENZIE :LECTOTYPE DESIGNATED BY WALCOTT,SEE NO $7760($ A):STUDIED 1972 BY AJ ROWELL ; 3 SPEC

ACROTRETA INFLATA (MATTHEW)

7760 (A) [130CM(A)] P-LECTO

LINGULELLA? INFLATA MATTHEW

LCA,

ST JOHN GP

[ST JOHN,ACADIAN,C1B1]

HANFORD BK, ST MARTINS,ST JOHN CO,NB

H276; MATTHEW GF, 1886,P33,PL5,FIG 7,7A; WALCOTT

CD, 1912,P691-692,PL76,FIG 2

REMARKS: C.GF MATTHEW:D-MACKENZIE :SPEC ' $F$ ' OF MATTHEW'S SYNTYPES;DESIGNATED LECTOTYPE BY WALCOTT:STUDIED 1972 BY AJ ROWELL; 1 SPEC

ACROTRETA INFLATA VAR OVALIS (MATTHEW)

8536 [906CM] P-SYN

LINGULELLA? INFLATA VAR OVALIS MATTHEW

LCA.

ST JOHN GP

[ST JOHN,ACADIAN,DIV 1B1]

HANFORD BK, ST MARTINS,ST JOHN CO,NB

H276; WALCOTT CD, 1912,P691,PL76,FIG 2A;MATTHEW

GF, 1895,NY,P127,PL5,FIG 4A-C

REMARKS: C-GF MATTHEW:D-MACKENZIE;STUDIED 1972 BY AJ ROWELL;SPEC 'A' FIG'D ; 4 SPEC

ACROTRETA MISERA (BILLINGS)

7833 [203CM ] REF SPEC-F

LINNARSSONIA MISERA BILLINGS

$M C A$.

ST JOHN GP

[ST JOHN,ACADIAN,DIV 1D]

PORTER'S BK, ST MARTINS.ST JOHN CO,NB

H276; WALCOTT CD,USGS MON 51.1912,P695:MATTHEW

GF, 1886,P35-36,PL5,FIG 12,12A-E

REMARKS: C.GF MATTHEW:D-MACKENZIE;SPEC

' $A$ " = FIGS 12C-E;SPEC ' $B$ ' = FIGS 12, 12A-B; 2 SPEC
ACROTRETA PAPILLATA MATTHEW

7693 [63CM ] P.SYN

$\mathrm{MCA}$,

BOURINOT GP.DUGALD FM

[LETCHEMINIAN,DIVE1D]

DUGALD BK, ESCASONIE,CAPE BRETON,NS

H276; WALCOTT CD, 1912, P7 18,PL80,FIG 6, 6", $6^{\prime \prime}$. MATTHEW GF, 1902,NB,P390-39 1,PL5,FIG 2A-F

REMARKS: C-GF MATTHEW:D-MACKENZIE :SPEC 'C' = FIG 2F; SPEC ' ${ }^{\prime}$ ' = FIG 2D;SPEC ' $E$ ' = FIG 2C; WALCOTT'S FIGS = SAME SPEC AS FIGS 2A-B;STRAT REV AS IN HUTCHINSON, 1952,GSC MEM 263 ; 5 SPEC

ACROTRETA PAPILLATA VAR LATA MATTHEW

7903 [273CM ] P-SYN

ACROTRETA PAPILLATA VAR

$M C A$,

BOURINOT GP,DUGALD FM

[L ETCHEMINIAN,DIV E1D]

DUGALD BK, ESCASONIE, CAPE BRETON,NS

H276; WALCOTT CD, 19 12,P7 18:MATTHEW

GF, 1902,P391,PL 15, FIG 3A-C

REMARKS: C-GF MATTHEW;D-MACKENZIE ;VARIETAL NAME MENTIONED ONLY IN PLATE CAPTION;

UNCERTAIN WHETHER THESE SPECS ARE TYPES;LOC

GIVEN AS DUGALD BK IN ROM RECORDS,BOUNDARY BK IN PUBL'N : 1 SLAB

ACROTRETA PAPILLATA MUT PRIMA MATTHEW

7709 [79CM ] P-HOLO

$\mathrm{MCA}$,

BOURINOT GP.ESKASONIE FM

[COLDBROOK]

DUGALD BK, ESCASONIE,CAPE BRETON,NS

H276;WALCOTT CD, 1912,P7 18;MATTHEW GF, 1902,P391392,PL 15,FIG 1A-C

REMARKS: C-GF MATTHEW;D-MACKENZIE ;LABELLED COTYPES; VAR BASED ON A SINGLE SPEC WHICH MUST BE THE HOLOTYPE-MAY BE SPEC 'E' OR 'F' BUT NOT CERT AIN:STUIDIED 1972 BY AJ ROWELL ; 6 SPEC

ACROTRETA SAGITTALIS TRANSVERSA (HARTT)

8337 [707CM] REF SPEC-F

LINNARSSONIA TRANSVERSA HARTT

$M C A$,

ST JOHN GP

[ST JOHN,DIV 1C]

PORTER'S BK, ST MARTINS,ST JOHNCO,NB

H276; WALCOTT CD, 1912,P708;MATTHEW GF.TRANS NY ACAD SCI, 1895,P125-126,PL5,FIG 1A-C

REMARKS: C-GF MATTHEW:D-MACKENZIE :NOTE DISCREPANCY IN LOC BETWEEN RECORDS (PORTER'S BK) AND PUBL'N (HANFORD BK); 2 SPEC

ACROTRETA SIPO MATTHEW

7956 [326CM ] P-SYN

O.TREMADOC

MCLEODBROOKFM

[ST JOHN,BRETONIAN,C3C2 ]

MCLEODBK,BOISDALE,NS

H276; WALCOTT CD, 1912,P679;MATTHEW

GF, 1902,NB,P406-407,PL 18,FIG 1,2

REMARKS: C.GF MATTHEW:D-MACKENZIE :LABELLED COTYPES; UNCERTAIN WHICH SPECS FIG'D;GSC ALSO CLAIMS TO HAVE SYNTYPES OF THIS SPECIES ; 4 SPEC

ACROTRETA? GULIELMI MATTHEW

REMARKS: SEE DISCINOPSIS GULIELMI (MATTHEW)

AMBOCOELIA ARCUATA GIRTY

6363 [2974CB ] P-PARA?

$P$

PARK CITYFM.PHOSPHATE BEDS

THOMASFORK,WYO,USA

H672;GIRTY GH,USGS BULL 436, 1910, P35

REMARKS: D-WF FERRIER, 1920 :LABELLED PARATYPE, SPEC NOT FIG'D \& NOT MENTIONED IN TEXT ; 1 SPEC 
ASPERLINUS ASPERULUS (WAAGEN)

32127 [B349] REF SPEC.F

UP, KAZAN

50 M FROM ROAD LEADING W FROM HWY, KHAO

PHRIK,NR RATBURI,THAILAND

H-..-WWATERHOUSE JB + PIYASIN

S, 1970, PALAEONTOGRAPHICA,BD 135,ABT A,P 132 133, PL23,FIG 4

REMARKS: C D-JB WATERHOUSE, 1964 .JBW NO B349.NEW GENUS:ORDER PRODUCTIDA ACCORDING TO

WATERHOUSE + PIYASIN; 1 SPEC (2 PCES)

ASPERLINUS ASPERULUS (WAAGEN)

32130 [B353] REF SPEC-M

UP.KAZAN

50 M FROM ROAD LEADING W FROM HWY, KHAO

PHRIK,NR RATBURI,THAILAND

H---:WATERHOUSE JB + PIYASIN

S, 1970,PALAEONTOGRAPHICA,BD 135,ABT A,P132-133

REMARKS: C,D-JB WATERHOUSE, 1964 ;JBW NO B353;NEW GENUS:ORDER PRODUCTIDA ACCORDING TO

WATERHOUSE + PIYASIN : 1 SPEC

ASPERLINUS ASPERULUS (WAAGEN)

32131 [B354] REF SPEC-F

UP, KAZAN

50 M FROM ROAD LEADING W FROM HWY, KHAO

PHRIK,NR RATBURI,THAILAND

H---;WATERHOUSE JB + PIYASIN

S, 1970,PALAEONTOGRAPHICA,BD 135,ABT A,P132133.PL23, FIG 2,3

REMARKS: C,D-JB WATERHOUSE, 1964 ;JBW NO B354;NEW GENUS:ORDER PRODUCTIDA ACCORDING TO

WATERHOUSE + PIYASIN ; 1 SPEC (2 PCES)

ASPERLINUS ASPERULUS (WAAGEN)

32124 [B346] REF SPEC-M

UP.KAZAN

50 M FROM ROAD LEADING W FROM HWY, KHAO

PHRIK, NR RATBURI, THAILAND

H---;WATERHOUSE JB + PIYASIN

S, 1970,PALAEONTOGRAPHICA,BD 135,ABT A.P 132-133

REMARKS: C.D-JB WATERHOUSE, 1964 .JBW NO B346: NEW

GENUS;ORDERPRODUCTIDA; 1 SPEC + 1 FRAG

ATHYRIS SPIRIFEROIDES EATON

12958 [2348D ] REF SPEC-F

$M D$,

KWATABOAHEGAN R,ONT

$\ldots$...;PARKS WA, ONT DEPT MINES, VOL 13,PT 1, 1904,PL8,FIG 2

AEMARKS: C-WA PARKS, 1903 :STORED UNDER

ORTHOCERAS LUXUM; 1 SLAB

ATRYPA RETICULARIS (LINNAEUS)

22451 [202S ] REF SPEC-F

MD,ERIAN

DETROIT RIVER GP,AMHERSTBURG FM

[UMONROE

LIVINGSTONE CUT, DETROIT R, AMHERSTBURG, ONT

H639; GRABAU AW, MICH GEOL\&BIOL SURV,PUBL 2, GEOL

SER 1,1909,P 162,PL20,FIG 1

REMARKS: D-REV. T NATTRESS, $1910 ; 1$ SPEC + 1 CAST

\section{ATRYPA SP INDET}

22653 [408S ] REF SPEC-M

$\mathrm{S}$

[SILURIAN ]

LIMESTONE RAPIDS, FAWN R,DIST OF PATRICIA.ONT

H639; PARKS WA, TRANS ROY CAN INST, VOL

$11, N 1,1915, P 48$

REMARKS: C-JB TYRRELL, SEPT 9,1912+;D-WH HEARST, $1915 ; 2$ SPEC

BARRANDELLA? MESOPLICATA PARKS

22635 [399S ] P-SYN

MS

ASSINA RAPIDS SEVERN R.DIST OF PATRICIA,ONT

H551:PARKS WA. TRANS ROY CAN INST, VOL

11.PT 1, 1915,P52,PL3,FIG 8- 10

REMARK S: C-JB TYRRELL, 1912 :D-WH HEARST, 1915:SPEC 'A' FIG'D : 2 SPEC
BILLINGSELLARETROFLEXA (VERNEUIL)

$7712(\mathrm{~A})[82 \mathrm{CM}(\mathrm{A})]$ REF SPEC-F

$\mathrm{MCA}$,

MACMULLIN FM

[L ETCHEMINIAN ]

YOUNG PT , GEORGE R,CAPE BRETON,NS

H306; WALCOTT CD,USGS MON 51,1912,P761,PL90,FIG 1E MATTHEW GF, 1903.P 148-151,PL 10,FIG 2B

REMARKS: C-GF MATTHEW;D-MACKENZIE ;STUDIED AND STRAT REV 1972 BY AJ ROWELL;CONSIDERED PARALECTOTYPE OF BILLINGSELLA RETROFLEXA (MATTHEW) BY WALCOTT;SPECS 'A', 'C'= FIGS $1 \mathrm{C}, 1 \mathrm{D}$ OF WALCOTT:LOC $307 D$ OF WALCOTT ; 2 SPEC

BILLINGSELLA RETROFLEXA (VERNEUIL)

7902 [272CM] REF SPEC.F

CLITAMBONITES PLANA VAR RETROFLEXA

(MATTHEW, 1895)

MCA

MACMULLIN FM

[LETCHEMINIAN]

YOUNG PT, GEORGE R CAPE BRETON NS

H306; WALCOTT CD,USGS MON 51,P761; MATTHEW GF, 1903,P 148-151,PL10,FIG 2A;MATTHEW

GF 1896 P267 FIG 1 A-C

REMARKS: C-GF MATTHEW;D-MACKENZIE ;STUDIED AND STRAT REV 1972 BY AJ ROWELL;CONSIDERED PARALECTOTYPE OF BILLINGSELLA RETROFLEXA (MATTHEW) BY WALCOTT;LOC 307D OF WALCOTT; SPEC

\section{BILLINGSELLA RETROFLEXA (VERNEUIL)}

7715 [85CM] REF SPEC-F

$M C A$.

MACMULLIN FM

[L ETCHEMINIAN,DIV E2A ]

YOUNG PT, GEORGE R,CAPE BRETON,NS

H306; WALCOTT CD,USGS MON 51,P761,PL90,FIG 1D; MATTHEW GF 1903,P 148-151

REMARKS: C-GF MATTHEW:D-MACKENZIE ;STUDIED AND STRAT REV 1972 BY AJ ROWELL;CONSIDERED PARALECTOTYPE OF BILLINGSELLA RETROFLEXA (MATTHEW) BY WALCOTT; SPEC 'B' FIG'D BY WALCOTT; LOC $307 D$ OF WALCOTT ; 2 SPEC

BILLINGSELLA RETROFLEXA (VERNEUIL)

7724 [94CM ] REF SPEC-F

CLITAMBONITES PLANA VARRETROFLEXA

(MATTHEW, 1895)

MCA,

MACMULLINFM

[L ETCHEMINIAN

YOUNG PT, GEORGE R, CAPE BRETON NS

H306; WALCOTT CD,USGS MON 51,1912,P761,PL90,FIG 1F; MATTHEW GF, 1903,P148-151,PL 10,FIG 2C

MATTHEW 1895,P267,PL2,FIG 1B

REMARKS: C-GF MATTHEW;D-MACKENZIE ;STUDIED AND STRAT REV 1972 BY AJ ROWELL;CONSIDERED

PARALECTOTYPE OF BILLINGSELLA RETROFLEXA (MATTHEW) BY WALCOTT;LOC 307D OF WALCOTT ; 1 SPEC

BILLINGSELLA RETROFLEXA (VERNEUIL) 8206 [576CM] REF SPEC-F

$\mathrm{MCA}$.

DIV E2A

[L ETCHEMINIAN,DIV E2A]

YOUNG PT, GEORGE R,CAPE BRETON,NS H306; MATTHEW GF,GSC, 1903,P 148-151; WALCOTT CD USGS MON 51,1912,P761.PL90,FIG 1A

REMARKS: C-GF MATTHEW;D-MACKENZIE;STUDIED AND STRAT REV 1972 BY AJ ROWELL;CONSIDERED

PARALECTOTYPE OF BILLINGSELLA RETROFLEXA

(MATTHEW) BY WALCOTT;SPEC 'A ' FIG'D BY WALCOTT; LOC $307 D$ OF WALCOTT ; 2 SPEC

BILLINGSELLARETROFLEXA (VERNEUIL)

$7700[70 \mathrm{CM}]$ REF SPEC-F

$\mathrm{MCA}$,

MACMULLINFM

[L ETCHEMINIAN ]

YOUNG PT, GEORGE R, CAPE BRETON,NS 
H306:WALCOTT CD,USGS MON 51, 1912,P761,PL90,FIG 1,1B;MATTHEW, 1903,P148-151

REMARKS: C-GF MATTHEW:D-MACKENZIE ;STUDIED AND STRAT REV 1972 BY AJ ROWELL:CONSIDERED PARALECTOTYPE OF BILLINGSELLA RETROFLEXA (MATTHEW) BY WALCOTT:SPECS ' $C^{\prime}, D^{\prime}=$ FIGS $1,1 \mathrm{~B}$ OF WALCOTT:LOC 307D OF WALCOTT ; 3 SPEC

BILLINGSELLA RETROFLEXA (VERNEUIL)

$7712(B)[82 C M(B)]$ REF SPEC-F

$M C A$

MACMULLIN FM

[LETCHEMINIAN]

YOUNG PT GEORGE R CAPE BRETON.NS

H306:WALCOTT CD,USGS MON 51, 1912,P761,PL90,FIG 1H; MATTHEW GF, 1903,P 148-151.PL 10,FIG 2B

REMARKS: C-GF MATTHEW:D-MACKENZIE :STUDIED AND STRAT REV 1972 BY AJ ROWELL:CONSIDERED LECTOTYPE OF BILLINGSELLA RETROFLEXA (MATTHEW) BY WALCOTT:SPEC 'B' OF MATTHEW'S SERIES:LOC 307D OF WALCOTT : 1 SPEC

\section{BOTSFORDIA PULCHRA (MATTHEW)}

$7794[164$ CM ] P-SYN

OBOLUS PULCHER MATTHEW

LCA.

HANFORD BROOK FM

[ST JOHN,ACADIAN]

CATONS IS, KINGS CO.NB

H282; WALCOTTCD, 1912,P607-608;MATTHEW

GF, 1890,P151-155,PL8,FIG 1A-M,2A-C:

MATTHEW 1889,P306-307, FIG 1-8

REMARKS: C-GF MATTHEW;D-MACKENZIE ;LABELLED COTYPES:UNCERTAIN WHICH SPECS FIG'D.STUDIED 1972 BY AJ ROWELL : 9 SPEC

\section{CAMERELLA PARVA BILLINGS}

$8058[428 \mathrm{CM}]$ REF SPEC-F

$M C A$.

ST JOHN GP

[ST JOHN,BRETONIAN,DIV 3A]

NAVY IS,ST JOHN,NB

H535:MATTHEW GF, TRANS ROY SOC CAN, VOL

10,1893,P103-104,PL7,FIG 9A-B

REMARKS: C-GF MATTHEW;D-MACKENZIE ; 1 SPEC

\section{CATAZYGA FILISTRIATA SPROULE}

39A P-LECTO

CATAZYGA HEADI FILISTRIATA SPROULE

MO.CHAMP.MOH,TRENT

TRENTON GP.COBOURG FM

CRAIGLEITH ONT

H634:SPROULE JC, 1936, GSC MEM 202,P 108 , PL 7,FIG 4-7: COPPER P, 1977, PALAEONT.VOL 20,PT2,PL39, FIG 1, 4

REMARKS: C-JC SPROULE, 1932 :SPEC A OF SPROULE'S SYNTYPES:DESIGNATED LECTOTYPE BY COPPER ; 1 SPEC

\section{CATAZYGA FILISTRIATA SPROULE}

39 P-PARAL

CATAZYGA HEADI FILISTRIATA SPROULE

MO.CHAMP.MOH.TRENT

TRENTON GP. COBOURG FM

CRAIGLEITH,ONT

H634:SPROULE JC, 1936, GSC MEM 202,P 108,PL7,FIG 4-7 COPPER P 1977, PALAEONT VOL 20,PT2,PL39,FIG 1,4

REMARKS: C-JC SPROULE, 1932 :SPECS B-F OF SPROULE'S SYNTYPES:LECTOTYPE DESIGNATED BY COPPER ; 5 SPEC

\section{CHONETES OSTIOLATUS GIRTY}

6374 [2988CB] P-PARA

$P$.

PARK CITY FM,PHOSPHATE BEDS

MONTPELIER IDAHO USA

H420:GIRTY GH.USGS BULL 436, 1910.P25-26

REMARKS: D-WF FERRIER, 1920 :LABELLED PARATYPES SPECS NOT FIG'D AND NOT MENTIONED IN PUBL' $N$ : 3 SPEC
CHONETES OSTIOLATUS VAR MINISCULUS GIRTY

6359 [2969CB] P-PARA

P.

PARK CITY FM.PHOSPHATE BEDS

SUBLETTE RANGE, WYO,USA

H306:GIRTY GH,USGS BULL 436, 1910.P27

REMARKS: D-WF FERRIER, 1920 :LABELLED PARATYPE SPEC NOT FIG'D AND NOT MENTIONED IN PUBL' $N$; 1 SPEC

CHONETES OSTIOLATUS VAR IMPRESSUS GIRTY

6358 [2968CB ] P-PARA

PARK CITY FM.PHOSPHATE BEDS

SUBLETTE RGE,WYO.USA

H420;GIRTY GH,USGS BULL 436, 1910,P26-27

REMARKS: D-WF FERRIER : LABELLED PARATYPE;SPEC NOT FIG'D AND NOT MENTIONED IN PUBL'N : 1 SPEC

CHONOSTEGES THAILANDICA WATERHOUSE + PIYASIN 31986 [B532] P-PARA

UP.KAZAN

50 M FROM ROAD LEADING W FROM HWY, KHAO

PHRIK, NR RATBURI,THAILAND

H457:WATERHOUSE JB + PIYASIN

S, 1970,PALAEONTOGRAPHICA,BD 135,ABT A,P118-119

REMARKS: C.D-JB WATERHOUSE, 1964 ; JBW NO B532

ORDER PRODUCTIDA ACCORDING TO WATERHOUSE + PIYASIN : 1 SPEC

CHONOSTEGES THAILANDICA WATERHOUSE + PIYASIN

31987 [B630] P-PARA

UP, KAZAN

50 M FROM ROAD LEADING W FROM HWY, KHAO

PHRIK, NR RATBURI, THAILAND

H457. WATERHOUSE JB + PIYASIN

S, 1970,PALAEONTOGRAPHICA,BD 135,ABT A,P118119,PL20,FIG 6.8

REMARK S: C.D-JB WATERHOUSE, 1964 :JBW NO B630 ORDER PRODUCTIDA ACCORDING TO WATERHOUSE + PIYASIN ; 5 FRAGS

CHONOSTEGES THAILANDICA WATERHOUSE + PIYASIN 31981 [B3 12] P-PARA

UP.KAZAN

$50 M$ FROM ROAD LEADING W FROM HWY, KHAO

PHRIK, NR RATBURI,THAILAND

H457: WATERHOUSE JB + PIYASIN

S, 1970,PALAEONTOGRAPHICA,BD 135,ABT A,P118119.PL20.FIG 3

REMARKS: C.D-JB WATERHOUSE, 1964 : JBW NO B312: ORDER PRODUCTIDA ACCORDING TO WATERHOUSE + PIYASIN : 1 SMALL SLAB

CHONOSTEGES THAILANDICA WATERHOUSE + PIYASIN

31982 [B311] P-PARA

UP.KAZAN

50 M FROM ROAD LEADING W FROM HWY, KHAO

PHRIK, NR RATBURI,THAILAND

H457: WATERHOUSE JB + PIYASIN

S, 1970,PALAEONTOGRAPHICA,BD 135,ABT A,P118119.PL20.FIG 4.7

REMARKS: C.D-JB WATERHOUSE, 1964 :JBW NO B311; ORDER PRODUCTIDA ACCORDING TO WATERHOUSE + PIYASIN : 1SPEC (2 PCES)

CHONOSTEGES THAILANDICA WATERHOUSE + PIYASIN

31983 [B313] P-HOLO

UP.KAZAN

50 M FROM ROAD LEADING W FROM HWY, KHAO

PHRIK. NR RATBURI,THAILAND

H457:WATERHOUSE JB + PIYASIN

S. 1970,PALAEONTOGRAPHICA,BD 135,ABT A.P118. 119.PL20.FIG 12

REMARKS: C.D-JB WATERHOUSE, 1964 : JBW NO B3 13 ORDER PRODUCTIDA ACCORDING TO WATERHOUSE + PIYASIN , ISPEC 
CHONOSTEGES THAILANDICA WATERHOUSE + PIYASIN 31984 [B315] REF SPEC-F

UP.KAZAN

50 M FROM ROAD LEADING W FROM HWY, KHAO

PHRIK.NR RATBURI,THAILAND

H457:WATERHOUSE JB + PIYASIN

S. 1970,PALAEONTOGRAPHICA,BD 135,ABT A,P118119,PL20,FIG 5

REMARKS: C.D-JB WATERHOUSE, 1964 : JBW NO B315: ORDER PRODUCTIDA ACCORDING TO WATERHOUSE + PIYASIN ; 1 SPEC

\section{CHONOSTEGES THAILANDICA WATERHOUSE + PIYASIN}

31985 [B531] P-PARA

UP.KAZAN

50 M FROM ROAD LEADING W FROM HWY, KHAO

PHRIK,NR RATBURI, THAILAND

H457:WATERHOUSE JB + PIYASIN

S, 1970,PALAEONTOGRAPHICA,BD 135,ABT A,P118-119 REMARKS: C,D-JB WATERHOUSE, 1964 ; JBW NO B531;

ORDER PRODUCTIDA ACCORDING TO WATERHOUSE + PIYASIN ; 1 SPEC

CLEIOTHYRIDINA PECTINIFERA (SOWERBY)

32041 [B644] REF SPEC-F

UP, KAZAN

$50 \mathrm{M}$ FROM ROAD LEADING W FROM HWY, KHAO

PHRIK,NR RATBURI,THAILAND

H662; WATERHOUSE JB + PIYASIN

S, 1970,PALAEONTOGRAPHICA,BD 135,ABT A,P142144,PL25,FIG 5,6

REMARKS: C,D-JB WATERHOUSE, 1964 ;JBW NO B644; 1 SPEC

CLEIOTHYRIDINA PECTINIFERA (SOWERBY)

32034 [B395 ] REF SPEC-M

UP,KAZAN

50 M FROM ROAD LEADING W FROM HWY, KHAO

PHRIK,NR RATBURI,THAILAND

H662; WATERHOUSE JB + PIYASIN

S, 1970,PALAEONTOGRAPHICA,BD 135, ABT A,P 142-144

REMARKS: C,D-JB WATERHOUSE, 1964 ;JBW NO B395; 1 SPEC

\section{CLEIOTHYRIDINA PECTINIFERA (SOWERBY}

32035 [B396 ] REF SPEC-F

UP, KAZAN

50 M FROM ROAD LEADING W FROM HWY, KHAO

PHRIK,NR RATBURI,THAILAND

H662; WATERHOUSE JB + PIYASIN

S, 1970,PALAEONTOGRAPHICA,BD 135,ABT A,P 142-

144,PL25, FIG 13,14

REMARKS: C,D-JB WATERHOUSE, 1964 ;JBW NO B396 ; 1 SPEC

\section{CLEIOTHYRIDINA PECTINIFERA (SOWERBY)}

32036 [B397 ] REF SPEC-F

UP,KAZAN

$50 M$ FROM ROAD LEADING W FROM HWY, KHAO

PHRIK,NR RATBURI, THAILAND

H662; WATERHOUSE JB + PIYASIN

S, 1970,PALAEONTOGRAPHICA,BD 135,ABT A,P 142 144.PL 25,FIG 4.8,12

REMARKS: C,D-JB WATERHOUSE, 1964 ;JBW NO B397 ; 1 SPEC

CLEIOTHYRIDINA PECTINIFERA (SOWERBY)

32037 [B398] REF SPEC-M

UP, KAZAN

50 M FROM ROAD LEADING W FROM HWY, KHAO

PHRIK,NR RATBURI, THAILAND

H662; WATERHOUSE JB + PIYASIN

S, 1970,PALAEONTOGRAPHICA,BD 135,ABT A,P 142-144 REMARKS: C,D-JB WATERHOUSE, 1964 :JBW NO B398: 4 FRAGS
CLEIOTHYRIDINA PECTINIFERA (SOWERBY)

32038 [B400] REF SPEC-F

UP.KAZAN

$50 \mathrm{M}$ FROM ROAD LEADING W FROM HWY, KHAO

PHRIK,NR RATBURI,THAILAND

H662; WATERHOUSE JB + PIYASIN

S, 1970,PALAEONTOGRAPHICA,BD 135,ABT A,P 142 144,PL25,FIG 9,10

REMARKS: C,D-JB WATERHOUSE, 1964 ;JBW NO B400; 1 SPEC

CLEIOTHYRIDINA PECTINIFERA (SOWERBY) 32039 [B401] REF SPEC-F

UP.KAZAN

$50 M$ FROM ROAD LEADING W FROM HWY, KHAO

PHRIK,NR RATBURI,THAILAND

H662; WATERHOUSE JB + PIYASIN

S, 1970,PALAEONTOGRAPHICA,BD 135,ABT A,P142-

144,PL25,FIG 7,11,15

REMARK S: C,D-JB WATERHOUSE, 1964 ;JBW NO B40 1; 1 SPEC ( 3 PCES)

\section{CONCHIDIUM CF DECUSSATUM (WHITEAVES)} 22638 [394S ] REF SPEC-M

$\mathrm{S}$

[SILURIAN,DRIFT ]

MOUTH OF NELSON R DIST OF PATRICIA ONT

H547:PARKS WA, TRANS ROY CAN INST, VOL 11, PT 1, 1915,P51

REMARKS: C-JB TYRRELL, 1912 ;D-WH HEARST, 1915 ; 1 SPEC

CONCHIDIUM CF DECUSSATUM (WHITEAVES) 22637 [392S ] REF SPEC-M

S.

[SILURIAN,DRIFT]

MOUTH OF NELSON R, DIST OF PATRICIA ONT

H547:PARKS WA TRANS ROY CAN INST VOL

11, PT 1,1915, P5 1

REMARKS: C-JB TYRRELL, 1912:D-WH HEARST, 1915 : 1 SPEC

\section{CONCHIDIUM CF DECUSSATUM (WHITEAVES)}

22636 [391S ] REF SPEC-M

[SILURIAN,DRIFT

MOUTH OF NELSON R, DIST OF PATRICIA,ONT

H547:PARKS WA TRANS ROY CAN INST, VOL

11, PT 1,1915, P5 1

REMARKS: C-JB TYRRELL, 1912;D-WH HEARST, 1915; SPEC

CONCHIDIUM CF DECUSSATUM (WHITEAVES)

22637 [393S ] REF SPEC-M

$S$,

[SILURIAN DRIFT]

MOUTH OF NELSON R, DIST OF PATRICIA, ONT

H547:PARKS WA, TRANS ROY CAN INST, VOL

11.PT1,1915.P51

REMARKS: C-JB TYRRELL, 19 12;D-WH HEARST, $1915 ; 1$ SPEC

\section{CRANIA SP}

12958 [2348D ] REF SPEC-F

$M D$

KWATABOAHEGAN R,ONT

---PARKS WA ONT DEPT MINES, VOL 13,PT1, 1904,PL8,FIG 2

REMARKS: C-WA PARKS, 1903 :STORED UNDER

ORTHOCERAS LUXUM;SPEC CIRCLED ON SLAB ; 1 SLAB

DALMANELLA RUGOSTRIATA PARKS\&DYER

17319 [1078HR] P-SYN

UO CINCIMAYSVILL

DUNDAS FM

DON VALLEY QUARRY,TORONTO,ONT

H333:PARKS \& DYER ONT DEPT MINES, VOL

30,PT7, 1922,P32-33,PL7,FIG 9, 10

REMARKS: C-H STEWART 'SPEC 'A' FIG'D ; 3 SPEC 
DELTHYRIS SP INDET

22642 [397S ] REF SPEC-F

ABOVE LIMESTONE RAPIDS, SEVERN R,DIST OF

PATRICIA.ONT

H680:PARKS WA TRANS ROY CAN INST VOL

11,PT1, 19 15,P53,PL3,FIG 13

REMARKS: C-J TYRRELL. 1912;D-1915 ;D-WH HEARST ; 1 SPEC

\section{DERBYIA? SP}

31754 [B569] REF SPEC-F

UP,KAZAN

50 M FROM ROAD LEADING W FROM HWY , KHAO

PHRIK,NR RATBURI,THAILAND

H411. WATERHOUSE JB + PIYASIN

S, 1970,PALAEONTOGRAPHICA.8D 135,ABT

A.P 105.PL14,FIG 19

REMARKS: C.D-JB WATERHOUSE, 1964 ;JBW NO B569; 1 SPEC

\section{DERBYIA ? SP}

31755 [8570] REF SPEC-F

UP, KAZAN

50 M FROM ROAD LEADING W FROM HWY, KHAO

PHRIK.NR RATBURI,THAILAND

H411:WATERHOUSE JB + PIYASIN

S. 1970,PALAEONTOGRAPHICA.BD 135,ABT

A.P105,PL14.FIG 20,21

REMARKS: C.D-JB WATERHOUSE, 1964 ;JBW NO B570 ; 1 SPEC + FRAGS

DIPLANUS MINUTA WATERHOUSE+PIYASIN

31699 [B125] P-PARA

DIPLANUS (MINIPLANUS) MINUTA W. +P

UP.KAZAN

$50 M$ FROM ROAD LEADING W FROM HWY, KHAO

PHRIK,NR RATBURI,THAILAND

H408:WATERHOUSE JB + PIYASIN

S, 1970,PALAEONTOGRAPHICA,BD 135,ABT A,P101-103

REMARKS: C D-JB WATERHOUSE, 1964 .JBW NO B 125

MINIPLANUS SUBGENUS OF DIPLANUS;NEW SUBGENUS 1 SPEC

DIPLANUS MINUTA WATERHOUSE+ PIYASIN

31700 [B 126] P-PARA

DIPLANUS (MINIPLANUS) MINUTA W. $+P$

UP.KAZAN

50 M FROM ROAD LEADING W FROM HWY, KHAO

PHRIK.NR RATBURI,THAILAND

H408.WATERHOUSE JB + PIYASIN

S, 1970,PALAEONTOGRAPHICA,BD 135,ABT A,P101-103

REMARKS: C,D-JB WATERHOUSE, 1964 ;JBW NO B 126 ;

MINIPLANUS SUBGENUS OF DIPLANUS;NEW SUBGENUS; 1 SPEC

DIPLANUS MINUTA WATERHOUSE+PIYASIN

31701 [B 127 ] P-PARA

DIPLANUS (MINIPLANUS) MINUTA W. +P

UP.KAZAN

50 M FROM ROAD LEADING W FROM HWY KHAO

PHRIK.NR RATBURI,THAILAND

H408; WATERHOUSE JB + PIYASIN

S. 1970 PALAEONTOGRAPHICA BD 135.ABT A P 101-103

REMARKS: C.D-JB WATERHOUSE, 1964 ;JBW NO B 127

MINIPLANUS SUBGENUS OF DIPLANUS;NEW SUBGENUS ;

1 SPEC

DIPLANUS MINUTA WATERHOUSE+PIYASIN

31702 [B128] P-PARA

DIPLANUS (MINIPLANUS) MINUTA W + $P$

UP.KAZAN

50 M FROM ROAD LEADING W FROM HWY, KHAO

PHRIK NR RATBURI,THAILAND

H408:WATERHOUSE JB + PIYASIN

S. 1970.PALAEONTOGRAPHICA.BD 135,ABT A,P 101-103

REMARKS: C.D-JB WATERHOUSE, 1964 ,JBW NO B 128

MINIPLANUS SUBGENUS OF DIPLANUS:NEW SUBGENUS

1 SPEC
DIPLANUS MINUTA WATERHOUSE + PIYASIN

31703 [B 129] P-PARA

DIPLANUS (MINIPLANUS) MINUTA W + $P$

UP.KAZAN

$50 M$ FROM ROAD LEADING W FROM HWY, KHAO

PHRIK,NR RATBURI THAILAND

H408:WATERHOUSE JB + PIYASIN

S, 1970.PALAEONTOGRAPHICA,BD 135,ABT A.P101103. PL 15. FIG 19,20

REMARKS: C,D-JB WATERHOUSE, 1964 ;JBW NO B 129

MINIPLANUS SUBGENUS OF DIPLANUS:NEW SUBGENUS. 1 SPEC

DIPLANUS MINUTA WATERHOUSE + PIYASIN

31704 [B 131] P-PARA

DIPLANUS (MINIPLANUS) MINUTA W. $+P$ UP.KAZAN

50 M FROM ROAD LEADING W FROM HWY, KHAO

PHRIK,NR RATBURI,THAILAND

H408:WATERHOUSE JB + PIYASIN

S. 1970 PALAEONTOGRAPHICA BD 135.ABT A P 101-103

REMARKS: C,D-JB WATERHOUSE, 1964 ; JBW NO B 131 ,

MINIPLANUS SUBGENUS OF DIPLANUS;NEW SUBGENUS 1 SPEC

DIPLANUS MINUTA WATERHOUSE+PIYASIN 31705 [B 132] P-PARA

DIPLANUS (MINIPLANUS) MINUTA W. $+P$.

UP.KAZAN

$5 O M$ FROM ROAD LEADING W FROM HWY, KHAO

PHRIK,NR RATBURI,THAILAND

H408:WATERHOUSE JB + PIYASIN

S, 1970,PALAEONTOGRAPHICA,BD 135,ABT A,P 101103, PL 15, FIG 25,26

REMARKS: C.D-JB WATERHOUSE, 1964 :JBW NO B132;

MINIPLANUS SUBGENUS OF DIPLANUS;NEW SUBGENUS ; 1 SPEC

DIPLANUS MINUTA WATERHOUSE + PIYASIN

31706 [B 133] P-PARA

DIPLANUS (MINIPLANUS) MINUTA W. $+P$ UP, KAZAN

50 M FROM ROAD LEADING W FROM HWY, KHAO

PHRIK, NR RATBURI, THAILAND

H408; WATERHOUSE JB + PIYASIN

S. 1970.PALAEONTOGRAPHICA BD 135 ABT A P 101-103

REMARKS: C,D-JB WATERHOUSE, 1964 ;JBW NO B 133;

MINIPLANUS SUBGENUS OF DIPLANUS;NEW SUBGENUS:

1 SPEC

DIPLANUS MINUTA WATERHOUSE + PIYASIN 31707 [B135] P-PARA

DIPLANUS (MINIPLANUS) MINUTA W. $+P$ UP, KAZAN

$50 M$ FROM ROAD LEADING W FROM HWY, KHAO

PHRIK,NR RATBURI,THAILAND

H408:WATERHOUSE JB + PIYASIN

S, 1970,PALAEONTOGRAPHICA,BD 135.ABT A,P 101 103,PL 15, FIG 17,18

REMARKS: C,D-JB WATERHOUSE, 1964 ; JBW NO B 135 MINIPLANUS SUBGENUS OF DIPLANUS;NEW SUBGENUS: 1 SPEC

DIPLANUS MINUTA WATERHOUSE + PIYASIN 31708 [B 137] P.PARA

DIPLANUS (MINIPLANUS) MINUTA $W .+P$ UP.KAZAN

50 M FROM ROAD LEADING W FROM HWY, KHAO

PHRIK,NR RATBURI, THAILAND H408; WATERHOUSE JB + PIYASIN

S. 1970.PALAEONTOGRAPHICA BD 135.ABT A P 101-103

REMARKS: C,D-JB WATERHOUSE, 1964 ; JBW NO B 137;

MINIPLANUS SUBGENUS OF DIPLANUS, NEW SUBGENUS 1 SPEC

DIPLANUS MINUTA WATERHOUSE+PIYASIN 31709 [B 139$]$ P-PARA DIPLANUS (MINIPLANUS) MINUTA $W+P$ UP.KAZAN

$50 \mathrm{M}$ FROM ROAD LEADING W FROM HWY, KHAO PHRIK, NR RATBURI,THAILAND 
H408; WATERHOUSE JB + PIYASIN

S. 1970,PALAEONTOGRAPHICA,BD 135,ABT A,P 101-103

REMARKS: C,D-JB WATERHOUSE, 1964 ;JBW NO B 139;

MINIPLANUS SUBGENUS OF DIPLANUS;NEW SUBGENUS ; 1 SPEC

DIPLANUS MINUTA WATERHOUSE+PIYASIN 31710 [B 140] P-PARA

DIPLANUS (MINIPLANUS) MINUTA W. + P.

UP,KAZAN

50 M FROM ROAD LEADING W FROM HWY, KHAO

PHRIK,NR RATBURI, THAILAND

H408; WATERHOUSE JB + PIYASIN

S, 1970,PALAEONTOGRAPHICA,BD 135,ABT A,P 101-103

REMARKS: C.D-JB WATERHOUSE, 1964 ;JBW NO B140;

MINIPLANUS SUBGENUS OF DIPLANUS;NEW SUBGENUS 2 SPEC

DIPLANUS MINUTA WATERHOUSE+PIYASIN

31711 [B525] P-PARA

DIPLANUS (MINIPLANUS) MINUTA W.+P.

UP,KAZAN

50 M FROM ROAD LEADING W FROM HWY, KHAO

PHRIK, NR RATBURI, THAILAND

H408; WATERHOUSE JB + PIYASIN

S, 1970,PALAEONTOGRAPHICA,BD 135,ABT A,P 101-103

REMARKS: C,D-JB WATERHOUSE, 1964 ;JBW NO B525;

MINIPLANUS SUBGENUS OF DIPLANUS;NEW SUBGENUS ; 1 SPEC

DIPLANUS MINUTA WATERHOUSE+PIYASIN

31712 [B526] P-PARA

DIPLANUS (MINIPLANUS) MINUTA W.+P

UP KAZAN

50 M FROM ROAD LEADING W FROM HWY, KHAO

PHRIK, NR RATBURI, THAILAND

H408; WATERHOUSE JB + PIYASIN

S, 1970,PALAEONTOGRAPHICA,BD 135,ABT A,P 101 103,PL 15, FIG 21,22

REMARKS: C,D-JB WATERHOUSE, 1964 ;JBW NO B526: MINIPLANUS SUBGENUS OF DIPLANUS;NEW SUBGENUS ; 1 SPEC

DIPLANUS MINUTA WATERHOUSE+PIYASIN

31713 [B527] P-PARA

DIPLANUS (MINIPLANUS) MINUTA W.+P.

UP,KAZAN

$50 \mathrm{M}$ FROM ROAD LEADING W FROM HWY, KHAO

PHRIK,NR RATBURI,THAILAND

H408; WATERHOUSE JB + PIYASIN

S, 1970,PALAEONTOGRAPHICA,BD 135,ABT A,P 101 103.PL 15, FIG 13,14

REMARKS: C,D-JB WATERHOUSE, 1964 ;JBW NO B527

MINIPLANUS SUBGENUS OF DIPLANUS;NEW SUBGENUS 1 SPEC

DIPLANUS MINUTA WATERHOUSE+PIYASIN

31714 [B528] P-PARA

DIPLANUS (MINIPLANUS) MINUTA W.+P

UP, KAZAN

50 M FROM ROAD LEADING W FROM HWY , KHAO

PHRIK,NR RATBURI, THAILAND

H408; WATERHOUSE JB + PIYASIN

S, 1970,PALAEONTOGRAPHICA,BD 135,ABT A,P 101-103

REMARKS: C.D-JB WATERHOUSE, 1964 ; JBW NO B528:

MINIPLANUS SUBGENUS OF DIPLANUS;NEW SUBGENUS ; 1 SPEC

DIPLANUS MINUTA WATERHOUSE+ PIYASIN 31715 [B561] P-PARA

DIPLANUS (MINIPLANUS) MINUTA W.+P

UP.KAZAN

50 M FROM ROAD LEADING W FROM HWY, KHAO

PHRIK, NR RATBURI, THAILAND

H408: WATERHOUSE JB + PIYASIN

S. 1970.PALAEONTOGRAPHICA.BD 135,ABT A.P 101-103

REMARKS: C.D-JB WATERHOUSE, 1964 ;JBW NO B561

MINIPLANUS SUBGENUS OF DIPLANUS:NEW SUBGENUS

1 SPEC + FRAG
DIPLANUS MINUTA WATERHOUSE+PIYASIN

31716 [B562] P-PARA

DIPLANUS (MINIPLANUS) MINUTA W.+P.

UP,KAZAN

50 M FROM ROAD LEADING W FROM HWY, KHAO

PHRIK,NR RATBURI, THAILAND

H408; WATERHOUSE JB + PIYASIN

S, 1970,PALAEONTOGRAPHICA,BD 135,ABT A.P101103,PL 15,FIG 23,24, TEXT FIG 3B

REMARKS: C,D-JB WATERHOUSE, 1964 ; JBW NO B562; MINIPLANUS SUBGENUS OF DIPLANUS;NEW SUBGENUS 1 SPEC

DIPLANUS MINUTA WATERHOUSE+PIYASIN 31717 [B563] P-PARA

DIPLANUS (MINIPLANUS) MINUTA W.+P.

UP,KAZAN

50 M FROM ROAD LEADING W FROM HWY, KHAO

PHRIK,NR RATBURI,THAILAND

H408; WATERHOUSE JB + PIYASIN

S, 1970,PALAEONTOGRAPHICA,BD 135,ABT A,P 101-103

REMARKS: C,D-JB WATERHOUSE, 1964 ;JBW NO B563;

MINIPLANUS SUBGENUS OF DIPLANUS;NEW SUBGENUS : 2 SPEC

DIPLANUS MINUTA WATERHOUSE+PIYASIN 31718 [B564] P-PARA

DIPLANUS (MINIPLANUS) MINUTA W.+P

UP, KAZAN

50 M FROM ROAD LEADING W FROM HWY, KHAO

PHRIK,NR RATBURI, THAILAND

H408; WATERHOUSE JB + PIYASIN

S, 1970,PALAEONTOGRAPHICA,BD 135,ABT A.P101-103

REMARKS: C,D-JB WATERHOUSE, 1964 ;JBW NO B564;

MINIPLANUS SUBGENUS OF DIPLANUS;NEW SUBGENUS ; 1 SPEC

DIPLANUS MINUTA WATERHOUSE+PIYASIN

31719 [B571] P-PARA

DIPLANUS (MINIPLANUS) MINUTA W. $+P$

UP.KAZAN

50 M FROM ROAD LEADING W FROM HWY, KHAO

PHRIK,NR RATBURI,THAILAND

H408:WATERHOUSE JB + PIYASIN

S, 1970,PALAEONTOGRAPHICA,BD 135,ABT A,P 101 103,PL 15, FIG 15,16

REMARKS: C.D-JB WATERHOUSE, 1964 :JBW NO B571: MINIPLANUS SUBGENUS OF DIPLANUS;NEW SUBGENUS 1 SPEC

DIPLANUS MINUTA WATERHOUSE+PIYASIN 31720 [B597] P-PARA

DIPLANUS (MINIPLANUS) MINUTA W.+P

UP,KAZAN

50 M FROM ROAD LEADING W FROM HWY, KHAO

PHRIK,NR RATBURI,THAILAND

H408; WATERHOUSE JB + PIYASIN

S, 1970.PALAEONTOGRAPHICA BD 135.ABT A.P 101-103

REMARKS: C.D-JB WATERHOUSE, 1964 ;JBW NO B597

MINIPLANUS SUBGENUS OF DIPLANUS; NEW SUBGENUS : 1 SPEC

DIPLANUS MINUTA WATERHOUSE+PIYASIN

31721 [B598,599] P-PARA

DIPLANUS (MINIPLANUS) MINUTA W. $+P$

UP, KAZAN

50 M FROM ROAD LEADING W FROM HWY, KHAO

PHRIK,NR RATBURI,THAILAND

H408:WATERHOUSE JB + PIYASIN

S, 1970,PALAEONTOGRAPHICA,BD 135,ABT A,P 101-103

REMARKS: C,D-JB WATERHOUSE, 1964 ; JBW NO B598,599 MINIPLANUS SUBGENUS OF DIPLANUS:NEW SUBGENUS 3 SPEC

DIPLANUS MINUTA WATERHOUSE+PIYASIN 31722 [B600] P-PARA

DIPLANUS (MINIPLANUS) MINUTA W. $+P$.

UP.KAZAN

50 M FROM ROAD LEADING W FROM HWY, KHAO

PHRIK, NR RATBURI, THAILAND

H408;WATERHOUSE JB + PIYASIN 
S. 1970.PALAEONTOGRAPHICA.BD 135.ABT A.P 101-103

REMARK S: C,D-JB WATERHOUSE, 1964 ; JBW NO B600;

MINIPLANUS SUBGENUS OF DIPLANUS:NEW SUBGENUS: 1 SPEC

DIPLANUS MINUTA WATERHOUSE+PIYASIN

31684 [B 108] P-PARA

DIPLANUS (MINIPLANUS) MINUTA W. + P

UP.KAZAN

50 M FROM ROAD LEADING W FROM HWY, KHAO

PHRIK, NR RATBURI, THAILAND

H408; WATERHOUSE JB + PIYASIN

S.1970,PALAEONTOGRAPHICA,BD 135,ABT A.P 101-103

REMARK S: C.D-JB WATERHOUSE, 1964 ;JBW NO B 108 ;

MINIPLANUS SUBGENUS OF DIPLANUS;NEW SUBGENUS; 1 SPEC

DIPLANUS MINUTA WATERHOUSE+PIYASIN

31685 [B1 10] P-PARA

DIPLANUS (MINIPLANUS) MINUTA W + P

UP.KAZAN

$50 M$ FROM ROAD LEADING W FROM HWY, KHAO

PHRIK, NR RATBURI, THAILAND

H408; WATERHOUSE JB + PIYASIN

S. 1970.PALAEONTOGRAPHICA,BD 135,ABT A.P 101-103

REMARKS: C,D-JB WATERHOUSE, 1964 ; JBW NO B 110 ;

MINIPLANUS SUBGENUS OF DIPLANUS:NEW SUBGENUS: 1 SPEC

DIPLANUS MINUTA WATERHOUSE+PIYASIN

31686 [B112] P-PARA

DIPLANUS (MINIPLANUS) MINUTA W $+P$

UP.KAZAN

50 M FROM ROAD LEADING W FROM HWY, KHAO

PHRIK, NR RATBURI, THAILAND

H408; WATERHOUSE JB + PIYASIN

S. 1970.PALAEONTOGRAPHICA,BD 135,ABT A.P101-103

REMARKS: C,D-JB WATERHOUSE, 1964 ; JBW NO B 112

MINIPLANUS SUBGENUS OF DIPLANUS:NEW SUBGENUS ;

1 SPEC

DIPLANUS MINUTA WATERHOUSE+PIYASIN

31687 [B113] P-HOLO

DIPLANUS (MINIPLANUS) MINUTA W. +P

UP.KAZAN

50 M FROM ROAD LEADING W FROM HWY, KHAO

PHRIK, NR RATBURI, THAILAND

H408; WATERHOUSE JB + PIYASIN

S, 1970,PALAEONTOGRAPHICA,BD 135,ABT A,P 101 103.PL 15. FIG 1-3

REMARKS: C.D-JB WATERHOUSE, 1964 ;JBW NO B 113 MINIPLANUS SUBGENUS OF DIPLANUS:NEW SUBGENUS 1 SPEC

DIPLANUS MINUTA WATERHOUSE+PIYASIN

31688 [B 114] P-PARA

DIPLANUS (MINIPLANUS) MINUTA W. $+P$

UP,KAZAN

$50 \mathrm{M}$ FROM ROAD LEADING W FROM HWY, KHAO

PHRIK NR RATBURI, THAILAND

H408; WATERHOUSE JB + PIYASIN

S. 1970.PALAEONTOGRAPHICA.BD 135,ABT A.P101-

103,PL15, FIG 4,5

REMARKS: C.D-JB WATERHOUSE, 1964 ;JBW NO B 114; MINIPLANUS SUBGENUS OF DIPLANUS:NEW SUBGENUS ; 1 SPEC

DIPLANUS MINUTA WATERHOUSE + PIYASIN

31689 [B115] P-PARA

DIPLANUS (MINIPLANUS) MINUTA $W .+P$

UP.KAZAN

50 M FROM ROAD LEADING W FROM HWY KHAO

PHRIK, NR RATBURI,THAILAND

H408: WATERHOUSE JB + PIYASIN

S, 1970,PALAEONTOGRAPHICA,BD 135,ABT A,P 101-103

REMARKS: C,D-JB WATERHOUSE, 1964 ;JBW NO B 115 MINIPLANUS SUBGENUS OF DIPLANUS; NEW SUBGENUS 1 SPEC
DIPLANUS MINUTA WATERHOUSE + PIYASIN

31690 [B 116 ] P.PARA

DIPLANUS (MINIPLANUS) MINUTA $W .+P$

UP, KAZAN

50 M FROM ROAD LEADING W FROM HWY , KHAO

PHRIK,NR RATBURI, THAILAND

H408:WATERHOUSE JB + PIYASIN

S, 1907,PALAEONTOGRAPHICA,BD 135,ABT A,P 101. 103,PL 15, FIG 7

REMARKS: C.D-JB WATERHOUSE, 1964 ; JBW NO B116: MINIPLANUS SUBGENUS OF DIPLANUS:NEW SUBGENUS : 1 SPEC

DIPLANUS MINUTA WATERHOUSE+PIYASIN

31691 [B117] P-PARA

DIPLANUS (MINIPLANUS) MINUTA W. $+P$

UP, KAZAN

50 M FROM ROAD LEADING W FROM HWY $K H A O$

PHRIK, NR RATBURI, THAILAND

H408; WATERHOUSE JB + PIYASIN

S, 1970,PALAEONTOGRAPHICA,BD 135,ABT A,P101103.PL 15,FIG 6.12

REMARKS: C,D-JB WATERHOUSE, 1964 ;JBW NO B 117 ; MINIPLANUS SUBGENUS OF DIPLANUS;NEW SUBGENUS ; 1 SPEC

DIPLANUS MINUTA WATERHOUSE+PIYASIN 31692 [B118] P-PARA

DIPLANUS (MINIPLANUS) MINUTA $W .+P$

UP,KAZAN

50 M FROM ROAD LEADING W FROM HWY, KHAO

PHRIK, NR RATBURI,THAILAND

H408: WATERHOUSE JB + PIYASIN

S, 1970,PALAEONTOGRAPHICA,BD 135,ABT A,P 101-103

REMARKS: C,D-JB WATERHOUSE, 1964 ;JBW NO B 118 :

MINIPLANUS SUBGENUS OF DIPLANUS:NEW SUBGENUS ; 1 SPEC (2 PCES)

DIPLANUS MINUTA WATERHOUSE+PIYASIN

31693 [B119] P-PARA

DIPLANUS (MINIPLANUS) MINUTA $W+P$

UP, KAZAN

$50 M$ FROM ROAD LEADING W FROM HWY, KHAO

PHRIK NR RATBURI, THAILAND

H408; WATERHOUSE JB + PIYASIN

S, 1970,PALAEONTOGRAPHICA,BD 135,ABT A,P 101-103

REMARKS: C.D-JB WATERHOUSE, 1964 ; JBW NO B 119

MINIPLANUS SUBGENUS OF DIPLANUS;NEW SUBGENUS

$1 \mathrm{SPEC}+\mathrm{FRAG}$

DIPLANUS MINUTA WATERHOUSE+PIYASIN

31694 [B 120] P-PARA

DIPLANUS (MINIPLANUS) MINUTA W.+P

UP.KAZAN

$50 M$ FROM ROAD LEADING W FROM HWY, KHAO

PHRIK, NR RATBURI,THAILAND

H408; WATERHOUSE JB + PIYASIN

S 1970 PALAEONTOGRAPHICA BD 135 ABT A.P 101-103

REMARKS: C,D-JB WATERHOUSE, 1964 ; JBW NO B 120;

MINIPLANUS SUBGENUS OF DIPLANUS;NEW SUBGENUS:

1 SPEC

DIPLANUS MINUTA WATERHOUSE+PIYASIN

31695 [B 121 ] P-PARA

DIPLANUS (MINIPLANUS) MINUTA W.+P

UP,KAZAN

50 M FROM ROAD LEADING W FROM HWY, KHAO

PHRIK,NR RATBURI,THAILAND

H408: WATERHOUSE JB + PIYASIN

S, 1970.PALAEONTOGRAPHICA,BD 135.ABT A.P 101-103

REMARK S: C.D-JB WATERHOUSE, 1964 ; JBW NO B 121:

MINIPLANUS SUBGENUS OF DIPLANUS:NEW SUBGENUS ; 1 SPEC

DIPLANUS MINUTA WATERHOUSE+PIYASIN

31696 [B 122] P.PARA

DIPLANUS (MINIPLANUS) MINUTA $W+P$

UP.KAZAN

50 M FROM ROAD LEADING W FROM HWY . KHAO

PHRIK, NR RATBURI,THAILAND

H408. WATERHOUSE JB + PIYASIN 
S, 1970,PALAEONTOGRAPHICA,BD 135,ABT A,P 101103,PL 15,FIG 10,11

REMARKS: C,D-JB WATERHOUSE, 1964 ;JBW NO B122; MINIPLANUS SUBGENUS OF DIPLANUS;NEW SUBGENUS : 1 SPEC

DIPLANUS MINUTA WATERHOUSE + PIYASIN

31697 [B 123 ] P.PARA

DIPLANUS (MINIPLANUS) MINUTA W. +P.

UP, KAZAN

$50 M$ FROM ROAD LEADING W FROM HWY, KHAO

PHRIK,NR RATBURI, THAILAND

H408:WATERHOUSE JB + PIYASIN

S. 1970,PALAEONTOGRAPHICA,BD 135,ABT A,P 101-103

REMARKS: C,D-JB WATERHOUSE, 1964 ; JBW NO B123;

MINIPLANUS SUBGENUS OF DIPLANUS;NEW SUBGENUS ; 1 SPEC

DIPLANUS MINUTA WATERHOUSE + PIYASIN

31698 [B124 ] P-PARA

DIPLANUS (MINIPLANUS) MINUTA W. +P.

UP,KAZAN

50 M FROM ROAD LEADING W FROM HWY, KHAO

PHRIK,NR RATBURI,THAILAND

H408; WATERHOUSE JB + PIYASIN

S, 1970,PALAEONTOGRAPHICA,BD 135,ABT A,P101103,PL 15, FIG 8,9

REMARKS: C.D-JB WATERHOUSE, 1964 ;JBW NO B4 12; MINIPLANUS SUBGENUS OF DIPLANUS;NEW SUBGENUS : 1 SPEC

\section{DISCINOPSIS GULIELMI (MATTHEW)}

8272(B) [642CM(B)] P-LECTO

ACROTRETA? GULIELMI MATTHEW

$M C A$,

ST JOHN GP

[ST JOHN,ACADIAN, DIV $1 C$ ]

SEELY ST,ST JOHN,NB

H276;MATTHEW GF, 1886,P37-39,PL5,FIG 14A-E;WALCOTT CD 1912,P720,PL82,FIG 5

REMARKS: C-GF MATTHEW;D-MACKENZIE :DESIGNATED LECTOTYPE BY WALCOTT:STUDIED 1972 BY AJ ROWELL;SPEC 'B' OF MATTHEW'S SYNTYPES ; 1 SPEC

DISCINOPSIS GULIELMI (MATTHEW)

8272(A) [642CM(A)] P-PARAL

ACROTRETA? GULIELMI MATTHEW

$M C A$

ST JOHN GP

[ST JOHN,ACADIAN]

SEELYST,ST JOHN,NB

H276;MATTHEW GF, TRANS ROY CAN INST, VOL 3,SECT

4,1886,P37-39,PL5,FIG 14A-E;WALCOTT

CD, 1912,P720,PL82,FIG 5A-C

REMARKS: C-GF MATTHEW:D-MACKENZIE :LECTOTYPE DESIGNATED BY WALCOTT;STUDIED 1972 BY AJ ROWELL:SPEC 'D' IS WALCOTT'S FIG $5 \mathrm{C}$; 9 SPEC

\section{DOLERORTHIS MINUTA SHAW}

5642 P-SYN

$M S, N I A G$

ERAMOSA FM

REFORMATORY QUARRY, GUELPH,ONT

H316; SHAW EW, TRANS ROY CAN INST, VOL

21.PT2, 1937, P357. PL24,FIG 4-6

REMARKS: C-EW SHAW; 3 SPEC

\section{ECHINAURIS SP}

31979 [B365] REF SPEC-F

UP, KAZAN

$50 M$ FROM ROAD LEADING W FROM HWY, KHAO

PHRIK,NR RATBURI,THAILAND

H480; WATERHOUSE JB + PIYASIN

S, 1970,PALAEONTOGRAPHICA,BD 135,ABT

A.P130,PL22,FIG 15, 16

REMARKS: C,D-JB WATERHOUSE, 1964 ;JBW NO B365; ORDER PRODUCTIDA ACCORDING TO WATERHOUSE + PIYASIN ; 1SPEC + 2 FRAGS

\section{ECHINAURIS SP}

31980 [B555] REF SPEC-F

UP,KAZAN

50 M FROM ROAD LEADING W FROM HWY, KHAO

PHRIK,NR RATBURI, THAILAND

H480; WATERHOUSE JB + PIYASIN

S, 1970,PALAEONTOGRAPHICA,BD 135 , ABT

A,P 130, PL22,FIG 17

REMARKS: C.D-JB WATERHOUSE, 1964 ;JBW NO B555 ORDER PRODUCTIDA ACCORDING TO WATERHOUSE + PIYASIN ; 1 SPEC

\section{EOORTHIS ATAVA (MATTHEW)}

7975 [345CM]P-SYN

STROPHOMENA ATAVA MATTHEW

UCA.

ST JOHN GP

[ST JOHN,BRETONIAN,DIV 3A]

NAVYIS,ST JOHN,NB

H307:WALCOTT CD, 1912,P74,PL95,FIG 7,7A-B;MATTHEW GF, 1893,P 102-103,PL7,FIG 8A-F

REMARKS: C-GF MATTHEW:D-MACKENZIE :LABELLED COTYPES;UNCERTAIN WHICH SPECS FIG'D; 7 SPEC

\section{EOORTHIS JOHANNENSIS (MATTHEW)}

8060 [430CM] P-SYN

ORTHISINA ? JOHANNENSIS MATTHEW

UCA.

ST JOHNGP

[ST JOHN,BRETONIAN,DIV 3A]

GERMAINE ST,ST JOHN,NB

H307:WALCOTT CD, 1912,P78 1-782,PL97,FIG 10, 10A:

MATTHEW GF, 1892,P49-50,PL 12,FIG 13A-C

REMARKS: C-GF MATTHEW;D-MACKENZIE ;SPECS 'A' 'B' $=$ FIGS $13 \mathrm{~A}, 13 \mathrm{C} ; 2$ SPEC

FARDENIA PLICATA (WAY) MS IN BOLTON

$7525[817 \mathrm{CL}$ ] P-SYN

LS,MED

CATARACT GP,MANITOULIN FM,U BIOHERMAL BEDS BIDWELL TP,LOT 24,CON 11, MANITOULIN IS,ONT H407:BOLTON TE GSC MEM 289, 1957,P70-71,PL11,FIG 11 15,23

REMARKS: C-HG WAY, 1931;4 SPEC

GLASSIA VARIABILIS PARKS

22645 [400S ] REF SPEC

MS

ABOVE LIMESTONE RAPIDS, SEVERN R.DIST OF

PATRICIA,ONT

H641;PARKS WA, TRANS ROY CAN INST, VOL

11.PT 1, 1915,P50,PL3,FIG 11

REMARKS: C-JB TYRRELL, 1912 ;D-WH HEARST, 1915;

SPEC MISSING, POSSIBLY THE FIG'D SPEC ; 2 SPEC

GLOBOSOBUCINA SCOPAE WATERHOUSE + PIYASIN 31974 [B538] P-HOLO

UP,KAZAN

50 M FROM ROAD LEADING W FROM HWY, KHAO

PHRIK,NR RATBURI, THAILAND

H---;WATERHOUSE JB + PIYASIN

S, 1970,PALAEONTOGRAPHICA,BD 135,ABT A,P123.

125,PL21,FIG 7-12, TEXT FIG 8

REMARK S: C,D-JB WATERHOUSE, 1964 ;JBW NO B538;NEW GENUS:ORDER PRODUCTIDA FAMILY FEMELLAROIIDAE ACCORDING TO WATERHOUSE + PIYASIN; 1 SMALL SLAB

HOWELLELLA? SUBMERSA (GRABAU)

22500 [252S ] P-LECTO

SPIRIFER SULCATA MUT SUBMERSA GRABAU

$M D, E R I A N$

DETROIT RIVER GP. AMHERSTBURG FM

[UMONROE]

LIVINGSTONE CUT,DETROIT R, AMHERSTBURG, ONT

H683;GRABAU AW, 1909,P 136-137,PL 18,FIG 6:

FAGERSTROM JA, GSC BULL 204, 1971,P44-45,PL7,FIG 22

REMARKS: D-T NATTRESS, 1914 ;PROBABLY LOC J OF

FAGERSTROM ; 1 SPEC 
HOWELLELLA ? SUBMERSA (GRABAU)

29575 P.PARAL

SPIRIFER SULCATA MUT SUBMERSA GRABAU

MD.ERIAN

DETROIT RIVER GP.AMHERSTBURG FM

LIVINGSTONE CUT,DETROIT R, AMHERSTBURG,ONT

H683:GRABAU AW, 1909,P 136-137,PL 18,FIG 4:

FAGERSTROM JA,GSC BULL 204, 1971,P44-45

REMARKS: D-T NATTRESS, 1914 ; CITED BY FAGERSTROM

AS NO 262S:PROBABLY LOC J OF FAGERSTROM ; 1

SPEC

HUSTEDIA RATBURIENSIS WATERHOUSE + PIYASIN

31969 [B390] P-PARA

UP.KAZAN

50 M FROM ROAD LEADING W FROM HWY, KHAO

PHRIK.NR RATBURI,THAILAND

H652:WATERHOUSE JB + PIYASIN

S, 1970,PALAEONTOGRAPHICA,BD 135,ABT A,P 137 140.PL23,FIG 24,25

REMARKS: C.D-JB WATERHOUSE, 1964 ;JBW NO B390; 1 SPEC

HUSTEDIA RATBURIENSIS WATERHOUSE + PIYASIN 31970 [B391] REF SPEC-F

UP.KAZAN

50 M FROM ROAD LEADING W FROM HWY, KHAO

PHRIK,NR RATBURI, THAILAND

H652; WATERHOUSE JB + PIYASIN

S, 1970,PALAEONTOGRAPHICA,BD 135, ABT A,P 137 140.PL23,FIG 19,20

REMARKS: C,D-JB WATERHOUSE, 1964 ;JBW NO B391; 1 SPEC

HUSTEDIA RATBURIENSIS WATERHOUSE + PIYASIN 31971 [B582] P-PARA

UP.KAZAN

50 M FROM ROAD LEADING W FROM HWY, KHAO

PHRIK, NR RATBURI,THAILAND

H652; WATERHOUSE JB + PIYASIN S, 1970 ,BD 135,ABT

A.P137-140,PL23,FIG 21

REMARKS: C,D-JB WATERHOUSE, 1964 ;JBW NO B582; 1 SPEC

HUSTEDIA RATBURIENSIS WATERHOUSE + PIYASIN 31972 [B583] P.PARA

UP.KAZAN

50 M FROM ROAD LEADING W FROM HWY, KHAO

PHRIK NR RATBURI,THAILAND

H652: WATERHOUSE JB + PIYASIN

S, 1970,PALAEONTOGRAPHICA,BD 135, ABT A,P 137-140

REMARKS: C,D-JB WATERHOUSE, 1964 :JBW NO B583: 1 SPEC

HUSTEDIA RATBURIENSIS WATERHOUSE + PIYASIN 31973 [B584] P-PARA

UP.KAZAN

$50 M$ FROM ROAD LEADING W FROM HWY, KHAO

PHRIK,NR RATBURI,THAILAND

H652:WATERHOUSE JB + PIYASIN

S, 1970,PALAEONTOGRAPHICA,BD 135,ABT A,P 137 140. TEXTFIG 13

REMARKS: C.D-JB WATERHOUSE, 1964 : JBW NO B584

LABELLED H. THAILANDICA N SP IN TEXT FIG; 1 SPEC

HUSTEDIA RATBURIENSIS WATERHOUSE + PIYASIN 31954 [B374 ] P.PARA

UP.KAZAN

$50 \mathrm{M}$ FROM ROAD LEADING W FROM HWY, KHAO

PHRIK.NR RATBURI,THAILAND

H652.WATERHOUSE JB + PIYASIN

S, 1970.PALAEONTOGRAPHICA,BD 135,ABT A,P 137-140

REMARKS: C.D-JB WATERHOUSE, 1964 ;JBW NO B374; 1

SPEC

HUSTEDIA RATBURIENSIS WATERHOUSE + PIYASIN

31955 [B375] P-PARA

UP,KAZAN

$50 M$ FROM ROAD LEADING W FROM HWY, KHAO

PHRIK,NR RATBURI,THAILAND
H652:WATERHOUSE JB + PIYASIN

S, 1970,PALAEONTOGRAPHICA,BD 135,ABT A,P 137 140,PL23,FIG 17,18

REMARKS: C.D-JB WATERHOUSE, 1964 ;JBW NO B375 ; 1 SPEC

HUSTEDIA RATBURIENSIS WATERHOUSE + PIYASIN 31956 [B376 ] P-PARA

UP.KAZAN

$50 \mathrm{M}$ FROM ROAD LEADING W FROM HWY, KHAO PHRIK.NR RATBURI,THAILAND H652:WATERHOUSE JB + PIYASIN

S, 1970,PALAEONTOGRAPHICA,BD 135, ABT A,P 137-140 REMARKS: C.D-JB WATERHOUSE, 1964 ;JBW NO B376; 1 SPEC

HUSTEDIA RATBURIENSIS WATERHOUSE + PIYASIN 31957 [B378 ] P-PARA

UP, KAZAN

50 M FROM ROAD LEADING W FROM HWY, KHAO

PHRIK,NR RATBURI, THAILAND H652; WATERHOUSE JB + PIYASIN

S, 1970,PALAEONTOGRAPHICA,BD 135, ABT A.P 137-140 REMARKS: C,D-JB WATERHOUSE, 1964 ;JBW NO B378; 1 SPEC

HUSTEDIA RATBURIENSIS WATERHOUSE + PIYASIN 31958 [B379] P-PARA

UP,KAZAN

$50 \mathrm{M}$ FROM ROAD LEADING W FROM HWY, KHAO PHRIK,NR RATBURI, THAILAND H652:WATERHOUSE JB + PIYASIN

S, 1970,PALAEONTOGRAPHICA,BD 135, ABT A,P 137-140 REMARKS: C,D-JB WATERHOUSE, 1964 ;JBW NO B379; 1 SPEC

HUSTEDIA RATBURIENSIS WATERHOUSE + PIYASIN 31959 [B380 ] P-PARA

UPKAZAN

$50 \mathrm{M}$ FROM ROAD LEADING W FROM HWY, KHAO PHRIK, NR RATBURI, THAILAND H652:WATERHOUSE JB + PIYASIN

S, 1970,PALAEONTOGRAPHICA,BD 135,ABT A,P 137-140 REMARKS: C.D.JB WATERHOUSE, 1964 ;JBW NO B380 ; 1 SPEC

HUSTEDIA RATBURIENSIS WATERHOUSE + PIYASIN 31960 [B381] P.PARA

UP, KAZAN

50 M FROM ROAD LEADING W FROM HWY, KHAO PHRIK,NR RATBURI,THAILAND

H652:WATERHOUSE JB + PIYASIN

S, 1970,PALAEONTOGRAPHICA,BD 135,ABT A,P 137 140.PL23,FIG 28-30

REMARKS: C,D-JB WATERHOUSE, 1964 ;JBW NO B38 1; 1 SPEC

HUSTEDIA RATBURIENSIS WATERHOUSE + PIYASIN 31961 [B382] P-PARA

U P,KAZAN

50 M FROM ROAD LEADING W FROM HWY, KHAO

PHRIK, NR RATBURI,THAILAND

H652; WATERHOUSE JB + PIYASIN

S, 1970,PALAEONTOGRAPHICA,BD 135.ABT A.P137140,PL23,FIG 22,23

REMARKS: C,D-JB WATERHOUSE, 1964 ;JBW NO B382 ; 1 SPEC

HUSTEDIA RATBURIENSIS WATERHOUSE + PIYASIN

31962 [B383] P-PARA

UP,KAZAN

50 M FROM ROAD LEADING W FROM HWY, KHAO

PHRIK,NR RATBURI,THAILAND

H652: WATERHOUSE JB + PIYASIN

S, 1970,PALAEONTOGRAPHICA,BD 135.ABT A.P 137-140 REMARKS: C,D-JB WATERHOUSE, 1964 ;JBW NO B383, 1 SPEC 
HUSTEDIA RATBURIENSIS WATERHOUSE + PIYASIN

31963 [B384] P-HOLO

UP.KAZAN

50 M FROM ROAD LEADING W FROM HWY, KHAO

PHRIK, NR RATBURI,THAILAND

H652; WATERHOUSE JB + PIYASIN

S, 1970,PALAEONTOGRAPHICA,BD 135,ABT A,P 137 140,PL23,FIG 15, 16

REMARKS: C,D-JB WATERHOUSE, 1964 ;JBW NO 8384; INCORRECTLY GIVEN AS B348 IN PLATE CAPTION ; 1 SPEC

HUSTEDIA RATBURIENSIS WATERHOUSE+PIYASIN 31964 [B385] P-PARA

UP, KAZAN

50 M FROM ROAD LEADING W FROM HWY, KHAO PHRIK,NR RATBURI,THAILAND H652; WATERHOUSE JB + PIYASIN

S, 1970,PALAEONTOGRAPHICA,BD 135,ABT A,P 137-140 REMARKS: C,D-JB WATERHOUSE, 1964 ;JBW NO $8385 ; 1$ SPEC

HUSTEDIA RATBURIENSIS WATERHOUSE + PIYASIN 31965 [B386] P-PARA

UP, KAZAN

50 M FROM ROAD LEADING W FROM HWY, KHAO

PHRIK,NR RATBURI,THAILAND

H652; WATERHOUSE JB + PIYASIN

S, 1970,PALAEONTOGRAPHICA,BD 135,ABT A,P $137-$

140,PL23, FIG 26, 27

REMARKS: C.D-JB WATERHOUSE, 1964 ;JBW NO 8386; 1 SPEC

HUSTEDIA RATBURIENSIS WATERHOUSE+PIYASIN 31966 [B387] P-PARA

UP.KAZAN

50 M FROM ROAD LEADING W FROM HWY, KHAO

PHRIK, NR RATBURI, THAILAND

H652; WATERHOUSE JB + PIYASIN

S, 1970,PALAEONTOGRAPHICA,BD 135,ABT A,P 137-140 REMARKS: C,D-JB WATERHOUSE, 1964 ;JBW NO B387 ; 1 SPEC

HUSTEDIA RATBURIENSIS WATERHOUSE + PIYASIN

31967 [B388] P-PARA

UP, KAZAN

50 M FROM ROAD LEADING W FROM HWY, KHAO

PHRIK,NR RATBURI,THAILAND

H652: WATERHOUSE JB + PIYASIN

S, 1970,PALAEONTOGRAPHICA,BD 135,ABT A,P 137-140

REMARKS: C,D-JB WATERHOUSE, 1964 ;JBW NO B388; 2 SPEC

HUSTEDIA RATBURIENSIS WATERHOUSE+PIYASIN

31968 [B389] P-PARA

UP,KAZAN

50 M FROM ROAD LEADING W FROM HWY, KHAO

PHRIK, NR RATBURI, THAILAND

H652; WATERHOUSE JB + PIYASIN

S, 1970,PALAEONTOGRAPHICA,BD 135,ABT A,P 137-140 REMARKS: C.D-JB WATERHOUSE, 1964 ;JBW NO B389 ; 1 SPEC

KUTORGINA GRANULATA MATTHEW

$8408[778 \mathrm{CM}]$ P-SYN

LCA

[ETCHEMINIAN]

SMITH SOUND, TRINITY BAY, NFLD

H297; WALCOTT CD, 1912,P582-583,PL5,FIG 5,5A-C; MATTHEW GF, 1899, NB,P 189,PL1,FIG 2A-D

REMARKS: C-GF MATTHEW:D-MACKENZIE :LABELLED COTYPES;UNCERTAIN WHICH SPECS FIG'D;WALCOTT'S FIGS 5,5B,5C=MATTHEW'S FIGS 2A,2C,2B ; 6 SPEC

KUTORGINA LATOURENSIS MATTHEW

REMARKS: SEE PROTORTHIS LATOURENSIS (MATTHEW)
KUTORGINA PTERINOIDES MATTHEW

$8274[644 \mathrm{CM}]$ P-HOLO

$\mathrm{MCA}$

ST JOHN GP

[ST JOHN GP,ACADIAN,DIV 1C]

HANFORD BK, ST MARTINS,ST JOHN CO,NB

H297:MATTHEW GF,TRANS ROY SOC CAN, VOL 3,SECT

4, 1886,P43,PL5,FIG 19

REMARKS: C-GF MATTHEW;D-MACKENZIE ; 1 SPEC

LEPTAENA RHOMBOIDALIS (WILCKENS)

17351 [1110HR] REF SPEC-M

UO, CINCI,MAYSVILL

DUNDAS FM

DON VALLEY QUARRY,TORONTO,ONT

H391:PARKS \& DYER, ONT DEPT MINES, VOL

30,PT7, 1922, P33-34

REMARK'S: C-J TOWNSEND ; THESE SPECS NOT FIG'D : 2 SPEC

LEPTAENA RHOMBOIDALIS (WILCKENS)

17350 [1109HR] REF SPEC-F

U O,CINCI,MAYSVILL

DUNDAS FM

DON VALLEY QUARRY,TORONTO,ONT

H391:PARKS \& DYER, ONT DEPT MINES, VOL 30,PT7, 1922,P33-34,PL7,FIG 12

REMARKS: C-JTOWNSEND :STORED WITH

PLECTAMBONITES SERICEUS ; 1 SPEC (SLAB)

LEPTOBOLUS ATAVUS MATTHEW

REMARK S: SEE LINGULELLA ATAVA (MATTHEW)

LEPTOBOLUS ATAVUS MUT INSULAE MATTHEW REMARKS: SEE LINGULELLA ATAVA INSULAE (MATTHEW)

LEPTOBOLUS ATAVUS MUT TRITAVUS MATTHEW

7823 [193CM ] P-SYN

$\mathrm{MCA}$,

BOURINOT GP.DUGALD FM

[LETCHEMINIAN]

DUGALD BK, ESCASONIE,CAPE BRETON,NS

H269;MATTHEW GF, GSC, 1903,P 109,PL6,FIG 5A

REMARKS: C-GF MATTHEW:D-MACKENZIE ·STRAT REV AS IN HUTCHINSON, 1952,GSC MEM 263; 1 SPEC

LEPTOBOLUS COLLICIA VAR COLLIS MATTHEW REMARKS: SEE LINGULELLA COLLICIA VAR COLLIS (MATTHEW)

LEPTOBOLUS FLUMENSIS MATTHEW REMARKS: SEE LINGULELLA FLUMENSIS (MATTHEW)

LEPTOBOLUS GEMMULUS (MATTHEW)

7958 [328CM ] P-SYN

OBOLELLA? GEMMULA MATTHEW

$M C A$.

ST JOHHN GP

MCLEOD BK,BOISDALE,NS

H269:WALCOTT CD 1912.P499,PL29,FIG 1U,V:MATTHEW GF, 1903,P 190,PL 14,FIG 1A-C; MATTHEW, 1891, P41,PL12,FIG 8A-C

REMARKS: C-GF MATTHEW:D-MACKENZIE :LABELLED COTYPES;UNCERTAIN WHICHSPECS FIG'D;WALCOTT'S FIGS $1 \mathrm{U}, \mathrm{V}=$ MATTHEW'S FIGS 8B,C ; 4 SPEC

LEPTOBOLUS GEMMULUS (MATTHEW)

25650 P-SYN

OBOLELLA? GEMMULA MATTHEW

$M C A$,

ST JOHN GP

[ST JOHN,BRETONIAN,DIV 3C]

NAVY IS,ST JOHN,NB

H269; WALCOTT CD, 1912,P499,PL29,FIG 1I,V;MATTHEW GF, 1903,P 190,PL 14,FIG 1A-C: MATTHEW, 1892,P41,PL12,FIG 8A-C

REMARKS: C-GF MATTHEW;D-MACKENZIE ;LABELLED COTYPES;UNCERTAIN WHICH SPECS FIG'D;WALCOTT'S FIGS $1 U, V=$ MATTHEW'S FIGS $8 B, C ; 4$ SPEC 
LEPTOBOLUS GRANDIS MATTHEW

REMARKS: SEE LINGULELLA GRANDIS (MATTHEW)

LEPTOBOLUS ? COLLICIA MATTHEW

REMARKS: SEE LINGULELLA COLLICIA (MATTHEW)

\section{LEPTODUS SP}

31734 [B356] REF SPEC-F

UP.KAZAN

50 M FROM ROAD LEADING W FROM HWY, KHAO

PHRIK, NR RATBURI, THAILAND

H518; WATERHOUSE JB + PIYASIN

S, 1970,PALAEONTOGRAPHICA,BD 135,ABT A,P 134-

145.PL24,FIG 1,2,TEXT FIG 10B

REMARKS: C,D-JB WATERHOUSE, 1964 ;JBW NO B356;

ORDER PRODUCTIDA ACCORDING TO WATERHOUSE + PIYASIN ; 1 SPEC (2 PCES)

\section{LEPTODUS SP}

31735 [B357 ] REF SPEC-F

UP.KAZAN

50 M FROM ROAD LEADING W FROM HWY, KHAO

PHRIK, NR RATBURI,THAILAND

H518: WATERHOUSE JB + PIYASIN

S, 1970,PALAEONTOGRAPHICA,BD 135,ABT A,P 134 135,PL24,FIG 8

REMARKS: C,D-JB WATERHOUSE, 1964 ; JBW NO B357; ORDER PRODUCTIDA ACCORDING TO WATERHOUSE + PIYASIN ; 1 SPEC

\section{LEPTODUS SP}

31737 [B359] REF SPEC-M

UP, KAZAN

50 M FROM ROAD LEADING W FROM HWY, KHAO

PHRIK, NR RATBURI, THAILAND

H518; WATERHOUSE JB + PIYASIN

S, 1970,PALAEONTOGRAPHICA.BD 135,ABT A.P 134-135

REMARKS: C,D-JB WATERHOUSE, 1964 ; JBW NO B359; ORDER PRODUCTIDA ACCORDING TO WATERHOUSE + PIYASIN : 1 SPEC

\section{LEPTODUS SP}

31738 [B360 ] REF SPEC-F

UP.KAZAN

50 M FROM ROAD LEADING W FROM HWY, KHAO

PHRIK, NR RATBURI,THAILAND

H5 18: WATERHOUSE JB + PIYASIN

S, 1970,PALAEONTOGRAPHICA,BD 135,ABT A,P134135 PL24, FIG 3,5, TEXT FIG $10 A$

REMARKS: C,D-JB WATERHOUSE, 1964 ; JBW NO B360; ORDER PRODUCTIDA ACCORDING TO WATERHOUSE + PIYASIN ; 1 SPEC

\section{LEPTODUS SP}

31739 [B361] REF SPEC-F

UP, KAZAN

$50 M$ FROM ROAD LEADING W FROM HWY, KHAO

PHRIK NR RATBURI, THAILAND

H5 18; WATERHOUSE JB + PIYASIN

S, 1970,PALAEONTOGRAPHICA,BD 135,ABT A,P 134 135. TEXT FIG 10C

REMARKS: C,D-JB WATERHOUSE, 1964 ;JBW NO B361; ORDER PRODUCTIDA ACCORDING TO WATERHOUSE + PIYASIN ; 1 SPEC

\section{LEPTODUS SP}

31740 [B362] REF SPEC-F

UP, KAZAN

50 M FROM ROAD LEADING W FROM HWY, KHAO

PHRIK, NR RATBURI, THAILAND

H5 18; WATERHOUSE JB + PIYASIN

S, 1970,PALAEONTOGRAPHICA.BD 135,ABT A.P 134135.PL24.FIG 4.6

REMARKS: C,D-JB WATERHOUSE, 1964 ;JBW NO B362 ORDER PRODUCTIDA ACCORDING TO WATERHOUSE + PIYASIN ; 1 SPEC
LEPTODUS SP

31741 [B363 ] REF SPEC-F

UP,KAZAN

50 M FROM ROAD LEADING W FROM HWY, KHAO

PHRIK, NR RATBURI, THAILAND

H5 18; WATERHOUSE JB + PIYASIN

S, 1970,PALAEONTOGRAPHICA,BD 135,ABT A.P 134 135,PL24,FIG 7,9

REMARKS: C,D-JB WATERHOUSE, 1964 : JBW NO B363: ORDER PRODUCTIDA ACCORDING TO WATERHOUSE + PIYASIN ; 1 SPEC

LINGULA NARRAWAYI WILSON

18900 P.SYN

MO.CHAMP,MOH,BLKRIV

BLACK RIVER GP, LOWVILLE FM

PARKDALE AVE,W OF MAIN OTTAWA SECTION

OTTAWA,ONT

H263;WILSON AE,GSC MUS BULL 33, 1921,P49-50,PL3,FIG 4 REMARKS: P-J NARRAWAY COLL ; 2 PCES ( 3 SPEC)

LINGULA CF PROGNE BILLINGS

17345 [1104HR] REF SPEC-F

U O,CINCI,MAYSVILL

DUNDAS FM

DON VALLEY QUARRY,TORONTO,ONT

H263;PARKS \& DYER,ONT DEPT MINES, VOL

30,PT7, 1922,P27,PL7,FIG 2

REMARKS: C-J TOWNSEND ; 2 SPEC

LINGULA RECTILATERALIS EMMONS

17346 [1105HR ] REF SPEC-F

U O,CINCI,MAYSVILL

DUNDAS FM

DON VALLEY QUARRY,TORONTO,ONT

H263; PARKS \& DYER,ONT DEPT MINES, VOL

30,PT7, 1922,P27-28,PL7,FIG 3

REMARKS: C-J TOWNSEND ; 2 SPEC

LINGULA ROTUNDA PARKS

24115 [918T] P-HOLO

MO,CHAMP,MOH,TRENT

TRENTON GP,COBOURG FM

[U COBOURG,BED 4 ]

E OF COLLINGWOOD.ONT

H263; PARKS WA,TRANS ROY SOC CAN,VOL 22,SECT

4,1928,P76,PL 1,FIG 25

REMARKS: C-WA PARKS, $1926 ; 1$ SPEC

LINGULA WESTONENSIS PARKS\&DYER

17320 [1079HR] P-HOLO

UO.CINCI,MAYSVILL

DUNDAS FM

HUMBER R, W SIDE, BELOW BRIDGE, WESTON, ONT

H263:PARKS \& DYER,ONT DEPT MINES, VOL

30,PT7, 1922,P26,PL7,FIG 1

REMARKS: C-WA PARKS, $1920 ; 1$ SPEC (CAST\&MOLD)

LINGULA ? LENS MATTHEW

REMARKS: SEE LINGULELLA LENS (MATTHEW)

LINGULELLA ATAVA (MATTHEW)

7766 [136CM ] P-PARAL

LEPTOBOLUS ATAVUS MATTHEW

$M C A$

BOURINOT GP,DUGALD FM

[U ETCHEMINIAN.DIVE3E]

DUGALD BK, ESCASONIE,CAPE BRETON,NS

H266:WALCOTT CD, 1912,P479-480,PL35,FIG 5;MATTHEW GF. 1899,P200,PL2,FIG 1A-C,E-F

REMARKS: C-GF MATTHEW:D-MACKENZIE LECTOTYPE DESIGNATED BY WALCOTT, SEE NO $7781(B)$;UNCERTAIN WHICH SPECS FIG'D; STRAT REV AS IN HUTCHINSON 1952, GSC MEM 263,6 SPEC 
LINGULELLA ATAVA (MATTHEW)

7781(A) [151CM(A)] P-PARAL

LEPTOBOLUS ATAVUS MATTHEW

$M C A$.

BOURINOT GP.DUGALD FM

[UETCHEMINIAN,DIVE3E]

DUGALD BK, ESCASONIE, CAPE BRETON,NS

H266:MATTHEW GF, 1899,P200,PL2,FIG 1A-F;WALCOTT CD,USGS MON 51,1912,P479-480

REMARKS: C-GF MATTHEW;D-MACKENZIE ;LECTOTYPE DESIGNATED BY WALCOTT, SEE NO 7781 (B);STRAT REV AS IN HUTCHINSON, 1952, GSC MEM $263 ; 4$ SPEC (OF 5)

LINGULELLA ATAVA (MATTHEW)

$7781(\mathrm{~B})[151 \mathrm{CM}(\mathrm{B})]$ P-LECTO

LEPTOBOLUS ATAVUS MATTHEW

$M C A$,

BOURINOT GP DUGALD FM

[UETCHEMINIAN,DIVE3E]

DUGALD BK, ESCASONIE, CAPE BRETON,NS

H266; WALCOTT CD, 1912,P479-480,PL35,FIG 5C;MATTHEW GF, 1899,NB,P200,PL2,FIG 1A-F

REMARKS: C-GF MATTHEW:D-MACKENZIE :SPEC 'B' OF MATTHEW'S SYNTYPES;DESIGNATED LECTOTYPE BY WALCOTT; STRAT REV AS IN HUTCHINSON. 1952,GSC MEM 263; 1SPEC (OF 5)

LINGULELLA ATAVA (MATTHEW)

7803 [173CM] P.PARAL

LEPTOBOLUS ATAVUS MATTHEW

$M C A$

BOURINOT GP,DUGALD FM

[U ETCHEMINIAN DIV E3D]

DUGALD BK, ESCASONIE,CAPE BRETON,NS

H266; WALCOTT CD, 19 12,P479-480,PL35,FIG 5;MATTHEW GF 1899 NB,P200 PL2 FIG 1A-C.E-F

REMARKS: C-GF MATTHEW;D-MACKENZIE ; LECTOTYPE DESIGNATED BY WALCOTT SEE NO 7881 (B):UNCERTAIN WHICH SPECS FIG'D; STRAT REV AS IN HUTCHINSON, 1952,GSC MEM 263; 7 SPEC

\section{LINGULELLA ATAVA INSULAE (MATTHEW)}

8309 [679CM]P-SYN

LEPTOBOLUS ATAVUS MUT INSULAE MATTHEW

$M C A$,

MACMULLIN FM

[LETCHEMINIAN]

YOUNG PT CAPE BRETON.NS

H266:WALCOTT CD,USGS MON 51, 1912,P480,PL35, FIG 8,8A;MATTHEW GF, 1903,P110,PL6,FIG 4A-C

REMARKS: C-GF MATTHEW:D-MACKENZIE ;YOUNG

PT = MCFEE'S PT;LABELLED COTYPES;UNCERTAIN

WHICH SPECS FIG'D ; 8 PCES

\section{LINGULELLA ATAVA INSULAE (MATTHEW)}

8548 [918CM]P-SYN

LEPTOBOLUS ATAVUS MUT INSULAE MATTHEW

$M C A$,

MACMULLIN FM

[LETCHEMINIAN,DIV 2A]

YOUNG PT CAPE BRETON NS

H266:WALCOTT CD, USGS MON 51, 1912,P480,PL35, FIG 8,8A:MATTHEW GF, 1903,P110,PL6,FIG 4A-C

REMARKS: C-GF MATTHEW:D-MACKENZIE :YOUNG

PT = MCFEE'S PT;LABELLED COTYPES;UNCERTAIN WHICH SPEC'S FIG'D ; 1 SPEC

LINGULELLA COLLICIA (MATTHEW)

7793(A) [163CM(A)]P-LECTO

LEPTOBOLUS ? COLLICIA MATTHEW

$M C A$

BOURINOT GP,DUGALDFM

[UETCHEMINTAN,DIV E3C]

DUGALD BK, ESCASONIE, CAPE BRETON,NS

H266;MATTHEW GF, 1899,P200,PL 1,FIG 3A-E;WALCOTT CD,USGS MON 51, 1912,P486,PL35,FIG 1

REMARKS: C-GF MATTHEW:D-MACKENZIE :SPEC 'A' OF MATTHEW'S SYNTYPES.DESIGNATED LECTOTYPE BY WALCOTT;STRAT REV AS IN HUTCHINSON 1952,GSC MEM 263:1 SPEC
LINGULELLA COLLICIA (MATTHEW)

7793(B) [163CM(B)] P-PARAL

LEPTOBOLUS? COLLCIA MATTHEW

$M C A$,

BOURINOT GP.DUGALD FM

[U ETCHEMINIAN, DIV E3C]

DUGALD BK, ESCASONIE, CAPE BRETON,NS

H266; WALCOTT CD, 1912,P486,PL35,FIG 1A;MATTHEW GF, 1899,NB,P200,PL 1,FIG 3A-E

REMARKS: C-GF MATTHEW:D-MACKENZIE :LECTOTYPE DESIGNATED BY WALCOTT,SEE NO 7793(A);SPEC 'D' FIG'D; STRAT REV AS IN HUTCHINSON, 1952, GSC MEM $263 ; 4$ SPEC

LINGULELLA COLLICIA (MATTHEW)

$8294[664$ CM] P-PARAL

LEPTOBOLUS COLLICIA MATTHEW

$M C A$

BOURINOT GP,DUGALD FM

[UETCHEMINIAN,DIV E3C]

DUGALD BK, ESCASONIE,CAPE BRETON,NS

H266; WALCOTT CD, 1912,P486,PL35,FIG 1B;MATTHEW GF, 1899,NB,P200,PL1,FIG 3A-E

REMARKS: C-GF MATTHEW:D-MACKENZIE :LECTOTYPE DESIGNATED BY WALCOTT,SEE NO 7793(A);SPEC 'B' FIG'D; STRAT REV AS IN HUTCHINSON, 1952, GSC MEM $263 ; 4$ SPEC

LINGULELLA COLLICIA VAR COLLIS (MATTHEW)

8481 [851CM] P-SYN

LEPTOBOLUS COLLICIA VAR COLLIS MATTHEW

$M C A$

BOURINOT GP,DUGALD FM

[U ETCHEMINIAN,DIVE3F]

INDIAN BK, ESCASONIE,CAPE BRETON,NS

H266;MATTHEW GF, 1903,P114-115;WALCOTT

CD, 1912,P486

REMARKS: C-GF MATTHEW;D-MACKENZIE ;THESE SPECS NOT FIG'D;NOT CONSIDERED A SEPARATE VAR OF L. COLLICIA BY WALCOTT ; 2 SPEC (CASTS\&MOLDS)

LINGULELLA CONCINNA MATTHEW

$7952[322 C M]$ P-LECTO

L ORD, TREM

MCLEOD BK FM

[ST JOHN,BRETONIAN,DIV 3C2]

MCLEOD BK,BOISDALE,NS

H266; WALCOTT CD, 1912,P486-488,PL33,FIG 2D;MATTHEW GF, 1900,NB,P273-274,PL5,FIG 2A-B

REMARKS: C-GF MATTHEW:D-MACKENZIE ;DESIGNATED LECTOTYPE BY WALCOTT;STRAT REV 1972 BY AJ ROWELL; 1 SPEC

LINGULELLA DAWSONI MATTHEW

$7741[111 \mathrm{CM}]$ P-SYN

$\mathrm{MCA}$,

ST JOHN GP

[ST JOHN,ACADIAN,DIV $1 C$ ]

ST JOHN,NB AND PORTLAND,NB

H266;MATTHEW GF, 1886,P33-34,PL5,FIG 9A-D;WALCOTT CD, 19 12,P497-498,PL29,FIG 1

REMARKS: C-GF MATTHEW:D-MACKENZIE ;LOC GIVEN AS ABOVE IN ROM RECORDS:PUBL'N MENTIONS ONLY PORTLAND + HANFORD BK ST MARTINS:SPECS LABELLED COTYPES;WALCOTT CONSIDERS THIS SPECIES $=$ L. FERRUGINEA ; 4 SPEC

LINGULELLA EXIGUA (MATTHEW)

7987 [357CM ] P-SYN

LINGULEPIS STARRI MUT EXIGUA MATTHEW

UCA,

ST JOHN GP

[ST JOHN, JOHANNIAN,BAND C2A]

MCLEAN BK, MIRA R, CAPE BRETON,NS

H266; WALCOTT CD, 1912,P551-554; MATTHEW GF,GSC, 1903,P 197-198,PL 14,FIG 3A-D

REMARKS:C-GF MATTHEW:D-MACKENZIE :SUBGENUS LINGULEPIS;THESE SPECS NOT FIG'D BY WALCOTT : 5 SPEC 
LINGULELLA FLUMENSIS (MATTHEW)

$8367(A)[737 \mathrm{CM}(\mathrm{A})]$ P-LECTO

LEPTOBOLUS FLUMENSIS MATTHEW

$M C A$,

[ACADIAN.DIV 2C]

MCNEIL BK.MIRA R.ON RD TO TROUT BK. CAPE BRETON,NS

H266:MATTHEW GF, 1903,P 189-190,PL11,FIG 7A-F: WALCOTT CD, 1912,P500-50 1,PL35,FIG 6

REMARKS: C-GF MATTHEW;D-MACKENZIE ; SPEC 'D OF MATTHEW'S SYNTYPES:DESIGNATED LECTOTYPE BY WALCOTT: 1 SPEC

LINGULELLA FLUMENSIS (MATTHEW)

$8367(\mathrm{~B})[737 \mathrm{CM}(\mathrm{B})]$ P.PARAL

LEPTOBOLUS FLUMENSIS MATTHEW

$M C A$,

[ACADIAN,DIV 2C]

MCNEIL BK, MIRA R, ON RD TO TROUT BK, CAPE

BRETON NS

H266:WALCOTT CD, 19 12,P500-591,PL35,FIG 6,6A-B;

MATTHEW GF, 1903,P 189.190,PL11,FIG 7A-F

REMARKS: C-GF MATTHEW:D-MACKENZIE:LECTOTYPE

DESIGNATED BY WALCOTT-SEE NO 8367(A):SPECS 'B' \& 'C' FIG'D ; 4 SPEC

LINGULELLA GRANDIS (MATTHEW)

$8105[475 \mathrm{CM}]$ P-SYN

LEPTOBOLUS GRANDIS MATTHEW

$M C A$.

ST JOHN GP

[ST JOHN,BRETONIAN,DIV 3E]

MCAPHEE'S CORNER, HARDINGVILLE,ST JOHN CO,NB

H266:WALCOTT CD, 19 12,P502-504,PL38,FIG 2B,E,F;

MATTHEW GF, 1894,P9 1-92,PL16,FIG 7A-C

REMARKS: C-GF MATTHEW;D-MACKENZIE;UNCERTAIN WHICH SPEC DESIGNATED LECTOTYPE BY WALCOTT 17 SPEC

LINGULELLA GREGWA MATTHEW

7729 [99CM ] P-SYN

$M C A$,

BOURINOT GP,DUGALD FM

[LETCHEMINIAN,DIVE1D]

BOUNDARY BK, ESCASONIE, CAPE BRETON,NS

H266: WALCOTT CD, 1912,P554-555; MATTHEW

GF, 1903,P 126-131,PL9,FIG 3A-F;MATTHEW

GF, 1899, NB,P 199-200,PL1,FIG 1A-F

REMARKS: C-GF MATTHEW:D-MACKENZIE :LABELLED COTYPES:UNCERTAIN WHICH SPECS FIG'D;STRAT REV AS IN HUTCHINSON, 1952, GSC MEM 263; 7 SPEC

\section{LINGULELLA GREGWA MATTHEW}

$7890[260 \mathrm{CM}]$ P-SYN

$M C A$.

BOURINOT GP.DUGALDFM

[LETCHEMINIAN,DIVE 1D]

DUGALD BK, ESCASONIE, CAPE BRETON, NS

H266; WALCOTT CD, 1912,P554-555:MATTHEW

GF, 1903,P126-131.PL9,FIG 3A-F:MATTHEW

GF, 1899,NB,P199-200,PL1,FIG 1A-F

REMARKS: C-GF MATTHEW:D-MACKENZIE:LABELLED COTYPES:UNCERTAIN WHICH SPECS FIG'D:STRAT REV AS IN HUTCHINSON, 1952,GSC MEM 263; 11 SPEC

LINGULELLA GREGWA MATTHEW

8555 [925CM ] P-SYN

$M C A$.

BOURINOT GP.DUGALD FM

[LETCHEMINIAN.DIV E 1D]

BOUNDARY BK, ESCASONIE,CAPE BRETON,NS

H266: WALCOTT CD, 1912.P554-555:MATTHEW GF, 1903,P126-131,PL9,FIG 3A-F:MATTHEW

GF, 1899,NB,P 199-200,PL1,FIG 1A-F

REMARKS: C-GF MATTHEW:D-MACKENZIE :LABELLED COTYPES:UNCERTAIN WHICH SPECS FIG'D;STRAT REV AS IN HUTCHINSON, 1952, GSC MEM 263: 6 SPEC
LINGULELLA GREGWA MATTHEW

8462 [832CM] P-SYN

$\mathrm{MCA}$.

BOURINOT GP.DUGALDFM

[LETCHEMINIAN,DIVE 1D]

BOUNDARY BK, ESCASONIE, CAPE BRETON, NS

H266:WALCOTT CD, 1912,P554-555;MATTHEW

GF, 1903,P126-131,PL9,FIG 3A-F:MATTHEW

GF, 1899,NB,P 199-200,PL1,FIG 1A-F

REMARKS: C-GF MATTHEW:D-MACKENZIE :LABELLED COTYPES:UNCERTAIN WHICH SPECS FIG'D:STRAT REV AS IN HUTCHINSON, 1952,GSC MEM 263; 3 SPEC

LINGULELLA GREGWA MATTHEW

7697 [67CM ] P-SYN

$M C A$,

BOURINOT GP.DUGALD FM

[LETCHEMINIAN,DIVE1D]

DUGALD BK, ESCASONIE,CAPE BRETON,NS

H266; WALCOTT CD, 1912,P554-555;MATTHEW GF, 1903,P126-131,PL9,FIG 3A-F;MATTHEW

GF, 1899,NB,P 199-200,PL1,FIG 1A-F

REMARKS: C-GF MATTHEW:D-MACKENZIE :LABELLED COTYPES:UNCERTAIN WHICH SPECS FIG'D;STRAT REV AS IN HUTCHINSON, 1952,GSC MEM 263; 6 SPEC

LINGULELLA GREGWA VAR ROBUSTA (MATTHEW)

7695 [65CM ] P-SYN

LINGULEPIS GREGWA VAR ROBUSTA MATTHEW

$M C A$,

BOURINOT GP,DUGALD FM

[LETCHEMINIAN,DIV E1C]

DUGALD BK, ESCASONIE, NS

H266:WALCOTT CD.USGS MON 51, 1912,P555:MATTHEW GF, 1903,P 131

REMARKS: C-GF MATTHEW:D-MACKENZIE :SUBGENUS LINGULEPIS IN WALCOTT; VAR NOT FIG'D; LABELLED COTYPES;STRAT REV AS IN HUTCHINSON, 1952,GSC MEM 263;4 SPEC

LINGULELLA GREGWA VAR ROBUSTA (MATTHEW)

7714 [84CM] P-SYN

LINGULEPIS GREGWA VAR ROBUSTA MATTHEW

$M C A$,

BOURINOT GP.DUGALD FM

[LETCHEMINIAN,DIVE1D]

DUGALD BK, ESCASONIE, CAPE BRETON,NS

H266:WALCOTT CD,USGS MON 51, 1912,P 155; MATTHEW GF, 1903,P131

REMARKS: C-GF MATTHEW;D-MACKENZIE;SUBGENUS LINGULEPIS IN WALCOTT:VAR NOT FIG'D:LABELLED COTYPES:STRAT REV AS IN HUTCHINSON, 1952, GSC MEM 263; 4 SPEC

LINGULELLA LAEVIS MATTHEW

7973(A) [343CM(A)] P-PARAL

U CA

ST JOHNGP

[ST JOHN GP,BRETONIAN,DIV C3A]

CARLETON SHORE, NEAR FALLS, ST JOHN NB

H266; WALCOTT CD, 1912,P5 12,PL 19,FIG 4A, B:MATTHEW GF, 1892,P39.PL 12,FIG 4A

REMARKS: C-GF MATTHEW:D-MACKENZIE;LECTOTYPE DESIGNATED BY WALCOTT-SEE NO 7973(B) : 2 SPEC

LINGULELLA LAEVIS MATTHEW

7973(B) [343CM(B)] P-LECTO

UCA.

ST JOHN GP

[ST JOHN GP,BRETONIAN, DIV C3A]

CARLETON SHORE, NEAR FALLS, ST JOHN NB

H266:MATTHEW GF, 1892,P39,PL 12,FIG 4B:WALCOTT

CD.USGS MON 51,1912.P5 12.PL 19,FIG 4

REMARKS: C-GF MATTHEW:D-MACKENZIE -SPEC ' $C$ ' OF MATTHEW'S SYNTYPES:DESIGNATED LECTOTYPE BY WALCOTT : 1 SPEC 
LINGULELLA LAEVIS VAR GRANDIS MATTHEW

7974 [344CM ] P-SYN

$\mathrm{MCA}$

ST JOHN GP

[ST JOHN GP,BRETONIAN,DIV 3A ]

CARLETON SHORE, NR SUSPENSION BRIDGE,ST JOHN, NB + ESCASONIE CAPE BRETON NS

H266; WALCOTT CD, USGS MON 51, 1912,P512,PL 19,FIG 4C; MATTHEW GF, 1903,P200-201,PL 15,FIG 1A,D

REMARKS: C-GF MATTHEW;D-MACKENZIE ;FIG 1A-SPEC B,ST JOHN;FIG 1D-SPEC A,ESCASONIE;SPEC A FIG'D BY WALCOTT: 2 SPEC

\section{LINGULELLA LENS (MATTHEW)}

7769 [139CM]P-SYN

LINGULA? LENS MATTHEW

$\mathrm{MCA}$,

BOURINOT GP DUGALD FM

[U ETCHEMINIAN,DIV E3E]

DUGALD BK, ESCASONIE,CAPE BRETON,NS

H266:WALCOTT CD, 1912,P512-513,PL33,FIG 1:MATTHEW GF, 1901,P274-275,PL5,FIG 3A-H

REMARKS: C-GF MATTHEW;D-MACKENZIE ;UNCERTAIN WHETHER LARGE SEPARATE SPEC IS THE ONE DESIGNATED LECTOTYPE BY WALCOTT:STRAT REV AS IN HUTCHINSON, 1952,GSC MEM 263; 2 SPEC

\section{LINGULELLA LINGULOIDES MATTHEW}

$7738[108 \mathrm{CM}] \mathrm{P}-\mathrm{SYN}$

$\mathrm{MCA}$

ST JOHN GP

[ST JOHN, ACADIAN,DIV 1D]

PORTER'S BK ST MARTINS,ST JOHN CO NB

H266; WALCOTT CD,USGS MON 51, 1912,P498;MATTHEW

GF, TRANS ROY SOC CAN,VOL 3,SECT 4, 1886,P34-

35. PL5, FIG 8,8A-B

REMARKS: C-GF MATTHEW;D-MACKENZIE ; 2 SPEC

\section{LINGULELLA LONGINERVIS (MATTHEW)}

7713 [83CM ] P-SYN

LINGULEPIS LONGINERVIS MATTHEW

$M C A$,

BOURINOT GP DUGALD FM

[L ETCHEMINIAN,DIV E2B]

DUGALD BK, ESCASONIE,CAPE BRETON,NS

H266; WALCOTT CD.USGS MON 51,1912,P555-556;

MATTHEW GF, 1903.P133-134.PL7,FIG 6A-C

REMARKS:C-GF MATTHEW;D-MACKENZIE;SUBGENUS

LINGULEPIS IN WALCOTT;SPEC 'C' FIG'D;STRAT REV AS

IN HUTCHINSON, 1952,GSC MEM $263 ; 5$ SPEC

\section{LINGULELLA LONGINERVIS (MATTHEW)}

7696 [66CM ] P-SYN

LINGULEPIS LONGINERVIS MATTHEW

$M C A$,

BOURINOT GP,DUGALD FM

[L ETCHEMINIAN,DIVE2B

DUGALD BK, ESCASONIE, CAPE BRETON,NS

H266; WALCOTT CD,USGS MON 51, 1912,P555-556,PL44,FIG 2E;MATTHEW GF, 1903,P133-135,PL7,FIG 6E-G

REMARKS: C-GF MATTHEW:D-MACKENZIE :SUBGENUS

LINGULEPIS IN WALCOTT;SEC 'C' FIG'D;STRAT REV AS

IN HUTCHINSON, 1952,GSC MEM $263 ; 4$ SPEC

\section{LINGULELLA CF LONGOVALIS MATTHEW}

$7732[102 \mathrm{CM}]$ REF SPEC-F

$M C A$,

BOURINOT GP,ESKASONIFM

[COLDBROOK TERRANE]

DUGALD BK, ESCASONIE, CAPE BRETON,NS

H266:MATTHEW GF, 1903,P75-76,PL7,FIG 2

REMARKS: C-GF MATTHEW:D-MACKENZIE :LABELLED

PLESIOTYPE;STRAT REV AS IN HUTCHINSON, 1952,GSC MEM 263; 1 SPEC

LINGULELLA LONGOVALIS MATTHEW

$7698[68 \mathrm{CM}]$ P-SYN

MCA

BOURINOT GP,DUGALD FM

[L ETCHEMINIAN DIV $1 C$ ]

DUGALD BK, ESCASONIE,CAPE BRETON,NS
H266.WALOCTT CD, USGS MON 51, 1912,P536, FIG 44H,H'; MATTHEW GF, 1903,P 123-125;PL 7,FIG 3A-F

REMARKS: C-GF MATTHEW;D-MACKENZIE :LABELLED COTYPES:UNCERTAIN WHICH SPECS FIG'D:STRAT REV AS IN HUTCHINSON, 1952,GSC MEM 263 ; 6 SPEC

LINGULELLA MARTINENSIS MATTHEW

7759 [129CM ] P-SYN

LCA.

ST JOHN GP

[ST JOHN GP,ACADIAN,DIV 1B1]

HANFORD BK, ST MARTINS, ST JOHN CO NB

H266; WALCOTT CD, 1912,P518-519,PL38,FIG 1,1A-E MATTHEW GF, 1895, NY,P113-114,PL2,FIG 6A-D;

1890.P 155-156.PL 8. FIG 4

REMARKS: C-GF MATTHEW:D-MACKENZIE ;LABELLED COTYPES;UNCERTAIN WHICH SPECS FIG'D;WALCOTT'S FIG $1 A, D=$ MATTHEW'S FIG 6D,6B; UNCERTAIN WHICH SPEC DESIGNATED LECTOTYPE BY WALCOTT ; 6 SPEC

\section{LINGULELLA MARTINENSIS MATTHEW}

$7684[54 \mathrm{CM}]$ P-SYN

$\mathrm{CAA}$,

ST JOHN GP

[ST JOHN,ACADIAN,DIV 1B]

HANFORD BK, ST MARTINS,ST JOHN CO,NB

H266; WALCOTT CD, 1912,P5 18-519,PL38,FIG 1,1A-E: MATTHEW GF, 1895, NY,P 113-114,PL2,FIG 6A-D; 1890,P 155-156, PL8,FIG 4

REMARKS: C-GF MATTHEW;D-MACKENZIE;LABELLED COTYPES;UNCERTAIN WHICH SPECS FIG'D;WALCOTT'S FIG $1 \mathrm{~A}, 1 \mathrm{D}=$ MATTHEW'S FIG 6D, 6B:UNCERTAIN WHICH SPEC DESIGNATED LECTOTYPE BY WALCOTT ; 1 SPEC (CAST\&MOLD)

LINGULELLA MINOR MATTHEW

8054(B) [424CM(B)] P-PARAL

LINGULELLA STARRI VAR MINOR MATTHEW

UCA,

BLACK SHALE BROOK FM

[ST JOHN, JOHANNIAN, C2B?]

LONG IS, KENNEBECASIS R, KINGS CO,NB

H266:MATTHEW GF, 1892,P58-59,PL12,FIG 5B;WALCOTT CD, 1912.P519.PL37.FIG 3A-B

REMARK S: C-GF MATTHEW:D-MACKENZIE ;LECTOTYPE DESIGNATED BY WALCOTT-SEE NO 8054(A): 1 SPEC

LINGULELLA MINOR MATTHEW

8054(A) [424CM(A)] P-LECTO

LINGULELLA STARRIVAR MINOR MATTHEW

UCA.

BLACK SHALE BROOK FM

[ST JOHN,JOHANNIAN,C2B?]

LONG IS, KENNEBECASIS R, KINGS CO,NB

H266:WALCOTT CD, 1912,P519,PL37,FIG 3;MATTHEW GF, 1892,P58-59,PL 12,FIG 5A

REMARK S: C-GF MATTHEW:D-MACKENZIE ;SPEC 'A' OF MATTHEW'S SYNTYPES:DESIGNATED LECTOTYPE BY WALCOTT ; 1 SPEC

LINGULELLA PUMILA (MATTHEW)

$7733($ A) [103CM(A)] P-LECTO

LINGULEPIS PUMILA MATTHEW

$M C A$,

BOURINOT GP,ESKASONI FM

[COLDBROOK TERRANE]

DUGALD BK, ESCASONIE,CAPE BRETON,NS

H266; WALCOTT CD, USGS MON 51, 1912 ,P556,PL 45, FIG 3 MATTHEW GF, 1903,P75,PL7,FIG 5A

REMARKS: C-GF MATTHEW:D-MACKENZIE ; SPEC 'A' OF MATTHEW'S SYNTYPES;DESIGNATED LECTOTYPE BY WALCOTT;STRAT REV AS IN HUTCHINSON, 1952,GSC MEM 263; 1 SPEC

LINGULELLA PUMILA (MATTHEW)

7733(B) [103CM(B)] P-PARAL

LINGULEPIS PUMILA MATTHEW

$M C A$

BOURINOT GP,ESKASONI FM

[COLDBROOK TERRANE ]

DUGALD BK, ESCASONIE,CAPE BRETON,NS 
H277:MATTHEW GF, 1903,P75,PL7,FIG 5B;WALCOTT CD.USGS MON 51, 1912 ,P556,PL45.FIG 3 A

REMARKS: C-GF MATTHEW:D-MACKENZIE;LECTOTYPE DESIGNATED BY WALCOTT,SEE NO 7733(A):SPEC 'C' FIG'D;STRAT REV AS IN HUTCHINSON, 1952, GSC MEM $263 ; 5$ SPEC

\section{LINGULELLA RADULA MATTHEW}

7989(B) [359CM(B)] P-PARAL

$M C A$.

ST JOHN GP

[ST JOHN, JOHANNIAN]

DUNNS, SIMONDS, COURTNEY BAY,NB

H266; WALCOTT CD, 1912,P529-530,PL45,FIG 1,1A-C,F; MATTHEW GF, 1891,P 147-148,PL 15,FIG 7A-B,8A-E

REMARKS: C-GF MATTHEW:D-MACKENZIE ;LECTOTYPE DESIGNATED BY WALCOTT,SEE NO $7989(A) ;$ SPEC ' $\mathrm{E}$ ' = FIG 1;5 SPEC

\section{LINGULELLA RADULA MATTHEW}

7989(A) [359CM(A)] P-LECTO

$\mathrm{MCA}$,

ST JOHN GP

[ST JOHN, JOHANNIAN]

DUNNS, SIMONDS, COURTNEY BAY,NB

H266:MATTHEW GF, 1891,P147-148, PL 15, FIG 7A-B,8A-E; WALCOTT CD, 1912,P529-530,PL45,FIG 1D

REMARKS: C-GF MATTHEW:D-MACKENZIE ;SPEC 'D' OF MATTHEW'S SYNTYPES;DESIGNATED LECTOTYPE BY WALCOTT : 1 SPEC

LINGULELLA RADULA VAR ASPERA MATTHEW

8053 [423CM] P-SYN

$\mathrm{MCA}$.

ST JOHN GP

[ST JOHN,JOHANNIAN,DIV C2C]

MC NEIL BK,MIRA R,NS

H266:WALCOTT CD, 1912,P519:MATTHEW

GF.GSC, 1903,P204-205,PL 15,FIG 2A-D

REMARKS: C-GF MATTHEW:D-MACKENZIE ;SPEC 'A'-

DORSAL, 'B'-VENTRAL;LABELLED COTYPES ; 2 SPEC

LINGULELLA ROBERTI MATTHEW

$7725(A)[95 \mathrm{CM}(\mathrm{A})]$ P-LECTO

$\mathrm{MCA}$.

MACMULLIN FM

[L ETCHEMINIAN,DIV E2A]

YOUNG'S PT, GEORGE R,NS

H266:MATTHEW GF, 1895.P256-257.PL1,FIG 2A-B

1903,P132,PL8,FIG5A-B;WALCOTT CD, 1912,P557558,PL37,FIG 4A

REMARKS: C-GF MATTHEW:D-MACKENZIE :SPEC ' $C$ ' OF MATTHEW'S SYNTYPES;DESIGNATED LECTOTYPE BY WALCOTT; STRAT REV AS IN HUTCHINSON, 1952,GSC MEM 263; 1 SPEC

LINGULELLA ROBERTI MATTHEW

$7725(B)[95 \mathrm{CM}(\mathrm{B})]$ P-PARAL

MCA.

MACMULLIN FM

[L ETCHEMINIAN,DIV E2A

YOUNG'S PT, GEORGE R, NS

H266:WALCOTT CD, 1912,P557-558,PL37.FIG 4,4B-D;

MATTHEW GF, 1903,P132,PL8,FIG 5A-B;

MATTHEW, 1895,P256-257,PL 1,FIG 2A-B

REMARKS: C-GF MATTHEW:D-MACKENZIE :LECTOTYPE DESIGNATED BY WALCOTT, SEE NO 7725(A);SPECS 'D', ' $\mathrm{E}^{\prime}=$ FIGS 4,4B OF WALCOTT:LOC $307 D$ OF WALCOTT; STRAT REV AS IN HUTCHINSON, 1952, GSC MEM 263; 5 SPEC

LINGULELLA SELWYNI MATTHEW

REMARKS: SEE OBOLUS SELWYNI (MATTHEW)

LINGULELLA STARRI (MATTHEW)

8045 [4 15CM ] REF SPEC-F

UCA

BLACK SHALE BROOK FM

[ST JOHN, JOHANNIAN,DIV 2B]

ST JOHN + PORTLAND, ST JOHN CO,NB
H266; WALCOTT CD, 1912.USGS MON 51,P559,PL37,FIG 2,2A-B

REMARKS: C-GF MATTHEW;D-W MACKENZIE;MATTHEW'S ORIGINAL TYPES LOST:SPECS FIG'D BY WALCOTT ARE MARKED ON SLABS; 7 SMALL SLABS

LINGULELLA STARRI VAR MINOR MATTHEW REMARKS: SEE LINGULELLA MINOR MATTHEW

LINGULELLA TORRENTIS (MATTHEW)

$7731[101 \mathrm{CM}]$ P-SYN

OBOLUS TORRENTIS MATTHEW

$M C A$,

BOURINOT GP,ESKASONI FM

[COLDBROOK TERRANE]

DUGALD BK, ESCASONIE,CAPE BRETON,NS

H266:WALCOTT CD.USGS MON 51,1912,P535-536,PL35,FIG 7; MATTHEW GF, 1903,P76,PL8,FIG 1

REMARKS: C-GF MATTHEW:D-MACKENZIE :UNCERTAIN WHICH SPEC DESIGNATED LECTOTYPE BY WALCOTT; STRAT REV AS IN HUTCHINSON, 1952, GSC MEM $263 ; 3$ SPEC

\section{LINGULELLA TRIPARILIS (MATTHEW)}

$8140[510 \mathrm{CM}]$ P-SYN

OBOLUS TRIPARILIS MATTHEW

$M C A$,

BOURINOT GP.DUGALD FM

[LETCHEMINIAN,DIVE1B]

DUGALD BK, ESCASONIE,CAPE BRETON,NS

H266; WALCOTT CD,USGS MON 51, 1912,P536-537,FIG 44B, B'; MATTHEW GF, 1903,P 136-137, PL8,FIG 4AB,PL9,FIG $1 A-B$

REMARKS: C-GF MATTHEW;D-MACKENZIE ;LABELLED COTYPES;SPEC ' $A$ ' = MATTHEW'S FIG $4 A=$ WALCOTT'S FIG 44B;STRAT REV AS IN HUTCHINSON, 1952, GSC MEM $263 ; 3$ SPEC

LINGULELLA TRIPARILIS (MATTHEW)

$8308[678 \mathrm{CM}]$ P-SYN

OBOLUS TRIPARILIS MATTHEW

$\mathrm{MCA}$,

BOURINOT GP,DUGALD FM

[L ETCHEMINIAN,DIVE1B]

DUGALD BK, ESCASONIE,CAPE BRETON,NS

H266; WALCOTT CD,USGS MON 51, 1912,P536-537,FIG 44B, B';MATTHEW GF, 1903,P 136-137,PL8,FIG 4A-

B.PL9,FIG 1A-B

REMARKS: C-GF MATTHEW;D-MACKENZIE ;LABELLED COTYPES; UNCERTAIN WHICH SPECS FIG'D;STRAT REV AS IN HUTCHINSON, 1952,GSC MEM 263; 5 SPEC

LINGULELLA TUMIDA MATTHEW

8178 [548CM ] P-SYN

MCA,

BOURINOT GP,DUGALD FM

[UETCHEMINIAN,DIV E3]

DUGALD BK? , ESCASONIE,CAPE BRETON,NS

H266:WALCOTT CD 1912.P537-538:MATTHEW

GF, 1903,P123,FIG 6A-C;MATTHEW

GF, 1899,NB,P200,PL1,FIG 2A-C

REMARKS: C-GF MATTHEW:D-MACKENZIE ; LOC GIVEN AS DUGALD BK, INDIAN BK, + GILLIS BK IN ROM RECORDSDUGALD BK IN PUBL'N;STRAT REV AS IN HUTCHINSON, 1952,GSC MEM $263 ; 3$ SPEC

LINGULELLA? CUNEATA MATTHEW

$8362[732 \mathrm{CM}]$ P-SYN

$\mathrm{MCA}$.

ST JOHN GP

[ACADIAN,DIV 3E]

MCAPHEE'S CORNER, HARDINGVILLE,ST JOHN CO,NB H266; WALCOTT CD, 19 12,P503,PL38,FIG 2,2A,C,D;

MATTHEW GF, 1894,P92-93,PL 16,FIG 5A-B

REMARKS: C-GF MATTHEW:D-MACKENZIE ;LABELLED

COTYPES:UNCERTAIN WHICH SPEC FIG'D ; 7 SPEC 
LINGULELLA? ESCASONIE MATTHEW

REMARKS: SEE OBOLUS ESCASONIE (MATTHEW)

LINGULELLA? INFLATA MATTHEW

REMARKS: ACROTRETA INFLATA (MATTHEW)

LINGULELLA? INFLATA VAR OVALIS MATTHEW

REMARKS: SEE ACROTRETA INFLATA VAROVALIS (MATTHEW)

LINGULEPIS LONGINERVIS MATTHEW REMARKS: SEE LINGULELLA LONGINERVIS (MATTHEW)

LINGULEPIS STARRI MATTHEW

REMARKS: SEE LINGULELLA STARRI (MATTHEW)

LINGULEPSIS GREGWA VAR ROBUSTA MATTHEW REMARKS: SEE LINGULELLA GREGWA VAR ROBUSTA (MATTHEW)

LINGULEPSIS PUMILA MATTHEW

REMARKS: SEE LINGULELLA PUMILA (MATTHEW)

LINGULEPSIS STARRI MUT EXIGUA MATTHEW

REMARKS: SEE LINGULELLA EXIGUA (MATTHEW)

LINGULODISCINA MISSOURIENSIS (SHUMARD) 6356 [2966CB ] REF SPEC-F

P

PARK CITY FM,PHOSPHATE BEDS

[PHOSPHATE BEDS,PARK CITY FM,U CARB

THOMAS FORK,WYO,USA

H285; GIRTY GH,USGS BULL 436, 19 10,P22-24,PL 1,FIG 10

REMARKS: D-WF FERRIER, 1920 ;LABELLED PARATYPES: NOT PRIMARY TYPES;UNCERTAIN WHICH SPEC FIG'D ; 3 SPEC

\section{LINGULODISCINA UTAHENSIS (MEEK)} 6357 [2967 CB ] REF SPEC?

P.

PARK CITY FM,PHOSPHATE BEDS

[PARK CITY FM,PHOSPHATE BEDS, U CARB]

WEBER CANYON,UTAH,USA

H285; GIRTY GH, USGS BULL 436,P24-25,PL1,FIG 11

REMARKS: D-WF FERRIER, 1920 ;LABELLED PARATYPE;

NOT A PRIMARY TYPE;UNCERTAIN WHETHER THIS SPEC FIG'D ; 1 SPEC

LINNARSSONIA MISERA BILLINGS

REMARKS: SEE ACROTRETA MISERA (BILLINGS)

LINNARSSONIA TRANSVERSA HARTT

REMARKS: SEE ACROTRETA TRANSVERSA (HARTT)

LINOPRODUCTUS SP

32165 [B342] REF SPEC-F

UP.KAZAN

50 M FROM ROAD LEADING W FROM HWY, KHAO

PHRIK, NR RATBURI, THAILAND

H500;WATERHOUSE JB + PIYASIN

S, 1970,PALAEONTOGRAPHICA,BD 135,ABT

A,P 131,PL22, FIG 18, 19

REMARKS: C,D-JB WATERHOUSE, 1964 ;JBW NO B342;

ORDER PRODUCTIDA ACCORDING TO WATERHOUSE + PIYASIN : 1 SPEC

\section{LINOPRODUCTUS SP}

32163 [B340] REF SPEC-F

UP.KAZAN

50 M FROM ROAD LEADING W FROM HWY, KHAO

PHRIK NR RATBURI, THAILAND

H500; WATERHOUSE JB + PIYASIN

S, 1970,PALAEONTOGRAPHICA,BD 135,ABT

A.P131,PL23,FIG 1
REMARKS: C.D-JB WATERHOUSE, 1964 ;JBW NO B340; ORDER PRODUCTIDA ACCORDING TO WATERHOUSE + PIYASIN ; 1 SPEC

MARGINIFERA AFF ELONGATA HUANG

32161 [B336] REF SPEC-M

UP, KAZAN

50 M FROM ROAD LEADING W FROM HWY, KHAO

PHRIK NR RATBURI, THAILAND

H477; WATERHOUSE JB + PIYASIN

S, 1970,PALAEONTOGRAPHICA,BD 135,ABT A,P 129-130

REMARKS: C,D-JB WATERHOUSE, 1964 ;JBW NO B336;

ORDER PRODUCTIDA ACCORDING TO WATERHOUSE + PIYASIN; 1 SMALL SLAB (2 PCE)

MARGINIFERA AFF ELONGATA HUANG

32158 [B22] REF SPEC-M

UP, KAZAN

50 M FROM ROAD LEADING W FROM HWY, KHAO

PHRIK, NR RATBURI, THAILAND

H477; WATERHOUSE JB + PIYASIN

S, 1970,PALAEONTOGRAPHICA,BD 135,ABT A.P 129-130

REMARKS: C,D-JB WATERHOUSE, 1964 ; JBW NO B22;

ORDER PRODUCTIDA ACCORDING TO WATERHOUSE + PIYASIN : 2 SPEC + 2 FRAGS

MARGINIFERA AFF ELONGATA HUANG

32159 [B23] REF SPEC-F

UP, KAZAN

50 M FROM ROAD LEADING W FROM HWY, KHAO

PHRIK,NR RATBURI,THAILAND

H477; WATERHOUSEJB + PIYASIN

S, 1970,PALAEONTOGRAPHICA,BD 135,ABT A,P129130.PL22, FIG 13,14

REMARKS: C.D-JB WATERHOUSE, 1964 ;JBW NO B23; ORDER PRODUCTIDA ACCORDING TO WATERHOUSE + PIYASIN : 1 SPEC

MARGINIFERA AFF ELONGATA HUANG

32160 [B24 ] REF SPEC-M

UP.KAZAN

50 M FROM ROAD LEADING W FROM HWY KHAO

PHRIK, NR RATBURI,THAILAND

H477: WATERHOUSE JB + PIYASIN

S, 1970,PALAEONTOGRAPHICA,BD 135,ABT A,P 129-130

REMARKS: C,D-JB WATERHOUSE, 1964 ; JBW NO B24;

ORDER PRODUCTIDA ACCORDING TO WATERHOUSE + PIYASIN ; 1 SPEC

MARGINIFERA TYPICA WAAGEN

32154 [B635] REF SPEC-F

UP,KAZAN

50 M FROM ROAD LEADING W FROM HWY, KHAO

PHRIK, NR RATBURI, THAILAND

H477: WATERHOUSE JB + PIYASIN

S, 1970,PALAEONTOGRAPHICA,BD 135,ABT A,P125129,PL22,FIG 2,3

REMARKS: C,D-JB WATERHOUSE, 1964 : JBW NO B365: ORDER PRODUCTIDA ACCORDING TO WATERHOUSE + PIYASIN ; 1 SPEC

MARGINIFERA TYPICA WAAGEN

32155 [B643] REF SPEC-F

UP,KAZAN

50 M FROM ROAD LEADING W FROM HWY, KHAO

PHRIK NR RATBURI, THAILAND

H477; WATERHOUSE JB + PIYASIN

S, 1970,PALAEONTOGRAPHICA,BD 135,ABT A,P 125 129, PI22, FIG 5,8

REMARKS: C,D-JB WATERHOUSE, 1964 ;JBW NO B643; ORDER PRODUCTIDA ACCORDING TO WATERHOUSE + PIYASIN : 1 SPEC

MARGINIFERA TYPICA WAAGEN

32142 [B20] REF SPEC-F

UP.KAZAN

50 M FROM ROAD LEADING W FROM HWY, KHAO

PHRIK, NR RATBURI, THAILAND

H477: WATERHOUSE JB + PIYASIN

S, 1970,PALAEONTOGRAPHICA,BD 135.ABT A,P125- 
129,PL22,FIG 1,4.6

REMARKS: C.D-JB WATERHOUSE, 1964 ;JBW NO B20; ORDER PRODUCTIDA ACCORDING TO WATERHOUSE + PIYASIN: 1 SPEC

MARGINIFERA TYPICA WAAGEN

32143 [B21] REF SPEC-M

UP,KAZAN

50 M FROM ROAD LEADING W FROM HWY, KHAO

PHRIK.NR RATBURI,THAILAND

H477:WATERHOUSE JB + PIYASIN

S, 1970,PALAEONTOGRAPHICA,BD 135,ABT A,P 125-129

REMARK S: C.D-JB WATERHOUSE, 1964 ; JBW NO B2 1

QUESTIONABLE WHETHER MATERIAL PRESENT IS THAT

REFERRED TO IN PAPER;ORDER PRODUCTIDA

ACCORDING TO WATERHOUSE + PIYASIN: 1 SPEC + 3 FRAGS

MARGINIFERA TYPICA WAAGEN

32144 [B331] REF SPEC-M

UP.KAZAN

50 M FROM ROAD LEADING W FROM HWY, KHAO

PHRIK NR RATBURI,THAILAND

H477; WATERHOUSE JB + PIYASIN

S. 1970 PALAEONTOGRAPHICA BD 135.ABT A P 125-129

REMARKS: C.D-JB WATERHOUSE, 1964 ; JBW NO B331;

ORDER PRODUCTIDA ACCORDING TO WATERHOUSE + PIYASIN : 1SPEC

MARGINIFERA TYPICA WAAGEN

32145 [B332] REF SPEC-M

U P.KAZAN

50 M FROM ROAD LEADING W FROM HWY, KHAO

PHRIK NR RATBURI,THAILAND

H477; WATERHOUSE JB + PIYASIN

S. 1970 PALAEONTOGRAPHICA BD 135,ABT A.P 125-129

REMARKS: C.D-JB WATERHOUSE, 1964 ;JBW NO B332

ORDER PRODUCTIDA ACCORDING TO WATERHOUSE + PIYASIN : 1 SPEC

MARGINIFERA TYPICA WAAGEN

32146 [B333 ] REF SPEC-M

UP.KAZAN

$50 M$ FROM ROAD LEADING W FROM HWY, KHAO

PHRIK NR RATBURI,THAILAND

H477; WATERHOUSE JB + PIYASIN

S. 1970 PALAEONTOGRAPHICA BD 135 ABT A P 125-129

REMARKS: C,D-JB WATERHOUSE, 1964 ;JBW NO B333;

ORDER PRODUCTIDA ACCORDING TO WATERHOUSE + PIYASIN : 1 SPEC

MARGINIFERA TYPICA WAAGEN

32147 [B334 ] REF SPEC-M

UP, KAZAN

$50 M$ FROM ROAD LEADING W FROM HWY, KHAO

PHRIK,NR RATBURI,THAILAND

H477; WATERHOUSE JB + PIYASIN

S. 1970 PALAEONTOGRAPHICA BD 135 ABT A P 125-129

REMARKS: C.D-JB WATERHOUSE, 1964 ;JBW NO B334;

ORDER PRODUCTIDA ACCORDING TO WATERHOUSE + PIYASIN : 1SPEC

MARGINIFERA TYPICA WAAGEN

32148 [B335] REF SPEC-M

UP,KAZAN

$50 M$ FROM ROAD LEADING W FROM HWY, KHAO

PHRIK,NR RATBURI,THAILAND

H477; WATERHOUSE JB + PIYASIN

S. 1970.PALAEONTOGRAPHICA BD 135 ABT A P125-129

REMARKS: C.D-JB WATERHOUSE, 1964 ;JBW NO B335;

ORDER PRODUCTIDA ACCORDING TO WATERHOUSE + PIYASIN : 1 SPEC

MARGINIFERA TYPICA WAAGEN

32150 [B534] REF SPEC-M

UP.KAZAN

50 M FROM ROAD LEADING W FROM HWY, KHAO

PHRIK NR RATBURI,THAILAND

H477; WATERHOUSE JB + PIYASIN

S, 1970,PALAEONTOGRAPHICA,BD 135,ABT A,P125-129
REMARKS: C D-JB WATERHOUSE 1964 . JBW NOB534 ORDER PRODUCTIDA ACCORDING TO WATERHOUSE + PIYASIN : 1 SPEC

MARGINIFERA TYPICA WAAGEN

32152 [B577] REF SPEC-F

UP.KAZAN

50 M FROM ROAD LEADING W FROM HWY KHAO

PHRIK, NR RATBURI, THAILAND

H477:WATERHOUSE JB + PIYASIN

S, 1970.PALAEONTOGRAPHICA,BD 135 ,ABT,P 125. 129,PL22,FIG 12

REMARKS: C.D-JB WATERHOUSE, 1964 ; JBW NO 8577 ORDER PRODUCTIDA ACCORDING TOWATERHOUSE + PIYASIN : 1 SPEC + 4 FRAGS

MARGINIFERA TYPICA WAAGEN 32153 [B578] REF SPEC-F

UP, KAZAN

$50 M$ FROM ROAD LEADING W FROM HWY, KHAO

PHRIK,NR RATBURI,THAILAND

H477; WATERHOUSE JB + PIYASIN

S, 1970.PALAEONTOGRAPHICA,BD 135,ABT A.P $125-$ 129, PL22, FIG 7,11

REMARKS: C,D-JB WATERHOUSE, 1964 ;JBW NO B578 ORDER PRODUCTIDA ACCORDING TOWATERHOUSE + PIYASIN ; 1 SPEC

MEEKELLA ? SP

31752 [B568 ] REF SPEC-M

UP, KAZAN

50 M FROM ROAD LEADING W FROM HWY KHAO

PHRIK, NR RATBURI,THAILAND

H405; WATERHOUSE JB + PIYASIN

S, 1970,PALAEONTOGRAPHICA,BD 135,ABT A.P99.100

REMARKS: C,D-JB WATERHOUSE, 1964 ;JBW NO B568; 3 SPEC + 3 FRAGS

MEEKELLA? SP

31753 [B652] REF SPEC-M

UP,KAZAN

50 M FROM ROAD LEADING W FROM HWY, KHAO

PHRIK,NR RATBURI,THAILAND

H405; WATERHOUSE JB + PIYASIN

S, 1970,PALAEONTOGRAPHICA.BD 135,ABT A.P99-100 REMARKS: C,D-JB WATERHOUSE, 1964 ;JBW NO B652 ; 1 SPEC

\section{MEEKELLA ? SP}

$31750[B 130]$ REF SPEC-F

UP,KAZAN

50 M FROM ROAD LEADING W FROM HWY, KHAO

PHRIK NR RATBURI,THAILAND

H405; WATERHOUSE JB + PIYASIN

S, 1970,PALAEONTOGRAPHICA,BD 135,ABT A.P99-

100, PL 14,FIG 22-23

REMARKS: C,D-JB WATERHOUSE, 1964 ;JBW NO B $130 ; 1$ SPEC + FRAG

MEEKELLA ? SP

31751 [B529] REF SPEC-M

UP,KAZAN

50 M FROM ROAD LEADING W FROM HWY KHAO

PHRIK,NR RATBURI, THAILAND

H405; WATERHOUSE JB + PIYASIN

S, 1970.PALAEONTOGRAPHICA,BD 135.ABT A.P99-100

REMARKS: C,D-JB WATERHOUSE, 1964 ;JBW NO B529; 2 SPEC + 2 FRAGS

\section{NEOSPIRIFER MOOSAKHAILENSIS (DAVIDSON)?}

32116 [B404] REF SPEC-F

UP,KAZAN

50 M FROM ROAD LEADING W FROM HWY, KHAO

PHRIK NR RATBURI,THAILAND

H706; WATERHOUSE JB + PIYASIN

S, 1970,PALAEONTOGRAPHICA,BD 135,ABT A,P147149,PL26,FIG 5,6

REMARKS:C,D-JB WATERHOUSE, 1964 ;JBW NO B404 : SLAB 
NEOSPIRIFER MOOSAKHAILENSIS (DAVIDSON)?

32119 [B403] REF SPEC-M

UP.KAZAN

50 M FROM ROAD LEADING W FROM HWY, KHAO

PHRIK,NR RATBURI,THAILAND

H706:WATERHOUSE JB + PIYASIN

S, 1970,PALAEONTOGRAPHICA,BD 135,ABT A,P 147-149

REMARK S: C,D-JB WATERHOUSE, 1964 ; JBW NO B403; 1

SPEC (2 PCES

NOTOTHYRIS PRAELECTA REED

32075 [B550] REF SPEC-F

UP.KAZAN

50 M FROM ROAD LEADING W FROM HWY, KHAO

PHRIK NR RATBURI, THAILAND

H758; WATERHOUSE JB + PIYASIN

S, 1970,PALAEONTOGRAPHICA,BD 135,ABT A,P 160162,PL30,FIG 13-15

REMARKS: C,D-JB WATERHOUSE, 1964 ;JBW NO B550 ; SPEC

NOTOTHYRIS PRAELECTA REED

32076 [B551] REF SPEC-F

UP.KAZAN

50 M FROM ROAD LEADING W FROM HWY, KHAO

PHRIK, NR RATBURI, THAILAND

H758; WATERHOUSE JB + PIYASIN

S, 1970,PALAEONTOGRAPHICA,BD 135,ABT A,P160162.PL30,FIG 4-6

REMARKS: C,D-JB WATERHOUSE, 1964 ;JBW NO B551; SPEC

\section{NOTOTHYRIS PRAELECTA REED}

32077 [B552] REF SPEC-M

UP, KAZAN

50 M FROM ROAD LEADING W FROM HWY, KHAO

PHRIK, NR RATBURI, THAILAND

H758; WATERHOUSE JB + PIYASIN

S, 1970,PALAEONTOGRAPHICA,BD 135,ABT A,P 160-162

REMARK S: C.D-JB WATERHOUSE, 1964 ;JBW NO B552 : 1 SPEC

\section{NOTOTHYRIS PRAELECTA REED}

32078 [B553 ] REF SPEC-M

UPKAZAN

50 M FROM ROAD LEADING W FROM HWY, KHAO

PHRIK,NR RATBURI,THAILAND

H758; WATERHOUSE JB + PIYASIN

S, 1970,PALAEONTOGRAPHICA,BD 135,ABT A,P 160-162 REMARKS: C D-JB WATERHOUSE, 1964 'JBW NO B553 * 1 SPEC

NOTOTHYRIS PRAELECTA REED

32081 [B645] REF SPEC-F

UP.KAZAN

50 M FROM ROAD LEADING W FROM HWY, KHAO

PHRIK,NR RATBURI,THAILAND

H758; WATERHOUSE JB + PIYASIN

S, 1970,PALAEONTOGRAPHICA,BD 135,ABT A,P 160162,PL31,FIG 2

REMARK S: C,D-JB WATERHOUSE, 1964 ;JBW NO B645 ; 1 SPEC

NOTOTHYRIS PRAELECTA REED

32082 [B646] REF SPEC-F

UP.KAZAN

50 M FROM ROAD LEADING W FROM HWY, KHAO

PHRIK,NR RATBURI, THAILAND

H758; WATERHOUSE JB + PIYASIN

S, 1970,PALAEONTOGRAPHICA,BD 135 , ABT A, P 160 162,PL30,FIG 19,20

REMARKS: C,D-JB WATERHOUSE, 1964 ;JBW NO B646; 1 SPEC

NOTOTHYRIS PRAELECTA REED

32083 [B648] REF SPEC-F

UP.KAZAN

50 M FROM ROAD LEADING W FROM HWY, KHAO

PHRIK, NR RATBURI, THAILAND

H758; WATERHOUSE JB + PIYASIN
S, 1970,PALAEONTOGRAPHICA,BD 135,ABT A,P 160 162.PL31,FIG 1,3

REMARK S: C,D-JB WATERHOUSE, 1964 ;JBW NO B648; 1 SPEC

\section{NOTOTHYRIS PRAELECTA REED}

32084 [B649 ] REF SPEC-F

UP, KAZAN

50 M FROM ROAD LEADING W FROM HWY, KHAO

PHRIK,NR RATBURI, THAILAND

H758; WATERHOUSE JB + PIYASIN

S, 1970,PALAEONTOGRAPHICA,BD 135,ABT A,P 160 162,PL29,FIG 16,17

REMARKS: C,D-JB WATERHOUSE, 1964 ;JBW NO B649; 1 SPEC

\section{NOTOTHYRIS PRAELECTA REED}

32085 [B650] REF SPEC-F

UP, KAZAN

50 M FROM ROAD LEADING W FROM HWY, KHAO

PHRIK,NR RATBURI, THAILAND

H758; WATERHOUSE JB + PIYASIN

S, 1970,PALAEONTOGRAPHICA,BD 135,ABT A,P 160162,PL29,FIG 18

REMARKS: C,D-JB WATERHOUSE, 1964 ;JBW NO B650 ; SPEC

\section{NOTOTHYRIS PRAELECTA REED}

32068 [B576] REF SPEC-M

UP.KAZAN

50 M FROM ROAD LEADING W FROM HWY, KHAO

PHRIK, NR RATBURI, THAILAND

H758: WATERHOUSE JB + PIYASIN

S, 1970,PALAEONTOGRAPHICA,BD 135,ABT A,P 160- 162 REMARKS: C D-JB WATERHOUSE, 1964 -JBW NO B576: 1 SMALLSLAB

\section{NOTOTHYRIS PRAELECTA REED}

32069 [B506] REF SPEC-M

UP,KAZAN

50 M FROM ROAD LEADING W FROM HWY, KHAO

PHRIK,NR RATBURI, THAILAND

H758:WATERHOUSE JB + PIYASIN

S, 1970,PALAEONTOGRAPHICA,BD 135,ABT A,P 160-162 REMARKS: C.D-JB WATERHOUSE, 1964 ;JBW NO B506 ; SPEC

\section{NOTOTHYRIS PRAELECTA REED}

32070 [B507 ] REF SPEC-M

UP,KAZAN

50 M FROM ROAD LEADING W FROM HWY, KHAO

PHRIK,NR RATBURI,THAILAND

H758: WATERHOUSE JB + PIYASIN

S, 1970,PALAEONTOGRAPHICA,BD 135,ABT A,P 160-162 REMARKS: C,D-JB WATERHOUSE, 1964 ;JBW NO B507 ; SPEC

NOTOTHYRIS PRAELECTA REED

32071 [B508] REF SPEC-F

UP.KAZAN

50 M FROM ROAD LEADING W FROM HWY, KHAO

PHRIK,NR RATBURI,THAILAND

H758.WATERHOUSE JB + PIYASIN

S, 1970,PALAEONTOGRAPHICA,BD 135,ABT A,P 160162,PL30,FIG 7-9

REMARKS: C.D-JB WATERHOUSE, 1964 ;JBW NO B508; 1 SPEC

\section{NOTOTHYRIS PRAELECTA REED}

32072 [B509] REF SPEC-F

UP,KAZAN

50 M FROM ROAD LEADING W FROM HWY, KHAO

PHRIK, NR RATBURI, THAILAND

H758; WATERHOUSE JB + PIYASIN

S, 1970,PALAEONTOGRAPHICA,BD 135,ABT A,P 160 162.PL29,FIG 19.20

REMARK S: C,D-JB WATERHOUSE, 1964 ;JBW NO B509 ; 1 SPEC 
NOTOTHYRIS PRAELECTA REED

32073 [B5 10 ] REF SPEC-M

UP.KAZAN

50 M FROM ROAD LEADING W FROM HWY, KHAO

PHRIK,NR RATBURI,THAILAND

H758; WATERHOUSE JB + PIYASIN

S, 1970,PALAEONTOGRAPHICA,BD 135, ABT A,P 160-162 REMARKS: C,D-JB WATERHOUSE, 1964 ;JBW NO B5 10; 1 SPEC

\section{NOTOTHYRIS PRAELECTA REED}

32074 [B511] REF SPEC-M

UP, KAZAN

$5 O M$ FROM ROAD LEADING W FROM HWY, KHAO

PHRIK,NR RATBURI,THAILAND

H758; WATERHOUSE JB + PIYASIN

S, 1970,PALAEONTOGRAPHICA,BD 135,ABT A,P160-162

REMARKS: C.D-JB WATERHOUSE, 1964 ;JBW NO B5 11; 1

SPEC (2 PCES)

\section{OBOLELLA NITIDA (FORD)}

8551 [92 1CM] REF SPEC?

LCA.

[ACADIAN.DIV 1B]

HANFORD BK, ST MARTINS,ST JOHN CO,NB

H292; MATTHEW GF,TRANS NY ACAD SCI,VOL

$14,1895, \mathrm{P} 125, \mathrm{PL} 2, \mathrm{FIG} 8$

REMARKS: C-GF MATTHEW;D-MACKENZIE;UNCERTAIN WHETHER SPEC IS FIG'D; LABELLED PLESIOTYPE;NOTE DISCREPANCY IN STRAT-PUBL'D (DIV 1B), ROM

RECORDS (B2-4); 1 SPEC

OBOLELLA SP

8245 [615CM ] REF SPEC-M

$M C A$.

BOURINOT GP,DUGALD FM

[UETCHEMINIAN]

DUGALD BK, ESCASONIE,CAPE BRETON,NS

H292;MATTHEW GH,TRANS ROY SOC CAN,VOL 3,SECT 4,1897,P170

REMARKS: C-GF MATTHEW;D-MACKENZIE ; LABELLED

PLESIOTYPE;STRAT REV AS IN HUTCHINSON, 1952,GSC MEM 263; 1 SPEC

\section{OBOLUS AEQUIPUTEIS MATTHEW}

8307 [677CM ] P-SYN

MCA,

MACMULLIN FM

[LETCHEMINIAN DIVE2A]

YOUNG PT, GEORGE R.CAPE BRETON,NS

H263: WALCOTT CD, 1912,P4 14,PL36,FIG 2,2A-B;MATTHEW GF, 1903,P 139,PL8,FIG 2A-D

REMARKS: C-GF MATTHEW;D-MACKENZIE ;SPECS 'A', 'B', 'D', 'E' FIG'D BY MATTHEW, FIGS 2B.C.A,D; STRAT REV AS IN HUTCHINSON, 1952,GSC MEM 263LOC 307D OF WALCOTT ; 5 SPEC

\section{OBOLUS DISCUS MATTHEW}

8236 [606CM ] P-SYN

$M C A$.

BOURINOT GP.DUGALDFM

[LETCHEMINIAN,DIVE1E]

DUGALD BK, ESCASONIE,CAPE BRETON,NS

H263:WALCOTT CD, 1912, P536,FIG 44A, A' MATTHEW GF, 1903,P138,PL8,FIG 3A-D

REMARKS: C-GF MATTHEW;D-MACKENZIE ;SPEC 'B' = FIG 3B, 'C' = FIG 3C, 'E' = FIG 3A; STRAT REV AS IN HUTCHINSON, 1952,GSC MEM 263; 5 SPEC

OBOLUS LENS MATTHEW

REMARKS: SEE OBOLUS (PALAEOBOLUS) BRETONENSIS LENS MATTHEW

OBOLUS LENS VAR LONGUS MATTHEW

REMARKS: SEE OBOLUS (PALAEOBOLUS) BRETONENSIS LENS LONGUS MATTHEW
OBOLUS PRISTINUS MATTHEW

REMARKS: SEE TREMATOBOLUS PRISTINUS (MATTHEW)

OBOLUS PULCHER MATTHEW

REMARKS: SEE BOTSFORDIA PULCHRA (MATTHEW)

OBOLUS REFULGENS MATTHEW

7954 [324CM] P-SYN

LO.

[BRETONIAN,DIV 3C]

NAVY IS,ST JOHN,NB

H263;WALCOTT CD, 1912,P409-410,PL9,FIG 2,2A-D; MATTHEW GF, 1892,P44-45,PL12,FIG 6A-D

REMARKS: C-GF MATTHEW:D-MACKENZIE :LABELLED COTYPES;UNCERTAIN WHICH SPECS FIG'D; WALCOTT'S FIG $2=$ MATTHEW'S FIG 6D(VENTRAL),FIG $2 A=$ FIG 6D(DORSAL) : 23 SPEC

OBOLUS SELWYNI (MATTHEW)

7727 [96CM ] P-SYN

LINGULELLA SELWYNI MATTHEW

CA.

MACMULLIN FM

[LETCHEMINIAN,DIV E2A]

YOUNG PT, GEORGE R,CAPE BRETON,NS

H263:WALCOTT CD 1912,P4 13-414,PL36,FIG 1-2B,PL37, FIG 1,1A-F;MATTHEW GF,1896,P255-256,PL1,FIG 1A-B

REMARKS: C-GF MATTHEW:D-MACKENZIE :LABELLED COTYPES:UNCERTAIN WHICH SPECS FIG'D;WALCOTT'S

FIG $1 C=$ MATTHEW'S FIG 1B:STRAT REV AS IN HUTCHINSON, 1952, GSC MEM 263; LOC 307D OF WALCOTT ; 6 SPEC

OBOLUS SELWYNI (MATTHEW)

7739 [109CM] P-SYN

LINGULELLA SELWYNI MATTHEW

$M C A$,

MACMULLIN FM

[LETCHEMINIAN,DIVE2A]

YOUNG PT , GEORGE R,CAPE BRETON,NS

H263; WALCOTT CD, 1912,P4 13-414,PL36,FIG 1-2B,PL37,FIG 1,1A-F;MATTHEW GF, 1896,P255-256,PL1,FIG 1A-B

REMARKS: C-GF MATTHEW;D-MACKENZIE ;LABELLED COTYPES;UNCERTAIN WHICH SPECS FIG'D;WALCOTT'S FIG $1 \mathrm{C}=$ MATTHEW'S FIG 1B:STRAT REV AS IN HUTCHINSON, 1952, GSC MEM 263; LOC 307D OF WALCOTT ; 3 SPEC

OBOLUS SELWYNI(MATTHEW)

7742 [112CM]P.SYN

LINGULELLA SELWYNI MATTHEW

$\mathrm{CA}$,

MACMULLIN FM

[LETCHEMINIAN,DIV E2A]

YOUNG PT, GEORGE R,CAPE BRETON,NS

H263:WALCOTT CD, 1912,P4 13-414,PL36,FIG 1-2B,PL37,FIG 1,1A-F:MATTHEW GF, 1896,P255-256,PL1,FIG 1A-B

REMARKS: C-GF MATTHEW;D-MACKENZIE :LABELLED COTYPES:UNCERTAIN WHICH SPEC'S FIG'D;WALCOTT'S FIG $1 C=$ MATTHEW'S FIG 1B;STRAT REV AS IN HUTCHINSON, 1952, GSC MEM 263; LOC 307D OF WALCOTT ; 3 SPEC

OBOLUS SELWYNI (MATTHEW)

8547 [9 17CM ] P-SYN

LINGULELLA SELWYNI MATTHEW

CA,

MACMULLIN FM

[LETCHEMINIAN,DIVE2A]

YOUNG PT, GEORGE R,CAPE BRETON,NS

H263.WALCOTT CD 1912,P413-313,PL36,FIG 1-2B,PL37.FIG 1,1A-F;MATTHEW GF, 1896,P255-256,PL1,FIG 1A-B

REMARKS: C-GF MATTHEW:D-MACKENZIE ; LABELLED COTYPES:UNCERTAIN WHICH SPECS FIG'D:WALCOTT'S FIG $1 C=$ MATTHEW'S FIG 1B;STRAT REV AS IN HUTCHINSON 1952, GSC MEM 263:LOC 307D OF WALCOTT ; 5 SPEC 
OBOLUS SEPTALIS WALCOTT

8579 [949CM ] P-SYN

OBOLUS (LINGULELLA) SEPTALIS WALCOTT

$\mathrm{MCA}$

STEPHEN FM

NW SLOPE, ABOVE FIELD, ON CPR, MT STEPHEN,BC

H263: WALCOTT CD,USGS MON 51, 1912,P4 14,PL34,FIG 2;

WALCOTT CD, 1905,P331

REMARKS: D-BE WALKER, 1914 ;LABELLED COTYPE ; SPEC

OBOLUS TORRENTIS MATTHEW

REMARKS: SEE LINGULELLA TORRENTIS (MATTHEW)

OBOLUS TRIPARILIS MATTHEW

REMARKS: SEE LINGULELLA TRIPARILIS (MATTHEW)

OBOLUS (PALAEOBOLUS)BRETONENSIS (MATTHEW)

7780 [150CM ] P-SYN

$\mathrm{MCA}$.

BOURINOT GP.DUGALDFM

[U ETCHEMINIAN,DIV E3D]

DUGALD BK, ESCASONIE,CAPE BRETON,NS

H263: WALCOTT CD, 1912,P426.PL32,FIG 5A:MATTHEW GF, 1899, NB,P202,PL2,FIG 2B,2E

REMARKS: C-GF MATTHEW;D-MACKENZIE :SPEC 'E' = FIG 2B. 'D' = FIG 2E, 'B' = FIG 5A:STRAT REV AS IN

HUTCHINSON, 1952,GSC MEM $263 ; 6$ SPEC

OBOLUS (PALAEOBOLUS)BRETONENSIS (MATTHEW)

7814 [184CM ] P-SYN

PALAEOBOLUS BRETONENSIS MATTHEW

$M C A$

BOURINOT GP,DUGALD FM

[U ETCHEMINIAN DIV E3D]

DUGALD BK, ESCASONIE, CAPE BRETON,NS

H263; WALCOTT CD,1912,P426,PL32,FIG 5D;MATTHEW

GF, 1899,NB,P202,PL2,FIG 2A,2D

REMARKS: C-GF MATTHEW;D-MACKENZIE ;SPEC ' $B$ ' = FIG

$2 D,{ }^{\prime} D^{\prime}=$ FIG $2 A{ }^{\prime}$ 'C' = FIG 5D;OLD RECORDS STATE 10 SPEC:STRAT REV AS IN HUTCHINSON, 1952, GSC MEM $263 ; 9$ SPEC

OBOLUS (PALAEOBOLUS)BRETONENSIS LENS MATTHEW

$8334[704 \mathrm{CM}]$ P-SYN

OBOLUS LENS MATTHEW

$\mathrm{MCA}$

BOURINOT GP,DUGALD FM

[U ETCHEMINIAN]

DUGALD BK, ESCASONIE,CAPE BRETON,NS

H263; WALCOTT CD, 1912,P427-428; MATTHEW

GF, 1903,P144-146.PL 10,FIG 1A-F:MATTHEW

GF, 1902,P94,PL 1,FIG 6A-E

REMARKS: C-GF MATTHEW;D-MACKENZIE :SPEC ' $C$ ' = FIG $1 A,{ }^{\prime}$ ' $=$ FIG 1E;STRAT REV AS IN HUTCHINSON, 1952,GSC MEM 263;6 SPEC

OBOLUS (PALAEOBOLUS)BRETONENSIS LENS LONGUS

8470 [840CM] P-SYN

OBOLUS LENS VAR LONGUS MATTHEW

$\mathrm{MCA}$

BOURINOT GP,DUGALD FM

[U ETCHEMINIAN,DIVE3E

DUGALD BK, ESCASONIE, CAPE BRETON,NS

H263; WALCOTT CD, 1912,P427-428; MATTHEW

GF 1903,P 146-147 PL7,FIG 4A B

REMARKS: C-GF MATTHEW:D-MACKENZIE ;SPEC ' $A$ ' = FIG 4 A $^{\prime}$ ' ' ' = FIG 4B;STRAT REV AS IN HUTCHINSON, 1952 GSC MEM $263 ; 4$ SPEC

OBOLUS (WESTONIA) ESCASONIE (MATTHEW)

8412 [782CM] P-SYN

LINGULELLA? ESCASONIE MATTHEW

UCA

ST JOHN GP

[ST JOHN,BRETONIAN,DIV C3B ]

MCADAM SHORE, ESCASONIE CAPE BRETON NS

H263; WALCOTT CD, 1912,P459-461,PL49,FIG 1,1A-F

MATTHEW GF, 190 1,NB,P270-273,PL5, FIG 1A-I

REMARKS: C-GF MATTHEW:D-MACKENZIE ·WALCOTT'S
FIGS $1 A, 1 B=$ MATTHEW'S FIGS 1G, 1D;UNCERTAIN WHICH SPEC DESIGNATED LECTOTYPE BY WALCOTT ; 6 SPEC

OBOLUS ? MAJOR MATTHEW

REMARKS: SEE RUSTELLA MAJOR (MATTHEW)

ORBICOELIA FRATERCULUS WATERHOUSE+PIYASIN 32053 [B484 ] P-PARA

UP,KAZAN

50 M FROM ROAD LEADING W FROM HWY, KHAO

PHRIK, NR RATBURI,THAILAND

H---;WATERHOUSE JB + PIYASIN

S, 1970, PALAEONTOGRAPHICA,BD 135,ABT A,P 144147,PL25,FIG 19,20

REMARKS: C.D-JB WATERHOUSE, 1964 ;JBW NO B484:NEW GENUS : 1 SPEC

ORBICOELIA FRATERCULUS WATERHOUSE+PIYASIN $32056[B 487$ ] P-PARA

UP.KAZAN

50 M FROM ROAD LEADING W FROM HWY, KHAO PHRIK,NR RATBURI,THAILAND H---:WATERHOUSE JB + PIYASIN

S, 1970, PALAEONTOGRAPHICA,BD 135,ABT A,P144147,PL26, FIG 1-3

REMARKS: C.D-JB WATERHOUSE, 1964 ; JBW NO B487: NEW GENUS : 1 SPEC

ORBICOELIA FRATERCULUS WATERHOUSE + PIYASIN 32060 [B573] P-HOLO

UP.KAZAN

50 M FROM ROAD LEADING W FROM HWY, KHAO PHRIK,NR RATBURI,THAILAND

H-..-WATERHOUSE JB + PIYASIN

S, 1970,PALAEONTOGRAPHICA,BD 135,ABT A,P 144 147.PL25,FIG 16-18

REMARKS: C.D-JB WATERHOUSE, 1964 :JBW NO B573:NEW GENUS : 1 SPEC

ORBICOELIA FRATERCULUS WATERHOUSE + PIYASIN 32061 [B574 ] P-PARA

UP KAZAN

$50 \mathrm{M}$ FROM ROAD LEADING W FROM HWY, KHAO PHRIK, NR RATBURI, THAILAND

H---;WATERHOUSE JB + PIYASIN

S, 1970,PALAEONTOGRAPHICA,BD 135,ABT A,P144147, PL25, FIG 21,22

REMARKS: C.D-JB WATERHOUSE, 1964 ;JBW NO B574: NEW GENUS : 1 SPEC

ORBICOELIA FRATERCULUS WATERHOUSE+PIYASIN 32062 [B575 ] P-PARA

UP,KAZAN

50 M FROM ROAD LEADING W FROM HWY, KHAO PHRIK, NR RATBURI,THAILAND

H---:WATERHOUSE JB + PIYASIN

S, 1970,PALAEONTOGRAPHICA,BD 135,ABT A,P144147,PL26, FIG 4,TEXT FIG 14

REMARKS: C D-JB WATERHOUSE, 1964 :JBW NO B575: NEW GENUS;LARGER OF 2 FRAGS FIG'D ; 2 FRAGS

ORBICOELIA FRATERCULUS WATERHOUSE + PIYASIN 32063 [B590] P-PARA

UP,KAZAN

50 M FROM ROAD LEADING W FROM HWY, KHAO

PHRIK, NR RATBURI,THAILAND

H.-.-WATERHOUSE JB + PIYASIN

S, 1970,PALAEONTOGRAPHICA,BD 135,ABT A,P 144147,PL25, FIG 25,26

REMARKS: C,D-JB WATERHOUSE, 1964 ;JBW NO B590;NEW GENUS ; 1 SPEC

ORBICOELIA FRATERCULUS WATERHOUSE+PIYASIN 32065 [B629 ] P-PARA

UP.KAZAN

50 M FROM ROAD LEADING W FROM HWY , KHAO

PHRIK, NR RATBURI, THAILAND

H---;WATERHOUSE JB + PIYASIN 
S. 1970,PALAEONTOGRAPHICA,BD 135.ABT A,P 144 147,PL25,FIG 23,24

REMARKS: C,D-JB WATERHOUSE, 1964 ;JBW NO B629;NEW GENUS ; 1 SPEC

ORBICOELIA FRATERCULUS WATERHOUSE + PIYASIN

32052 [B483] P.PARA

UP,KAZAN

50 M FROM ROAD LEADING W FROM HWY , KHAO

PHRIK,NR RATBURI, THAILAND

H---;WATERHOUSE JB + PIYASIN

S, 1970,PALAEONTOGRAPHICA,BD 135,ABT A,P 144-147

REMARKS: C,D-JB WATERHOUSE, 1964 ;JBW NO B483;NEW GENUS ; 1 SPEC + FRAGS

ORBICOELIA FRATERCULUS WATERHOUSE + PIYASIN 32054 [B485] P.PARA

UP, KAZAN

$50 \mathrm{M}$ FROM ROAD LEADING W FROM HWY, KHAO

PHRIK, NR RATBURI, THAILAND

H-..-WWTERHOUSE JB + PIYASIN

S, 1970,PALAEONTOGRAPHICA,BD 135,ABT A,P 144-147

REMARKS: C,D-JB WATERHOUSE, 1964 ; JBW NO B485; NEW GENUS : 1 SPEC

ORBICOELIA FRATERCULUS WATERHOUSE+PIYASIN 32055 [B486] P-PARA

UP, KAZAN

50 M FROM ROAD LEADING W FROM HWY, KHAO

PHRIK,NR RATBURI, THAILAND

H---;WATERHOUSE JB + PIYASIN

S, 1970.PALAEONTOGRAPHICA,BD 135,ABT A,P 144-147

REMARKS: C,D-JB WATERHOUSE, 1964 ;JBW NO B486; NEW GENUS ; 1 SPEC

ORBICOELIA FRATERCULUS WATERHOUSE + PIYASIN 32057 [B493] P-PARA

UP.KAZAN

50 M FROM ROAD LEADING W FROM HWY, KHAO

PHRIK,NR RATBURI,THAILAND

H---;WATERHOUSE JB + PIYASIN

S, 1970,PALAEONTOGRAPHICA,BD 135,ABT A,P 144-147

REMARKS: C,D-JB WATERHOUSE, 1964 ;JBW NO B493;NEW GENUS ; 1 SPEC

ORBICOELIA FRATERCULUS WATERHOUSE + PIYASIN 32058 [8539] P-PARA

UP, KAZAN

50 M FROM ROAD LEADING W FROM HWY, KHAO

PHRIK,NR RATBURI,THAILAND

H---:WATERHOUSE JB + PIYASIN

S, 1970,PALAEONTOGRAPHICA,BD 135,ABT A,P 144-147

REMARKS: C,D-JB WATERHOUSE, 1964 ;JBW NO B539;NEW GENUS ; 2 SPEC

ORBICOELIA FRATERCULUS WATERHOUSE+PIYASIN 32059 [B572] P-PARA

UP, KAZAN

50 M FROM ROAD LEADING W FROM HWY, KHAO

PHRIK, NR RATBURI, THAILAND

H---;WATERHOUSE JB + PIYASIN

S, 1970,PALAEONTOGRAPHICA,BD 135,ABT A,P144-147

REMARKS: C,D-JB WATERHOUSE, 1964 ;JBW NO B572;NEW GENUS ; 1 SPEC

ORBICOELIA FRATERCULUS WATERHOUSE+PIYASIN 32066 [B644] P.PARA

UP, KAZAN

50 M FROM ROAD LEADING W FROM HWY, KHAO

PHRIK, NR RATBURI,THAILAND

H---;WATERHOUSE JB + PIYASIN

S, 1970,PALAEONTOGRAPHICA,BD 135,ABT A,P 144-147

REMARKS: C,D-JB WATERHOUSE, 1964 ;JBW NO B644;NEW GENUS : 1 SPEC
ORTHIS CARAUSII SALTER

8059 [429CM] REF SPEC-F

UCA.

ST JÖHN GP

[BRETONIAN,DIV C3A]

NAVYIS.ST JOHN,NB

H311; MATTHEW GF, TRANS ROY SOC CAN VOL 10,SECT 4,1893,P102,PL7,FIG 7A

REMARKS: C-GF MATTHEW:D-MACKENZIE ; 1 SPEC

ORTHIS ELECTRA VAR MAJOR MATTHEW

$7971[341 \mathrm{CM}]$ P-SYN

$\mathrm{MCA}$,

ST JOHN GP

[ST JOHN,BRETONIAN,DIV 3D ]

FALLS, ST JOHN,NB

H311:MATTHEW GF, TRANS ROY SOC CAN, VOL 10,SECT 4, 1893.P 100,PL7,FIG 3A-C

REMARKS: C-GF MATTHEW:D-MACKENZIE;LABELLED COTYPES:UNCERTAIN WHICH SPEC IS FIG'D; 11 SPEC

ORTHIS EURYONE BILLINGS

7970 [340CM] REF SPEC-F

$\mathrm{MCA}$,

ST JOHHN GP

[ST JOHN,BRETONIAN,DIV 3D]

SUSPENSION BRIDGE,FALLS, ST JOHN,NB

H311:MATTHEW GF,TRANS ROY SOC CAN, VOL 10,SECT 4,1893,P101,PL7,FIG 5

REMARKS: C-GF MATTHEW;D-MACKENZIE ;SPEC 'B' FIG'D 1 SPEC (CAST\&MOLD)

ORTHIS LENTICULARIS (WAHLENBERG)

7978 [348CM] REF SPEC-F

UCA, OR LO,

ST JOHN GP

[ST JOHN,BRETONIAN ]

LOC UNCERTAIN

H311:MATTHEW GF, TRANS ROY SOC CAN, VOL 9,SECT 4, 1892,P46-48,PL12,FIG 9A-D

REMARKS: C-GF MATTHEW;D-MACKENZIE ;LOC GIVEN IN ROM RECORDS AS MCLEOD BK,BOISDALE,NS;IN PUBL'N AS GERMAINEST,ST JOHN,NB;LABELLED PLESIOTYPE IN OLD RECORDS ; 1 SPEC

ORTHIS ORTHAMBONITES PANDEF

7969 [339CM ] REF SPEC-F

L O,

[BRETONIAN,DIV 3D]

SUSPENSION BRIDGE, REVERSIBLE FALLS, ST JOHN,NB H311:MATTHEW GF. TRANS ROY SOC CAN, VOL 10,SECT 4, 1893,P101,PL7,FIG 4

REMARKS: C-GF MATTHEW:D-MACKENZIE ;ENTEREDIN ROM RECORDS AND FRITZ'S TYPE CAT AS ORTHIS PANDERIANA HALL\&CLARKE;SPEC LABELLED PLESIOTYPE ; 1 SPEC

ORTHIS QUACOENSIS MATTHEW

REMARKS: SEE PROTORTHIS QUACOENSIS (MATTHEW)

ORTHISINA? JOHANNENSIS MATTHEW

REMARKS: SEE EOORTHIS JOHANNENSIS (MATTHEW)

ORTHORHYNCULA BIDWELLENSIS WAY IN BOLTON 7518 [810CL ] P-SYN

S,MED

CATARACT GP,MANITOULIN FM

[MANITOULIN FM, U BIOHERMAL BEDS

CENTER OF LOT 24,CON 11, BIDWELL TP,MANITOULIN IS,ONT

H557;BOLTON TE,GSC MEM 289, 1957,P69-70,PL11,FIG 16 22

REMARKS: C-HG WAY, $1932 ; 27$ SPEC

ORTHOTETES ? SP

31998 [B530] REF SPEC-F

UP.KAZAN

50 M FROM ROAD LEADING W FROM HWY, KHAO

PHRIK,NR RATBURI, THAILAND 
H409;WATERHOUSE JB + PIYASIN

S, 1970,PALAEONTOGRAPHICA,BD 135,ABT

A.P105.PL 14.FIG 24.25

REMARKS: C.D-JB WATERHOUSE, 1964 ;JBW NO B530:

LARGER OF 2 SPEC FIG'D ; 2 SPEC

ORTHOTICHIA INDICA (WAAGEN)

32025 [B 105] REF SPEC-F

UP,KAZAN

50 M FROM ROAD LEADING W FROM HWY , KHAO

PHRIK,NR RATBURI,THAILAND

H332: WATERHOUSE JB + PIYASIN

S, 1970,PALAEONTOGRAPHICA,BD 135,ABT A,P95-

98,PL 14,FIG 10,11

REMARKS: C,D-JB WATERHOUSE, 1964 ;JBW NO B 105; 1 SPEC

ORTHOTICHIA INDICA (WAAGEN)

32026 [B 106] REF SPEC-F

UP,KAZAN

50 M FROM ROAD LEADING W FROM HWY, KHAO

PHRIK,NR RATBURI,THAILAND

H332: WATERHOUSE JB + PIYASIN

S, 1970,PALAEONTOGRAPHICA,BD 135, ABT A,P9598,PL14,FIG 12,13

REMARKS: C,D-JB WATERHOUSE, 1964 ;JBW NO B106; 1 SPEC

\section{ORTHOTICHIA INDICA (WAAGEN)}

32029 [B546] REF SPEC-F

UP,KAZAN

50 M FROM ROAD LEADING W FROM HWY, KHAO

PHRIK, NR RATBURI,THAILAND

H332; WATERHOUSE JB + PIYASIN

S, 1970,PALAEONTOGRAPHICA,BD 135, ABT A,P9598.PL14.FIG 14

REMARKS: C,D-JB WATERHOUSE, 1964 ;JBW NO B546; 1 SPEC

\section{ORTHOTICHIA INDICA (WAAGEN)}

32030 [B580] REF SPEC-F

UP, KAZAN

50 M FROM ROAD LEADING W FROM HWY, KHAO

PHRIK, NR RATBURI,THAILAND

H332: WATERHOUSE JB + PIYASIN

S, 1970,PALAEONTOGRAPHICA,BD 135,ABT A,P9598.PL14,FIG 18

REMARKS: C,D-JB WATERHOUSE, 1964 ;JBW NO B580 ; 1 SPEC

\section{ORTHOTICHIA INDICA (WAAGEN)}

32031 [B581] REF SPEC-F

UP.KAZAN

50 M FROM ROAD LEADING W FROM HWY, KHAO

PHRIK, NR RATBURI, THAILAND

H332. WATERHOUSE JB + PIYASIN

S, 1970,PALAEONTOGRAPHICA,BD 135,ABT A,P9598,PL14,FIG 16

REMARKS: C,D-JB WATERHOUSE, 1964 ;JBW NO B581; 1 SPEC

ORTHOTICHIA INDICA (WAAGEN)

32032 [B641] REF SPEC-F

UP.KAZAN

50 M FROM ROAD LEADING W FROM HWY, KHAO

PHRIK, NR RATBURI, THAILAND

H332; WATERHOUSE JB + PIYASIN

S, 1970 PALAEONTOGRAPHICA,BD 135.ABT A.P9598,PL 14,FIG 17

REMARKS: C,D-JB WATERHOUSE, 1964 ;JBW NO B641;2 SPEC

ORTHOTICHIA INDICA (WAAGEN)

32023 [B 102] REF SPEC-M

UP.KAZAN

50 M FROM ROAD LEADING W FROM HWY KHAO

PHRIK NR RATBURI THAILAND

H332; WATERHOUSE JB + PIYASIN

S. 1970.PALAEONTOGRAPHICA BD 135,ABT A.P95-98

REMARKS: C,D-JB WATERHOUSE, 1964 ;JBW NO B 102 ; 1 SPEC
ORTHOTICHIA INDICA (WAAGEN)

32024 [B 103] REF SPEC-M

UP, KAZAN

50 M FROM ROAD LEADING W FROM HWY, KHAO

PHRIK,NR RATBURI,THAILAND

H332; WATERHOUSE JB + PIYASIN

S, 1970,PALAEONTOGRAPHICA,BD 135,ABT A,P95-98

REMARKS: C,D-JB WATERHOUSE, 1964 ;JBW NO B103; 1 SPEC

PALAEOBOLUS BRETONENSIS MATTHEW

REMARKS: SEE OBOLUS (PALAEOBOLUS) BRETONENSIS (MATTHEW)

PHOLIDOPS CINCINNATIENSIS HALL

17349 [1108HR] REF SPEC-F

U O,CINCI,MAYSVILL

DUNDAS FM

DON VALLEY QUARRY,TORONTO,ONT

H273;PARKS \& DYER,ONT DEPT MINES, VOL

30,PT7, 1922, P31-32,PL7, FIG 6

REMARKS: C-H STEWART ;SPEC 'A' FIG'D ; 3 SPEC

PHRICODOTHYRIS ATTENUATA REED

32099 [B488 ] REF SPEC-F

UP, KAZAN

50 M FROM ROAD LEADING W FROM HWY, KHAO

PHRIK,NR RATBURI,THAILAND

H724; WATERHOUSE JB + PIYASIN

S, 1970,PALAEONTOGRAPHICA,BD 135,ABT A,P157160,PL26, FIG 14,18

REMARKS: C,D-JB WATERHOUSE, 1964 ;JBW NO B488; 1 SPEC

PHRICODOTHYRIS ATTENUATA REED

32100 [B489] REF SPEC-F

UP,KAZAN

50 M FROM ROAD LEADING W FROM HWY, KHAO

PHRIK,NR RATBURI, THAILAND

H724; WATERHOUSE JB + PIYASIN

S, 1970,PALAEONTOGRAPHICA,BD 135,ABT A,P157 160,PL26, FIG 12

REMARKS: C,D-JB WATERHOUSE, 1964 ;JBW NO B489; 1 SPEC

\section{PHRICODOTHYRIS ATTENUATA REED}

32101 [B490] REF SPEC-M

UP, KAZAN

50 M FROM ROAD LEADING W FROM HWY, KHAO

PHRIK,NR RATBURI, THAILAND

H724; WATERHOUSE JB + PIYASIN

S, 1970,PALAEONTOGRAPHICA,BD 135,ABT A,P 157-160

REMARKS: C,D-JB WATERHOUSE, 1964 ;JBW NO B490; 1 SPEC

PHRICODOTHYRIS ATTENUATA REED

32103 [B492] REF SPEC-M

UP,KAZAN

50 M FROM ROAD LEADING W FROM HWY, KHAO

PHRIK,NR RATBURI,THAILAND

H724; WATERHOUSE JB + PIYASIN

S, 1970,PALAEONTOGRAPHICA,BD 135,ABT A,P 157-160

REMARKS: C, D-JB WATERHOUSE, 1964 -JBW NO B492 : 1 SPEC (2 PCES)

\section{PHRICODOTHYRIS ATTENUATA REED}

32104 [B494] REF SPEC-F

UP, KAZAN

50 M FROM ROAD LEADING W FROM HWY, KHAO

PHRIK,NR RATBURI,THAILAND

H724; WATERHOUSE JB + PIYASIN

S, 1970,PALAEONTOGRAPHICA,BD 135,ABT A.P157160, PL26,FIG 13,17

REMARKS: C,D-JB WATERHOUSE, 1964 ;JBW NO B494; 1 SPEC 
PHRICODOTHYRIS ATTENUATA REED

32105 [B495] REF SPEC-F

UP, KAZAN

50 M FROM ROAD LEADING W FROM HWY, KHAO

PHRIK,NR RATBURI,THAILAND

H724: WATERHOUSE JB + PIYASIN

S, 1970,PALAEONTOGRAPHICA.BD 135,ABT A,P 157 160,PL26,FIG 9,15

REMARKS: C,D-JB WATERHOUSE, 1964 ;JBW NO B495; 1 SPEC

PHRICODOTHYRIS ATTENUATA REED

32106 [B497] REF SPEC-F

UP.KAZAN

50 M FROM ROAD LEADING W FROM HWY, KHAO

PHRIK,NR RATBURI,THAILAND

H724: WATERHOUSE JB + PIYASIN

S, 1970,PALAEONTOGRAPHICA,BD 135,ABT A,P157160,PL26,FIG 16

REMARKS: C,D-JB WATERHOUSE, 1964 ;JBW NO B497 ; 1 SPEC

\section{PHRICODOTHYRIS ATTENUATA REED}

32107 [B540] REF SPEC-F

UP,KAZAN

50 M FROM ROAD LEAOING W FROM HWY, KHAO

PHRIK,NR RATBURI,THAILAND

H724:WATERHOUSE JB + PIYASIN

S, 1970,PALAEONTOGRAPHICA,BD 135, ABT A,P157160,PL26,FIG 7.8

REMARKS: C,D-JB WATERHOUSE, 1964 ;JBW NO B540 ; 1 SPEC

\section{PHRICODOTHYRIS ATTENUATA REED}

32108 [B542] REF SPEC-F

UP KAZAN

$50 \mathrm{M}$ FROM ROAD LEAOING W FROM HWY, KHAO

PHRIK,NR RATBURI,THAILAND

H724:WATERHOUSE JB + PIYASIN

S, 1970,PALAEONTOGRAPHICA,BD 135,ABT A,P157160.PL26, FIG 10,11

REMARKS: C,D-JB WATERHOUSE, 1964 ;JBW NO B542; 1

SPEC + 11FRAGS

\section{PHRICODOTHYRIS ATTENUATA REED}

32095 [B 496] REF SPEC-M

U P.KAZAN

$50 M$ MROM ROAO LEADING W FROM HWY, KHAO

PHRIK.NR RATBURI,THAILAND

H724: WATERHOUSE JB + PIYASIN

S. 1970,PALAEONTOGRAPHICA,BD 135,ABT A,P 157-160

REMARKS: C.D-JB WATERHOUSE, 1964 ;JBW NO B496; 1 SMALL SLAB

\section{PLECTAMBONITES CF RUGOSUS (JAMES)}

17352 [1111HR] REF SPEC-F

PLECTAMBONITES CF RUGOSUS (MEEK)

UO.CINCI,MAYSVILL

DUNDAS FM

DON VALLEY QUARRY,TORONTO,ONT

H370:PARKS \& DYER,ONT DEPT MINES, VOL

30,PT7, 1922, P35-36, PL 7, FIG 11

REMARKS: C-H STEWART, 1920 ;UNCERTAIN WHICH SPEC FIG'D ; 4 SPEC

PLECTAMBONITES SERICEUS (SOWERBY)

$17350[1109 \mathrm{HR}]$ REF SPEC-F

UO,CINCI,MAYSVILL

DUNDASFM

DON VALLEY QUARRY TORONTO ONT

H378;PARKS \& DYER,ONT DEPT MINES, VOL

30.PT7, 1922,P35,PL 7,FIG 15, 16

REMARKS: C-J TOWNSEND; 1 SLAB

\section{PLECTAMBONITES TRANSVERSALIS WAHLENBERG} 22652 [407S ] REF SPEC-M

$\mathrm{S}$

[SILURIAN]

LIMESTONE RAPIDS, FAWN R, DIST OF PATRICIA, ONT

H370; PARKS WA,TRANS ROY CAN INST, VOL

11,PT 1, 1915,P48

REMARKS: C-JB TYRRELL, $1912+$;D-WH HEARST ; 1 SPEC
PRODUCTUS EUCHARIS GIRTY

6360 [2970CB] P-PARA?

$P$,

PARK CITY FM, PHOSPHATE BEDS

MONTPELIER, IDAHO,USA

H484;GIRTY GH,USGS BULL 436, 19 10,P28-29

REMARKS: D-WF FERRIER, $1920 ; 1$ SPEC

PRODUCTUS MONTPELIERENSIS GIRTY

$6361[2971$ CB] P-PARA?

P,

PARK CITY FM,PHOSPHATE BEDS

MONTPELIER,IDAHO,USA

H484; GIRTY GH,USGS BULL 436, 1910, P30

REMARKS: D-WF FERRIER, 1920 ;LABELLED PARATYPE NOT MENTIONED IN TEXT; 1 SPEC

PRODUCTUS PHOSPHATICUS GIRTY

6373 [2987CB] P-PARA?

P.

PARK CITY FM,PHOSPHATE BEDS

MONTPELIER,IDAHO,USA

H484;GIRTY GH,USGS BULL 436, 1910,P29-30

REMARKS: D-WF FERRIER, 1920 ;LABELLED PARATYPE: NOT MENTIONED IN TEXT ; 1 SPEC

PROTORTHIS LATOURENSIS (MATTHEW)

7877 [246CM] P-SYN

KUTORGINA LATOURENSIS MATTHEW

$M C A$,

ST JOHN GP

[ST JOHN,ACADIAN]

SEELY ST,ST JOHN,NB

H309;WALCOTT CD, 1912,P74 1-742,PL99,FIG 3,3B,3C;

MATTHEW GF, 1886,P42-43,PL5,FIG 18,18A-D

REMARKS: C-GF MATTHEW;D-MACKENZIE ;SPECS 'D','H', ' $N$ ' $=$ FIGS $3,3 \mathrm{~B}, 3 \mathrm{C} ; 13$ SPEC

PROTORTHIS QUACOENSIS (MATTHEW)

$7871(B)[241 \mathrm{CM}(\mathrm{B})]$ P-LECTO

ORTHIS QUACOENSIS MATTHEW

$M C A$,

ST JOHN GP

[ST JOHN, ACADIAN,DIV $1 \mathrm{C}$ ]

PORTER'S BK, ST MARTINS,ST JOHN CO,NB

H309;MATTHEW GF, 1886,P43-44,PL5,FIG 20B,C;WALCOTT CD, 1912,P742-743,PL99,FIG 2B

REMARKS: C-GF MATTHEW;D-MACKENZIE ;DESIGNATED LECTOTYPE BY WALCOTT;SPEC 'B' OF MATTHEW'S SYNTYPES ; 1 SPEC

PROTORTHIS QUACOENSIS (MATTHEW)

7871 (A) [241CM(A)] P-PARAL

ORTHIS QUACOENSIS MATTHEW

$M C A$,

ST JOHNGP

[ST JOHN, ACADIAN, DIV $1 \mathrm{C}$ ]

PORTER'S BK, ST MARTINS,ST JOHN CO,NB

H309;WALCOTT CD, 19 12,P742-743,PL99, FIG 2,2A,2C-E; MATTHEW GF, 1886,P43-44,PL5,FIG 20,20A

REMARKS: C-GF MATTHEW:D-MACKENZIE;LECTOTYPE DESIGNATED BY WALCOTT-SEE NO $7871(\mathrm{~B}) ; 1$ SPEC

PROTOSIPHON KEMPANUM MATTHEW

REMARKS: SEE TREMATOBOLUS KEMPANUM (MATTHEW

PUGNAX WEEKSI GIRTY

6354 [2961CB] P-PARA

P.

PARK CITY FM,PHOSPHATE BEDS

MONTPELIER,IDAHO,USA

H572; GIRTY GH,USGS BULL 436, 1910,P31-32

REMARKS: D-WF FERRIER ;LABELLED PARATYPES : 9 SPEC 
PUGNAX WEEKSI VAR NOBILIS GIRTY

$6362[2973$ CB ] P-PARA?

$P$.

PARK CITY FM,PHOSPHATE BEDS

MONTPELIER,IDAHO,USA

H572:GIRTY GH.USGS BULL 436, 1910,P32-33

REMARKS: D-WF FERRIER, 1920 ;LABELLED PARATYPE ; 1 SPEC

\section{PUNCTOSPIRIFER ? SP}

32115 [B634] REF SPEC-M

UP,KAZAN

50 M FROM ROAD LEADING W FROM HWY, KHAO

PHRIK, NR RATBURI, THAILAND

H714; WATERHOUSE JB + PIYASIN

S, 1970,PALAEONTOGRAPHICA,BD 135,ABT A,P155-156 REMARKS: C,D-JB WATERHOUSE, 1964 ;JBW NO B634; 1 SPEC

\section{PUNCTOSPIRIFER? SP}

32114 [B6 19] REF SPEC-F

UP.KAZAN

50 M FROM ROAD LEADING W FROM HWY , KHAO

PHRIK, NR RATBURI, THAILAND

H714:WATERHOUSE JB + PIYASIN

S, 1970,PALAEONTOGRAPHICA,BD 135,ABT A,P155156,PL29,FIG 6,7

REMARKS: C,D-JB WATERHOUSE, 1964 ;JBW NO B619; LARGER OF 2 SPECS FIG'D; 2 SPEC

RAFINESQUINA ALTERNATA (EMMONS)

17353 [1112HR] REF SPEC-F

U O.CINCI,MAYSVILL

DUNDAS FM

BLOOR ST CROSSING, HUMBER R,TORONTO,ONT

H388:PARKS \& DYER ONT DEPT MINES, VOL

30,PT7, 1922,P36-37,PL7,FIG 18

REMARKS: C-WA PARKS, 1921; 1 SPEC

RAFINESQUINA ALTERNATA VAR SUBCIRCULARIS DYER 12173 P-HOLO

U O,CINCI,MAYSVILL.

MEAFORD FM,ERINDALE MBR

NE OF HOME OF WM CROZIER CREDIT

R,STREETSVILLE,ONT

H388;DYER WS,ONT DEPT MINES, VOL 32,SECT

7,1925,P73,PL7,FIG 14

REMARKS: C-WS DYER;LOC-SEC 10 OF DYER; 1 SPEC

RAFINESQUINA MUCRONATA VAR TORONTONENSIS P +D 17355 [1114HR] P-SYN

U O,CINCI,MAYSVILL

DUNDAS FM

MILL RACE, NRHUMBERVALE QUARRY,ONT

H388:PARKS \& DYER, ONT DEPT MINES, VOL

30,PT 7, 1922,P38,PL7,FIG 17

REMARKS: C-WA PARKS, $1921 ; 1$ SPEC

RAFINESQUINA MUCRONATA VAR TORONTONENSIS P\&D

17354 [1113HR] P-SYN

U O,CINCI,MAYSVILL

DUNDAS FM

NR WATER LEVEL, BELOW ROAD BRIDGE, WESTON, ONT

H388;PARKS + DYER,ONT DEPT MINES, VOL

30,PT7, 1922, P38,PL7,FIG 13,14

REMARKS: C-WAPARKS, 1921 : SPEC 'A' = FIG 13:SPEC

' ${ }^{\prime}=$ FIG 14;PUBL'N GIVES LOC AS LAMBTON ; 2 SPEC

RAMAVECTUS N SP

32001 [B3 14] REF SPEC-F

UP.KAZAN

50 M FROM ROAD LEADING W FROM HWY, KHAO

PHRIK, NR RATBURI, THAILAND

H492; WATERHOUSE JB + PIYASIN

S, 1970,PALAEONTOGRAPHICA,BD 135,ABT A,P119120,PL20,FIG 12,22

REMARKS: C.D-JB WATERHOUSE, 1964 : JBW NO B314:

CONSIDERED BY WATERHOUSE + PIYASIN AS

SUPERFAMILY STROPHALOSIACEA,ORDER

PRODUCTIDA: 1 SPEC
RAMAVECTUS N SP

32002 [B316] REF SPEC-F

UP.KAZAN

50 M FROM ROAD LEADING W FROM HWY, KHAO

PHRIK,NR RATBURI,THAILAND

H492; WATERHOUSE JB + PIYASIN

S, 1970,PALAEONTOGRAPHICA,BD 135,ABT A,P119120,PL20,FIG 11

REMARKS: C,D-JB WATERHOUSE, 1964 ;JBW NO B3 16; CONSIDERED BY WATERHOUSE + PIYASIN AS SUPERFAMILY STROPHALOSIACEA,ORDER PRODUCTIDA;PL20,FIG 13 LISTED AS B3 16;SHOULD BE B3 $18 ; 1$ SPEC

\section{RAMAVECTUSN SP}

32003 [B3 17] REF SPEC-F

UP.KAZAN

50 M FROM ROAD LEADING W FROM HWY, KHAO

PHRIK,NR RATBURI,THAILAND

H492; WATERHOUSE JB + PIYASIN

S, 1970,PALAEONTOGRAPHICA,BD 135,ABT A,P119-120

REMARKS: C,D-JB WATERHOUSE, 1964 ;JBW NO B317;

CONSIDERED BY WATERHOUSE + PIYASIN AS

SUPERFAMILY STROPHALOSIACEA,ORDER

PRODUCTIDA : 1 SPEC

\section{RAMAVECTUSN SP}

32004 [B3 18] REF SPEC-F

UP KAZAN

50 M FROM ROAD LEADING W FROM HWY, KHAO

PHRIK, NR RATBURI,THAILAND

H492; WATERHOUSE JB + PIYASIN

S, 1970,PALAEONTOGRAPHICA,BD 135,ABT A,P119120,PL20,FIG 13

REMARKS: C,D-JB WATERHOUSE, 1964 ; JBW NO B318; CONSIDERED BY WATERHOUSE + PIYASIN AS SUPERFAMILY STROPHALOSIACEA,ORDER PRODUCTIDA;PL20,FIG 13 LISTED AS B316, ILLUSTRATES SPEC B318;PL20,FIG 9 LISTED AS B318, DOES NOT RESEMBLE SPEC ; 1 SPEC

\section{RAMAVECTUS N SP}

32005 [B3 19] REF SPEC-F

UP.KAZAN

50 M FROM ROAD LEADING W FROM HWY, KHAO

PHRIK, NR RATBURI,THAILAND

H492; WATERHOUSE JB + PIYASIN

S, 1970,PALAEONTOGRAPHICA,BD 135,ABT A,P119120.PL20,FIG 16,17

REMARKS: C,D-JB WATERHOUSE, 1964 ;JBW NO B3 19; CONSIDERED BY WATERHOUSE + PIYASIN AS SUPERFAMILY STROPHALOSIACEA,ORDER PRODUCTIDA; 1 SPEC

\section{RAMAVECTUS N SP}

32006 [B320 ] REF SPEC-F

$\cup P, K A Z A N$

50 M FROM ROAD LEADING W FROM HWY, KHAO

PHRIK, NR RATBURI, THAILAND

H492; WATERHOUSE JB + PIYASIN

S, 1970,PALAEONTOGRAPHICA.BD 135,ABT A,P119120,PL20,FIG 18

REMARKS: C,D-JB WATERHOUSE, 1964 ;JBW NO B320; CONSIDERED BY WATERHOUSE + PIYASIN AS SUPERFAMILY STROPHALOSIACEA,ORDER PRODUCTIDA ; 1 SPEC

RAMAVECTUS N SP

32007 [B322] REF SPEC-F

UP, KAZAN

50 M FROM ROAD LEADING W FROM HWY, KHAO

PHRIK, NA RATBURI, THAILAND

H492; WATERHOUSE JB + PIYASIN

S, 1970,PALAEONTOGRAPHICA,BD 135,ABT A,P 119 120,PL20,FIG 10,21

REMARK S: C.D-JB WATERHOUSE, 1964 ;JBW NO B322;

CONSIDERED BY WATERHOUSE + PIYASIN AS

SUPERFAMILY STROPHALOSIACEA,ORDER

PRODUCTIDA: 1 SPEC 
RAMAVECTUS N SP

32013 [B328] REF SPEC-F

UP KAZAN

50 M FROM ROAD LEADING W FROM HWY, KHAO

PHRIK, NR RATBURI,THAILAND

H492; WATERHOUSE JB + PIYASIN

S, 1970,PALAEONTOGRAPHICA,BD 135,ABT A,P119120.PL20,FIG 15,20

REMARKS: C.D-JB WATERHOUSE, 1964 ;JBW NO B328;

CONSIDERED BY WATERHOUSE + PIYASIN AS

SUPERFAMILY STROPHALOSIACEA,ORDER

PRODUCTIDA ; 1 SPEC

\section{RHIPIDOMELLA? SP}

31747 [B502] REF SPEC-F

UP, KAZAN

50 M FROM ROAD LEADING W FROM HWY, KHAO

PHRIK.NR RATBURI,THAILAND

H341; WATERHOUSE JB + PIYASIN

S. 1970.PALAEONTOGRAPHICA,BD 135,ABT A,P98-

99.PL14.FIG 1-3

REMARKS: C,D-JB WATERHOUSE, 1964 ;JBW NO B502; 1 SPEC

\section{RHIPIDOMELLA ? SP}

31748 [B586] REF SPEC-F

UP, KAZAN

50 M FROM ROAD LEADING W FROM HWY, KHAO

PHRIK,NR RATBURI,THAILAND

H341; WATERHOUSE JB + PIYASIN

S, 1970,PALAEONTOGRAPHICA,BD 135,ABT A,P98-

99, PL 14,FIG 4

REMARKS: C.D-JB WATERHOUSE, 1964 ;JBW NO B586; 1 SPEC

RHYNCHOSPIRA LOWI WHITEAVES

22634 [389S ] REF SPEC-M

$\mathrm{S}$.

[SILURIAN]

LIMESTONE RAPIDS, SEVERN R,DIST OF PATRICIA,ONT H652:PARKS WA, TRANS ROY CAN INST, VOL

11, PT 1, 1915, P50

REMARKS: C-JB TYRRELL, $1912+$ D-WH HEARST, $1915 ; 1$ SPEC

\section{RICHTHOFENIA SP}

31976 [B338 ] REF SPEC-F

UP.KAZAN

$50 \mathrm{M}$ FROM ROAD LEADING W FROM HWY, KHAO

PHRIK,NR RATBURI,THAILAND

H462.WATERHOUSE JB + PIYASIN

S, 1970,PALAEONTOGRAPHICA, BD 135,ABT A.P122123,PL21,FIG 1,3,4

REMARKS: C,D-JB WATERHOUSE, 1964 ;JBW NO B338 ORDER PRODUCTIDA ACCORDING TO WATERHOUSE + PIYASIN; 1 SPEC

\section{RICHTHOFENIA SP}

31975 [B337 ] REF SPEC-F

UP, KAZAN

50 M FROM ROAD LEADING W FROM HWY, KHAO

PHRIK,NR RATBURI,THAILAND

H462: WATERHOUSE JB + PIYASIN

S, 1970,PALAEONTOGRAPHICA,BD 135,ABT A,P122-

123,PL21,FIG 5,6

REMARKS: C.D-JB WATERHOUSE. 1964 ;JBW NO B337

ORDER PRODUCTIDA ACCORDING TO WATERHOUSE + PIYASIN ; 1 SPEC

RORESPIRIFER RUINOSUS WATERHOUSE+PIYASIN 32140 [B545] P-HOLO

UP.KAZAN

50 M FROM ROAD LEADING W FROM HWY, KHAO

PHRIK NR RATBURI,THAILAND

H---:WATERHOUSE JB + PIYASIN

S, 1970,PALAEONTOGRAPHICA,BD 135,ABT A,P156-

157. PL 29, FIG $10,14,15$

REMARKS: C.D-JB WATERHOUSE, 1964 ;JBW NO B545;NEW GENUS ; 1 SPEC (3 PCES)
RORESPIRIFER RUINOSUS WATERHOUSE+PIYASIN

32135 [B 499 ] P-PARA

UP.KAZAN

50 M FROM ROAD LEADING W FROM HWY , KHAO

PHRIK,NR RATBURI,THAILAND

H---:WATERHOUSE JB + PIYASIN

S, 1970,PALAEONTOGRAPHICA,BD 135,ABT A,P156-

157,PL29, FIG 8

REMARKS: C,D-JB WATERHOUSE, 1964 ;JBW NO B499;NEW

GENUS ; 1 SPEC

RORESPIRIFER RUINOSUS WATERHOUSE + PIYASIN 32136 [B500] P-PARA

UP.KAZAN

50 M FROM ROAD LEADING W FROM HWY, KHAO

PHRIK,NR RATBURI,THAILAND

H--.;WATERHOUSE JB + PIYASIN

S, 1970,PALAEONTOGRAPHICA,BD 135,ABT A,P156157.PL29,FIG 11

REMARKS: C.D-JB WATERHOUSE, 1964 ;JBW NO B500; NEW GENUS ; 1 SPEC (2 PCES)

RORESPIRIFER RUINOSUS WATERHOUSE + PIYASIN 32137 [B50 1] P-PARA

UP.KAZAN

50 M FROM ROAD LEADING W FROM HWY, KHAO

PHRIK,NR RATBURI,THAILAND

H---;WATERHOUSE JB + PIYASIN

S, 1970,PALAEONTOGRAPHICA,BD 135,ABT A,P 156-157

REMARKS: C,D-JB WATERHOUSE, 1964 ;JBW NO B50 1;NEW GENUS; ABOUT 10 FRAGS

RORESPIRIFER RUINOSUS WATERHOUSE + PIYASIN

32138 [B503] P.PARA

$\cup P, K A Z A N$

$50 M$ FROM ROAD LEADING W FROM HWY, KHAO

PHRIK,NR RATBURI,THAILAND

H---;WATERHOUSE JB + PIYASIN

S, 1970,PALAEONTOGRAPHICA,BD 135,ABT A,P156-

157,PL29,FIG 9,13

REMARKS: C,D-JB WATERHOUSE, 1964 ;JBW NO B503;NEW GENUS ; 1 SPEC

RORESPIRIFER RUINOSUS WATERHOUSE + PIYASIN 32139 [B544] P-PARA

UP,KAZAN

$50 M$ FROM ROAD LEADING W FROM HWY, KHAO

PHRIK,NR RATBURI,THAILAND

H---;WATERHOUSE JB + PIYASIN

S, 1970,PALAEONTOGRAPHICA,BD 135,ABT A,P156157.PL29,FIG 12

REMARKS: C,D-JB WATERHOUSE, 1964 ;JBW NO B544; NEW GENUS ; 1 SPEC

RUSTELLA MAJOR (MATTHEW)

8490 [860CM] P-HOLO

OBOLUS ? MAJORMATTHEW

$\mathrm{LCA}$

[U ETCHEMINIAN]

HANFORD BK, ST MARTINS,ST JOHN CO,NB

H297; WALCOTT CD, 1912,P328,FIG 19:MATTHEW

GF, 1890,P155,PL8,FIG 3

REMARKS: C-GF MATTHEW;D-MACKENZIE ; 1 SPEC

SCHIZAMBON PRISCUS MATTHEW

7950 [320CM ] P-SYN

$\mathrm{LO}$,

ST JOHN GP

[ST JOHN,BRETONIAN,DIV 3C2 ]

MCLEOD BK. BOISDALE,CAPE BRETON,NS

H288; WALCOTT CD, 1912,P623-624; MATTHEW GF, 1901,NB,P277-278,PL5,FIG 4A-D

REMARKS: C-GF MATTHEW:D-MACKENZIE :LABELLED

COTYPES:UNCERTAIN WHICH SPECS FIG'D ; 6 SPEC

SCHIZOCRANIA FILOSA (HALL)

17347 [1106HR] REF SPEC-F

U O.CINCI,MAYSVILL

DUNDAS FM

DON VALLEY QUARRY,TORONTO,ONT 
H283;PARKS \& DYER,ONT DEPT MINES, VOL

30,PT7, 1922,P28-29,PL7,FIG 4-5

REMARKS: C-J TOWNSEND; 2 SPEC

SEPTASTEGES ACANTHUS WATERHOUSE + PIYASIN 31991 [B302 ] P-PARA

UP, KAZAN

50 M FROM ROAD LEADING W FROM HWY, KHAO PHRIK,NR RATBURI,THAILAND

H---;WATERHOUSE JB + PIYASIN

S, 1970,PALAEONTOGRAPHICA,BD 135,ABT A,P 120 . 122.PL 19, FIG 17,20

REMARKS: C,D-JB WATERHOUSE, 1964 ; JBW NO B302; CONSIDERED BY WATERHOUSE + PIYASINAS

SUPERFAMILY STROPHALOSIACEA,ORDER

PRODUCTIDA;NEW GENUS ; 1 SPEC (2 PCES)

SEPTASTEGES ACANTHUS WATERHOUSE + PIYASIN 31992 [B303] P-PARA

$\cup P, K A Z A N$

50 M FROM ROAD LEADING W FROM HWY, KHAO

PHRIK, NR RATBURI,THAILAND H-..; WATERHOUSE JB + PIYASIN

S, 1970.PALAEONTOGRAPHICA,BD 135,ABT A,P 120-122

REMARKS: C,D-JB WATERHOUSE, 1964 ;JBW NO B303; CONSIDERED BY WATERHOUSE + PIYASIN AS

SUPERFAMILY STROPHALOSIACEA,ORDER

PRODUCTIDA;NEW GENUS ; 1 SPEC (2 PCES)

SEPTASTEGES ACANTHUS WATERHOUSE+PIYASIN 31993 [B304] P-PARA

UP, KAZAN

50 M FROM ROAD LEADING W FROM HWY, KHAO

PHRIK, NR RATBURI,THAILAND

H---;WATERHOUSE JB + PIYASIN

S, 1970,PALAEONTOGRAPHICA,BD 135,ABT A,P 120

122,PL19,FIG 15, 16

REMARKS: C,D-JB WATERHOUSE, 1964 ;JBW NO B304

CONSIDERED BY WATERHOUSE + PIYASIN AS

SUPERFAMILY STROPHALOSIACEA,ORDER

PRODUCTIDA;NEW GENUS ; 1 SPEC

\section{SEPTASTEGES ACANTHUS WATERHOUSE + PIYASIN}

31994 [B305] P-PARA

UP, KAZAN

50 M FROM ROAD LEADING W FROM HWY, KHAO

PHRIK,NR RATBURI, THAILAND

H---;WATERHOUSE JB + PIYASIN

S, 1970,PALAEONTOGRAPHICA,BD 135,ABT A,P 120. 122,PL 19, FIG 18

REMARKS: C.D-JB WATERHOUSE, 1964 ; JBW NO B305;

CONSIDERED BY WATERHOUSE + PIYASIN AS

SUPERFAMILY STROPHALOSIACEA,ORDER

PRODUCTIDA;NEW GENUS ; 1 SPEC

SEPTASTEGES ACANTHUS WATERHOUSE + PIYASIN 31995 [B306 ] P-PARA

UP,KAZAN

50 M FROM ROAD LEADING W FROM HWY, KHAO

PHRIK,NR RATBURI,THAILAND

H-..-WATERHOUSE JB + PIYASIN

S, 1970,PALAEONTOGRAPHICA,BD 135,ABT A,P120-122 REMARKS: C,D-JB WATERHOUSE, 1964 ;JBW NO B306;

CONSIDERED BY WATERHOUSE + PIYASIN AS

SUPERFAMILY STROPHALOSIACEA,ORDER

PRODUCTIDA;NEW GENUS ; 5 FRAGS

SEPTASTEGES ACANTHUS WATERHOUSE + PIYASIN

31996 [B307] P.PARA

UP,KAZAN

50 M FROM ROAD LEADING W FROM HWY, KHAO

PHRIK, NR RATBURI,THAILAND

H--;WATERHOUSE JB + PIYASIN

S, 1970 PALAEONTOGRAPHICA,BD 135,ABT A,P 120-122

REMARKS: C,D-JB WATERHOUSE, 1964 ; JBW NO B307;

CONSIDERED BYWATERHOUSE + PIYASINAS

SUPERFAMILY STROPHALOSIACEA,ORDER

PRODUCTIDA; NEW GENUS ; 1 SPEC
SEPTASTEGES ACANTHUS WATERHOUSE + PIYASIN 31997 [B308 ] P-PARA

UP,KAZAN

$50 M$ FROM ROAD LEADING W FROM HWY, KHAO PHRIK,NR RATBURI,THAILAND

H---;WATERHOUSE JB + PIYASIN

S, 1970,PALAEONTOGRAPHICA,BD 135,ABT A,P 120-122

REMARKS: C,D-JB WATERHOUSE, 1964 ; JBW NO B308

CONSIDERED BY WATERHOUSE + PIYASIN AS

SUPERFAMILY STROPHALOSIACEA, ORDER

PRODUCTIDA;NEW GENUS : 1 SPEC

SEPTASTEGES ACANTHUS WATERHOUSE + PIYASIN 31990 [B300] P-HOLO

$\cup P, K A Z A N$

$50 M$ FROM ROAD LEADING W FROM HWY, KHAO PHRIK,NR RATBURI,THAILAND

H-.-; WATERHOUSE JB + PIYASIN

S, 1970, PALAEONTOGRAPHICA,BD 135,ABT A,P 120

122. PL 19, FIG 13,14

REMARK S: C,D-JB WATERHOUSE, 1964 ;JBW NO B300

CONSIDERED BYWATERHOUSE + PIYASINAS

SUPERFAMILY STROPHALOSIACEA,ORDER

PRODUCTIDA;NEW GENUS ; 1 SPEC

SPIRIFER SULCATA MUT SUBMERSA GRABAU

REMARKS: SEE HOWELLELLA SUBMERSA (GRABAU)

SPIRIFERELLINA ADUNCATA WATERHOUSE + PIYASIN 31662 [B639] P-PARA

UP, KAZAN

$50 M$ FROM ROAD LEADING W FROM HWY, KHAO

PHRIK,NR RATBURI,THAILAND

H7 14; WATERHOUSE JB + PIYASIN

S, 1970,PALAEONTOGRAPHICA,BD 135,ABT A,P149-155

REMARKS: C,D-JB WATERHOUSE, 1964 ; JBW NO B639;

FAMILY SPIRIFERIDAE,SUBFAMILY NEOSPIRIFERINAE ACCORDING TO WATERHOUSE + PIYASIN; 1 SPEC (2 PCES)

SPIRIFERELLINA ADUNCATA WATERHOUSE + PIYASIN 31584 [B406 ] P.PARA

UP, KAZAN

50 M FROM ROAD LEADING W FROM HWY, KHAO

PHRIK,NR RATBURI,THAILAND

H714; WATERHOUSE JB + PIYASIN

S, 1970 PALAEONTOGRAPHICA BD 135 ABT A.P 149-155 REMARKS: C,D-JB WATERHOUSE, 1964 ;JBW NO B406;

FAMILY SPIRIFERIDAE,SUBFAMILY NEOSPIRIFERINAE ACCORDING TOWATERHOUSE + PIYASIN : 1 SPEC

SPIRIFERELLINA ADUNCATA WATERHOUSE + PIYASIN 31585 [B407] P-PARA

UP, KAZAN

50 M FROM ROAD LEADING W FROM HWY, KHAO

PHRIK NR RATBURI, THAILAND

H714; WATERHOUSE JB + PIYASIN

S, 1970.PALAEONTOGRAPHICA,BD 135, ABT A.P 149-155 REMARK S: C.D-JB WATERHOUSE, 1964 ; JBW NO B407: FAMILY SPIRIFERIDAE,SUBFAMILY NEOSPIRIFERINAE ACCORDING TO WATERHOUSE + PIYASIN ; 1 SPEC

SPIRIFERELLINA ADUNCATA WATERHOUSE + PIYASIN 31637 [B461] P-PARA

UP, KAZAN

$50 M$ FROM ROAD LEADING W FROM HWY, KHAO

PHRIK NR RATBURI, THAILAND

H7 14; WATERHOUSE JB + PIYASIN

S, 1970.PALAEONTOGRAPHICA.BD 135,ABT A.P149-155 REMARKS: C D-JB WATERHOUSE, 1964 ;JBW NO B461; FAMILY SPIRIFERIDAE,SUBFAMILY NEOSPIRIFERINAE ACCORDING TO WATERHOUSE + PIYASIN; 1 SPEC

SPIRIFERELLINA ADUNCATA WATERHOUSE + PIYASIN 31638 [B462] P-PARA

UP,KAZAN

50 M FROM ROAD LEADING W FROM HWY, KHAO

PHRIK NR RATBURI, THAILAND

H7 14: WATERHOUSE JB + PIYASIN

S, 1970,PALAEONTOGRAPHICA,BD 135,ABT A,P 149-155 
REMARKS: C.D-JB WATERHOUSE, 1964 : JBW NO B462: FAMILY SPIRIFERIDAE.SUBFAMILY NEOSPIRIFERINAE ACCORDING TO WATERHOUSE + PIYASIN ; 1 SPEC

SPIRIFERELLINA ADUNCATA WATERHOUSE + PIYASIN $31639[B 463]$ P.PARA

UP.KAZAN

50 M FROM ROAD LEADING W FROM HWY , KHAO PHRIK,NR RATBURI,THAILAND

H714:WATERHOUSE JB + PIYASIN

S. 1970,PALAEONTOGRAPHICA.BD 135,ABT A,P 149-155 REMARK S: C.D-JB WATERHOUSE, 1964 ; JBW NO B463: FAMILY SPIRIFERIDAE,SUBFAMILY NEOSPIRIFERINAE ACCORDING TO WATERHOUSE + PIYASIN : 1 SPEC

SPIRIFERELLINA ADUNCATA WATERHOUSE+PIYASIN

31640 [B464] P-PARA

UP KAZAN

50 M FROM ROAD LEADING W FROM HWY, KHAO

PHRIK,NR RATBURI,THAILAND

H714: WATERHOUSE JB + PIYASIN

S. 1970.PALAEONTOGRAPHICA.BD 135,ABT A.P149-155

REMARKS: C.D-JB WATERHOUSE, 1964 ; JBW NO B464; FAMILY SPIRIFERIDAE, SUBFAMILY NEOSPIRIFERINAE ACCORDING TO WATERHOUSE + PIYASIN ; 1 SPEC

SPIRIFERELLINA ADUNCATA WATERHOUSE+PIYASIN

31641 [B465 ] P-PARA

UP,KAZAN

50 M FROM ROAD LEADING W FROM HWY, KHAO

PHRIK, NR RATBURI,THAILAND

H7 14: WATERHOUSE JB + PIYASIN

S, 1970,PALAEONTOGRAPHICA,BD 135,ABT A,P 149-155

REMARKS: C.D-JB WATERHOUSE, 1964 ; JBW NO B465:

FAMILY SPIRIFERIDAE,NEOSPIRIFERINAE ACCORDING TO WATERHOUSE + PIYASIN ; 1 SPEC

SPIRIFERELLINA ADUNCATA WATERHOUSE+PIYASIN 31642 [B466] P-PARA

UP,KAZAN

$50 M$ FROM ROAD LEADING W FROM HWY, KHAO

PHRIK, NR RATBURI,THAILAND

H7 14: WATERHOUSE JB + PIYASIN

S, 1970.PALAEONTOGRAPHICA,BD 135,ABT A,P 149-155

REMARKS: C.D-JB WATERHOUSE, 1964 .JBW NO B466. FAMILY SPIRIFERIDAE,SUBFAMILY NEOSPIRIFERINAE ACCORDING TO WATERHOUSE + PIYASIN; 1 SPEC

SPIRIFERELLINA ADUNCATA WATERHOUSE + PIYASIN 31643 [B467] P-PARA

UP.KAZAN

$5 O M$ FROM ROAD LEADING W FROM HWY, KHAO

PHRIK, NR RATBURI,THAILAND

H7 14:WATERHOUSE JB + PIYASIN

S. 1970,PALAEONTOGRAPHICA,BD 135,ABT A,P 149-155

REMARK S: C,D-JB WATERHOUSE, 1964 ; JBW NO B467;

FAMILY SPIRIFERIDAE,SUBFAMILY NEOSPIRIFERINAE

ACCORDING TO WATERHOUSE + PIYASIN ; 1 SPEC

SPIRIFERELLINA ADUNCATA WATERHOUSE + PIYASIN

31644 [B468] P-PARA

UP.KAZAN

50 M FROM ROAD LEADING W FROM HWY, KHAO

PHRIK,NR RATBURI,THAILAND

H714. WATERHOUSE JB + PIYASIN

S. 1970,PALAEONTOGRAPHICA.BD 135,ABT A,P 149-155

REMARKS: C,D-JB WATERHOUSE, 1964 ; JBW NO B468;

FAMILY SPIRIFERIDAE,SUBFAMILY NEOSPIRIFERINAE

ACCORDING TO WATERHOUSE + PIYASIN:UNCERTAIN WHICH OF 2 SPEC MEASURED : 2 SPEC

SPIRIFERELLINA ADUNCATA WATERHOUSE + PIYASIN 31645 [B469] P-PARA

UP.KAZAN

$50 M$ FROM ROAD LEADING W FROM HWY, KHAO

PHRIK. NR RATBURI, THAILAND

H714:WATERHOUSE JB + PIYASIN

S, 1970.PALAEONTOGRAPHICA,BD 135,ABT A,P149-155

REMARKS: C.D-JB WATERHOUSE, 1964 ; JBW NO 8469;

FAMILY SPIRIFERIDAE, SUBFAMILY NEOSPIRIFERINAE

ACCORDING TOWATERHOUSE + PIYASIN , 1 SPEC
SPIRIFERELLINA ADUNCATA WATERHOUSE + PIYASIN

$31646[B 470]$ P-PARA

UP.KAZAN

50 M FROM ROAD LEADING W FROM HWY, KHAO

PHRIK,NRRATBURI,THAILAND

H7 14; WATERHOUSE JB + PIYASIN

S, 1970,PALAEONTOGRAPHICA.BD 135,ABT A.P 149

155.PL28,FIG 6,7,TEXT FIG 16A

REMARKS: C.D-JB WATERHOUSE, 1964 ; JBW NO B470;

FAMILY SPIRIFERIDAE SUBFAMILY NEOSPIRIFERINAE ACCORDING TO WATERHOUSE + PIYASIN : 1 SPEC

SPIRIFERELLINA ADUNCATA WATERHOUSE + PIYASIN

31647 [B471] P-PARA

UP.KAZAN

$50 \mathrm{M}$ FROM ROAD LEADING W FROM HWY , KHAO

PHRIK,NR RATBURI,THAILAND

H7 14:WATERHOUSE JB + PIYASIN

S, 1970,PALAEONTOGRAPHICA,BD 135,ABT A,P149-155

REMARKS: C,D-JB WATERHOUSE, 1964 : JBW NO B471

FAMILY SPIRIFERIDAE,SUBFAMILY NEOSPIRIFERINAE ACCORDING TO WATERHOUSE + PIYASIN ; 1 SPEC (3 PCES)

SPIRIFERELLINA ADUNCATA WATERHOUSE + PIYASIN

31648 [B472] P-PARA

UP,KAZAN

50 M FROM ROAD LEADING W FROM HWY, KHAO

PHRIK,NR RATBURI, THAILAND

H714; WATERHOUSE JB + PIYASIN

S, 1970.PALAEONTOGRAPHICA,BD 135,ABT A.P 149-155

REMARKS: C,D-JB WATERHOUSE, 1964 ; JBW NO B472; FAMILY SPIRIFERIDAE, SUBFAMILY NEOSPIRIFERINAE ACCORDING TOWATERHOUSE + PIYASIN ; 1 SPEC

SPIRIFERELLINA ADUNCATA WATERHOUSE + PIYASIN

31649 [B473] P-PARA

UP,KAZAN

50 M FROM ROAD LEADING W FROM HWY, KHAO

PHRIK, NR RATBURI, THAILAND

H714: WATERHOUSE JB + PIYASIN

S, 1970,PALAEONTOGRAPHICA,BD 135,ABT A,P149155,PL26, FIG 21,22

REMARKS: C.D-JB WATERHOUSE, 1964 ;JBW NO B473; FAMILY SPIRIFERIDAE,SUBFAMILY NEOSPIRIFERINAE ACCORDING TO WATERHOUSE + PIYASIN ; 1 SPEC

SPIRIFERELLINA ADUNCATA WATERHOUSE + PIYASIN 31650 [B474] P-PARA

UP.KAZAN

$5 O M$ FROM ROAD LEADING W FROM HWY, KHAO

PHRIK, NR RATBURI,THAILAND

H7 14: WATERHOUSE JB + PIYASIN

S, 1970,PALAEONTOGRAPHICA,BD 135,ABT A,P 149- 155

REMARKS: C,D-JB WATERHOUSE, 1964 ; JBW NO B474:

FAMILY SPIRIFERIDAE, SUBFAMILY NEOSPIRIFERINAE ACCORDING TO WATERHOUSE + PIYASIN; 1 SPEC

SPIRIFERELLINA ADUNCATA WATERHOUSE+PIYASIN 31651 [B475] P-PARA

UP.KAZAN

$50 M$ FROM ROAD LEADING W FROM HWY , KHAO

PHRIK,NR RATBURI,THAILAND

H714: WATERHOUSE JB + PIYASIN

S, 1970,PALAEONTOGRAPHICA,BD 135,ABT A,P 149155 PL29,FIG 1,2

REMARKS: C.D-JB WATERHOUSE, 1964 ;JBW NO B475; FAMILY SPIRIFERIDAE,SUBFAMILY NEOSPIRIFERINAE ACCORDING TOWATERHOUSE + PIYASIN ; 1 SPEC

SPIRIFERELLINA ADUNCATA WATERHOUSE + PIYASIN

31652 [B476] P-PARA

U P.KAZAN

50 M FROM ROAD LEADING W FROM HWY, KHAO

PHRIK,NR RATBURI,THAILAND

H714; WATERHOUSE JB + PIYASIN

S. 1970 PALAEONTOGRAPHICA.BD 135 ABT A.P 149-155

REMARKS: C.D-JB WATERHOUSE, 1964 ; JBW NO B476; FAMILY SPIRIFERIDAE,SUBFAMILY NEOSPIRIFERINAE ACCORDING TOWATERHOUSE + PIYASIN , 1 SPEC 
SPIRIFERELLINA ADUNCATA WATERHOUSE + PIYASIN 31653 [B477] P-PARA

UP.KAZAN

50 M FROM ROAD LEADING W FROM HWY, KHAO

PHRIK, NR RATBURI, THAILAND

H7 14: WATERHOUSE JB + PIYASIN

S, 1970,PALAEONTOGRAPHICA.BD 135,ABT A,P149155,PL28,FIG 1,3,TEXT FIG 16B

REMARKS: C.D-JB WATERHOUSE, 1964 ;JBW NO B477;

FAMILY SPIRIFERIDAE, SUBFAMILY NEOSPIRIFERINAE

ACCORDING TO WATERHOUSE + PIYASIN; 1 SPEC

SPIRIFERELLINA ADUNCATA WATERHOUSE + PIYASIN

31654 [B478] P-PARA

UP.KAZAN

50 M FROM ROAD LEADING W FROM HWY, KHAO

PHRIK,NR RATBURI, THAILAND

H714:WATERHOUSE JB + PIYASIN

S. 1970 PALAEONTOGRAPHICA,BD 135,ABT A.P149.155

REMARKS: C,D-JB WATERHOUSE, 1964 ; JBW NO B478:

FAMILY SPIRIFERIDAE,SUBFAMILY NEOSPIRIFERINAE

ACCORDING TO WATERHOUSE + PIYASIN ; 1 SPEC

SPIRIFERELLINA ADUNCATA WATERHOUSE + PIYASIN

31655 [B479] P-PARA

UP, KAZAN

50 M FROM ROAD LEADING W FROM HWY, KHAO

PHRIK,NR RATBURI, THAILAND

H714; WATERHOUSE JB + PIYASIN

S, 1970,PALAEONTOGRAPHICA,BD 135,ABT A,P 149-155

REMARKS: C.D-JB WATERHOUSE, 1964 :JBW NO B479;

FAMILY SPIRIFERIDAE, SUBFAMILY NEOSPIRIFERINAE

ACCORDING TO WATERHOUSE + PIYASIN; 1 SPEC

SPIRIFERELLINA ADUNCATA WATERHOUSE+PIYASIN 31657 [B481] P-PARA

UP.KAZAN

50 M FROM ROAD LEADING W FROM HWY, KHAO

PHRIK, NR RATBURI,THAILAND

H714. WATERHOUSE JB + PIYASIN

S, 1970,PALAEONTOGRAPHICA,BD 135,ABT A,P149155,PL28,FIG 5

REMARKS: C D-JB WATERHOUSE, 1964 -JBW NO B481; FAMILY SPIRIFERIDAE,SUBFAMILY NEOSPIRIFERINAE ACCORDING TO WATERHOUSE;SPEC FIG'D BUT NOT LISTED AS PARATYPE ; 1 SPEC (2 PCES)

SPIRIFERELLINA ADUNCATA WATERHOUSE+PIYASIN 31658 [B482] P-PARA?

UP,KAZAN

50 M FROM ROAD LEADING W FROM HWY, KHAO

PHRIK,NR RATBURI,THAILAND

H714:WATERHOUSE JB + PIYASIN

S, 1970,PALAEONTOGRAPHICA,BD 135,ABT A,P149-155

REMARKS: C,D-JB WATERHOUSE, 1964 ; JBW NO B482;

FAMILY SPIRIFERIDAE,SUBFAMILY NEOSPIRIFERINAE

ACCORDING TO WATERHOUSE;SPEC MAY BE FIG'D PL27,FIG 6;PLATE CAPTION GIVES NO NUMBER FOR THIS SPEC ; 1 SPEC (2 PCES)

SPIRIFERELLINA ADUNCATA WATERHOUSE + PIYASIN

31659 [B504] P-PARA

UP,KAZAN

50 M FROM ROAD LEADING W FROM HWY, KHAO

PHRIK, NR RATBURI, THAILAND

H7 14; WATERHOUSE JB + PIYASIN

S, 1970,PALAEONTOGRAPHICA,BD 135,ABT A,P149-155

REMARKS: C.D-JB WATERHOUSE, 1964 .JBW NO B504; FAMILY SPIRIFERIDAE,SUBFAMILY NEOSPIRIFERINAE ACCORDING TO WATERHOUSE;SPEC MEASURED BUT NOT LISTED AS PARATYPE : 1 SPEC

SPIRIFERELLINA ADUNCATA WATERHOUSE+PIYASIN

31660 [B505] P.PARA

U P,KAZAN

50 M FROM ROAD LEADING W FROM HWY, KHAO

PHRIK NR RATBURI, THAILAND

H7 14; WATERHOUSE JB + PIYASIN

S, 1970,PALAEONTOGRAPHICA,BD 135,ABT A,P149-155

REMARKS: C,D-JB WATERHOUSE, 1964 ;JBW NO B505;

FAMILY SPIRIFERIDAE, SUBFAMILY NEOSPIRIFERINAE

ACCORDING TO WATERHOUSE;SPEC MEASURED BUT

NOT LISTED AS PARATYPE ; 1 SPEC
SPIRIFERELLINA ADUNCATA WATERHOUSE+PIYASIN

$31661[B 638]$ P.PARA

UP.KAZAN

$50 \mathrm{M}$ FROM ROAD LEADING W FROM HWY, KHAO

PHRIK, NR RATBURI, THAILAND

H7 14; WATERHOUSE JB + PIYASIN

S, 1970,PALAEONTOGRAPHICA,BD 135,ABT A,P 149155,PL27,FIG 8,11

REMARKS: C,D-JB WATERHOUSE, 1964 ;JBW NO B638; FAMILY SPIRIFERIDAE, SUBFAMILY NEOSPIRIFERINAE ACCORDING TO WATERHOUSE + PIYASIN; 1SPEC

SPIRIFERELLINA ADUNCATA WATERHOUSE+PIYASIN 31586 [B408] P-PARA

$\cup P, K A Z A N$

50 M FROM ROAD LEADING W FROM HWY, KHAO

PHRIK,NR RATBURI,THAILAND

H7 14; WATERHOUSE JB + PIYASIN

S, 1970,PALAEONTOGRAPHICA,BD 135,ABT A,P149-155

REMARKS: C,D-JB WATERHOUSE, 1964 ;JBW NO B408;

FAMILY SPIRIFERIDAE, SUBFAMILY NEOSPIRIFERINAE

ACCORDING TO WATERHOUSE + PIYASIN; 1 SPEC

SPIRIFERELLINA ADUNCATA WATERHOUSE+PIYASIN 31587 [B409] P-PARA

UP,KAZAN

50 M FROM ROAD LEADING W FROM HWY, KHAO

PHRIK,NR RATBURI,THAILAND

H714; WATERHOUSE JB + PIYASIN

S, 1970,PALAEONTOGRAPHICA,BD 135,ABT A,P149-155

REMARKS: C,D-JB WATERHOUSE, 1964 ;JBW NO B409;

FAMILY SPIRIFERIDAEXSUBFAMILY NEOSPIRIFERINAE ACCORDING TO WATERHOUSE + PIYASIN ; 1 SPEC

SPIRIFERELLINA ADUNCATA WATERHOUSE+PIYASIN 31588 [B4 10] P-PARA

U P KAZAN

50 M FROM ROAD LEADING W FROM HWY, KHAO PHRIK,NR RATBURI,THAILAND

H714; WATERHOUSE JB + PIYASIN

S, 1970,PALAEONTOGRAPHICA,BD 135,ABT A,P149-155

REMARKS: C,D-JB WATERHOUSE, 1964 ;JBW NO B410; FAMILY SPIRIFERIDAE,SUBFAMILY NEOSPIRIFERINAE ACCORDING TO WATERHOUSE + PIYASIN ; 1 SPEC

SPIRIFERELLINA ADUNCATA WATERHOUSE+PIYASIN

31589 [B411] P-PARA

UP,KAZAN

$50 \mathrm{M}$ FROM ROAD LEADING W FROM HWY, KHAO PHRIK,NR RATBURI,THAILAND

H7 14: WATERHOUSE JB + PIYASIN

S, 1970,PALAEONTOGRAPHICA,BD 135,ABT A,P 149-155 REMARKS: C,D-JB WATERHOUSE, 1964 ;JBW NO B411; FAMILY SPIRIFERIDAE, SUBFAMILY NEOSPIRIFERINAE ACCORDING TO WATERHOUSE + PIYASIN ; 1 SPEC

SPIRIFERELLINA ADUNCATA WATERHOUSE+PIYASIN $31590[B 412]$ P-PARA

UP,KAZAN

50 M FROM ROAD LEADING W FROM HWY, KHAO

PHRIK NR RATBURI, THAILAND

H7 14; WATERHOUSE JB + PIYASIN

S, 1970,PALAEONTOGRAPHICA BD 135,ABT A.P 149-155

REMARK S: C,D-JB WATERHOUSE, 1964 ;JBW NO B412; FAMILY SPIRIFERIDAE, SUBFAMILY NEOSPIRIFERINAE ACCORDING TO WATERHOUSE + PIYASIN ; 1 SPEC

SPIRIFERELLINA ADUNCATA WATERHOUSE+PIYASIN

31591 [B4 13] P-PARA

UP.KAZAN

50 M FROM ROAD LEADING W FROM HWY, KHAO PHRIK, NR RATBURI,THAILAND

H7 14: WATERHOUSE JB + PIYASIN

S, 1970,PALAEONTOGRAPHICA,BD 135,ABT A,P 149-155

REMARKS: C.D-JB WATERHOUSE, 1964 ;JBW NO B4 13: FAMILY SPIRIFERIDAE,SUBFAMILY NEOSPIRIFERINAE ACCORDING TO WATERHOUSE + PIYASIN ; 1 SPEC 
SPIRIFERELLINA ADUNCATA WATERHOUSE + PIYASIN 31592 [B4 14] P.PARA

UP.KAZAN

50 M FROM ROAD LEADING W FROM HWY, KHAO

PHRIK,NR RATBURI,THAILAND

H7 14:WATERHOUSE JB + PIYASIN

S. 1970.PALAEONTOGRAPHICA.BD 135.ABT A.P 149-155

REMARKS: C,D-JB WATERHOUSE, 1964 ; JBW NO B4 14;

FAMILY SPIRIFERIDAE, SUBFAMILY NEOSPIRIFERINAE

ACCORDING TO WATERHOUSE + PIYASIN ; 1 SPEC

SPIRIFERELLINA ADUNCATA WATERHOUSE + PIYASIN

31593 [B415] P-PARA

UP.KAZAN

50 M FROM ROAD LEADING W FROM HWY, KHAO

PHRIK,NR RATBURI,THAILAND

H7 14:WATERHOUSE JB + PIYASIN

S, 1970,PALAEONTOGRAPHICA,BD 135,ABT A,P 149-155

REMARKS: C,D-JB WATERHOUSE, 1964 ; JBW NO B4 15:

FAMILY SPIRIFERIDAE, SUBFAMILY NEOSPIRIFERINAE

ACCORDING TO WATERHOUSE + PIYASIN ; 1 SPEC

SPIRIFERELLINA ADUNCATA WATERHOUSE+PIYASIN 31594 [B4 16 ] P-PARA

UP, KAZAN

$50 M$ FROM ROAD LEADING W FROM HWY, KHAO

PHRIK,NR RATBURI,THAILAND

H714:WATERHOUSE JB + PIYASIN

S, 1970,PALAEONTOGRAPHICA,BD 135,ABT A,P149-155

REMARKS: C,D-JB WATERHOUSE, 1964 ; JBW NO B4 16;

FAMILY SPIRIFERIDAE, SUBFAMILY NEOSPIRIFERINAE

ACCORDING TO WATERHOUSE + PIYASIN ; 1 SPEC

SPIRIFERELLINA ADUNCATA WATERHOUSE + PIYASIN 31595 [B4 17 ] P-PARA

UP, KAZAN

$50 M$ FROM ROAD LEADING W FROM HWY, KHAO

PHRIK,NR RATBURI,THAILAND

H7 14; WATERHOUSE JB + PIYASIN

S, 1970,PALAEONTOGRAPHICA,BD 135,ABT A,P149-155

REMARKS: C.D-JB WATERHOUSE, 1964 ; JBW NO B4 17;

FAMILY SPIRIFERIDAE,SUBFAMILY NEOSPIRIFERINAE

ACCORDING TO WATERHOUSE + PIYASIN ; 1 SPEC

SPIRIFERELLINA ADUNCATA WATERHOUSE+PIYASIN 31596 [B4 18 ] P-PARA

UP, KAZAN

$50 M$ FROM ROAD LEADING W FROM HWY, KHAO

PHRIK,NR RATBURI,THAILAND

H714; WATERHOUSE JB + PIYASIN

S. 1970,PALAEONTOGRAPHICA,BD 135,ABT A,P 149-155

REMARKS: C,D-JB WATERHOUSE, 1964 ; JBW NO B4 18

FAMILY SPIRIFERIDAE, SUBFAMILY NEOSPIRIFERINAE

ACCORDING TO WATERHOUSE + PIYASIN ; 1 SPEC

SPIRIFERELLINA ADUNCATA WATERHOUSE + PIYASIN 31597 [B4 19 ] P-PARA

UP,KAZAN

$50 M$ FROM ROAD LEADING W FROM HWY, KHAO

PHRIK, NR RATBURI, THAILAND

H7 14:WATERHOUSE JB + PIYASIN

S, 1970,PALAEONTOGRAPHICA,BD 135.ABT A.P149-155

REMARKS: C,D-JB WATERHOUSE, 1964 ; JBW NO B4 19;

FAMILY SPIRIFERIDAE,SUBFAMILY NEOSPIRIFERINAE

ACCORDING TO WATERHOUSE + PIYASIN ; 1 SPEC

SPIRIFERELLINA ADUNCATA WATERHOUSE+PIYASIN

31598 [B420] P.PARA

UP.KAZAN

$5 O M$ FROM ROAD LEADING W FROM HWY, KHAO

PHRIK NR RATBURI, THAILAND

H714; WATERHOUSE JB + PIYASIN

S. 1970,PALAEONTOGRAPHICA,BD 135,ABT A,P 149-155

REMARKS: C,D-JB WATERHOUSE, 1964 ;JBW NO B420;

FAMILY SPIRIFERIDAE,SUBFAMILY NEOSPIRIFERINAE

ACCORDING TO WATERHOUSE + PIYASIN ; 1 SPEC
SPIRIFERELLINA ADUNCATA WATERHOUSE + PIYASIN $31599[B 421]$ P-PARA

UP.KAZAN

50 M FROM ROAD LEADING W FROM HWY, KHAO

PHRIK.NR RATBURI, THAILAND

H714; WATERHOUSE JB + PIYASIN

S, 1970,PALAEONTOGRAPHICA.BD 135,ABT A.P 149-155

REMARKS: C,D-JB WATERHOUSE, 1964 ;JBW NO B421;

FAMILY SPIRIFERIDAE, SUBFAMILY NEOSPIRIFERINAE

ACCORDING TO WATERHOUSE + PIYASIN ; 1 SPEC

SPIRIFERELLINA ADUNCATA WATERHOUSE + PIYASIN

31600 [B422] P-PARA

UP.KAZAN

$50 M$ FROM ROAD LEADING W FROM HWY , KHAO

PHRIK NR RATBURI,THAILAND

H714:WATERHOUSE JB + PIYASIN

S, 1970,PALAEONTOGRAPHICA,BD 135.ABT A,P 149-155 REMARKS: C.D-JB WATERHOUSE, 1964 ; JBW NO B422:

FAMILY SPIRIFERIDAE,SUBFAMILY NEOSPIRIFERINAE

ACCORDING TO WATERHOUSE + PIYASIN ; 1SPEC

SPIRIFERELLINA ADUNCATA WATERHOUSE + PIYASIN

31601 [B423] P-PARA

UP,KAZAN

50 M FROM ROAD LEADING W FROM HWY, KHAO

PHRIK,NR RATBURI,THAILAND

H714.WATERHOUSE JB + PIYASIN

S, 1970,PALAEONTOGRAPHICA,BD 135,ABT A,P 149-155

REMARKS: C.D-JB WATERHOUSE, 1964 :JBW NO B423.

FAMILY SPIRIFERIDAE, SUBFAMILY NEOSPIRIFERINAE

ACCORDING TO WATERHOUSE + PIYASIN ; 1 SPEC

SPIRIFERELLINA ADUNCATA WATERHOUSE + PIYASIN

31602 [B424] P-PARA

UP,KAZAN

50 M FROM ROAD LEADING W FROM HWY, KHAO

PHRIK,NR RATBURI,THAILAND

H7 14. WATERHOUSE JB + PIYASIN

S, 1970,PALAEONTOGRAPHICA,BD 135,ABT A,P149-155 REMARKS: C,D-JB WATERHOUSE, 1964 ; JBW NO B424;

FAMILY SPIRIFERIDAE, SUBFAMILY NEOSPIRIFERINAE

ACCORDING TO WATERHOUSE + PIYASIN ; 1 SPEC

SPIRIFERELLINA ADUNCATA WATERHOUSE + PIYASIN 31603 [B425] P-PARA

UP, KAZAN

$50 M$ FROM ROAD LEADING W FROM HWY, KHAO

PHRIK,NR RATBURI, THAILAND

H7 14; WATERHOUSE JB + PIYASIN

S, 1970,PALAEONTOGRAPHICA,BD 135,ABT A,P 149155,PL29,FIG 5

REMARKS: C,D-JB WATERHOUSE, 1964 ;JBW NO B425

FAMILY SPIRIFERIDAE.SUBFAMILY NEOSPIRIFERINAE ACCORDING TO WATERHOUSE + PIYASIN; 1 SPEC

SPIRIFERELLINA ADUNCATA WATERHOUSE+PIYASIN 31604 [B426] P-PARA

UP, KAZAN

50 M FROM ROAD LEADING W FROM HWY, KHAO

PHRIK,NR RATBURI,THAILAND H714: WATERHOUSE JB + PIYASIN

S, 1970,PALAEONTOGRAPHICA,BD 135, ABT A P 149-155

REMARKS: C,D-JB WATERHOUSE, 1964 ;JBW NO B426

FAMILY SPIRIFERIDAE,SUBFAMILY NEOSPIRIFERINAE

ACCORDING TO WATERHOUSE + PIYASIN ; 1 SPEC

SPIRIFERELLINA ADUNCATA WATERHOUSE + PIYASIN 31605 [B427] P-PARA

UP.KAZAN

50 M FROM ROAD LEADING W FROM HWY, KHAO

PHRIK NR RATBURI, THAILAND

H7 14: WATERHOUSE JB + PIYASIN

S, 1970,PALAEONTOGRAPHICA,BD 135,ABT A,P 149-155

REMARKS: C.D-JB WATERHOUSE, 1964 ; JBW NO B427

FAMILY SPIRIFERIDAE, SUBFAMILY NEOSPIRIFERINAE

ACCORDING TO WATERHOUSE + PIYASIN; 1 SPEC 
SPIRIFERELLINA ADUNCATA WATERHOUSE+ PIYASIN 31606 [B428 ] P-PARA

UP, KAZAN

50 M FROM ROAD LEADING W FROM HWY, KHAO

PHRIK, NR RATBURI, THAILAND

H714; WATERHOUSE JB + PIYASIN

S, 1970,PALAEONTOGRAPHICA,BD 135,ABT A,P149-155

REMARKS: C.D-JB WATERHOUSE, 1964 ; JBW NO B428;

FAMILY SPIRIFERIDAE, SUBFAMILY NEOSPIRIFERINAE ACCORDING TO WATERHOUSE + PIYASIN ; 1 SPEC (BROKEN)
SPIRIFERELLINA ADUNCATA WATERHOUSE + PIYASIN 31613 [B435] P-PARA

U P, KAZAN

50 M FROM ROAD LEADING W FROM HWY, KHAO

PHRIK,NR RATBURI,THAILAND

H714; WATERHOUSE JB + PIYASIN

S, 1970,PALAEONTOGRAPHICA,BD 135,ABT A,P149155, PL27, FIG 1-5

REMARKS: C,D-JB WATERHOUSE, 1964 ; JBW NO B435; FAMILY SPIRIFERIDAE, SUBFAMILY NEOSPIRIFERINAE ACCORDING TO WATERHOUSE + PIYASIN ; 1 SPEC

SPIRIFERELLINA ADUNCATA WATERHOUSE + PIYASIN 31614 [B436] P-PARA

U P, KAZAN

50 M FROM ROAD LEADING W FROM HWY, KHAO PHRIK, NR RATBURI,THAILAND H714; WATERHOUSE JB + PIYASIN

S, 1970,PALAEONTOGRAPHICA,BD 135,ABT A.P 149-155 REMARKS: C,D-JB WATERHOUSE, 1964 ; JBW NO B436; FAMILY SPIRIFERIDAE, SUBFAMILY NEOSPIRIFERINAE ACCORDING TO WATERHOUSE + PIYASIN; 1 SPEC

SPIRIFERELLINA ADUNCATA WATERHOUSE + PIYASIN 31615 [B437] P-PARA

UP, KAZAN

50 M FROM ROAD LEADING W FROM HWY, KHAO PHRIK,NR RATBURI,THAILAND H714:WATERHOUSE JB + PIYASIN

S, 1970,PALAEONTOGRAPHICA,BD 135,ABT A,P 149-155

REMARKS: C.D-JB WATERHOUSE, 1964 ; JBW NO B437; FAMILY SPIRIFERIDAE, SUBFAMILY NEOSPIRIFERINAE ACCORDING TO WATERHOUSE;UNCERTAIN WHICH SPEC MEASURED ; 2 SPEC

SPIRIFERELLINA ADUNCATA WATERHOUSE + PIYASIN 31616 [B438 ] P-PARA

UP,KAZAN

50 M FROM ROAD LEADING W FROM HWY, KHAO PHRIK,NR RATBURI,THAILAND

H714: WATERHOUSE JB + PIYASIN

S, 1970,PALAEONTOGRAPHICA,BD 135,ABT A,P149-155 REMARKS: C,D-JB WATERHOUSE, 1964 ;JBW NO B438;

FAMILY SPIRIFERIDAE, SUBFAMILY NEOSPIRIFERINAE ACCORDING TO WATERHOUSE + PIYASIN ; 1 SPEC

SPIRIFERELLINA ADUNCATA WATERHOUSE + PIYASIN 31617 [B440] P-PARA

UP,KAZAN

$50 M$ MROM ROAD LEADING W FROM HWY, KHAO PHRIK,NR RATBURI,THAILAND H714; WATERHOUSE JB + PIYASIN

S, 1970, PALAEONTOGRAPHICA,BD 135, ABT A,P 149-155 REMARKS: C.D-JB WATERHOUSE, 1964 ; JBW NO B440; FAMILY SPIRIFERIDAE, SUBFAMILY NEOSPIRIFERINAE ACCORDING TO WATERHOUSE + PIYASIN ; 1 SPEC

\section{SPIRIFERELLINA ADUNCATA WATERHOUSE+PIYASIN} 31618 [B441] P-PARA UP.KAZAN 50 M FROM ROAD LEADING W FROM HWY, KHAO PHRIK,NR RATBURI,THAILAND H714:WATERHOUSE JB + PIYASIN

S. 1970,PALAEONTOGRAPHICA,BD 135,ABT A,P 149-155 REM ARK S: C,D-JB WATERHOUSE, $1964 ;$ JBW NO B441 FAMILY SPIRIFERIDAE, SUBFAMILY NEOSPIRIFERINAE ACCORDING TO WATERHOUSE + PIYASIN ; 1 SPEC

SPIRIFERELLINA ADUNCATA WATERHOUSE + PIYASIN 31619 [B442] P-PARA

UP, KAZAN

$50 M$ FROM ROAD LEADING W FROM HWY, KHAO PHRIK,NR RATBURI,THAILAND H7 14:WATERHOUSE JB + PIYASIN

S, 1970,PALAEONTOGRAPHICA,BD 135,ABT A,P149-155 REMARKS: C D-JB WATERHOUSE, 1964 : JBW NO B442; FAMILY SPIRIFERIDAE,SUBFAMILY NEOSPIRIFERINAE ACCORDING TO WATERHOUSE + PIYASIN ; 1 SPEC 
SPIRIFERELLINA ADUNCATA WATERHOUSE + PIYASIN 31620 [B443 ] P-PARA

UP,KAZAN

50 M FROM ROAD LEADING W FROM HWY, KHAO

PHRIK.NR RATBURI,THAILAND

H7 14:WATERHOUSE JB + PIYASIN

S. 1970.PALAEONTOGRAPHICA,BD 135,ABT A.P 149- 155 REMARKS: C.D-JB WATERHOUSE, 1964 ; JBW NO B443; FAMILY SPIRIFERIDAE.SUBFAMILY NEOSPIRIFERINAE ACCORDING TO WATERHOUSE + PIYASIN; 1 SPEC

SPIRIFERELLINA ADUNCATA WATERHOUSE + PIYASIN 31621 [B445] P-PARA

UP,KAZAN

50 M FROM ROAD LEADING W FROM HWY, KHAO

PHRIK,NR RATBURI, THAILAND

H714:WATERHOUSE JB + PIYASIN

S. 1970.PALAEONTOGRAPHICA,BD 135,ABT A.P 149-155 REMARKS: C,D-JB WATERHOUSE, 1964 ;JBW NO B445; FAMILY SPIRIFERIDAE, SUBFAMILY NEOSPIRIFERINAE ACCORDING TO WATERHOUSE + PIYASIN ; 1 SPEC

SPIRIFERELLINA ADUNCATA WATERHOUSE + PIYASIN 31622 [B446] P-PARA

UP KAZAN

$50 M$ FROM ROAD LEADING W FROM HWY, KHAO

PHRIK.NR RATBURI,THAILAND

H714; WATERHOUSE JB + PIYASIN

S. 1970,PALAEONTOGRAPHICA.BD 135,ABT A.P 149-155

REMARKS: C.D-JB WATERHOUSE, 1964 ;JBW NO B446; FAMILY SPIRIFERIDAE, SUBFAMILY NEOSPIRIFERINAE ACCORDING TO WATERHOUSE + PIYASIN ; 1 SPEC

SPIRIFERELLINA ADUNCATA WATERHOUSE + PIYASIN

31623 [B447] P.PARA

UP, KAZAN

50 M FROM ROAD LEADING W FROM HWY, KHAO

PHRIK,NR RATBURI, THAILAND

H714: WATERHOUSE JB + PIYASIN

S, 1970,PALAEONTOGRAPHICA,BD 135,ABT A,P 149-155

REMARKS: C.D-JB WATERHOUSE, 1964 ; JBW NO B447;

FAMILY SPIRIFERIDAE,SUBFAMILY NEOSPIRIFERINAE

ACCORDING TO WATERHOUSE + PIYASIN ; 1 SPEC

SPIRIFERELLINA ADUNCATA WATERHOUSE+PIYASIN

31624 [B448] P-PARA

U P.KAZAN

50 M FROM ROAD LEADING W FROM HWY, KHAO

PHRIK,NR RATBURI,THAILAND

H714; WATERHOUSE JB + PIYASIN

S. 1970,PALAEONTOGRAPHICA,BD 135,ABT A,P149-155

REMARKS: C,D-JB WATERHOUSE, 1964 ;JBW NO B448;

FAMILY SPIRIFERIDAE,SUBFAMILY NEOSPIRIFERINAE

ACCORDING TO WATERHOUSE + PIYASIN; 1 SPEC

SPIRIFERELLINA ADUNCATA WATERHOUSE + PIYASIN

31625 [B449] P-PARA

UP,KAZAN

$5 O M$ MROM ROAD LEADING W FROM HWY, KHAO

PHRIK NR RATBURI, THAILAND

H7 14:WATERHOUSE JB + PIYASIN

S. 1970,PALAEONTOGRAPHICA,BD 135,ABT A,P 149-155

REMARKS: C,D-JB WATERHOUSE, 1964 ;JBW NO B449;

FAMILY SPIRIFERIDAE,SUBFAMILY NEOSPIRIFERINAE

ACCORDING TO WATERHOUSE + PIYASIN ; 1 SPEC

SPIRIFERELLINA ADUNCATA WATERHOUSE+PIYASIN

$31626[B 450]$ P-PARA

UP.KAZAN

50 M FROM ROAD LEADING W FROM HWY, KHAO

PHRIK,NR RATBURI,THAILAND

H714: WATERHOUSE JB + PIYASIN

S. 1970,PALAEONTOGRAPHICA,BD 135, ABT A,P149155.PL29,FIG 3,4

REMARKS:C.D.JB WATERHOUSE, 1964 :JBW NO B450; FAMILY SPIRIFERIDAE, SUBFAMILY NEOSPIRIFERINAE ACCORDING TOWATERHOUSE + PIYASIN ; 1 SPEC
SPIRIFERELLINA ADUNCATA WATERHOUSE + PIYASIN 31627 [B451] P.PARA

UP.KAZAN

50 M FROM ROAD LEADING W FROM HWY, KHAO

PHRIK, NR RATBURI, THAILAND

H7 14:WATERHOUSE JB + PIYASIN

S, 1970,PALAEONTOGRAPHICA,BD 135,ABT A,P149. 155,PL27,FIG 7,9

REMARKS: C.D-JB WATERHOUSE, 1964 ;JBW NO B451; FAMILY SPIRIFERIDAE, SUBFAMILY NEOSPIRIFERINAE ACCORDING TO WATERHOUSE + PIYASIN ; 1 SPEC

SPIRIFERELLINA ADUNCATA WATERHOUSE + PIYASIN 31628 [B452] P-PARA

UP.KAZAN

$50 M$ FROM ROAD LEADING W FROM HWY, KHAO PHRIK,NR RATBURI,THAILAND H7 14; WATERHOUSE JB + PIYASIN

S, 1970,PALAEONTOGRAPHICA,BD 135,ABT A,P 149-155

REMARKS: C,D-JB WATERHOUSE, 1964 ;JBW NO B452; FAMILY SPIRIFERIDAE, SUBFAMILY NEOSPIRIFERINAE ACCORDING TO WATERHOUSE + PIYASIN ; 1 SPEC

SPIRIFERELLINA ADUNCATA WATERHOUSE + PIYASIN 31629 [B453 ] P-HOLO

UP, KAZAN

$50 M$ FROM ROAD LEADING W FROM HWY KHAO

PHRIK,NR RATBURI,THAILAND

H714; WATERHOUSE JB + PIYASIN

S, 1970,PALAEONTOGRAPHICA,BD 135,ABT A,P149155,PL27,FIG 13,15

REMARKS: C,D-JB WATERHOUSE, 1964 ;JBW NO B453; FAMILY SPIRIFERIDAE,SUBFAMILY NEOSPIRIFERINAE ACCORDING TO WATERHOUSE + PIYASIN; 1 SPEC + FRAG

SPIRIFERELLINA ADUNCATA WATERHOUSE + PIYASIN 31630 [B454] P-PARA

UP, KAZAN

50 M FROM ROAD LEADING W FROM HWY, KHAO PHRIK,NR RATBURI,THAILAND H714; WATERHOUSE JB + PIYASIN

S, 1970 PALAEONTOGRAPHICA BD 135,ABT A P 149-155 REMARKS: C,D-JB WATERHOUSE, 1964 ; JBW NO B454; FAMILY SPIRIFERIDAE, SUBFAMILY NEOSPIRIFERINAE ACCORDING TO WATERHOUSE + PIYASIN ; 1SPEC

SPIRIFERELLINA ADUNCATA WATERHOUSE+PIYASIN 31631 [B455] P-PARA

UP,KAZAN

50 M FROM ROAD LEADING W FROM HWY, KHAO

PHRIK,NR RATBURI,THAILAND

H7 14:WATERHOUSE JB + PIYASIN

S, 1970,PALAEONTOGRAPHICA,BD 135,ABT A,P 149-155 REMARKS: C,D-JB WATERHOUSE, 1964 ;JBW NO B455: FAMILY SPIRIFERIDAE,SUBFAMILY NEOSPIRIFERINAE ACCORDING TO WATERHOUSE + PIYASIN ; 1 SPEC

SPIRIFERELLINA ADUNCATA WATERHOUSE+PIYASIN 31632 [B456] P-PARA

UP. KAZAN

$50 M$ FROM ROAD LEADING W FROM HWY, KHAO PHRIK,NR RATBURI,THAILAND H714:WATERHOUSE JB + PIYASIN

S, 1970,PALAEONTOGRAPHICA,BD 135,ABT A,P 149-155 REMARKS: C.D-JB WATERHOUSE, 1964 .JBW NO B456: FAMILY SPIRIFERIDAE, SUBFAMILY NEOSPIRIFERINAE ACCORDING TO WATERHOUSE + PIYASIN : 1 SPEC

SPIRIFERELLINA ADUNCATA WATERHOUSE + PIYASIN 31633 [B457] P-PARA

UP.KAZAN

50 M FROM ROAD LEADING W FROM HWY, KHAO PHRIK,NR RATBURI,THAILAND H7 14; WATERHOUSE JB + PIYASIN

S. 1970,PALAEONTOGRAPHICA.BD 135.ABT A.P 149-155 REMARKS: C.D-JB WATERHOUSE, 1964 .JBW NO B457: FAMILY SPIRIFERIDAE, SUBFAMILY NEOSPIRIFERINAE ACCORDING TO WATERHOUSE + PIYASIN : 1SPEC 
SPIRIFERELLINA ADUNCATA WATERHOUSE + PIYASIN

31634 [B458 ] P.PARA

UP.KAZAN

50 M FROM ROAD LEADING W FROM HWY, KHAO

PHRIK,NR RATBURI,THAILAND

H714: WATERHOUSE JB + PIYASIN

S, 1970,PALAEONTOGRAPHICA,BD 135.ABT A,P149-155

REMARKS: C.D-JB WATERHOUSE, 1964 ; JBW NO B458

FAMILY SPIRIFERIDAE.SUBFAMILY SPIRIFERINAE

ACCORDING TO WATERHOUSE + PIYASIN; 1 SPEC

SPIRIFERELLINA ADUNCATA WATERHOUSE + PIYASIN

31635 [B459] P-PARA

UP,KAZAN

50 M FROM ROAD LEADING W FROM HWY, KHAO

PHRIK,NR RATBURI,THAILAND

H7 14:WATERHOUSE JB + PIYASIN

S, 1970,PALAEONTOGRAPHICA,BD 135,ABT A,P 149-155 REMARKS: C,D-JB WATERHOUSE, 1964 ;JBW NO B459;

FAMILY SPIRIFERIDAE, SUBFAMILY NEOSPIRIFERINAE

ACCORDING TO WATERHOUSE + PIYASIN; 1 SPEC

SPIRIFERELLINA ADUNCATA WATERHOUSE + PIYASIN

31636 [B460] P-PARA

UP,KAZAN

$50 M$ FROM ROAD LEADING W FROM HWY, KHAO

PHRIK,NR RATBURI,THAILAND

H714:WATERHOUSE JB + PIYASIN

S, 1970,PALAEONTOGRAPHICA,BD 135,ABT A,P149-155 REMARKS: C,D-JB WATERHOUSE, 1964 ;JBW NO B460;

FAMILY SPIRIFERIDAE, SUBFAMILY NEOSPIRIFERINAE

ACCORDING TO WATERHOUSE + PIYASIN : 1 SPEC

\section{STREPTORHYNCHUS SP}

31729 [B 138] REF SPEC-F

UP, KAZAN

50 M FROM ROAD LEADING W FROM HWY, KHAO

PHRIK,NR RATBURI,THAILAND

H409:WATERHOUSE JB + PIYASIN

S, 1970,PALAEONTOGRAPHICA,BD 135,ABT A,P103105,PL 15,FIG 27,28

REMARKS: C,D-JB WATERHOUSE, 1964 ;JBW NO B138; 1 SPEC

\section{STREPTORHYNCHUS SP}

31730 [B 189] REF SPEC-F

UP,KAZAN

50 M FROM ROAD LEADING W FROM HWY, KHAO

PHRIK, NR RATBURI, THAILAND

H409. WATERHOUSE JB + PIYASIN

S, 1970,PALAEONTOGRAPHICA,BD 135,ABT A,P103105, PL 15, FIG 32,33

REMARKS: C,D-JB WATERHOUSE, 1964 ;JBW NO B 189; 1 SPEC + FRAG

\section{STREPTORHYNCHUS SP}

31731 [B565] REF SPEC-F

UP, KAZAN

50 M FROM ROAD LEADING W FROM HWY, KHAO

PHRIK, NR RATBURI, THAILAND

H409:WATERHOUSE JB + PIYASIN

S, 1970,PALAEONTOGRAPHICA,BD 135,ABT A,P 103105,PL15,FIG 29

REMARKS: C,D-JB WATERHOUSE, 1964 ;JBW NO B565; 1 SPEC

\section{STREPTORHYNCHUS SP}

31733 [B567] REF SPEC-F

UP, KAZAN

50 M FROM ROAD LEADING W FROM HWY, KHAO

PHRIK,NR RATBURI,THAILAND

H409: WATERHOUSE JB + PIYASIN

S. 1970,PALAEONTOGRAPHICA BD 135,ABT A.P 103105. PL 15,FIG 30,31, TEXT FIG 3C

REMARKS: C.D-JB WATERHOUSE, 1964 :JBW NO B567 : 1 SPEC
STREPTORHYNCHUS SP

31727 [B 134] REF SPEC-M

UP.KAZAN

$50 M$ FROM ROAD LEADING W FROM HWY, KHAO

PHRIK, NR RATBURI,THAILAND

H409; WATERHOUSE JB + PIYASIN

S. 1970,PALAEONTOGRAPHICA,BD 135,ABT A,P 103-105

REMARK S: C.D-JB WATERHOUSE, 1964 . JBW NO B 134

UNCERTAIN WHICH OF 2 SPEC MEASURED ; 2 SPEC IN MATRIX

\section{STREPTORHYNCHUS SP}

31728 [B 136] REF SPEC-F

UP.KAZAN

$50 \mathrm{M}$ FROM ROAD LEADING W FROM HWY, KHAO

PHRIK,NR RATBURI,THAILAND

H409:WATERHOUSE JB + PIYASIN

S. 1970,PALAEONTOGRAPHICA,BD 135,ABT A,P 103. 105.PL 15, FIG 34,35

REMARKS: C,D-JB WATERHOUSE, 1964 ;JBW NO B 136 ; 1 SPEC

STRIOCHONETES SCUTELLA WATERHOUSE + PIYASIN

31781 [B240] P-PARA

UP KAZAN

50 M FROM ROAD LEADING W FROM HWY, KHAO

PHRIK, NR RATBURI,THAILAND

H-...WATERHOUSE JB + PIYASIN

S. 1970,PALAEONTOGRAPHICA,BD 135,ABT A,P106-

112,PL $16, F I G 27,28$

REMARKS: C,D-JB WATERHOUSE, 1964 ;JBW NO B240;NEW

GENUS:ORDER PRODUCTIDA,SUBFAMILY

STRIOCHONETINAE ACCORDING TO WATERHOUSE +

PIYASIN : 1 SPEC

STRIOCHONETES SCUTELLA WATERHOUSE + PIYASIN 31782 [B241] P-PARA

U P.KAZAN

50 M FROM ROAD LEADING W FROM HWY, KHAO

PHRIK,NR RATBURI,THAILAND

H--.;WATERHOUSE JB + PIYASIN

S, 1970,PALAEONTOGRAPHICA,BD 135,BAT A,P 106-112

REMARK S: C.D-JB WATERHOUSE, 1964 :JBW NO B241:NEW

GENUS;ORDER PRODUCTIDA,SUBFAMILY

STRIOCHONETINAE ACCORDING TO WATERHOUSE + PIYASIN ; 2 SPEC

STRIOCHONETES SCUTELLA WATERHOUSE+PIYASIN 31783 [B242] P-PARA

UP.KAZAN

50 M FROM ROAD LEADING W FROM HWY, KHAO

PHRIK,NR RATBURI,THAILAND

H---:WATERHOUSE JB + PIYASIN

S, 1970,PALAEONTOGRAPHICA BD 135,ABT A.P 106-112 REMARKS: C.D-JB WATERHOUSE, 1964 ; JBW NO B242;NEW

GENUS:ORDER PRODUCTIDA, SUBFAMILY

STRIOCHONETINAE ACCORDING TO WATERHOUSE +

PIYASIN : 1 SPEC

STRIOCHONETES SCUTELLA WATERHOUSE + PIYASIN

31784 [B243] P-PARA

UP,KAZAN

50 MFROM ROAD LEADING W FROM HWY KHAO

PHRIK,NR RATBURI, THAILAND

H--.;WATERHOUSE JB + PIYASIN

S. 1970 PALAEONTOGRAPHICA.BD 135 ABT A.P106.112 REMARK S: C,D-JB WATERHOUSE, 1964 ;JBW NO B243;NEW GENUS;ORDER PRODUCTIDA,SUBFAMILY

STRIOCHONETINAE ACCORDING TO WATERHOUSE + PIYASIN; 1 SPEC

STRIOCHONETES SCUTELLA WATERHOUSE + PIYASIN 31785 [B244] P-PARA

UPKAZAN

50 M FROM ROAD LEADING W FROM HWY, KHAO PHRIK,NR RATBURI,THAILAND H--.-WATERHOUSE JB + PIYASIN

S. 1970 PALAEONTOGRAPHICA BD 135,ABT A.P 106-112

REMARKS: C,D-JB WATERHOUSE, 1964 ; JBW NO B244; NEW GENUS:ORDER PRODUCTIDA.SUBFAMILY

STRIOCHONETINAE ACCORDING TO WATERHOUSE + PIYASIN ; 1 SPEC 
STRIOCHONETES SCUTELLA WATERHOUSE + PIYASIN 31786 [B246] P-PARA

UP.KAZAN

50 M FROM ROAD LEADING W FROM HWY, KHAO

PHRIK, NR RATBURI,THAILAND

H---;WATERHOUSE JB + PIYASIN

S. 1970,PALAEONTOGRAPHICA,BD 135,ABT A,P 106-112

REMARKS: C,D-JB WATERHOUSE, 1964 :JBW NO B246; NEW

GENUS:ORDER PRODUCTIDA.SUBFAMILY

STRIOCHONETINAE ACCORDING TO WATERHOUSE +

PIYASIN ; 1 SPEC

STRIOCHONETES SCUTELLA WATERHOUSE + PIYASIN

31787 [B247] P-PARA

UP.KAZAN

50 M FROM ROAD LEADING W FROM HWY , KHAO

PHRIK,NR RATBURI,THAILAND

H...: WATERHOUSE JB + PIYASIN

S, 1970,PALAEONTOGRAPHICA,BD 135,ABT A,P $106-$ 112,PL 16.FIG 3

REMARKS: C.D-JB WATERHOUSE, 1964 ;JBW NO B247; NEW GENUS:ORDER PRODUCTIDA,SUBFAMILY

STRIOCHONETINAE ACCORDING TO WATERHOUSE + PIYASIN; 1 SPEC

STRIOCHONETES SCUTELLA WATERHOUSE+PIYASIN 31788 [B248] P-PARA

UP.KAZAN

50 M FROM ROAD LEADING W FROM HWY, KHAO

PHRIK.NR RATBURI,THAILAND

H---:WATERHOUSE JB + PIYASIN

S. 1970.PALAEONTOGRAPHICA,BD 135,ABT A.P 106-112

REMARKS: C,D-JB WATERHOUSE, 1964 ; JBW NO B248; NEW

GENUS;ORDER PRODUCTIDA.SUBFAMILY

STRIOCHONETINAE ACCORDING TO WATERHOUSE +

PIYASIN : 1 SPEC

STRIOCHONETES SCUTELLA WATERHOUSE + PIYASIN 31789 [B249] P-PARA

UP.KAZAN

50 M FROM ROAD LEADING W FROM HWY, KHAO

PHRIK,NR RATBURI,THAILAND

H---;WATERHOUSE JB + PIYASIN

S, 1970,PALAEONTOGRAPHICA,BD 135,ABT A,P 106 112.PL 16.FIG 16,17

REMARKS: C.D-JB WATERHOUSE, 1964 ; JBW NO B249; NEW GENUS;ORDER PRODUCTIDA,SUBFAMILY STRIOCHONETINAE ACCORDING TO WATERHOUSE + PIYASIN ; 1 SPEC

STRIOCHONETES SCUTELLA WATERHOUSE+PIYASIN 31790 [B250] P-PARA

UP.KAZAN

50 M FROM ROAD LEADING W FROM HWY, KHAO

PHRIK, NR RATBURI, THAILAND

H---;WATERHOUSE JB + PIYASIN

S, 1970,PALAEONTOGRAPHICA,BD 135,ABT A,P106112.PL 16, FIG 14,15

REMARKS: C.D-JB WATERHOUSE, 1964 ; JBW NO B250:NEW GENUS;ORDER PRODUCTIDA.SUBFAMILY STRIOCHONETINAE ACCORDING TO WATERHOUSE + PIYASIN ; 1 SPEC

STRIOCHONETES SCUTELLA WATERHOUSE+PIYASIN 31791 [B251] P-PARA

UP.KAZAN

$50 M$ FROM ROAD LEADING W FROM HWY, KHAO

PHRIK, NR RATBURI,THAILAND

H...;WATERHOUSE JB + PIYASIN

S. 1970 PALAEONTOGRAPHICA BD 135 ABT A.P 106-112

REMARKS: C.D-JB WATERHOUSE, 1964 ; JBW NO B251; NEW

GENUS:ORDER PRODUCTIDA.SUBFAMILY

STRIOCHONETINAE ACCORDING TO WATERHOUSE +

PIYASIN ; 1 SPEC

STRIOCHONETES SCUTELLA WATERHOUSE + PIYASIN

31792 [B252] P-PARA

UP.KAZAN

$50 M$ FROM ROAD LEADING W FROM HWY, KHAO

PHRIK,NR FATBURI,THAILAND

H---;WATERHOUSE JB + PIYASIN
S, 1970,PALAEONTOGRAPHICA,BD 135,ABT A,P 106-112

REMARKS: C.D-JB WATERHOUSE, 1964 ,JBW NO B252; NEW

GENUS;ORDER PRODUCTIDA,SUBFAMILY

STRIOCHONETINAE ACCORDING TO WATERHOUSE +

PIYASIN: 1 SPEC

STRIOCHONETES SCUTELLA WATERHOUSE + PIYASIN

31793 [B253] P-PARA

UP, KAZAN

$50 M$ FROM ROAD LEADING W FROM HWY, KHAO

PHRIK, NR RATBURI,THAILAND

H--;WATERHOUSE JB + PIYASIN

S, 1970,PALAEONTOGRAPHICA,BD 135,ABT A,P 106-112

REMARKS: C,D-JB WATERHOUSE, 1964 ;JBW NO B253; NEW

GENUS:ORDER PRODUCTIDA,SUBFAMILY

STRIOCHONETINAE ACCORDING TO WATERHOUSE +

PIYASIN; 1 SPEC

STRIOCHONETES SCUTELLA WATERHOUSE+ PIYASIN 31794 [B254 ] P-PARA

UP,KAZAN

50 M FROM ROAD LEADING W FROM HWY , KHAO

PHRIK, NR RATBURI,THAILAND

H-.-;WATERHOUSE JB + PIYASIN

S. 1970,PALAEONTOGRAPHICA,BD 135,ABT A,P 106

112.PL 16.FIG 18, 19

REMARKS: C,D-JB WATERHOUSE, 1964 ;JBW NO B254;NEW

GENUS:ORDER PRODUCTIDA,SUBFAMILY

STRIOCHONETINAE ACCORDING TO WATERHOUSE +

PIYASIN ; 1 SPEC

STRIOCHONETES SCUTELLA WATERHOUSE+ PIYASIN

31795 [B255 ] P-PARA

UP.KAZAN

50 M FROM ROAD LEADING W FROM HWY, KHAO

PHRIK, NR RATBURI,THAILAND

H---;WATERHOUSE JB + PIYASIN

S, 1970,PALAEONTOGRAPHICA,BD 135,ABT A,P $106-$ 112,PL 16,FIG 20,21

REMARKS: C.D-JB WATERHOUSE, 1964 ;JBW NO B255:NEW

GENUS:ORDER PRODUCTIDA,SUBFAMILY

STRIOCHONETINAE ACCORDING TO WATERHOUSE +

PIYASIN: 1 SPEC

STRIOCHONETES SCUTELLA WATERHOUSE+PIYASIN

31796 [B256] P-PARA

UP.KAZAN

50 M FROM ROAD LEADING W FROM HWY, KHAO

PHRIK, NR RATBURI,THAILAND

H---.WATERHOUSE JB + PIYASIN

S, 1970.PALAEONTOGRAPHICA,BD 135.ABT A.P 106 112, PL 16, FIG 22

REMARKS: C.D-JB WATERHOUSE, 1964 :JBW NO B256:NEW GENUS:ORDER PRODUCTIDA,SUBFAMILY

STRIOCHONETINAE ACCORDING TO WATERHOUSE +

PIYASIN ; 1 SPEC

STRIOCHONETES SCUTELLA WATERHOUSE+PIYASIN

31797 [B257] P-PARA

UP, KAZAN

$50 M$ FROM ROAD LEADING W FROM HWY, KHAO

PHRIK,NR RATBURI, THAILAND

H---;WATERHOUSE JB + PIYASIN

S, 1970 PALAEONTOGRAPHICA BD 135 ABT A.P 106-112

REMARKS: C.D-JB WATERHOUSE, 1964 ;JBW NO B257;NEW

GENUS:ORDER PRODUCTIDA,SUBFAMILY

STRIOCHONETINAE ACCORDING TO WATERHOUSE +

PIYASIN; 1 SPEC

STRIOCHONETES SCUTELLA WATERHOUSE+PIYASIN 31798 [B258] P-PARA

UP.KAZAN

$50 M$ FROM ROAD LEADING W FROM HWY, KHAO

PHRIK,NR RATBUAI,THAILAND

H---;WATERHOUSE JB + PIYASIN

S, 1970,PALAEONTOGRAPHICA,BD 135,ABT A,P 106-112

REMARKS: C.D-JB WATERHOUSE, 1964 ; JBW NO B258:NEW

GENUS:ORDER PRODUCTIDA, SUBFAMILY

STRIOCHONETINAE ACCORDING TO WATERHOUSE +

PIYASIN : 1 SPEC 
STRIOCHONETES SCUTELLA WATERHOUSE+PIYASIN 31799 [B259] P-PARA

UP, KAZAN

$50 M$ MROM ROAD LEADING W FROM HWY, KHAO

PHRIK,NR RATBURI,THAILAND

H-.-;WATERHOUSE JB + PIYASIN

S. 1970,PALAEONTOGRAPHICA,BD 135,ABT A.P 106-112

REMARKS: C.D-JB WATERHOUSE, 1964 ;JBW NO B259;NEW

GENUS;ORDER PRODUCTIDA,SUBFAMILY

STRIOCHONETINAE ACCORDING TO WATERHOUSE +

PIYASIN ; 1 SPEC

\section{STRIOCHONETES SCUTELLA WATERHOUSE + PIYASIN}

31800 [B260] P-PARA

UP.KAZAN

50 M FROM ROAD LEADING W FROM HWY, KHAO

PHRIK,NR RATBURI,THAILAND

H---;WATERHOUSE JB + PIYASIN

S, 1970,PALAEONTOGRAPHICA,BD 135,ABT A,P 106-112

REMARKS: C,D-JB WATERHOUSE, 1964 ;JBW NO B260;NEW

GENUS:ORDER PRODUCTIDA,SUBFAMILY

STRIOCHONETINAE ACCORDING TO WATERHOUSE +

PIYASIN ; 1 SPEC

STRIOCHONETES SCUTELLA WATERHOUSE + PIYASIN

31801 [B261] P-PARA

UP.KAZAN

50 M FROM ROAD LEADING W FROM HWY, KHAO

PHRIK,NR RATBURI,THAILAND

H---:WATERHOUSE JB + PIYASIN

S. 1970, PALAEONTOGRAPHICA.BD 135,ABT A.P 106-112

REMARKS: C,D-JB WATERHOUSE, 1964 ;JBW NO B261;NEW

GENUS:ORDER PRODUCTIDA,SUBFAMILY

STRIOCHONETINAE ACCORDING TO WATERHOUSE +

PIYASIN; 1 SPEC

STRIOCHONETES SCUTELLA WATERHOUSE + PIYASIN 31802 [B262] P-PARA

UP.KAZAN

50 M FROM ROAD LEADING W FROM HWY, KHAO

PHRIK,NR RATBURI,THAILAND

H-..-WATERHOUSE JB + PIYASIN

S, 1970,PALAEONTOGRAPHICA,BD 135,ABT A,P 106-112

REMARKS: C.D-JB WATERHOUSE, 1964 ; JBW NO B262;NEW

GENUS:ORDER PRODUCTIDA,SUBFAMILY

STRIOCHONETINAE ACCORDING TO WATERHOUSE +

PIYASIN ; 1 SPEC (2 PCES)

STRIOCHONETES SCUTELLA WATERHOUSE + PIYASIN

31803 [B263] P-PARA

UP.KAZAN

50 M FROM ROAD LEADING W FROM HWY, KHAO

PHRIK,NR RATBURI, THAILAND

H---;WATERHOUSE JB + PIYASIN

S, 1970,PALAEONTOGRAPHICA,BD 135,ABT A,P106-

112,PL 16,FIG 23,24

REMARKS: C,D-JB WATERHOUSE, 1964 ;JBW NO B263;NEW GENUS:ORDER PRODUCTIDA.SUBFAMILY

STRIOCHONETINAE ACCORDING TO WATERHOUSE + PIYASIN ; 1 SPEC

STRIOCHONETES SCUTELLA WATERHOUSE + PIYASIN 31804 [B264 ] P-PARA

UP.KAZAN

50 M FROM ROAD LEADING W FROM HWY, KHAO

PHRIK, NR RATBURI,THAILAND

H---:WATERHOUSE JB + PIYASIN

S, 1970,PALAEONTOGRAPHICA,BD 135,ABT A,P106112,PL 16,FIG 25,26

REMARKS: C,D-JB WATERHOUSE, 1964 .JBW NO B264:NEW

GENUS;ORDER PRODUCTIDA, SUBFAMILY

STRIOCHONETINAE ACCORDING TO WATERHOUSE +

PIYASIN ; 1 SPEC

STRIOCHONETES SCUTELLA WATERHOUSE + PIYASIN

31805 [B265 ] P.PARA

UP,KAZAN

50 M FROM ROAD LEADING W FROM HWY KHAO

PHRIK,NR RATBURI,THAILAND

H---;WATERHOUSE JB + PIYASIN

S, 1970,PALAEONTOGRAPHICA,BD 135,ABT A,P 106-112
REMARKS: C,D-JB WATERHOUSE, 1964 ;JBW NO B265;NEW GENUS:ORDER PRODUCTIDA,SUBFAMILY

STRIOCHONETINAE ACCORDING TO WATERHOUSE + PIYASIN ; 1 SPEC (3 PCES)

STRIOCHONETES SCUTELLA WATERHOUSE + PIYASIN

31806 [B266 ] P-PARA

UP.KAZAN

50 M FROM ROAD LEADING W FROM HWY, KHAO

PHRIK,NR RATBURI,THAILAND

H-.-:WATERHOUSE JB + PIYASIN

S, 1970,PALAEONTOGRAPHICA,BD 135,ABT A,P 106-112

REMARKS: C,D-JB WATERHOUSE, 1964 ;JBW NO B266;NEW

GENUS;ORDER PRODUCTIDA,SUBFAMILY

STRIOCHONETINAE ACCORDING TO WATERHOUSE + PIYASIN ; 1 SPEC

STRIOCHONETES SCUTELLA WATERHOUSE + PIYASIN

31807 [B267] P-PARA

UP,KAZAN

50 M FROM ROAD LEADING W FROM HWY, KHAO PHRIK,NR RATBURI,THAILAND

H---;WATERHOUSE JB + PIYASIN

S, 1970,PALAEONTOGRAPHICA,BD 135,ABT A,P106-112

REMARKS: C,D-JB WATERHOUSE, 1964 ;JBW NO B267;NEW

GENUS;ORDER PRODUCTIDA,SUBFAMILY

STRIOCHONETINAE ACCORDING TO WATERHOUSE + PIYASIN ; 1 SPEC

STRIOCHONETES SCUTELLA WATERHOUSE+PIYASIN 31808 [B268] P-PARA

UP,KAZAN

50 M FROM ROAD LEADING W FROM HWY, KHAO

PHRIK, NR RATBURI, THAILAND

H---;WATERHOUSE JB + PIYASIN

S.1970,PALAEONTOGRAPHICA,BD 135,ABT A,P 106-112

REMARKS: C,D-JB WATERHOUSE, 1964 ;JBW NO B268;NEW GENUS;ORDER PRODUCTIDA,SUBFAMILY

STRIOCHONETINAE ACCORDING TO WATERHOUSE + PIYASIN : 1 SPEC

STRIOCHONETES SCUTELLA WATERHOUSE + PIYASIN 31809 [B269] P-PARA

UP.KAZAN

50 M FROM ROAD LEADING W FROMHWY, KHAO PHRIK,NR RATBURI,THAILAND

H---:WATERHOUSE JB + PIYASIN

S, 1970,PALAEONTOGRAPHICA,BD 135,ABT A,P 106-112

REMARKS: C.D-JB WATERHOUSE, 1964 :JBW NO B269;NEW

GENUS;ORDER PRODUCTIDA,SUBFAMILY

STRIOCHONETINAE ACCORDING TO WATERHOUSE + PIYASIN ; 1 SPEC

STRIOCHONETES SCUTELLA WATERHOUSE + PIYASIN 31810 [B270] P-PARA

UP,KAZAN

$50 M$ FROM ROAD LEADING W FROM HWY, KHAO

PHRIK,NR RATBURI, THAILAND

H---:WATERHOUSE JB + PIYASIN

S, 1970,PALAEONTOGRAPHICA,BD 135,ABT A,P 106-112

REMARKS: C,D-JB WATERHOUSE, 1964 ;JBW NO B270;NEW

GENUS:ORDER PRODUCTIDA,SUBFAMILY

STRIOCHONETINAE ACCORDING TO WATERHOUSE +

PIYASIN ; 1 SPEC

STRIOCHONETES SCUTELLA WATERHOUSE + PIYASIN

$31811[B 271]$ P-PARA

UP,KAZAN

50 M FROM ROAD LEADING W FROM HWY, KHAO

PHRIK,NR RATBURI, THAILAND

H-.-:WATERHOUSE JB + PIYASIN

S, 1970,PALAEONTOGRAPHICA,BD 135,ABT A,P 106-112

REMARKS: C,D-JB WATERHOUSE, 1964 ; JBW NO B271;NEW GENUS:ORDER PRODUCTIDA.SUBFAMILY

STRIOCHONETINAE ACCORDING TO WATERHOUSE +

PIYASIN : 1 SPEC 
STRIOCHONETES SCUTELLA WATERHOUSE + PIYASIN

31812 [B272] P-PARA

UP.KAZAN

50 M FROM ROAD LEADING W FROM HWY, KHAO

PHRIK,NR RATBURI, THAILAND

H--.;WATERHOUSE JB + PIYASIN

S, 1970,PALAEONTOGRAPHICA,BD 135,ABT A,P106112.PL 16,FIG 29,30

REMARKS: C.D-JB WATERHOUSE, 1964 ; JBW NO B272;NEW GENUS:ORDER PRODUCTIDA.SUBFAMILY

STRIOCHONETINAE ACCORDING TO WATERHOUSE + PIYASIN; 1 SPEC

STRIOCHONETES SCUTELLA WATERHOUSE + PIYASIN

31813 [B273] P-PARA

UP.KAZAN

$50 \mathrm{M}$ FROM ROAD LEADING W FROM HWY, KHAO

PHRIK.NR RATBURI,THAILAND

H....WATERHOUSE JB + PIYASIN

S. 1970,PALAEONTOGRAPHICA,BD 135, ABT A,P106-

112.PL 16,FIG 31,32

REMARKS: C.D-JB WATERHOUSE, 1964 :JBW NO B273:NEW

GENUS:ORDER PRODUCTIDA, SUBFAMILY

STRIOCHONETINAE ACCORDING TO WATERHOUSE + PIYASIN ; 2 SPEC

STRIOCHONETES SCUTELLA WATERHOUSE + PIYASIN

31814 [B274] P-PARA

$\cup P, K A Z A N$

50 M FROM ROAD LEADING W FROM HWY, KHAO

PHRIK,NR RATBURI,THAILAND

H---;WATERHOUSE JB + PIYASIN

S. 1970,PALAEONTOGRAPHICA,BD 135,ABT A.P106-112

REMARKS: C,D-JB WATERHOUSE, 1964 ; JBW NO B274;NEW

GENUS; ORDER PRODUCTIDA,SUBFAMILY

STRIOCHONETINAE ACCORDING TO WATERHOUSE +

PIYASIN ; 1 SPEC

STRIOCHONETES SCUTELLA WATERHOUSE + PIYASIN 31816 [B276] P-PARA

UP.KAZAN

50 M FROM ROAD LEADING W FROM HWY, KHAO

PHRIK,NR RATBURI,THAILAND

H---;WATERHOUSE JB + PIYASIN

S. 1970,PALAEONTOGRAPHICA BD 135,ABT A.P106-112

REMARKS: C,D-JB WATERHOUSE, 1964 ; JBW NO B276; NEW GENUS:ORDER PRODUCTIDA,SUBFAMILY

STRIOCHONETINAE ACCORDING TO WATERHOUSE PIYASIN; 1 SPEC

STRIOCHONETES SCUTELLA WATERHOUSE + PIYASIN 31818 [B278] P-PARA

UP.KAZAN

$50 M$ FROM ROAD LEADING W FROM HWY, KHAO PHRIK, NR RATBURI,THAILAND

H--.-WATERHOUSE JB + PIYASIN

S. 1970.PALAEONTOGRAPHICA,BD 135,ABT A,P 106-112 REMARKS: C.D-JB WATERHOUSE, 1964 ;JBW NO B278;NEW GENUS:ORDER PRODUCTIDA.SUBFAMILY

STRIOCHONETINAE ACCORDING TO WATERHOUSE + PIYASIN; 1 SPEC

STRIOCHONETES SCUTELLA WATERHOUSE + PIYASIN 31819 [B279] P-PARA

UP.KAZAN

50 M FROM ROAD LEADING W FROM HWY, KHAO PHRIK NR RATBURI,THAILAND

H---;WATERHOUSE JB + PIYASIN

S. 1970,PALAEONTOGRAPHICA,BD 135,ABT A,P106-112

REMARKS: C,D-JB WATERHOUSE, 1964 ;JBW NO B279;NEW

GENUS:ORDER PRODUCTIDA,SUBFAMILY

STRIOCHONETINAE ACCORDING TO WATERHOUSE + PIYASIN: 1 SPEC

STRIOCHONETES SCUTELLA WATERHOUSE + PIYASIN

31820 [B280] P-PARA

UP.KAZAN

$5 O M$ FROM ROAD LEADING W FROM HWY, KHAO

PHRIK, NR RATBURI,THAILAND

H---:WATERHOUSE JB + PIYASIN

S. 1970,PALAEONTOGRAPHICA.BD 135,ABT A,P106-112
REMARKS: C.D-JB WATERHOUSE, 1964 :JBW NO B280;NEW GENUS;ORDER PRODUCTIDA, SUBFAMILY STRIOCHONETINAE ACCORDING TO WATERHOUSE + PIYASIN ; 1 SPEC

STRIOCHONETES SCUTELLA WATERHOUSE + PIYASIN

31821 [B281] P.PARA

UP, KAZAN

$50 \mathrm{M}$ FROM ROAD LEADING W FROM HWY, KHAO

PHRIK, NR RATBURI, THAILAND

H---;WATERHOUSE JB + PIYASIN

S, 1970,PALAEONTOGRAPHICA,BD 135,ABT A,P 106-112

REMARKS: C,D-JB WATERHOUSE, 1964 ;JBW NO B281;NEW GENUS:ORDER PRODUCTIDA, SUBFAMILY

STRIOCHONETINAE ACCORDING TO WATERHOUSE + PIYASIN ; 1 SPEC

STRIOCHONETES SCUTELLA WATERHOUSE + PIYASIN 31823 [B283] P-PARA

UP, KAZAN

50 M FROM ROAD LEADING WFROM HWY, KHAO

PHRIK,NR RATBURI,THAILAND

H---;WATERHOUSE JB + PIYASIN

S, 1970,PALAEONTOGRAPHICA,BD 135,ABT A,P 106-112

REMARKS: C,D-JB WATERHOUSE, 1964 ;JBW NO B283;NEW GENUS:ORDER PRODUCTIDA SUBFAMILY

STRIOCHONETINAE ACCORDING TO WATERHOUSE + PIYASIN ; 1 SPEC

STRIOCHONETES SCUTELLA WATERHOUSE + PIYASIN

31825 [B285] P-PARA

UP,KAZAN

50 M FROM ROAD LEADING W FROM HWY, KHAO

PHRIK, NR RATBUR!, THAILAND

H.--WATERHOUSE JB + PIYASIN

S, 1970,PALAEONTOGRAPHICA,BD 135,ABT A,P 106-112 REMARKS: C,D-JB WATERHOUSE, 1964 ; JBW NO B285; NEW GENUS;ORDER PRODUCTIDA,SUBFAMILY STRIOCHONETINAE ACCORDING TO WATERHOUSE + PIYASIN ; 1 SPEC

STRIOCHONETES SCUTELLA WATERHOUSE+PIYASIN 31826 [B286] P.PARA

UP, KAZAN

$50 M$ FROM ROAD LEADING W FROM HWY, KHAO PHRIK,NR RATBURI,THAILAND

H---;WATERHOUSE JB + PIYASIN

S, 1970,PALAEONTOGRAPHICA,BD 135,ABT A,P106-112

REMARKS: C,D-JB WATERHOUSE, 1964 ;JBW NO B286; NEW GENUS:ORDER PRODUCTIDA,SUBFAMILY

STRIOCHONETINAE ACCORDING TO WATERHOUSE + PIYASIN; 1 SPEC

STRIOCHONETES SCUTELLA WATERHOUSE + PIYASIN

31828 [B288] P-PARA

UP, KAZAN

50 M FROM ROAD LEADING W FROM HWY, KHAO

PHRIK, NR RATBURI,THAILAND

H---;WATERHOUSE JB + PIYASIN

S. 1970 PALAEONTOGRAPHICA BD 135 ABT A.P 106-112

REMARKS: C,D-JB WATERHOUSE, 1964 ;JBW NO B288;NEW GENUS;ORDER PRODUCTIDA,SUBFAMILY

STRIOCHONETINAE ACCORDING TO WATERHOUSE + PIYASIN ; 1 SPEC

STRIOCHONETES SCUTELLA WATERHOUSE + PIYASIN

31830 [B290 ] P-PARA

UP.KAZAN

50 M FROM ROAD LEADING W FROM HWY, KHAO

PHRIK,NR RATBURI, THAILAND

H---:WATERHOUSE JB + PIYASIN

S, 1970.PALAEONTOGRAPHICA BD 135,ABT A.P 106-112

REMARKS: C.D-JB WATERHOUSE, 1964 ; JBW NO B290;NEW GENUS ORDER PRODUCTIDA SUBFAMILY

STRIOCHONETINAE ACCORDING TO WATERHOUSE + PIYASIN : 1 SPEC 
STRIOCHONETES SCUTELLA WATERHOUSE+PIYASIN 31833 [B392] P.PARA

UP,KAZAN

50 M FROM ROAD LEADING W FROM HWY, KHAO

PHRIK, NR RATBURI, THAILAND

H---:WATERHOUSE JB + PIYASIN

S. 1970 PALAEONTOGRAPHICA,BD 135,ABT A.P 106-112

REMARK S: C,D-JB WATERHOUSE, 1964 ; JBW NO B392; NEW GENUS:ORDER PRODUCTIDA,SUBFAMILY

STRIOCHONETINAE ACCORDING TO WATERHOUSE + PIYASIN; 1 SPEC

STRIOCHONETES SCUTELLA WATERHOUSE + PIYASIN

31834 [B393] P-PARA

UP.KAZAN

50 M FROM ROAD LEADING W FROM HWY, KHAO

PHRIK,NR RATBURI,THAILAND

H--. WATERHOUSE JB + PIYASIN

S. 1970,PALAEONTOGRAPHICA,BD 135,ABT A,P 106-112

REMARKS: C.D-JB WATERHOUSE, 1964 ; JBW NO B393; NEW GENUS:ORDER PRODUCTIDA,SUBFAMILY

STRIOCHONETINAE ACCORDING TO WATERHOUSE + PIYASIN; 1 SPEC

STRIOCHONETES SCUTELLA WATERHOUSE + PIYASIN 31839 [B6 15] P-PARA

UP,KAZAN

$50 M$ FROM ROAD LEADING W FROM HWY, KHAO

PHRIK,NR RATBURI,THAILAND

H---;WATERHOUSE JB + PIYASIN

S, 1970,PALAEONTOGRAPHICA,BD 135,ABT A,P 106-112

REMARK S: C.D-JB WATERHOUSE, 1964 ; JBW NO B615; NEW

GENUS:ORDER PRODUCTIDA,SUBFAMILY

STRIOCHONETINAE ACCORDING TO WATERHOUSE +

PIYASIN; 1 SPEC

STRIOCHONETES SCUTELLA WATERHOUSE + PIYASIN

31840 [B6 16] P-PARA

UP,KAZAN

$50 \mathrm{M}$ FROM ROAD LEADING W FROM HWY, KHAO

PHRIK,NR RATBURI,THAILAND

H---;WATERHOUSE JB + PIYASIN

S, 1970,PALAEONTOGRAPHICA,BD 135,ABT A,P 106-112

REMARK S: C,D-JB WATERHOUSE, 1964 ;JBW NO B616;NEW

GENUS;ORDER PRODUCTIDA,SUBFAMILY

STRIOCHONETINAE ACCORDING TO WATERHOUSE +

PIYASIN : 1 SPEC

STRIOCHONETES SCUTELLA WATERHOUSE + PIYASIN

31841 [B6 17 ] P.PARA

UP.KAZAN

$50 M$ FROM ROAD LEADING W FROM HWY, KHAO

PHRIK, NR RATBURI,THAILAND

H---:WATERHOUSE JB + PIYASIN

S, 1970.PALAEONTOGRAPHICA.BD 135,ABT A,P 106-112

REMARK S: C,D-JB WATERHOUSE, 1964 ; JBW NO B617;NEW GENUS:ORDER PRODUCTIDA,SUBFAMILY

STRIOCHONETINAE ACCORDING TO WATERHOUSE + PIYASIN ; 1 SPEC

STRIOCHONETES SCUTELLA WATERHOUSE+PIYASIN

31815 [B275 ] P-PARA

UP,KAZAN

50 M FROM ROAD LEADING W FROM HWY, KHAO

PHRIK, NR RATBURI,THAILAND

H---; WATERHOUSE JB + PIYASIN

S, 1970,PALAEONTOGRAPHICA,BD 135,ABT A,P 106-

112,PL 16, FIG 33,34

REMARK S: C,D-JB WATERHOUSE, 1964 ;JBW NO B275:NEW

GENUS:ORDER PRODUCTIDA,SUBFAMILY

STRIOCHONETINAE ACCORDING TO WATERHOUSE +

PIYASIN : 1 SPEC

STRIOCHONETES SCUTELLA WATERHOUSE + PIYASIN

31817 [B277 ] P.PARA

UP.KAZAN

$50 M$ FROM ROAD LEADING W FROM HWY KHAO

PHRIK, NR RATBURI, THAILAND

H---; WATERHOUSE JB + PIYASIN

S, 1970,PALAEONTOGRAPHICA,BD 135,ABT A,P 106-

112,PL 16, FIG 37
REMARKS: C D-JB WATERHOUSE 1964.JBW NO B277: NEW GENUS;ORDER PRODUCTIDA,SUBFAMILY STRIOCHONETINAE ACCORDING TO WATERHOUSE + PIYASIN: 1 SPEC

STRIOCHONETES SCUTELLA WATERHOUSE + PIYASIN

31822 [B282] P.PARA

UP, KAZAN

$50 \mathrm{M}$ FROM ROAD LEADING W FROM HWY, KHAO

PHRIK, NR RATBURI,THAILAND

H---;WATERHOUSE JB + PIYASIN

S, 1970,PALAEONTOGRAPHICA,BD 135,ABT A,P106112.PL 16, FIG 35,36

REMARKS: C,D-JB WATERHOUSE, 1964 ; JBW NO B282; NEW GENUS;ORDER PRODUCTIDA,SUBFAMILY

STRIOCHONETINAE ACCORDING TOWATERHOUSE + PIYASIN ; 1 SPEC

STRIOCHONETES SCUTELLA WATERHOUSE + PIYASIN

31824 [B284] P-PARA

UP KAZAN

50 M FROM ROAD LEADING W FROM HWY, KHAO

PHRIK,NR RATBURI,THAILAND

H...:WATERHOUSE JB + PIYASIN

S, 1970, PALAEONTOGRAPHICA,BD 135,ABT A,P106112,PL 16, FIG 38,39

REMARK S: C.D-JB WATERHOUSE, 1964 ; JBW NO B284;NEW GENUS:ORDER PRODUCTIDA,SUBFAMILY

STRIOCHONETINAE ACCORDING TO WATERHOUSE + PIYASIN : 1 SPEC

STRIOCHONETES SCUTELLA WATERHOUSE + PIYASIN 31827 [B287 ] P-PARA

UP,KAZAN

$50 M$ FROM ROAD LEADING W FROM HWY, KHAO

PHRIK, NR RATBURI,THAILAND

H-..-;WATERHOUSE JB + PIYASIN

S, 1970,PALAEONTOGRAPHICA,BD 135,ABT A,P 106

112.PL 17. FIG 1,2

REMARKS: C.D-JB WATERHOUSE, 1964 ;JBW NO B287;NEW GENUS;ORDER PRODUCTIDA,SUBFAMILY

STRIOCHONETINAE ACCORDING TO WATERHOUSE + PIYASIN : 1 SPEC

STRIOCHONETES SCUTELLA WATERHOUSE + PIYASIN 31829 [B289] P-PARA

UP,KAZAN

$50 \mathrm{M}$ FROM ROAD LEADING W FROM HWY, KHAO

PHRIK,NR RATBURI,THAILAND

H---;WATERHOUSE JB + PIYASIN

S, 1970,PALAEONTOGRAPHICA,BD 135,ABT A,P 106

112,PL 17, FIG 7

REMARKS: C,D-JB WATERHOUSE, 1964 ;JBW NO B289;NEW GENUS:ORDER PRODUCTIDA,SUBFAMILY

STRIOCHONETINAE ACCORDING TO WATERHOUSE + PIYASIN: 1 SPEC

STRIOCHONETES SCUTELLA WATERHOUSE + PIYASIN 31831 [B291] P.PARA

UP.KAZAN

50 M FROM ROAD LEADING W FROM HWY, KHAO

PHRIK,NR RATBURI,THAILAND

H---WATERHOUSE JB + PIYASIN

S, 1970, PALAEONTOGRAPHICA,BD 135,ABT A,P 106 112,PL 17, FIG 8

REMARKS: C.D-JB WATERHOUSE, 1964 ;JBW NO B291;NEW GENUS;ORDER PSODUCTIDA,SUBFAMILY STRIOCHONETINAE ACCORDING TO WATERHOUSE + PIYASIN: 1 SPEC

STRIOCHONETES SCUTELLA WATERHOUSE + PIYASIN

31832 [B292] P-PARA

UP, KAZAN

50 M FROM ROAD LEADING W FROM HWY, KHAO

PHRIK, NR RATBURI, THAILAND

H---;WATERHOUSE JB + PIYASIN

S, 1970,PALAEONTOGRAPHICA,BD 135,ABT A,P 106 112.PL 16, FIG 3,4,6

REMARK S:C.D-JB WATERHOUSE, 1964 ;JBW NO B292;NEW GENUS;ORDER PRODUCTIDA.SUBFAMILY

STRIOCHONETINAE ACCORDING TO WATERHOUSE + PIYASIN; 1 SPEC 
STRIOCHONETES SCUTELLA WATERHOUSE + PIYASIN

31835 [B394] P.PARA

UP.KAZAN

$50 M$ FROM ROAD LEADING W FROM HWY, KHAO

PHRIK.NR RATBURI,THAILAND

H---;WATERHOUSE JB + PIYASIN

S. 1970.PALAEONTOGRAPHICA,BD 135,ABT A,P 106 112, PL 17, FIG 5

REMARKS: C.D-JB WATERHOUSE, 1964 :JBW NO B394:NEW GENUS:ORDER PRODUCTIDA.SUBFAMILY

STRIOCHONETINAE ACCORDING TO WATERHOUSE + PIYASIN : 1 SPEC

STRIOCHONETES SCUTELLA WATERHOUSE + PIYASIN 31836 [B587] REF SPEC-M

UP.KAZAN

$50 M$ FROM ROAD LEADING W FROM HWY, KHAO

PHRIK NR RATBURI,THAILAND

H---:WATERHOUSE JB + PIYASIN

S. 1970,PALAEONTOGRAPHICA,BD 135.ABT A.P 106-112

REMARKS: C,D-JB WATERHOUSE, 1964 ;JBW NO B587; NEW GENUS:ORDER PRODUCTIDA.SUBFAMILY

STRIOCHONETINAE ACCORDING TO WATERHOUSE + PIYASIN : 1 SPEC

STRIOCHONETES SCUTELLA WATERHOUSE+PIYASIN

31837 [B588] REF SPEC-M

UP,KAZAN

50 M FROM ROAD LEADING W FROM HWY, KHAO

PHRIK,NR RATBURI,THAILAND

H---;WATERHOUSE JB + PIYASIN

S, 1970,PALAEONTOGRAPHICA,BD 135,ABT A,P106-112

REMARKS: C,D-JB WATERHOUSE, 1964 ; JBW NO B588;NEW

GENUS;ORDER PRODUCTIDA, SUBFAMILY

STRIOCHONETINAE ACCORDING TO WATERHOUSE +

PIYASIN : 1 SPEC

STRIOCHONETES SCUTELLA WATERHOUSE + PIYASIN

31838 [B6 14] P-PARA

UP.KAZAN

$50 M$ FROM ROAD LEADING W FROM HWY, KHAO

PHRIK.NR RATBURI.THAILAND

H---:WATERHOUSE JB + PIYASIN

S. 1970.PALAEONTOGRAPHICA,BD 135,ABT A,P106-

112, PL 16, FIG 4,5

REMARKS: C,D-JB WATERHOUSE, 1964 ; JBW NO B6 14:NEW

GENUS:ORDER PRODUCTIDA, SUBFAMILY

STRIOCHONETINAE ACCORDING TO WATERHOUSE + PIYASIN ; 1 SPEC

STRIOCHONETES SCUTELLA WATERHOUSE + PIYASIN 31842 [B6 18] P-PARA

UP,KAZAN

$50 M$ FROM ROAD LEADING W FROM HWY, KHAO

PHRIK NR RATBURI,THAILAND

H---;WATERHOUSE JB + PIYASIN

S, 1970,PALAEONTOGRAPHICA,BD 135,ABT A,P 106-112

REMARKS: C,D-JB WATERHOUSE, 1964 ;JBW NO B618;NEW GENUS;ORDER PRODUCTIDA.SUBFAMILY

STRIOCHONETINAE ACCORDING TO WATERHOUSE; SPEC MEASURED BUT NOT LISTED AS PARATYPE : 1 SPEC

STRIOCHONETES SCUTELLA WATERHOUSE + PIYASIN 31843 [B237] P-PARA

UP.KAZAN

$50 M$ FROM ROAD LEADING W FROM HWY, KHAO

PHRIK,NR RATBURI,THAILAND

H---.WATERHOUSE JB + PIYASIN

S, 1970,PALAEONTOGRAPHICA,BD 135,ABT A,P 106-112

REMARKS: C.D-JB WATERHOUSE, 1964 ;JBW NO B237; NEW

GENUS:ORDER PRODUCTIDA,SUBFAMILY

STRIOCHONETINAE ACCORDING TO WATERHOUSE + PIYASIN ; 1 SMALL SLAB

STRIOCHONETES SCUTELLA WATERHOUSE + PIYASIN 31844 [B245] P-PARA

UP.KAZAN

50 M FROM ROAD LEADING W FROM HWY, KHAO

PHRIK NR RATBURITHAILAND

H---;WATERHOUSE JB + PIYASIN
S, 1970,PALAEONTOGRAPHICA,BD 135.ABT A,P 106-112

REMARKS: C,D-JB WATERHOUSE, 1964 ;JBW NO B254;NEW GENUS:ORDER PRODUCTIDA SUBFAMILY

STRIOCHONETINAE ACCORDING TO WATERHOUSE + PIYASIN : 1 SMALL SLAB

STRIOCHONETES SCUTELLA WATERHOUSE + PIYASIN $31756[B 211]$ P-PARA

UP,KAZAN

$50 M$ FROM ROAD LEADING W FROM HWY KHAO

PHRIK.NR RATBURI,THAILAND

H---:WATERHOUSE JB + PIYASIN

S, 1970,PALAEONTOGRAPHICA,BD 135,ABT A.P 106-112

REMARKS: C.D-JB WATERHOUSE, 1964 ; JBW NO B211;NEW GENUS;ORDER PRODUCTIDA, SUBFAMILY

STRIOCHONETINAE ACCORDING TO WATERHOUSE + PIYASIN; 1 SPEC

STRIOCHONETES SCUTELLA WATERHOUSE + PIYASIN

31757 [B212] P-PARA

UP, KAZAN

$50 \mathrm{M}$ FROM ROAD LEADING W FROM HWY, KHAO

PHRIK.NR RATBURI,THAILAND

H---:WATERHOUSE JB + PIYASIN

S. 1970.PALAEONTOGRAPHICA BD 135,ABT A P 106-112

REMARKS: C,D-JB WATERHOUSE, 1964 ;JBW NO B212;NEW

GENUS:ORDER PRODUCTIDA,SUBFAMILY

STRIOCHONETINAE ACCORDING TO WATERHOUSE + PIYASIN; 1 SPEC

STRIOCHONETES SCUTELLA WATERHOUSE + PIYASIN 31758 [B2 13] P-PARA

UP, KAZAN

$5 O M$ FROM ROAD LEADING W FROM HWY, KHAO

PHRIK,NR RATBURI,THAILAND

H---;WATERHOUSE JB + PIYASIN

S, 1970,PALAEONTOGRAPHICA,BD 135,ABT A,P106-

112, PL 16,FIG 12,13

REMARKS: C,D-JB WATERHOUSE, 1964 :JBW NO B213;NEW

GENUS;ORDER PRODUCTIDA,SUBFAMILY

STRIOCHONETINAE ACCORDING TO WATERHOUSE +

PIYASIN ; 1 SPEC

STRIOCHONETES SCUTELLA WATERHOUSE + PIYASIN

31759 [B2 15] P-PARA

UP,KAZAN

50 M FROM ROAD LEADING W FROM HWY KHAO

PHRIK,NR RATBURI,THAILAND

H---;WATERHOUSE JB + PIYASIN

S. 1970.PALAEONTOGRAPHICA BD 135,ABT A P 106-112

REMARKS: C,D-JB WATERHOUSE, 1964 ; JBW NO B2 15; NEW

GENUS:ORDER PRODUCTIDA SUBFAMILY

STRIOCHONETINAE ACCORDING TO WATERHOUSE +

PIYASIN; 1 SPEC

STRIOCHONETES SCUTELLA WATERHOUSE + PIYASIN 31760 [B2 16] P-PARA

UP, KAZAN

$50 M$ FROM ROAD LEADING W FROM HWY, KHAO

PHRIK NR RATBURI,THAILAND

H---:WATERHOUSE JB + PIYASIN

S. 1970,PALAEONTOGRAPHICA,BD 135, ABT A.P 106-112

REMARK S: C.D-JB WATERHOUSE, 1964 :JBW NO B2 16:NEW

GENUS;ORDER PRODUCTIDA, SUBFAMILY

STRIOCHONETINAE ACCORDING TO WATERHOUSE +

PIYASIN; 1 SPEC

STRIOCHONETES SCUTELLA WATERHOUSE + PIYASIN

$31761[$ [B217] P-PARA

UP KAZAN

50 M FROM ROAD LEADING W FROM HWY, KHAO

PHRIK, NR RATBURI, THAILAND

H---:WATERHOUSE JB + PIYASIN

S, 1970,PALAEONTOGRAPHICA,BD 135.ABT A.P 106-112

REMARKS: C,D-JB WATERHOUSE, 1964 ; JBW NO B2 17; NEW

GENUS:ORDER PRODUCTIDA, SUBFAMILY

STRIOCHONETINAE ACCORDING TO WATERHOUSE +

PIYASIN : 1 SPEC 
STRIOCHONETES SCUTELLA WATERHOUSE + PIYASIN

31762 [B218] P.PARA

UP, KAZAN

$50 \mathrm{M}$ FROM ROAD LEADING W FROM HWY, KHAO

PHRIK, NR RATBURI, THAILAND

H-..;WATERHOUSE JB + PIYASIN

S, 1970,PALAEONTOGRAPHICA,BD 135,ABT A,P 106 -

112.PL 16.FIG 10,11

REMARKS: C,D-JB WATERHOUSE, 1964 ;JBW NO B218;NEW GENUS:ORDER PRODUCTIDA,SUBFAMILY

STRIOCHONETINAE ACCORDING TO WATERHOUSE + PIYASIN ; 1 SPEC

STRIOCHONETES SCUTELLA WATERHOUSE + PIYASIN

31763 [B219] P-PARA

UPKAZAN

50 M FROM ROAD LEADING W FROM HWY, KHAO

PHRIK,NR RATBURI,THAILAND

H---:WATERHOUSE JB + PIYASIN

S. 1970,PALAEONTOGRAPHICA,BD 135,ABT A,P 106-112

REMARKS: C,D-JB WATERHOUSE, 1964 ;JBW NO B2 19;NEW GENUS:ORDER PRODUCTIDA, SUBFAMILY

STRIOCHONETINAE ACCORDING TO WATERHOUSE + PIYASIN ; 1 SPEC

STRIOCHONETES SCUTELLA WATERHOUSE + PIYASIN

31764 [B220] P-PARA

UP, KAZAN

50 M FROM ROAD LEADING W FROM HWY, KHAO

PHRIK, NR RATBURI,THAILAND

H---:WATERHOUSE JB + PIYASIN

S, 1970,PALAEONTOGRAPHICA,BD 135,ABT A,P 106112,PL 16, FIG 8,9

REMARKS: C.D-JB WATERHOUSE, 1964 ; JBW NO B220;NEW GENUS;ORDER PRODUCTIDA,SUBFAMILY STRIOCHONETINAE ACCORDING TO WATERHOUSE + PIYASIN : 1 SPEC

STRIOCHONETES SCUTELLA WATERHOUSE + PIYASIN 31765 [B221] P-PARA

UP.KAZAN

50 M FROM ROAD LEADING W FROM HWY, KHAO

PHRIK, NR RATBURI,THAILAND

H-.-; WATERHOUSE JB + PIYASIN

S, 1970.PALAEONTOGRAPHICA,BD 135,ABT A,P 106-112

REMARKS: C,D-JB WATERHOUSE, 1964 ; JBW NO B221;NEW GENUS,ORDER PRODUCTIDA,SUBFAMILY

STRIOCHONETINAE ACCORDING TO WATERHOUSE + PIYASIN ; 1 SPEC

STRIOCHONETES SCUTELLA WATERHOUSE+PIYASIN

31766 [B222] P-PARA

UP.KAZAN

50 M FROM ROAD LEADING W FROM HWY, KHAO

PHRIK,NR RATBURI,THAILAND

H-.. WATERHOUSE JB + PIYASIN

S, 1970,PALAEONTOGRAPHICA,BD 135,ABT A,P 106-112

REMARKS: C,D-JB WATERHOUSE, 1964 ; JBW NO B222; NEW

GENUS,ORDER PRODUCTIDA SUBFAMILY

STRIOCHONETINAE ACCORDING TO WATERHOUSE +

PIYASIN ; 1 SPEC

STRIOCHONETES SCUTELLA WATERHOUSE + PIYASIN

31767 [B223 ] P.PARA

UP,KAZAN

50 M FROM ROAD LEADING WFROM HWY, KHAO

PHRIK, NR RATBURI THAILAND

H---;WATERHOUSE JB + PIYASIN

S, 1970,PALAEONTOGRAPHICA,BD 135,ABT A,P 106-112

REMARKS: C.D-JB WATERHOUSE, 1964 ; JBW NO B223; NEW

GENUS:ORDER PRODUCTIDA,SUBFAMILY

STRIOCHONETINAE ACCORDING TO WATERHOUSE +

PIYASIN : 1 SPEC

STRIOCHONETES SCUTELLA WATERHOUSE + PIYASIN

31768 [B224] P-PARA

UP,KAZAN

50 M FROM ROAD LEADING W FROM HWY, KHAO

PHRIK, NR RATBURI,THAILAND

H---;WATERHOUSE JB + PIYASIN

S, 1970,PALAEONTOGRAPHICA.BD 135.ABT A.P 106-112
REMARKS: C.D-JB WATERHOUSE, 1964 ; JBW NO B224: NEW GENUS;ORDER PRODUCTIDA,SUBFAMILY STRIOCHONETINAE ACCORDING TO WATERHOUSE + PIYASIN: 1SPEC

STRIOCHONETES SCUTELLA WATERHOUSE + PIYASIN

31769 [B226] P-PARA

UP.KAZAN

50 M FROM ROAD LEADING W FROM HWY, KHAO

PHRIK,NR RATBURI,THAILAND

H---; WATERHOUSE JB + PIYASIN

S, 1970,PALAEONTOGRAPHICA,BD 135,ABT A,P 106-112 REMARKS: C.D-JB WATERHOUSE, 1964 ;JBW NO B226;NEW GENUS,ORDER PRODUCTIDA,SUBFAMILY STRIOCHONETINAE ACCORDING TO WATERHOUSE + PIYASIN : 1 SPEC

STRIOCHONETES SCUTELLA WATERHOUSE + PIYASIN

31770 [B227 ] P-PARA

UP.KAZAN

50 M FROM ROAD LEADING W FROM HWY, KHAO PHRIK, NR RATBURI,THAILAND

H-..;WATERHOUSE JB + PIYASIN

S, 1970,PALAEONTOGRAPHICA,BD 135,ABT A,P 106-112

REMARKS: C,D-JB WATERHOUSE, 1964 ;JBW NO B227;NEW GENUS;ORDER PRODUCTIDA,SUBFAMILY

STRIOCHONETINAE ACCORDING TO WATERHOUSE + PIYASIN; 1 SPEC

STRIOCHONETES SCUTELLA WATERHOUSE+PIYASIN 31771 [B228] P-PARA

UP.KAZAN

50 M FROM ROAD LEADING W FROM HWY, KHAO

PHRIK,NR RATBURI,THAILAND

H---;WATERHOUSE JB + PIYASIN

S, 1970,PALAEONTOGRAPHICA,BD 135,ABT A,P 106-112

REMARKS: C,D-JB WATERHOUSE, 1964 ;JBW NO B228; NEW GENUS;ORDER PRODUCTIDA.SUBFAMILY

STRIOCHONETINAE ACCORDING TO WATERHOUSE + PIYASIN; 1 SPEC

STRIOCHONETES SCUTELLA WATERHOUSE+PIYASIN

31772 [B229] P-PARA

UP,KAZAN

$50 M$ FROM ROAD LEADING W FROM HWY, KHAO

PHRIK, NR RATBURI,THAILAND

H---;WATERHOUSE JB + PIYASIN

S, 1970,PALAEONTOGRAPHICA, BD 135, ABT A,P 106-112

REMARKS: C,D-JB WATERHOUSE, 1964 ; JBW NO B229;NEW GENUS:ORDER PRODUCTIDA,SUBFAMILY

STRIOCHONETINAE ACCORDING TO WATERHOUSE + PIYASIN ; 1 SPEC

STRIOCHONETES SCUTELLA WATERHOUSE + PIYASIN

31773 [B230] P.PARA

UP,KAZAN

50 M FROM ROAD LEADING W FROM HWY, KHAO

PHRIK,NR RATBURI,THAILAND

H--.;WATERHOUSE JB + PIYASIN

S, 1970,PALAEONTOGRAPHICA,BD 135,ABT A,P 106-112

REMARKS: C.D-JB WATERHOUSE, 1964 ;JBW NO B230;NEW

GENUS:ORDER PRODUCTIDA,SUBFAMILY

STRIOCHONETINAE ACCORDING TO WATERHOUSE + PIYASIN; 1 SPEC

STRIOCHONETES SCUTELLA WATERHOUSE + PIYASIN 31774 [B231] P-PARA

UP,KAZAN

50 M FROM ROAD LEADING W FROM HWY, KHAO PHRIK,NR RATBURI, THAILAND

H---;WATERHOUSE JB + PIYASIN

S, 1970,PALAEONTOGRAPHICA,BD 135,ABT A.P 106

112,PL 16,FIG 6,7

REMARKS: C,D-JB WATERHOUSE, 1964 ;JBW NO B231; NEW GENUS:ORDER PRODUCTIDA,SUBFAMILY

STRIOCHONETINAE ACCORDING TO WATERHOUSE + PIYASIN ; 1 SPEC 
STRIOCHONETES SCUTELLA WATERHOUSE + PIYASIN 31775 [B233] P-PARA

UP.KAZAN

50 M FROM ROAD LEADING W FROM HWY, KHAO

PHRIK,NR RATBURI,THAILAND

H-.--WATERHOUSE JB + PIYASIN

S. 1970.PALAEONTOGRAPHICA,BD 135,ABT A.P 106-112

REMARKS: C,D-JB WATERHOUSE, 1964 ; JBW NO B233; NEW GENUS:ORDER PRODUCTIDA.SUBFAMILY

STRIOCHONETINAE ACCORDING TO WATERHOUSE +

PIYASIN ; 6 PCES

STRIOCHONETES SCUTELLA WATERHOUSE + PIYASIN 31776 [8234] P-PARA

UP.KAZAN

50 M FROM ROAD LEADING W FROM HWY, KHAO

PHRIK,NR RATBURI,THAILAND

H---;WATERHOUSE JB + PIYASIN

S. 1970.PALAEONTOGRAPHICA.BD 135.A8T A.P 106-112

REMARKS: C,D-JB WATERHOUSE, 1964 ;JBW NO B234;NEW

GENUS:ORDER PRODUCTIDA.SUBFAMILY

STRIOCHONETINAE ACCORDING TO WATERHOUSE +

PIYASIN; 1 SPEC

STRIOCHONETES SCUTELLA WATERHOUSE + PIYASIN

31777 [B235] P-HOLO

UP,KAZAN

$50 M$ FROM ROAD LEADING W FROM HWY, KHAO

PHRIK NR RATBURI,THAILAND

H...:WATERHOUSE JB + PIYASIN

S, 1970,PALAEONTOGRAPHICA,BD 135,ABT A,P 106-

112.PL 16.FIG 1.2

REMARKS: C.D-J8 WATERHOUSE, 1964 ;JBW NO B235; NEW GENUS:ORDER PRODUCTIDA, SUBFAMILY

STRIOCHONETINAE ACCORDING TO WATERHOUSE + PIYASIN : 1 SPEC

STRIOCHONETES SCUTELLA WATERHOUSE + PIYASIN 31778 [B236] P-PARA

UP.KAZAN

$50 M$ FROM ROAD LEADING W FROM HWY, KHAO

PHRIK,NR RATBURI,THAILAND

H---:WATERHOUSE JB + PIYASIN

S. 1970.PALAEONTOGRAPHICA BD 135.ABT A.P 106-112

REMARKS: C,D-JB WATERHOUSE, 1964 ;JBW NO B236; NEW GENUS:ORDER PRODUCTIDA,SUBFAMILY

STRIOCHONETINAE ACCORDING TO WATERHOUSE + PIYASIN : 1 SPEC

STRIOCHONETES SCUTELLA WATERHOUSE + PIYASIN

31779 [B238] P-PARA

UP,KAZAN

50 M FROM ROAD LEADING W FROM HWY, KHAO

PHRIK.NR RATBURI,THAILAND

H---:WATERHOUSE JB + PIYASIN

S. 1970.PALAEONTOGRAPHICA,BD 135,ABT A,P106-112

REMARKS: C.D-J8 WATERHOUSE, 1964 ;JBW NO B238;NEW

GENUS,ORDER PRODUCTIDA,SUBFAMILY

STRIOCHONETINAE ACCORDING TO WATERHOUSE +

PIYASIN; 1 SPEC

STRIOCHONETES SCUTELLA WATERHOUSE + PIYASIN

31780 [B239] P.PARA

UP, KAZAN

50 M FROM ROAD LEADING W FROM HWY, KHAO

PHRIK NR RATBURI,THAILAND

H---:WATERHOUSE JB + PIYASIN

S. 1970.PALAEONTOGRAPHICA,8D 135.ABT A.P 106-112

REMARKS:C.D-JB WATERHOUSE, 1964 J JBW NO B239;NEW

GENUS:ORDER PRODUCTIDA, SUBFAMILY

STRIOCHONETINAE ACCORDING TO WATERHOUSE +

PIYASIN: 1SPEC

STROPHEODONTA DEMISSA MUT HOMALOSTRIATUS G. 22492 [244S] P-SYN

MD.ERIAN

DETROIT RIVER GP,AMHERSTBURG FM

UMONROE

LIVINGSTONE CUT, DETROIT R, AMHERSTBURG, ONT

H395:GRABAU AW, MICHIGAN GEOL \& BIOL SURV,PUBL

2.GEOL SER 1,1909,P126,PL 17,FIG 6

REMARKS: D-T NATTRESS, 1914 :LARGER SPEC FIG'D : 2 SPEC
STROPHEODONTA SP INDET 22658 [4 14S ] REF SPEC-F

$\mathrm{MS}$

ABOVE LIMESTONE RAPIDS, SEVERN R,DIST OF

PATRICIA.ONT

H395:PARKS WA TRANS ROY CAN INST, VOL

11,PT 1, 1915,P49,PL4,FIG 18

REMARKS: C-JB TYRRELL, 1912 ;D-WH HEARST. $1915 ; 1$ SPEC

STROPHEODONTA SP INDET

22657 [4 13S ] REF SPEC-F

MS,

LIMESTONE RAPIDS, FAWN R DIST OF PATRICIA ONT

H395:PARKS WA,TRANS ROY CAN INST, VOL

11,PT 1, 1915,P49,PL4,FIG 17

REMARKS: C-JB TYRRELL, 1912 ;D-WH HEARST, $1915 ; 1$ SPEC

STROPHEODONTA VASCULOSA GRABAU

22439 [190S ] P-SYN

$M D$.ERIAN

DETROIT RIVER GP, AMHERSTBURG FM

[UMONROE]

LIVINGSTONE CUT,DETROIT R AMHERSTBURG ONT

H395;GRABAU AW, MICHIGAN GEOL \& BIOL SURV,PUBL

2,GEOL SER 1,1909,P124-126,PL 17,FIG 8,9

REMARKS: D-T NATTRESS, $1914 ; 1$ SPEC

STROPHOMENA ATAVA MATTHEW

REMARKS: SEE EOORTHIS ATAVA (MATTHEW)

STROPHOMENA PLANUMBONA VAR ERINDALENSIS DYER

12174 P-HOLO

UO.CINCI,MAYSVILL

MEAFORD FM ERINDALE MBR

MULLET CK,ERINDALE,ONT

H384:DYER WS, ONT DEPT MINES, VOL

32,PT7,1925,P74,PL 7,FIG 11,12,16

REMARKS: C-WS DYER, 1923 ;SPEC 'B';SECT 2,INT 8 OF DYER ; 1 SPEC

\section{STROPHOMENA VARSENSIS DYER}

12174 P-HOLO

U O,CINCI,MAYSVILL

MEAFORD FM,ERINDALE MBR

MULLET CK ERINDALE,ONT

H384;DYER WS, ONT DEPT MINES, VOL

32,PT7, 1925, P75,PL7,FIG 13

REMARKS: C-WS DYER, 1923 'SPEC 'A'.SECT 8 INT 2 OF DYER;STORED UNDER S. PLANUMBONA ERINDALENSIS 1 SPEC

STROPHOMENA? TETRASTRIATA PARKS

$22648[403 S$ ] P-SYN

O.

LOWER RAPIDS,GODS R,MAN

H384.WA PARKS, TRANS ROY CAN INST, VOL

11.PT 1, 1915,P 16-17,PL4,FIG 16

REMARKS: C-JB TYRRELL, 1912 ;D-WH HEARST, 1915;SPEC 'A' FIG'D:LOC GIVEN AS SHAMATTAWA R-NOW CALLED GODS R,TRIBUTARY OF HAYES R ; 3 SPEC

TREMATIS MILLEPUNCTATA HALL

17348 [1107HR] REF SPEC-F

U O.CINCI.MAYSVILL

DUNDAS FM

DON VALLEY QUARRY,TORONTO,ONT

H283:PARKS \& DYER,ONT DEPT MINES, VOL

30.PT7, 1922,P30,PL6.FIG 1,PL7,FIG 7.8

REMARKS: C-J TOWNSEND;FIGS ARE COMPOSITE DRAWINGS : 4 SPEC

TREMATOBOLUS INSIGNIS MATTHEW

8432(B) [802CM(B)] P.PARAL

LCA.

ST JOHN GP

[ACADIAN,DIV 1B2]

HANFORD BK, ST MARTINS,ST JOHN CO.NB 
H293:WALCOTT CD, 19 12,P617-619:MATTHEW GF CAN REC SCI. 1893.P276-279,FIG 1A-D; 1894,TRSC,P88,PL 16,FIG $4 A-D$

REMARKS: C-GF MATTHEW:D-MACKENZIE ;LECTOTYPE DESIGNATED BY WALCOTT-SEE NO 8432(A); 1 SPEC

TREMATOBOLUS INSIGNIS MATTHEW

8476 [846CM ] P-PARAL

LCA,

[ACADIAN,DIV 1B2]

HANFORD BK. ST MARTINS,ST JOHN CO,NB

H293: WALCOTT CD, 19 12,P617-619,PL84,FIG 5B:MATTHEW GF.CAN REC SCI, 1893,P276-279,FIG 1A;

1894,TRSC,P88,PL 16, FIG 4A-D

REMARKS: C-GF MATTHEW:D-MACKENZIE ;SPEC 'A' = FIG $5 B$ OF WALCOTT,FIG $1 A+4 A$ OF MATTHEW; LECTOTYPE DESIGNATED BY WALCOTT,SEE NO 8432(A) ; 2 SPEC

TREMATOBOLUS INSIGNIS MATTHEW

8432(A) [802CM(A)] P-LECTO

LCA.

ST JOHN GP

[ACADIAN,DIV 1B2]

HANFORD BK, ST MARTINS,ST JOHN CO,NB

H293:MATTHEW GF, 1894,P88,PL 16,FIG 4B; 1893,P276279.FIG 1B:WALCOTT CD, 19 12,P617-619,PL84,FIG 5,5A

REMARKS: C-GF MATTHEW;D-MACKENZIE ;SPEC 'A' OF MATTHEW'S SYNTYPES;DESIGNATED LECTOTYPE BY WALCOTT : 1 SPEC

\section{TREMATOBOLUS KEMPANUM (MATTHEW)}

$7816[186 \mathrm{CM}]$ P-SYN

PROTOSIPHON KEMPANUM MATTHEW

LCA,

ST JOHN GP

[ST JOHN,ACADIAN,C 1B2-4]

LONG IS, KINGS CO,NB

H293; WAL.COTT CD, 1912,P619-621,PL83,FIG 1B, $1 \mathrm{H}$; MATTHEW GF,GEOL MAG,VOL 4, 1897,P70-7 1,FIG 1-4 REMARKS: C-GF MATTHEW:D-MACKENZIE :LABELLED COTYPES;UNCERTAIN WHICH SPECS FIG'D ; 9 SPEC

\section{TREMATOBOLUS KEMPANUM (MATTHEW)}

$7806[176 \mathrm{CM}] \mathrm{P}-\mathrm{SYN}$

LCA,

ST JOHNGP

[ST JOHN,ACADIAN,C 1B2-4]

LONG IS, KINGS CO,NB

H293.WALCOTT CD 1912, P6 19-621,PL 83, FIG 1B, $1 \mathrm{H}$

MATTHEW GF,GEOL MAG, 1897,P70-71,FIG 1-4

REMARKS: C-GF MATTHEW:D-MACKENZIE :LABELLED

COTYPES:UNCERTAIN WHICH SPECS FIG'D ; 13 SPEC

TREMATOBOLUS PRISTINUS (MATTHEW)

7685 [55CM ] P-PARAL

OBOLUS PRISTINUS MATTHEW

$\mathrm{LCA}$,

ST JOHN GP

[ST JOHN,ACADIAN,DIV 1B2]

HANFORD BK, ST MARTINS,ST JOHN CO,NB

H293; WALCOTT CD, 1912,P621-622,PL83,FIG 2E-H;

MATTHEW GF, 1895,NY,P 121-122,PL4,FIG 1A,B

REMARKS: C-GF MATTHEW:D-MACKENZIE :LECTOTYPE

DESIGNATED BY WALCOTT-SEE NO 7686;UNCERTAIN

WHETHER THIS SPEC FIG'D; 1 SPEC

TREMATOBOLUS PRISTINUS (MATTHEW)

7686 [56CM ] P-LECTO

OBOLUS PRISTINUS MATTHEW

LCA

ST JOHN GP

[ST JOHN,ACADIAN,DIV 1B2]

HANFORD BK, ST MARTINS, ST JOHN CO NB

H293; WALCOTT GF, 1912,P621-622,PL83,FIG 2F;MATTHEW GF 1895 , NY.P 121-122.PL 4.FIG 1C

REMARKS: C-GF MATTHEW:D-MACKENZIE ;DESIGNATED LECTOTYPE BY WALCOTT; 1 SPEC
TREMATOBOLUS PRISTINUS (MATTHEW)

7812 [182CM] P-PARAL

OBOLUS PRISTINUS MATTHEW

LCA.

ST JOHN GP

[ST JOHN ACADIAN DIV 1B2]

HANFORD BK, ST MARTINS,ST JOHN CO,NB

H293; WALCOTT CD, 1912,P621-622,PL83,FIG 2E-H

MATTHEW GF, 1895,NY,P 121-122,PL4,FIG 1E

REMARKS: C-GF MATTHEW;D-MACKENZIE ;LECTOTYPE DESIGNATED BY WALCOTT, SEE NO 7686:SPEC ' $C$ ' ' $B$ ', ' $A$ ' = FIGS 2E, G, H OF WALCOTT ; 3 SPEC

UNCINELLA CF INDICA WAAGEN

31749 [B520] REF SPEC-F

$\cup P, K A Z A N$

50 M FROM ROAD LEADING W FROM HWY, KHAO

PHRIK, NR RATBURI, THAILAND

H654; WATERHOUSE JB + PIYASIN

S, 1970.PALAEONTOGRAPHICA,BD 135,ABT A.P141-

142,PL25, FIG 1-3

REMARKS: C,D-JB WATERHOUSE, 1964 ;JBW NO B520 ; 1 SPEC

WAAGENITES SPECIOSUS WATERHOUSE + PIYASIN 31857 [B 147] P-PARA

UP.KAZAN

50 M FROM ROAD LEADING W FROM HWY, KHAO PHRIK,NR RATBURI,THAILAND

H433: WATERHOUSE JB + PIYASIN

S, 1970,PALAEONTOGRAPHICA,BD 135,ABT A,P112-117

REMARKS: C,D-JB WATERHOUSE, 1964 ; JBW NO B 147 ;

ORDER PRODUCTIDA ACCORDING TO WATERHOUSE + PIYASIN ; 1 SPEC

WAAGENITES SPECIOSUS WATERHOUSE+PIYASIN 31858 [B148] P-PARA

UP.KAZAN

50 M FROM ROAD LEADING W FROM HWY, KHAO

PHRIK, NR RATBURI,THAILAND

H433; WATERHOUSE JB + PIYASIN

S, 1970,PALAEONTOGRAPHICA,BD 135,ABT A,P112-117

REMARKS: C,D-JB WATERHOUSE, 1964 ; JBW NO B148;

ORDER PRODUCTIDA ACCORDING TO WATERHOUSE + PIYASIN ; 1 SPEC

WAAGENITES SPECIOSUS WATERHOUSE+PIYASIN 31859 [B 149] P-PARA

UP.KAZAN

50 M FROM ROAD LEADING W FROM HWY, KHAO

PHRIK, NR RATBURI, THAILAND

H433; WATERHOUSE JB + PIYASIN

S, 1970,PALAEONTOGRAPHICA,BD 135,ABT A,P112117, PL 18,FIG 8,9

REMARKS: C,D-JB WATERHOUSE, 1964 ;JBW NO B 149; ORDER PRODUCTIDA ACCORDING TO WATERHOUSE + PIYASIN ; 1 SPEC

WAAGENITES SPECIOSUS WATERHOUSE + PIYASIN 31860 [B 150] P-PARA

UP.KAZAN

50 M FROM ROAD LEADING W FROM HWY, KHAO PHRIK, NR RATBURI,THAILAND H433:WATERHOUSE JB + PIYASIN

S, 1970.PALAEONTOGRAPHICA,BD 135, ABT A,P 112-117

REMARKS: C,D-JB WATERHOUSE, 1964 ;JBW NO B 150 ; ORDER PRODUCTIDA ACCORDING TO WATERHOUSE + PIYASIN : 1 SPEC

WAAGENITES SPECIOSUS WATERHOUSE + PIYASIN 31861 [B151] P-PARA

UP,KAZAN

50 M FROM ROAD LEADING W FROM HWY, KHAO

PHRIK, NR RATBURI,THAILAND

H433.WATERHOUSE JB + PIYASIN

S, 1970,PALAEONTOGRAPHICA,BD 135,ABT A,P 112 117,PL 18,FIG 4,5

REMARKS: C,D-JB WATERHOUSE, 1964 ; JBW NO B 151 . ORDER PRODUCTIDA ACCORDING TO WATERHOUSE + PIYASIN : 1 SPEC 
WAAGENITES SPECIOSUS WATERHOUSE + PIYASIN 31862 [B152] P-PARA

UP.KAZAN

$50 M$ FROM ROAD LEADING W FROM HWY, KHAO

PHRIK,NR RATBURI,THAILAND

H433; WATERHOUSE JB + PIYASIN

S. 1970,PALAEONTOGRAPHICA.BD 135,ABT A.P 112-117

REMARKS: C.D-JB WATERHOUSE, 1964 ;JBW NO B 152;

ORDER PRODUCTIDA ACCORDING TO WATERHOUSE + PIYASIN : 1 SPEC + FRAG

WAAGENITE S SPECIOSUS WATERHOUSE + PIYASIN 31863 [B153] P.PARA

UP.KAZAN

50 M FROM ROAD LEADING W FROM HWY, KHAO

PHRIK.NR RATBURI,THAILAND

H433:WATERHOUSE JB + PIYASIN

S, 1970,PALAEONTOGRAPHICA,BD 135,ABT A,P112-117

REMARKS: C.D-JB WATERHOUSE, 1964 ;JBW NO B 153;

ORDER PRODUCTIDA ACCORDING TO WATERHOUSE + PIYASIN ; 1 SPEC

WAAGENITES SPECIOSUS WATERHOUSE + PIYASIN

31864 [B 154] P-PARA

UP, KAZAN

50 M FROM ROAD LEADING W FROM HWY, KHAO

PHRIK,NR RATBURI, THAILAND

H433:WATERHOUSE JB + PIYASIN

S, 1970,PALAEONTOGRAPHICA,BD 135,ABT A,P112-117

REMARKS: C,D-JB WATERHOUSE, 1964 ; JBW NO B154;

ORDER PRODUCTIDA ACCORDING TO WATERHOUSE + PIYASIN ; 1 SPEC

WAAGENITES SPECIOSUS WATERHOUSE + PIYASIN 31865 [B 155] P-PARA

UP, KAZAN

50 M FROM ROAD LEADING W FROM HWY, KHAO

PHRIK NR RATBURI,THAILAND

H433; WATERHOUSE JB + PIYASIN

S. 1970,PALAEONTOGRAPHICA BD 135,ABT A P112-117

REMARKS: C,D-JB WATERHOUSE, 1964 ;JBW NO B 155;

ORDER PRODUCTIDA ACCORDING TO WATERHOUSE + PIYASIN; 1 SPEC

WAAGENITES SPECIOSUS WATERHOUSE + PIYASIN 31866 [B156] P-HOLO

UP.KAZAN

50 M FROM ROAD LEADING W FROM HWY, KHAO

PHRIK,NR RATBURI,THAILAND

H433; WATERHOUSE JB + PIYASIN

S, 1970,PALAEONTOGRAPHICA,BD 135,ABT A,P112117.PL 18.FIG 1,3

REMARKS: C.D-JB WATERHOUSE, 1964 ;JBW NO B156; ORDER PRODUCTIDA ACCORDING TO WATERHOUSE + PIYASIN ; 1 SPEC

WAAGENITES SPECIOSUS WATERHOUSE + PIYASIN 31867 [B157] ] -PARA

UP,KAZAN

$5 O M$ FROM ROAD LEADING W FROM HWY, KHAO

PHRIK,NR RATBURI,THAILAND

H433; WATERHOUSE JB + PIYASIN

S, 1970,PALAEONTOGRAPHICA,BD 135,ABT A,P 112-117

REMARKS: C.D-JB WATERHOUSE, 1964 ; JBW NO B 157;

ORDER PRODUCTIDA ACCORDING TO WATERHOUSE + PIYASIN : 1 SPEC

WAAGENITES SPECIOSUS WATERHOUSE + PIYASIN

31868 [B158] P-PARA

UP,KAZAN

50 M FROM ROAD LEADING W FROM HWY, KHAO

PHRIK, NR RATBURI, THAILAND

H433: WATERHOUSE JB + PIYASIN

S, 1970,PALAEONTOGRAPHICA,BD 135,ABT A,P 112-117

REMARKS: C.D-JB WATERHOUSE, 1964 ; JBW NO B 158;

ORDER PRODUCTIDA ACCORDING TO WATERHOUSE +

PIYASIN ; 1 SPEC
WAAGENITES SPECIOSUS WATERHOUSE + PIYASIN

31869 [B159] P.PARA

UP.KAZAN

$50 \mathrm{M}$ FROM ROAD LEADING W FROM HWY, KHAO

PHRIK,NR RATBURI,THAILAND

H433; WATERHOUSE JB + PIYASIN

S. 1970,PALAEONTOGRAPHICA,BD 135,ABT A,P112-117

REMARKS: C,D-JB WATERHOUSE, 1964 :JBW NO B159:

ORDER PRODUCTIDA ACCORDING TO WATERHOUSE +

PIYASIN : 1 SPEC

WAAGENITES SPECIOSUS WATERHOUSE + PIYASIN

31870 [B 160] P-PARA

UP, KAZAN

50 M FROM ROAD LEADING W FROM HWY KHAO

PHRIK,NR RATBURI,THAILAND

H433: WATERHOUSE JB + PIYASIN

S, 1970,PALAEONTOGRAPHICA,BD 135,ABT A,P112-117

REMARKS: C.D-JB WATERHOUSE, 1964 ; JBW NO B 160

ORDER PRODUCTIDA ACCORDING TO WATERHOUSE +

PIYASIN : 1 SPEC

WAAGENITES SPECIOSUS WATERHOUSE + PIYASIN

31871 [B 161] P-PARA

UP, KAZAN

$50 \mathrm{M}$ FROM ROAD LEADING W FROM HWY, KHAO

PHRIK, NR RATBURI, THAILAND

H433; WATERHOUSE JB + PIYASIN

S, 1970,PALAEONTOGRAPHICA,BD 135,ABT A,P112-117

REMARKS: C.D-JB WATERHOUSE, 1964 ; JBW NO B 161

ORDER PRODUCTIDA ACCORDING TO WATERHOUSE + PIYASIN ; 1 SPEC

WAAGENITES SPECIOSUS WATERHOUSE + PIYASIN

31872 [B 162] P.PARA

UP.KAZAN

$50 \mathrm{M}$ FROM ROAD LEADING W FROM HWY, KHAO

PHRIK, NR RATBURI, THAILAND

H433: WATERHOUSE JB + PIYASIN

S, 1970,PALAEONTOGRAPHICA,BD 135,ABT A,P112 117,PL17,FIG 18,19

REMARKS: C,D-JB WATERHOUSE, 1964 ; JBW NO B 162; ORDER PRODUCTIDA ACCORDING TO WATERHOUSE + PIYASIN ; 1 SPEC

WAAGENITES SPECIOSUS WATERHOUSE + PIYASIN

31873 [B 163 ] P.PARA

UP, KAZAN

$50 M$ FROM ROAD LEADING W FROM HWY, KHAO

PHRIK,NR RATBURI,THAILAND

H433; WATERHOUSE JB + PIYASIN

S, 1970,PALAEONTOGRAPHICA,BD 135,ABT A.P112-117

REMARKS: C,D-JB WATERHOUSE, 1964 ; JBW NO B 163

ORDER PRODUCTIDA ACCORDING TO WATERHOUSE + PIYASIN ; 1 SPEC

WAAGENITES SPECIOSUS WATERHOUSE + PIYASIN

31874 [B 164] P-PARA

UP.KAZAN

50 M FROM ROAD LEADING W FROM HWY, KHAO

PHRIK, NR RATBURI, THAILAND

H433; WATERHOUSE JB + PIYASIN

S. 1970,PALAEONTOGRAPHICA,BD 135,ABT A,P112-117

REMARKS: C.D-JB WATERHOUSE, 1964 : JBW NO B 164;

ORDER PRODUCTIDA ACCORDING TO WATERHOUSE + PIYASIN ; 1 SPEC

WAAGENITES SPECIOSUS WATERHOUSE + PIYASIN

31875 [B165] P-PARA

U P, KAZAN

$50 \mathrm{M}$ FROM ROAD LEADING W FROM HWY, KHAO

PHRIK,NR RATBURI,THAILAND

H433; WATERHOUSE JB + PIYASIN

S, 1970,PALAEONTOGRAPHICA,BD 135,ABT A,P112

117.PL17,FIG 26,27

REMARKS: C,D-JB WATERHOUSE, 1964 ; JBW NO B 165 ORDER PRODUCTIDA ACCORDING TO WATERHOUSE + PIYASIN ; 1 SPEC 
WAAGENITES SPECIOSUS WATERHOUSE + PIYASIN 31876 [B 166] P-PARA

UP,KAZAN

50 M FROM ROAD LEADING W FROM HWY , KHAO PHRIK,NR RATBURI,THAILAND H433; WATERHOUSE JB + PIYASIN

S, 1970,PALAEONTOGRAPHICA,BD 135,ABT A, $9112 \cdot 117$ REMARK S: C.D-JB WATERHOUSE, 1964 ;JBW NO B 166

ORDER PRODUCTIDA ACCORDING TO WATERHOUSE + PIYASIN : 1 SPEC

WAAGENITES SPECIOSUS WATERHOUSE + PIYASIN 31877 [B167] P.PARA

UP,KAZAN

50 M FROM ROAD LEADING WFROM HWY, KHAO

PHRIK,NR RATBURI,THAILAND

H433; WATERHOUSE JB + PIYASIN

S, 1970.PALAEONTOGRAPHICA,BD 135,ABT A.P112-117

REMARKS: C,D-JB WATERHOUSE, 1964 ; JBW NO B167;

ORDER PRODUCTIDA ACCORDING TO WATERHOUSE + PIYASIN: 1 SPEC

WAAGENITES SPECIOSUS WATERHOUSE+PIYASIN 31878 [B 168 ] P-PARA

UP,KAZAN

50 M FROM ROAD LEADING W FROM HWY, KHAO PHRIK, NR RATBURI, THAILAND

H433; WATERHOUSE JB + PIYASIN

S, 1970,PALAEONTOGRAPHICA,BD 135,ABT A,P112-

117,PL 18, FIG 19

REMARKS: C,D-JB WATERHOUSE, 1964 ;JBW NO B 168; ORDER PRODUCTIDA ACCORDING TO WATERHOUSE + PIYASIN; 1 SPEC

WAAGENITES SPECIOSUS WATERHOUSE+PIYASIN

31879 [B 169 ] P-PARA

$\cup P, K A Z A N$

50 M FROM ROAD LEADING W FROM HWY, KHAO

PHRIK,NR RATBURI,THAILAND

H433; WATERHOUSE JB + PIYASIN

S, 1970,PALAEONTOGRAPHICA,BD 135,ABT A,P112-117

REMARKS: C,D-JB WATERHOUSE, 1964 ; JBW NO B 169;

ORDER PRODUCTIDA ACCORDING TO WATERHOUSE + PIYASIN; 1 SPEC

WAAGENITES SPECIOSUS WATERHOUSE+PIYASIN $31880[8170]$ P-PARA

UP,KAZAN

50 M FROM ROAD LEADING W FROM HWY, KHAO

PHRIK, NR RATBURI, THAILAND

H433; WATERHOUSE JB + PIYASIN

S, 1970,PALAEONTOGRAPHICA BD 135,ABT A,P112-117

REMARKS: C,D-JB WATERHOUSE, 1964 ; JBW NO B 170;

ORDER PRODUCTIDA ACCORDING TO WATERHOUSE +

PIYASIN : 1 SPEC

WAAGENITES SPECIOSUS WATERHOUSE+PIYASIN

31881 [B171] P-PARA

UP, KAZAN

$50 \mathrm{M}$ FROM ROAD LEADING W FROM HWY, KHAO

PHRIK, NR RATBURI,THAILAND

H433: WATERHOUSE JB + PIYASIN

S, 1970,PALAEONTOGRAPHICA,BD 135,ABT A,P112-117

REMARKS: C,D-JB WATERHOUSE, 1964 ;JBW NO B 171 ;

ORDER PRODUCTIDA ACCORDING TO WATERHOUSE + PIYASIN : SPEC IN MATRIX,FRAGS

WAAGENITES SPECIOSUS WATERHOUSE+PIYASIN

31882 [B172] P-PARA

UP, KAZAN

50 M FROM ROAD LEADING W FROM HWY, KHAO

PHRIK, NR RATBURI, THAILAND

H433; WATERHOUSE JB + PIYASIN

S, 1970,PALAEONTOGRAPHICA,BD 135 ABT A.P 112-117

REMARKS: C,D-JB WATERHOUSE, 1964 ; JBW NO B 172 ;

ORDER PRODUCTIDA ACCORDING TO WATERHOUSE +

PIYASIN; 1 SPEC + FRAG
WAAGENITES SPECIOSUS WATERHOUSE + PIYASIN

31883 [B173] P-PARA

UP,KAZAN

50 M FROM ROAD LEADING W FROM HWY, KHAO

PHRIK, NR RATBURI,THAILAND

H433; WATERHOUSE JB + PIYASIN

S, 1970,PALAEONTOGRAPHICA,BD 135,ABT A,P112117,PL 18,FIG 10,11

REMARKS: C,D-JB WATERHOUSE, 1964 ; JBW NO B173; ORDER PRODUCTIDA ACCORDING TO WATERHOUSE + PIYASIN:SPEC BROKEN SINCE PHOTOGRAPHED:PL 19 FIG 2,3,8 LISTED AS B 173 BUT NOT SAME SPEC AS PL 18 , FIG 10,$11 ; 1$ SPEC

WAAGENITES SPECIOSUS WATERHOUSE + PIYASIN 31884 [B 174 ] P-PARA

UP, KAZAN

50 M FROM ROAD LEADING W FROM HWY, KHAO

PHRIK, NR RATBURI, THAILAND

H433; WATERHOUSE JB + PIYASIN

S, 1970,PALAEONTOGRAPHICA,BD 135,ABT A,P 112 -

117 PL 18,FIG 18

REMARKS: C,D-JB WATERHOUSE, 1964 ;JBW NO B 174 ORDER PRODUCTIDA ACCORDING TO WATERHOUSE + PIYASIN ; 1 SPEC + FRAG

WAAGENITES SPECIOSUS WATERHOUSE+PIYASIN 31885 [B175] P-PARA

UP.KAZAN

50 M FROM ROAD LEADING W FROM HWY, KHAO

PHRIK, NR RATBURI,THAILAND

H433; WATERHOUSE JB + PIYASIN

S, 1970,PALAEONTOGRAPHICA,BD 135,ABT A,P112117,PL 17, FIG 28,30

REMARKS: C,D-JB WATERHOUSE, 1964 ; JBW NO B 175 ORDER PRODUCTIDA ACCORDING TO WATERHOUSE + PIYASIN ; 1 SPEC

WAAGENITES SPECIOSUS WATERHOUSE+PIYASIN 31886 [B176] P.PARA

UP.KAZAN

50 M FROM ROAD LEADING W FROM HWY, KHAO PHRIK, NR RATBURI,THAILAND H433: WATERHOUSE JB + PIYASIN

S, 1970,PALAEONTOGRAPHICA,BD 135,ABT A,P112. 117,PL 18,FIG 2,13

REMARK S: C.D-JB WATERHOUSE, 1964 ;JBW NO B 176 ORDER PRODUCTIDA ACCORDING TO WATERHOUSE + PIYASIN;2 SPEC NUMBERED B 176;THIS SPEC MEASURED P $114 ; 1$ SPEC

WAAGENITES SPECIOSUS WATERHOUSE+PIYASIN

31887 [B176] P-PARA

UP.KAZAN

50 M FROM ROAD LEADING W FROM HWY, KHAO PHRIK,NR RATBURI,THAILAND H433: WATERHOUSE JB + PIYASIN

S 1970 PALAEONTOGRAPHICA BD 135 ABT A.P 112-117 REMARKS: C.D-JB WATERHOUSE, 1964 ; JBW NO B176; ORDER PRODUCTIDA ACCORDING TO WATERHOUSE + PIYASIN:2 SPEC NUMBERED B 176;THIS SPEC MEASURED P $113 ; 1$ SPEC

WAAGENITES SPECIOSUS WATERHOUSE + PIYASIN 31888 [B 177 ] P-PARA

UP.KAZAN

50 M FROM ROAD LEADING W FROM HWY, KHAO PHRIK,NR RATBURI,THAILAND H433.WATERHOUSE JB + PIYASIN

S, 1970,PALAEONTOGRAPHICA,BD 135,ABT A,P 112-117 REMARKS: C.D-JB WATERHOUSE, 1964 ; JBW NO 8177 ORDER PRODUCTIDA ACCORDING TO WATERHOUSE + PIYASIN; 1 SPEC

WAAGENITES SPECIOSUS WATERHOUSE + PIYASIN 31889 [B 178] P-PARA

UP,KAZAN

50 M FROM ROAD LEADING W FROM HWY . KHAO PHRIK, NR RATBURI,THAILAND H433: WATERHOUSE JB + PIYASIN

S, 1970,PALAEONTOGRAPHICA,BD 135,ABT A,P112- 
117, PL 17,FIG 12,13

REMARKS: C,D-JB WATERHOUSE, 1964 ; JBW NO B 178 ORDER PRODUCTIDA ACCORDING TO WATERHOUSE + PIYASIN; 1 SPEC

WAAGENITES SPECIOSUS WATERHOUSE + PIYASIN

31890 [B 179] P.PARA

UP.KAZAN

$50 \mathrm{M}$ FROM ROAD LEADING W FROM HWY, KHAO

PHRIK,NR RATBURI,THAILAND

H433: WATERHOUSE JB + PIYASIN

S. 1970,PALAEONTOGRAPHICA,BD 135, ABT A,P 112 117. PL 17. FIG 14,15

REMARKS: C,D-JB WATERHOUSE, 1964 ; JBW NO B 179 ORDER PRODUCTIDA ACCORDING TO WATERHOUSE + PIYASIN; 1 SPEC

WAAGENITES SPECIOSUS WATERHOUSE + PIYASIN 31891 [B180] P-PARA

UP,KAZAN

50 M FROM ROAD LEADING W FROM HWY , KHAO

PHRIK,NR RATBURI,THAILAND

H433: WATERHOUSE JB + PIYASIN

S. 1970.PALAEONTOGRAPHICA,BD 135,ABT A,P112-117

REMARKS: C.D-JB WATERHOUSE, 1964 ; JBW NO B 180

ORDER PRODUCTIDA ACCORDING TO WATERHOUSE + PIYASIN ; 1 SPEC

WAAGENITES SPECIOSUS WATERHOUSE + PIYASIN 31892 [B181] P-PARA

UP,KAZAN

50 M FROM ROAD LEADING WFROM HWY, KHAO PHRIK,NR RATBURI,THAILAND

H433: WATERHOUSE JB + PIYASIN

S, 1970.PALAEONTOGRAPHICA,BD 135,ABT A,P112-117

REMARKS: C.D-JB WATERHOUSE, 1964 ; JBW NO B 181

ORDER PRODUCTIDA ACCORDING TO WATERHOUSE + PIYASIN ; 1 SPEC

WAAGENITES SPECIOSUS WATERHOUSE + PIYASIN 31893 [B 182 ] P-PARA

UP, KAZAN

$50 \mathrm{M}$ FROM ROAD LEADING W FROM HWY, KHAO

PHRIK,NR RATBURI,THAILAND

H433: WATERHOUSE JB + PIYASIN

S, 1970,PALAEONTOGRAPHICA,BD 135,ABT A.P112-117

REMARKS: C.D-JB WATERHOUSE, 1964 ; JBW NO B 182

ORDER PRODUCTIDA ACCORDING TO WATERHOUSE + PIYASIN; 1 SPEC

WAAGENITES SPECIOSUS WATERHOUSE + PIYASIN 31894 [B183] P.PARA

UP.KAZAN

$50 M$ MROM ROAD LEADING W FROM HWY, KHAO

PHRIK,NR RATBURI, THAILAND

H433: WATERHOUSE JB + PIYASIN

S, 1970,PALAEONTOGRAPHICA,BD 135,ABT A,P112-117

REMARKS: C.D-JB WATERHOUSE, 1964 : JBW NO B 183

ORDER PRODUCTIDA ACCORDING TO WATERHOUSE + PIYASIN; 1 SPEC

WAAGENITES SPECIOSUS WATERHOUSE + PIYASIN 31895 [B 184] P.PARA

UP.KAZAN

50 M FROM ROAD LEADING W FROM HWY, KHAO

PHRIK, NR RATBURI, THAILAND

H433; WATERHOUSE JB + PIYASIN

S 1970.PALAEONTOGRAPHICA BD 135 ABT A.P112-117

REMARKS: C,D-JB WATERHOUSE, 1964 ; JBW NO B 184

ORDER PRODUCTIDA ACCORDING TO WATERHOUSE + PIYASIN : 1 SPEC

WAAGENITES SPECIOSUS WATERHOUSE + PIYASIN

31897 [B 186 ] P.PARA

UP, KAZAN

50 M FROM ROAD LEADING W FROM HWY, KHAO

PHRIK,NR RATBURI, THAILAND

H433: WATERHOUSE JB + PIYASIN

S, 1970,PALAEONTOGRAPHICA,BD 135,ABT A,P 112-117

REMARKS: C.D-JB WATERHOUSE, 1964 ; JBW NO B186;

ORDER PRODUCTIDA ACCORDING TO WATERHOUSE +

PIYASIN, 1 SPEC
WAAGENITES SPECIOSUS WATERHOUSE + PIYASIN 31898 [B187] P.PARA

UP, KAZAN

50 M FROM ROAD LEADING W FROM HWY, KHAO PHRIK, NR RATBURI, THAILAND

H433: WATERHOUSE JB + PIYASIN

S, 1970.PALAEONTOGRAPHICA,BD 135,ABT A,P 112-117

REMARKS: C,D-JB WATERHOUSE, 1964 ;JBW NO B 187

ORDER PRODUCTIDA ACCORDING TO WATERHOUSE + PIYASIN : 1 SPEC

WAAGENITES SPECIOSUS WATERHOUSE + PIYASIN 31900 [B 189 ] P-PARA

UP, KAZAN

50 M FROM ROAD LEADING W FROM HWY, KHAO PHRIK NR RATBURI, THAILAND

H433: WATERHOUSE JB + PIYASIN

S, 1970,PALAEONTOGRAPHICA,BD 135,ABT A,P 112-117

REMARKS: C,D-JB WATERHOUSE, 1964 ; JBW NO B 189;

ORDER PRODUCTIDA ACCORDING TO WATERHOUSE + PIYASIN: 1 SPEC

WAAGENITES SPECIOSUS WATERHOUSE + PIYASIN 31901 [B 190] P.PARA

UP,KAZAN

50 M FROM ROAD LEADING W FROM HWY, KHAO PHRIK, NR RATBURI, THAILAND

H433: WATERHOUSE JB + PIYASIN

S, 1970,PALAEONTOGRAPHICA,BD 135,ABT A,P 112-117

REMARKS: C.D-JB WATERHOUSE, 1964 .JBW NO B 190.

ORDER PRODUCTIDA ACCORDING TO WATERHOUSE + PIYASIN ; 1 SPEC (2 PCES)

WAAGENITES SPECIOSUS WATERHOUSE + PIYASIN 31903 [B 192 ] P-PARA

UP,KAZAN

50 M FROM ROAD LEADING W FROM HWY, KHAO PHRIK, NR RATBURI, THAILAND

H433; WATERHOUSE JB + PIYASIN

S, 1970,PALAEONTOGRAPHICA,BD 135,ABT A,P 112-117

REMARKS: C.D-JB WATERHOUSE, 1964 ;JBW NO B 192;

ORDER PRODUCTIDA ACCORDING TO WATERHOUSE + PIYASIN; 1SPEC

WAAGENITES SPECIOSUS WATERHOUSE + PIYASIN 31904 [B 193 ] P.PARA

UP,KAZAN

50 M FROM ROAD LEADING W FROM HWY, KHAO PHRIK,NR RATBURI,THAILAND H433: WATERHOUSE JB + PIYASIN

S, 1970,PALAEONTOGRAPHICA,BD 135,ABT A,P 112-117 REMARKS: C.D-JB WATERHOUSE, 1964 ; JBW NO B 193;

ORDER PRODUCTIDA ACCORDING TO WATERHOUSE + PIYASIN; 1 SPEC

WAAGENITES SPECIOSUS WATERHOUSE + PIYASIN 31905 [B 194 ] P.PARA

UP,KAZAN

50 M FROM ROAD LEADING W FROM HWY, KHAO PHRIK,NR RATBURI,THAILAND H433. WATERHOUSE JB + PIYASIN

S, 1970,PALAEONTOGRAPHICA,BD 135,ABT A,P 112-117 REM ARKS: C.D-JB WATERHOUSE, 1964 ; JBW NO B 194

ORDER PRODUCTIDA ACCORDING TO WATERHOUSE + PIYASIN; 1 SPEC

WAAGENITES SPECIOSUS WATERHOUSE + PIYASIN

31906 [B 195 ] P.PARA

UP,KAZAN

$50 M$ FROM ROAD LEADING W FROM HWY KHAO

PHRIK, NR RATBURI,THAILAND

H433: WATERHOUSE JB + PIYASIN

S. 1970.PALAEONTOGRAPHICA,BD 135,ABT A.P 112-117 REMARKS: C.D-JB WATERHOUSE, 1964 ; JBW NO B 195

ORDER PRODUCTIDA ACCORDING TO WATERHOUSE + PIYASIN; 1 SPEC 
WAAGENITES SPECIOSUS WATERHOUSE + PIYASIN 31907 [B196] P-PARA

UP, KAZAN

50 M FROM ROAD LEADING W FROM HWY, KHAO

PHRIK, NR RATBURI, THAILAND

H433; WATERHOUSE JB + PIYASIN

S, 1970,PALAEONTOGRAPHICA,BD 135.ABT A,P 112-117 REMARKS: C,D-JB WATERHOUSE, 1964 ;JBW NO B 196;

ORDER PRODUCTIDA ACCORDING TO WATERHOUSE + PIYASIN; 1 SPEC

WAAGENITES SPECIOSUS WATERHOUSE + PIYASIN 31908 [B 197] P-PARA

U P,KAZAN

50 M FROM ROAD LEADING W FROM HWY, KHAO

PHRIK,NR RATBURI, THAILAND

H433:WATERHOUSE JB + PIYASIN

S, 1970,PALAEONTOGRAPHICA,BD 135,ABT A,P112-117

REMARKS: C,D-JB WATERHOUSE, 1964 ;JBW NO B 197;

ORDER PRODUCTIDA ACCORDING TO WATERHOUSE + PIYASIN; 1 SPEC

WAAGENITES SPECIOSUS WATERHOUSE + PIYASIN 31909 [B 198] P-PARA

UP, KAZAN

50 M FROM ROAD LEADING W FROM HWY, KHAO

PHRIK,NR RATBURI,THAILAND

H433; WATERHOUSE JB + PIYASIN

S, 1970,PALAEONTOGRAPHICA,BD 135,ABT A,P112-117

REMARKS: C,D-JB WATERHOUSE, 1964 ; JBW NO B 198;

ORDER PRODUCTIDA ACCORDING TO WATERHOUSE + PIYASIN; 1 SPEC

WAAGENITES SPECIOSUS WATERHOUSE + PIYASIN 31910 [B 199 ] P-PARA

U P,KAZAN

$50 \mathrm{M}$ FROM ROAD LEADING W FROM HWY, KHAO

PHRIK,NR RATBURI,THAILAND

H433; WATERHOUSE JB + PIYASIN

S, 1970,PALAEONTOGRAPHICA,BD 135,ABT A,P112-117

REMARKS: C,D-JB WATERHOUSE, 1964 ;JBW NO B 199 ;

ORDER PRODUCTIDA ACCORDING TO WATERHOUSE + PIYASIN ; 1 SPEC (2 PCES)

WAAGENITES SPECIOSUS WATERHOUSE + PIYASIN $31911[$ B200] P-PARA

U P,KAZAN

$50 \mathrm{M}$ FROM ROAD LEADING W FROM HWY , KHAO

PHRIK,NR RATBURI,THAILAND

H433; WATERHOUSE JB + PIYASIN

S, 1970,PALAEONTOGRAPHICA,BD 135,ABT A,P112-117

REMARKS: C,D-JB WATERHOUSE, 1964 ;JBW NO B200;

ORDER PRODUCTIDA ACCORDING TO WATERHOUSE + PIYASIN ; 1 SPEC

WAAGENITES SPECIOSUS WATERHOUSE+PIYASIN

31912 [B201] P-PARA

$U P, K A Z A N$

50 M FROM ROAD LEADING W FROM HWY, KHAO

PHRIK,NR RATBURI,THAILAND

H433; WATERHOUSE JB + PIYASIN

S, 1970,PALAEONTOGRAPHICA,BD 135,ABT A,P112-117

REMARKS: C,D-JB WATERHOUSE, 1964 ; JBW NO B201;

ORDER PRODUCTIDA ACCORDING TO WATERHOUSE + PIYASIN; 1 SPEC

WAAGENITES SPECIOSUS WATERHOUSE + PIYASIN

31914 [B203]P-PARA

UP.KAZAN

50 M FROM ROAD LEADING W FROM HWY, KHAO

PHRIK,NR RATBURI,THAILAND

H433; WATERHOUSE JB + PIYASIN

S, 1970,PALAEONTOGRAPHICA, BD 135,ABT A,P112-117

REMARKS: C,D-JB WATERHOUSE, 1964 ; JBW NO B203

ORDER PRODUCTIDA ACCORDING TO WATERHOUSE +

PIYASIN : 1 SPEC
WAAGENITES SPECIOSUS WATERHOUSE + PIYASIN 31918 [B207] P-PARA

UP,KAZAN

50 M FROM ROAD LEADING W FROM HWY, KHAO PHRIK,NR RATBURI, THAILAND H433:WATERHOUSE JB + PIYASIN

S, 1970,PALAEONTOGRAPHICA,BD 135,ABT A,P 112-117 REMARKS: C,D-JB WATERHOUSE, 1964 ;JBW NO B207 ORDER PRODUCTIDA ACCORDING TO WATERHOUSE + PIYASIN; 1 SPEC

WAAGENITES SPECIOSUS WATERHOUSE + PIYASIN 31920 [B209] P-PARA

UP.KAZAN

50 M FROM ROAD LEADING W FROM HWY, KHAO PHRIK,NR RATBURI,THAILAND H433; WATERHOUSE JB + PIYASIN

S, 1970,PALAEONTOGRAPHICA,BD 135,ABT A,P112-117

REMARKS: C,D-JB WATERHOUSE, 1964 ;JBW NO B209; ORDER PRODUCTIDA ACCORDING TO WATERHOUSE + PIYASIN; 1 SPEC

WAAGENITES SPECIOSUS WATERHOUSE + PIYASIN 31921 [B210] P-PARA

U P, KAZAN

$50 \mathrm{M}$ FROM ROAD LEADING W FROM HWY, KHAO PHRIK,NR RATBURI,THAILAND H433; WATERHOUSE JB + PIYASIN

S, 1970,PALAEONTOGRAPHICA,BD 135,ABT A,P112-117

REMARKS: C,D-JB WATERHOUSE, 1964 ;JBW NO B210; ORDER PRODUCTIDA ACCORDING TO WATERHOUSE + PIYASIN; 1 SPEC

WAAGENITES SPECIOSUS WATERHOUSE + PIYASIN 31922 [B521] P-PARA

UP, KAZAN

$50 M$ FROM ROAD LEADING W FROM HWY, KHAO

PHRIK,NR RATBURI,THAILAND H433; WATERHOUSE JB + PIYASIN

S, 1970,PALAEONTOGRAPHICA,BD 135,ABT A,P112-117

REMARKS: C,D-JB WATERHOUSE, 1964 ;JBW NO B521; ORDER PRODUCTIDA ACCORDING TO WATERHOUSE + PIYASIN; 1 SPEC

WAAGENITES SPECIOSUS WATERHOUSE+PIYASIN 31923 [B522] P-PARA

UP,KAZAN

$50 M$ FROM ROAD LEADING W FROM HWY, KHAO PHRIK,NR RATBURI,THAILAND H433; WATERHOUSE JB + PIYASIN

S, 1970,PALAEONTOGRAPHICA,BD 135 , ABT A,P112-117

REMARKS: C,D-JB WATERHOUSE, 1964 ;JBW NO B522; ORDER PRODUCTIDA ACCORDING TO WATERHOUSE + PIYASIN; 1 SPEC

WAAGENITES SPECIOSUS WATERHOUSE + PIYASIN 31924 [B523] P-PARA

UP,KAZAN

$50 M$ FROM ROAD LEADING W FROM HWY, KHAO PHRIK,NR RATBURI,THAILAND H433; WATERHOUSE JB + PIYASIN

S, 1970 PALAEONTOGRAPHICA BD 135 ABT A,P112-117 REMARKS: C,D-JB WATERHOUSE, 1964 ;JBW NO B523; ORDER PRODUCTIDA ACCORDING TO WATERHOUSE + PIYASIN ; 1 SPEC

WAAGENITES SPECIOSUS WATERHOUSE + PIYASIN 31925 [B524] P-PARA

U P.KAZAN

50 M FROM ROAD LEADING W FROM HWY, KHAO PHRIK,NR RATBURI,THAILAND H433; WATERHOUSE JB + PIYASIN

S, 1970,PALAEONTOGRAPHICA,BD 135,ABT A,P112-117

REMARKS: C.D-JB WATERHOUSE, 1964 ; JBW NO B524;

ORDER PRODUCTIDA ACCORDING TO WATERHOUSE + PIYASIN ; 1 SPEC 
WAAGENITES SPECIOSUS WATERHOUSE + PIYASIN 31896 [B 185] P.PARA

UP.KAZAN

50 M FROM ROAD LEADING W FROM HWY , KHAO

PHRIK.NR RATBURI.THAILAND

H433: WATERHOUSE JB + PIYASIN

S, 1970,PALAEONTOGRAPHICA,BD 135,ABT A,P112. 117,PL17,FIG 20,21

REMARKS: C.D-JB WATERHOUSE, 1964 ;JBW NO B185: ORDER PRODUCTIDA ACCORDING TO WATERHOUSE + PIYASIN; 1 SPEC

WAAGENITES SPECIOSUS WATERHOUSE + PIYASIN

31899 [B188 ] P-PARA

UP.KAZAN

50 M FROM ROAD LEADING W FROM HWY, KHAO

PHRIK,NR RATBURI, THAILAND

H433: WATERHOUSE JB + PIYASIN

S. 1970,PALAEONTOGRAPHICA,BD 135,ABT A,P112. 117.PL17,FIG 24,25

REMARKS: C.D-JB WATERHOUSE, 1964 :JBW NO B 188 : ORDER PRODUCTIDA ACCORDING TO WATERHOUSE + PIYASIN : 1 SPEC

WAAGENITES SPECIOSUS WATERHOUSE + PIYASIN 31902 [B 191] P.PARA

UP.KAZAN

50 M FROM ROAD LEADING W FROM HWY, KHAO

PHRIK.NR RATBURI,THAILAND

H433; WATERHOUSE JB + PIYASIN

S, 1970,PALAEONTOGRAPHICA,BD 135,ABT A,P112117, PL 17.FIG 22,23

REMARKS: C,D-JB WATERHOUSE, 1964 ;JBW NO B191; ORDER PRODUCTIDA ACCORDING TO WATERHOUSE + PIYASIN: 1SPEC

WAAGENITES SPECIOSUS WATERHOUSE + PIYASIN 31913 [B202] P-PARA

UP.KAZAN

50 M FROM ROAD LEADING W FROM HWY, KHAO

PHRIK,NR RATBURI,THAILAND

H433; WATERHOUSE JB + PIYASIN

S, 1970,PALAEONTOGRAPHICA,BD 135,ABT A,P112117. PL 18. FIG 12,14

REMARKS: C,D-JB WATERHOUSE, 1964 :JBW NO B202: ORDER PRODUCTIDA ACCORDING TO WATERHOUSE + PIYASIN : 1 SPEC

WAAGENITES SPECIOSUS WATERHOUSE + PIYASIN 31915 [B204] P.PARA

UP.KAZAN

50 M FROM ROAD LEADING W FROM HWY, KHAO

PHRIK NR RATBURI,THAILAND

H433:WATERHOUSE JB + PIYASIN

S, 1970,PALAEONTOGRAPHICA,BD 135,ABT A,P112117.PL 19.FIG 17

REMARKS: C.D-JB WATERHOUSE, 1964 ;JBW NO B204; ORDER PRODUCTIDA ACCORDING TO WATERHOUSE + PIYASIN: 1 SPEC

WAAGENITES SPECIOSUS WATERHOUSE+PIYASIN 31916 [B205] P-PARA

UP.KAZAN

$50 M$ FROM ROAD LEADING W FROM HWY, KHAO

PHRIK.NR RATBURI,THAILAND

H433:WATERHOUSE JB + PIYASIN

S, 1970,PALAEONTOGRAPHICA,BD 135,ABT A,P112117.PL 18,FIG 16,17

REMARKS: C.D-JB WATERHOUSE, 1964 ;JBW NO B205: ORDER PRODUCTIDA ACCORDING TO WATERHOUSE + PIYASIN : 1 SPEC

WAAGENITES SPECIOSUS WATERHOUSE + PIYASIN

31917 [B206]P-PARA

UP.KAZAN

50 M FROM ROAD LEADING W FROM HWY , KHAO

PHRIK, NR RATBURI, THAILAND

H433: WATERHOUSE JB + PIYASIN

S, 1970,PALAEONTOGRAPHICA,BD 135,ABT A,P112.

117 PL 18.FIG 2021

REMARKS: C.D-JB WATERHOUSE, 1964 ; JBW NO B206 ORDER PRODUCTIDA ACCORDING TO WATERHOUSE + PIYASIN: 1 SPEC
WAAGENITES SPECIOSUS WATERHOUSE + PIYASIN 31919 [B208] P-PARA

UP,KAZAN

$50 M$ FROM ROAD LEADING W FROM HWY, KHAO PHRIK,NR RATBURI,THAILAND

H433; WATERHOUSE JB + PIYASIN

S. 1970,PALAEONTOGRAPHICA,BD 135,ABT A,P112117.PL 18. FIG 15.PL 19. FIG 4,5

REMARKS: C.D-JB WATERHOUSE, 1964 :JBW NO B208: ORDER PRODUCTIDA ACCORDING TO WATERHOUSE + PIYASIN ; 1 SPEC

WAAGENITES SPECIOSUS WATERHOUSE + PIYASIN 31926 [B556 ] P.PARA

UP, KAZAN

50 M FROM ROAD LEADING W FROM HWY, KHAO PHRIK, NR RATBURI,THAILAND

H433; WATERHOUSE JB + PIYASIN

S. 1970,PALAEONTOGRAPHICA BD 135 ABT A P 112-117

REMARKS: C,D-JB WATERHOUSE, 1964 ;JBW NO B556;

ORDER PRODUCTIDA ACCORDING TO WATERHOUSE + PIYASIN:SPEC SMASHED SINCE MEASUREMENT ; 1 SPEC

WAAGENITES SPECIOSUS WATERHOUSE + PIYASIN 31928 [B558] P-PARA

UP,KAZAN

$50 M$ FROM ROAD LEADING W FROM HWY, KHAO PHRIK,NR RATBURI,THAILAND

H433:WATERHOUSE JB + PIYASIN

S, 1970,PALAEONTOGRAPHICA,BD 135,ABT A,P 112-117

REMARKS: C.D-JB WATERHOUSE, 1964 ; JBW NO B558:

ORDER PRODUCTIDA ACCORDING TO WATERHOUSE + PIYASIN ; 1 SPEC

WAAGENITES SPECIOSUS WATERHOUSE + PIYASIN 31929 [B559] P-PARA

UP, KAZAN

50 M FROM ROAD LEADING W FROM HWY, KHAO PHRIK,NR RATBURI,THAILAND H433; WATERHOUSE JB + PIYASIN

S, 1970,PALAEONTOGRAPHICA,BD 135,ABT A,P 112-117 REMARKS: C,D-JB WATERHOUSE, 1964 : JBW NO B559; ORDER PRODUCTIDA ACCORDING TO WATERHOUSE + PIYASIN; 1 SPEC

WAAGENITES SPECIOSUS WATERHOUSE + PIYASIN 31930 [B560]P-PARA

UP.KAZAN

$50 M$ FROM ROAD LEADING W FROM HWY, KHAO

PHRIK NR RATBURI,THAILAND

H433; WATERHOUSE JB + PIYASIN

S, 1970,PALAEONTOGRAPHICA,BD 135,ABT A,P112117. PL17.FIG 9,10,11

REMARKS: C,D-JB WATERHOUSE, 1964 ;JBW NO B560; ORDER PRODUCTIDA ACCORDING TO WATERHOUSE + PIYASIN ; 1 SPEC

WAAGENITES SPECIOSUS WATERHOUSE + PIYASIN 31931 [B601] P-PARA

UP KAZAN

$50 M$ FROM ROAD LEADING W FROM HWY, KHAO PHRIK, NR RATBURI, THAILAND H433:WATERHOUSE JB + PIYASIN

S. 1970,PALAEONTOGRAPHICA,BD 135,ABT A,P112-117 REMARKS: C,D-JB WATERHOUSE, 1964 ; JBW NO B601; ORDER PRODUCTIDA ACCORDING TO WATERHOUSE + PIYASIN ; 1 SPEC

WAAGENITES SPECIOSUS WATERHOUSE + PIYASIN 31932 [B602] P-PARA

UP.KAZAN

$50 M$ FROM ROAD LEADING W FROM HWY, KHAO PHRIK,NR RATBURI, THAILAND

H433: WATERHOUSE JB + PIYASIN

S. 1970,PALAEONTOGRAPHICA,BD 135,ABT A,P112117,PL 19, FIG 6

REMARKS: C.D-JB WATERHOUSE, 1964 ;JBW NO B602 ORDER PRODUCTIDA ACCORDING TO WATERHOUSE + PIYASIN ; 1 SPEC 
WAAGENITES SPECIOSUS WATERHOUSE+PIYASIN 31933 [B603 ] P-PARA

UP.KAZAN

50 M FROM ROAD LEADING W FROM HWY, KHAO PHRIK, NR RATBURI,THAILAND H433: WATERHOUSE JB + PIYASIN

S, 1970,PALAEONTOGRAPHICA,BD 135,ABT A,P 112-117

REMARKS: C.D-JB WATERHOUSE, 1964 ;JBW NO B603;

ORDER PRODUCTIDA ACCORDING TO WATERHOUSE + PIYASIN : 1 SPEC

WAAGENITES SPECIOSUS WATERHOUSE + PIYASIN

31934 [B604] P-PARA

UP, KAZAN

50 M FROM ROAD LEADING W FROM HWY, KHAO

PHRIK, NR RATBURI,THAILAND

H433: WATERHOUSE JB + PIYASIN

S, 1970,PALAEONTOGRAPHICA,BD 135,ABT A,P112-117

REMARKS: C.D-JB WATERHOUSE, 1964 ; JBW NO B604

ORDER PRODUCTIDA ACCORDING TO WATERHOUSE + PIYASIN ; 1 SPEC

WAAGENITES SPECIOSUS WATERHOUSE+PIYASIN

31935 [B605] P-PARA

UP,KAZAN

50 M FROM ROAD LEADING W FROM HWY, KHAO

PHRIK, NR RATBURI,THAILAND

H433. WATERHOUSE JB + PIYASIN

S, 1970,PALAEONTOGRAPHICA,BD 135,ABT A,P112-117

REMARKS: C,D-JB WATERHOUSE, 1964 ;JBW NO B605;

ORDER PRODUCTIDA ACCORDING TO WATERHOUSE +

PIYASIN; 1 SPEC

WAAGENITES SPECIOSUS WATERHOUSE + PIYASIN

31936 [B606 ] P.PARA

UP,KAZAN

50 M FROM ROAD LEADING W FROM HWY, KHAO

PHRIK, NR RATBURI,THAILAND

H433; WATERHOUSE JB + PIYASIN

S, 1970,PALAEONTOGRAPHICA,BD 135,ABT A,P 112-117

REMARKS: C,D-JB WATERHOUSE, 1964 ; JBW NO B606;

ORDER PRODUCTIDA ACCORDING TO WATERHOUSE

UNCERTAIN WHICH SPEC MEASURED; 6 SPEC + FRAGS

WAAGENITES SPECIOSUS WATERHOUSE + PIYASIN

31937 [B607] P.PARA

UP,KAZAN

50 M FROM ROAD LEADING W FROM HWY, KHAO

PHRIK, NR RATBURI, THAILAND

H433; WATERHOUSE JB + PIYASIN

S, 1970,PALAEONTOGRAPHICA,BD 135,ABT A,P112-117

REMARKS: C,D-JB WATERHOUSE, 1964 ; JBW NO B607;

ORDER PRODUCTIDA ACCORDING TO WATERHOUSE +

PIYASIN ; 1 SPEC

WAAGENITES SPECIOSUS WATERHOUSE + PIYASIN

31938 [B608 ] P-PARA

UP,KAZAN

50 M FROM ROAD LEADING W FROM HWY, KHAO

PHRIK, NR RATBURI, THAILAND

H433; WATERHOUSE JB + PIYASIN

S, 1970,PALAEONTOGRAPHICA,BD 135,ABT A.P112-117

REMARKS: C,D-JB WATERHOUSE, 1964 ; JBW NO B608;

ORDER PRODUCTIDA ACCORDING TO WATERHOUSE +

PIYASIN ; 1 SPEC

WAAGENITES SPECIOSUS WATERHOUSE+PIYASIN

31939 [B609 ] P-PARA

UP.KAZAN

50 M FROM ROAD LEADING W FROM HWY, KHAO

PHRIK, NR RATBURI, THAILAND

H433; WATERHOUSE JB + PIYASIN

S, 1970,PALAEONTOGRAPHICA,BD 135,ABT A.P112-117

REMARKS: C,D-JB WATERHOUSE, 1964 ; JBW NO B609;

ORDER PRODUCTIDA ACCORDING TO WATERHOUSE +

PIYASIN : 1 SPEC
WAAGENITES SPECIOSUS WATERHOUSE + PIYASIN

31940 [B610] P-PARA

UP.KAZAN

50 M FROM ROAD LEADING W FROM HWY, KHAO

PHRIK, NR RATBUAI,THAILAND

H433:WATERHOUSE JB + PIYASIN

S, 1970,PALAEONTOGRAPHICA,BD 135,ABT A,P112-117

REMARKS: C,D-JB WATERHOUSE, 1964 ; JBW NO B6 10;

ORDER PRODUCTIDA ACCORDING TOWATERHOUSE + PIYASIN: 1 SPEC

WAAGENITES SPECIOSUS WATERHOUSE+PIYASIN

31941 [B611] P-PARA

UP,KAZAN

$50 \mathrm{M}$ FROM ROAD LEADING W FROM HWY, KHAO

PHRIK, NR RATBUAI,THAILAND

H433: WATERHOUSE JB + PIYASIN

S, 1970,PALAEONTOGRAPHICA,BD 135,ABT A,P112-117

REMARKS: C,D-JB WATERHOUSE, 1964 ; JBW NO B611;

ORDER PRODUCTIDA ACCORDING TO WATERHOUSE + PIYASIN; 1 SPEC

WAAGENITES SPECIOSUS WATERHOUSE+PIYASIN

31942 [B6 12] P-PARA

UP,KAZAN

50 M FROM ROAD LEADING W FROM HWY, KHAO

PHRIK, NR RATBURI,THAILAND

H433; WATERHOUSE JB + PIYASIN

S, 1970,PALAEONTOGRAPHICA,BD 135,ABT A,P112-117

REMARKS: C,D-JB WATERHOUSE, 1964 ;JBW NO B612

ORDER PRODUCTIDA ACCORDING TO WATERHOUSE + PIYASIN: 1 SPEC

WAAGENITES SPECIOSUS WATERHOUSE + PIYASIN 31943 [B613] P-PARA

UP, KAZAN

50 M FROM ROAD LEADING W FROM HWY, KHAO

PHRIK, NR RATBURI,THAILAND

H433; WATERHOUSE JB + PIYASIN

S, 1970,PALAEONTOGRAPHICA,BD 135, ABT A,P112-117

REMARKS: C,D-JB WATERHOUSE, 1964 ; JBW NO B6 13

ORDER PRODUCTIDA ACCORDING TO WATERHOUSE + PIYASIN ; 1 SPEC

WAAGENITES SPECIOSUS WATERHOUSE+PIYASIN 31944 [B614] P-PARA

UP,KAZAN

50 M FROM ROAD LEADING W FROM HWY, KHAO

PHRIK, NR RATBURI,THAILAND

H433; WATERHOUSE JB + PIYASIN

S, 1970,PALAEONTOGRAPHICA,BD 135,ABT A,P112-117

REMARKS: C,D-JB WATERHOUSE, 1964 ; JBW NO B6 14

ORDER PRODUCTIDA ACCORDING TO WATERHOUSE + PIYASIN ; 2 SPEC + FRAG

WAAGENITES SPECIOSUS WATERHOUSE+PIYASIN 31850 [B 139] P-PARA

UP,KAZAN

50 M FROM ROAD LEADING W FROM HWY, KHAO

PHRIK, NR RATBURI, THAILAND

H433; WATERHOUSE JB + PIYASIN

S, 1970,PALAEONTOGRAPHICA,BD 135, ABT A.P112-117

REMARKS: C,D-JB WATERHOUSE, 1964 ; JBW NO B 139;

ORDER PRODUCTIDA ACCORDING TO WATERHOUSE + PIYASIN ; 1 SPEC

WAAGENITES SPECIOSUS WATERHOUSE + PIYASIN 31851 [B 141] P-PARA

UP,KAZAN

50 M FROM ROAD LEADING W FROM HWY, KHAO

PHRIK, NA RATBURI,THAILAND

H433; WATERHOUSE JB + PIYASIN

S, 1970,PALAEONTOGRAPHICA,BD 135, ABT A,P 112-117

REMARKS: C,D-JB WATERHOUSE, 1964 ; JBW NO B 141

ORDER PRODUCTIDA ACCORDING TO WATERHOUSE + PIYASIN : 1 SPEC 
WAAGENITES SPECIOSUS WATERHOUSE + PIYASIN 31852 [B 142] P-PARA

UP.KAZAN

$50 M$ FROM ROAD LEADING W FROM HWY, KHAO PHRIK,NR RATBURI,THAILAND

H433; WATERHOUSE JB + PIYASIN

S, 1970,PALAEONTOGRAPHICA,BD 135,ABT A,P112-

117. PL 17,FIG 16,17

REMARKS: C.D-JB WATERHOUSE, 1964 ;JBW NO B 142; ORDER PRODUCTIDA ACCORDING TO WATERHOUSE + PIYASIN; 1 SPEC

WAAGENITES SPECIOSUS WATERHOUSE + PIYASIN 31853 [B 143] P-PARA

UP.KAZAN

50 M FROM ROAD LEADING W FROM HWY, KHAO

PHRIK,NR RATBURI,THAILAND

H433. WATERHOUSE JB + PIYASIN

S, 1970,PALAEONTOGRAPHICA,BD 135,ABT A,P112.

117,PL17,FIG 29

REMARKS: C,D-JB WATERHOUSE, 1964 ;JBW NO B 143; ORDER PRODUCTIDA ACCORDING TO WATERHOUSE + PIYASIN; 1 SPEC

WAAGENITES SPECIOSUS WATERHOUSE + PIYASIN 31854 [B144] P-PARA

UP,KAZAN

50 M FROM ROAD LEADING W FROM HWY, KHAO

PHRIK,NRRATBURI,THAILAND

H433; WATERHOUSE JB + PIYASIN

S, 1970,PALAEONTOGRAPHICA,BD 135 ABT A P112-117

REMARKS: C,D-JB WATERHOUSE, 1964 ; JBW NO B 144;

ORDER PRODUCTIDA ACCORDING TO WATERHOUSE + PIYASIN; 1 SPEC

WAAGENITES SPECIOSUS WATERHOUSE + PIYASIN 31855 [B 145] P-PARA

UP.KAZAN

$50 \mathrm{M}$ FROM ROAD LEADING W FROM HWY, KHAO

PHRIK,NR RATBURI,THAILAND

H433:WATERHOUSE JB + PIYASIN

S. 1970,PALAEONTOGRAPHICA,BD 135,ABT A.P112-117

REMARK S: C,D-JB WATERHOUSE, 1964 ; JJBW NO B 145

ORDER PRODUCTIDA ACCORDING TO WATERHOUSE + PIYASIN ; 1 SPEC

WAAGENITES SPECIOSUS WATERHOUSE + PIYASIN 31856 [B 146] P-PARA

UP.KAZAN

50 M FROM ROAD LEADING W FROM HWY, KHAO PHRIK, NR RATBURI,THAILAND

H433: WATERHOUSE JB + PIYASIN

S, 1970,PALAEONTOGRAPHICA,BD 135,ABT A,P112-

117. PL18,FIG 67

REMARKS: C,D-JB WATERHOUSE, 1964 ;JBW NO B146: ORDER PRODUCTIDA ACCORDING TO WATERHOUSE + PIYASIN: 1 SPEC

WELLERELLA NALIENSIS REED

32045 [B370] REF SPEC-F

UP.KAZAN

50 M FROM ROAD LEADING W FROM HWY KHAO

PHRIK, NR RATBURI, THAILAND

H590:WATERHOUSE JB + PIYASIN

S, 1970,PALAEONTOGRAPHICA,BD 135, ABT A,P 135 137.PL23,FIG 12

REMARKS: C.D-JB WATERHOUSE, 1964 :JBW NO B370; SPEC

WELLERELLA NALIENSIS REED

32047 [B547] REF SPEC-F

UP.KAZAN

$50 M$ FROM ROAD LEADING W FROM HWY, KHAO

PHRIK, NR RATBURI, THAILAND

H590:WATERHOUSE JB + PIYASIN

S. 1970,PALAEONTOGRAPHICA,BD 135, ABT A.P135-

137, PL23,FIG 8,11,13

REMARKS: C.D-JB WATERHOUSE, 1964 ;JBW NO B547, 1 SPEC
WELLERELLA NALIENSIS REED

32049 [B666] REF SPEC-M

UP KAZAN

50 M FROM ROAD LEADING W FROM HWY, KHAO

PHRIK, NR RATBURI,THAILAND

H590:WATERHOUSE JB + PIYASIN

S, 1970,PALAEONTOGRAPHICA,BD 135.ABT A,P 135- 137

REMARKS: C.D-JB WATERHOUSE, 1964 ;JBW NO B $666 ; 1$ SPEC

WELLERELLA NALIENSIS REED

32043 [B367] REF SPEC-M

$U P, K A Z A N$

$50 M$ FROM ROAD LEADING W FROM HWY, KHAO

PHRIK, NR RATBURI,THAILAND

H590:WATERHOUSE JB + PIYASIN S, 1970,BD 135,ABT

A.P135-137, PL23,FIG 9, 10, 14

REMARKS: C,D-JB WATERHOUSE, 1964 ;JBW NO B367; 1 SPEC

WELLERELLA NALIENSIS REED

32044 [B368] REF SPEC-F

UP, KAZAN

50 M FROM ROAD LEADING W FROM HWY, KHAO

PHRIK, NR RATBURI,THAILAND

H590:WATERHOUSE JB + PIYASIN

S, 1970,PALAEONTOGRAPHICA,BD 135,ABT A,P 135 137. TEXT FIG 11

REMARKS: C,D-JB WATERHOUSE, 1964 ; JBW NO B368 ; 1 SPEC IN MATRIX

WHITFIELDELLA PARVUS SHAW

5643 P-SYN

MS,NIAG

ALBEMARLE GP,ERAMOSA FM

GUELPH,ONT

H658:SHAW EW, TRANS ROY CAN INST, VOL

21,PT2, 1937,P357-358,PL24,FIG 4-6

REMARKS: C-EW SHAW;FIG 4=LARGER SPEC;FIG 5 .

$6=$ SMALLER SPEC ; 2 SPEC

XENOSTEGES ? SP

31989 [B366] REF SPEC-F

UP.KAZAN

50 M FROM ROAD LEADING W FROM HWY, KHAO

PHRIK, NR RATBURI,THAILAND

H455; WATERHOUSE JB + PIYASIN

S. 1970 PALAEONTOGRAPHICA,BD 135.ABT A.P117

118,PL19,FIG 11, 12

REMARKS: C,D-JB WATERHOUSE, 1964 ;JBW NO B366; ORDER PRODUCTIDA ACCORDING TO WATERHOUSE + PIYASIN; 1 SPEC

XENOSTEGES ? SP

31988 [B293] REF SPEC-F

UP.KAZAN

50 M FROM ROAD LEADING W FROM HWY, KHAO

PHRIK, NR RATBURI, THAILAND

H455: WATERHOUSE JB + PIYASIN

S, 1970,PALAEONTOGRAPHICA,BD 135,ABT A,P 117 118,PL 19,FIG 9,10

REMARKS: C.D-JB WATERHOUSE 1964 ·JBW NO B293:

ORDER PRODUCTIDA ACCORDING TO WATERHOUSE + PIYASIN ; 1 SPEC

\section{ZYGOSPIRA CINCINNATIENSIS MEEK}

16848 [558HR] REF SPEC-F

U O, CINCI,MAYSVILL

DUNDAS FM

DON VALLEY OUARRY, TORONTO ONT

H634:PARKS \& DYER.ONT DEPT MINES, VOL

30,PT7, 1922, P4 1-42,PL7,FIG 20

REMARKS: D-BE WALKER :UNCERTAIN WHICH SPEC FIG'D 11 SPEC

ZYGOSPIRA CINCINNATIENSIS MEEK

16889 [601HR] REF SPEC-F

U O.CINCI,MAYSVILL

DUNDAS FM

DON VALLEY QUARRY?, TORONTO,ONT 
H634:PARKS \& DYER, ONT DEPT MINES, VOL

30,PT7, 1922,P41-42,PL7,FIG 23

REMARKS: C-J TOWNSEND ; 1 SPEC

ZYGOSPIRA CINCINNATIENSIS MEEK

17356 [1115HA ] REF SPEC-F

U O.CINCI,MAYSVILL

DUNDAS FM

DON VALLEY QUARRY,TORONTO,ONT

H634:PARKS \& DYER,ONT DEPT MINES, VOL

30,PT7, 1922,P4 1-42,PL7,FIG 21

REMARKS: C-JTOWNSEND ; 1 SPEC

ZYGOSPIRA ERRATICA HALL

17357 [1116HR] REF SPEC-F

U O,CINCI,MAYSVILL

DUNDASFM

HUMBER R,TORONTO,ONT

H634;PARKS + DYER, ONT DEPT MINES,VOL

30,PT 7, 1922,P42-43,PL7,FIG 24, 25

REMARKS: C-J TOWNSEND ;SPEC 'A' FIG'D ; 3 SPEC

ZYGOSPIRA MODESTA HALL

16888 [600HR ] REF SPEC-F

U O.CINCI,MAYSVILL

DUNDAS FM

DON VALLEY QUARAY,TORONTO,ONT

H634;PARKS \& DYER,ONT DEPT MINES, VOL

30,PT7, 1922,P40-41,PL7,FIG 19

REMARKS: C-J TOWNSEND; SPEC 'A' $=$ FIG $19 ; 2$ SPEC

ZYGOSPIRA MODESTA HALL

$16874^{\prime}$ ' 5584HR] REF SPEC-F

U O, CINCI,RICHMOND

RICHMOND GP

[RICHMOND]

MANITOWANING, MANITOULIN IS, ONT

H634;PARKS \& DYER,ONT DEPT MINES,VOL

30,PT7, 1922, P40-41, PL7,FIG 22

REMARKS: D-BE WALKER, 1905 ;RECORDS STATE 6

SPECS;UNCERTAIN WHICH SPEC FIG'D ; 4 SPEC

\section{ZYGOSPIRA RICHMONDENSIS CALEY}

12448 P-HOLO

U O.CINCI,RICHMOND

KAGAWONG FM

HIGH FALLS, MANITOULIN IS, ONT

H634:CALEY JF,GSC MEM 202, 1936,P78,PL 1,FIG 4,6

REMARKS: C-JF CALEY, $1932 ; 1$ SPEC

\section{UNDETERMINED RHYNCHONELLID}

31746 [B623 ] REF SPEC-F

UP, KAZAN

$50 \mathrm{M}$ FROM ROAD LEADING W FROM HWY, KHAO

PHRIK,NR RATBURI,THAILAND

H---;WATERHOUSE JB + PIYASIN

S. 1970,PALAEONTOGRAPHICA,BD 135,ABT

A,P 137,PL23,FIG 6,7

REMARKS: C,D-JB WATERHOUSE, 1964 :JBW NO B623 : 1 SPEC

\section{MOLLUSCA,MONOPLACOPHORA}

ARCHINACELLA PULASKIENSIS FOERSTE

17450 [1211HR] REF SPEC-F

U O,CINCI,MAYSVILL

DUNDAS FM

DON VALLEY QUARRY,TORONTO,ONT

181; PARKS \& FRITZ, ONT DEPT MINES, VOL 31,PT9, 1923,P2-

3.PL 1,FIG 1,2

REMARK S: C-J TOWNSEND ; 1 SPEC
PARMOPHORELLA ACADICA (DAWSON)

26017 REF SPEC-F

STENOTHECA (PARMOPHORELLA) ACADICA (DAWSON) $M C A$.

ST JOHN GP

[ST JOHN,ACADIAN,DIV 1C2]

PORTER'S BK, ST MARTINS,ST JOHN CO,NB

177;MATTHEW GF, 1886,P59-60,PL6,FIG 10,10A-C;KNIGHT

JB,GSA SPEC PAP 32, 194 1,P234-235,PL2,FIG 6C-D

REMARKS: C-GF MATTHEW:D-MACKENZIE ; 2 SPEC

PARMOPHORELLA ACADICA (DAWSON)

7827 [197CM] REF SPEC?

STENOTHECA (PARMOPHORELLA) ACADICA DAWSON

$M C A$.

ST JOHN GP

[ST JOHN,ACADIAN]

RATCLIFFE'S MILLSTREAM, ST JOHN,NB

177:MATTHEW GF, 1886,P59-60; KNIGHT JB,GSA SPEC PAP $32,1941, P 234-235$

REMARKS: C-GF MATTHEW:D-MACKENZIE :LABELLED PLESIOTYPES;UNCERTAIN WHETHER FIG'D,BUT NOT MENTIONED SPECIFICALLY; 7 SPEC

\section{MOLLUSCA,GASTROPODA}

\section{ACANTHONEMA HOLOPIFORMIS GRABAU}

22485 [237S ] P-SYN

$M$ D.ERIAN

DETROIT RIVER GP.AMHERSTBURG FM

[UMONROE]

LIVINGSTONE CUT, DETROIT R, AMHERSTBURG, ONT

1317:GRABAU AW, MICH GEOL \& BIOL SURV,PUB 2, GEOL SER 1,1910,P182-183,PL23,FIG 6.7?

REMARKS: D-T NATTRESS, 1914 ;LABELLED PARATYPES; UNCERTAIN WHETHER SPECS ARE FIG'D ; 4 SPEC

BELLEROPHON BRETONENSIS MATTHEW

8428 [798CM]P-SYN

UCA, ORLO

[BRETONIAN]

MCLEOD BK, BOISDALE,CAPE BRETON,NS

1182:MATTHEW GF, BULL NAT HIST SOC NB, VOL 4,N20,1902,P409-410,PL18,FIG 4A-D

REMARKS: C-GF MATTHEW;D-MACKENZIE ;SPEC ' $B$ ' = FIG $4 B ; 2$ SPEC

BELLEROPHON SEMISCULPTUS MATTHEW $8427[797 \mathrm{CM}]$ P-SYN

$\mathrm{MCA}$,

[BRETONIAN]

MCLEOD BK, BOISDALE,CAPE BRETON,NS

1182; MATTHEW GF,BULL NAT HIST SOC NB, VOL

4,N20,1902,P4 10,PL 18,FIG 5

REMARKS: C-GF MATTHEW:D-MACKENZIE ; 1 SPEC

\section{BELLEROPHON SP INDET}

22602 [355S ] REF SPEC-F

BELLEROPHON (CYMBULARIA) SP INDET

$S$

LIMESTONE RAPIDS, SEVERN R,DIST OF PATRICIA, ONT

1182; PARKS WA, TRANS ROY CAN INST, VOL 11, PT 1, 1915,P56,PL4, FIG 6,7

REMARKS: C-JB TYRRELL, 1912 :D-WH HEARST, $1914: 3$ SPEC

\section{BELLEROPHON SP}

12958 [2348D] REF SPEC-F

$M D$

KWATABOAHEGAN R,ONT

I182;PARKS WA ONT DEPT MINES, VOL

13,PT 1, 1904,PL8,FIG 2

REMARKS: C-WA PARKS, 1903 ;STORED UNDER

ORTHOCERAS LUXUM ; 1 SLAB 
BELLEROPHON SP

22603 [356S ] REF SPEC-F

S.

LIMESTONE RAPIDS, SEVERN R,DIST OF PATRICIA, ONT

1182; PARKS WA, TRANS ROY CAN INST, VOL

11.PT 1, 19 15,P55-56,PL4,FIG 5

REMARKS: C-JB TYRRELL; D-WH HEARST, 1914: 1 SPEC

CAMPELOMA NEBRASCENSIS NEBRASCENSIS (M\&H)

$9710[4446 C T$ ] REF SPEC-F

PALEOC.

RAVENSCRAG FM

NRESTEVAN,SASK

I---;TOZER ET,GSC MEM 280, 1956,P61,PL5,FIG 11A,B

REMARKS: C.D-A MACLEAN : SUBSPECIES AUTHORS

(MEEK + HAYDEN):MORE COMPLETE SPEC FIG'D;PLATE

CAPTION GIVES STRAT AS LRPT EDMONTON FM;SPEC

RECORDS AND TEXT STATE RAVENSCRAG FM ; 2 SPEC

CLATHROSPIRA SUBCONICA HALL

23603 [292T] REF SPEC-F

MO.CHAMP, MOH.TRENT

TRENTON GP

[TRENTON (DRIFT)]

TORONTO.ONT

1206:PARKS \& FRITZ,ONT DEPT MINES,VOL

31,PT9, 1923,P5-6,PL1,FIG 9

REMARKS: D-WH HEARST, $1919 ; 1$ SPEC

\section{COELIDIUM SPINDET}

22593 [346S ] REF SPEC-F

S.

LIMESTONE RAPIDS, SEVERN R, DIST OF PATRICIA, ONT

I293; PARKS WA,TRANS ROY CAN INST, VOL

11,PT 1, 1915,P58-59,PL 1,FIG 6

REMARKS: C-JB TYRRELL;D-1914;D-WH HEARST; 1 SPEC

\section{CF CYCLONEMA? SP INDET}

17457 [1218HR] REF SPEC-F

U O, CINCI,MAYSVILL

DUNDAS FM

[DUNDAS ]

DON VALLEY QUARRY, TORONTO,ONT

1240; PARKS \& FRITZ, ONT DEPT MINES, VOL

31,PT9, 1923,P12,PL 1,FIG 18

REMARKS: C-JTOWNSEND ; 1 SPEC

\section{CYRTOLITES INFLATA SHAW}

5646 P-HOLO

MS,NIAG

GUELPH FM

[GUELPH DOLOMITE]

TOBERMORY,ONT

1320; SHAW EW, TRANS ROY CAN INST, VOL

21,PT2, 1937,P361,PL24,FIG 12

REMARKS: C-EW SHAW.1934; 1 SPEC

\section{CYRTOLITES ORNATUS CONRAD}

17448 [1209HR] REF SPEC-F

U O.CINCI,MAYSVILL

DUNDASFM

DON VALLEY QUARRY, TORONTO,ONT

1175:PARKS \& FRITZ,ONT DEPT MINES, VOL

31,PT9, 1923,P3-4,PL 1,FIG 5

REMARKS: C-J TOWNSEND ·FIG 4 SAID TO BE SPEC

1209HR,LOOKS MORE LIKE 1208HR; 1 SPEC

CYRTOLITES ORNATUS CONRAD

17447 [1208HR] REF SPEC-F

U O.CINCI,MAYSVILL

DUNDAS

HUMBERVALE QUARRY, 6 FT LEVEL, TORONTO,ONT

1175:PARKS \& FRITZ, ONT DEPT MINES, VOL

31,PT9, 1923,P3-4,PL1,FIG 3,4

REMARKS: C-H STEWART, 1919 ;FIG 4 SAID TO BE SPEC

1209HR,LOOKS MORE LIKE 1208HR; 2 SPEC
CYRTOLITHES ATLANTOIDES MATTHEW

REMARKS: SEE PELAGIELLA ATLANTOIDES (MATTHEW)

EOTOMARIA? HOPKINSI PARKS

22609 [362S ] P-HOLO

S.

LIMESTONE RAPIDS, SEVERN R,DIST OF PATRICIA, ONT 1204:PARKS WA, TRANS ROY CAN INST, VOL

11,PT 1, 1915,P60,PL 1,FIG 5

REMARKS: C-JB TYRRELL, 1912 ;D-WH HEARST, 1915; 1 SPEC

EUOMPHALOPTERUS TYRRELLI PARKS

22591 [344S ] P-HOLO

$\mathrm{S}$.

LIMESTONE RAPIDS, SEVERN R,DIST OF PATRICIA, ONT

1212; PARKS WA, TRANS ROY CAN INST, VOL

11,PT 1, 1915,P63-64,PL3,FIG 15, 16

REMARKS: C-JB TYRRELL;D- 1914 ;D-WH HEARST ; 1 SPEC (SEV PCES)

\section{EUOMPHALUS MINOR PARKS}

22641 [396S ] P-SYN

S.

LIMESTONE RAPIDS, SEVERN R,DIST OF PATRICIA, ONT

I192; PARKS WA, TRANS ROY CAN INST, VOL 11,PT 1,1915,P61-62,PL4,FIG 8.9

REMARKS: C-JB TYRRELL, 1912:D-1915 D-WH HEARST: INTERNAL CAST FIG'D;CAST AND MOULD ARE NOT OF SAME SPEC : 2 SPEC

EUOMPHALUS ROTUNDUS PARKS

22595 [348S ] P-SYN

$\mathrm{S}$,

LIMESTONE RAPIDS SEVERN R DIST OF PATRICIA ONT

1192; PARKS WA, TRANS ROY CAN INST, VOL

11, PT 1, 1915,P61,PL7,FIG 6,7

REMARKS: C-JB TYRRELL, 1912;D-WH HEARST, 1915:2 SPEC ( 4 PCES)

EUOMPHALUS SP

22616 [369S ] REF SPEC-F

$\mathrm{S}$.

LIMESTONE RAPIDS, SEVERN R,DIST OF PATRICIA, ONT 1192: PARKS WA,TRANS ROY CAN INST, VOL

11,PT 1, 1915,P62-63,PL7,FIG 10

REMARKS: C-JB TYRRELL, 1912 ;D-WH HEARST, $1915 ; 1$ SPEC (2 PCES)

HOLOPEA MEDIA PARKS

22608 [361S] P.HOLO

O.

LOWER RAPIDS, GODS R, MAN

1239; PARKS WA, TRANS ROY CAN INST, VOL

11,PT 1, 1915,P18-19,PL4,FIG 4

REMARKS: C-JB TYRRELL, 1912 ;D-WH HEARST, 1915;LOC GIVEN AS SHAMATTAWA R-NOW CALLED GODS R, TRIBUTARY OF HAYES R: 1 SPEC

HORMOTOMA ACUMINATA PARKS

22610 [363S ] P-HOLO

O.

LOWER RAPIDS, GODS R.MAN

1291; PARKS WA, TRANS ROY CAN INST, VOL

11.PT 1, 1915,P17-18,PL4, FIG 3

REMARKS: C-JB TYRRELL, 1912 :D-WH HEARST, 1915:LOC

GIVEN AS SHAMATTAWA R-NOW CALLED GODS R,

TRIBUTARY OF HAYES R: 1 SPEC

HORMOTOMA GRACILIS (HALL)

17452 [ 1213HR] REF SPEC-F

U O, CINCI,MAYSVILL

DUNDASFM

DON VALLEY QUARRY,TORONTO,ONT

1291:PARKS \& FRITZ,ONT DEPT MINES, VOL

31.PT9, 1923,P6-7,PL 1,FIG 10

REMARKS: C-J TOWNSEND ; 2 SPEC 
HORMOTOMA PATRICIAENSIS PARKS

22592 [345S] P-SYN

$\mathrm{S}$,

LIMESTONE RAPIDS, SEVERN R,DIST OF PATRICIA,ONT I291; PARKS WA, TRANS ROY CAN INST, VOL

11,PT1,1915,P57-58,PL 1,FIG 8

REMARKS: C-JB TYRRELL, 1912;D-1914;D-WH HEARST:

LARGER SPEC FIG'D;2 SPEC

\section{HORMOTOMA SPINDET}

22629 [384S ] REF SPEC-M

O.

LOWER RAPIDS, GODS R,MAN

I291; PARKS WA, TRANS ROY CAN INST, VOL

11,PT $1,1915, \mathrm{P} 18$

REMARKS: C-JB TYRRELL, 1912 ;D-WH HEARST, 1915;SPEC MENTIONED IN CONJUNCTION WITHH. ACUMINATA:LOC GIVEN AS SHAMATTAWA R-NOW CALLED GODS R, TRIBUTARY OF HAYES R ; 1 SPEC

\section{HORMOTOMA SP}

$22610(A)[363 S(A)]$ REF SPEC-M

0 .

LOWER RAPIDS,GODS R,MAN

1239:PARKS WA,TRANS ROY CAN INST, VOL

11,PT 1,1915, P 18

REMARKS: C-JB TYRRELL, 1912 ;D-WH HEARST, 1915;

MENTIONED IN CONJUNCTION WITH H. ACUMINATA;LOC

GIVEN AS SHAMATTAWA R-NOW CALLED GODS R,

TRIBUTARY OF HAYES R; 1 SPEC

\section{HYDROBIA WARRENANA (MEEK+HAYDEN)}

29885 REF SPEC-F

PALEOC,

RAVENSCRAG FM

LS 1,SEC 20,TP 8,RGE 4,W 4TH MERID, S OF BULLSHEAD

CK,ALTA

1319:RUSSELL LS, 1974,ROM,LIFE SCI CONTR,N102,P15,FIG 2I,J

REMARKS: C,D-LS RUSSELL;C-1937; 1 SPEC

\section{LIOSPIRA CF HELENA (BILLINGS)}

17442 [1202HR] REF SPEC-F

U O,CINCI,MAYSVILL

DUNDAS FM

1 MI ABOVE RLWY, ETOBICOKE R.ONT

1201:PARKS \& FRITZ,ONT DEPT MINES,VOL

31.PT9,1923,P7-8,PL1,FIG 11

REMARKS: C-WA PARKS, $1920 ; 1$ SPEC

\section{LIOSPIRA STEVENSONI PARKS}

22646 [401S] P-SYN

$\mathrm{S}$

LIMESTONE RAPIDS, SEVERN R,DIST OF PATRICIA,ONT

1201:PARKS WA, TRANS ROY CAN INST, VOL

11.PT1, 1915,P68,PL 4,FIG 10,11

REMARKS: C-JB TYRRELL, 1912 ;D-WH HEARST, 1914;SPEC

'A'=FIG 10;SPEC 'B' $=$ FIG $11 ; 2$ SPEC

\section{LOPHOSPIRA BEATRICE FOERSTE}

17437 [1197HR] REF SPEC-F

U O,CINCI,MAYSVILL

DUNDAS FM

[DUNDAS ]

W SIDE,CUT 1 MI BELOW LAMBTON BR, 15 FT ABOVE

WATER, HUMBER R, LAMBTON ONT

1208:PARKS \& FRITZ,ONT DEPT MINES, VOL

31,PT9, 1923,P8-10,PL1,FIG 13

REMARKS: C-WAPARKS, $1920 ; 1$ SPEC

\section{LOPHOSPIRA BEATRICE VAR PARVA PARKS\&FRITZ}

17439 [1199HR] P-SYN

U O,CINCI,MAYSVILL

DUNDAS FM

DON VALLEY QUARRY, TORONTO.ONT

1208;PARKS \& FRITZ,ONT DEPT MINES,VOL

31.PT9, 1923.P10.PL 1.FIG 14

REMARKS: C-J TOWNSEND ;SPEC ON LARGER SLAB FIG'D 2 SPEC
LOPHOSPIRA DEPRESSA SHAW

5644 P-HOLO

MS,NIAG

GUELPHFM

GAULT,ONT

1208: SHAW EW, TRANS ROY CAN INST, VOL

21,PT2, 1937,P359,PL24,FIG 11

REMARKS: C-EW SHAW, 1934 ;WAX CAST FIG'D ; 1 SPEC \& 2 CASTS

\section{LOPHOSPIRA ELORA SHAW}

5645 P-HOLO

MS,NIAG

GUELPHFM

ELORA,ONT

1208; SHAW EW, TRANS ROY CAN INST, VOL

21,PT2, 1937,P359,PL24,FIG 11

REMARKS: C-EW SHAW, 1934 ;WAX CAST FIG'D ; 1 SPEC \& WAX CAST

LOPHOSPIRA TROPIDOPHORA (MEEK)

17446 [1206HR] REF SPEC-F

UO,CINCI,MAYSVILL

DUNDAS FM

HUMBERVALE QUARRY , TORONTO,ONT

1208;PARKS \& FRITZ,ONT DEPT MINES,VOL

31,PT9, 1923,P 10-11,PL1,FIG 15

REMARKS: C-J TOWNSEND, $1921 ; 1$ SPEC

\section{LOPHOSPIRA TROPIDOPHORA (MEEK)}

17475 [1236HR] REF SPEC-F

UO,CINCI,MAYSVILL

DUNDAS FM

HUMBERVALE QUARRY, TORONTO ONT

1208; PARKS \& FRITZ,ONT DEPT MINES,VOL

31,PT9, 1923, P 10-11,PL 1, FIG 16

REMARKS: C-JTOWNSEND; 1 SPEC

LOPHOSPIRA XANTHIPPE (BILLINGS)

15349 [842G] REF SPEC-F

$M S, N I A G$

GUELPH FM

FERGUS,ONT

1208;SHAW EW, TRANS ROY CAN INST, VOL

21,PT2, 1937,P358,PL24,FIG 8

REMARKS: D-J MALCOLM, $1915 ; 2$ SPEC

LOPHOSPIRA XANTHIPPE (BILLINGS)

15355 [848G] REF SPEC-F

MS,NIAG

GUELPHFM

FERGUS,ONT

1208:SHAW EW TRANS ROY CAN INST, VOL

21,PT2, 1937,P358,PL24,FIG 9

REMARKS: D-J MOFFAT, $1915 ; 2$ SPEC

\section{MACLUREA SUBOVATA PARKS}

22598 [351S] P-SYN

0 .

LOWWR RAPIDS, GODS R,MAN

189:TYRRELL JB, 1913 P 190:PARKS WA TRANS ROY CAN INST, VOL 11,PT 1, 19 15,P 19-20,PL 7, FIG 4,5

REMARKS: C-JB TYRRELL, 1912 ;D-WH HEARST, 1915;LOC GIVEN AS SHAMATTAWA R-NOW CALLED GODS R, TRIBUTARY OF HAYES R ; 3 SPEC (4 PCES)

MACLURINA MANITOBENSIS VAR ACUTA PARKS REMARKS: SEE MACLURITES ACUTA (PARKS

MACLURITES ACUTA (PARKS)

22597 [350S ] P-HOLO

MACLURINA MANITOBENSIS VAR ACUTA PARKS $\mathrm{O}$

LOWER RAPIDS, GODS R,MAN

1189; TYRRELL JB, 1913,P 190;PARKS WA, TRANS ROY CAN INST, VOL 11,PT 1, 1915,P21-22,PL 7,FIG 1,2

REMARKS: C-JB TYRRELL, 1912 :D-WH HEARST, 1915:LOC GIVEN AS SHAMATTAWA R-NOW CALLED GODS R,

TRIBUTARY OF HAYES R: 1 SPEC 
METOPTOMA AMII MATTHEW

$8048[418 C M]$ P.SYN

$\mathrm{MCA}$.

MT STEPHEN,FIELD,BC

1232:MATTHEW GF,TRANS ROY SOC CAN,VOL 8,SEC 4,1902,P111,PL1,FIG 12A-B

REMARKS: C-GF MATTHEW;D-MACKENZIE;LABELLED HOLOTYPE ; 1 SPEC

OMPHALOTROCHUS CONOIDEUS GIRTY

6352 [2959CB ] P-PARA?

P

PARK CITY FM,PHOSPHATE BEDS

MONTPELIER,IDAHO

| 196:GIRTY GH,USGS BULL 436, 1910,P50-51

REMARKS: D-W FERRIER, 1920 ;LABELLED PARATYPES; SPECS NOT FIG'D; 4 SPEC

OMPHALOTROCHUS FERRIERI GIRTY

6353 [2960CB ] P-PARA ?

$P$

PARK CITY FM,PHOSPHATE BEDS

MONTPELIER,IDAHO

I196; GIRTY GH, USGS BULL 436, 19 10,P49-50

REMARKS: D-W FERRIER, 1920 ;LABELLED PARATYPES: SPECS NOT FIG'D; 4 SPEC

PELAGIELLA ATLANTOIDES (MATTHEW)

26018 P-PARAL

CYRTOLITHES ATLANTOIDES MATTHEW

$M C A$,

ST JOHN GP

[ST JOHN,ACADIAN]

HANFORD BK ST MARTINS,ST JOHN CO,NB

1323:MATTHEW GF, 1894,P94,PL 16,FIG 18B;KNIGHT JB, GSA SPEC PAP 32,P237-238, 1941,PL63,FIG 6A-B

REMARKS: C-GF MATTHEW:D-MACKENZIE :FREE SPEC FIG'D;ORIG INCLUDED WITH LECTOTYPE AS NO 123CM ; 2 SPEC

PELAGIELLA ATLANTOIDES (MATTHEW)

7753 [123CM] P-LECTO

CYRTOLITHES ATLANTOIDES MATTHEW

$M C A$,

ST JOHN GP

[ST JOHN, ACADIAN]

HANFORD BK, ST MARTINS,ST JOHN CO,NB

1323:MATTHEW GF, 1904,P94,PL16,FIG 18A; KNIGHT JB, GSA SPEC PAP 32, 1941,P237-238,PL63,FIG 6C

REMARKS: C-GF MATTHEW:D-MACKENZIE ;DESIGNATED LECTOTYPE BY KNIGHT; 1 SPEC

PLANORBIS SPECTABILIS MEEK

27174 REF SPEC-F

EOC

BRIDGER FM

N OF LONE TREE,E SIDE NW 1/4.SEC 9,T 13N,R113W, UINTA CO.WYO.USA

1---;RUSSELL LS,CAN NAT MUS BULL

126, 1952,P 131,PL19,FIG 2

REMARKS: C-GF STERNBERG, 1950 ;GENUS NOT

DISCUSSED INTREATISE ; 1 SPEC

PLATYCERAS APERTA MATTHEW

7895 [265CM ] P-SYN

$\mathrm{MCA}$,

ST JOHN GP

[ST JOHN,ACADIAN]

SEELY ST, ST JOHN,NB

1240:MATTHEW GF, TRANS ROY SOC CAN, VOL 8, SECT

4.1891,P132,PL 11,FIG 4A-D

REMARKS: C-GF MATTHEW:D-MACKENZIE :SPEC ' $A$ ' = FIG $4 A-C \cdot$ SPEC $^{\prime} B^{\prime}=$ FIG $4 \mathrm{D}: 2$ SPEC
PLEUROTOMARIA ADJUSTOR HALL

12958 [2348D ] REF SPEC-F

M D.

KWATABOAHEGAN R,ONT

12 19:PARKS WA.ONT DEPT MINES, VOL

13,PT 1, 1904,PL8,FIG 2

REMARKS: C-WA PARKS, 1903 ;STORED UNDER

ORTHOCERAS LUXUM ; 1 SLAB

PLEUROTOMARIA DELICATULA VAR CAMERA PARKS

25580 P-SYN

$M D$

KWATABOAHEGAN R,ONT

I219;PARKS WA,ONT DEPT MINES, VOL

13,PT 1, 1904,P187,PL7,FIG 3

REMARKS: C-WA PARKS, 1903 ;CAST OF SPEC 'A' FIG'D : 2 SPEC \& 1 CAST

POLEUMITA HUDSONICA PARKS

22618[37 1S ]P-SYN

S.

LIMESTONE RAPIDS, SEVERN R,DIST OF PATRICIA,ONT

I192; PARKS WA, TRANS ROY CAN INST, VOL

11,PT 1, 1915,P67,PL4,FIG 1,2

REMARKS: C-JB TYRRELL, 1912;D-1915;D-WH HEARST;

LARGER SPEC FIG'D;2 SPEC

PROGRANGERELLA SPERATA

23283 P-HOLO

UK.

OLDMAN FM,COMREY SS

LS 2,SEC 3,TP 2,RGE 6,W 4TH MERID, MILK R VALLEY, NR COMREY ALTA

RUSSELL LS,JOUR PALEONT, VOL 15, 1941,P3 10-311,FIG 1 REMARKS: C-LS RUSSELL, 1940 ;NOT IN TREATISE; 1 SPEC

PROGRANGERELLA SPERATA RUSSELL

23286 P-PARA

U K.

OLDMAN FM,COMREY SS

LS 2,SEC 3,TP 2 RGE 6,W 4TH MERID,MILK R VALLEY, NR COMREY,ALTA

RUSSELL LS,JOUR PALEONT, VOL 15, 1941 , P310-311,FIG 4 REMARKS: C-LS RUSSELL, 1940 ;NOT IN TREATISE ; 1 SPEC

PROGRANGERELLA SPERATA RUSSELL

23285 P-PARA

UK.

OLDMAN FM COMREY SS

LS 2,SEC 3,TP 2,RGE 6,W 4 TH MERID,MILK R VALLEY, NR COMREY,ALTA

RUSSELLLS, JOUR PALEONT, VOL 15, 1941,P310-311,FIG 3

REMARKS: C-LS RUSSELL, 1940 ;NOT IN TREATISE; 1 SPEC

PROGRANGERELLA SPERATA RUSSELL

23284 P-PARA

UK,

OLDMAN FM, COMREY SS

LS 2,SEC 3,TP 2,RGE 6,W 4TH MERID, MILK R VALLEY, NR COMREY,ALTA

RUSSELL LS,JOUR PALEONT, VOL 15, 1941,P310-311,FIG 2

REMARKS: C-LS RUSSELL, 1940 ;NOT IN TREATISE; 1 SPEC

PYCNOMPHALUS COLEMANI PARKS

22594 [347S ] P-HOLO

S.

LIMESTONE RAPIDS, SEVERN R,DIST OF PATRICIA,ONT 1244:PARKS WA, TRANS ROY CAN INST, VOL

11,PT 1, 19 15,P65,PL 1,FIG 9, 10,PL7,FIG 3

REMARKS: C-JB TYRRELL, 1912 ;D-WH HEARST, $1915 ; 1$ SPEC (2 PCES

PYCNOMPHALUS DAPHNE (BILLINGS)

15343 [832G] REF SPEC-F

MS,NIAG

GUELPHFM

[GUELPH]

BELWOOD,ONT

1244: SHAW EW, TRANS ROY CAN INST, VOL 
21,PT2, 1937,P360-361,PL24,FIG 7

REMARKS: C-J TOWNSEND ;SPEC 'A' FIG'D;RECORDS STATE 3 SPECS ; 4 SPEC

PYCNOMPHALUS SP INDET

22615 [368S ] REF SPEC-F

ASSINA RAPIDS, SEVERN R,DIST OF PATRICIA,ONT 1244:PARKS WA.TRANS ROY CAN INST, VOL 11.PT 1.1911.P65-66,PL7,FIG 9

REMARKS: C-JB TYRRELL, 1912 ;D-1915;D-WH HEARST. SECTIONED SPEC FIG'D ; 2 SPEC (3 PCES)

RAPHISTOMA? KELLIENSIS MATTHEW

8289 [659CM ] P-HOLO

UCA ORLO.

[BRETONIAN]

KELLY'S IS, CONCEPTION BAY,NFLD

1201:MAT THEW GF, TRANS ROY SOC CAN,VOL 5,SEC

4, 1899,P70-71,PL3,FIG 4A-B

REMARKS: C-GF MATTHEW;D-MACKENZIE ; 1 SPEC

STENOTHECA ACADICA (DAWSON)

REMARKS: SEE PARMOPHORELLA ACADICA (DAWSON)

STROPHOSTYLUS CYCLOSTOMUS HALL

22506 [258S ] REF SPEC-F

MD.

DETROIT RIVER GP,AMHERSTBURG FM

[UMONROE]

LIVINGSTONE CUT,DETROIT R, AMHERSTBURG,ONT

1242; GRABAU AW, MICH BIOL \& GEOL SURV,PUB 2, GEOL

SER 1, 1910.P185.FIG 9-11

REMARKS: D-T NATTRESS, $1914 ; 1$ SPEC

\section{TROCHUS SPINDET}

22614 [367S ] REF SPEC-F

$\mathrm{S}$,

LIMESTONE RAPIDS, SEVERN R,DIST OF PATRICIA, ONT 1259; PARKS WA, TRANS ROY CAN INST, VOL

11,PT 1, 1915,P69,PL3,FIG 7

REMARKS: C-JB TYRRELL, 1912 ;D-WH HEARST, $1915 ; 1$ SPEC

\section{TROCHUS SP}

22647 [402S ] REF SPEC-F

S.

LIMESTONE RAPIDS, SEVERN R,DIST OF PATRICIA,ONT 1259; PARKS WA,TRANS ROY CAN INST, VOL

11.PT 1, 1915.PL69,P3,FIG 14

REMARKS: C-JB TYRRELL, 1912 ;D-WH HEARST, $1915 ; 1$ SPEC

\section{MOLLUSCA,BIVALVIA}

\section{AVICULOPECTEN ? MONTPELIERENSIS GIRTY}

6367 [2978CB ] P-PARA?

$P$,

PARK CITY FM,PHOSPHATE BEDS

MONTPELIER,IDAHO,USA

N336;GIRTY GH,USGS BULL 436, 1910,P42-43

REMARKS: D-WF FERRIER, 1920 ;LABELLED PARATYPE ; 1 SPEC

BYSSONYCHIA ALVEOLATA ULRICH

17225 [983HR] REF SPEC-F

U O,CINCI,MAYSVILL

DUNDAS FM

[LORRAINE,DUNDAS]

16 FT LEVEL, HUMBERVALE QUARRY, TORONTO,ONT

N285; STEWART H,ONT DEPT MINES,VOL 29,PT6, 1920,P2425, PL4,FIG 1,2

REMARKS: C-H STEWART, $1920 ; 2$ SPEC
BYSSONYCHIA PRAECURSA ULRICH

17226 [984HR ] REF SPEC-F

U O,CINCI,MAYSVILL

DUNDASFM

16 FT LEVEL, HUMBERVALE QUARRY, TORONTO,ONT N285;STEWART H,ONT DEPT MINES, VOL

29,PT6, 1920,P24,PL1,FIG 27

REMARKS: C-H STEWART, $1920 ; 1$ SPEC

BYSSONYCHIA RADIATA (HALL)

17243 [ 100 1HR ] REF SPEC-F

U O.CINCI,MAYSVILL

DUNDAS FM

5 FT LEVEL, HUMBERVALE QUARRY, TORONTO,ONT

N285; STEWART H,ONT DEPT MINES, VOL 29,PT6, 1920,P2324,PL4,FIG 3

REMARKS: C-H STEWART, 1920 ;SPEC 'A' FIG'D ; 1 SPEC \& CAST

BYSSONYCHIA VERA ULRICH

17227 [985HR] REF SPEC-F

U O,CINCI,MAYSVILL

DUNDAS FM

[DUNDAS]

6 FT LEVEL, HUMBERVALE QUARRY, TORONTO,ONT N285; STEWART H,ONT DEPT MINES,VOL

29,PT6, 1920,P25,PL1,FIG 23,24

REMARKS: C-H STEWART, 1920 ;SPEC 'A' FIG'D ; 2 SPEC

BYSSONYCHIA VERA VAR PLANA STEWART

16682 [345HR] P-HOLO

U O,CINCI,MAYSVILL

DUNDAS FM

[DUNDAS]

HUMBER R,TORONTO,ONT

N285;STEWART H,ONT DEPT MINES,VOL 29,PT6, 1920,P2526,PL1,FIG 26

REMARKS: C-J TOWNSEND, $1905 ; 1$ SPEC

CLIDOPHORUS CF FABERI MILLER

17239 [997HR] REF SPEC-F

UO,CINCI,MAYSVILL

DUNDAS FM

HUMBER R CUT, 25 FT LEVEL, HUMBER R, TORONTO,ONT

N233;STEWART H,ONT DEPT MINES,VOL

29,PT6, 1920,P 13,PL 1,FIG 6

REMARKS: C-H STEWART, $1920 ; 1$ SPEC

CLIDOPHORUS FABULUS (HALL)

17260 [1019HR] REF SPEC-F

U O,CINCI,MAYSVILL

DUNDAS FM

15 FT LEVEL, HUMBERVALE QUARRY, TORONTO,ONT

N233; STEWART H,ONT DEPT MINES,VOL

29,PT6, 1920,P 13,PL 1,FIG 8

REMARKS: C-H STEWART, $1920 ; 1$ SPEC

CLIDOPHORUS OBLIQUUS STEWART

17259 [1018HR] P-HOLO

U O,CINCI,MAYSVILL

DUNDAS FM

HUMBER R CUT, TORONTO,ONT

N233:STEWART H.ONT DEPT MINES, VOL 19,PT6, 1920,P12-

13,PL1,FIG 9

REMARKS: C-H STEWART, $1920 ; 1$ SPEC

CLIDOPHORUS PLANULATUS (CONRAD)

17230 [988HR] REF SPEC-F

UO,CINCI,MAYSVILL

DUNDAS FM

3 FT LEVEL, DON VALLEY QUARRY, TORONTO,ONT

N233;STEWART H,ONT DEPT MINES,VOL 19,PT6, 1920,P1112,PL1,FIG 12

REMARKS: C-H STEWART, 1920:1 SPEC 
CLIDOPHORUS PRAEVOLUTUS FOERSTE

17269 [1028HR] REF SPEC-F

U O,CINCI,MAYSVILL

DUNDAS FM

DON VALLEY QUARRY.TORONTO.ONT

N233:STEWART H,ONT DEPT MINES, VOL

29.PT6, 1920,P12,PL 1,FIG 10

REMARKS: C-J TOWNSEND :LARGER SPEC FIG'D ; 2 SPEC

COLPOMYA FABA PUSILLA FOERSTE

17222 [980HR] REF SPEC-F

U O.CINCI,MAYSVILL

DUNDASFM

BELOW 8 FT LEVEL, DON VALLEY QUARRY, TORONTO,ONT N394:STEWART H.ONT DEPT MINES, VOL

29.PT6, 1920,P28,PL5,FIG 2

REMARKS: C-H STEWART, 1920; 1 SPEC

COLPOMYA FABA PUSILLA FOERSTE

25154 [744U] REF SPEC-F

UOCINCIEDEN

COLLINGWOODFM

CRAIGLEITH.ONT

N394:PARKS WA,TRANS ROY SOC CAN, VOL 22,SECT

4, 1928,P83,PL 1,FIG 22

REMARKS: C-J TOWNSEND : 1 SPEC

\section{CORBICULA OCCIDENTALIS VAR VENTRICOSA}

10835[5296CT] P-SYN

UK,SENON,MAAST

EDMONTON FM.DRUMHELLER MARINE TONGUE

SE 1/4,SEC 33,TP 28,RGE 21, HORSESHOE CANYON , 8 MI W DRUMHELLER,ALTA

N666: ALLAN \& SANDERSON RES COUNCIL ALTA,REPT

13,1944, P90,PL5,FIG $18,19,25,26$

REMARKS: C-JOG SANDERSON, $1926: 4$ SPEC

CORBULA MACTRIFORMIS MEEK + HAYDEN

29884 REF SPEC-F

PALEOC,

RAVENSCRAG FM

OLD RD S OF SHAND COAL MINE,E SIDE COULEE

ROCHE PERCEE AREA,SASK

N692:RUSSEL LS, 1974, ROM, LIFE SCI CONTR, N102,FIG 2E,F

REMARKS: C,D-LS RUSSELL ;LOC-LS 5,SEC 3,TP 2,RGE 7 ,

W 2 ND MERID:LT BR CARB + SILTY BUFF CLAY 12 IN,

BENEATH 8 IN IRONSTONE BAND,32 FT ABOVE ESTEVAN

COAL SEAM ; 1 SPEC

CORBULA SPHERIOIDES RUSSELL

24831 P-HOLO

UK

EASTEND FM,LR PART

SEC 30.TP 6.RGE 21,W 3RD MERID:ROAD CUT ON HWY 13 S OF EASTEND,SASK

N692; RUSSELL LS,JOUR PALEONT, VOL 17, N3, 1943,P287-

288.PL49,FIG 4

REMARKS: C-LS RUSSELL, $1940 ; 1$ SPEC

CORBULA SPHERIOIDES RUSSELL

24833 P-PARA

UK

EASTEND FM.LRPART

SEC 30,TP 6,RGE 21,W 3RD MERID;ROAD CUT ON HWY 13 S OF EASTEND,SASK

N692; RUSSELL LS,JOUR PALEONT, VOL 17,N3, 1943,P287-

288,PL49,FIG 5

REMARKS: C-LS RUSSELL, $1940 ; 1$ SPEC

CORBULA SPHERIOIDES RUSSELL

24834 P-PARA

UK

EASTEND FM,LRPART

SEC 30,TP 6,RGE 21,W 3RD MERID:ROAD CUT ON HWY 13 SOF EASTEND SASK

N692:RUSSELL LS,JOUR PALEONT, VOL 17,N3, 1943,P287-

288. PL49,FIG 6

REMARKS:C-LS RUSSELL, $1940 ; 1$ SPEC
CORBULA SPHERIOIDES RUSSELL

24832 P-PARA

UK

EASTEND FM,LRPART

SEC 30,TP 6. RGE 21,W 3RD MERID;ROAD CUT ON HWY 13

S OF EASTEND SASK

N692; RUSSELL LS,JOUR PALEONT, VOL 17,N3, 1943,P287288

REMARKS: C-LS RUSSELL, 1940 ;THIS SPEC NOT FIG'D : 1 SPEC

CTENODONTA CABOTENSIS JOHNSON IN BOLTON

7416 [651CL] P-HOLO

L S.MED

CATARACT GP. CABOT HEAD FM

FORKS OF THE CREDIT R, ONT

N228;BOLTON TE,GSC MEM 289, 1957,P71-72,PL11,FIG 6

REMARKS: C-H JOHNSON, 1930:1 SPEC

CTENODONTA CINGULATA ULRICH

17265 [1024HR] REF SPEC-F

U O.CINCI,MAYSVILL

DUNDAS FM

17 FT LEVEL, HUMBERVALE OUARRY TORONTO ONT

N228:STEWART H.ONT DEPT MINES, VOL 29,PT6, 1920,P89.PL 1, FIG 25

REMARKS: C-H STEWART ; 1 SPEC

CTENODONTA ELONGATA PARKS

$25146[736 \mathrm{U}] \mathrm{P}-\mathrm{HOLO}$

U O,CINCI,EDEN

COLLINGWOOD FM

CRAIGLEITH ONT

N228:PARKS WA, TRANS ROY SOC CAN, VOL 22,SECT

4,1928,P80,PL 1,FIG 23

REMARKS: C-WA PARKS, $1927 ; 1$ SPEC

CTENODONTA FILISTRIATA ULRICH

17258 [1017HR] REF SPEC-F

U O,CINCI,MAYSVILL

DUNDASFM

HUMBERRCUT, TORONTO ONT

N228; STEWART H,ONT DEPT MINES, VOL

29,PT6, 1920,P9,PL1,FIG 5

REMARK S: C-H STEWART, 1920:1 SPEC

CTENODONTA GEORGINA PARKS

25147 [737U] P-SYN

U O.CINCI,EDEN

COLLINGWOOD FM

CRAIGLEITH ONT

N228;PARKS WA,TRANS ROY SOC CAN.VOL 22,SECT

4,1928,P80,PL1,FIG 5,6

REMARKS: C-WA PARKS, $1927: 2$ SPEC

CTENODONTA GLENNA BOLTON

26478 P-PARA

S.MED

CATARACT GP.POWER GLEN FM

POWER GLEN , DECEW FALLS. ONT

N228;BOLTON TE, GSC MEM 289, 1957,P72,PL 11,FIG 3,4

REMARKS: C-TE BOLTON :SMALLER 2 OF 3 SPECS WITH

THIS NUMBER:OTHER IS HOLOTYPE ; 2 SPEC

CTENODONTA GLENNA BOLTON

26478 P-HOLO

S,MED

CATARACT GP.POWER GLEN FM

POWER GLEN , DECEW FALLS, ONT

N228;BOLTON'TE,GSC MEM 289, 1957,P72,PL 11,FIG 5

REMARK S: C-TE BOLTON : LARGEST OF 3 SPECS WITH

THIS NUMBER:OTHER 2 ARE PARATYPES : 1 SPEC

CTENODONTA MYALTA STEWART

17267 [ 1026HR] P-SYN

U O.CINCI,MAYSVILL

DUNDASFM

BELOW 8 FT LEVEL, DON VALLEY QUARRY TORONTO, ONT

N228;STEWART H,ONT DEPT MINES, VOL

29.PT6, 1920, P 10,PL1,FIG 11

REMARKS: C-H STEWART, 1920: 1 SPEC 
CTENODONTA MYALTA STEWART

$17266[1025$ HR ] P-SYN

U O.CINCI,MAYSVILL

DUNDAS FM

15 FT LEVEL, HUMBERVALE QUARRY, TORONTO,ONT

N228:STEWART H,ONT DEPT MINES,VOL

29, PT6, 1920, P 10,PL 1, FIG 7

REMARKS: C-HSTEWART, $1920 ; 1$ SPEC

CTENODONTA PULCHELLA (HALL)

25145 [735U] REF SPEC-F

U O,CINCI.EDEN

COLLINGWOOD FM

CRAIGLEITH,ONT

N228:PARKS WA,TRANS ROY SOC CAN, VOL 22,SECT

4,1928,P79-80,PL1,FIG 19

REMARKS: C-WA PARKS, $1927 ; 1$ SPEC

CUNEAMYA NEGLECTA (HALL\&WHITFIELD)

17263 [1022HR] REF SPEC-F

U O,CINCI,MAYSVILL

DUNDAS FM

25 FT LEVEL, HUMBERVALE QUARRY , TORONTO,ONT

N820:STEWART H.ONT DEPT MINES, VOL

29,PT6, 1920,P6,PL 1,FIG 4

REMARKS: C-H STEWART, $1920 ; 1$ SPEC

CUNEAMYA SCAPHA HALL\&WHITFIELD

17262 [1021HR] REF SPEC-F

U O, CINCI,MAYSVILL

DUNDAS FM

25 FT LEVEL, HUMBERVALE QUARRY, TORONTO,ONT N820:STEWART H,ONT DEPT MINES, VOL

29.PT6, 1920,P7, PL1,FIG 2

REMARKS: C-H STEWART, $1920 ; 1$ SPEC

\section{CUNEAMYA SCAPHA BREVIOR FOERSTE}

17229 [987HR] REF SPEC-F

UO.CINCI,MAYSVILL

DUNDAS FM

DON VALLEY QUARRY,TORONTO

N820:STEWART H,ONT DEPT MINES, VOL 29,PT6, 1920, P7-

8.PL1.FIG 1

REMARKS: C-H STEWART, 1920 ;SPEC 'A' FIG'D ; 2 SPEC

CYMATONATA PARALLELA (HALL)

$17223[981 \mathrm{HR}]$ REF SPEC-F

U O.CINCI,MAYSVILL

DUNDAS FM

4 FT LEVEL, OLD SHALE PITS, LAMBTON,ONT

N394: STEWART H, ONT DEPT MINES, VOL 29, PT6, 1920, P30-

31,PL4,FIG 10

REMARKS: C-HSTEWART, $1920 ; 1$ SPEC

\section{CYMATONATA PARALLELA (HALL)}

17224 [982HR] REF SPEC-F

U O,CINCI,MAYSVILL

DUNDAS FM

HUMBER R, TORONTO ONT

N394; STEWART H,ONT DEPT MINES, VOL 29,PT6, 1920,P3031, PL4,FIG 11

REMARKS: C-H STEWART, $1920 ; 1$ SPEC

\section{CYMATONATA PHOLADIS (CONRAD)}

17237 [995HR] REF SPEC-F

U O,CINCI,MAYSVILL

DUNDAS FM

DON VALLEY QUARRY,TORONTO,ONT

N394;STEWART H,ONT DEPT MINES, VOL 29,PT6, 1920,P2930,PL4,FIG 6

REMARKS: C-J TOWNSEND ; 1 SPEC

CYMATONATA RECTA ULRICH

16934 [654HR] REF SPEC-F

U O,CINCI,MAYSVILL

DUNDAS FM

DON VALLEY QUARRY, TORONTO,ONT

N394; STEWART H,ONT DEPT MINES, VOL 29,PT6, 1920,P3132.PL 4.FIG 8

REMARKS: C-J TOWNSEND; 1 SPEC
CYMBOPHORA GRATIAE RUSSELL

24844 P-HOLO

U K.

EAŚTEND FM,LR PART

SEC 30,TP 6,RGE 21,W 3RD MERID;ROAD CUT ON HWY 13 SOF EASTEND,SASK

N598; RUSSELL LS,JOUR PALEONT, VOL 17,N3, 1943,P283-

284,FIG 1,PL47,FIG 1

REMARKS: C-LS RUSSELL, $1940 ; 1$ SPEC

\section{CYMBOPHORA GRATIAE RUSSELL}

24845 P-PARA

UK,

EASTEND FM,LR PART

SEC 30,TP 6,RGE 21,W 3RD MERID;ROAD CUT ON HWY 13 , $S$ OF EASTEND,SASK

N598:RUSSELL LS, JOUR PALEONT, VOL 17, N3, 1943,P283.

284 , FIG 1,PL47,FIG 2

REMARKS: C-LS RUSSELL, $1940 ; 1$ SPEC

\section{CYMBOPHORA GRATIAE RUSSELL}

24846 P-PARA

UK,

EASTEND FM,LR PART

SEC 30,TP 6,RGE 21,W 3RD MERID;ROAD CUT ON HWY 13 , S OF EASTEND,SASK

N598:RUSSELL LS, JOUR PALEONT, VOL 17, N3, 1943,P283284, FIG 1,PL47,FIG 3,4

REMARKS: C-LS RUSSELL, $1940 ; 1$ SPEC

CYMBOPHORA GRATIAE RUSSELL

24847 P-PARA

U K

EASTENDFM,LR PART

SEC 30,TP 6,RGE 21,W 3RD MERID;ROAD CUT ON HWY 13. S OF EASTEND,SASK

N598; RUSSELL LS,JOUR PALEONT, VOL 17,N3, 1943,P283284,FIG 1,PL47,FIG 5

REMARKS: C-LS RUSSELL, $1940 ; 1$ SPEC

\section{CYPRICARDINIA CANADENSIS GRABAU}

22489 [241S] P-SYN

$M D$

DETROIT RIVER GP,AMHERSTBURG FM

[U MONROE]

DETROIT R, LIVINGSTONE CUT, AMHERSTBURG,ONT N579:GRABAU AW MICH GEOL \& BIOL SURV,PUB2,GEOL SER 1,1910,P170,PL23,FIG 15

REMARKS: D-T NATTRESS, 1914 ;LABELLED COTYPE ; 1 SPEC

CYPRICARDINIA CF INDENTATA HALL

12958 [2348D] REF SPEC-F

$M D$,

KWATABOAHEGAN R,ONT

N579;PARKS WA, ONT DEPT MINES, VOL

13,PT1, 1904,PL8,FIG 2

REMARKS: C-WA PARKS, 1903 ;STORED UNDER

ORTHOCERAS LUXUM (HALL); 1 SLAB

EXOGYRA INCIPIENS SANDERSON

10803 [5264CT ] P-SYN

U K,SENON,MAAST

EDMONTON FM,PIERRE-ST MARY RIVER BEDS

OLDMAN R, MONARCH,ALTA

N1115;ALLAN \& SANDERSON,RES COUNCIL ALTA,REPT

13,1944, P87-88,PL5, FIG 9,10,14

REMARKS: C-WA PARKS ; 2 SPEC

GERVILLIA BIRUGATA RUSSELL

24835 P-HOLO

UK

EASTEND FM,LRPART

SEC 30,TP 6,RGE 21,W 3RD MERID;ROAD CUT ON HWY 13 S OFEASTEND,SASK

N308; RUSSELL LS,JOUR PALEONT, VOL

17, N3, 1943, P286, PL49,FIG 1

REMARKS: C-LS RUSSELL, $1940 ; 1$ SPEC 
GRAMMYSIA ARCUATA (CONRAD)

26879 REF SPEC-F

MD.ERIAN

HAMILTON GP

[HAMILTON]

PRATT'S FALLS,NY,USA

N8 19; WATERHOUSE JB, 1969, LETHAIA, VOL 2,N2,P116,FIG $12 \mathrm{~A}$

REMARK S: D-COBOURG Cl, 1949 ;SPEC 'A' FIG'D;

ERRONEOUSLY LABELLED IN FIG CAPTION AS NO.

1497D,G. LIRATA : 5 SPEC

GRAMMYSIA BISULCATA (CONRAD)

26875 REF SPEC-F

MD,ERIAN

HAMILTONGP

[HAMILTON]

CAYUGACO.NY.USA

N8 19; WATERHOUSE JB, 1969, LETHAIA, VOL 2,N2,P116, FIG $12 \mathrm{~B}$

REMARKS: D-COBOURG CI, 1949 ;SPEC 'B' FIG'D

ERRONEOUSLY LABELLED IN FIG CAPTION AS NO.

1497D.G. LIRATA ; 2 SPEC

ISCHYRODONTA ELONGATA ULRICH

17231 [989HR] REF SPEC-F

U O,CINCI,MAYSVILL

DUNDAS FM

HUMBER R REGION, TORONTO,ONT

N400:STEWART H,ONT DEPT MINES.VOL 29,PT6, 1920,P15-

16,PL3,FIG 4

REMARKS: C-J TOWNSEND : 1 SPEC

\section{ISCHYRODONTA UNIONOIDES}

17241 [999HR] REF SPEC?

U O CINCIMAYSVILL

DUNDAS FM

[DUNDAS]

HUMBER R,TORONTO,ONT

N400:STEWARD BH,ONT DEPT MINES, VOL 29,PT

VI, 1920,P 14

REMARKS: C-J TOWNSEND :LABELLED PLESIOTYPE;MAY BE ONE SPEC OF TWO FROM HUMBER R REGION MENTIONEDIN TEXT; 1 SPEC

\section{ISCHYRODONTA UNIONOIDES (MEEK)}

17247 [1006HR] REF SPEC-F

UO.CINCI,MAYSVILL

DUNDAS FM

DON VALLEY QUARRY.TORONTO ONT

N400:STEWART H.ONT DEPT MINES, VOL 19,PT6, 1920.P14-

15, PL1, FIG 13

REMARKS: C-J TOWNSEND; 1 SPEC

LAEVICARDIUM HOLMESI RUSSELL

24851 P.PARA

UK.

EASTENDFM,LR PART

SEC 30,TP 6.RGE 21,W 3RD MERID;ROAD CUT ON HWY 13. S OF EASTEND,SASK

N585:RUSSELL LS,JOUR PALEONT, VOL 17,N3, 1943,P284285.PL49,FIG 7

REMARKS: C-LS RUSSELL, $1940 ; 1$ SPEC

LAEVICARDIUM HOLMESI RUSSELL

24848 P-HOLO

U K,

EASTEND FM,LR PART

SEC 30,TP 6.RGE 21,W 3RD MERID;ROAD CUT ON HWY 13 SOF EASTEND SASK

N589;RUSSELL LS.JOUR PALEONT,VOL 17,N3, 1943,P284285,PL47,FIG 6

REMARKS: C-LS RUSSELL, $1940 ;$ RIGHT VALVE ; 1 SPEC

\section{LAEVICARDIUM HOLMESI RUSSELL}

24849 P-PARA

UK.

EASTEND FM,LR PART

SEC 30.TP 6.RGE 21.W 3RD MERID;ROAD CUT ON HWY 13 S OF EASTEND.SASK
N589;RUSSELL LS,JOUR PALEONT, VOL 17,N3, 1943,P284-

285.PL47. FIG 8.9. PL 49, FIG 8

REMARKS: C-LS RUSSELL, 1940 ;RIGHT VALVE ; 1 SPEC

LAEVICARDIUM HOLMESI RUSSELL

24850 P-PARA

UK.

EASTEND FM,LR PART

SEC 30,TP 6,RGE 21,W 3RD MERID;ROAD CUT ON HWY 13 S OF EASTEND,SASK

N589; RUSSELL LS,JOUR PALEONT, VOL 17, N3, 1943,P284. 285, PL 47. FIG 7

REMARKS: C-LS RUSSELL, 1940 ;LEFT VALVE ; 1 SPEC

LYRODESMA CINCINNATIENSE HALL

$17228[986 \mathrm{HR}] \mathrm{REF}$ SPEC-F

U O.CINCI,MAYSVILL

DUNDAS FM

HUMBER R REGION , TORONTO,ONT

N471; STEWART H,ONT DEPT MINES, VOL

29.PT6, 1920,P27. PL 4. FIG 7

REMARKS: C-J TOWNSEND; 1 SPEC

LYRODESMA POSTSTRIATUM (EMMONS)

16843 [553HR] REF SPEC-F

U O. CINCI,MAYSVILL

DUNDAS FM

DON VALLEY QUARRY, TORONTO,ONT

N47 1; STEWART H,ONT DEPT MINES, VOL 29,PT6, 1920,P26 27.PL4,FIG 5

REMARKS: C-J TOWNSEND ; 1 SPEC

LYRODESMA POSTSTRIATUM VAR ELONGATUM STEW.

17240 [998HR ] P-HOLO

U O,CINCI,MAYSVILL

DUNDAS FM

17 FT LEVEL,HUMBER R CUT, TORONTO,ONT

N471; STEWART H,ONT DEPT MINES,VOL

29.PT6, 1920,P27,PL4,FIG 4

REMARKS: C-H STEWART, $1920 ; 1$ SPEC

MODIOLODON SUBOVALIS ULRICH

$17246[1004 \mathrm{HR}] \mathrm{REF}$ SPEC-F

UO.CINCI,MAYSVILL

DUNDAS FM

HUMBER R REGION, TORONTO,ONT

N397:STEWART H,ONT DEPT MINES,VOL

29,PT6, 1920,P33,PL4,FIG 12

REMARKS: C-J TOWNSEND : 1 SPEC

MODIOLOPSIS BOREALIS FOERSTE

17516 [1277HR] REF SPEC-F

UO,CINCI,MAYSVILL

DUNDAS FM,CREDIT MBR

CREDIT R,ERINDALE,ONT

N397;FRITZ MA,ONT DEPT MINES, VOL 32,PT 7, 1925,P3738,PL4,FIG 8

REMARKS: C-WS DYER, 1922 :SECT 6 OF DYER:

INCORRECTLY PUBLISHED AS NO $1278 \mathrm{HR}$; 1 SPEC

MODIOLOPSIS BREVIS PARKS

25150 [740U] P-SYN

UO.CINCI.EDEN

COLLINGWOODFM

CRAIGLEITH,ONT

N397:PARKS WA,TRANS ROY SOC CAN, VOL 22, SECT 4.1928,P81-82,PL 1,FIG 20,21

REMARKS: C-WA PARKS, 1927 ;SPEC 'A' = FIG 20;SPEC

'B' = FIG 21; PUBL'D AS 'NOM PROV'; 3 SPEC

MODIOLOPSIS CONCENTRICA HALL\&WHITFIELD

17236 [994HR] REF SPEC-F

U O,CINCI,MAYSVILL

DUNDAS FM

17 FT LEVEL, HUMBER R CUT, TORONTO,ONT

N397:STEWART H,ONT DEPT MINES,VOL

29.PT6, 1920,P35. PL5. FIG 4

REMARKS: C-H STEWART, 1920, 1 SPEC 
MODIOLOPSIS CREDITENSIS JOHNSON IN BOLTON

7409 [644CL] P-HOLO

LS,MED

CATARACT GP CABOT HEAD FM

HAMILTON.ONT

N397:BOLTON TE,GSC MEM 289, 1957,P74,PL11,FIG 10

REMARKS: C-H JOHNSON, $1930 ; 1$ SPEC \& 1 CAST

MODIOLOPSIS MODIOLARIS (CONRAD)

17270 [1029HR] REF SPEC-F

U O.CINCI,MAYSVILL

DUNDAS FM

DON VALLEY QUARRY, TORONTO, ONT

N397:STEWART H,ONT DEPT MINES,VOL

29, PT 6, 1920,P34,PL3,FIG 1

REMARKS: C-J TOWNSEND ; 1 SPEC

\section{MODIOLOPSIS OVATA (CONRAD)}

17513 [1274HR] REF SPEC-F

U O,CINCI,MAYSVILL

DUNDAS FM

PRISON FARM QUARRY, MIMICO ONT

N397; PARKS WA,ONT DEPT MINES,VOL

32, PT 7, 1925,P37,PL4,FIG 2,6,9

REMARKS: C-WA PARKS, 1922 :SPECS 'A' ' $B$ ' ' 'C' = FIGS 2, 6 ,

$$
9: 4 \text { SPEC }
$$

MODIOLOPSIS STRIATA PARKS

17514 [1275HR] P-HOLO

U O,CINCI,MAYSVILL

DUNDAS FM

HUMBER R AT ROAD BRIDGE, WESTON,ONT

N397:PARKS WA,ONT DEPT MINES, VOL

32, PT7, 1925, P37, PL 4,FIG 3

REMARKS: C-WA PARKS, $1922 ; 1$ SPEC

\section{MODIOMORPHA SP}

12974 [2364D ] REF SPEC-F

$M D$

[MDEVONIAN]

KWATABOAHEGAN R,ONT

REMARKS: C-WA PARKS, $1903 ; 1$ SPEC

\section{MODIOMORPHA ? ACUMINATA PARKS}

22663 [419S] P-SYN

$\mathrm{S}$.

LIMESTONE RAPIDS, SEVERN R,DIST OF PATRICIA,ONT

N393; PARKS WA,IN TYRRELL, 1913,P194;PARKS

WA, TRANS ROY CAN INST, VOL 11,PT1,1915,P54-

55,PL4,FIG 12-14

REMARKS: C-JB TYRRELL, 1912 ;D-WH HEARST 1915;

SPECS ' $A$ ', 'B', 'C' = FIGS 12, 13,14; OLD RECORDS STATE 9 SPECS;SPECIES FIRST MENTIONED 1913,NOT

DESCRIBED UNTIL $1915 ; 8$ SPEC

\section{NUCULA MONTPELIERENSIS GIRTY}

6364 [2975CB] P-PARA

$P$.

PARK CITY FM,PHOSPHATE BEDS

MONTPELIER IDAHO

N230;GIRTY GH,USGS BULL 436, 1910,P38-39

REMARKS: D-WF FERRIER, 1920 ;LABELLED PARATYPES ; 3 SPEC

\section{ORTHODESMA APPROXIMATUM FOERSTE}

17196 [954HR] REF SPEC-F

U O,CINCI,MAYSVILL

DUNDAS FM

HUMBER R REGION TORONTO ONT

N397:STEWART H,ONT DEPT MINES, VOL

29,PT6, 1920,P37,PL5,FIG 10

REMARKS: C-J TOWNSEND; 1 SPEC

\section{ORTHODESMA NASUTUM (CONRAD)}

17235 [993HR] REF SPEC-F

U O.CINCI,MAYSVILL

DUNDAS FM

10 FT LEVEL, HUMBERVALE QUARRY, TORONTO,ONT

N397; STEWART H,ONT DEPT MINES, VOL 29,PT6, 1920,P38,PL5,FIG 3

REMARKS: C-H STEWART, $1920 ; 1$ SPEC \& CAST
OSTREA AURICULATA SANDERSON

10795 [5255CT ] P-SYN

UK,SENON,MAAST

EDMONTON FM, PIERRE-ST MARY RIVER BEDS

OLDMAN R, MONARCH,ALTA

N1138; ALLAN \& SANDERSON,RES COUNCIL ALTA,REPT

13,1944,P87,PL5,FIG 15,16

REMARKS: C-WA PARKS ; 2 SPEC

OSTREA GLABRA VAR LATA SANDERSON

10804 [5265CT ] P-SYN

UK,CAMPAN

FOREMOST FM

[FOREMOST BEDS,BELLY RIVER]

BOW IS SOUTH SASKATCHEWAN R,ALTA

N1138; ALLAN \& SANDERSON,RES COUNCIL ALTA,REPT 13,1944, P 86, PL5, FIG $1,3,6,7$

REMARKS: C-WA PARKS ; 4 SPEC

OSTREA GLABRA COALVILLENSIS MEEK

10798 [5258CT ] REF SPEC-F

K,SENON,MAAST

EDMONTON FM,BASE OF DRUMHELLER MARINE TONGUE

SE 1/4 SEC 31,TP 27,RGE 20,DEBRINSKI COULEE, NR BEYNON ALTA

N1138;ALLAN \& SANDERSON,RES COUNCIL ALTA,REPT 13,1944,P85,PL6,FIG 11,14

REMARKS: D-JOG SANDERSON, $1927 ; 2$ SPEC

OSTREA GLABRA VAR PONDEROSA SANDERSON

10792 [5252CT] P-SYN

UK,SENON,MAAST

EDMONTON FM.PIERRE-ST MARY RIVER BEDS

OLDMAN R, MONARCH,ALTA

N1138:ALLAN \& SANDERSON, RES COUNCIL ALTA REPT

13,1944,P86-87,PL5,FIG $8,11,12,13,17$

REMARKS: C-WA PARKS : 5 SPEC

OSTREA GLABRA VAR EXPANSA SANDERSON

10799 [5259CT] P-SYN

K,SENON,MAAST

EDMONTON FM,DRUMHELLER MARINE TONGUE

NW 1/4 SEC 4,TP 30,RGE 20,1-1/4 MI S OF MUNSON,ALTA N1138;ALLAN \& SANDERSON,RES COUNCIL ALTA,REPT

13, 1944, P85-86, PL6, FIG 13,15, 16

REMARKS: C-WA PARKS ; 3 SPEC

OXYTOMA NEBRASKANA (EVENS\&SHUMARD) VAR

24830 REF SPEC-F

UK,

EAŚTEND FM,LR PART

SEC 30,TP 6,RGE 21,W 3RD MERID;ROAD CUT ON HWY 13 S OF EASTEND,SASK

N344;RUSSELL LS,JOUR PALEONT, VOL 17,N3, 1943,P285286, PL48,FIG 5

REMARKS: C-LS RUSSELL, $1940 ; 1$ SPEC

PLESIELLIPTIO POSTBIPLICATUS (WHITFIELD) 32182 REF SPEC-F

UK,

HELL CREEK FM

[HELL CREEK FM,CRETACEOUS ]

SEC 30/31,T21N,R36E, SNOW CK, GARFIELD

CO,MONT,USA

N431:RUSSELL LS, 1976, CAN J EARTH SCI, VOL

13,N2,P368,PL 1,FIG 1

REMARKS: C-DC BECKMAN, 1972 ;D-LS RUSSELL, 1976 ; 1 SPEC

PLESIELLIPTIO PRISCUS (MEEK + HAYDEN)

29284 REF SPEC-F

PALEOC,

RAVENSCRAG FM

SW CORNER,SEC 2,TP 8,RGE 21,W 3RD MERID , PINE CREE PARK,SASK

N431;RUSSELL LS, 1974,ROM,LIFE SCI

CONTR,N $102, P 11, F I G 2 A$

REMARKS:C.D-LS RUSSELL:C- 1972; 1SPEC 
PSILOCONCHA INORNATA ULRICH

17221 [979HR] REF SPEC-F

U O.CINCI,MAYSVILL

DUNDAS FM

15 FT LEVEL, HUMBERVALE QUARRY, TORONTO.ONT

N394:STEWART H,ONT DEPT MINES.VOL 29,PT6, 1920,P40-

41.PL5,FIG 11

REMARK S: C-H STEWART, 1920 : 1 SPEC

PSILOCONCHA SINUATA ULRICH

17219 [977HR] REF SPEC-F

U O,CINCI,MAYSVILL

DUNDAS FM

DON VALLEY QUARRY,TORONTO,ONT

N394:STEWART H,ONT DEPT MINES, VOL

29,PT6, 1920,P40,PL5,FIG 8

REMARKS: C-H STEWART, 1920; 1 SPEC

PSILOCONCHA SINUATA BOREALIS FOERSTE

17271 [1030HR] REF SPEC-F

UO,CINCI,MAYSVILL

DUNDASFM

DON VALLEY QUARRY,TORONTO,ONT

N394:STEWART H.ONT DEPT MINES, VOL

29.PT6, 1920,P40,PL5, FIG 7

REMARKS: C-H STEWART, 1920; 1 SPEC

PSILOCONCHA SINUATA VAR BREVIS FRITZ

17551 [ 1318HR] P-HOLO

U O.CINCI,MAYSVILL

DUNDAS FM, HUMBER MBR

WORKMAN'S CK.ONT

N394; FRITZ MA,TRANS ROY SOC CAN,VOL 20,SECT

4.P106-107,PL4.FIG 6

REMARKS: C-MA FRITZ, $1925 ; 1$ SPEC

PSILOCONCHA SUBOVALIS ULRICH

17220 [978HR] REF SPEC-F

U O,CINCI,MAYSVILL

DUNDASFM

DON VALLEY QUARRY,TORONTO,ONT

N394:STEWART H,ONT DEPT MINES,VOL

29,PT6, 1920,P41,PL5,FIG 10

REMARKS: C-J TOWNSEND; 1 SPEC

PSILOCONCHA SUBRECTA ULRICH

17219 [976HR] REF SPEC-F

U O, CINCI,MAYSVILL

DUNDAS FM

HUMBERVALE QUARRY, 10 FT LEVEL, TORONTO,ONT

N394:STEWART H.ONT DEPT MINES, VOL 29.PT6, 1920,P41.

42.PL5. FIG 5

REMARKS: C-H STEWART, $1920 ; 1$ SPEC

PTERINEA CINCINNATIENSIS MILLER\&FABER

17245 [ 1003 HR ] REF SPEC-F

U O.CINCI,MAYSVILL

DUNDAS FM

HUMBER R CUT, 25 FT LEVEL, TORONTO,ONT

N299:STEWART H,ONT DEPT MINES, VOL 29,PT6, 1920,P22

23. PL 1.FIG 14,15

REMARK S: C-H STEWART, $1920 ; 2$ SPEC ON SAME PCE,

BOTH FIG'D; 1 SPEC

PTERINEA DEMISSA (CONRAD

16924 [652HR] REF SPEC-F

U O.CINCI,MAYSVILL

DUNDAS FM

HUMBER R REGION, TORONTO,ONT

N299:STEWART H ONT DEPT MINES, VOL

29.PT6, 1920,P22,PL 1.FIG 28

REMARKS: C-J TOWNSEND; 1 SPEC

PTERINEA PARVA PARKS

25066 [456U] P-SYN

$\mathrm{O} . \mathrm{CINCl}$

GLOUCESTER FM

DRAIN CUT,RIDEAU R, OTTAWA,ONT

N299:PARKS WA TRANS ROY SOC CAN VOL 22.SECT

4.1928,P83-84,PL 1,FIG 7

REMARKS: C-MA FRITZ, $1927 ; 3$ SPEC (1 SLAB)
RHYTIMYA COLEMANI STEWART

17272 [1031HR] P-HOLO

U O.CINCI.MAYSVILL

DUNDAS FM

DON VALLEY QUARRY,TORONTO,ONT

N818:STEWART H.ONT DEPT MINES.VOL

29,PT6, 1920,P46,PL5,FIG 13

REMARKS: C-J TOWNSEND ; GENUS NOT DISCUSSED IN TREATISE : 1 SPEC

RHYTIMYA COMPRESSA ULRICH

17233 [991HR] REF SPEC-F

U O.CINCI,MAYSVILL

DUNDAS FM

DON VALLEY QUARRY,TORONTO,ONT

N8 18:STEWART H.ONT DEPT MINES,VOL 29,PT6, 1920,P4546.PL5,FIG 14

REMARKS: C-H STEWART, 1920 :GENUS NOT DISCUSSED IN TREATISE; UNCERTAIN WHICH SPEC FIG'D : 4 SPEC \& 3 CASTS

RHYTIMYA CURTA PARKS

25153 [743U] P-HOLO

U O,CINCI,EDEN

COLLINGWOOD FM

RIDEAU R,CUMMINGS BRIDGE, OTTAWA,ONT

N818:PARKS WA,TRANS ROY SOC CAN,VOL 22,SECT 4, 1928,P82,PL 1,FIG 24

REMARKS: C-J TOWNSEND ;GENUS NOT DISCUSSED IN TREATISE : 1 SPEC

RHYTIMYA GRANULOSA WILSON

17232 [990HR] REF SPEC-F

U O.CINCI,MAYSVILL

DUNDAS FM

DON VALLEY QUARRY, TORONTO,ONT

N818:STEWART H,ONT DEPT MINES,VOL

29,PT6, 1920,P44,PL5,FIG 12

REMARKS: C-H STEWART, 1920 ;GENUS NOT DISCUSSED IN TREATISE ; 1 SPEC

RHYTIMYA OEHANA ULRICH

17261 [ 1020HR] REF SPEC-F

U O,CINCI,MAYSVILL

DUNDAS FM

LR 8 FT, DON VALLEY QUARRY,TORONTO,ONT

N818; STEWART H,ONT DEPT MINES, VOL

29,PT6, 1920,P45,PL5, FIG 15

REMARKS: C-H STEWART, 1920 ;GENUS NOT DISCUSSED INTREATISE; 1 SPEC

SAXICAVA LEVIS RUSSELL

24840 P-PARA

UK,

EASTEND FM,LR PART

SEC 30,TP 6, RGE 21,W 3RD MERID;ROAD CUT ON HWY 13 S OF EASTEND.SASK

N700;RUSSELL LS,JOUR PALEONT,VOL 17,N3, 1943,P287

REMARK S: C-LS RUSSELL, 1940 ;SPEC NOT FIG'D ; 1 SPEC

SAXICAVA LEVIS RUSSELL

24836 P-HOLO

UK

EASTEND FM,LR PART

SEC 30.TP 6.RGE 21,W 3RD MERID;ROAD CUT ON HWY 13. S OF EASTEND,SASK

N700:RUSSELL LS,JOUR PALEONT, VOL

17, N3, 1943,P287, PL49,FIG 2

REMARKS: C-LS RUSSELL, $1940 ; 1$ SPEC

SAXICAVALEVIS RUSSELL

24837 P-PARA

UK,

EASTEND FM,LR PART

SEC 30,TP 6, RGE 21,W 3RD MERID;ROAD CUT ON HWY 13 , S OF EASTEND,SASK

N700:RUSSELL LS, JOUR PALEONT,VOL

17, N3, 1943,P287, PL49,FIG 3

REMARKS: C-LS RUSSELL, $1940 ; 1$ SPEC 
SAXICAVA LEVIS RUSSELL

24838 P-PARA

UK,

EASTEND FM,LR PART

SEC 30, TP 6, RGE 21, W 3RD MERID;ROAD CUT ON HWY 13 S OF EASTEND,SASK

N700; RUSSELL LS,JOUR PALEONT, VOL 17, N3, 1943,P287

REMARKS: C-LS RUSSELL, 1940 ;SPEC NOT FIG'D ; 1 SPEC

SAXICAVA LEVIS RUSSELL

24839 P-PARA

UK

EASTEND FM,LR PART

SEC 30,TP 6, RGE 21,W 3RD MERID;ROAD CUT ON HWY 13 SOF EASTEND,SASK

N700;RUSSELL LS, JOUR PALEONT, VOL 17,N3,1943,P287

REMARKS: C-LS RUSSELL, 1940 ;SPEC NOT FIG'D; 1 SPEC

\section{SCHIZODUS FERRIERI GIRTY}

6366 [2977CB ] P-PARA?

P.

PARK CITY FM,PHOSPHATE BEDS

MONTPELIER,IDAHO,USA

N475;GIRTY GH,USGS BULL 436,1910,P41-42

REMARKS: D-WF FERRIER, 1920 ;LABELLED PARATYPE ; 1 SPEC

TANCREDIA AMERICANA CUPRESSENSIS LANDES

24843 REF SPEC-F

UK,

EASTEND FM,LR PART

SEC 30, TP 6,RGE 21,W 3RD MERID;ROAD CUT ON HWY 13. S OF EASTEND,SASK

N640;RUSSELL LS, JOUR PALEONT, VOL

17, N3, 1943,P285, FIG 3,PL48, FIG 4

REMARKS: C-LS RUSSELL, $1940 ; 1$ SPEC

TANCREDIA AMERICANA CUPRESSENSIS LANDES

24841 REF SPEC-F

UK,

EASTEND FM,LR PART

SEC 30,TP 6,RGE 21,W 3RD MERID;ROAD CUT ON HWY 13 , SOF EASTEND,SASK

N640;RUSSELL LS,JOUR PALEONT, VOL

17,N3, 1943,P285, FIG 3,PL48,FIG 1,2

REMARKS: C-LS RUSSELL, 1940; 1 SPEC

TANCREDIA AMERICANA CUPRESSENSIS LANDES 24842 REF SPEC-F

UK.

EASTENDFM,LR PART

SEC 30,TP 6, RGE 21, W 3RD MERID;ROAD CUT ON HWY 13 S OF EASTEND,SASK

N640;RUSSELL LS,JOUR PALEONT, VOL

17, N3, 1943,P285, FIG 3,PL48,FIG 3

REMARKS: C-LS RUSSELL, $1940 ; 1$ SPEC

\section{VANUXEMIA SP}

24357 [1199T ] REF SPEC-F

MO,CHAMP,MOH,TRENT

TRENTON GP

RLWY CUT,LITTLE CURRENT, MANITOULIN IS, ONT

N250;CALEY JF,GSC MEM 202, 1936,P79,PL1,FIG 2,7,8

REMARKS: C-JF CALEY, 1932 ;SPECS 'A', 'B', 'C' = FIGS 2,7,8

6 SPEC

\section{? VLASTA AMERICANA FRITZ}

26646 P-SYN

UO,CINCI,MAYSVILL

DUNDAS FM, HUMBER MBR

THISTLETOWN ONT

N247;FRITZ MA.JOUR PALEONT, VOL 25,N5, 1951,P697. 698,FIG 4

REMARK S: C,D-B METCALFE, $1950 ; 1$ SPEC
? VLASTA AMERICANA FRITZ

26647 P.SYN

U O,CINCI,MAYSVILL

DUNDAS FM, HUMBER MBR

THISTLETOWN,ONT

N247; FRITZ MA,JOUR PALEONT, VOL 25,N5, 1951, P697698. FIG 1

REMARK S: C,D-B METCALFE, 1950; 1 SPEC

? VLASTA AMERICANA FRITZ

26648 P-SYN

U O,CINCI,MAYSVILL

DUNDAS FM, HUMBER MBR

THISTLETOWN,ONT

N247;FRITZ MA,JOUR PALEONT,VOL 25,N5, 1951,P697. 698,FIG 2

REMARKS: C,D-B METCALFE, 1950; 1 SPEC

? VLASTA AMERICANA FRITZ

26649 P-SYN

U O,CINCI,MAYSVILL

DUNDAS FM, HUMBER MBR

THISTLETOWN,ONT

N247;FRITZ MA,JOUR PALEONT, VOL 25, N5, 1951,P697. 698,FIG 3

REMARKS: C.D-B METCALFE, $1950 ; 1$ SPEC

WHITELLA ACUTIUMBONIS STEWART

$17256[1015$ HR ] P-SYN

U O,CINCI,MAYSVILL

DUNDAS FM

DON VALLEY QUARRY, TORONTO,ONT

N249; STEWART H,ONT DEPT MINES, VOL

29,PT6, 1920,P20,PL1,FIG 20,21

REMARKS: C-H STEWART, 1920;2 SPEC

WHITELLA ACUTIUMBONIS STEWART

$17255[1014 \mathrm{HR}]$ P-SYN

U O,CINCI,MAYSVILL

DUNDASFM

LR 8 FT, DON VALLEY QUARRY, TORONTO,ONT

N249;ST'EWART H,ONT DEPT MINES, VOL

29,PT6, 1920,P20,PL1,FIG 19

REMARKS: C-H STEWART, 1920 ;SPEC 'A' FIG'D ; 2 SPEC

WHITELLA GONIUMBONATA FOERSTE

$17251[1010 \mathrm{HR}] \mathrm{REF}$ SPEC-F

U O,CINCI,MAYSVILL

DUNDAS FM

LR 8 FT, DON VALLEY QUARRY, TORONTO,ONT

N249;STEWART H,ONT DEPT MINES, VOL

29,PT6, 1920,P 18,PL 1,FIG 16

REMARKS: C-H STEWART, 1920; 1 SPEC

WHITELLA GONIUMBONATA FOERSTE

17250 [1009HR ] REF SPEC-F

UO,CINCI,MAYSVILL

DUNDAS FM

LR 8 FT, DON VALLEY QUARRY,TORONTO,ONT

N249;STEWART H,ONT DEPT MINES,VOL

29,PT6, 1920,P 18,PL 1,FIG 22

REMARKS: C-HSTEWART, 1920; 1 SPEC

WHITELLA CF HINDI (BILLINGS)

16784 [490HR] REF SPEC-F

U O,CINCI,MAYSVILL

DUNDAS FM

[LORRAINE FM]

HUMBER R,TORONTO,ONT

N249:STEWART BH.ONT DEPT OF MINES,VOL 29,PT

VI, 1920,P 16,PL2, FIG1

REMARKS: C-J TOWNSEND, $1913 ; 1$ SPEC

WHITELLA CF HINDI (BILLINGS)

17248 [1007HR] REF SPEC-F

UO.CINCI,MAYSVILL

DUNDAS FM

HUMBER R, TORONTO,ONT

N249:STEWART H,ONT DEPT MINES,VOL 29,PT6, 1920,P16 17.PL2,FIG 4

REMARKS: C-J TOWNSEND, $1913 ; 1$ SPEC 
WHITELLA IMPRESSATA STEWART

$17252[1011 \mathrm{HR}]$ P-SYN

UO,CINCI,MAYSVILL

DUNDAS FM

LR 8 FT , DON VALLEY QUARRY,TORONTO,ONT

N249:STEWART H.ONT DEPT MINES, VOL

29.PT6, 1920,P 19,PL2, FIG 2,3,6

REMARKS: C-H STEWART, 1920 ;ALL SPECS FIG'D ; 3 SPEC

WHITELLA LATA STEWART

17253[1012HR]P-HOLO

U O.CINCI,MAYSVILL

DUNDAS FM

LR 8 FT, DON VALLEY QUARRY, TORONTO,ONT

N249:STEWART H,ONT DEPT MINES, VOL

29. PT6, 1920, P 19, PL3,FIG 7

REMARKS: C-H STEWART, 1920; 1 SPEC

\section{WHITELLA PARKSI STEWART}

17254 [1013HR]P-SYN

U O.CINCI,MAYSVILL

DUNDAS FM

6 FT LEVEL, DON VALLEY QUARRY TORONTO ONT

N249:STEWART H,ONT DEPT MINES,VOL 29,PT6,1920,P1920,PL1,FIG 17

REMARKS: C-H STEWART, 1920 ;LARGER SPEC FIG'D ; 2 SPEC

\section{WHITELLA RADIATA STEWART}

17244 [ 1002HR ] P-HOLO

U O.CINCI,MAYSVILL

DUNDAS FM

LOC UNCERTAIN-PROBABLY DON VALLEY QUARRY,

TORONTO,ONT

N249:STEWART H,ONT DEPT MINES, VOL

29, PT6, 1920,P21,PL3,FIG 3

REMARKS: C-H STEWART, $1920 ; 1$ SPEC

WHITELLA TORONTONENSIS STEWART

17274 [1033HR]P-SYN

UO.CINCI,MAYSVILL

DUNDAS FM

LOC UNCERTAIN-PROBABLY DON VALLEY QUARRY,

TORONTO,ONT

N249:STEWART H.ONT DEPT MINES, VOL

29,PT6, 1920,P17,PL2,FIG 5

REMARKS: C-J TOWNSEND; 1 SPEC

\section{MOLLUSCA,CEPHALOPODA}

ACLEISTOCERAS ? LOUISENSE FOERSTE

12906 [22960] P-HOLO

MD.

ABITIBI RIVER FM

[ABITIBI RIVER (CORAL BEDS)]

HEAD OF LOUISE IS, MOOSE R, ONT

K308;FOERSTE AF,ONT DEPT MINES, VOL 37,PT6, 1928,P73-

74.PL1,FIG 4A-B

REMARKS: C,D-WS DYER, 1927 ; 1 SPEC

ACLEISTOCERAS ? SP (A)

12907 [2297D] REF SPEC-F

$\mathrm{MD}$

ABITIBI RIVER FM

[ABITIBI RIVER (CORAL BEDS)]

GRAND RAPIDS, MATTAGAMI R,ONT

K308;FOERSTE AF.ONT DEPT MINES, VOL

37.PT6, 1928.P73,PL2,FIG 2A.B

REMARKS: C,D-WS DYER, 1927 ; 1 SPEC
W308:FOERSTE AF,ONT DEPT MINES, VOL

37. PT6, 1928,P73,PL2,FIG 3

REMARKS: C,D-WS DYER, 1927 ; 1 SPEC

ACTINOCERAS CF BIGSBYIBRONN

REMARKS: SEE KOCHOCERAS SHAMATTAWAENSE FOERSTE + SAVAGE

ACTINOCERAS CF CLOUEI (BARRANDE)

17467 [1228HR] REF SPEC-F

U O,CINCI,MAYSVILL

DUNDAS FM

[DUNDAS]

HUMBERVALE QUARRY, TORONTO,ONT

K204:PARKS \& FRITZ ONT DEPT MINES, VOL

31,PT9, 1923,P27-28,PL4,FIG 5

REMARKS: C-WA PARKS, 1920 ; 1 SPEC (2 PCES)

ACTINOCERAS CF CLOUEI PARKS

REMARKS: SEE ARMENOCERAS SP

ACTINOCERAS CREBRISEPTUM (HALL)

17461 [1222HR] REF SPEC-F

U O,CINCI,MAYSVILL

DUNDAS FM

[DUNDAS ]

PRISON FARM QUARRY, MIMICO,ONT

K204:PARKS \& FRITZ ONT DEPT MINES, VOL

31,PT9, 1923,P20-27,PL3,FIG 9

REMARKS: C-WA PARKS, $1920 ; 1$ SPEC

ACTINOCERAS CREBRISEPTUM (HALL)

$17460[1221 \mathrm{HR}]$ REF SPEC-F

U O.CINCI,MAYSVILL

DUNDAS FM

[DUNDAS ]

PRISON FARM QUARRY MIMICO ONT

K204:PARKS \& FRITZ,ONT DEPT MINES, VOL

31,PT9, 1923,PL3,FIG 3

REMARKS: C-WA PARKS, $1920 ; 1$ SPEC

ACTINOCERAS CREBRISEPTUM (HALL)

17465 [1226HR] REF SPEC-F

U O,CINCI,MAYSVILL

DUNDAS FM

[DUNDAS ]

PRISON FARM QUARRY, MIMICO, ONT

K204:PARKS \& FRITZ, ONT DEPT MINES, VOL

31,PT9, 1923,P20-27,PL3,FIG 6

REMARKS: C-WA PARKS, 1920 ; 1 SPEC (3 PCES)

ACTINOCERAS CREBRISEPTUM (HALL)

17468 [1229HR] REF SPEC-F

U O,CINCI,MAYSVILL

DUNDAS FM

[DUNDAS ]

PRISON FARM QUARRY, MIMICO,ONT

K204:PARKS \& FRITZ, ONT DEPT MINES, VOL

31,PT9, 1923,P20-27,PL3,FIG 7,7A

REMARKS: C-WA PARKS, 1920 ; 1 SPEC (2 PCES)

ACTINOCERAS CREBRISEPTUM (HALL)

17469 [1230HR] REF SPEC-F

UO.CINCI,MAYSVILL

DUNDAS FM

[DUNDAS ]

ESIDE,LR 4 FT AT S END OF BIG EXPOSURE , HUMBER

R,TORONTO ONT

K204;PARKS \& FRITZ,ONT DEPT MINES, VOL

31.PT9, 1923,P20-27, PL3,FIG 8

REMARKS: C-WA PARKS, $1920 ; 1$ SPEC

ACTINOCERAS CREBRISEPTUM (HALL)

17185 [941HR] REF SPEC-F

U O.CINCI,MAYSVILL

DUNDASFM

[DUNDAS ]

HUMBER R,TORONTO.ONT 
K204:PARKS \& FRITZ,ONT DEPT MINES, VOL

31.PT9, 1923,P20-27,PL3,FIG 1

REMARKS: D-MISS DELAPORTE, $1913 ; 1$ SPEC

ACTINOCERAS CREBRISEPTUM (HALL)

17464 [1225HR ] REF SPEC-F

U O,CINCI,MAYSVILL

DUNDAS FM

[DUNDAS]

PRISON FARM QUARRY, MIMICO,ONT

K204:PARKS \& FRITZ, ONT DEPT MINES, VOL

31,PT9, 1923,P20-27,PL3,FIG 4

REMARKS: C-WA PARKS, $1920 ; 1$ SPEC

ACTINOCERAS CREBRISEPTUM (HALL)

17458 [1219HR ] REF SPEC-F

U O,CINCI,MAYSVILL.

DUNDAS FM

[DUNDAS]

PRISON FARM QUARRY, MIMICO,ONT

K204;PARKS \& FRITZ,ONT DEPT MINES, VOL

31,PT9, 1923,P20-27,PL3,FIG 2

REMARKS: C-WA PARKS ; 1 SPEC (2 PCES)

ACTINOCERAS CREBRISEPTUM (HALL)

17459 [1220HR] REF SPEC-F

U O,CINCI,MAYSVILL

DUNDAS FM

[DUNDAS]

PRISON FARM QUARRY, MIMICO,ONT

K204;PARKS \& FRITZ,ONT DEPT MINES, VOL

31, PT9, 1923, P20-27, PL3,FIG 5

REMARKS: C-WA PARKS, $1920 ; 1$ SPEC

ACTINOCERAS HEARSTI PARKS

REMARKS: SEE ARMENOCERAS HEARSTI (PARKS)

ACTINOCERAS PARKSI FOERSTE

22556 [308S ] P-HOLO

$\mathrm{O}$

[SHAMATTAWA LS]

LOWER RAPIDS, GODS R MAN

K204;FOERSTE AF,DEN UNIV BULL, VOL 19, 1921 ,P297299, PL35,FIG 3

REMARKS: C-JB TYRRELL, 1912 ;D-WH HEARST, 1914;LOC GIVEN AS SHAMATTAWA R-NOW CALLED GODS R, TRIBUTARY OF HAYES R ; 1 SPEC (2 PCES)

ACTINOCERAS RICHARDSONI VAR MAGNUM PARKS REMARKS: SEE ARMENOCERAS MAGNUM (PARKS)

ANTIPLECTOCERAS SHAMATTAWENSE (PARKS)

22572 [325S ] P-HOLO

DISCOCERAS? SHAMATTAWENSE PARKS

$\mathrm{O}$

[SHAMATTAWA LS

LOWER RAPIDS,GODS R.MAN

K372;PARKS WA, 1915,P31,PL 1,FIG 1; FOERSTE \&

SAVAGE,DEN UNIV BULL,J SCI LAB,VOL 22, 1927,P58.

59,PL24,FIG 2

REMARKS: C-JB TYRRELL, 1912 ;D-WH HEARST, 1914;LOC

GIVEN AS SHAMATTAWA R-NOW CALLED GODS R,

TRIBUTARY OF HAYES R ; 1 SPEC

ARMENOCERAS HEARSTI (PARKS)

22562 [314S] P-HOLO

ACTINOCERAS HEARSTIPARKS

S,

LIMESTONE RAPIDS, SEVERN R,DIST OF PATRICIA,ONT

K206;PARKS WA, 1915,P73-74,PL6,FIG 5;FOERSTE \&

SAVAGE, 1927,P68-69,PL8,FIG 4,PL24,FIG 5

REMARKS: C-JB TYRRELL, 1912 ;D-WH HEARST, 1915; 1

SPEC (2 PCES)
ARMENOCERAS MAGNUM (PARKS)

22557 [309S ] P-HOLO

ACTINOCERAS RICHARDSONI VAR MAGNUM PARKS $\mathrm{O}$

[SHAMATTAWA LS

LOWER RAPIDS,GODS R,MAN

K206:PARKS WA, 1915,P25-27,PL2,FIG 1;FOERSTE \& SAVAGE, 1927,P64-65,PL8,FIG 1

REMARKS: C-JB TYRRELL, 1912 ;D-WH HEARST, 1915;LOC GIVEN AS SHAMATTAWA R-NOW CALLED GODS R, TRIBUTARY OF HAYES R ; 1 SPEC (4 PCES)

\section{ARMENOCERAS SP}

22559 [311S] REF SPEC-F

ACTINOCERAS CF CLOUEI PARKS

O, ? (DRIFT)

[ORDOVICIAN? (DRIFT)]

MOUTH OF NELSON R YORK FACTORY MAN

K206;PARKS WA, 1915,P86,PL2,FIG 6,PL6,FIG 5;FOERSTE + SAVAGE, 1927,P65-66,PL9,FIG 7A,B

REMARKS: C-JB TYRRELL, 1912 ;D-WH HEARST, 1914; 1 SPEC (2 PCES)

ASCOCERAS BOREALE PARKS

REMARKS: SEE BILLINGSITES BOREALE (PARKS)

\section{BARRANDEOCERAS ? SP INDET} 22589 [342S ] REF SPEC-F

$\mathrm{S}$,

LIMESTONE RAPIDS, SEVERN R,DIST OF PATRICIA, ONT K372;PARKS WA, TRANS ROY CAN INST, VOL

11,PT 1, 1915,P79,PL5,FIG 11,PL7,FIG 8

REMARKS: C-JB TYRRELL, 1912 ;D-WH HEARST, $1915 ; 1$ SPEC

BILLINGSITES BOREALE (PARKS)

22573 [326S ] P-HOLO

ASCOCERAS BOREALE PARKS

$\mathrm{O}$

[SHAMATTAWA LS ]

LOWER RAPIDS,GODS R,MAN

K273:PARKS, IN TYRRELL, 1913,P 192:PARKS, 1915 P32

33,PL2,FIG 8,9;FOERSTE SAVAGE, 1927,P30-31,PL3,FIG $4 \mathrm{~A}-\mathrm{D}$

REMARKS: C-JB TYRRELL, 1912 :D-WH HEARST, 1915:LOC GIVEN AS SHAMATTAWA R-NOW CALLED GODS R, TRIBUTARY OF HAYES R; 1 SPEC (2 PCES)

BILLINGSITES PRIMUS FRITZ

REMARKS: SEE PROBILLINGSITES PRIMUS (FRITZ)

CHICAGOOCERAS ? LONGIDOMUM FOERSTE\&SAVAGE 22582 [335S ] P-HOLO ORTHOCERAS SP INDET PARKS (1915)

$\mathrm{S}$,

LIMESTONE RAPIDS, SEVERN R,DIST OF PATRICIA,ONT K259;PARKS, 1915,P73,PL5,FIG 12; FOERSTE \&

SAVAGE,DEN UNIV BULL, J SCI LAB, VOL 22, 1927, P48-

50, PL 18, FIG 2B

REMARKS: C-JB TYRRELL, 1912 ;D-WH HEARST, 1914 ; 1 SPEC (2 PCES)

CYRTOCERAS ORODES BILLINGS

22453 [204S] REF SPEC-F

CYRTOCERAS (CYCLOSTOMICERAS) ORODES BILLINGS

D.

DETROIT RIVER GP,AMHERSTBURG FM

[U MONROE FM ]

LIVINGSTONE CUT DETROIT R AMHERSTBURG.ONT

K---;GRABAU AW,MICH GEOL + BIOL SUR,PUB 2, GEOL

SER 1,1910,P 197-198,PL29,FIG 3

REMARKS: D-T NATTRESS, $1914 ; 1$ SPEC

CYRTOCERAS ORODES BILLINGS

22454 [205S] REF SPEC-F

CYRTOCERAS (CYCLOSTOMICERAS) ORODES BILLINGS

D.

DETROIT RIVERGP,AMHERSTBURG FM

[U MONROE FM] 
LIVINGSTONE CUT, DETROIT R, AMHERSTBURG, ONT K...:GRABAU AW.MICH GEOL + BIOL SUR,PUB 2,GEOL

SER 1, 1910,P 197-198,PL29,FIG 2

REMARKS: D-T NATTRESS, $1914 ; 1$ SPEC

\section{CYRTORIZOCERAS? SP}

22583(B) [336S (B)] REF SPEC-F

OOCERAS SP INDET PARKS (1915)

$\mathrm{S}$,

[EKWAN OR ATTAWAPISKAT FM]

LIMESTONE RAPIDS, SEVERN R, DIST OF PATRICIA,ONT

K284; PARKS WA, 1915.P76; FOERSTE \& SAVAGE,DEN UNIV

BULL.J SCI LAB, VOL 22, 1927,P52-53,PL5,FIG 6A, B

REMARKS: C-JB TYRRELL, 1912 ;D-WH HEARST, $1914 ; 1$ SPEC

DAWSONOCERAS ANNULATUM VAR AMERICANUM FOORD 22505 [257S ] REF SPEC-F

D.

DETROIT RIVER GP.AMHERSTBURG FM

[UMONROE FM]

LIVINGSTONE CUT,DETROIT R, AMHERSTBURG, ONT

K238;GRABAU AW,MICH GEOL \& BIOL SUR,PUB 2,GEOL

SER 1,1910,P196-197,PL28,FIG 8

REMARKS: D-T NATTRESS, $1914 ; 1$ SPEC

DAWSONOCERAS ANNULATUM VAR AMERICANUM FOORD 22455 [206S ] REF SPEC-F

D.

DETROIT RIVER GP,AMHERSTBURG FM

[U MONROE FM]

LIVINGSTONE CUT,DETROIT R, AMHERSTBURG, ONT

K238:GRABAU AW, MICH GEOL \& BIOL SUR,PUB 2,GEOL

SER 1,1910,P196-197,PL29,FIG 1

REMARKS: D-T NATTRESS, $1914 ; 1$ SPEC

\section{DIESTOCERAS TYRRELLI (PARKS)}

22568 [320S] P-SYN

POTERIOCERAS TYRRELLI PARKS

O.

LOWER RAPIDS, GODS R,MAN

K293; PARKS,IN TYRRELL, 1913,P191;PARKS, 1915,P29-

30,PL2,FIG 2;FOERSTE SAVAGE, 1927,P86-87,PL 16,FIG

$1 A-C$

REMARKS: C-JB TYRRELL, 1912 ;D-WH HEARST, 1914;SPEC '1' FIG'D:LOC GIVEN AS SHAMATTAWA R-NOW CALLED GODS R,TRIBUTARY OF HAYES R ; 2 SPEC

\section{DISCOCERAS? SHAMATTAWAENSE PARKS}

REMARKS: SEE ANTIPLECTOCERAS SHAMATTAWAENSE (PARKS)

\section{DISCOSORUS PARKSI FOERSTE + SAVAGE}

22561 [313S] REF SPEC-M

ACTINOCERAS SP (PARKS, 1915)

S.

ASSINA RAPIDS, SEVERN R,DIST OF PATRICIA,ONT K336:PARKS, 1915,P74,PL2,FIG 3;FOERSTE \& SAVAGE,DEN UNIV BULL,J SCI LAB, VOL 22, 1927,P75-76,PL8,FIG 3,PL23,FIG 8

REMARKS: C-JB TYRRELL, 1912 ;D-WH HEARST, 1914; 1 SPEC

\section{DUNLEITHOCERAS CORDATUM (PARKS)}

22576 [329S ] P-HOLO

OOCERAS CORDATUM PARKS

$\mathrm{S}$, ?

[NELSON LS?,DRIFT]

DRIFT, NELSON R,ONT

K284;PARKS WA, 19 15,P86-87,PL3,FIG 3;FOERSTE \& SAVAGE,DEN UNIV BULL,J SCI LAB, VOL 22, 1927, P53-

54, PL 15, FIG 1A-D

REMARKS: C-JB TYRRELL, 1912 ;D-WH HEARST, 1914 ; 1 SPEC
ENDOCERAS PROTEIFORME HALL

17474 [1235HR] REF SPEC-F

U O.CINCI,MAYSVILL

DUNDAS FM

[DUNDAS]

HUMBER R, TORONTO,ONT

K173:PARKS \& FRITZ,ONT DEPT MINES,VOL

31.PT9, 1923,P14-16.PL 4.FIG 3

REMARKS: OLD COM INST COLL: 1 SPEC.

ENDOCERAS PROTEIFORME HALL

17194 [952HR] REF SPEC-F

U O.CINCI,MAYSVILL

DUNDAS FM

[DUNDAS]

COLLINGWOOD ONT

K173:PARKS \& FRITZ,ONT DEPT MINES, VOI

31,PT9, 1923,P14-16, PL4,FIG 2,4

REMARKS: OLD COLL; 1 SPEC (2 PCES)

\section{ENDOCERAS ? HUDSONICUM PARKS}

22570 [322S] P-HOLO

$\mathrm{S}$

LIMESTONE RAPIDS, SEVERN R,DIST OF PATRICIA,ONT K173;PARKS, 1913,P 195;PARKS, 1915,P72,PL5,FIG 10; FOERSTE \& SAVAGE, 1927,P28-29,PL2.FIG 4

REMARKS: C-JB TYRRELL, 1912 ;D-WH HEARST, $1914 ; 1$ SPEC (3 PCES)

EPHIPPIOCERAS SIGMOIDALE FRITZ

25161[751U]P-SYN

$\cup O, \mathrm{CINCI}, \mathrm{EDEN}$

L COLLINGWOOD FM

BETWEEN COLLINGWOOD \& CRAIGLEITH, ONT

K448:PARKS WA,TRANS ROY SOC CAN, VOL 22, SEC 4,1928,P86-87,PL1,FIG 26,27

REMARKS: C-MA FRITZ, 1927 ;LABELLED COTYPES;

LARGEST SPEC FIG'D; 3 SPEC

GEISONOCERAS SP

$22580(B)[333 S(B)]$ REF SPEC-F

ORTHOCERAS SPINDET (PARKS, 19 15,2ND SPEC)

(O)

LOWER RAPIDS, GODS R MAN

K236;PARKS WA, 1915,P23;FOERSTE \& SAVAGE,DEN UNIV BULL,J SCI LAB, VOL 22, 1927, P34,PL4,FIG 2

REMARKS: C-JB TYRRELL, 1912 ;D-WH HEARST, 1914:LOC GIVEN AS SHAMATTAWA R-NOW CALLED GODS R, TRIBUTARY OF HAYES R; 1 SPEC

\section{? GOMPHOCERAS SP}

22578 [331S] REF SPEC-M

$M S$

[EKWAN OR ATTAWAPISKAT LS

ABOVE LIMESTONE RAPIDS, SEVERN R,DIST OF PATRICIA ONT

K3 18;PARKS WA, 1915,P79;FOERSTE + SAVAGE,DEN UNIV BULL, J SCI LAB, VOL 22, 1927, P 101-102

REMARKS: C-JB TYRRELL, 1912 :D-WH HEARST, 1914: SPEC

HERCOCERAS AURICULUM PARKS

25385 [863UH] P-SYN

$M D$

KWATABOAHEGAN R.ONT

K418;PARKS WA,ONT DEPT MINES, VOL

13.PT1,1904,P 190,PL6,FIG 5.6

REMARKS: D-WAPARKS : 2 SPEC

HURONIA INFLECTA PARKS

REMARKS: SEE HURONIELLA INFLECTA (PARKS)

HURONIA SEPTATA PARKS

22560 [312S] P-HOLO

0

MOUTH OF GODS R.MAN

K2 10; PARKS WA, 19 15,P27-28,PL5,FIG 8; FOERSTE \& SAVAGE, 1927,P71-72,PL12,FIG 2

REMARKS: C-JB TYRRELL, $1912 \cdot$ D-WH HEARST, 1914:LOC GIVEN AS SHAMATTAWA R-NOW CALLED GODS R TRIBUTARY OF HAYES R; 1 SPEC 
HURONIELLA INFLECTA (PARKS)

22558 [310S ] P.HOLO

HURONIA INFLECTA PARKS

$\mathrm{S}$.

LIMESTONE RAPIDS, SEVERN R,DIST OF PATRICIA,ONT K210:PARKS WA, 1915,P75,PL6,FIG 4;FOERSTE \&

SAVAGE, 1927,P70-71,PL11,FIG 1A,B

REMARKS: C-JB TYRRELL, 1912 ;D-WH HEARST, $1914 ; 1$ SPEC ( 3 PCES)

KIONOCERAS DYERI FOERSTE

12909 [2299D ] P-HOLO

$M D$.

ABITIBI RIVER FM (CORAL BEDS)

21 MI UPSTREAM,AT PRECAMB CONTACT , W

BRANCH,FRENCH R,ONT

K229;FOERSTE AF, ONT DEPT MINES, VOL 37,PT6, 1928,P7172,PL 1,FIG 5

REMARKS: C,D-WS DYER, $1927 ; 1$ SPEC

KOCHOCERAS SHAMATTAWAENSE FOERSTE\&SAVAGE

22563 [315S ] P-HOLO

ACTINOCERAS CF BIGSBYI BRONN. (PARKS, 1915)

O

LOWER RAPIDS, GODS R,MAN

K206;PARKS WA, 1915,P25,PL6,FIG 6;FOERSTE \&

SAVAGE, 1927,P62-63,PL7,FIG 2

REMARKS: C-JB TYRRELL, 1912 ;D-WH HEARST, 1914;LOC

GIVEN AS SHAMATTAWA R-NOW CALLED GODS R,

TRIBUTARY OF HAYES R; 1 SPEC (2 PCES)

\section{LYTOCERAS BATESII TRASK}

3588 REF SPEC-F

$\mathrm{K}$

COTTONWOOD CK,CALIF,USA

L194;WESTERMANN GEG, 197 1,LIFE SCI

CONTR,ROM,N78,P3,FIG 2

REMARKS: P-; 1 SPEC

\section{LYTOCERAS CORNUCOPIAE SOWERBY}

19083 [2136L ] REF SPEC-M

L J,TOARC

ILMINSTER,ENG

L194; WESTERMANN GEG, 1971,LIFE SCI

CONTR,ROM,N78,P3

REMARKS: P-STURTZ: 1 SPEC, 1 TS, 1 PEEL

MELOCERAS SP INDET

REMARKS: SEE OOCERINA SPINDET

\section{NAUTILOID OF UNKNOWN GENERIC AFFINITIES}

12910 [2300D] REF SPEC

MD,ERIAN

ABITIBI RIVER FM CORAL BEDS

GRAND RAPIDS, MATTAGAMI R,ONT

FOERSTE AF,ONT DEPT MINES, VOL

37,PT6, 1928,P75,PL2,FIG 4

REMARKS: C,D-WS DYER, $1927 ; 1$ SPEC

\section{OCTAMEROCERAS WALKERI FOERSTE\&SAVAGE}

22574 [327S ] P-HOLO

SEPTAMEROCERAS SP INDET (PARKS, 19 15)

[EKWAN OR ATTAWAPISKAT FM ]

LIMESTONE RAPIDS,BOULDER, SEVERN R,DIST OF

PATRICIA,ONT

K295:PARKS WA, 1915,P79;FOERSTE \&

SAVAGE, 1927,P 104,PL23,FIG 5A,B

REMARKS: C-JB TYRRELL, 1912 ;D-WH HEARST, $1914 ; 1$ SPEC

\section{OOCERAS CORDATUM PARKS}

REMARKS: SEE DUNLEITHOCERAS CORDATUM (PARKS)
OOCERAS SP

SEE CYRTORIZOCERAS SP OOCERINA SEVERNENSIS

FOERSTE + SAVAGE,OOCERINA SP, AND

PENTAMEROCERAS SP

OOCERINA SEVERNENSE FOERSTE\&SAVAGE

22577(A) [330S(A)] P-HOLO

OOCERAS ? SP NOV (PARKS, 1915)

$\mathrm{S}$

[ATTAWAPISKAT LS ?]

LIMESTONE RAPIDS SEVERN R DIST OF PATRICIA,ONT

K288;PARKS WA, 1915,P76,PL3,FIG 4,PL6,FIG 3;FOERSTE \& SAVAGE, 1927,P79-80,PL 14,FIG 2A-C

REMARKS: C-JB TYRRELL, 1912 ;D-WH HEARST, $1915 ; 1$ SPEC

OOCERINA SHAMATTAWAENSE FOERSTE\&SAVAGE 22580 (A) [333S(A)] P-HOLO

ORTHOCERAS SP INDET

O

LOWER RAPIDS,GODS R,MAN

K288;PARKS WA, 1915,P23;FOERSTE \& SAVAGE, 1927,P7879, PL 15, FIG 2A,B

REMARKS: C-JB TYRRELL, 1912 ;D-WH HEARST, 19 14;LOC GIVEN AS SHAMATTAWA R-NOW CALLED GODS R, TRIBUTARY OF HAYES R; 1 SPEC

OOCERINA SP

$22577(B)[330 S(B)]$ REF SPEC-F

OOCERAS ? SP (PARKS, 1915)

$\mathrm{S}$,

SEVERN R,DIST OF PATRICIA,ONT

K288;PARKS WA, 1915,P76,PL6,FIG 8;FOERSTE \&

SAVAGE, 1927,P80-81,PL 14,FIG 4

REMARKS: C-JB TYRRELL, 1912 ;D-WH HEARST, $1914 ; 1$ SPEC

\section{OOCERINA ? SPINDET}

22575 [328S] REF SPEC-F

MELOCERAS SP INDET (PARKS, 1915)

O

[SHAMATTAWA LS ]

LOWER RAPIDS GODS R MAN

K288;PARKS WA, 19 15,P29,PL2,FIG 5;FOERSTE \& SAVAGE, 1927,P79,PL 15,FIG 3A,B

REMARKS: C-JB TYRRELL, 1912 ;D-WH HEARST, 1914;CUT SPEC FIG'D; LOC GIVEN AS SHAMATTAWA R-NOW CALLED GODS R,TRIBUTARY OF HAYES R ; 2 SPEC (3 PCES)

\section{ORTHOCERAS ALGOMENSE PARKS}

25389 [867UH] P-HOLO

$M D$

KWATABOAHEGAN R,ONT

K224;PARKS WA,ONT DEPT MINES, VOL

13,1904,P 189,PL6,FIG 1,3

REMARKS: D-WA PARKS ; 1 SPEC

ORTHOCERAS DUSERI HALL\&WHITFIELD

17462 [1223HR] REF SPEC-F

UO,CINCI,MAYSVILL

DUNDAS FM

E SIDE, 10 FT BELOW BASE OF BIG SCARP, HUMBER

R,WESTON,ONT

K224;PARKS \& FRITZ,ONT DEPT MINES, VOL

31,PT9, 1922,P16-18,PL4,FIG 8

REMARKS: C-WA PARKS, $1921 ; 3$ PCES

ORTHOCERAS DUSERI HALL\&WHITFIELD

12903 REF SPEC-F

U O.CINCI,RICHMOND

RICHMOND GP. WAYNESVILLE FM

CLARKSVILLE,OHIO,USA

K224:PARKS \& FRITZ,ONT DEPT MINES, VOL

31,PT9, 1922,PL4,FIG 7

REMARKS: D-AF FOERSTE, 1921; 1 SPEC (3 PCES) 
ORTHOCERAS EXTREMUM PARKS

25387 [865UH ] P-HOLO

MD.

KWATABOAHEGAN R,ONT

K224;PARKS WA.ONT DEPT MINES, VOL

13, 1904,P 189,PL6.FIG 7

REMARKS: D-WAPARKS ; 1 SPEC

\section{ORTHOCERAS LAMELLOSUM HALL}

17472 [1233HR] REF SPEC-F

U O.CINCI,MAYSVILL

DUNDAS FM

DOUNDAS

PFISON FARM QUARRY MIMICO ONT

K224:PARKS \& FRITZ,ONT DEPT MINES, VOL

31,PT9, 1922, P 18-19,PL4,FIG 6

REMARKS: C-WA PARKS, $1920 ; 1$ SPEC

ORTHOCERAS LEPIDODENDROIDES PARKS

REMARK S: SEE PARKSOCERAS LEPIDODENDROIDES (PARKS)

ORTHOCERAS LUXUM (HALL)

12958 [2348D ] REF SPEC-F

$M D$.

KWATABOAHEGAN R,ONT

K224;PARKS WA,ONT DEPT MINES, VOL

13.PT 1, 1904,PL8,FIG 2

REMARKS: C-WA PARKS, $1903 ; 1$ SLAB

\section{ORTHOCERAS ONTARIOENSIS FOERSTE}

12911 [2301D ] P-HOLO

$M D, E R I A N$

ABITIBI RIVER FM

21 MIUPSTREAM,AT PRECAMB CONTACT, W

BRANCH,FRENCH R,ONT

K224,FOERSTE AF, ONT DEPT MINES, VOL 37,PT6, 1928,P70-

71.PL1,FIG 1A-C

REMARKS: C.D-WS DYER, $1927 ; 1$ SPEC

\section{ORTHOCERAS PULCHER PARKS}

25386 [864UH] P-HOLO

$M D$

KWATABOAHEGAN R,ONT

K224:PARKS WA, ONT DEPT MINES, VOL 13,PT6, 1904,P 189 190. PL6.FIG 2

REMARKS: D-WAPARKS ; 1 SPEC

\section{ORTHOCERAS SP INDET}

REMARK S: SEE OOCERINA SHAMATTAWAENSE

FOERSTE + SAVAGE AND CHICAGOOCERAS

LONGIDOMUM FOERSTE + SAVAGE

ORTHOCERAS CF THOAS (HALL)

25388 [866UH] REF SPEC-M

MD.

KWATABOAHEGAN R,ONT

K224:PARKS WA,ONT DEPT MINES, VOL 13,PT 1, 1904,P 189 REMARK S: C-WA PARKS ; 1 SPEC

\section{ORTHOCERAS SP}

12975 [2365D ] REF SPEC-F

MD

KWATABOAHEGAN R,ONT

K224:PARKS WA.ONT DEPT MINES, VOL

13.PT 1, 1904.P 189,PL6, FIG 4

REMARKS: C-WA PARKS, $1903 ; 1$ SPEC

PARKSOCERAS LEPIDODENDROIDES (PARKS)

22569 [321S ] P-HOLO

ORTHOCERAS LEPIDODENDROIDES PARKS, 1913

O

ORDOVICIAN, SHAMATTAWA LS ]

LOWER RAPIDS.GODS R.MAN

K488:PARKS WA, IN TYRRELL, 1913,P190;PARKS

WA 1915,P22-23,PL2,FIG 4:FOERSTE +

SAVAGE, 1927.P45-46.PL 18,FIG 3A.B

REMARKS: C-JB TYRRELL, 1912 ;D-WH HEARST, 19 15;LOC

GIVEN AS SHAMATTAWA R-NOW CALLED GODS R

TRIBUTARY OF HAYES R, 1 SPEC
PENTAMEROCERAS RARUM PARKS

22571 [324S ] P-HOLO

$\mathrm{S}$,

[EKWAN OR ATTAWAPISKAT LS:CF E2]

ABOVE LIMESTONE RAPIDS, SEVERN R,DIST OF

PATRICIA.ONT

K299;PARKS WA, 1915,P78-79,PL3,FIG 6;FOERSTE \&

SAVAGE, 1927,P 102-103,PL23,FIG 7A-C

REM ARK S: C-JB TYRRELL, 1912 ;D-WH HEARST, 1914 ; 1 SPEC

PENTAMEROCERAS ? SP

22583(A) [3365(A)] REF SPEC-F

OOCERAS SPINDET (PARKS, 1915)

S.

[EKWAN OR ATTAWAPISKAT FM]

LIMESTONE RAPIDS, SEVERN R,DIST OF PATRICIA,ONT

K299;PARKS WA, 1915,P76;FOERSTE \& SAVAGE, 1927,P103104.PL23,FIG 6A-C

REMARKS: C-JB TYRRELL, 1912 ;D-WH HEARST, $1914 ; 1$ SPEC

PHRAGMOCERAS LINEOLATUM WHITEAVES REMARKS: SEE PHRAGMOCERAS SP

PHRAGMOCERAS NELSONENSE PARKS 22564 [316S ] P-PARAL

MS

EKWAN RIVER FM

[EKWAN LS ]

NR MOUTH OF SEAL R, NELSON R,ONT

K338:PARKS WA, 19 15,P83-84,PL3,FIG 1-2; FOERSTE \&

SAVAGE, 1927,P93-94,PL 19,FIG 4-6

REMARK S: C-JB TYRRELL, $1912 ;$ D- 1915 ; LECTOTYPE

DESIGNATED BY FOERSTE \& SAVAGE; 3 SPEC

PHRAGMOCERAS NELSONENSE PARKS

22564(A) [316S(A)] P-LECTO

$\mathrm{MS}$

EKWAN RIVER FM

[EKWAN LS ]

NR MOUTH OF SEAL R, NELSON R,ONT

K338;PARKS WA, 1915,P83-84,PL1,FIG 7;FOERSTE \&

SAVAGE, 1927,P93-94.PL 19,FIG 3

REMARKS: C-JB TYRRELL, 1912; LECTOTYPE

DESIGNATED BY FOERSTE \& SAVAGE:D-WH HEARST, 1915; 1 SPEC

PHRAGMOCERAS WHITNEYI PARKS

22566 [318S ] P-SYN

MS,

EKWAN RIVER FM

[EKWAN LS]

LIMESTONE RAPIDS, SEVERN R,DIST OF PATRICIA,ONT

K338:PARKS WA 1915,P77.PL3,FIG 5;FOERSTE \&

SAVAGE, 1927,P99-100,PL22,FIG 1

REMARKS: C-JB TYRRELL, 1912 ;D-WH HEARST, $1914 ; 1$ SPEC

PHRAGMOCERAS WHITNEYI PARKS

22567 [319S ] P-SYN

$M S$

EKWAN RIVER FM

[EKWAN LS ]

ASSINA RAPIDS, SEVERN R,DIST OF PATRICIA,ONT

K338;PARKS WA, TRANS ROY CAN INST, VOL

11,PT 1, 1915,P77,PL6,FIG 2

REMARKS: C-JB TYRRELL, 1912 :D-WH HEARST 1914:1 SPEC

PHRAGMOCERAS SP

22565 [317S ] REF SPEC-F

PHRAGMOCERAS LINEOLATUM WHITEAVES (PARKS, 1915)

$M S$

[EKWAN OR ATTAWAPISKAT LS ]

LIMESTONE RAPIDS, SEVERN R.DIST OF PATRICIA ONT

K338:PARKS WA, 19 15.P78;FOERSTE \& SAVAGE, 1927,P9495,PL20,FIG 4

REMARKS: C-JB TYRRELL, 1912 :D-WH HEARST, 1914

LARGEST SPEC FIG'D : 3 SPEC 
PHYLLOCERAS HETEROPHYLLUM (SOWERBY)

18965 [2005L] REF SPEC-M

AMALTHEUS HETEROPHYLLUM SOWERBY

LJ,

[ULIASSIC]

WHITBY, YORKS,ENG

L187;WESTERMANN GEG, 1971,LIFE SCI

CONTR,ROM, N78,P3

REMARKS: OLD COLL ;IDENTIFICATION REVISED BY WESTERMANN ; 1 SPEC, 1 PEEL

POLYGRAMMOCERAS SP

$19212[2302 D]$ REF SPEC-F

MD,ERIAN

ABITIBI RIVER FM

50 MI UPSTREAM, KWATABOAHEGAN R,ONT

K230;FOERSTE AF,ONT DEPT MINES, VOL

37.PT6, 1928,P71,PL2,FIG 1

REMARKS: C,D-WS DYER, 1927 ; 1 SPEC

POTERIOCERAS CF SAURIDENS CLARKE\&RUEDEMANN 22456 [207S ] REF SPEC-F

$M D, E R I A N$

DETROIT RIVER GP. AMHERSTBURG FM

[U MONROE FM]

LIVINGSTONE CUT,DETROIT R, AMHERSTBURG,ONT

....:GRABAU AW,MICH GEOL \& BIOL SUR,PUB 2, GEOL SER

1,P198-199,PL29,FIG 4

REMARKS: D-T NATTRESS, $1910 ; 1$ SPEC

\section{POTERIOCERAS TYRRELLI PARKS}

REMARKS: SEE DIESTOCERAS TYRRELLI (PARKS)

PROBILLINGSITES PRIMUS (FRITZ)

25159 [749U] P-HOLO

BILLINGSITES PRIMUS FRITZ

MO,CHAMP,MOH,TRENT

TRENTON GP, U COBOURG FM

[U COBOURG]

E OF COLLINGWOOD,ONT

K276:PARKS WA, TRANS ROY SOC CAN VOL 22, SECT

4,1928,P85-86,PL1,FIG 8-10;FOERSTE AF, 1928,P3 18 REMARKS: C-MA FRITZ; 1 SPEC

SACTOCERAS TYPICALE

25160 [750U] P-SYN

U O,CINCI,EDEN

COLLINGWOOD FM

BETWEEN COLLINGWOOD \& CRAIGLEITH, NR

COLLINGWOOD,ONT

K211; PARKS WA, TRANS ROY SOC CAN, VOL 22,SECT

4,1928,P87-88,PL 1,FIG 1

REMARKS: C-MA FRITZ :LARGER SPEC FIG'D : 2 SPEC

\section{SACTOCERAS SP}

12913 [2303D] REF SPEC-F

$M D$ ERIAN

ABITIBI RIVER FM

GRAND RAPIDS, MATTAGAMI R,ONT

K211:FOERSTE AF ONT DEPT MINES, VOL

37,PT6, 1928,P72,PL2,FIG 5

REMARKS: C,D-WS DYER, $1927 ; 1$ SPEC

\section{SCAPHITES CF HIPPOCREPIS (DEKAY)}

26029 REF SPEC- $M$

UK,GULF

MONTANA GP PIERRE FM

BLACK HILLS,S DAK,USA

L228;WESTERMANN GEG, 1971, LIFE SCI

CONTR,ROM,N78,P3

REMARKS: P-WARD, $1943 ; 1$ SPEC, 2 PEELS

\section{SEPTAMEROCERAS SP INDET}

REMARKS: SEE OCTAMEROCERAS WALKERI

FOERSTE + SAVAGE
SPHYRADOCERAS CF CLIO HALL

12914 [2304D] REF SPEC-F

MD,ERIAN

ABITIBI RIVER FM

GRAND RAPIDS, MATTAGAMI R,ONT

K380;FOERSTE AF, ONT DEPT MINES, VOL 37,PT6, 1928,P74.

75. PL 1,FIG 2A,B

REMARKS: C,D-WS DYER, $1927 ; 1$ SPEC

TRIPLEUROCERAS BOREALE FOERSTE

12915 [2305D ] P-HOLO

$M D, E R I A N$

ABITIBI RIVER FM

OPP LETTER N IN 'FRENCH' ON MAP FRENCH R ONT

K302;FOERSTE AF,ONT DEPT MINES,VOL

37,PT6, 1928,P72,PL 1,FIG 3A-C

REMARKS: C,D-WS DYER, 1927 ;LOC-SEE MAP IN PUBL' N ; 1 SPEC

TROCHOCERAS ANDERDONENSE GRABAU 22498 [250S ] P-SYN

D.

DETROIT RIVER GP,AMHERSTBURG FM

[U MONROE FM]

LIVINGSTONE CUT,DETROIT R AMHERSTBURG, ONT

K422; GRABAU AW, MICH GEOL \& BIOL SUR,PUB 2, GEOL

SER 1,1910,P200,PL28,FIG 9,PI 29,FIG 5,6

REMARKS: D-T NATTRESS, 1914 ;LABELLED COTYPE ; 1 SPEC

\section{ZITTELOCERAS FIMBRIATUM FRITZ}

24192 [1029T] P-HOLO

MO,CHAMP,MOH,BLKRIV

BLACK RIVER GP

PAQUETTE'S RAPIDS OTTAWA ONT

K288;FRITZ MA, TRANS ROY CAN INST, VOL

17,PT2, 1930,P223-224,PL1,FIG 1,2

REMARKS: C-J TOWNSEND; 1 SPEC

\section{MOLLUSCA,MISCELLANEOUS}

DIPLOTHECA ACADICA (HARTT)

7876 [245CM ] REF SPEC-F

$\mathrm{MCA}$

ST JOHNGP

[ST JOHN, ACADIAN, DIV 1D]

PORTER'S BK, ST MARTINS, ST JOHN CO,NB

W 130;MATTHEW GF,TRANS ROY SOC CAN, VOL 3,SECT 4,1886, P54-55 PL6.FIG 6, 6A

REMARKS: C-GF MATTHEW:D-MACKENZIE :SPEC MARKED ' $X$ ' = FIG 6;2 SPECS MARKED 'A' MAY BE FROM HANFORD $\mathrm{BK} ; 6$ SPEC

DIPLOTHECA ACADICA VAR OBTUSUS MATTHEW

$7692[62 \mathrm{CM}]$ P-HOLO

$\mathrm{MCA}$,

ST JOHN GP

[ST JOHN, DIV 1C,P. ABENACUS SUBFAUNA HANFORD BK, ST MARTINS,ST JOHN CO,NB W130:MATTHEW GF, TRANS ROY SOC CAN, VOL 3,SECT

4.1886,P55,PL6,FIG 8

REMARKS: C-GFMATTHEW;D-MACKENZIE 1 SPEC

DIPLOTHECA ACADICA VAR SERICEA MATTHEW

7836 [206CM ] P.SYN

$\mathrm{MCA}$

ST JOHN GP

[ST JOHN,ACADIAN] 
PORTER'S BK, ST MARTINS.ST JOHN CO,NB

W130:MATTHEW GF,TRANS ROY SOC CAN,VOL 3,SECT 4,1886, P55,PL6,FIG 7,7A-8

REMARKS: C-GF MATTHEW:D-MACKENZIE;RECORDS

STATE 14 SPEC;LABELLED COTYPES;UNCERTAIN

WHICH SPECS FIG'D; 13 SPEC

DIPLOTHECA ACADICA VAR CRASSA MATTHEW

$7811[181 \mathrm{CM}]$ P-HOLO

CA.

ST JOHN GP

[ST JOHN,ACADIAN,DIV 182']

HANFORD BK, ST MARTINS.ST JOHN CO,NB

W 130:MATTHEW GF, TRANS ROY SOC CAN, VOL 3,SECT 4, 1886, P55-56,PL6, FIG 9

REMARKS: C-GF MATTHEW;D-MACKENZIE; 1 SPEC

DIPLOTHECA HYATTIANA MATTHEW

$8271[641 \mathrm{CM}]$ P-SYN

$\mathrm{MCA}$,

ST JÖHN GP

[ST JOHN, ACADIAN,DIV 1C]

HANFORD BK. ST MARTINS,ST JOHN CO,NB

W 130 ;MATTHEW GF, TRANS ROY SOC CAN, VOL 3,SECT

4, 1886, P52-53,PL6, FIG 4,4A

REMARKS: C-GF MATTHEW;D-MACKENZIE ; 2 SPEC

DIPLOTHECA HYATTIANA VAR CAUDATA MATTHEW

8273 [643CM] P-SYN

MCA,

ST JOHN GP

[ST JOHN, ACADIAN.DIV 1D]

PORTER'S BK, ST MARTINS, ST JOHN CO,NB

W130;MATTHEW GF, TRANS ROY SOC CAN, VOL 3,SECT 4.1886,P53-54,PL6, FIG 5,5A

REMARKS: C-GF MATTHEW;D-MACKENZIE ;LABELLED COTYPES:UNCERTAIN WHICH SPECS FIG'D; 10 SPEC

HELENIA GRANULATA MATTHEW

8344 [7 14CM] P-SYN

LCA.

ADEYTON GP,BONAVISTA FM

[ETCHEMINIAN]

SMITH SD,NFLD

W124:MATTHEW GF,8ULL NAT HIST SOC NB,VOL 4.1899,P192,PL2,FIG 7

REMARKS: C-GF MATTHEW:D-MACKENZIE :LABELLED COTYPES ; UNCERTAIN WHICH SPECS FIG'D;STRAT REV AS IN HUTCHINSON, 1962,GSC BULL 88; 2 SPEC

\section{HYOLITHES CARINATUS MATTHEW}

8463 [833CM] P-SYN

MCA.

MT STEVEN,BC

W124:MATTHEW GF, TRANS ROY SOC CAN, VOL 5,SECT 4, 1899,P 42,PL 1,FIG 5A-B

REMARKS: C-GF MATTHEW-D-MACKENZIE - LABELLED COTYPES;UNCERTAIN WHICH SPEC FIG'D ; 5 SPEC

HYOLITHES DANIANUM WALCOTT

7826 [196CM] REF SPEC?

HYOLITHES (CAMEROTHECA) DANIANUM WALCOTT

$M C A$.

ST JOHN GP,DIV ID2

[ST JOHN, ACADIAN]

PORTER'S BK, ST MARTINS ST JOHNCO,NB

W 124;MATTHEW GF.TRANS ROY SOC CAN, VOL 3,SECT 4.1886,P49-50,PL6, FIG 1,1A-C

REMARKS: C-GF MATTHEW:D-MACKENZIE ;LISTED AS COTYPES IN FRIT'S TYPE CATALOGUE ; 6 SPEC

HYOLITHES DECIPIENS MATTHEW

$7800[170 \mathrm{CM}]$ P-SYN

LCA.

ZST JOHN GP

[ST JOHN, ACADIAN,DIV 182]

HANFORD BK, ST MARTINS.ST JOHN CO NB

W 124:MATTHEW GF, TRANS ROY SOC CAN, VOL 11,SECT 4.1894,P96,PL 16. FIG 11A-D

REMARKS: C-GF MATTHEW:D-MACKENZIE ; SPEC ' $A$ ' = FIG

$11 \mathrm{D} ; \mathrm{SPEC}^{\prime} \mathrm{B}$ ' = FIG $11 \mathrm{~A}-\mathrm{C} ; 3$ SPEC
HYOLITHES GRACILIS MATTHEW

7829[199CM ]P-SYN

HYOLITHES (CAMEROTHECA) GRACILIS MATTHEW

$M C A$,

ST JOHN GP

[ST JOHN ACADIAN DIV 1D]

PORTER'S BK, ST MARTINS,ST JOHN CO,NB

W124:MATTHEW GF, TRANS ROY SOC CAN, VOL 3,SECT 4, 1886,P50-51,PL6,FIG 2,2A-C

REMARKS: C-GF MATTHEW:D-MACKENZIE ;SPEC ' $A$ ' $=$ FIG $2,{ }^{\prime} \mathrm{B}^{\prime}=\mathrm{FIG} 2 \mathrm{~B},{ }^{\prime} \mathrm{C}^{\prime}+{ }^{\prime} \mathrm{G}^{\prime}=2 \mathrm{~A} ; 8$ SPEC

HYOLITHES GRACILIS MUT GRACILLIMUS MATTHEW

7870 [240CM ] P-SYN

$\mathrm{MCA}$,

ST JOHN GP

[ST JOHN, ACADIAN,DIV 1C]

GILFORD ST, ST JOHN, NB

W124;MATTHEW GF, TRANS ROY SOC CAN, VOL 7,SECT

4, 1901,P109-110,FIG 3

REMARKS: C-GF MATTHEW;D-MACKENZIE ; 4 SPEC

HYOLITHES MICMAC MATTHEW

8247 [617CM ] P-SYN

HYOLITHES (CAMEROTHECA) MICMAC MATTHEW

$\mathrm{MCA}$,

ST JOHN GP.DIV $1 \mathrm{C}$

HANFORD BK, ST MARTINS,ST JOHN CO,NB

W124; MATTHEW GF, TRANS ROY SOC CAN,VOL 3,SECT 4,1886,P51,PL6,FIG 3,3A

REMARKS: C-GF MATTHEW;D-MACKENZIE ;SPEC ' $A$ ' = FIG 3; LABELLED COTYPES ; 9 SPEC

HYOLITHES RUGOSUS MATTHEW

$8121[491 \mathrm{CM}]$ P-SYN

LCA.

ADEYTONGP,BONAVISTA FM

[UETCHEMINIAN]

SMITH SD, NFLD

W124;MATTHEW GF,BULL NAT HIST SOC NB,VOL 4, 1899,P194,PL3,FIG 4A-8

REMARKS: C-GF MATTHEW;D-MACKENZIE ;LABELLED HOLOTYPE;STRAT REV AS IN HUTCHINSON, 1962,GSC BULL $88 ; 1$ SPEC

ORTHOTHECA BAYONET MATTHEW

7797 [167CM ] P-SYN

LCA,

ADEYTON GP,BONAVISTA FM

[U ETCHEMINIAN]

FOSTER'S PT, SMITH SD,NFLD

W125;MATTHEW GF,BULL NAT HIST SOC NB,VOL 4, 1899,P193-194,PL3,FIG 1A-F

REMARKS: C-GF MATTHEW;D-MACKENZIE ;LABELLED COTYPES:STRAT REV AS IN HUTCHINSON, 1962, GSC BULL 88;6 SPEC

ORTHOTHECA CORRUGATA MATTHEW

8596 [966CM] P-HOLO

MCA

STEPHEN FM

MT STEPHEN $B C$

W125;MATTHEW GF, 1899,TRANS ROY SOC CAN, VOL 5,SECT 4,P42,PL 1,FIG 3

REMARKS: D-BE WALKER ; 1 SPEC

ORTHOTHECA PUGIO MATTHEW

$8363[733 \mathrm{CM}]$ P-SYN

LCA.

ADEYTONGP,BONAVISTA FM

[U ETCHEMINIAN]

SMITH SD,NFLD

W125;MATTHEW GF.8ULL NAT HIST SOC NB,VOL 4, 1899,P 193,PL2,FIG 4A-D

REMARKS: C-GF MATTHEW;D-MACKENZIE;LABELLED COTYPES;STRAT REV AS IN HUTCHINSON, 1962, GSC BULL $88 ; 8$ SPEC 
ORTHOTHECA SICA MATTHEW

8107 [477CM] P-SYN

LCA,

ADEYTON GP.BONAVISTA FM

[U ETCHEMINIAN]

SMITH SD,NFLD

W 125;MATTHEW GF.BULL NAT HIST SOC NB,VOL 4.1899,P193,PL2,FIG 5A-E

REMARKS: C-GF MATTHEW;D-MACKENZIE ;LABELLED COTYPES:STRAT REV AS IN HUTCHINSON, 1962,GSC BULL 88:SPEC 'B' = FIG 5E; 12 SPEC

ORTHOTHECA SICA MUT MATTHEW

26015 REF SPEC-F

LCA,

ADEYTON GP,BONAVISTA FM

[UETCHEMINIAN]

SMITH SD, NFLD

W125:MATTHEW GF.BULL NAT HIST SOC NB, VOL 4.1899.P 193,PL2,FIG 6A-B

REMARKS: C-GF MATTHEW;D-MACKENZIE ;LABELLED COTYPES:NEW TAXON NOT NAMED:STRAT REV AS IN HUTCHINSON, 1962, GSC BULL $88 ; 6$ SPEC

\section{ORTHOTHECA STILETTO MATTHEW}

$8280[650 \mathrm{CM}]$ P-SYN

LCA

ADEYTON GP,BONAVISTAFM

[UETCHEMINIAN]

FOSTER'S PT , SMITH SD,NFLD

W125; MATTHEW GF, BULL NAT HIST SOC NB,VOL

4,1899.P 193,PL2,FIG 3A-B

REMARKS: C-GFMATTHEW;D-MACKENZIE ;LABELLED COTYPES;STRAT REV AS IN HUTCHSINON, 1962, GSC BULL $88 ; 3$ SPEC

RIBEIRIA CALCIFERA BILLINGS

442 [13CAL] REF SPEC-F

LO,CANAD

BEEKMANTOWN GP

[CALCIFEROUS,BEEKMANTOWN]

LOTS 17 18, MARLBOROUGH TP,CARLETON CO,ONT

POJETA \& RUNNEGAR, 1976, USGS PROF PAP

968,P51,PL4,FIG 13

REMARKS: C-J TOWNSEND, 1906 ;D-W BILLINGS ; 1 SPEC

RIBEIRINA LONGIUSCULA(BILLINGS)

455 [26CAL] REF SPEC-F

LO.CANAD

BEEKMANTOWNGP

[CALCIFEROUS,BEEKMANTOWN]

LOT 20,CON 7, MARLBOROUGH TP,CARLETON CO,ONT

POJETA \& RUNNEGAR, 1976,USGS PROF PAP

966,P55,PL3,FIG 19,20

REMARKS: C-J TOWNSEND, $1909 ; 1$ SPEC

STYLIOLA PRIMAEVA MATTHEW

$7964[334 \mathrm{CM}]$ P-SYN

L

ST JOHN GP

[ST JOHN,BRETONIAN,DIV 3D ]

SUSPENSION BRIDGE, ST JOHN NB

W110;MATTHEW GF, TRANS ROY SOC CAN,VOL 10,SECT 4, 1893,P 104,PL7,FIG 10A-B

REMARKS: C-GF MATTHEW:D-MACKENZIE :LABELLED HOLOTYPE; 1 SPEC

\section{ARTHROPODA,TRILOBITA}

\section{ACANTHOLENUS SPINIGER (MATTHEW)}

7967 [337CM]P-SYN?

LEPTOPLASTUS SPINIGER MATTHEW

UCA ORLO

[BRETONIAN L ORD DIV 2]

LONG IS, KINGS CO,NB

O000:MATTHEW GF, 1889,P487,FIG 2A-D;

MATTHEW, 1892,P61-62,PL 13,FIG 4A-E:MATTHEW
1899,P 142-143,PL2,FIG 4A-E

REMARKS: C-GF MATTHEW:D-MACKENZIE :LABELLED

COTYPES;UNCERTAIN WHICH SPECS FIG'D; 18 SPEC

ACROLICHAS CUCULLUS OTTAWAENSIS FOERSTE

18791 P-SYN

MO.CHAMP, MOH,TRENT

TRENTON GP

[TRENTON]

HULL, QUE

O498;FOERSTE AF, 1919,BULL DEN UNIV SCI LAB,VOL 19,P73,PL17,FIG 4A,B;FOERSTE AF, 1920,JOUR

SCI,PL1,FIG 1A,B,E

REMARKS: P-JE NARRAWAY COLL, $1938 ; 2$ SPEC

\section{ACROLICHAS NARRAWAYI FOERSTE}

18793 P-HOLO

MO.CHAMP,MOH,TRENT

TRENTON GP

[TRENTON]

WRIGHT'S QUARRY NR HULL, QUE

O498;FOERSTE AF,AM JOUR SCI,VOL

49,N289, 1920,P31,PL 1,FIG 2

REMARKS: P-JE NARRAWAY COLLN, 1938; 1 SPEC

\section{AGNOSTUS ACADICUS HARTT}

8117 [487CM] REF SPEC-F

$\mathrm{MCA}$,

ST JOHN GP

[ST JOHN,ACADIAN,DIV 1C]

SEELY ST,ST JOHN,NB

0172:MATTHEW GF, TRANS ROY SOC CAN, VOL 3,SECT

4,1886,P70,PL7,FIG 5A,B

REMARKS: C-GF MATTHEW;D-MACKENZIE ; 2 SPEC

\section{AGNOSTUS ACADICUS VAR DECLIVIS MATTHEW}

8324 [694CM] P-SYN

$M C A$

ST JOHN GP

[ST JOHN,ACADIAN, DIV $1 C$ ]

PORTER'S BK, ST MARTINS,ST JOHN CO,NB

O 172; MATTHEW GF, TRANS ROY SOC CAN.VOL 3,SECT 4.1886,P70-71,PL7,FIG 6A,B

REMARKS: C-GF MATTHEW:D-MACKENZIE :LABELLED

COTYPES;UNCERTAIN WHICH SPECS FIG'D; 12 SPEC

\section{AGNOSTUS BISECTUS MATTHEW}

7977 [347CM ] P-SYN

UCA,

[BRETONIAN,L ORDOVICIAN,DIV 3B]

NAVY IS.ST JOHN NB

O172; MATTHEW GF, TRANS ROY SOC CAN, VOL 9,SECT 4,1891,P50-51,PL13,FIG 2A,B

REMARKS: C-GF MATTHEW:D-MACKENZIE :LABELLED COTYPES:UNCERTAIN WHICH SPECS FIG'D : 4 SPEC

\section{AGNOSTUS FALLAX VAR VIR MATTHEW}

26213 P-HOLO

AGNOSTUS VIR MATTHEW

MCA.

ST JOHN GP

[ST JOHN, ACADIAN,DIV 1C1]

ST JOHN NB

O 172;MATTHEW GF, 1886,P69-70,PL7,FIG 3;MATTHEW

GF, TRANS NY ACAD SCI,VOL 15, 1896,P215-216,PL 15,FIG

REMARKS: C-GF MATTHEW:D-MACKENZIE ; 1 SPEC

\section{AGNOSTUS FALLAX VAR CONCINNUS MATTHEW}

8302 [672CM ] P-SYN

AGNOSTUS VIR VAR CONCINNUS MATTHEW

$M C A$.

ST JOHN GP

[ST JOHN ACADIAN DIV 1D1]

PORTER'S BK. STMARTINS, ST JOHN CO,NB

C.172:MATTHEW GF, TRANS NY ACAD SCI, VOL

15, 1896,P216,PL 15,FIG 7A-C;MATTHEW

GF, 1886,P70,PL7,FIG 4A-C

REMARKS: C-GF MATTHEW:D-MACKENZIE :LABELLED

COTYPES;UNCERTAIN WHICH SPECS FIG'D ; 14 SPEC 
AGNOSTUS FALLAX VAR TRILOBATA MATTHEW

7843 [2 13CM ]P-SYN

AGNOSTUS PARTITUS MATTHEW

$M C A$.

ST JOHN GP

[ST JOHN,ACADIAN,DIV 1C1]

HANFORD BK,ST MARTINS AND SEELY ST,ST JOHN, ST

JOHN CONB

O 172;MATTHEW GF, 1886,P68,PL7,FIG 2B;MATTHEW GF, TRANS NY ACAD SCI,VOL 15, 1896,P216-217,PL 15, FIG 9

REMARKS: C-GF MATTHEW;D-MACKENZIE ;LABELLED COTYPES ; 4 SPEC

AGNOSTUS FISSUS VAR TRIFISSUS MATTHEW

8329 [699CM ] P-SYN

$\mathrm{MCA}$.

ST JOHN GP

[ST JOHN,ACADIAN,DIV 1D1]

PORTER'S BK, ST MARTINS, ST JOHN CO,NB

0172:MATTHEW GF,TRANS NY ACAD SCI,VOL 15, 1896,P231-232,PL 16,FIG 10

REMARKS: C-GF MATTHEW:D-MACKENZIE :LABELLED COTYPES;FIG'D SPEC IS MARKED ; 9 SPEC

\section{AGNOSTUS FISSUS VAR TRIFISSUS MATTHEW}

26104 P-SYN

$M C A$,

ST JOHN GP

[ST JOHN,ACADIAN,DIV 1D]

CHAPEL ARM, TRINITY BAY, NFLD

0172; MATTHEW GF,TRANS NY ACAD SCI,VOL 15,1896, P23 1-232

REMARKS: C-GF MATTHEW;D-MACKENZIE ; LABELLED COTYPES;THESE SPECS NOT FIG'D ; 3 SPEC

\section{AGNOSTUS GIBBUS VAR PARTITUS MATTHEW}

8167 [537CM] P-SYN

$\mathrm{MCA}$.

ST JOHN GP

[ST JOHN,ACADIAN,DIV 1C1]

PORTER'S BK, ST MARTINS.ST JOHN CO,NB

0172; MATTHEW GF, TRANS NY ACAD SCI,VOL

15, 1896, P227,PL 16, FIG 7

REMARKS: C-GF MATTHEW:D-MACKENZIE ;SPEC 'C' FIG'D 9 SPEC

\section{AGNOSTUS GIBBUS VAR ACUTILOBUS MATTHEW}

$8331[701 \mathrm{CM}]$ P-SYN

MCA.

ST JOHN GP

[ST JOHN,ACADIAN,DIV 1D1]

PORTER'S BK , ST MARTINS, ST JOHN CO NB

0172; MATTHEW GF, TRANS NY ACAD SCI, VOL 15, 1896,P227-228,PL 16, FIG 8

REMARKS: C-GF MATTHEW:D-MACKENZIE :LABELLED

COTYPES;ONE SPEC LABELLED 'FIG 10'-POSSIBLY

SHOULDBE 'FIG 8' AS FIG 10 IS A. FISSUS: 10 SPEC

\section{AGNOSTUS LAEVIGATUS VAR MAMILLA MATTHEW}

27293 P-SYN

$\mathrm{MCA}$

ST JOHN GP

[ST JOHN ACADIAN,DIV 1D2]

HIGHLAND COVE, TRINITY BAY, NFLD

0172; MATTHEW GF,TRANS NY ACAD SCI,VOL

15, 1896 P234-235 PL17 FIG 2B

REMARKS: C-GF MATTHEW:D-MACKENZIE ; 1 SPEC

\section{AGNOSTUS LAEVIGATUS VAR TERRANOVICUS $M$.}

7930 [300CM] P-SYN

$M C A$,

ST JOHN GP

[ST JOHN,ACADIAN,DIV 1D]

HIGHLAND COVE, TRINITY BAY,NFLD

O172;MATTHEW GF, NY, 1896,P233-234,PL17,FIG 2A,B: HUTCHINSON RD.GSC BULL 88, 1962,P85-86

REMARKS: C-GF MATTHEW:D-MACKENZIE :SPECS MENTIONED BY HUTCHINSON AS HYPOTYPES OF PTYCHAGNOSTUS CICEROIDES (MATTHEW) ; 2 SPEC
AGNOSTUS LAEVIGATUS VAR CICEROIDES MATT. REMARKS: SEE PTYCHAGNOSTUS CICEROIDES (MATTHEW)

AGNOSTUS MONTIS MATTHEW

7981 [35 1CM] P-HOLO

MCA,

MT STEPHEN,FIELD,BC

O 172; MATTHEW GF, TRANS ROY SOC CAN,VOL 5,SECT 4.1899,P43-44,PL 1,FIG 6

REMARKS: C-GF MATTHEW:D-MACKENZIE ; 1 SPEC

\section{AGNOSTUS NATHORSTI VAR CONFLUENS MATTHEW}

V26 105 P-SYN

$M C A$,

ST JOHN GP

[ST JOHN, ACADIAN]

PORTER'S BK, ST MARTINS,ST JOHN GP.NB

O172:MATTHEW GF. TRANS NY ACAD SCI,VOL

15, 1896,P230,PL6,FIG 9A,B

REMARKS: C-GF MATTHEW;D-MACKENZIE ;LABELLED COTYPES ; 7 SPEC

? AGNOSTUS NUDUS BEYRICH

8200 [570CM ] REF SPEC-M

$\mathrm{MCA}$.

ST JOHN GP

[ST JOHN,ACADIAN,DIV 1D]

CHAPEL ARM, TRINITY BAY,NFLD

O172:MATTHEW GF, TRANS NY ACAD SCI, VOL 15,1896, P235

REMARKS: C-GF MATTHEW;D-MACKENZIE ;LABELLED PLESIOTYPE;NOT FIG'D ; 1 SPEC

AGNOSTUS OBTUSILOBUS MATTHEW

7839 [209CM ] P-SYN

$\mathrm{MCA}$,

ST JOHN GP

[ST JOHN,ACADIAN]

PORTER'S BK, ST MARTINS,ST JOHN CO,NB

O 172:MATTHEW GF, TRANS ROY SOC CAN,VOL 3,SECT 4,1886, P72-73,PL7,FIG 9

REMARKS: C-GF MATTHEW;D-MACKENZIE :LABELLED COTYPES;UNCERTAIN WHICH SPEC FIG'D ; 4 SPEC

AGNOSTUS PARTITUS MATTHEW

REMARKS: SEE AGNOSTUS FALLAX VAR TRILOBATA MATTHEW

AGNOSTUS PARVIFRONS VAR TESSELLA MATTHEW

7844 [214CM] P-SYN

$\mathrm{MCA}$

ST JOHN GP

[ST JOHN ACADIAN DIV 10 ]

PORTER'S BK ST MARTINS.ST JOHN CO NB

O172;MATTHEW GF, TRANS NY ACAD SCI,VOL 15, 1896, P221-222, PL 16,FIG 3A-C

REMARKS: C-GF MATTHEW;D-MACKENZIE ;LABELLED COTYPES;UNCERTAIN WHICH SPECS FIG'D; 30 SPEC

AGNOSTUS PARVIFRONS VAR TRUNCATUS MATTHEW $8338[708 \mathrm{CM}]$ P-SYN

$M C A$

[ACADIAN,DIV 1D]

PORTER'S BK ST MARTINS, ST JOHN CONB

O172:MATTHEW GF, TRANS NY ACAD SCI, VOL $15,1896, P 222$

REMARKS: C-GF MATTHEW:D-MACKENZIE :LABELLED PLESIOTYPES:NOT FIG'D ; 11 SPEC

AGNOSTUS PISIFORMIS VAR A MATTHEW

8366 [736CM] REF SPEC-F

$\mathrm{UCA}$

[BRETONIAN,DIV 3A, L ORDOVICIAN]

LONG IS, KINGS CO,NB

O172:MATTHEW GF, TRANS ROY SOC CAN, VOL 4.SECT 4.1899.P 136-137. PL2.FIG 1A-C

REMARKS: C-GF MATTHEW;D-MACKENZIE :LABELLED COTYPES : 1 SLAB 
AGNOSTUS PISIFORMIS VAR VALIDA MATTHEW

$8365[735 \mathrm{CM}]$ P-SYN

UCA

[BRETONIAN,DIV 3A,L ORDOVICIAN]

LONG IS, KINGS CO,NB

O 172: MATTHEW GF, TRANS ROY SOC CAN, VOL 4,SECT 4.1899,P $137-138$

REMARKS: C-GF MATTHEW;D-MACKENZIE ;LABELLED COTYPES ; 8 SPEC

AGNOSTUS PISIFORMIS MUT AFFINIS MATTHEW

$8364[734 \mathrm{CM}]$ P-SYN

UCA

[BRETONIAN,DIV 3A,L ORDOVICIAN]

LONGIS,KINGS CO,NB

O172; MATTHEW GF, TRANS ROY SOC CAN, VOL 4,SECT 4, 1899,P137,PL2,FIG 3

REM ARKS: C-GF MATTHEW:D-MACKENZIE ;LABELLED COTYPES ; 15 SPEC

\section{AGNOSTUS PISIFORMIS MUT RUGULOSUS MATTHEW}

$8400[770 \mathrm{CM}]$ P.SYN

UCA,

[BRETONIAN,L ORDOVICIAN]

LONG IS, KINGS CO,NB

O172;MATTHEW GF,TRANS ROY SOC CAN,VOL 4,SECT 4,1899,P 137,PL2,FIG 2

REMARKS: C-GF MATTHEW;D-MACKENZIE ;LABELLED COTYPES;OLD RECORDS STATE 5 PCES ; 4 SPEC

\section{AGNOSTUS REGULUS MATTHEW}

8323 [693CM ] P-SYN

$\mathrm{MCA}$

ST JOHN GP

[ST JOHN,ACADIAN,DIV 1C]

HANFORD BK, ST MARTINS,ST JOHN CO,NB

O 172; MATTHEW GF, TRANS ROY SOC CAN, VOL 3, SECT 4, 1886,P67-68,PL7,FIG 1A-C

REMARKS: C-GF MATTHEW;D-MACKENZIE ; 5 SPEC

\section{AGNOSTUS REX VAR TRANSECTUM MATTHEW}

8484 [854CM] P-HOLO

MCA,

[DIV 1D,ACADIAN]

PORTER'S BK, ST MARTINS,ST JOHN CO,NB

0172; MATTHEW GF, TRANS NY ACAD SCI,VOL

15,1896, P2 14, PL 16, FIG 2

REMARKS: C-GF MATTHEW;D-MACKENZIE ; 1 SPEC

\section{AGNOSTUS UMBO MATTHEW}

7848 [218CM] P-SYN

$\mathrm{MCA}$

ST JOHN GP

[ST JOHN, ACADIAN,DIV 1D]

PORTER'S BK, ST MARTINS,ST JOHN CO,NB

O 172; MATTHEW GF, TRANS ROY SOC CAN, VOL 3,SECT 4,1886,P71-72,PL7,FIG 8A,B

REMARKS: C-GF MATTHEW:D-MACKENZIE ;LABELLED COTYPES;UNCERTAIN WHICH SPECS FIG'D ; 7 SPEC

AGNOSTUS VIR MATTHEW

REMARKS: SEE AGNOSTUS FALLAX VAR VIR MATTHEW

AGNOSTUS VIR VAR CONCINNUS MATTHEW

REMARKS: SEE AGNOSTUS FALLAXVAR CONCINNUS MATTHEW

AGRAULOS ARIELI MATTHEW

$8116[486 C M]$ P-SYN ?

MCA

ST JOHN GP

[ST JOHN,ACADIAN,DIV 1D3 ]

HASTINGS COVE,KINGS CO,NB

REMARKS: C-GF MATTHEW:D-MACKENZIE ;LABELLED COTYPES:REF NOT KNOWN ; 4 SPEC
AGRAULOS CETICEPHALUS VAR CARINATUS MATT.

7845 [215CM ] P-SYN

$\mathrm{MCA}$

ST JOHN GP

[ST JOHN, ACADIAN,DIV 1D3

HASTINGS COVE, KINGS CO,NB

O278; MATTHEW GF, TRANS ROY SOC CAN, VOL 3,SECT 4,1897,P176,PL2,FIG 2A,B

REMARKS: C-GF MATTHEW;D-MACKENZIE ;LABELLED COTYPES ; 2 SPEC

AGRAULOS SOCIALIS BILLINGS 8185 [555CM ] REF SPEC-F

MCA,

ST JOHN GP

[ST JOHN ACADIAN DIV 1C ORD]

CONCEPTION BAY NFLD

O278;MATTHEW GF, TRANS ROY SOC CAN, VOL 4,SECT 4,1887,P151-153,FIG 1A-C

REMARKS: C-GF MATTHEW:D-MACKENZIE :LABELLED PLESIOTYPES IN OLD RECORDS;NO SPECS COMPLETE 3 SPEC

AGRAULOS ? ARCTICEPHALUS MATTHEW

REMARKS: SEE BERGERONIA ARCTICEPHALA (MATTHEW)

AGRAULOS ? HOLOCEPHALUS MATTHEW

$8114[484 \mathrm{CM}]$ P-SYN

$\mathrm{MCA}$,

ST JOHN GP

[ST JOHN,ACADIAN,DIV 1D3

HASTINGS COVE,KINGS CO,NB

O278:MATTHEW GF TRANS ROY SOC CAN, VOL 8 SECT 4,1891,P138-139,PL11,FIG 5A-D

REMARKS: C-GF MATTHEW;D-MACKENZIE ;LABELLED COTYPES;PUBL'N GIVES LOC AS TORRYBURN COVE ; 3 SPEC

AGRAULOS? NANUS MATTHEW

8113 [483CM] P-SYN

$\mathrm{MCA}$

ST JOHN GP

[ST JOHN,ACADIAN,DIV 1D3

HASTINGS COVE, KINGS CO,NB

O278:MATTHEW GF, TRANS ROY SOC CAN, VOL 3,SECT 4,1897,P 176,PL2, FIG 2A,B

REMARKS: C-GF MATTHEW;D-MACKENZIE;LABELLED COTYPES : 4 SPEC

AGRAULOS ? PUSILLUS MATTHEW

$8115[485 \mathrm{CM}]$ P-SYN

$\mathrm{MCA}$,

ST JOHN GP

[ST JOHN,ACADIAN,DIV 1D3

HASTINGS COVE, KINGS CO,NB

O278:MATTHEW GF TRANS ROY SOC CAN VOL 3 SECT 4,1897,P178-179,PL2,FIG 6A,B

REMARKS: C-GF MATTHEW;D-MACKENZIE ;LABELLED COTYPES : 2 SPEC

AGRAULOS? WHITFIELDIANUS MATTHEW

7838 [208CM] P-SYN

$\mathrm{MCA}$,

ST JOHN GP

[ST JOHN, ACADIAN,DIV $1 \mathrm{C}$

PORTER'S BK, ST MARTINS,ST JOHN CO,NB

O278:MATTHEW GF, TRANS ROY SOC CAN, VOL 5, SECT 4, 1888,P 130-131,PL1,FIG 1A-F

REMARKS: C-GF MATTHEW:D-MACKENZIE ;LABELLED COTYPES : 4 SPEC

ANOMOCARE MAGNUM BROGGER? VAR

8128 [498CM] REF SPEC-F

$\mathrm{MCA}$,

ST JOHH GP

[ST JOHN, ACADIAN,DIV 1D3 ]

HASTINGS COVE,KINGS CO,NB

O286:MATTHEW GF, TRANS ROY SOC CAN, VOL 3,SECT 4,1897,P184,PL3,FIG 5A-E

REMARKS: C-GF MATTHEW:D-MACKENZIE;LABELLED COTYPES;UNCERTAIN WHICH SPECS FIG'D; 13 SPEC 
ANOMOCARE STENOTOIDES (MATTHEW)

$8353[723 \mathrm{CM}]$ P-SYN

LEPTOPLASTUS STENOTOIDES MATTHEW

UCA

[BRETONIAN,DIV 3A,L ORDOVICIAN]

LONG IS. KINGS CONB

O286;MATTHEW GF, 1889,P486-487;MATTHEW, 1892,P61; MATTHEW, 1898,P139-141

REMARKS: C-GF MATTHEW:D-MACKENZIE :LABELLED COTYPES;THESE SPECS NOT FIG'D;SEE NO 26 109; 42 SPEC

\section{ANOMOCARE STENOTOIDES (MATTHEW)}

26109 P-SYN

LEPTOPLASTUS STENOTOIDES MATTHEW

UCA.

[BRETONIAN,DIV 3A,L ORDOVICIAN]

LONG IS. KINGS CO.NB

O286:MATTHEW GF, 1889,P486-487,FIG 2A-D;

MATTHEW, 1892,P61,PL 13,FIG 3A-D

MATTHEW 1899.P 139-141.PL2,FIG 5A-I

REMARKS: C-GF MATTHEW:D-MACKENZIE ; 9 SPEC

ASAPHELLUS HOMFRAYISALTER VAR

$8012[382 C M]$ REF SPEC-F

LO

MCLEOD BKFM

[BRETONIAN,DIV 3C2, L ORDOVICIAN]

MCLEOD BK, BOISDALE CAPE BRETON, NS

0346; MATTHEW GF,BULL NAT HIST SOC NB,VOL

4. 1902,P4 13-4 18,PL 18,FIG 10A-E

REMARKS: C-GF MATTHEW;D-MACKENZIE ;LABELLED

COTYPES;STRAT REV BY AJ ROWELL, $1972 ; 4$ SPEC

ASAPHELLUS? PLANUS MATTHEW

7951 [32 1CM ] P-SYN

L O.

MCLEOD BROOK FM

[BRETONIAN, DIV 3C2, L ORDOVICIAN]

MCLEOD BK, BOISDALE,CAPE BRETON,NS

O346; MATTHEW GF,BULL NAT HIST SOC NB,VOL 4, 1902,P419-420,PL18,FIG 11

REMARKS: C-GF MATTHEW:D-MACKENZIE :LABELLED COTYPES;STRAT REV BY AJ ROWELL, $1972 ; 4$ SPEC

AVALONIA ACADICA MATTHEW

$7813[183 \mathrm{CM}]$ P-HOLO

$\mathrm{MCA}$

ST JOHN GP

[ST JOHN,ACADIAN]

HANFORD BK ST MARTINS ST JOHN CO NB

O5 12; MATTHEW GF,TRANS NY ACAD SCI,VOL 14, 1895, P 140-141, PL9,FIG 5

REMARKS: C-GF MATTHEW:D-MACKENZIE ; 1 SPEC

AVALONIA PLANA MATTHEW

7763 [ $133 \mathrm{CM}]$ P-HOLO

CA

[UETCHEMINIAN

MANUEL'S BK, CONCEPTION BAY,NFLD

O5 12:MATTHEW GF, TRANS ROY SOC CAN, VOL 5, SECT

4, 1899, P81-82, PL4,FIG

REMARKS: C-GF MATTHEW;D-MACKENZIE ; 1 SPEC

BASILICUS ROMINGERI (WALCOTT)

18695 REF SPEC-F

MO.CHAMP MOH,BLKRIV

BLACK RIVER GP

[BLACK RIVER]

MECHANICSVILLE,ONT

O336:RAYMOND \& NARRAWAY,ANN CARNEGIE MUS,VOL

7.N1, 19 10.P49-50.PL15,FIG 9

REMARKS: P-JE NARRAWAY COLLN, 1938 ; 1 SPEC

BATHYURUS EXTANS (HALL)

18755 REF SPEC-F

M O.CHAMP.MOH,BLKRIV

BLACK RIVER GP

[BIRDS EYE.BLACK RIVER]

MECHANICSVILLE,ONT
O376:RAYMOND \& NARRAWAY,ANN CARNEGIE MUS.VOL 7.N1, 1910,P46-47,PL15, FIG 8

REMARK S: P-JE NARRAWAY COLLN, 1938 ;LARGER SPEC FIG'D; 2 SPEC

BATHYURUS SUPERBUS RAYMOND

18788 REF SPEC-F

MO.CHAMP,MOH,BLKRIV

BLACK RIVER GP.PAMELIA FM

[PAMELIA,BLACK RIVER]

NR MECHANICSVILLE,ONT

O376; WHITTINGTON HB, 1953,J PALEONT, VOL

27.N5,P653,PL69,FIG 28

REMARKS: P-JE NARRAWAY COLL, $1938 ; 1$ SPEC

BERGERONIA ARCTICEPHALA (MATTHEW)

7751 [121CM ] P-SYN

AGRAULOS? ARCTICEPHALUS MATTHEW

$M C A$.

ST JOHN GP

[ST JOHN,ACADIAN]

HANFORD BK, ST MARTINS,ST JOHN CO NB

O211:MATTHEW GF, 1886, VOL 3,P75-76,PL7,FIG 14A,B

MATTHEW GF, TRANS NY ACAD SCI,VOL

14, 1895,P 147,PL 10,FIG 5A,B

REMARKS: C-GF MATTHEW:D-MACKENZIE ; 8 SPEC

\section{BERGERONIA ELEGANS (MATTHEW}

7795 [165CM ] P-SYN

PROTOLENUS ELEGANS MATTHEW

$\mathrm{MCA}$,

ST JOHN GP

[ST JOHN, ACADIAN]

HANFORD BK ST MARTINS, ST JOHN CO NB

O211;MATTHEW GF, 1894,TRANS ROY SOC CAN,VOL 11,P 100; 1902,NB,P35;MATTHEW GF, VOL 14, 1895.P 147. PL 11,FIG 3A-E

REMARKS: C-GF MATTHEW;D-MACKENZIE ;LABELLED COTYPES IN OLD RECORDS;SHOULD BE 12 SPECS ; 11 SPEC

BONNIA SENECTA (BILLINGS)

26468 REF SPEC?

LCA.

FORTEAUFM

DEER ARM,OPP INNER BAYS, BONNE BAY,NFLD

REMARKS: D-BF HOWELL, 1948 ;LABELLED PLESIOTYPE REFERENCE NOT KNOWN : 2 SPEC

BUMASTUS BILLINGSI RAYMOND\&NARRAWAY

18792 P-HOLO

MO,CHAMP,MOH,TRENT

TRENTONGP

[TRENTONLS ]

NR MATTHEWS FACTORY, HULL, QUE

O374:RAYMOND \& NARRAWAY,ANN CARNEGIE MUS, VOL 4, 1908,P250-252,PL62,FIG 1,2

REMARKS: P-JE NARRAWAY COLL 1938; 1 SPEC

BUMASTUS INDETERMINATUS (WALCOTT)

18700 REF SPEC-F

MO.CHAMP, MOH,BLKRIV

BLACK RIVER GP

[BLACK RIVER]

MECHANICSVILLE ONT

O374; RAYMOND \& NARRAWAY,ANN CARNEGIE MUS, VOL 4, 1908,P253-254,PL62,FIG 8,9

REMARKS: P-JE NARRAWAY COLL, $1938 ; 2$ SPEC

BUMASTUS CF IOXUS (HALL) 22669 [426S ] REF SPEC-F

LIMESTONE RAPIDS, SEVERN R,DIST OF PATRICIA,ONT O374:PARKS WA.TRANS ROY CAN INST.VOL

11.PT 1, 1915,P80 PL4.FIG 19

REMARKS: C-JB TYRRELL;D-WH HEARST, 1915; 1 SPEC 
BUMASTUS MILLERI (BILLINGS)

18688 REF SPEC-F

MO,CHAMP,MOH,BLKRIV

BLACK RIVER GP,LOWVILLE FM

[LOWVILLE LS ]

HOGS BACK,OTTAWA,ONT

O374;RAYMOND \& NARRAWAY,ANN CARNEGIE MUS,VOL 4, 1908,P249-250,PL62,FIG 3

REMARKS: P-JE NARRAWAY COLLN, $1938 ; 1$ SPEC

CALLIOPS ALATUS OKULITCH

25763 P-HOLO

MO.CHAMP,MOH,TRENT

TRENTON GP

HULL,QUE

O492; OKULITCH VJ,TRANS ROY SOC CAN, VOL 36,SECT

4,1942,P101-102,PL1,FIG 4

REMARKS: P-JE NARRAWAY COLL, $1938 ; 1$ SPEC

\section{CALLIOPS HURONENSIS OKULITCH}

25764 P-HOLO

M O,CHAMP,MOH,TRENT

TRENTON GP

ST JOSEPH IS, L HURON,ONT

O492; OKULITCH VJ,TRANS ROY SOC CAN, VOL 36,SECT

4,P99-100,PL1,FIG 7,8,9

REMARKS: C-RS MARTIN, $1941 ; 1$ SPEC

\section{CALLIOPS NARRAWAYI OKULITCH}

\section{P-SYN}

M O.CHAMP,MOH,TRENT

TRENTON GP

HULL,QUE

O492; OKULITCH VJ,TRANS ROY SOC CAN, VOL 36,SECT 4,1942,P100-101,PL1,FIG 5,6

REMARKS: P-JE NARRAWAY COLL, 1938 :LARGEST +

SMALLEST SPECS ARE FIG'D ; 3 SPEC

\section{CALYMENE MEEKI FOERSTE}

183 REF SPEC-F

U O,CINCI,MAYSVILL

DUNDAS FM

DON VALLEY QUARRY,TORONTO,ONT

O452; FRITZ MA,ONT DEPT MINES,VOL 32,PT7, 1925,P2425. PL2, FIG 6

REMARKS: C-J TOWNSEND ;FIGS 6 AND 9 MIS-LABELLED ONPLATE ; 1 SPEC

\section{CAL YMENE PLATYS GREEN}

25582 REF SPEC-F

$M D, E R I A N$

ONONDAGA FM

HAGERSVILLE, WALPOLE TP,ONT

O452; HALL J, 1888,PAL NEW YORK, VOL 7,P1-4,PL1,FIG 2

REMARKS: D-BE WALKER; 1 SPEC

\section{CALYPTAULAX OTTAWANUS OKULITCH}

25766 P-SYN

M O.CHAMP,MOH,TRENT

TRENTON GP

[TRENTON]

HULL,QUE

O492; OKULITCH VJ,TRANS ROY SOC CAN, VOL 36,SECT 4, 1942,P 102,PL1,FIG 2,3

REMARKS: P-JE NARRAWAY COLLN, 1938 ;BOTH SPECS FIG'D; 2 SPEC

\section{CERAURINUS MARGINATUS BARTON}

23963 [762T] REF SPEC-F

M O,CHAMP,MOH,TRENT

TRENTON GP.COBOURG FM

COBOURG,ONT

O432:LUDVIGSEN R, 1977,J PALEO,VOL 51,N5,P965966. PL1,FIG 7

REMARKS: D-VICTORIA COLLEGE ; 1 SPEC
CERAURINUS MARGINATUS BARTON

32419 REF SPEC-F

M O.CHAMP.MOH,TRENT

TRENTONGP COBOURG FM

[MID COBOURG FM ]

COLBORNE QUARRY,ONT

O432; LUDVIGSEN R, 1977,J PALEO,VOL 51,N5,P965966.PL 1.FIG 4

REMARKS: C-J BALOGH;D-R LUDVIGSEN : 1 SPEC

\section{CERAURINUS MARGINATUS BARTON}

32420 REF SPEC-F

M O.CHAMP,MOH,TRENT

TRENTON GP,COBOURG FM

[MID COBOURG FM]

COLBORNE QUARRY,ONT

O432:LUDVIGSEN R, 1977,J PALEO,VOL 51,N5,P965 $966, P L 1, F I G 2$

REMARKS: C-J BALOGH:D-R LUDVIGSEN ; 1 SPEC

\section{CERAURINUS MARGINATUS BARTON}

32421 REF SPEC-F

MO,CHAMP,MOH, TRENT

TRENTON GP.COBOURG FM

[U-MOST COBOURG FM]

S SIDE HWY 26,3 KM E OF COLLINGWOOD,ONT

O432; LUDVIGSEN R, 1977,J PALEO,VOL 51,N5,P965966.PL1,FIG 6

REMARKS: D-R LUDVIGSEN ;C-PALEO CLASS,UNIV WEST ONT, 1972; 1 SPEC

\section{CERAURINUS MARGINATUS BARTON}

32418 REF SPEC-F

MO,CHAMP,MOH,TRENT

TRENTON GP,COBOURG FM

[U-MOST COBOURG FM]

S SIDE HWY 26, 3 KM E OF COLLINGWOOD,ONT

O432; LUDVIGSEN R, 1977,J PALEO,VOL 51,N5,P965966 PL 1.FIG 5

REMARKS: D-R LUDVIGSEN, 1977 ;C-PALEO CLASS,UNIV WEST ONT, 1972; 1 SPEC

CERAURUS BISPINOSUS RAYMOND\&BARTON 18683 P-PARA

MO.CHAMP,MOH,BLKRIV

BLACK RIVER GP

[BLACK RIVER LS]

TETREAUVILLE, QUE

O433; RAYMOND \& BARTON,BULL MUS COMP ZOOL

HARV,VOL 54,N20,1913,P536-538,PL1, FIG 3

REMARKS: P-JE NARRAWAY COLLN, 1938; 1 SPEC

CERAURUS BISPINOSUS RAYMOND\&BARTON

18682 P-HOLO

MO,CHAMP,MOH,BLKRIV

BLACK RIVERGP

[BLACK RIVER LS ]

TETREAUVILLE, QUE

O433:RAYMOND \& BARTON BULL MUS COMP ZOOL

HARV, VOL 54,N20,1913,P536-538,PL 1,FIG 4

REMARKS: P-JE NARRAWAY COLLN $1938 ; 1$ SPEC

\section{CHASMOPS SIGMOOCULARIS OKULITCH}

25767 P-HOLO

U O.CINCI,RICHMOND

[NELSON LS]

BELOW MOUTH OF WABASSI R,200 MI W OF FT ALBANY,

ALBANY R,ONT

O493;OKULITCH VJ,TRANS ROY SOC CAN, VOL 36.SECT

4.1942,P103-104,PL1,FIG 11,12

REMARKS: C-VK PREST : 1 SPEC

CONOCORYPHE BAILEYI(HARTT)

7866 [236CM ] REF SPEC-F

$\mathrm{MCA}$,

ST JOHN GP

[ST JOHN,ACADIAN,DIV 1C]

SEELY ST,ST JOHN,NB

0242:MATTHEW GF,TRANS ROY SOC CAN, VOL 8,SECT

4,1891,P135. PL 11, FIG 10

REMARKS: C-GF MATTHEW;D-MACKENZIE ; 3 SPEC 
CONOCORYPHE BAILEYI(HARTT)

7869 [239CM] REF SPEC

$\mathrm{MCA}$

ST JOHNGP

[ST JOHN,ACADIAN,DIV 1C]

SEELY ST.ST JOHN,NB

O242:MATTHEW GF, TRANS ROY SOC CAN, VOL 2,SECT

4, 1885, P 111-114.PL 1,FIG 22,24-27

REMARKS: C-GF MATTHEW;D-MACKENZIE :UNCERTAIN WHICH SPECS FIG'D; 15 SPEC

\section{CONOCORYPHE BAILEYI VAR ARCUATA MATTHEW}

7835 [205CM] P-SYN

MCA.

ST JOHN GP

[ST JOHN,ACADIAN,DIV 1C2]

SEELY ST,ST JOHN,NB

O242:MATTHEW GF, TRANS ROY SOC CAN,VOL 2,SECT 4,1885,P113,PL 1,FIG 23,23B

REMARKS: C-GF MATTHEW;D-MACKENZIE ;LABELLED HOLOTYPE ; 1 SPEC, 2 CASTS

\section{CONOCORYPHE BAILEYI VAR ARCUATA MATTHEW}

26106 P-SYN

$M C A$.

ST JOHN GP

[ST JOHN,ACADIAN,DIV 1C2]

SEELY ST,ST JOHN,NB

O242; MATTHEW GF,TRANS ROY SOC CAN,VOL 2,SECT 4,1885,P 113

REMARKS: C-GF MATTHEW;D-MACKENZIE ;LABELLED PARATYPES ; 4 SPEC

\section{CONOCORYPHE ELEGANS (HARTT)}

8152 [522CM] REF SPEC?

$M C A$.

ST JOHN GP

[ST JOHN,ACADIAN.DIV $1 C$ ]

CATON'S IS, KINGS CO,NB

O242:MATTHEW GF,TRANS ROY SOC CAN,VOL 2,SECT 4, 1885,P115-119,PL1,FIG 28-34

REMARKS: C-GF MATTHEW;D-MACKENZIE :LABELLED PLESIOTYPE;DOES NOT RESEMBLE FIGS ; 1 SPEC

\section{CONOCORYPHE WALCOTTIMATTHEW}

8120 [490CM] P-SYN

$\mathrm{MCA}$,

ST JOHN GP

[ST JOHN,ACADIAN,DIV $1 \mathrm{C} 1$ ]

ST JOHN,NB

O242:MATTHEW GF,TRANS ROY SOC CAN,VOL 2,SECT 4,1885,P119-120,PL1,FIG 36,368

REMARKS: C-GF MATTHEW;D-MACKENZIE ;LABELLED COTYPE ; 1 SPEC-CAST\&MOULD

\section{CONOCORYPHE WALCOTTI MATTHEW}

7860 [230CM] REF SPEC-F

$\mathrm{MCA}$

ST JOHN GP

[ST JOHN,ACADIAN,DIV $1 C$ ]

SEELY ST,ST JOHN,NB

O242;MATTHEW GF, TRANS ROY SOC CAN, VOL 8,SECT 4. 1891.P134-135.PL11,FIG 7A-C

REMARKS: C-GF MATTHEW;D-MACKENZIE ;LABELLED HEAUTOTYPES ; 2 SPEC, 2 MOULDS

\section{CONOCORYPHE WALCOTTIMATTHEW}

8122 [492CM] P-SYN

$M C A$,

ST JOHN GP

[ST JOHN,ACADIAN,DIV $1 \mathrm{C} 1$ ]

ST JOHN NB

O242; MATTHEW GF, TRANS ROY SOC CAN, VOL 2,SECT 4.1885, P1 19- 120

REMARKS: C-GF MATTHEW;D-MACKENZIE :LABELLED COTYPES : 10 SPEC
CTENOCEPHALUS MATTHEWI (HARTT)

7881 [251CM] REF SPEC-F

$\mathrm{MCA}$

ST JOHN GP

SEELYST,ST JOHN,NB

O242; MATTHEW GF, TRANS ROY SOC CAN VOL 2, SECT 4. 1885,P 103-111,PL 1,FIG 14-19

REMARKS: C-GF MATTHEW:D-MACKENZIE :GROWTH STAGES;UNCERTAIN WHICH SPECS FIG'D, 17 SPEC

CTENOCEPHALUS MATTHEWI (HARTT)

$7880[250 \mathrm{CM}]$ REF SPEC-F

MCA

ST JOHN GP

[ST JOHN.ACADIAN]

SEELY ST.ST JOHN,NB

O242:MATTHEW GF, TRANS ROY SOC CAN, VOL 2,SECT 4.1885,P 103-111.PL1,FIG 6-11,13.20

REMARKS: C-GF MATTHEW;D-MACKENZIE :OLD RECORDS STATE 11 SPECS;UNCERTAIN WHICH SPECS FIG'D ; 12 SPEC

CTENOCEPHALUS MATTHEWI VAR GEMINISPINOSUS (H.) 7852 [222CM ] REF SPEC-F

$\mathrm{MCA}$,

ST JOHN GP

[ST JOHN,ACADIAN,DIV 1C]

SEELY ST,ST JOHN,NB

0242; MATTHEW GF,TRANS ROY SOC CAN, VOL 2,SECT 4,1885, P 106,PL 1,FIG 21

REMARKS: C-GF MATTHEW:D-MACKENZIE :SP AND VAR AUTHOR-HARTT:UNCERTAIN WHICH SPEC FIG'D ; 4 SPEC

CTENOCEPHALUS MATTHEWI VAR PERHISPIDUS (HARTT) 8341 [711CM ] REF SPEC-F

MCA,

ST JOHN GP

[ST JOHN, ACADIAN, DIV 1C]

SEELY ST,ST JOHN,NB

O242;MATTHEW GF,TRANS ROY SOC CAN,VOL 2,SECT 4, 1885,P 107,PL1,FIG 12

REMARKS: C-GF MATTHEW:D-MACKENZIE ;LABELLED COTYPES ; 2 SPEC

CTENOPYGE ACADICA MATTHEW

8015 [385CM] P-SYN

CTENOPYGE SPECTABILIS BROG VAR

$\mathrm{LO}$,

[BRETONIAN, L ORDOVICIAN]

KING ST, ST JOHN NB

O264;MATTHEW GF, 1892,P57,PL 13,FIG 13A,B;MATTHEW GF,TRANS ROY SOC CAN, VOL 11,SECT 4, 1894,P 109 110.PL17.FIG 13A-E

REMARKS: C-GF MATTHEW:D-MACKENZIE ;LABELLED COTYPES;UNCERTAIN WHICH SPECS FIG'D; 7 SPEC

\section{CTENOPYGE FLAGILLIFER ANG. VAR}

$8368[738 \mathrm{CM})$ REF SPEC-F

L O,

[BRETONIAN,L ORDOVICIAN]

KING ST,ST JOHN,NB

O264:MATTHEW GF, TRANS ROY SOC CAN, VOL 9,SECT 4, 1892,P56-57,PL13, FIG 12A,B

REMARKS: C-GF MATTHEW:D-MACKENZIE :LABELLED COTYPES;NOT A NEW TAXON;UNCERTAIN WHICH SPECS FIG'D; 10 SPEC

CTENOPYGE SPECTABILIS BROG. VAR REMARKS: SEE CTENOPYGE ACADICA MATTHEW

CYBELE ELLA NARRAWAY\&RAYMOND

18696 P-HOLO

M O,CHAMP,MOH,BLKRIV

BLACK RIVER GP

[BLACK RIVER]

MECHANICSVILLE,ONT

P448:NARRAWAY\&RAYMOND, ANN CARN MUS,VOL

3,N4, 1906,P600-604,FIG

REMARKS: P-JE NARRAWAY; 1 SPEC 
CYCLOGNATHUSROTUNDIFRONS MATTHEW

7976 [346CM ] P-SYN

UCA,

[BRETONIAN.DIV 3D,L ORDOVICIAN]

SUSPENSION BRIDGE,ST JOHN,NB

O266:MATTHEW GF.TRANS ROY SOC CAN,VOL 10,SECT 4.1893,P 107-108,PL7,FIG 16A,B

REMARKS: C-GF MATTHEW;D-MACKENZIE ;LABELLED COTYPES:UNCERTAIN WHICH SPECS FIG'D ; 3 SPEC

\section{CYCLOGNATHUS ROTUNDIFRONS MATTHEW}

7968 [338CM] P-SYN

UCA.

[BRETONIAN,DIV 3D,L ORDOVICIAN]

SUSPENSION BRIDGE,ST JOHN,NB

O266;MATTHEW GF,TRANS ROY SOC CAN,VOL 10,SECT 4, 1893,P107-108,PL7,FIG 16A,B

REMARK S: C-GF MATTHEW;D-MACKENZIE ;LABELLED

COTYPES;UNCERTAIN WHICH SPECS FIG'D ; 13 SPEC

\section{DOLICHOMETOPUS ACADICUS MATTHEW}

7834 [204CM] P-SYN

$M C A$

ST JOHN GP

[ST JOHN,ACADIAN,DIV 1D3]

HASTINGS COVE, KINGS CO,NB

O221; MATTHEW GF,TRANS ROY SOC CAN, VOL 3,SECT 4, 1897,P185-186,PL3,FIG 6A-D

REMARKS: C-GF MATTHEW:D-MACKENZIE ; LABELLED COTYPES ; 5 SPEC

\section{DOLICHOMETOPUS OCCIDENTALIS MATTHEW} 8027 [397CM] P-HOLO

MCA,

MT STEPHEN,FIELD,BC

O221:MATTHEW GF,TRANS ROY SOC CAN, VOL 5, SECT 4, 1899,P49-50,PL1,FIG 2

REMARKS: C-GF MATTHEW;D-MACKENZIE ; 1 SPEC \& CAST

DORYPYGE DAWSONI WALCOTT 8042 [412CM] REF SPEC-F

$M C A$,

STEPHEN FM

MT STEPHEN,FIELD,BC

O217:MATTHEW GF, TRANS ROY SOC CAN, VOL 5,SECT 4, 1899,P56-57,PL3,FIG 1 REMARKS: C-GF MATTHEW;D-MACKENZIE ; 2 SPEC

\section{DORYPYGE HORRIDA MATTHEW}

8410 [780CM] P-HOLO

$\mathrm{MCA}$,

ST JOHN GP.DIV $1 D 3$

[ST JOHN, ACADIAN,DIV 103

HASTINGS COVE, KINGS CO,NB

O2 17:MATTHEW GF, TRANS ROY SOC CAN, VOL 3,SECT 4,1897,P 190,PL4,FIG 3A,B

REMARKS: C-GF MATTHEW;D-MACKENZIE ; 1 SPEC

\section{DORYPYGE QUADRICEPS VAR VALIDA MATTHEW}

8413 [783CM] P-HOLO

$\mathrm{MCA}$,

[ACADIAN]

HASTINGS COVE, KINGS CO,NB

O217; MATTHEW GF, TRANS ROY SOC CAN, VOL 3,SECT 4,1897,P189,PL4,FIG 2C.D

REMARKS: C-GF MATTHEW;D-MACKENZIE; 1 SPEC

DORYPYGE WASATCHENSIS VAR ACADICA MATTHEW 8414 [784CM ] P-SYN

$\mathrm{MCA}$.

[ACADIAN]

HASTINGS COVE, KINGS CO,NB

O217: MATTHEW GF,TRANS ROY SOC CAN,VOL 3,SECT 4, 1897,P 188-189,PL4,FIG 1

REMARKS: C-GF MATTHEW:D-MACKENZIE :LABELLED HOLOTYPE;SPECIES APPEARS TO BE BASED ON 2 SPECS ; 1 SPEC
ELLIPSOCEPHALUS GALEATUS MATTHEW

7758 [128CM ] P-SYN

LCA.

ST JOHN GP

[ST JOHN,ACADIAN,DIV 1B3

HANFORD BK, ST MARTINS,ST JOHN CO,NB

O207: MATTHEW GF, 1892,P249-250,PL1,FIG 4A-D

MATTHEW GF, TRANS ROY SOC CAN, VOL 11, SECT 4, 1894,P103,PL17,FIG 7A-D

REMARKS: C-GF MATTHEW:D-MACKENZIE ;LABELLED COTYPES;UNCERTAIN WHICH SPECS FIG'D ; 5 SPEC

ELLIPSOCEPHALUS GALEATUS VAR A GRAULOIDES

7755 [125CM] P-SYN

$\mathrm{MCA}$,

ST JOHN GP

[ST JOHN,ACADIAN]

HANFORD BK, ST MARTINS ST JOHN CO NB

O207; MATTHEW GF, 1893,P250,PL 1,FIG 4E;MATTHEW GF,TRANS ROY SOC CAN,VOL 11,SECT

4,1894,P103,PL17,FIG 7E

REMARKS: C-GF MATTHEW;D-MACKENZIE ;LABELLED COTYPES ; 2 SPEC

\section{ELLIPSOCEPHALUS GRANDIS MATTHEW}

$7752[122 \mathrm{CM}]$ P-SYN

$\mathrm{MCA}$

ST JÖHN GP

[ST JOHN,ACADIAN ]

HANFORD BK, ST MARTINS,ST JOHN CO,NB

O207; MATTHEW GF,TRANS ROY SOC CAN, VOL 11,SECT 4,1894,P 105-106 PL17.FIG 6A-C

REMARKS: C-GF MATTHEW;D-MACKENZIE ;LABELLED COTYPES;UNCERTAIN WHICH SPECS FIG'D; 7 SPEC

\section{ENCRINURUS CF PUNCTATUS BRUNNICH} 22670 [427S ] REF SPEC-F

S,

EKWAN R,ONT

O446;PARKS WA, TRANS ROY CAN INST, VOL

11,PT 1, 1915,P80-81,PL4,FIG 20

REMARKS: C-JB TYRRELL, 1912 ;D-WHHEARST, 19 15;LOC GIVEN IN PUBL'N AS LIMESTONE RAPIDS,FAWN R;ROM RECORDS STATE EKWAN R; 1 SPEC

EOBRONTEUS LUNATUS (BILLINGS)

18753 REF SPEC-F

MO,CHAMP,MOH,TRENT

TRENTONGP

[TRENTON]

CHAUDIERE FALLS,QUE

O371; SINCLAIR GW, 1949,J PALEO, VOL 23,N1,P5253.PL 14, FIG 1,4

REMARKS: P-JE NARRAWAY,APRIL $1938 ; 1$ SPEC

EOBRONTEUS LUNATUS (BILLINGS)

18753 REF SPEC-F

M O,CHAMP,MOH,TRENT

TRENTON GP

[TRENTON]

CHAUDIERE FALLS,QUE

O371:SINCLAIR GW, 1949,J PALEO,VOL 23,N1,P52-

53,PL 14,FIG 1,4

REMARKS: P-JE NARRAWAY,APRIL 1938; 1 SPEC

\section{EOBRONTEUS SCULPTUS SINCLAIR}

18734 P-SYN

M O,CHAMP,MOH,TRENT

TRENTON GP

NR ST LOUIS DAM,OTTAWA,ONT

O371; SINCLAIR GW,JOUR PAL, VOL

23, N 1, 1949,P53,PL 13, FIG 3-6

REMARKS: P-JE NARRAWAY COLLN, 1938 ;LARGER SPEC FIG'D; 2 SPEC

HEMIARGES PAULIANUS (CLARKE)

18794 REF SPEC-F

$M$ O.CHAMP, MOH,TRENT

TRENTONGP

[TRENTON 
NR MATTHEWS FACTORY, HULL,QUE

O503:FOERSTE AF, AM JOUR SCI,VOL

49, N289, 1920,PL2, FIG 6A-C

REMARKS: P-JE NARRAWAY COLLN, 1938 ; 2 SPEC

HOLASAPHUS CENTROPYGE MATTHEW

7894 [264CM] P-SYN?

LCA

[LETCHEMINIAN.DIVE2A]

YOUNG PT, GEORGE R,NS

O261;MATTHEW GF.1896,P268,PL2,FIG 4A,B:MATTHEW GF.GSC, 1903,P174-175.PL 10,FIG 3A-C;DEAN WT, $1972, \mathrm{P} 269.271$

REMARKS: C-GF MATTHEW:D-MACKENZIE ;LABELLED COTYPES:DEAN STATES THESE SPECS ARE NOT SYNTYPES : 10 SPEC

\section{ILLAENUS ANGUSTICOLLIS BILLINGS}

18684 REF SPEC-F

MO,CHAMP,MOH,BLKRIV

BLACK RIVER GP

[BASAL 8EDS OF BLACK RIVER]

PELTON'S QUARRY, 6 MIS OF OTTAWA,ONT

O372; RAYMOND \& NARRAWAY,ANN CARNEGIE MUS, VOL

4, 1908,P245-247,PL61,FIG 1,2

REMARKS: P-JE NARRAWAY COLLN, $1938 ; 1$ SPEC

ILLAENUS CONRADI BILLINGS

18691 REF SPEC-F

M O,CHAMP,MOH,8LKRIV

BLACK RIVER GP

[BLACK RIVER LS]

VAL TETREAU,NR HULL,QUE

O372; RAYMOND \& NARRAWAY,ANN CARNEGIE MUS, VOL 4.1908,P245,PL60,FIG 9,10

REMARKS: P-JE NARRAWAY COLLN, 1938 ;OLD RECORDS GIVE LOC AS VAL TETREAU;PUBL'N STATES

TETREAUVILLE ; 1 SPEC

ILLAENUS LATIAXIATUS RAYMOND\&NARRAWAY

18754 P-SYN

M O.CHAMP,MOH,8LKRIV

BLACK RIVER GP

[BLACK RIVER LS]

MECHANICSVILLE,ONT + TETREAUVILLE,QUE, NR

OTTAWA,ONT

O372; RAYMOND \& NARRAWAY,ANN CARNEGIE MUS, VOL 4,1908,P243-245,PL60,FIG 4-8

REMARKS: P-JE NARRAWAY COLLN ;HEAD FROM MECHANICSVILLE;PYGIDIUM \& THORAXFROM TETREAUVILLE; 3 SPEC

ISOTELUS GIGAS DEKAY

18689 REF SPEC-F

MOCHAMP, MOH,BLKRIV

BLACK RIVER GP

[BLACK RIVER]

VAL TETREAU,NR HULL,QUE

O339; RAYMOND \& NARRAWAY, ANN CARNEGIE MUS, VOL

7.N1, 1910,P53-56, PL 15, FIG 2

REMARKS: P-JE NARRAWAY COLLN, 1938 ;SMALLER SPEC FIG'D ; 2 SPEC

ISOTELUS IOWENSIS (OWEN)

18698 REF SPEC-F

ISOTELUS SP (RAYMOND\&NARRAWAY, 1910)

MO,CHAMP,MOH, BLKRIV

BLACK RIVER GP

[LOWVILLE AND BLACK RIVER]

MECHANICSVILLE,ONT

O339;RAYMOND \& NARRAWAY, 19 10,P56-57,PL 15,FIG 3; RAYMOND PE, BULL MUS COMP ZOO, HARVARD, VOL $58, N 5,1914$, P256

REMARKS: P-JE NARRAWAY COLLN, 1938 ;LARGER SPEC FIG'D; 2 SPEC
ISOTELUS MAXIMUS LOCKE

26319 REF SPEC-F

U O,CINCI,MAYSVILL

DUNDASFM

[DUNDAS]

DON VALLEY QUARRY TORONTO ONT

O339:FRITZ MA.TRANS ROY SOC CAN,VOL 40 , SECT 4,1946,P27-32,PL 1,FIG 1

REMARKS: D-EE WATSON, 1946 ; REPLICAS OF SPEC NO $26353 ; 4$ PLASTER CASTS

ISOTELUS MAXIMUS LOCKE

26352 REF SPEC-F

UO.CINCI,MAYSVILL

DUNDASFM

[DUNDAS]

DON VALLEY QUARRY, TORONTO ONT

O339;FRITZ MA,TRANS ROY SOC CAN, VOL 40 ,SECT 4, 1946,P27-32,PL1,FIG 3

REMARKS: D-EE WATSON, $1946 ; 3$ PLASTER REPLICAS MADE-SEE NO 26318; 1 SPEC

ISOTELUS MAXIMUS LOCKE

26353 REF SPEC-F

UO.CINCI,MAYSVILL

DUNDASFM

[DUNDAS]

DON VALLEY QUARRY,TORONTO,ONT

O339:FRITZ MA,TRANS ROY SOC CAN,VOL 40,SECT 4,1946,P27-32,PL1,FIG 1

REMARKS: D-EE WATSON, $1946 ; 3$ PLASTER REPLICAS MADE-SEE NO 26319;1 SPEC

ISOTELUS MAXIMUS LOCKE

17608 [1375HR] REF SPEC-F

U O.CINCI,MAYSVILL

DUNDAS FM

[DUNDAS]

DON VALLEY QUARRY,TORONTO,ONT

O339:FRITZ MA,TRANS ROY SOC CAN,VOL 40,SECT

4, 1946,P27-32,PL 1,FIG 2

REMARKS: C-MA FRITZ, $1925 ; 1$ SPEC \& 3 REPLICAS

ISOTELUS MAXIMUS LOCKE

26318 REF SPEC-F

U O.CINCI,MAYSVILL

DUNDAS FM

[DUNDAS]

DON VALLEY QUARRY,TORONTO.ONT

O339;FRITZ MA, TRANS ROY SOC CAN, VOL 40,SECT 4,1946,P27-32,PL1,FIG 3

REMARKS: D-EE WATSON :REPLICAS OF SPEC NO 26352 3 PLASTER CASTS

ISOTELUS MAXIMUS LOCKE

17107 [855HR] REF SPEC-F

U O.CINCI,MAYSVILL

DUNDAS FM

[DUNDAS]

HUMBERVALE QUARRY, TORONTO,ONT

O339;FRITZ MA,ONT DEPT MINES,VOL 32,PT7, 1925,P2324.PL2,FIG 17

REMARKS: C-J TOWNSEND ? ; 1 SPEC

LEPTOPLASTUS LATUS MATTHEW

7963 [333CM ] P.SYN

UCA, ORLO

ST JOHN GP?

[ST JOHN,BRETONIAN,L ORDOVICIAN]

NAVYIS,ST JOHN NB

O263;MATTHEW GF,CAN REC SCI, 1891,P461-462; MATTHEW GF, TRANS ROY SOC CAN, VOL $9, S E C T$ 4,1892,P54-55,PL13,FIG 10A-C

REMARKS: C-GF MATTHEW;D-MACKENZIE ;LABELLED COTYPES:UNCERTAIN WHICH SPECS FIG'D; 17 SPEC 
LEPTOPLASTUS SPINIGER MATTHEW

REMARKS: SEE ACANTHOLENUS SPINIGER (MATTHEW)

LEPTOPLASTUS SPINOSUM MATTHEW

7955 [325CM ] P-SYN

UCA, ORLO

ST JOHN GP

[ST JOHN,BRETONIAN, L ORDOVICIAN]

KING ST,ST JOHN,NB

O263:MATTHEW GF, TRANS ROY SOC CAN,VOL 11,SECT 4,1894,P106-107, PL 17,FIG 14A-E

REMARKS: C-GF MATTHEW:D-MACKENZIE ;LABELLED

COTYPES:UNCERTAIN WHICH SPECS FIG'D ; 13 SPEC

LEPTOPLASTUS STENOTOIDES MATTHEW

REMARKS: SEE ANOMOCARE STENOTOIDES (MATTHEW)

LIOSTRACUS OUANGONDIANUS (HARTT)

8298 [668CM ] REF SPEC-F

$\mathrm{MCA}$

ST JOHN GP

[ST JOHN,ACADIAN,DIV $1 \mathrm{C}$ ]

PORTER'S BK, ST MARTINS.ST JOHN CO NB

O261; MATTHEW GF, TRANS ROY SOC CAN,VOL 5,SECT

4,1888,P138-139,PL1,FIG 4A

REMARKS: C-GF MATTHEW;D-MACKENZIE ;LABELLED

PLESIOTYPES:UNCERTAIN WHICH SPEC FIG'D; 11 SPEC

LIOSTRACUS OUANGONDIANUS VAR GIBBA (HARTT)

$7858[228 \mathrm{CM}]$ REF SPEC-F

$\mathrm{MCA}$,

ST JOHN GP

[ST JOHN,ACADAAN,DIV $1 \mathrm{C} 1$ ]

PORTER'S BK, ST MARTINS,ST JOHN CO,NB

O261; MATTHEW GF,TRANS ROY SOC CAN,VOL 5,SECT

4, 1888, P 140,PL 1,FIG 4H-K

REMARKS: C-GF MATTHEW;D-MACKENZIE ;LABELLED

COTYPES;VAR AUTHOR GIVEN AS MATTHEW IN FRITZ'S

TYPE CAT + ROM RECORDS ; 2 SPEC

LIOSTRACUS OUANGONDIANUS VAR PLANA (HARTT)

$8300[670 \mathrm{CM}]$ REF SPEC-F

$\mathrm{MCA}$

ST JOHN GP

[ST JOHN,ACADIAN,DIV $1 \mathrm{C}$ ]

ST MARTINS.ST JOHN CO,NB

O261:MATTHEW GF,TRANS ROY SOC CAN,VOL 5,SECT

4,1888,P140,PL 1,FIG 4E-G

REMARK S: C-GF MATTHEW:D-MACKENZIE :LABELLED

COTYPES;VAR AUTHOR GIVEN AS MATTHEW IN FRITZ'S

TYPE CAT + ROM RECORDS ; 2 SPEC

\section{LIOSTRACUS OUANGONDIANUS VAR IMMARGINATA}

$7851[221 \mathrm{CM}]$ REF SPEC-F

$M C A$,

ST JOHN GP

[ST JOHN, ACADIAN,DIV 1C1]

SEELY ST,ST JOHN,NB

O261; MATTHEW GF, TRANS ROY SOC CAN,VOL 5,SECT

4.1888,P139,PL 1,FIG 4B-D

REMARKS: C-GF MATTHEW;D-MACKENZIE ;LABELLED

COTYPES:VAR AUTHOR (HARTT)-GIVEN AS MATTHEW IN FRITZ'S TYPE CAT + ROM RECORDS ; 3 SPEC

LIOSTRACUS OUANGONDIANUS VAR AURORA (HARTT)

$8332[702 \mathrm{CM}]$ REF SPEC-F

$\mathrm{MCA}$

ST JOHN GP

[ST JOHN,ACADIAN,DIV $1 \mathrm{C}$ ]

PORTER'S BK,ST MARTINS \& SEELY ST,ST JOHN, ST JOHN CO.NB

O261; MATTHEW GF, TRANS ROY SOC CAN,VOL 5,SECT

4. 1888,P 139-140 PI 2.FIG 7A-F

REMARKS: C-GF MATTHEW;D-MACKENZIE ; 4 SPEC
LIOSTRACUS TENER (HARTT)

$7840[210 \mathrm{CM}]$ REF SPEC-F

$\mathrm{MCA}$

ST JOHN GP

[ST JOHN, ACADIAN,DIV $1 \mathrm{C}$ ]

SEELY ST.ST JOHN.NB

O261:MATTHEW GF TRANS ROY SOC CAN, VOL 5,SECT 4,1888,P 137-138,PL 1,FIG 3A-C

REMARKS: C-GF MATTHEW;D-MACKENZIE ;LABELLED PLESIOTYPES ; 9 SPEC

LIOSTRACUS VALIDUS MATTHEW

7678 [48CM] P-HOLO

$\mathrm{MCA}$.

ST JOHN GP

[ST JOHN,ACADIAN]

HASTINGS COVE, KINGS CO,NB

O261; MATTHEW GF,TRANS ROY SOC CAN,VOL 3,SECT 4.1897.P 179.PL2.FIG 7A B

REMARKS: C-GFMATTHEW;D-MACKENZIE; LABELLED HOLOTYPE IN OLD RECORDS ; 1 SPEC

\section{METADOXIDES MAGNIFICUS MATTHEW}

7824 [194CM ] P-SYN

$\mathrm{LCA}$,

[UETCHEMINIAN]

RLWY CUT,MANUEL'S STN , CONCEPTION BAY,NFLD

O2 14:MATTHEW GF,BULL NAT HIST SOC NB,VOL 4,N17,P137-141,PL3

REMARKS: C-GF MATTHEW;D-MACKENZIE ; 5 SLABS

MICMACCA ANGIMARGO MATTHEW

$7782[152 \mathrm{CM}] \mathrm{P} \cdot \mathrm{HOLO}$

$\mathrm{CA}$,

[UETTCHEMINIAN]

MANUEL'S BK . CONCEPTION BAY NFLD

O208:MATTHEW GF, TRANS ROY SOC CAN,VOL 5,SECT 4,1899,P80-81,PL4,FIG 6

REMARKS: C-GF MATTHEW;D-MACKENZIE ; 1 SPEC

MICMACCA MATTHEVIMATTHEW

$7746[116 \mathrm{CM}]$ P-HOLO

$M C A$.

ST JOHN GP

[ST JOHN, ACADIAN, DIV 1D3]

HANFORD BK, ST MARTINS,ST JOHN CO,NB O208:MATTHEW GF, TRANS NY ACAD SCI,VOL 14.1895, P 141-142,PL 10,FIG 1A.B

REMARKS: C-GF MATTHEW;D-MACKENZIE ; 1 SPEC(CAST\&MOULD)

MICMACCA PLANA MATTHEW

$7754[124 \mathrm{CM}] \mathrm{P}-\mathrm{HOLO}$

$M C A$.

ST JOHHN GP

[ST JOHN ACADIAN ]

HANFORD BK, ST MARTINS,ST JOHN CO,NB

O208:MATTHEW GF, TRANS NY ACAD SCI,VOL

14, 1895, P 143-144, PL 11, FIG 2A, B

REMARKS: C-GF MATTHEW;D-MACKENZIE ; 1 SPEC

MICMACCA VANINGENI MATTHEW

$7802[172 \mathrm{CM}]$ P-SYN

LCA,

ST JOHN GP

[ST JOHN,ACADIAN,DIV 1B3]

HANFORD BK, ST MARTINS, ST JOHN CO,NB

O208:MATTHEW GF, TRANS NY ACAD SCI,VOL

14,1895, P142,PL11,FIG 1A,B

REMARK S: C-G VAN INGEN ; LABELLED COTYPES;SPEC 'A FIG'D ; 4 SPEC

MICMACCA WALCOTTIMATTHEW

7783 [153CM] P-SYN

LCA

[UETCHEMINIAN SHALE]

MANUELS BK CONCEPTION BAY NFLD

O208:MATTHEW GF, TRANS ROY SOC CAN,VOL 5,SECT 4,1899,P79-80,PL4,FIG 5A-D

REMARKS: C-GF MATTHEW:D-MACKENZIE :LABELLED COTYPES ; 10 SPEC 
MICRODISCUS BELLIMARGINATUS MUT INSULARIS M. 7775 [145CM]P-SYN

LCA,

[UETCHEMINIAN ]

MANUELS BK, CONCEPTION BAY,NFLD

O188:MATTHEW GF,TRANS ROY SOC CAN, VOL 5.SECT 4. 1899,P75,PL 17,FIG 4A,B

REMARKS: C-GF MATTHEW;D-MACKENZIE ;LABELLED COTYPES ; 2 SPEC

MICRODISCUS PRECURSOR MATTHEW

7677 [47CM] P-SYN

MICRODISCUS PUNCTATUS VAR PRECURSOR MATTHEW $M C A$

[ACADIAN,GRAY SHALES

HANFORD BK, ST MARTINS,ST JOHN CO,NB

0188; MATTHEW GF, 1886,P75,PL7,FIG 13:MATTHEW

GF,TRANS NY ACAD SCI, VOL 15, 1896,P239-240

REMARKS: C-GF MATTHEW;D-MACKENZIE ; 2 SPEC

MICRODISCUS PUNCTATUS VAR PRECURSOR MATTHEW REMARKS: SEE MICRODISCUS PRECURSOR MATTHEW

MICRODISCUS PUNCTATUS VAR PULCHELLUS (HARTT)

8188 [558CM ] REF SPEC-F

MCA

ST JOHN GP

[ST JOHN,ACADIAN,DIV 1D]

PORTER'S BK, ST MARTINS,ST JOHN CO,NB

0188:MATTHEW GF, TRANS ROY SOC CAN, VOL 3,SECT 4.1886,P74-75,PL7,FIG 12A-C

REMARKS: C-GF MATTHEW;D-MACKENZIE ;LABELLED PLESIOTYPES;UNCERTAIN WHICH SPECS FIG'D : 16 SPEC

MICRODISCUS SCHUCHERTI MATTHEW

7768 [138CM] P-SYN

CA

[ETCHEMINIAN]

TROY,NY,USA

0188:MATTHEW GF TRANS NY ACAD SCI,VOL

15. 1896.P238-239,PL 17,FIG 4A,B

REMARKS: C-GF MATTHEW;D-MACKENZIE ; 6 SPEC

PARABOLINA HERES VAR LATA MATTHEW

$7972[342 \mathrm{CM}]$ P-SYN

UCA

[DIV 3B,BRETONIAN,L ORDOVICIAN]

NAVY IS,ST JOHN,NB

O262; MAT THEW GF, TRANS ROY SOC CAN, VOL 9,SECT

4, 1892,P5 1-52,PL 13,FIG 6A-F

REMARKS: C-GF MATTHEW;D-MACKENZIE ;LABELLED

COTYPES:UNCERTAIN WHICH SPECS FIG'D;OLD

RECORDS STATE 19 SPECS; 16 SPEC

PARABOLINA HERES VAR GRANDIS MATTHEW

$7961[331 \mathrm{CM}]$ P.HOLO

UCA.

[BRETONIAN, L ORDOVICIAN,DIV 3B]

NAVY IS.ST JOHN,NB

O262:MATTHEW GF,TRANS ROY SOC CAN,VOL 9,SECT

4, 1892,P52,PL 13,FIG 7

REMARKS: C-GF MATTHEW:D-MACKENZIE ; 1 SPEC

PARABOLINA SPINULOSA (WAHLENBERG)

$8043[413 \mathrm{CM}]$ REF SPEC-F

UCA,

[DIV 3B,BRETONIAN,L ORDOVICIAN]

GERMAIN ST,ST JOHN,NB

O262; MATTHEW GF,TRANS ROY SOC CAN, VOL

9.1892,P51,PL13, FIG 5A-D

REMARKS: C-GF MATTHEW:D-MACKENZIE ;LABELLED

PLESIOTYPES:UNCERTAIN WHICH SPECS FIG'D : 6 SPEC

PARABOLINELLA POSTHUMA MATTHEW

$7960[330$ CM] P-SYN

UCA

[DIV 3D.BRETONIAN,L ORDOVICIAN]

SUSPENSION BRIDGE,ST JOHN,NB
O267:MATTHEW GF, TRANS ROY SOC CAN, VOL 10,SECT 4.1893.P107.PL7.FIG 15A,B

REMARKS: C-GF MATTHEW:D-MACKENZIE ; 3 SPEC

PARABOLINELLA QUADRATA MATTHEW

7962 [332CM] P-SYN

UCA OR LO

[BRETONIAN,L ORDOVICIAN]

MCLEOD BK, BOISDALE,NS

O267:MATTHEW GF.BULL NAT HIST SOC NB,VOL

4.1902.P4 11.412. PL 18,FIG 7

REMARKS: C-GF MATTHEW;D-MACKENZIE ;LABELLED

COTYPES;SPEC 'A ' FIG'D ; 3 SPEC

PARADOXIDES ABENACUS MATTHEW

$7821[191 \mathrm{CM}]$ P-SYN

$\mathrm{MCA}$,

ST JOHN GP

IST JOHN,ACADIAN,DIV 10

PORTER'S BK, ST MARTINS,ST JOHN CO,NB

O213:MATTHEW GF, TRANS ROY SOC CAN, VOL 3,SECT

4,1886,P78-80,PL7,FIG 17,17B-D

REMARKS: C-GF MATTHEW:D-MACKENZIE :LABELLED

COTYPES;UNCERTAIN WHICH SPECS FIG'D; 16 SPEC

PARADOXIDES ABENACUS MATTHEW

$7850[220 \mathrm{CM}]$ P-SYN

$M C A$

ST JOHN GP,DIV 10

[ST JOHN ACADIAN]

PORTER'S BK, ST MARTINS,ST JOHN CO,NB

O2 13; MATTHEW GF, TRANS ROY SOC CAN,VOL 3,SECT

4, 1886,P78-80,PL7,FIG 17A

REMARKS: C-GF MATTHEW;D-MACKENZIE ; 1 SPEC

PARADOXIDES ABENACUS MATTHEW

7884 [254CM] P-SYN

$\mathrm{MCA}$

ST JOHN GP

[ST JOHN, ACADIAN, DIV 1D]

PORTER'S BK, ST MARTINS,ST JOHN CO,NB

O213;MATTHEW GF,TRANS ROY SOC CAN, VOL 3,SECT

4, 1886,P78-80,PL7,FIG 17,17B-D

REMARKS: C-GF MATTHEW:D-MACKENZIE :2

LITHOLOGIES PRESENT-BLACK SHALE \& GRAY LS:

LABELLED COTYPES:UNCERTAIN WHICH SPECS FIG'D ; 20 SPEC

PARADOXIDES ABENACUS MATTHEW MUT

7886 [256CM ] REF SPEC-F

$\mathrm{MCA}$

ST JOHN GP

[ST JOHN,ACADIAN,DIV $10+$ BLACK SH]

HASTINGS COVE,S SHORE KENNEBECASIS R, ST JOHN CO.NB

O2 13:MATTHEW GF,TRANS ROY SOC CAN, VOL 3,SECT

4,1897,P175-176,PL 1,FIG 9A-C,PL2,FIG 1A-D

REMARKS: C-GF MATTHEW:D-MACKENZIE:2

LITHOLOGIES PRESENT: GREY CALCAREOUS MATERIAL (DIV 1D) FIG'D ON PL 1:BLACK SHALE MATERIAL. DESCRIBED AS 'FORM 2',FIG'D ON PL2;BLACK SPECS

'B', 'G' = FIGS 1A,1D;LABELLED COTYPES IN OLD RECORDS;NOT FROM TYPE LOC; 26 SPEC

PARADOXIDES ACADICUS MATTHEW

$7818[188 \mathrm{CM}]$ P-SYN

$\mathrm{MCA}$

ST JOHN GP

[ST JOHN, ACADIAN

SEELY ST, ST JOHN, NB

O2 13; MATTHEW GF, TRANS ROY SOC CAN,VOL 1,SECT 4, 1883,P103-104,PL9,FIG 16,17,18

REMARKS: C-GF MATTHEW:D-MACKENZIE :LABELLED

COTYPES:SPEC 'B' = FIG $16 ; 13$ SPEC

PARADOXIDES ACADICUS VAR SURICUS MATTHEW

$7817[187 \mathrm{CM}]$ P-SYN

MCA.

ST JOHN GP

[ST JOHN,ACADIAN,DIV 1C1] 
SEELY ST,ST JOHN, NB

O2 13:MATTHEW GF, TRANS ROY SOC CAN, VOL 3,SECT 4,1886,P77-78,PL7,FIG 16

REMARKS: C-GF MATTHEW:D-MACKENZIE ; 3 SPEC

PARADOXIDES ETEMINICUS MATTHEW

7873 [243CM ] P-SYN

$\mathrm{MCA}$

ST JOHN GP

[ST JOHN, ACADIAN ]

SEELY ST,ST JOHN,NB

O2 13:MATTHEW GF. TRANS ROY SOC CAN.VOL 1,SECT 4.1883.P92,PL9,FIG 11,12

REMARKS: C-GF MATTHEW:D-MACKENZIE ;LABELLED COTYPES:SPECS 'A'\&'G' FIG'D; 6 SPEC

\section{PARADOXIDES ETEMINICUS MATTHEW}

$7900[270 \mathrm{CM}]$ P-SYN

$\mathrm{MCA}$,

ST JOHN GP,DIV $1 \mathrm{C}$

SEELY ST.ST JOHN.NB

O2 13;MATTHEW GF, TRANS ROY SOC CAN, VOL 1,SECT 4, 1883.P92-97

REMARKS: D-W MACKENZIE ;LABELLED COTYPE,G STEAD COLL;THIS SPEC NOT FIG'D; 1 SPEC

PARADOXIDES ETEMINICUS MATTHEW

8199 [569CM] P-SYN

$\mathrm{MCA}$

ST JOHN GP,DIV $1 \mathrm{C}$

[ST JOHN, ACADIAN]

SEELY ST, \& GILFORD ST ST JOHN WEST, ST JOHN,NB O2 13;MATTHEW GF, TRANS ROY SOC CAN, VOL 1,SECT 4, 1883,P92-97,PL9,FIG 8,9

REMARKS: C-GF MATTHEW:D-MACKENZIE ; 4 SPECS ARE OF DIFF LITHOLOGY;LABELLED 'CARLETON' PLUS A DATE-1891 OR 1889;THESE 4 MAY NOT BE TYPES ; 10 SPEC

PARADOXIDES ETEMINICUS MATTHEW

$7868[238 \mathrm{CM}]$ P-SYN

$\mathrm{MCA}$,

ST JOHN GP

[ST JOHN, ACADIAN]

SEELY ST,ST JOHN,NB

O213:MATTHEW GF,TRANS ROY SOC CAN, VOL 1,SECT 4, 1883,P92-97

REMARKS: C-GF MATTHEW;D-MACKENZIE ;LABELLED COTYPES:UNCERTAIN WHETHER THESE SPECS FIG'D: OLD RECORDS STATE 8 SPECS ; 7 SPEC

\section{PARADOXIDES ETEMINICUS VAR MALITICUS MATT.}

7825 [195CM ] P-HOLO

$\mathrm{MCA}$

ST JOHN GP

[ST JOHN,ACADIAN ]

SEELY ST, ST JOHN, NB

O2 13-MATTHEW GF, TRANS ROY SOC CAN, VOL 1,SECT 4, 1883,P 101-102, PL9,FIG 13,13A

REMARKS: C-GF MATTHEW:D-MACKENZIE ; 1 SPEC \& MOULD

PARADOXIDES ETEMINICUS VAR PONTIFICALISM.

7883 [253CM ] P-SYN?

$M C A$

ST JOHN GP

[ST JOHN, ACADIAN ]

SEELY ST ST JOHN NB

O2 13:MATTHEW GF,TRANS ROY SOC CAN, VOL 1,SECT 4, 1883,P 102-103,PL9,FIG 15,15A

REMARKS: C-GF MATTHEW:D-MACKENZIE : ENTERED IN

OLD RECORDS AS P. MICMAC PONTIFICALIS.COTYPES

FROM ABOVE LOC; TYPE LOC PUBL'D AS PORTLAND,NB;

SPECS DO NOT RESEMBLE FIGS : 5 SPEC

PARADOXIDES ETEMINICUS VAR QUACOENSIS MATT.

8299 [669CM] P-HOLO

$\mathrm{MCA}$

ST JOHN GP,DIV $1 \mathrm{C} 1$

[ST JOHN, ACADIAN]
HANFORD BK, ST MARTINS,ST JOHN CO,NB

O2 13:MATTHEW GF,TRANS ROY SOC CAN.VOL 1,SECT

4,1883,P102,PL9,FIG 14,14A

REMARKS: C-GF MATTHEW;D-MACKENZIE : 1 SPEC

PARADOXIDES ETEMINICUS VAR SURICOIDES MATT.

8198 [568CM ] P-SYN?

MCA,

ST JOHN GP

[ST JOHN,ACADIAN,DIV 1C]

SEELY ST,ST JOHN,NB

O2 13:MATTHEW GF,TRANS ROY SOC CAN, VOL 1,SECT

4,1883,P97-99,PL9,FIG 4,6

REMARKS: C-GF MATTHEW;D-MACKENZIE ;LABELLED

COTYPES:PUBL'N GIVES LOC AS PORTLAND, NB AND

STATES 'HAS NOT BEEN FOUND ELSEWHERE' ; 3 SPEC

PARADOXIDES LAMELLATUS HARTT

$7819[189 \mathrm{CM}] \mathrm{REF}$ SPEC-F

$M C A$,

ST JOHN GP

[ST JOHN,ACADIAN]

PORTER'S BK, ST MARTINS,ST JOHN CO,NB

O2 13:MATTHEW GF, TRANS ROY SOC CAN, VOL 8,SECT 4, 1891,P135-136,PL11,FIG

REMARKS: C-WD MATTHEW:D-MACKENZIE ; SPEC 'C' FIG'D ; 6 SPEC

PARADOXIDES LAMELLATUS VAR LORICATUS MATTHEW 7874 [244CM ] P-SYN?

$\mathrm{MCA}$

ST JOHN GP

[ST JOHN,ACADIAN,DIV 1C1]

SEELY ST,ST JOHN,NB

O213:MATTHEW GF,TRANS ROY SOC CAN, VOL 1,SECT

4, 1883,P106,PL9,FIG 19

REMARKS: C-GF MATTHEW;D-MACKENZIE ;SPECS

LABELLED COTYPES; ONE SPEC LABELLED SEELY ST PUBL'N MENTIONS JUST 2 SPECS,FROM PORTLAND,NB 10 SPEC

PARADOXIDES MICMAC HARTT

7837 [207CM] REF SPEC ?

$\mathrm{MCA}$,

ST JOHN GP

[ST JOHN, ACADIAN]

SEELY ST,ST JOHN,NB

O213:MATTHEW GF, TRANS ROY SOC CAN, VOL 3, 1886,P808

REMARKS: C-GF MATTHEW:D-MACKENZIE :LABELLED PLESIOTYPES IN OLD RECORDS;LOC IN PUBL'N GIVEN AS PORTLAND AND HANFORD BK;THESE SPECS NOT FIG'D; 14 SPEC

PARADOXIDES MICMAC HARTT

7896 [266CM ] REF SPEC?

$\mathrm{MCA}$

ST JOHN GP

[ST JOHN,ACADIAN]

SEELY ST,ST JOHN,NB

O2 13:MATTHEW GF, TRANS ROY SOC CAN, VOL 3, 1886,P8081

REMARKS: C-GF MATTHEW:D-MACKENZIE :LABELLED

PLESIOTYPES IN OLD RECORD; LOC IN PUBL'N GIVEN AS PORTLAND + HANFORD BK;THESE SPECS NOT FIG'D ; 8 SPEC

PARADOXIDES REGINA MATTHEW

7899 [269CM] P.SYN

$\mathrm{MCA}$

ST JOHN GP

[ST JOHN, ACADIAN]

PORTLAND,ST JOHN,NB

O2 13; MATTHEW GF.TRANS ROY SOC CAN, VOL 5,SECT 4, 1888,P119-121,PL3,FIG 1

REMARKS: C-GF MATTHEW:D-MACKENZIE ;PART OF THIS SPEC DESTROYED IN A FIRE IN ST JOHN:LABELLED HOLOTYPE IN OLD RECORDS ; 1 SPEC (INCOMPLETE) 
PARADOXIDES REGINA MATTHEW

26212 P.SYN

$M C A$.

ST JOHN GP

[ST JOHN,ACADIAN,DIV $1 \mathrm{C}$ ]

ST JOHN.NB

O213:MATTHEW GF, TRANS ROY SOC CAN, VOL 5,SECT 4,1888,P119-121

REMARKS: C-GF MATTHEW:D-MACKENZIE :LABELLED

PARATYPES IN OLD RECORDS; THESE SPECS NOT FIG 'D 6 PCES

\section{PARADOXIDOID TRILOBITE}

8195 [565CM ] REF SPEC-F

CA.

[LETCHEMINIAN DIVE2A]

YOUNG PT,GEORGE R,NS

O2 13:MATTHEW GF GSC, 1903,P176,PL11,FIG 1A-E

REMARKS: C-GF MATTHEW;D-MACKENZIE ; 4 SPEC

PELTURA SCARABEOIDES (WAHLENBERG)

8001 [371CM] REF SPEC?

LO,

[BRETONIAN,L ORDOVICIAN]

MCADAM'S BK, ESCASONIE,CAPE BRETON,NS

O264:MATTHEW GF, GSC, 1903,P230

REMARKS:C-GF MATTHEW;D-MACKENZIE :LABELLED

PLESIOTYPES;NOT FIG'D : 8 SPEC

PROETUS CRASSIMARGINATUS HALL

22504 [256S ] REF SPEC-F

$M D$ ERIAN

DETROIT RIVER GP,AMHERSTBURG FM

[UMONROE]

LIVINGSTONE CUT,DETROIT R, AMHERSTBERG ONT

O384:GRABAU AW,MICH GEOL \& BIOL SUR,PUB 2, GEOL SER 1,1909,P207,PL20,FIG 18

REMARKS: D-T NATTRESS, $1914 ; 1$ SPEC

PROETUS CRASSIMARGINATUS HALL

22457 [208S ] REF SPEC-F

MD.ERIAN

DETROIT RIVER GP,AMHERSTBURG FM

[AMHERSTBERG,UMONROE

LIVINGSTONE CUT,DETROIT R , AMHERSTBERG, ONT O384;GRABAU AW, MICH GEOL \& BIOL SUR,PUB 2,GEOL

SER 1, 1909,P207,PL20,FIG 16

REMARKS: D-T NATTRESS, $1914 ; 1$ SPEC

PROETUS CRASSIMARGINATUS HALL

22502 [254S ] REF SPEC-F

$M D E R I A N$

DETROIT RIVER GP,AMHERSTBURG FM

[UMONROE]

LIVINGSTONE CUT,DETROIT R, AMHERSTBERG, ONT

O384; GRABAU AW, MICH GEOL \& BIOL SUR,PUB 2, GEOL

SER 1.1909,P207.PL20.FIG 17

REMARKS: D-T NATTRESS, $1914 ; 1$ SPEC

PROETUS SP

12977 [2367D ] REF SPEC-F

$M D$,

KWATABOAHEGAN R,ONT

O384:PARKS WA, ONT BUR MINES, VOL

13,PT1,1904,P191,PL8,FIG 1

REMARKS: C-WA PARKS, $1903 ; 1$ SPEC

PROTAGRAULOS PRISCUS MATTHEW

7809 [179CM]P-SYN

LCA.

ST JOHN GP

[ST JOHN. ACADIAN.DIV 1B3]

HANFORD BK, ST MARTINS.ST JOHN CO,NB

O207. MATTHEW GF, TRANS NY ACAD SCI, VOL 14, 1895,P139,PL9,FIG 1

REMARKS: C-GF MATTHEW:D-MACKENZIE, LABELLED COTYPES:UNCERTAIN WHICH SPEC FIG'D ; 4 SPEC
PROTOLENUS BITUBERCULATUS MATTHEW

8322 [692CM] P-SYN?

LCA.

ST JOHN GP

[ST JOHN,ACADIAN,DIV 1B3]

HANFORD BK ST MARTINS ST JOHNCONB

REMARKS: C-GF MATTHEW;D-MACKENZIE ; LABELLED COTYPES IN OLD RECORDS;REFERENCE NOT KNOWN ; 2 SPEC

PROTOLENUS ELEGANS MATTHEW

REMARKS: SEE BERGERONIA ELEGANS (MATTHEW)

PROTOLENUS PARADOXOIDES MATTHEW

8321 [691CM] P-SYN

$\mathrm{MCA}$.

ST JOHN GP

[ST JOHN,ACADIAN

HANFORD BK, ST MARTINS,ST JOHN CO,NB

O211; MATTHEW GF, TRANS ROY SOC CAN, VOL 11,SECT 4.1894 P 101-102 PL 17 FIG 10A B

REMARKS: C-GFMATTHEW:D-MACKENZIE :LABELLED COTYPES:UNCERTAIN WHICH SPECS FIG'D : 16 SPEC

PROTOPELTURA ACANTHURA VAR TETRACANTHURA M 7995 [365CM ] P-SYN

$\cup C A$,

[BRETONIAN,L ORDOVICIAN,DIV 3B]

NAVYIS.ST JOHN NB

O266:MATTHEW GF, TRANS ROY SOC CAN, VOL 9 ,SECT 4,1892,P53,PL 13,FIG 8A-C

REMARKS: C-GF MATTHEW:D-MACKENZIE :SPEC ' $C$ ' $=F I G$ $8 A ; S^{S P E C}{ }^{\prime} B^{\prime}=F I G ~ 8 B ; S P E C ~ ' D '=F I G ~ 8 C ; 5$ SPEC

PSEUDOGYGITES LATIMARGINATUS (HALL)

27759 REF SPEC-F

M O,CINCI,EDEN

COLLINGWOODFM

[CRAIGLEITH FM]

CRAIGLEITH,ONT

O343;FRITZ MA,CAN JOUR ZOOL,VOL 37, 1959,P1117.

1120,PL1,FIG 1,PL2,FIG

REMARKS: C-MA FRITZ, $1959 ; 1$ SPEC

PTYCHAGNOSTUS CICEROIDES (MATTHEW

26117 P-LECTO

AGNOSTUS LAEVIGATUS VAR CICEROIDES MATTHEW

$M C A$.

ST JOHN GP

[ST JOHN,ACADIAN,DIV 1D]

HIGHLAND COVE TRINITY BAY NFLD

0179:MATTHEW GF, NY, 1896,P234,PL 17, FIG 3A,B;

HUTCHINSON RD,GSC BULL 88, 1962,P85-86, PL9, FIG 20

REMARKS: C-GF MATTHEW:D-MACKENZIE :DESIGNATED LECTOTYPE BY HUTCHINSON:WAS ON SLAB WITH NO $8202 ; 1$ SPEC

PTYCHAGNOSTUS CICEROIDES (MATTHEW)

8202 [572CM] P.PARAL

AGNOSTUS LAEVIGATUS VAR CICEROIDES MATTHEW

$M C A$,

ST JOHN GP

[ST JOHN, ACADIAN,DIV 1D2]

HASTINGS COVE. TRINITY BAY,NFLD

0179;MATTHEW GF, NY, 1896,P234,PL 17, FIG 3B

HUTCHINSON RD GSC BULL 88, 1962,P85-86

REMARKS: C-GF MATTHEW;D-MACKENZIE;LECTOTYPE DESIGNATED BY HUTCHINSON,NO $26117 ; 1$ SPEC

PTYCHAGNOSTUS PUNCTUOSUS (ANGELIN)

27292 REF SPEC-M

AGNOSTUS LAEVIGATUS VAR TERRANOVICUS MATTHEW

$M C A$.

ST JOHN GP

[ST JOHN ACADIAN DIV 1D]

HIGHLAND COVE, TRINITY BAY, NFLD

0 179:HUTCHINSON RD.GSC BULL 88, 1962,P85

REMARKS:C-GF MATTHEW-D.MACKENZIE-THIS SPEC WAS ORIGINALLY ON A SLAB WITH 2 OTHER SPECS, COTYPES OF PTYCHAGNOSTUS CICEROIDES (MATTHEW),NO 8202; REIDENTIFIED BY HUTCHINSON : 1 SPEC 
PTYCHOPARIA ADAMSI (BILLINGS) NARROW FORM 8373 [743CM ] REF SPEC-F

$M C A$,

ST JOHN GP

[ST JOHN,ACADIAN,DIV 1D3 ]

HASTINGS COVE, KINGS CO,NB

O233; MATTHEW GF, TRANS ROY SOC CAN, VOL 3,SECT

4,1897,P182,PL3,FIG 2A,B

REMARKS: C-GF MATTHEW:D-MACKENZIE ; 5 SPEC

PTYCHOPARIA LINNARSSONI (BROGGER)

8205 [575CM] REF SPEC-F

MCA.

ST JOHN GP

[ST JOHN,ACADIAN,DIV 1D]

PORTER'S BK, ST MARTINS,ST JOHN CO,NB

O233: MATTHEW GF,TRANS ROY SOC CAN, VOL 5,SECT 4,1888,P143-147,PL2,FIG 1A-M

REMARKS: C-GF MATTHEW:D-MACKENZIE ;LABELLED

PLESIOTYPES;UNCERTAIN WHICH SPECS FIG'D; 9 SPEC

\section{PTYCHOPARIA LINNARSSONI VAR ALATA MATTHEW}

7867 [237CM ] P-SYN

$\mathrm{MCA}$,

ST JOHN GP

[ST JOHN,ACADIAN ]

PORTER'S BK, ST MARTINS,ST JOHN CO,NB

O233:MATTHEW GF,TRANS ROY SOC CAN, VOL 5,SECT 4,1888,P147-149,PL2,FIG 2A-F

REMARK S: C-GF MATTHEW;D-MACKENZIE ;LABELLED COTYPES;UNCERTAIN WHETHER THESE SPECS FIG'D ; 25 SPEC

\section{PTYCHOPARIA LINNARSSONI VAR ALATA MATTHEW}

8325 [695CM ] P-SYN

$\mathrm{MCA}$,

ST JOHN GP

[ST JOHN,ACADIAN,DIV 1D ]

PORTER'S BK, ST MARTINS,ST JOHN CO,NB

O233:MATTHEW GF,TRANS ROY SOC CAN, VOL 5,SECT 4, 1888,P147-149,PL2,FIG 2A-F

REMARKS: C-GF MATTHEW;D-MACKENZIE ;LABELLED COTYPES;UNCERTAIN WHETHER THESE SPECS FIG'D ; 8 SPEC

PTYCHOPARIA LINNARSSONI VAR ALATA MATTHEW

7856 [226CM ] P-SYN

$M C A$,

ST JOHN GP

[ST JOHN, ACADIAN,DIV 1D]

PORTER'S BK, ST MARTINS,ST JOHN CO,NB

O233; MATTHEW GF,TRANS ROY SOC CAN, VOL 5,SECT 4,1888,P147-149,PL2,FIG 2A-F

REMARKS: C-GF MATTHEW:D-MACKENZIE ;LABELLED COTYPES:UNCERTAIN WHETHER THESE SPECS FIG'D ; 11 SPEC

PTYCHOPARIA MINORA MATTHEW

$8335[705 \mathrm{CM}]$ P-SYN?

$\mathrm{MCA}$,

ST JOHN GP

[ST JOHN,ACADIAN]

PORTER'S BK, ST MARTINS,ST JOHN CO,NB

REMARK S: C-GF MATTHEW;D-MACKENZIE ;LABELLED COTYPES;REFERENCE NOT KNOWN ; 8 SPEC

\section{? SCUTELLUM REGALE FRITZ}

28075 P-HOLO

MS

[LOWER MIDDLE SILURIAN]

MOUTH OF OWL R,MAN

O369;FRITZ MA,PROC GAC,VOL 15,PT2, 1964,P91-95,FIG 1,PL 1,FIG 1,PL2,FIG 1,2

REMARKS: C-G MARLBOROUGH, 1962; 1 SPEC (CAST\& MOULD)
SENELOPLEURA BOMBIFRONS MATTHEW

$7790[160 \mathrm{CM}] \mathrm{P}-\mathrm{HOLO}$

LCA.

[U ETCHEMINIAN]

TOPSAIL HEAD, CONCEPTIONBAY,NFLD

O275; MATTHEW GF, TRANS ROY SOC CAN, VOL 4,SECT

4,1887,P156-157,FIG 5,5A,

REMARKS: C-GF MATTHEW;D-MACKENZIE ; 1 SPEC

SOLENOPLEURA ACADICA WHITEAVES

$7885[255 \mathrm{CM}$ ] REF SPEC-F

$\mathrm{MCA}$,

ST JOHN GP

[ST JOHN, ACADIAN,DIV 1D]

PORTER'S BK, ST MARTINS,ST JOHN CO,NB

O275:MATTHEW GF, TRANS ROY SOC CAN, VOL 5, SECT 4,1888,P157-159,PL2,FIG 5A,B

REMARKS: C-GF MATTHEW;D-MACKENZIE ; LABELLED

PLESIOTYPES IN OLD RECORDS ; 10 SPEC

SOLENOPLEURA ACADICA WHITEAVES

8130 [500CM] REF SPEC-M

MCA,

ST JOHN GP

[ST JOHN,ACADIAN]

PORTER'S BK, ST MARTINS,ST JOHN CO,NB

O275:MATTHEW GF, TRANS ROY SOC CAN, VOL 5,SECT 4, 1888,P $157-159$

REMARKS: C-GF MATTHEW:D-MACKENZIE :LABELLED

PLESIOTYPE IN OLD RECORDS ; 1 SPEC

SOLENOPLEURA ARENOSA VAR ANGILIMBATA MATTHEW 8328 [698CM ] P-HOLO

$\mathrm{MCA}$,

ST JOHN GP

[ST JOHN,ACADIAN]

HASTINGS COVE, KINGS CO,NB

O275:MATTHEW GF, TRANS ROY SOC CAN, VOL 3,SECT

4,1897,P 183,PL3,FIG 3A,B

REMARKS: C-GF MATTHEW;D-MACKENZIE ; 1 SPEC

SOLENOPLEURA BRETONENSIS MATTHEW

26216 P-SYN

$\mathrm{MCA}$,

BOURINOT GP.DUGALD FM

[U ETCHEMINIAN]

DUGALD BK, ESCASONIE,CAPE BRETON,NS

O275:MATTHEW GF,GSC, 1903,P 176-177,PL 11,FIG 2B,C

REMARKS: C-GF MATTHEW:D-MACKENZIE :SPECS 'E' 'C' = FIGS 2B,C;STRAT REV AS IN HUTCHINSON, 1952 , GSC MEM 263;2 SPEC

SOLENOPLEURA BRETONENSIS MATTHEW

7773 [143CM ] P-SYN

$\mathrm{MCA}$

[UETCHEMINIAN]

GILLIS,INDIAN BK, ESCASONIE,CAPE BRETON,NS

O275:MATTHEW GF, GSC, 1903,P176-177,PL 11,FIG 2A

REMARKS: C-GF MATTHEW:D-MACKENZIE ;SPEC 'B' FIG'D 2 SPEC

SOLENOPLEURA ROBBII (HARTT)

7853 [223CM] REF SPEC-F

$\mathrm{MCA}$

ST JOHN GP

[ST JOHN,ACADIAN

SEELY ST,ST JOHN \& HANFORD BK,ST MARTINS, ST JOHN CONB

O275:MATTHEW GF,TRANS ROY SOC CAN,VOL 5,SECT

4,1888,P 153-157,PL2,FIG 3G-L

REMARKS: C-GF MATTHEW:D-MACKENZIE :LABELLED PLESIOTYPES ; 7 SPEC

SOLENOPLEURA ROBBII MUT PARVA (HARTT)

8303 [673CM] REF SPEC-F

$M C A$

ST JÖHN GP

[ST JOHN,ACADIAN,DIV 1D3]

HASTINGS COVE, KINGS CO NB

O275; MATTHEW GF, TRANS ROY SOC CAN,VOL 3,SECT 
4. 1898,P 183.PL3,FIG 4A.B

REMARKS: C-GF MATTHEW;O-MACKENZIE ;UNCERTAIN WHICH SPECS FIG'D; 4 SPEC

SOLENOPLEURA ROBBII VAR ORESTES (HARTT)

7832 [202CM ] REF SPEC-F

$\mathrm{MCA}$,

ST JOHN GP

[ST JOHN, ACADIAN.DIV 1C]

RATCLIFFE STREAM, SIMOND,NB

O275:MATTHEW GF, TRANS ROY SOC CAN, VOL 5,SECT 4.1888,P154-155,PL2,FIG 4A-E

REMARKS: C-GF MATTHEW:D-MACKENZIE :LABELLED PLESIDTYPES;UNCERTAIN WHICH SPECS FIG'D;LOC IN RECOROS IS 'SEELY ST,ST JOHN \& RATCLIFFE STREAM'; ST JOHN NOT MENTIONED IN PUBL' $N$; 5 SPEC

SOLENOPLEURA ROBBII VAR ORESTES (HARTT)

7846 [216CM ] REF SPEC-F

$\mathrm{MCA}$.

ST JOHN GP

[ST JOHN, ACADIAN]

HANFORD BK, ST MARTINS, ST JOHN CO,NB

O275:MATTHEW GF, TRANS ROY SOC CAN, VOL 5,SECT 4, 1888,P 154-155,PL2,FIG 4A-E

REMARKS: C-GF MATTHEW;D-MACKENZIE ;LABELLED PLESIOTYPES;UNCERTAIN WHICH SPECS FIG'D : 10 SPEC

SPHAEROPHTHALMUS ALATUS VAR CANADENSIS MATTHEW 8461 [831CM] P-SYN

UCA, ORLO

[BRETONIAN.LORDOVICIAN

KING ST.ST JOHN,NB

O264;MATTHEW GF.TRANS ROY SOC CAN,VOL 11,SECT 4, 1894,P107-109, PL 17,FIG 12A,B

REMARKS: C-GF MATTHEW:D-MACKENZIE ;LABELLED

COTYPES:UNCERTAIN WHICH SPECS FIG'D; 19 SPEC

SPHAEROPHTHALMUS ALATUS VAR CANADENSIS MATTHEW 7997 [367CM] P-SYN

UCA, ORLO.

[BRETONIAN,L ORDOVICIAN]

KING ST,ST JOHN,NB

O264:MATTHEW GF,TRANS ROY SOC CAN,VOL 11,SECT 4, 1894,P107-109,PL17,FIG 11A.B

REMARKS: C-GF MATTHEW:D-MACKENZIE ;LABELLED COTYPES;UNCERTAIN WHICH SPECS FIG'D; 5 SPEC

STRENUELLA ATTLEBORENSIS MUT VIGILANS MATT.

$7764[134 \mathrm{CM}]$ P-HOLO

LCA,

[UETCHEMINIAN]

MANUEL'S BK, CONCEPTION BAY NFLD

O207; MATTHEW GF, TRANS ROY SOC CAN, VOL 5,SECT 4.1899,P78-79,PL4,FIG 4A,B

REMARKS: C-GF MATTHEW;D-MACKENZIE; 1 SPEC

STRENUELLA? HALLIANA MATTHEW

7842 [212CM ] P-SYN

$\mathrm{MCA}$

ST JOHN GP

[ST JOHN,ACADIAN,DIV 1C2]

HANFORD BK, ST MARTINS.ST JOHN CO.NB

O207:MATTHEW GF, TRANS ROY SOC CAN, VOL 5,SECT 4. 1888, P132-133, PL 1,FIG 2A-M

REMARKS: C-GF MATTHEW:D-MACKENZIE ;LABELLED COTYPES ; 6 SPEC

TRIARTHRUS CANADENSIS SMITH

25094 [484U] REF SPEC-F

UO.CINCI.EDEN

GLOUCESTER FM

[GLOUCESTER]

EAST BRANCH ROUGE R.ONT

O267, PARKS WA, TRANS ROY SOC CAN, VOL 22, SECT

41928 PQO FIG 27

REMARK S: C-WA PARKS, 1926, 1 SPEC
TRIARTHRUS CANADENSIS SMITH

25180 [801U] REF SPEC-F

U O.CINCI,EDEN

GLOUCESTER FM

[GLOUCESTER]

E BRANCH,W SIDE,3/4 MI ABOVE KINGSTON RD BRIDGE ROUGE R,ONT

O267:PARKS WA, TRANS ROY SOC CAN, VOL 15,SECT

4, 1921,P49-50,PL1,FIG 4

REMARKS: C-WA PARKS, 1920 ;CRANIDIUM ; 1 SPEC

TRIARTHRUS CANADENSIS SMITH

25181 [802U] REF SPEC-F

U O,CINCI,EDEN

GLOUCESTER FM

[GLOUCESTER]

E BRANCH,W SIDE,3/4 MI ABOVE KINGSTON RD BRIDGE ROUGE R.ONT

O267:PARKS WA, TRANS ROY SOC CAN, VOL 15.SECT

4,1921,P51,PL1,FIG 10

REMARKS: C-WA PARKS, 1920 ;PYGIDIUM ; 1 SPEC

TRIARTHRUS CANADENSIS SMITH

25182 [803U] REF SPEC-F

U O,CINCI,EDEN

GLOUCESTER FM

[GLOUCESTER ]

W BRANCH, ROUGE R,ONT

O267:PARKS WA,TRANS ROY SOC CAN, VOL 15,SECT 4,1921,P49,PL1,FIG 3

REMARKS: C-WA PARKS, 1920 ;FREE CHEEK ; 1 SPEC (CAST\&MOULD)

TRIARTHRUS CANADENSIS SMITH 25183 [804U] REF SPEC-F

U O,CINCI,EDEN

BLUE MOUNTAIN FM

[BLUE MT]

CRAIGLEITH,ONT

O267:PARKS WA, TRANS ROY SOC CAN, VOL 15,SECT 4,1921,P49,PL1,FIG 1

REMARKS: C-WA PARKS, 1920 ;FREE CHEEK ; 1 SPEC

TRIARTHRUS CANADENSIS SMITH

25188 [815U] REF SPEC-F

U O,CINCI,EDEN

GLOUCESTER FM

[GLOUCESTER]

E BRANCH,W SIDE,3/4 MI ABOVE KINGSTON RD BRIDGE ROUGE R,ONT

O267:PARKS WA, TRANS ROY SOC CAN, VOL 15,SECT

4, 1921,P48-49,PL 1,FIG 2

REMARKS: C-WA PARKS, $1920 ; 1$ SPEC (CAST\&MOULD)

TRIARTHRUS CF CANADENSIS,CF GLABER

25096 [486U] REF SPEC-F

U O CINCI EDEN

GLOUCESTER FM

[GLOUCESTER]

WHITBY,ONT

O267:PARKS WA, TRANS ROY SOC CAN, VOL 22, SECT 4, 1928,P90,FIG 28

REMARKS: C-WA PARKS, 1926 ;SPEC REFERRED TO BOTH SPECIES;T. CF CANADENSIS BILLINGS,T. CF GLABER SMITH ; 1 SPEC

TRIARTHRUS EATONI HALL

25097 [487U] REF SPEC-F

U O.CINCI,EDEN

COLLINGWOOD FM

[COLLINGWOOD]

CAMPERDOWN ONT

O267:PARKS WA, TRANS ROY SOC CAN,VOL 22,SECT

4, 1928,P88,FIG 24

REMARK S: C-WA PARKS. $1927 ; 1$ SLAB 
TRIARTHRUS GLABER BILLINGS

25095 [485U] REF SPEC-F

L GLOUCESTER FM

[L GLOUCESTER]

WHITBY,ONT

O267:PARKS WA, TRANS ROY SOC CAN, VOL 22,SECT

4, 1928,P89,FIG 26

REMARKS: C-WA PARKS, $1926 ; 1$ SPEC

TRIARTHRUS SPINOSUS VAR NARRAWAYI PARKS

18802 P-HOLO

U O,CINCI,EDEN

UGLOUCESTER FM

[U GLOUCESTER]

BILLINGS BRIDGE,OTTAWA,ONT

O267:PARKS WA, TRANS ROY SOC CAN, VOL 22,SECT 4, 1928,P89

REMARKS: P-JE NARRAWAY COLLN, 1938 ;NOT FIG'D ; 1 SPEC

25189 [816U] P-PARA

U O CINCI,EDEN

GLOUCESTER FM

[GLOUCESTER]

E BRANCH,W SIDE, 3/4 MI ABOVE KINGSTON RD BRIDGE, ROUGE R,ONT

O267; PARKS WA, TRANS ROY SOC CAN, VOL 15,SECT

4, 1921,P51,PL1,FIG 11

REMARKS: C-WA PARKS, 1920 ;PYGIDIUM ; 1 SPEC

TRIARTHRUS SPINOSUS ROUGENSIS PARKS

25093 [483U] REF SPEC-F

$\mathrm{U} O, \mathrm{CINCI}, \mathrm{EDEN}$

GLOUCESTER FM

[GLOUCESTER]

EAST BRANCH, ROUGE R,ONT

O267; PARKS WA,TRANS ROY SOC CAN, VOL 22,SECT

4, 1928,P89,FIG 25

REMARKS: C-WA PARKS, $1926 ; 1$ SPEC

TRIARTHRUS SPINOSUS ROUGENSIS PARKS

$25170[789$ U] P-HOLO

U O,CINCI,EDEN

GLOUCESTER FM

[GLOUCESTER]

E BRANCH,W SIDE,3/4 MI ABOVE KINGSTON RD BRIDGE, ROUGE R.ONT

O267;PARKS WA,TRANS ROY SOC CAN,VOL 15,SECT

4,1921,P51,PL1,FIG 8

REMARKS: C-WA PARKS, $1920 ; 1$ SPEC

TRIARTHRUS SPINOSUS ROUGENSIS PARKS

25171 [790U] P-PARA

U O,CINCI,EDEN

GLOUCESTERFM

[GLOUCESTER]

E BRANCH,W SIDE,3/4 MI ABOVE KINGSTON RD BRIDGE, ROUGE R,ONT

O267; PARKS WA,TRANS ROY CAN INST, VOL 15, SECT

4,1921,P51,PL 1,FIG 7

REMARKS: C-WA PARKS, 1920 ;CRANIDIUM ; 1 SPEC
U O.CINCI,EDEN

TRIARTHRUS SPINOSUS ROUGENSIS PARKS

APARCHITES SECUNDA MATTHEW

REMARKS: SEE INDIANA SECUNDA (MATTHEW)

APARCHITES ? ROBUSTUS MATTHEW

REMARKS: SEE BRADORIA ROBUSTA (MATTHEW)

BEYRICHIA PRIMAEVA MATTHEW

REMARKS: SEE BRADORIA PRIMAEVA (MATTHEW)

BEYRICHIA TRICEPS MATTHEW

REMARKS: SEE BEYRICHONA TRICEPS (MATTHEW)

BEYRICHONA OVATA MATTHEW

7767 [137CM] P-HOLO

LCA

ST JOHN GP

[ST JOHN, HANFORDIAN,DIV C1B2]

HANFORD BK, ST MARTINS,ST JOHN CONB

Q 102;MATTHEW GF,NY, 1895,P 135,PL7,FIG 8;ULRICH \&

BASSLER,PROC USNM, VOL 78,ART 4, 1931,P47,PL7,FIG 10

REMARKS: C-GF MATTHEW;D-MACKENZIE ; 1 SPEC

BEYRICHONA OVATA MATTHEW

26287 REF SPEC?

LCA,

ST JOHN GP

[ST JOHN, HANFORDIAN,DIV C 1B2]

HANFORD BK, ST MARTINS, ST JOHN CO,NB

Q 102; ULRICH \& BASSLER,PROC USNM,VOL 78,ART $4,1931, P 47$

REMARKS: C-GF MATTHEW:D-MACKENZIE :ENTEREDIN OLD RECORDS AS AN ONOMATYPE; 1 SPEC

BEYRICHONA PAPILIO MATTHEW

7744 [114CM ] P-HOLO

$\mathrm{LCA}$,

ST JOHN GP

[ST JOHN,HANFORDIAN,DIV C1B4]

HANFORD BK, ST MARTINS, ST JOHN CO, NB

Q 102; MATTHEW GF,TRSC, 1886,P65,PL6,FIG 20,20A,B;

ULRICH \& BASSLER,PRO'C USNM,VOL 78,ART

4, 1931,P43,PL7,FIG 5

REMARKS: C-GF MATTHEW;D-MACKENZIE;STRAT REV

FROM C1B2 TOC1B4 BY MATTHEW, $1895 ; 1$ SPEC

(CAST\&MOULD)

BEYRICHONA PLANATA MATTHEW

REMARKS: SEE BEYRICHONA TINEA PLANATA MATTHEW

BEYRICHONA ROTUNDATA MATTHEW

7750 (B) [120CM(B)] P-PARAL

LCA,

ST JOHNGP

[ST JOHN,HANFORDIAN,DIV C1B2]

HANFORD BK, ST MARTINS, ST JOHN CO,NB

Q 102; MATTHEW GF,NY, 1895,P136,PL7,FIG 9;ULRICH \&

BASSLER,PROC USNM, VOL 78,ART 4, 1931,P46

REMARKS: C-GF MATTHEW;D-MACKENZIE :SPEC (A)

DESIGNATED LECTOTYPE;SEE NO 26290-B. CF TINEA ; 1 SPEC

BEYRICHONA ROTUNDATA MATTHEW

REMARKS: SEE BEYRICHONA CF TINEA MATTHEW

\section{ARTHROPODA,OSTRACODA}

\section{ALUTA FLEXILIS MATTHEW}

8112 [482CM] P-HOLO

$M C A$

ST JOHN GP

[ST JOHN ACADIAN DIV 1C1]

PORTER'S BK, ST MARTINS, ST JOHNCO,NB

Q 102; MATTHEW GF, NY, 1896,P 198,PL 15, FIG 4;ULRICH \&

BASSLER,PROC USNM, VOL 78,ART 4,1931,P52,PL8,FIG 13,14

REMARKS: C-GF MATTHEW;D-MACKENZIE ; 1 SPEC

\section{BEYRICHONA ROTUNDATA MATTHEW}

$7750(A)[120 C M(A)]$ P-LECTO

LCA,

ST JOHN GP

[ST JOHN, HANFORDIAN, DIV C1B2]

HANFORD BK, ST MARTINS,ST JOHN CO,NB

Q102; MATTHEW GF,NY, 1895.P 136.PL7,FIG 9;ULRICH \& BASSLER,PROC USNM, VOL 78,ART 4, 1931,P46,PL6,FIG I2',PL7, FIG 11,12

REMARKS: C-GF MATTHEW:D-MACKENZIE :DESIGNATED LECTOTYPE BY ULRICH + BASSLER;SEE NO 26290,B. CF TINEA ; 1 SPEC 
BEYRICHONA TINEA MATTHEW

$7748[118 \mathrm{CM}]$ P.SYN

LCA

ST JOHN,HANFORDIAN,DIV C 184

[ST JOHN.HANFORDIAN.DIV C 1B2]

HANFORD BK ST MARTINS ST JOHNCO NB

O 102:MATTHEW GF, 1886,P66,PL6.FIG 21,21A,B;ULRICH \& BASSLER,PROC USNM, VOL 78,ART 4, 1931,P43-

44. PL7.FIG 4

REMARKS: C-GF MATTHEW;D-MACKENZIE ;SPEC ' $B$ ' IS CAST + MOULD;UNCERTAIN WHICH SPEC IS FIG'D + DESIGNATED LECTOTYPE BY ULRICH + BASSLER: STRAT REV FROM C $1 B 2$ TO C 184 BY MATTHEW, $1895 ; 5$ SPEC

\section{BEYRICHONA CF TINEA MATTHEW}

26290 REF SPEC-M

BEYRICHONA ROTUNDATA MATTHEW

LCA.

ST JOHNGP

[ST JOHN,HANFORDIAN,C $1 B 3$

HANFORD BK, ST MARTINS,ST JOHN CO,NB

Q 102; ULRICH \& BASSLER,PROC USNM,VOL 78,ART 4, 1931,P46

REMARKS: C-GF MATTHEW:D-MACKENZIE ;ORIGINALLY WITH SYNTYPES OF B. ROTUNDATA, NO 7750; IDENT REVISED BY ULRICH \& BASSLER; 1 SPEC

BEYRICHONA TINEA PLANATA MATTHEW

8243 (A) [613CM(A)] P-PARAL

BEYRICHONA PLANATA MATTHEW

LCA.

ST JOHN GP

IST JOHN.HANFORDIAN,C1B2

HANFORD BK, ST MARTINS,ST JOHN CO.NB

O 102:MATTHEW GF,NY, 1895,P 134-135; ULRICH \&

BASSLER,PROC USNM, VOL 78,ART 4,1931,P45 46,PL7,FIG 2

REMARKS: C-GF MATTHEW; D-MACKENZIE ;SPECS 'A', 'C' 'D' OF MATTHEW'S SYNTYPIC SUITE;SPEC 'D' FIG'D ; 3 SPEC

BEYRICHONA TINEA PLANATA MATTHEW

$8243(B)[613 \mathrm{CM}(\mathrm{B})]$ P-LECTO

BEYRICHONA PLANATA MATTHEW

LCA.

ST JOHNGP

[ST JOHN.HANFORDIAN C1B2

HANFORD BK, ST MARTINS, ST JOHN CO,NB

O 102:MATTHEW GF,NY, 1895,P 134-135,PL 7, FIG 7; ULRICH \& BASSLER,PROC USNM,VOL 78,ART 4,1931,P4546,PL7.FIG 1,3

REMARKS: C-GF MATTHEW;D-MACKENZIE ;DESIGNATED LECTOTYPE BY ULRICH + BASSLER;SPEC 'B' OF MATTHEW'S SYNTYPIC SUITE; 1 SPEC

BEYRICHONA TINEA TRIANGULA MATTHEW

$7749[119 \mathrm{CM}]$ P-SYN

BEYRICHONA TRIANGULA MATTHEW

LCA.

ST JOHN GP

[ST JOHN HANFORDIAN C 183

HANFORD BK, ST MARTINS,ST JOHN CO,NB

Q 102;MATTHEW GF,NY, 1895,P 135,PL7,FIG 5; ULRICH \&

BASSLER,PROC USNM.VOL 78,ART 4, 1931,P44,PL 7,FIG 8

REMARKS: C-GF MATTHEW;D-MACKENZIE ; SPEC ' $A$ ' OF

MATTHEW'S SYNTYPIC SUITE;SEE ALSO NO 26288,B.

TRIANGULA ; 1 SPEC

BEYRICHONA TRIANGULA MATTHEW

26288 P-SYN

LCA.

ST JOHN GP

[ST JOHN,HANFORDIAN,C 183

HANFORD BK, ST MARTINS,ST JOHN CO,NB

0102:MATTHEW GF.NY, 1895,P135.PL7, FIG 5:ULRICH \& BASSLER,PROC USNM,VOL 78,ART 4, 1931,P43,PL7,FIG 6.7

REMARKS: C-GF MATTHEW:D-MACKENZIE ;SPECS 'B', 'C OF MATTHEW'S SYNTYPIC SUITE FOR B. TRIANGULA; IDENT REV BY ULRICH + BASSLER TO P. PAPILIO MATTHEW : 2 SPEC
BEYRICHONA TRIANGULA MATTHEW

REMARKS: SEE ALSO BEYRICHONA TINEA TRIANGULA MATTHEW

BEYRICHONA TRICEPS (MATTHEW)

$7690[60 \mathrm{CM}]$ ] -SYN

BEYRICHIA TRICEPS MATTHEW

$M C A$,

ST JOHN GP

[JOHANNIAN.DIV C2B]

MCLEAN BK, NR MIRA R,CAPE BRETON,NS

O102:MATTHEW GF, GSC, 1903,P2 19-220.PL 16. FIG 4A-C.

ULRICH \& BASSLER,PROC USNM, VOL 78,ART 4,1931,P46-47

REMARKS: C-GF MATTHEW:D-MACKENZIE :UNCERTAIN WHETHER THIS SPEC FIG'D; 1 SPEC

BEYRICHONA TRICEPS (MATTHEW)

8535 [905CM] P-SYN

BEYRICHIA TRICEPS MATTHEW

$M C A$,

ST JOHN GP

[JOHANNIAN]

MCLEAN BK, NR MIRA R,CAPE BRETON,NS

O 102; MATTHEW GF, GSC, 1903,P219-220,PL 16,FIG 4A-C; ULRICH \& BASSLER,PROC USNM, VOL 78,ART 4.1931,P46-47

REMARKS: C-GF MATTHEW;D-MACKENZIE ;LABELLED COTYPE IN OLD RECORDS ; 1 SMALL SLAB

BEYRICHONA TRICEPS (MATTHEW)

7687 [57CM] P-SYN

BEYRICHIA TRICEPS MATTHEW

$M C A$,

ST JOHN GP

[JOHANNIAN,DIV C2B]

MCLEAN BK, NR MIRA R,CAPE BRETON,NS

Q 102:MATTHEW GF, GSC, 1903,P2 19-220 PL 16 FIG 4A-C: ULRICH \& BASSLER,PROC USNM, VOL 78,ART 4,1931,P46-47

REMARKS: C-GF MATTHEW:D-MACKENZIE :UNCERTAIN WHETHER THIS SPEC FIG'D ; 1 SPEC

BEYRICHONA TRICEPS (MATTHEW)

7689 [59CM] P-SYN

BEYRICHIA TRICEPS MATTHEW

MCA.

ST JO'HN GP

[JOHANNIAN, DIV C2B]

MCLEAN BK, NR MIRA R,CAPE BRETON,NS

Q102;MATTHEW GF, GSC, 1903,P2 19-220,PL 16,FIG 4A-C; ULRICH \& BASSLER,PROC USNM, VOL 78,ART 4.1931,P $46-47$

REMARKS: C-GF MATTHEW;D-MACKENZIE ;UNCERTAIN WHETHER THIS SPEC FIG'D; 1 SPEC

BEYRICHONA ? RUTELLUM (MATTHEW)

7785 [155CM]P-SYN

ESCASONA RUTELLUM MATTHEW

$M C A$

BOURINOT GP,DUGALD FM

[UETCHEMINIAN,E3F]

GILLIS, INDIAN BK,CAPE BRETON,NS

0102:MATTHEW GF 1902.P458.PL2 FIG 5A-C MATTHEW, 1903,P 167:ULRICH\&BASSLER,PROC USNM,VOL 78, 1931,P48,PL 1,FIG 32,PL3,FIG 21

REMARKS: C-GF MATTHEW:D-MACKENZIE :STRAT REV AS IN HUTCHINSON, 1952,GSC MEM 263; 1SPEC

BRADORIA ACUTA (MATTHEW)

7789 [159CM]P-SYN

SCHMIDTELLA ACUTA MATTHEW

$M C A$

BOURINOT GP,DUGALD FM

UU ETCHEMINIAN

DUGALD BK, ESCASONIE, CAPE BRETON.NS

O 102; MATTHEW GF, 1899,P206-207,PL4,FIG 4A-C: 1903,P 173-174; ULRICH \& BASSLER, 193 1,P 19-20,PL 1, FIG 25

REMARKS: C-GF MATTHEW:D-MACKENZIE ; UNCERTAIN WHICH SPEC FIG'D; STRAT REV AS IN HUTCHINSON, 1952.GSC MEM 263;2 SPEC 
BRADORIA BENEPUNCTA (MATTHEW)

$7701[71 \mathrm{CM}]$ P-SYN

BRADORONA OBSERVATOR VAR BENEPUNCTA MATTHEW $M C A$,

BOURINOT GP,DUGALD FM

[L ETCHEMINIAN,DIV E1D]

BOUNDARY BK, ESCASONIE,CAPE BRETON,NS

Q 102;MATTHEW GF, 1902,P449,PL1,FIG 16;

1903,P 161,PL 12,FIG 16:ULRICH \& BASSLER,PROC USNM, 1931,P 18,PL 1,FIG 16

REMARKS: C-GF MATTHEW;D-MACKENZIE ;SPEC 'B' FIG'D; STRAT REV AS IN HUTCHINSON, 1952, GSC MEM $263 ; 2$ SPEC

\section{BRADORIA CAMBRICA (MATTHEW)}

7745 [115CM ] P-SYN

SCHMIDTELLA CAMBRICA MATTHEW

$\mathrm{LCA}$,

ST JOHN GP

[ST JOHN, HANFORDIAN,C1B3]

HANFORD BK, ST MARTINS,ST JOHN CO,NB

Q 102:MATTHEW GF,NY, 1895,P 137,PL7,FIG 10A-B;ULRICH \& BASSLER,PROC USNM,VOL 78,ART 4,1931,P28,PL1,FIG 10

REMARKS: C-GF MATTHEW:D-MACKENZIE ;SPEC 'A' FIG'D 2 SPEC

\section{BRADORIA CONCINNA (MATTHEW)}

$7718[88 \mathrm{CM}]$ P-LECTO

SCHMIDTELLA? PERVETUS MUT CONCINNA MATTHEW $\mathrm{MCA}$,

BOURINOT GP.DUGALD FM

[LETCHEMINIAN,E1D]

BOUNDARY BK, ESCASONIE, CAPE BRETON,NS

Q 102; MATTHEW GF, 1902,P464-465;ULRICH \&

BASSLER,PROC USNM, VOL 78,ART 4,1931,P28,PL3,FIG 9

REMARKS: C-GF MATTHEW;D-MACKENZIE ;STRAT REV AS

IN HUTCHINSON, 1952,GSC MEM $263 ; 1$ SPEC

\section{BRADORIA MINOR (MATTHEW)}

8348 [718CM ] P-SYN

LEPERDITIA? MINOR MATTHEW

LCA

ST JOHNGP

[ST JOHN, HANFORDIAN,C1B3]

HANFORD BK, ST MARTINS,ST JOHN CO,NB

Q102:MATTHEW GF,NY 1895,P 138,PL8,FIG 4A-B;ULRICH

BASSLER,PROC USNM, VOL 78,ART 4, 1931,P29,PL 1,FIG 22

REMARKS: C-GF MATTHEW;D-MACKENZIE ; 1 SPEC

BRADORIA OBESA MATTHEW

8319 [689CM] P-HOLO

BRADORIA VIGILANS MUT OBESA MATTHEW

MCA.

BOURINOT GP.DUGALD FM

[L ETCHEMINIAN,E1B]

DUGALD BK , ESCASONIE,CAPE BRETON,NS

Q 102: MATTHEW GF, 1902,P455:ULRICH \& BASSLER PROC USNM, VOL 78,ART 4,1931,P27,PL1,FIG 27,PL3,FIG 13

REMARKS: C-GF MATTHEW;D-MACKENZIE ;STRAT REV AS IN HUTCHINSON, 1952,GSC MEM 263; 1 SPEC

BRADORIA OBESA MATTHEW

REMARKS: SEE ALSO BRADORIA VIGILANS MATTHEW,NO 26292

BRADORIA OCULATA (MATTHEW)

8347 [717CM ] P-HOLO

PRIMITIA OCULATA MATTHEW

LCA.

ST JOHN GP.DIV C1B3

[ST JOHN, HANFORDIAN, C 1B3]

HANFORD BK, ST MARTINS,ST JOHN CO,NB

Q102; MATTHEW GF, 1895,P 136,PL8,FIG 2A,B;

MATTHEW 1899,P204:ULRICH + BASSLER,PROC

USNM, VOL 78,ART 4,1931,P 16,PL1,FIG 13,14

REMARKS: C-GF MATTHEW;D-MACKENZIE; 1 SPEC
BRADORIA OVALIS (MATTHEW)

7717 [87CM]P-SYN

INDIANA OVALIS MATTHEW

MCA

BOURINOT GP,DUGALDFM

[L ETCHEMINIAN,DIV E1E]

DUGALD BK, ESCASONIE,CAPE BRETON,NS

Q 102; MATTHEW GF, 1902,P461-462,PL2,FIG 8A-C

1903,P 170-171;ULRICH \& BASSLER,PROC USNM VOL

78,ART 4,1931,P21,PL1,FIG 18

REMARKS: C-GF MATTHEW;D-MACKENZIE ;STRAT REV AS

IN HUTCHINSON, 1952, GSC MEM 263; 1SPEC

BRADORIA CF OVALIS (MATTHEW)

7791 [161CM] REF SPEC-M

INDIANA CF OVALIS MATTHEW

$M C A$,

BOURINOT GP,DUGALDFM

[LETCHEMINIAN,DIV E3E ]

DUGALD BK, ESCASONIE,CAPE BRETON,NS

Q102; MATTHEW GF, 1902,P462; 1903,P 171; ULRICH \& BASSLER,PROC USNM, VOL 78, 1931,P21

REMARKS:C-GF MATTHEW:D-MACKENZIE :THIS SPEC

CONSIDERED BY ULRICH + BASSLER TO BE B.

SCRUTATOR OR B. VIGILANS;STRAT REV AS IN

HUTCHINSON, 1952,GSC MEM 263; 1 SPEC

BRADORIA OVALIS MUT PRIMA (MATTHEW)

8317 [687CM ] P-SYN?

INDIANA OVALIS MUT PRIMA MATTHEW

$M C A$.

BOURINOT GP,ESKASONIFM

[COLDBROOK GP ]

DUGALD BK \& INDIAN BK, ESCASONIE,CAPE BRETON,NS

Q102; MATTHEW GF, 1902,P462-463,PL2,FIG 9A-C; 1903,P 171; ULRICH \& BASSLER,PROC USNM, VOL 78, 1931,P21

REMARKS: C-GF MATTHEW:D-MACKENZIE ;UNCERTAIN WHETHER THESE ARE MATTHEW'S TYPES;SPEC 'A' FROM INDIAN BK ACCORDING TO OLD RECORDS ; 3 SPEC

BRADORIA PERSPICATOR (MATTHEW)

7723 (B) [93CM(B)] P-PARAL

BRADORONA PERSPICATORMATTHEW

$M C A$

BOURINOT GP,DUGALD FM

[L ETCHEMINIAN,DIV E1D]

BOUNDARY BK, ESCASONIE, CAPE BRETON,NS

Q 102; MATTHEW GF, 1902,P444-445; 1902,P 156-157;ULRICH \& BASSLER,PROC USNM, VOL 78,ART 4,1931,P24-25

REMARKS: C-GF MATTHEW;D-MACKENZIE ;SPECS 'B', 'C', 'D' OF MATTHEW'S SYNTYPES;STRAT REV AS IN HUTCHINSON, 1952,GSC MEM $263 ; 3$ SPEC

BRADORIA PERSPICATOR (MATTHEW)

7723 (A) [93CM(A)] P-LECTO

BRADORONA PERSPICATOR MATTHEW

$M C A$

BOURINOT GP.DUGALD FM

[LETCHEMINIAN,DIVE1D]

BOUNDARY BK, ESCASONIE,CAPE BRETON,NS

Q102:MATTHEW GF, 1902,P444.PL1,FIG 8A-D;1903.P 156; ULRICH \& BASSLER,PROC USNM, VOL 78, 1931,P2425,PL 1,FIG 6,8,9,PL3,FIG $12^{\prime}$

REMARKS: C-GF MATTHEW:D-MACKENZIE :SPEC 'A' OF MATTHEW'S SYNTYPES;DESIGNATED LECTOTYPE BY ULRICH + BASSLER:STRAT REV AS IN HUTCHINSON, 1952,GSC MEM 263; 1 SPEC

BRADORIA PERSPICATOR (MATTHEW)

REMARKS: SEE ALSO BRADORONA OBERSVATOR MATTHEW

BRADORIA PRIMAEVA (MATTHEW)

7757 [127CM]P-HOLO

BEYRICHIA PRIMAEVA MATTHEW

LCA.

ST JOHN GP

[ST JOHN, HANFORDIAN,DIV C 1B]

HANFORD BK, ST MARTINS,ST JOHN CO,NB 
Q 102: MATTHEW GF, 1899,P 133-134,PL 1,FIG 2A-C;ULRICH \& BASSLER,PROC USNM, VOL 78,ART 4,1931,P29

REMARKS: C-GF MATTHEW:D-MACKENZIE :ULRICH + BASSLER STATE SPECIES CANNOT BE DETERMINED FROM THIS SPEC ; 1 SPEC

BRADORIA ROBUSTA (MATTHEW)

REMARKS: SEE ALSO BRADORONA PERSPICATOR MUT MAGMA MATTHEW, B. PERSPICATOR MUT MAJOR,AND B.PERSPICATORMUT MAXIMA

\section{BRADORIA ROBUSTA (MATTHEW)}

8354(B) [724CM(B)] P-PARAL

BRADORONAROBUSTA MATTHEW

LCA

ST JOHN GP

[ST JOHN, HANFORDIAN, DIV C 18

LONG IS, KENNEBACASIS R,NB

Q 102; MATTHEW GF, 1899,P 132-133;ULRICH \&

BASSLER,PROC USNM VOL 78, ART 4, 1931,P22-24

REMARKS: C-GF MATTHEW:D-MACKENZIE ;SPECS 'B', 'C' OF MATTHEW'S SYNTYPES;SPEC 'A' DESIGNATED LECTOTYPE BY ULRICH \& BASSLER ; 2 SPEC

BRADORIA ROBUSTA (MATTHEW)

8354(A) [724CM(A)] P-LECTO

BRADORONA ROBUSTA MATTHEW

LCA.

ST JOHN GP

[ST JOHN,HANFORDIAN,DIV C 1B

LONGIS KENNEBACASIS R NB

Q102; MATTHEW GF, 1899,P132-133,PL1,FIG 4A-C;ULRICH \& BASSLER,PROC USNM,VOL 78,ART 4,1931,P2224.PL1,FIG

REMARKS: C-GF MATTHEW;D-MACKENZIE ;SPEC 'A' OF MATTHEW'S SYNTYPES:DESIGNATED LECTOTYPE BY ULRICH \& BASSLER; 1 SPEC

BRADORIA RUGULOSA (MATTHEW)

REMARKS: SEE ALSO BRADORONA PERSPICATOR MUT MAJOR MATTHEW AND BRADORONA OBSERVATOR MUT LAEVIS MATTHEW

BRADORIA RUGULOSA MATTHEW

7737 [107CM ] P-SYN

$\mathrm{MCA}$,

BOURINOT GP,DUGALD FM

[U ETCHEMINIAN,DIV E3E]

DUGALD BK, ESCASONIE,CAPE BRETON,NS

Q 102:MATTHEW GF 1899 P205 PL3,FIG 3A-D 1902,P456: 1903,P 166;ULRICH \& BASSLER,PROC USNM, 1931,P2122,PL 1,FIG 17

REMARKS: C-GF MATTHEW:D-MACKENZIE :SPEC 'A' FIG'D; STRAT REV AS IN HUTCHINSON, 1952,GSC MEM 263;3 SPEC

\section{BRADORIA SCRUTATOR MATTHEW}

$7772[142 \mathrm{CM}]$ P-SYN

$\mathrm{MCA}$.

BOURINOT GP DUGALD FM

[UETCHEMINIAN DIV E3E]

DUGALD BK, ESCASONIE,CAPE BRETON,NS

Q 102; MATTHEW GF, 1899,P204-205,PL4,FIG 1A-C 1903,P 163; ULRICH \& BASSLER,PROC USNM, VOL 78,1931,P14-16,PL1,FIG 11

REMARKS: C-GF MATTHEW;D-MACKENZIE ;SPEC 'A CONSIDERED BY ULRICH + BASSLEA TO BE B. BENEPUNCTATA:STRAT REV AS IN HUTCHINSON, 1952, GSC MEM $263 ; 3$ SPEC

\section{BRADORIA SPECTATOR (MATTHEW)}

7735 [105CM ] P-SYN

BRADORONA SPECTATOR MATTHEW

$M C A$.

BOURINOT GP,DUGALD FM

[L ETCHEMINIAN,DIVE1D]

BOUNDARY BK \& DUGALD BK, ESCASONIE, CAPE

BRETON.NS

Q 102:MATTHEW GF, 1902.P447.PL 1,FIG 12A-D; 1903.P 158

ULRICH \& BASSLER,PROC USNM, VOL $78,1931, P 25-$
26,PL1,FIG 19

REMARKS: C-GF MATTHEW:D-MACKENZIE ; OLD RECORDS STATE 8 SPECS:LOC GIVEN AS BOUNDARY BK;SPEC 'E' MARKED DUGALD BK,DIV E3D;STRAT REV AS IN HUTCHINSON, 1952,GSC MEM 263; 7 SPEC

BRADORIA SPECTATOR VAR ACUTA (MATTHEW)

7706 [76CM ] P-SYN

BRADORONA SPECTATOR VAR ACUTA MATTHEW $M C A$,

BOURINOT GP,DUGALD FM

[LETCHEMINIAN,DIV E1D]

DUGALD BK (SPEC A) \& BOUNDARY BK (SPEC B),

ESCASONIE,CAPE BRETON,NS

Q 102;MATTHEW GF, 1902,P447-448; 1903,P 159;ULRICH \& BASSLER,PROC USNM, VOL 78, 1931,P20,PL $3, F I G 3$

REMARKS: C-GF MATTHEW:D-MACKENZIE ;THESE ARE 2 OF MATTHEW'S 3 SYNTYPES:ALSO SYNTYPES OF B.

SUBQUADRATA ULRICH + BASSLER:SPEC 'A ' FIG'D;

STRAT REV AS IN HUTCHINSON, 1952,GSC MEM $263 ; 2$ SPEC

BRADORIA SPECTATOR VAR ACUTA (MATTHEW)

26291 P-SYN

BRADORONA SPECTATOR VAR ACUTA MATTHEW

$M C A$,

BOURINOT GP.DUGALD FM

[LETCHEMINIAN ]

DUGALD BK? , ESCASONIE,CAPE BRETON, NS

Q 102;MATTHEW GF, 1902,P447-448; 1903,P 159;ULAICH \& BASSLER,PROC USNM, VOL 78,1931,P20

REMARKS: C-GF MATTHEW:D-MACKENZIE :SPEC 'C' OF MATTHEW'S SYNTYPES (SEE NO 7706); IDENT REV BY ULRICH + BASSLER TO BRADORIA SP (REF SPEC-M); STRAT REV AS IN HUTCHINSON, 1952,GSC MEM 263; SPEC

\section{BRADORIA SPECTATOR MUT AEQUATA (MATTHEW)}

8181 [551CM] P-SYN

BRADORONA SPECTATOR MUT AEQUATA MATTHEW $M C A$,

BOURINOT GP DUGALD FM

[UETCHEMINIÁN,DIV E3D]

DUGALD BK, ESCASONIE,CAPE BRETON,NS

Q102; MATTHEW GF, 1902,P448,PL1,FIG 14A,B; 1903,P 160 ; ULRICH \& BASSLER,PROC USNM,VOL

78, 1931,P26,PL1,FIG 21

REMARKS: C-GF MATTHEW:D-MACKENZIE :CONSIDERED BY ULRICH + BASSLER TO BE SYNONYM OF B. SPECTATOR SPINOSA;STRAT REV AS IN HUTCHINSON, 1952, GSC MEM $263 ; 2$ SPEC

BRADORIA SPECTATOR SPINOSA (MATTHEW)

7708 [78CM ] P-HOLO

BRADORONA SPECTATOR MUT SPINOSA MATTHEW

$M C A$,

BOURINOT GP,DUGALDFM

[LETCHEMINIAN DIV E1E

DUGALD BK, ESCASONIE,CAPE BRETON,NS

Q 102:MATTHEW GF, 1902,P448,PL 1,FIG 13A,8;1903,P159;

ULRICH \& BASSLER,PROC USNM,VOL

78, 1931,P26,PL 1,FIG 20

REMARKS: C-GF MATTHEW:D-MACKENZIE :STRAT REV AS IN HUTCHINSON, 1952,GSC MEM 263; 1 SPEC

BRADORIA SUBQUADRATA ULRICH+BASSLER

7706 [76CM ] P-SYN

BRADORONA SPECTATOR VAR ACUTA MATTHEW (PART)

M CA.

BOURINOT GP,DUGALD FM

[LETCHEMINIAN,DIV E1D]

DUGALD BK (SPEC A) \& BOUNDARY BK (SPEC B).

ESCASONIE CAPE BRETON,NS

Q 102; MATTHEW GF, 1902,P447-448; 1903,P 159;ULRICH \& BASSLER,PROC USNM, VOL 78, 1931,P20,PL3,FIG 3

REMARKS: C-GF MATTHEW:D-MACKENZIE ·THESE ARE 2 OF MATTHEW'S 3 SYNTYPES FOR B. SPECTATOR ACUTA;ALSO SYNTYPES OF B. SUBQUADRATA; SPEC 'A' FIG'D; STRAT REV AS IN HUTCHINSON, 1952, GSC MEM $263 ; 2$ SPEC 
BRADORIA VIGILANS MATTHEW

26292 P-PARAL

$M C A$.

BOURINOT GP,DUGALD FM

[UETCHEMINIAN,DIVE3E]

DUGALD BK, ESCASONIE, CAPE BRETON,NS

Q 102:MATTHEW GF,NB, 1899,P205;ULRICH \&

BASSLER,PROC USNM, VOL 78,ART 4, 1931,P 16, 27

REMARK S: C-GF MATTHEW;D-MACKENZIE ;ORIGINALLY

SPECS 'A' + 'B' OF MATTHEW'S SYNTYPES, NO 7774:

CONSIDERED BY ULRICH + BASSLER TO BE B. OBESA:

STRAT REV AS IN HUTCHINSON, 1952, GSC MEM $263 ; 2$

SPEC

BRADORIA VIGILANS MATTHEW

$7774(\mathrm{~A})[144 \mathrm{CM}(\mathrm{A})]$ P-LECTO

MCA.

BOURINOT GP.DUGALD FM

[U ETCHEMINIÁN,DIVE3E]

DUGALD BK, ESCASONIE,CAPE BRETON,NS

Q102:MATTHEW GF.NB 1899,P205.PL4,FIG 2A-C:ULRICH \&

BASSLER,PROC USNM, VOL 78,ART 4,1931,P16-

17,PL1,FIG 15

REMARKS: C-GF MATTHEW'D-MACKENZIE 'SPEC 'E' OF MATTHEW'S SYNTYPES;DESIGNATED LECTOTYPE BY ULRICH + BASSLER;STRAT REV AS IN HUTCHINSON, 1952,GSC MEM 263; 1SPEC

BRADORIA VIGILANS MATTHEW

7774(B) [144CM(B)] P-PARAL

$\mathrm{MCA}$,

BOURINOT GP,DUGALD FM

[U ETCHEMINIAN,DIV E3E]

DUGALD BK, ESCASONIE,CAPE BRETON,NS

Q 102:MATTHEW GF NB 1899.P205:ULRICH \&

BASSLER,PROC USNM VOL 78,ART 4, 1931,P 16-17

REMARKS: C-GF MATTHEW;D-MACKENZIE ;SPEC 'D' OF MATTHEW'S SYNTYPES;STRAT REV AS IN HUTCHINSON, 1952,GSC MEM 263; 1 SPEC

\section{BRADORIA VIGILANS CONCENTRICA U.\&B.}

26293 P-SYN

BRADORIA VIGILANS MATTHEW (PARALECTOTYPE)

$M C A$,

BOURINOT GP,DUGALD FM

[UETCHEMINIÁN,DIV E3E]

DUGALD BK, ESCASONIE,CAPE BRETON,NS

Q102;MATTHEW GF,NB, 1899,P205;ULRICH \&

BASSLER,PROC USNM,VOL 78,ART 4,1931,P 17

REMARKS: C-GF MATTHEW:D-MACKENZIE;THIS IS

MATTHEW'S SYNTYPE 'C' FOR B. VIGILANS,ORIGINALLY

WITH NO 7774; STRAT REV AS IN HUTCHINSON, 1952, GSC

MEM 263; 1 SPEC

BRADORIA VIGILANS MUT OBESA MATTHEW

REMARKS: SEE BRADORIA OBESA MATTHEW

BRADORIA ? ORNATA MATTHEW

7703 [73CM] P-HOLO

$\mathrm{MCA}$,

BOURINOT GP,DUGALDFM

[LETCHEMINIAN,DIV E1C ]

DUGALD BK, ESCASONIE,CAPE BRETON,NS

Q102:MATTHEW GF, 1902,P456-457,PL2,FIG 4A-C

1903,P 166;ULRICH \& BASSLER,PROC USNM, VOL 78,ART 4.1931,P30,PL1,FIG 24

REMARKS: C-GF MATTHEW;D-MACKENZIE ;STRAT REV AS IN HUTCHINSON, 1952, GSC MEM 263; 1 SPEC

BRADORONA OBSERVATOR MATTHEW

7705 [75CM] P-SYN

MCA.

BOURINOT GP,DUGALDFM

[L ETCHEMINIÁN,DIVE1D]

BOUNDARY BK, ESCASONIE,CAPE BRETON,NS

Q 102; MATTHEW GF, 1902,P448-449,PL1,FIG 15A-C

1903,P 160; ULRICH \& BASSLER,PROC USNM, VOL 78,ART 4 1931,P25,PL1,FIG 7

REMARKS: C-GF MATTHEW;D-MACKENZIE ;CONSIDERED BY ULRICH + BASSLER TO BE BRADORIA

PERSPICATOR:STRAT REV AS IN HUTCHINSON, 1952

GSC MEM 263; 7 SPEC
BRADORONA OBSERVATOR VAR BENEPUNCTATA

REMARKS: SEE BRADORIA BENEPUNCTATA (MATTHEW)

BRADORONA OBSERVATOR MUT LAEVIS MATTHEW

7707 [77CM ] P-SYN

MCA,

BOURINOT GP,DUGALD FM

[L ETCHEMINIAN,DIV E1B]

DUGALD BK , ESCASONIE,CAPE BRETON,NS

Q102:MATTHEW GF, 1902,P450-451; 1903,P 161;ULRICH \& BASSLER,PROC USNM,VOL 78,ART 4,1931,P21-22

REMARKS: C-GF MATTHEW;D-MACKENZIE ;CONSIDERED

BY ULRICH + BASSLER TO BE BRADORIA RUGULOSA

(REF SPEC); STRAT REV AS IN HUTCHINSON, 1952, GSC

MEM 263;3 SPEC

BRADORONA PERSPICATOR MATTHEW

REMARKS: SEE BRADORIA PERSPICATOR (MATTHEW)

BRADORONA PERSPICATOR MUT MAGNA MATTHEW

7722 [92CM] P-SYN

$\mathrm{MCA}$,

BOURINOT GP,DUGALD FM

[LETCHEMINIAN,DIV E2B]

DUGALD BK, ESCASONIE,CAPE BRETON,NS

Q 102:MATTHEW GF, 1902,P446,PL1,FIG 11A,B; 1903, P 158 ULRICH \& BASSLER,PROC USNM, VOL 78, 1931,P2223,PL1,FIG 5

REMARK S: C-GF MATTHEW:D-MACKENZIE :CONSIDERED BY ULRICH + BASSLER TO BE BRADORIA ROBUSTA (REF SPEC):STRAT REV AS IN HUTCHINSON, 1952, GSC MEM 263; 1SPEC

BRADORONA PERSPICATOR MUT MAJOR MATTHEW $7761[131 \mathrm{CM}]$ P-SYN

$M C A$

BOURINOT GP,DUGALDFM

[UETCHEMINIAN,E3F ]

DUGALD BK, ESCASONIE, CAPE BRETON, NS

Q 102; MATTHEW GF, 1902,P446-447,PL1,FIG 10A-B; 1903,P 158; ULRICH \& BASSLER,PROC USNM, 1931,P2223,PL1,FIG 4

REMARKS: C-GF MATTHEW;D-MACKENZIE ;CONSIDERED BY ULRICH + BASSLER TO BE BRADORIA ROBUSTA (REF SPEC);STRAT REV AS IN HUTCHINSON, 1952, GSC MEM 263; 1 SPEC

BRADORONA PERSPICATOR MUT MAXIMA MATTHEW 7720 [90CM] P-SYN

$\mathrm{MCA}$,

BOURINOT GP,DUGALD FM

[LETCHEMINIAN,DIVE1C]

DUGALD BK, ESCASONIE,CAPE BRETON,NS

Q 102:MATTHEW GF, 1902,P445-446,PL1,FIG 9A,B 1903,P 157; ULRICH \& BASSLER,PROC USNM, VOL 78,ART 4, 1931,P22-23,PL1,FIG 3

REMARKS: C-GF MATTHEW:D-MACKENZIE :CONSIDERED BY ULRICH + BASSLER TO BE BRADORIA ROBUSTA (REF SPEC); STRAT REV AS IN HUTCHINSON, 1952, GSC MEM 263; 1 SPEC

BRADORONA SPECTATOR MATTHEW REMARKS: SEE BRADORIA SPECTATOR (MATTHEW)

BRADORONA SPECTATOR VAR ACUTA MATTHEW REMARK S: SEE BRADORIA SPECTATOR VAR ACUTA (MATTHEW) AND BRADORIA SUBQUADRATA ULRICH+BASSLER

BRADORONA SPECTATOR MUT AEQUATA MATTHEW REMARKS: SEE BRADORIA SPECTATOR MUT AEQUATA (MATTHEW)

BRADORONA SPECTATOR MUT SPINOSA MATTHEW REMARKS: SEE BRADORIA SPECTATOR SPINOSA (MATTHEW) 


\section{ESCASONA VETUS MATTHEW}

$7704[74 \mathrm{CM}] \mathrm{P}-\mathrm{HOLO}$

$M C A$.

BOURINOT GP.DUGALD FM

[LETCHEMINIAN, DIV E 1D]

DUGALD BK. ESCASONIE, CAPE BRETON,NS

Q 102:MATTHEW GF, 1902,P458-459,PL2,FIG 6A.B:

1903.P 168; ULRICH \& BASSLER,PROC USNM, VOL

78.1931,P49,PL 1,FIG 33

REMARKS:C-GF MATTHEW:D-MACKENZIE :STRAT REV AS

IN HUTCHINSON, 1952, GSC MEM 263; 1 SPEC

\section{ESCASONA ? INGENS MATTHEW}

$8316[686 \mathrm{CM}]$ P.HOLO

$M C A$

ESKASONIFM?

[COLDBROOKIAN. FINE GREY SHALE]

DUGALD BK ESCASONIE, CAPE BRETON,NS

Q 102;MATTHEW GF, 1902,P459-460,PL2,FIG 7A-C:

1903 P 168:ULRICH \& BASSLER,PROC USNM VOL

$78,1931, \mathrm{P} 48, \mathrm{PL} 1, \mathrm{FIG} 31$

REMARKS: C-GF MATTHEW:D-MACKENZIE;STRAT REV BY AJ ROWELL. 1972:1SPEC

\section{HIPPONICHARION CAVATUM MATTHEW}

$8425[795 \mathrm{CM}]$ P.SYN

LCA.

ST JOHN GP

[ST JOHN,HANFORDIAN,C 1B1]

HANFORD BK, ST MARTINS, ST JOHN CO,NB

Q102:MATTHEW GF, 1894.P99, PL 17.FIG 3A B; ULRICH

BASSLER,PROC USNM, VOL 78, 1931,P63,PL7,FIG 32

REMARKS: C-GF MATTHEW:D-MACKENZIE ; 1 SPEC

(CAST\&MOULD)

HIPPONICHARION EOS MATTHEW

7897 [267CM] P-SYN

$\mathrm{LCA}$

ST JOHN GP

[ST JOHN.HANFORDIAN.C 1B1]

HANFORD BK, ST MARTINS,ST JOHN CO,NB

Q 102:MATTHEW GF, 1886,P64-65,PL6,FIG 19,19A,B;ULRICH + BASSLER,PROC USNM, VOL 78, 1931,P62-63,PL7,FIG 31

REMARKS: C-GF MATTHEW;D-MACKENZIE ; 1 SPEC

\section{HIPPONICHARION MINUS MATTHEW}

7898 [268CM] P-SYN

$\mathrm{LCA}$,

ST JOHN GP

[ST JOHN, HANFORDIAN]

HANFORD BK, ST MARTINS,ST JOHN CO,NB

Q 102:MATTHEW GF, 1893,P99,PL 17,FIG 4A,B:ULRICH \&

BASSLER,PROC USNM VOL $78,1931, \mathrm{P} 63$, PL 7 FIG 30

REMARKS: C-WD MATTHEW;D-MACKENZIE ; 3 SPEC

HOLLINA EMACIATA VAR OCCIDENTALIS GIRTY

6370 [2984CB] P-PARA

$P$.

PARK CITY FM,PHOSPHATE BEDS

THOMAS FORK, WYO,USA

Q137:GIRTY GH,USGS BULL 436, 1910,P55

REMARKS: D-WF FERRIER, 1920 ;LABELLED PARATYPE: THIS SPEC NOT FIG'D ; 1 SPEC

INDIANA LIPPA MATTHEW

$7792[162 C M]$ P-LECTO

$\mathrm{MCA}$

BOURINOT GP,DUGALD FM

[UETCHEMINIAN DIV E3F]

DUGALD BK, ESCASONIE.CAPE BRETON,NS

Q 103;MATTHEW GF, 1902,P463,PL2,FIG 10A-D; 1903, P 171 ULRICH \& BASSLER,PROC USNM, VOL 78, 1931,P70-

71,PL9,FIG 1.4

REMARKS: C-GF MATTHEW:D-MACKENZIE ; STRAT REV AS IN HUTCHINSON, 1957, GSC MEM 263; 1 SPEC (2 PCES)
INDIANA OVALIS MATTHEW

REMARKS: SEE BRADORIA OVALIS (MATTHEW)

INDIANA PRIMAEVA (MATTHEW)

7683 [53CM ] P-HOLO

LEPERDITIA? PRIMAEVA MATTHEW

LCA

ST JOHN GP

[ST JOHN,HANFORDIAN,C 1B3]

ST MARTINS.ST JOHN CO.NB

Q103:MATTHEW GF, 1895,P 138,PL8,FIG 6A,B:ULRICH \&

BASSLER,PROC USNM,VOL 78, 1931,P77-78,PL9,FIG 9

REMARK S: C-GF MATTHEW:D-MACKENZIE ; 1 SPEC

INDIANA PYRIFORMIS (MATTHEW)

$7799[169 C M] P-S Y N$

PRIMITIA PYRIFORMIS (MATTHEW)

LCA

ST JOHN GP

[ST JOHN,HANFORDIAN,C 1B3]

LONG IS.KING'S CO,NB

Q 103:MATTHEW GF, 1899,P132,PL1,FIG 3A-C; 1902, P 461

ULRICH \& BASSLER,PROC USNM, VOL 78, 1931,P72-

73.PL9.FIG 11

REMARKS: C-GF MATTHEW;D-MACKENZIE ; 2 SPEC

INDIANA SECUNDA (MATTHEW)

7679 [49CM]P-LECTO

APARCHITES SECUNDA MATTHEW

LCA,

ST JOHN GP

[ST JOHN, HANFORDIAN,DIV C1B3]

HANFORD BK, ST MARTINS, ST JOHN CO,NB

Q103;MATTHEW GF, 1895,P 136,PL7,FIG 11A,B; ULRICH \&

BASSLER,PROC USNM, VOL 78, 1931,P71-72,PL9,FIG 5-8

REMARKS: C-GF MATTHEW;D-MACKENZIE ;DESIGNATED LECTOTYPE BY ULRICH + BASSLER; 1 SPEC

INDIANA ? ACADICA (MATTHEW)

8249 [619CM]P-HOLO

PRIMITIA ACADICA MATTHEW

$M C A$.

ST JOHN GP

[ST JOHN,ACADIAN,C1C1]

PORTER'S BK, ST MARTINS,ST JOHN CO,NB

Q 103:MATTHEW GF, 1886,P66-67,PL6,FIG 22,22A,B;ULRICH

\& BASSLER,PROC USNM, VOL 78, 1931,P82-83,PL9,FIG 13 REMARKS: C-GF MATTHEW;D-MACKENZIE ; 1 SPEC

JONESINA CARBONIFERA GIRTY

$6371[2985 C B]$ P-PARA

$P$

PARK CITY FM,PHOSPHATE BEDS

THOMAS FORK, WYO, USA

Q413:GIRTY GH,USGS BULL 436, 1910,P56

REMARKS: D-WF FERRIER, 1920 ;LABELLED PARATYPE:

THIS SPEC NOT FIG'D : 1 SPEC

LEPERDITIA VENTRICOSA MATTHEW

$8434[804 C M]$ P.SYN

LCA

ST JOHN GP

[ST JOHN,HANFORDIAN,DIV C1B1]

HANFORD BK, ST MARTINS,ST JOHN CO,NB

Q 106:MATTHEW GF, 1890,P159-160,PL7,FIG 12A-D;ULRICH

\& BASSLER,PROC USNM, VOL 78, 1931,P 100-101,PL7,FIG 35

REMARKS: C-GF MATTHEW;D-MACKENZIE ; 1 SPEC

LEPERDITIA? MINOR MATTHEW

REMARKS: SEE BRADORIA MINOR (MATTHEW)

LEPERDITIA ? PRIMAEVA MATTHEW

REMARKS: SEE INDIANA PRIMAEVA (MATTHEW) 
LEPIDILLA ANOMALA MATTHEW

8191 [561CM]P-SYN

$M C A$,

ST JOHNGP

[ST JOHN, ACADIAN, DIV 1C2]

HANFORD BK, ST MARTINS,ST JOHN CO,NB

Q4 14:MATTHEW GF, 1886,P62-63,PL6,FGI 18A-C; 1890,1891, PL $11, F I G$ 2; ULRICH \& BASSLER,PROC USNM, 1931,P 100

REMARKS: C-GF MATTHEW;D-MACKENZIE ;AFFINITIES UNCERTAIN;CONSIDERED OSTRACOD BY MATTHEW, CONCHOSTRACAN BY ULRICH \& BASSLER ; 3 SPEC

LEPIDITTA ALATA MATTHEW

8119 [489CM] P-SYN

$M C A$,

ST JOHN GP

[ST JOHN,ACADIAN, DIV C 1C]

HANFORD BK, ST MARTINS, ST JOHN CO,NB

Q414;MATTHEW GF, 1886,P61-62,PL6,FIG 16,16A;

1896, P 194-195; ULRICH \& BASSLER,PROC USNM, VOL

78,1931, P94-95,PL7,FIG 24,25

REMARKS: C-GF MATTHEW;D-MACKENZIE;AFFINITIES UNCERTAIN;CONSIDERED OSTRACOD BY MATTHEW, CONCHOSTRACAN BY ULRICH \& BASSLER ; 2 SPEC

\section{LEPIDITT A AURICULATA MATTHEW}

$8287[657 \mathrm{CM}]$ P-HOLO

$\mathrm{MCA}$,

ST JOHN GP

[ST JOHN,ACADIAN,DIV C 1C1]

HANFORD BK, ST MARTINS, ST JOHN CO, NB

Q4 14;MATTHEW GF, 1894;P99,PL17,FIG 2A,B; 1896,P196; ULRICH \& BASSLER,PROC USNM, VOL

78, 1931,P96,PL5,FIG 20,PL7,FIG 26

REMARKS: C-GFMATTHEW;D-MACKENZIE ;AFFINITIES UNCERTAIN;CONSIDERED OSTRACOD BY MATTHEW, CONCHOSTRACAN BY ULRICH \& BASSLER; 1 SPEC

\section{LEPIDITTA CURTA MATTHEW}

8118 [488CM] P-SYN

$M C A$,

ST JOHNGP

[ST JOHN,ACADIAN, DIV C 1D1]

PORTER'S BK, ST MARTINS, ST JOHN CO,NB

Q4 14;MATTHEW GF, 1886,P62,PL6,FIG 17; 1896,P 195;

ULRICH \& BASSLER,PROC USNM, VOL 78, 1931,PL5, FIG 18, 19,PL7,FIG 29

REMARKS: C-GF MATTHEW;D-MACKENZIE;AFFINITIES UNCERTAIN;CONSIDERED OSTRACOD BY MATTHEW, CONCHOSTRACAN BY ULRICH \& BASSLER ; 2 SPEC

LEPIDITTA SIGILLATA MATTHEW

$7743[113 \mathrm{CM}]$ P-LECTO

LCA,

ST JOHNGP

[ST JOHN,HANFORDIAN,DIV C 1B3]

HANFORD BK, ST MARTINS, ST JOHN CO,NB

Q414;MATTHEW GF, 1894,P98,PL17,FIG 1; 1895,P138;

ULRICH \& BASSLER, PROC USNM, VOL 78, 1931 ,P9697,PL5,FIG 21,PL7,FIG 27

REMARKS: C-WD MATTHEW:D-MACKENZIE ;AFFINITES UNCERTAIN;CONSIDERED OSTRACOD BY MATTHEW ; 1 SPEC

MONONOTELLA FUSIFORMIS (MATTHEW)

$7762[132 \mathrm{CM}]$ P-LECTO

PRIMITIA ? FUSIFORMIS MATTHEW

LCA,

ST JOHN GP

[ST JOHN, HANFORDIAN, DIV C 1B3]

HANFORD BK, ST MARTINS, ST JOHN CO,NB

Q103;MATTHEW GF, 1895,P137,PL8,FIG 3A,B;ULRICH \& BASSLER,PROC USNM, VOL 78, 1931,P92-93,PL8,FIG 22

REMARKS: C-GF MATTHEW:D-MACKENZIE :LOC GIVEN AS

ST MARTINS IN PUBL' $N$; LONG IS,KENNEBACASIS R IN

OLD RECORDS ; 1 SPEC
PRIMITIA ACADICA MATTHEW

REMARKS: SEE INDIANA? ACADICA (MATTHEW)

PRIMITIA OCCULATA MATTHEW

REMARKS: SEE BRADORIA OCCULATA (MATTHEW)

PRIMITIA PYRIFORMIS MATTHEW

REMARKS: SEE INDIANA PYRIFORMIS (MATTHEW)

PRIMITIA ? FUSIFORMIS MATTHEW

REMARKS: SEE MONONOTELLA FUSIFORMIS (MATTHEW)

SCHMIDTELLA ACUTA MATTHEW

REMARKS: SEE BRADORIA ACUTA (MATTHEW)

SCHMIDTELLA CAMBRICA MATTHEW

REMARKS: SEE BRADORIA CAMBRICA (MATTHEW)

SCHMIDTELLA ? PERVETUS MATTHEW

7788 [158CM]P-SYN

$\mathrm{MCA}$.

BOURINOT GP,DUGALDFM

[UETCHEMINIAN,DIVE3E]

DUGALD BK , ESCASONIE,CAPE BRETON, NS

Q193;MATTHEW GF, 1899,P206,PL4,FIG 3A-C; 1903,P 172;

ULRICH \& BASSLER,PROC USNM, VOL

78, 1931,P27,PL1,FIG 26

REMARKS: C-GFMATTHEW;D-MACKENZIE ;STRAT REV AS IN HUTCHINSON, 1952,GSC MEM 263;2 SPEC

SCHMIDTELLA ? PERVETUS MUT CONCINNA MATTHEW REMARKS: SEE BRADORIA CONCINNA (MATTHEW)

\section{ARTHROPODA,INSECTA}

ACRIDIDAE INDET

30735 REF SPEC-F

PLEIST,

TALARA TAR PITS,PERU

CHURCHER CS, 1966, CAN JOUR ZOOL, VOL 44,P985993,PL2,FIG 21

REMARKS: D-CS CHURCHER,VERT PALAEO;D-1974; DISTALPORTION OF METATARSUS ; 1 SLIDE

\section{CARABIDAEINDET}

30568 REF SPEC-F

PLEIST,

TALARA TAR PITS,PERU

CHURCHER CS, 1966,CAN JOUR ZOOL, VOL 44,P985993,PL 1,FIG 5

REMARKS: D-CS CHURCHER,VERT PALAEO:D-1974:LEFT SPEC OF 3 MOUNTED ON SAME SLIDE;DORSAL VIEW OF RIGHT ELYTRON; 1 SLIDE

\section{CURCULIONIDAE INDET I}

30730 REF SPEC-F

PLEIST,

TALARA TAR PITS,PERU

CHURCHER CS, 1966, CAN JOUR ZOOL, VOL 44,P985993,PL2,FIG 16

REMARKS: D-CS CHURCHER, VERT PALAEO;D-1974; BOTTOM, 2ND FROM LEFT OF 8 SPECS ON SAME SLIDE: DORSAL VIEW OF FUSED ELYTRA;SAME SLIDE AS 30731 ISLIDE

\section{CURCULIONIDAE INDET II}

30731 REF SPEC-F

PLEIST,

TALARAं TAR PITS,PERU

CHURCHER CS, 1966, CAN JOUR ZOOL, VOL 44,P985993,PL2,FIG 17

REMARKS: D-CS CHURCHER, VERT PALAEO;D-1974; 
BOTTOM 2ND FROM RIGHT OF 8 SPECS ON SAME SLIDE; DORSAL VIEW OF FUSED ELYTRA;SAME SLIDE AS 30730 1 SLIDE

\section{CURCULIONIDAE INDET}

30734 REF SPEC-F

PLEIST.

TALARA TAR PITS.PERU

CHURCHER CS, 1966, CAN JOUR ZOOL, VOL 44,P985993.PL2, FIG 20

REMARKS: D-CS CHURCHER,VERT PALAEO:D-1974;RIGHT SPEC OF 8 ON SAME SLIDE:LATERAL VIEW OF HEAD \& PROBOSCIS;SAME SLIDE AS 30733;SPEC DAMAGED ; 1 SPEC

\section{CURCULIONIDAE INDET}

30733 REF SPEC-F

PLEIST.

TALARA TAR PITS.PERU

CHURCHER CS, 1966.CAN JOUR ZOOL,VOL 44,P985993,PL2,FIG 19

REMARKS: D-CS CHURCHER,VERT PALAEO;D-1974;2ND FROM RIGHT OF 8 SPECS ON SAME SLIDE;LATERAL VIEW OF HEAD \& PROBOSCIS:SAME SLIDE AS $30734 ; 1$ SPEC

\section{CURCULIONIDAE INDET}

30736 REF SPEC-F

PLEIST,

TALARA TAR PITS,PERU

CHURCHER CS, 1966,CAN JOUR ZOOL, VOL 44,P985993,PL2,FIG 22

REMARKS: D-CS CHURCHER,VERT PALAEO;D-1974;2ND FROM RIGHT OF 4 SPEC ON SAME SLIDE;DORSAL VIEW OF FUSED ELYTRA ; 1 SLIDE

\section{DYTISCIDAE INDET}

30573 REF SPEC-F

PLEIST,

TALARA TAR PITS,PERU

CHURCHER CS, 1966. CAN JOUR ZOOL,VOL 44,P985993,PL1,FIG 10

REMARKS: D-CS CHURCHER,VERT PALAEO:D- 1974:RIGHT SPEC OF 2 ON SAME SLIDE;VENTRAL VIEW OF STERNITE : 1 SLIDE

\section{DYTISCIDAE INDET}

30566 REF SPEC-F

PLEIST.

TALARA TAR PITS PERU

CHURCHER CS, 1966.CAN JOUR ZOOL,VOL 44,P985993. PL 1,FIG 3

REMARKS: D-CS CHURCHER,VERT PALAEO;D-1974;TOP OF 2 SPECS MOUNTED ON SAME SLIDE;DORSAL VIEW OF LEFT ELYTRON : 1SLIDE

\section{HYDROPHILUS SP}

30569 REF SPEC-F

PLEIST,

TALARA TAR PITS,PERU

CHURCHER CS, 1966. CAN JOUR ZOOL, VOL 44,P985993,PL 1,FIG 6

REMARKS: D-CS CHURCHER, VERT PALAEO;D-1974;TOP 2ND FROM LEFT. OF 8 SPECS MOUNTED ON SAME SLIDE: DORSAL VIEW OF HEAD; 1 SLIDE

\section{HYDROPHILUS SP}

30572 REF SPEC-F

PLEIST.

TALARA TAR PITS PERU

CHURCHER CS, 1966,CAN JOUR ZOOL, VOL 44,P985993.PL 1.FIG 9

REMARKS: D-CS CHURCHER,VERT PALAEO;D-1974:TOP LEFT OF 3 SPECS ON SAME SLIDE;DORSAL VIEW OF PRONOTUM : 1 SLIDE
$?$ HYDROPHILUS SP

30575 REF SPEC-F

PLEIST.

TALARA TAR PITS, PERU

CHURCHER CS, 1966.CAN JOUR ZOOL,VOL 44,P985993,PL1,FIG 12

REMARKS: D-CS CHURCHER VERT PALAEO'D- 1974:TOP RIGHT OF 6 SPECS ON SAME SLIDE:VENTRAL VIEW OF PROSTERNUM ; 1 SLIDE

HYDROPHILUS SP

30727 REF SPEC-F

PLEIST,

TALARA TAR PITS.PERU

CHURCHER CS, 1966.CAN JOUR ZOOL,VOL 44,P985993,PL1,FIG 13

REMARKS: D-CS CHURCHER,VERT PALAEO;D- 1974 VENTRAL VIEW OF METASTERNUM : 1 SPEC

\section{HYDROPHILUS SP}

30565 REF SPEC-F

PLEIST,

TALARA TAR PITS, PERU

CHURCHER CS, 1966, CAN JOUR ZOOL, VOL 44,P985993.PL1,FIG 1,2

REMARKS: D-CS CHURCHER, VERT PALAEO;D-1974 : 1 SPEC

\section{HYDROPHILUS SP}

30567 REF SPEC-F

PLEIST

TALARA TAR PITS,PERU

CHURCHER CS, 1966,CAN JOUR ZOOL, VOL 44,P985993.PL 1,FIG 4

REMARKS: D-CS CHURCHER VERT PALAEO-D-1974, BOTTOM OF 2 SPECS MOUNTED ON SAME SLIDE; DORSAL VIEW OF LEFT ELYTRON ; 1 SLIDE

\section{INCERTAE SEDIS}

31027 REF SPEC-F

$M E O C$,

[DRIFTWOOD CREEK FOSSIL BEDS ]

S SIDE DRIFTWOOD CK,DRIFTWOOD CANYON PROV PARK E OF SMITHERS,BC

WILSON MVH, 1977,CAN J EARTH SCI,VOL 14,N5,P1152,FIG $4 A$

REMARKS: C.D-MVH WILSON : 1 SPEC

\section{INCERTAE SEDIS}

31029 REF SPEC-F

$M E O C$,

[DRIFTWOOD CREEK FOSSIL BEDS

S SIDE DRIFTWOOD CK,DRIFTWOOD CANYON PROV PARK E OF SMITHERS,BC

WILSON MVH, 1977,CAN J EARTH SCI,VOL 14,N5,P1152,FIG

REMARKS: C.D-MVH WILSON ; 1 SPEC

\section{INCERTAE SEDIS}

31318 REF SPEC-F

$M E O C$.

IDRIFTWOOD CK FOSSIL BEDS

S SIDE DRIFTWOOD CK,DRIFTWOOD CANYON PROV PARK E OF SMITHERS,BC

WILSON MVH, 1977,CAN J EARTH SCI,VOL 14,N5,P1152,FIG 3H

REMARKS: C.D-MVH WILSON ; 1 SPEC

\section{INSECT-MYCETOPHILIDAE}

31302 REF SPEC-F

EOC

4.7 MI E OF HORSEFLY R BRIDGE ALONG BLACK CK RD , N BANK HORSEFLY R,BC

WILSON MVH, 1977,CAN J EARTH SCI,VOL

14.N5,P1149, 1150,FIG 5H,6B

REMARKS: C.D-MVH WILSON : 1 SPEC 
INSECT-MASTOTERMITIDAE

31308 REF SPEC-F

$M E O C$

6 MI N OF HORSEFLY BY QUESNEL L RD, NE BANK

HORSEFLYR,BC

WILSON MVH, 1977,CAN J EARTH SCI,VOL 14,N5,P1141,FIG $2 A, 3 A$

REMARKS: C.D-MVH WILSON;WING; 1 SPEC

\section{INSECT-MYCETOPHILIDAE}

31056 REF SPEC-F

$M E O C$

[DRIFTWOOD CREEK FOSSIL BEDS]

S SIDE DRIFTWOOD CK,DRIFTWOOD CANYON PROV PARK, E OF SMITHERS,BC

WILSON MVH, 1977,CAN J EARTH SCI, VOL

14. N5.P 1149,1150 FIG 5G, 6D

REMARKS: C,D-MVH WILSON ; 1 SPEC

\section{INSECT-BIBIONIDAE}

31070 REF SPEC-M

$M E O C$,

[DRIFTWOOD CREEK FOSSIL BEDS ]

S SIDE DRIFTWOOD CK,DRIFTWOOD CANYON PROV PARK, E OF SMITHERS,BC

WILSON MVH, 1977,CAN J EARTH SCI,VOL 14,N5,P1146

REMARKS: C,D-MVH WILSON ; 1 SPEC

\section{INSECT-BIBIONIDAE}

31072 REF SPEC-F

EOC

BELLOW MILEPOST 39A ON HORSEFLY-BLACK CK RD, HORSEFLY R,BC

WILSON MVH, 1977,CAN J EARTH SCI,VOL 14,N5,P1146,FIG

$7 A$

REMARKS: C,D-MVH WILSON; 1 SPEC

\section{INSECT-BIBIONIDAE}

31073 REF SPEC-M

$M E O C$.

6 MIN OF HORSEFLY BY QUESNEL L RD, NE BANK HORSEFLY R,BC

WILSON MVH, 1977, CAN J EARTH SCI, VOL 14,N5,P1146

REMARKS: C,D-MVH WILSON ; 1 SPEC

\section{INSECT-BIBIONIDAE}

31074 REF SPEC-M

EOC

4.7 MI E OF HORSEFLY R BRIDGE ALONG BLACK CK RD, N BANK HORSEFLY R,BC

WILSON MVH, 1977,CAN J EARTH SCI,VOL 14,N5,P1146 REMARKS: C,D-MVH WILSON ; 1 SPEC

INSECT-BIBIONIDAE

31075 REF SPEC-M

EOC

4.7 MI E OF HORSEFLY R BRIDGE ALONG BLACK CK RD , N BANK HORSEFLY R,BC

WILSON MVH, 1977,CAN J EARTH SCI,VOL 14,N5,P1146 REMARKS: C,D-MVH WILSON ; 1 SPEC

INSECT-BIBIONIDAE

31301 REF SPEC-M

EOC.

$4.7 \mathrm{MIE}$ OF HORSEFLY R BRIDGE ALONG BLACK CK RD, N BANK HORSEFLY R,BC

WILSON MVH, 1977,CAN J EARTH SCI,VOL 14,N5,P1146 REMARKS: C,D-MVH WILSON : 1 SPEC

INSECT-BIBIONIDAE

31032 REF SPEC-M

$M E O C$

[DRIFTWOOD CREEK FOSSIL BEDS ]

S SIDE DRIFTWOOD CK, DRIFTWOOD CANYON PROV PARK, E OF SMITHERS,BC

WILSON MVH, 1977.CAN J EARTH SCI VOL 14,N5,P1146

REMARKS: C,D-MVH WILSON ; 1 SPEC
INSECT-BIBIONIDAE

31305 REF SPEC-M

$M E O C$

6 MI N OF HORSEFLY BY QUESNEL L RD, NE BANK

HORSEFLY R,BC

WILSON MVH, 1977,CAN J EARTH SCI,VOL 14,N5,P1146

REMARKS: C,D-MVH WILSON ; 1 SPEC

INSECT-BIBIONIDAE

31307 REF SPEC-M

$M E O C$

6 MIN OF HORSEFLY BY QUESNEL L RD, NE BANK

HORSEFLYR,BC

WILSON MVH, 1977,CAN J EARTH SCI,VOL 14,N5,P1146 REMARKS: C.D-MVH WILSON ; 1 SPEC

\section{INSECT-BIBIONIDAE}

31035 REF SPEC-M

$M E O C$,

[DRIFTWOOD CREEK FOSSIL BEDS

S SIDE DRIFTWOOD CK,DRIFTWOOD CANYON PROV PARK, E OF SMITHERS, BC

WILSON MVH, 1977,CAN J EARTH SCI,VOL 14,N5,P1146 REMARKS: C,D-MVH WILSON ; 1 SPEC

INSECT-BIBIONIDAE

31310 REF SPEC-M

$M E O C$

$6 \mathrm{MIN}$ OF HORSEFLY BY QUESNEL L RD, NE BANK

HORSEFLY R,BC

WILSON MVH, 1977,CAN J EARTH SCI,VOL 14,N5,P 1146

REMARKS: C,D-MVH WILSON; 1 SPEC

\section{INSECT-BIBIONIDAE}

31317 REF SPEC-M

MEOC

[DRIFTWOOD CK FOSSIL BEDS ]

S SIDE DRIFTWOOD CK,DRIFTWOOD CANYON PROV PARK, E OF SMITHERS,BC

WILSON MVH,1977,CAN J EARTH SCI,VOL 14,N5,P11451146

REMARK S: C,D-MVH WILSON ; 1 SPEC

INSECT-BIBIONIDAE

31039 REF SPEC-M

$M E O C$

[DRIFTWOOD CREEK FOSSIL BEDS

S SIDE DRIFTWOOD CK,DRIFTWOOD CANYON PROV PARK, E OF SMITHERS, BC

WILSON MVH, 1977,CAN J EARTH SCI,VOL 14,N5,P1146 REMARKS: C,D-MVH WILSON ; 1 SPEC

INSECT-BIBIONIDAE

31323 REF SPEC-M

$M E O C$

PRINCETON GP ALLENBY FM

N BANK TULAMEEN R,E END VERMILLION BLUFFS, $1.5 \mathrm{M}$ W OF PRINCETON,BC

WILSON MVH, 1977,CAN J EARTH SCI, VOL 14, N5,P1146

REMARKS: C,D-MVH WILSON ; 1 SPEC

\section{INSECT-BIBIONIDAE}

31040 REF SPEC-M

$M E O C$

[DRIFTWOOD CREEK FOSSIL BEDS]

S SIDE DRIFTWOOD CK,DRIFTWOOD CANYON PROV PARK E OF SMITHERS,BC

WILSON MVH, 1977,CAN J EARTH SCI,VOL 14,N5,P1146 REMARKS: C,D-MVH WILSON ; 1 SPEC

INSECT-BIBIONIDAE

31041 REF SPEC-M

$M E O C$

[DRIFTWOOD CREEK FOSSIL BEDS

S SIDE DRIFTWOOD CK,DRIFTWOOD CANYON PROV PARK, E OF SMITHERS,BC

WILSON MVH, 1977. CAN J EARTH SCI,VOL 14,N5,P1146

REMARKS: C,D-MVH WILSON ; 1 SPEC 
INSECT-BIBIONIDAE

31042 REF SPEC-M

$M E O C$,

[DRIFTWOOD CREEK FOSSIL BEDS ]

S SIDE DRIFTWOOD CK.DRIFTWOOD CANYON PROV PARK,

E OF SMITHERS,BC

WILSON MVH, 1977.CAN J EARTH SCI,VOL 14,N5,P1146

REMARKS: C.D-MVH WILSON ; 1 SPEC

\section{INSECT-BIBIONIDAE}

31046 REF SPEC-M

$M E O C$.

[DRIFTWOOD CREEK FOSSIL BEDS ]

S SIDE DRIFTWOOD CK,DRIFTWOOD CANYON PROV PARK, E OF SMITHERS,BC

WILSON MVH, 1977.CAN J EARTH SCI,VOL 14,N5,P1144

REMARKS: C,D-MVH WILSON: 1 SPEC

\section{INSECT-BIBIONIDAE}

31031 REF SPEC-M

MEOC.

[DRIFTWOOD CREEK FOSSIL BEDS]

S SIDE DRIFTWOOD CK,DRIFTWOOD CANYON PROV PARK, E OF SMITHERS.BC

WILSON MVH, 1977,CAN J EARTH SCI,VOL 14,N5,P1146

REMARKS: C,D-MVHWILSON; 1 SPEC

\section{INSECT-BIBIONIDAE}

31058 REF SPEC- $M$

MEOC,

[DRIFTWOOD CREEK FOSSIL BEDS ]

S SIDE DRIFTWOOD CK,DRIFTWOOD CANYON PROV PARK, E OF SMITHERS,BC

WILSON MVH, 1977,CAN J EARTH SCI,VOL 14,N5,P1146

REMARK S: C,D-MVHWILSON ; 1 SPEC

\section{INSECT-BRACONIDAE}

31030 REF SPEC-F

MEOC,

[DRIFTWOOD CREEK FOSSIL BEDS ]

S SIDE DRIFTWOOD CK,DRIFTWOOD CANYON PROV PARK E OF SMITHERS.BC

WILSON MVH, 1977,CAN J EARTH SCI,VOL 14,N5,P1151-

1152. FIG 8B

REMARKS: C,D-MVH WILSON; 1 SPEC

\section{INSECT-BRACONIDAE}

31324 REF SPEC-F

$M E O C$.

[DRIFTWOOD CREEK FOSSIL BEDS]

S SIDE DRIFTWOOD CK,DRIFTWOOD CANYON PROV PARK, E OF SMITHERS,BC

WILSON MVH, 1977.CAN J EARTH SCI,VOL 14,N5,P1151.

1152, FIG 8 A

REMARKS: C,D-MVHWILSON ; 1 SPEC

INSECT-CERCOPIDAE

31061 REF SPEC-F

$M$ EOC

[DRIFTWOOD CREEK FOSSIL BEDS ]

S SIDE DRIFTWOOD CK,DRIFTWOOD CANYON PROV PARK E OF SMITHERS, BC

WILSON MVH, 1977,CAN J EARTH SCI,VOL 14,N5,P1144,FIG 28.3F

REMARKS: C.D-MVH WILSON ; 1 SPEC

\section{INSECT-CERCOPIDAE}

31028 REF SPEC-F

$M E O C$.

[DRIFTWOOD CREEK FOSSIL BEDS ]

S SIDE DRIFTWOOD CK,DRIFTWOOD CANYON PROV PARK E OF SMITHERS.BC

WILSON MVH, 1977.CAN JEARTH SCI,VOL 14,N5,P1144,FIG 3E

REMARKS: C.D-MVHWILSON : 1 SPEC
INSECT-CICADELLIDAE

31051 REF SPEC-F

MEOC

[DRIFTWOOD CREEK FOSSIL BEDS ]

S SIDE DRIFTWOOD CK,DRIFTWOOD CANYON PROV PARK, EOF SMITHERS. BC

WILSON MVH, 1977,CAN J EARTH SCI,VOL 14,N5,P1145,FIG $3 G$

REMARKS: C,D-MVHWILSON ; 1 SPEC

INSECT-EMPIDIDAE

31050 REF SPEC.F

$M E O C$

[DRIFTWOOD CREEK FOSSIL BEDS]

S SIDE DRIFTWOOD CK,DRIFTWOOD CANYON PROV PARK E OF SMITHERS, BC

WILSON MVH, 1977,CAN J EARTH SCI,VOL 14,N5,P1150,FIG 78

REMARKS: C.D-MVH WILSON ; 1 SLAB

INSECT-EMPIDIDAE

31053 REF SPEC-F

$M E O C$,

[DRIFTWOOD CREEK FOSSIL BEDS ]

S SIDE DRIFTWOOD CK,DRIFTWOOD CANYON PROV PARK E OF SMITHERS,BC

WILSON MVH, 1977,CAN J EARTH SCI,VOL 14,N5,P1150,FIG $6 \mathrm{H}$

REMARKS: C,D-MVHWILSON ; 1 SPEC

INSECT-GERRIDAE

31045 REF SPEC-M

$M E O C$,

[DRIFTWOOD CREEK FOSSIL BEDS ]

S SIDE DRIFTWOOD CK,DRIFTWOOD CANYON PROV PARK, E OF SMITHERS,BC

WILSON MVH, 1977,CAN J EARTH SCI,VOL 14,N5,P1144

REMARKS: C,D-MVH WILSON ; 1 SPEC

INSECT-GERRIDAE

31320 REF SPEC-M

$M E O C$,

PRINCETON GP

SITE OF BLAKEBURN NO 3 MINE,S OF COALMONT , W OF PRINCETON,BC

WILSON MVH, 1977,CAN J EARTH SCI,VOL 14,N5,P1141. 1144

REMARKS: C,D-MVHWILSON : 1 SPEC

INSECT-GERRIDAE

31062 REF SPEC-M

$M E O C$,

[DRIFTWOOD CREEK FOSSIL BEDS ]

S SIDE DRIFTWOOD CK,DRIFTWOOD CANYON PROV PARK E OF SMITHERS, BC

WILSON MVH, 1977,CAN J EARTH SCI,VOL 14,N5,P11411144

REMARKS: C,D-MVH WILSON ; 1 SPEC

INSECT-GERRIDAE

31064 REF SPEC-M

$M E O C$,

[DRIFTWOOD CREEK FOSSIL BEDS ]

S SIDE DRIFTWOOD CK,DRIFTWOOD CANYON PROV PARK E OF SMITHERS,BC

WILSON MVH, 1977.CAN J EARTH SCI,VOL 14,N5,P1141 1144

REMARKS: C.D-MVH WILSON : 1 SPEC

INSECT-GERRIDAE

31065 REF SPEC-F

$M E O C$,

[DRIFTWOOD CREEK FOSSIL BEDS ]

S SIDE DRIFTWOOD CK,DRIFTWOOD CANYON PROV PARK E OF SMITHERS, BC

WILSON MVH, 1977. CAN J EARTH SCI,VOL 14,N5,P11411144 ,FIG 3C

REMARKS: C.D-MVH WILSON : 1 SPEC 
INSECT-GERRIDAE

31067 REF SPEC-M

MEOC,

[DRIFTWOOD CREEK FOSSIL BEDS ]

S SIDE DRIFTWOOD CK,DRIFTWOOD CANYON PROV PARK,

E OF SMITHERS,BC

WILSON MVH, 1977,CAN J EARTH SCI,VOL 14,N5,P11411144

REMARKS: C,D-MVH WILSON ; 1 SPEC

\section{INSECT-GERRIDAE}

31068 REF SPEC-M

$M E O C$.

[DRIFTWOOD CREEK FOSSIL BEDS ]

S SIDE DRIFTWOOD CK,DRIFTWOOD CANYON PROV PARK,

E OF SMITHERS.BC

WILSON MVH, 1977,CAN J EARTH SCI,VOL 14,N5,P11411144

REMARKS: C,D-MVH WILSON ; 1 SPEC

\section{INSECT-GERRIDAE}

31036 REF SPEC-F

$M$ EOC

[DRIFTWOOD CREEK FOSSIL BEDS ]

S SIDE DRIFTWOOD CK,DRIFTWOOD CANYON PROV PARK, E OF SMITHERS,BC

WILSON MVH, 1977,CAN J EARTH SCI,VOL 14,N5,P1141-

1144,FIG $3 B$

REMARKS: C.D-MVHWILSON : 1 SPEC

INSECT-GERRIDAE

31048 REF SPEC-M

$M E O C$,

[DRIFTWOOD CREEK FOSSIL BEDS ]

S SIDE DRIFTWOOD CK,DRIFTWOOD CANYON PROV PARK, E OF SMITHERS,BC

WILSON MVH, 1977,CAN J EARTH SCI,VOL 14,N5,P11411144

REMARKS: C,D-MVH WILSON ; 1 SPEC

\section{INSECT-GERRIDAE}

31038 REF SPEC-M

$M E O C$,

[DRIFTWOOD CREEK FOSSIL BEDS ]

S SIDE DRIFTWOOD CK,DRIFTWOOD CANYON PROV PARK, E OF SMITHERS,BC

WILSON MVH, 1977,CAN J EARTH SCI,VOL 14,N5,P11411144

REMARKS: C,D-MVH WILSON; 1 SPEC

\section{INSECT-ICHNEUMONIDAE}

31049 REF SPEC-F

$M$ EOC

[DRIFTWOOD CREEK FOSSIL BEDS ]

S SIDE DRIFTWOOD CK,DRIFTWOOD CANYON PROV PARK, E OF SMITHERS,BC

WILSON MVH,1977,CAN J EARTH SCI,VOL 14,N5,P1150,FIG $7 \mathrm{~F}$

REMARKS: C,D-MVHWILSON ; 1 SPEC

\section{INSECT-ICHNEUMONIDAE}

31054 REF SPEC-F

MEOC

[DRIFTWOOD CREEK FOSSIL BEDS ]

S SIDE DRIFTWOOD CK,DRIFTWOOD CANYON PROV PARK, E OF SMITHERS.BC

WILSON MVH, 1977,CAN J EARTH SCI,VOL 14,N5,P1150,FIG $5 \mathrm{D}, 8 \mathrm{D}$

REMARKS: C.D-MVH WILSON :ON SLAB WITH TWO TIPULIDS ; 1 SLAB

\section{INSECT-ICHNEUMONIDAE}

31072 REF SPEC-F

EOC

BELOW MILPOST 39A ON HORSEFLY-BLACK CK RD

HORSEFLYR,BC

WILSON MVH, 1977, CAN J EARTH SCI, VOL 14,N5,P1150,FIG $8 \mathrm{~F}$

REMARKS: C.D-MVHWILSON : 1 SPEC
INSECT-ICHNEUMONIDAE

31055 REF SPEC-F

$M$ EOC,

[DRIFTWOOD CREEK FOSSIL BEDS ]

S SIDE DRIFTWOOD CK,DRIFTWOOD CANYON PROV PARK E OF SMITHERS, BC

WILSON MVH, 1977,CAN J EARTH SCI,VOL 14,N5,P11451146 , FIG 5C

REMARKS: C,D-MVH WILSON ; 1 SPEC

\section{INSECT-ICHNEUMONIDAE}

31047 REF SPEC-F

$M E O C$,

[DRIFTWOOD CREEK FOSSIL BEDS ]

S SIDE DRIFTWOOD CK,DRIFTWOOD CANYON PROV PARK, E OF SMITHERS,BC

WILSON MVH, 1977,CAN J EARTH SCI,VOL 14,N5,P 1150,FIG 7D

REMARKS: C,D-MVH WILSON ; 1 SPEC

INSECT-ICHNEUMONIDAE

31033 REF SPEC-F

$M E O C$,

[DRIFTWOOD CREEK FOSSIL BEDS]

S SIDE DRIFTWOOD CK,DRIFTWOOD CANYON PROV PARK, E OF SMITHERS,BC

WILSON MVH, 1977,CAN J EARTH SCI,VOL 14,N5,P1 150,FIG $8 \mathrm{C}$

REMARKS: C,D-MVH WILSON ; 1 SPEC

\section{INSECT-MEGALOPTERA}

31313 REF SPEC-F

EOC,

4.7 MI E OF HORSEFLY R BRIDGE ALONG BLACK CK RD, $N$ BANK HORSEFLY R,BC

WILSON MVH, 1977,CAN J EARTH SCI,VOL 14,N5,P1145,FIG $2 \mathrm{C}$

REMARKS: C,D-MVH WILSON ; 1 SPEC

\section{INSECT-PENTATOMIDAE}

31321 REF SPEC-F

MEOC,

PRINCETON GP,ALLENBY FM

N BANK TULAMEEN R,E END VERMILLION BLUFFS, $1.5 \mathrm{MI}$ W OF PRINCETON,BC

WILSON MVH, 1977,CAN J EARTH SCI, VOL 14,N5,P1144,FIG $4 \mathrm{~B}$

REMARKS: C.D-MVH WILSON ; 1 SPEC

INSECT-SCIARIDAE

31050 REF SPEC-F

$M E O C$,

[DRIFTWOOD CREEK FOSSIL BEDS ]

S SIDE DRIFTWOOD CK,DRIFTWOOD CANYON PROV PARK, E OF SMITHERS,BC

WILSON MVH, 1977,CAN J EARTH SCI,VOL

$14, N 5, P 1146,1149, F I G 6 E$

REMARK S: C.D-MVH WILSON : 1 SLAB

INSECT-SCIARIDAE

31309 REF SPEC-F

MEOC,

6 MI N OF HORSEFLY BY QUESNEL L RD , NE BANK HORSEFLYR,BC

WILSON MVH, 1977,CAN J EARTH SCI,VOL

$14, N 5, P 1146,1149, \mathrm{FIG} 5 \mathrm{~F}, 6 \mathrm{~F}$

REMARKS: C.D-MVH WILSON ; 1 SPEC

INSECT-SYRPHIDAE

31071 REF SPEC-F

$M E O C$,

6 MIN OF HORSEFLY BY QUESNEL L RD, NE BANK HORSEFLY R,BC

WILSON MVH, 1977, CAN J EARTH SCI,VOL 14,N5,P1150,FIG $6 \mathrm{G}, 7 \mathrm{C}$

REMARKS: C.D-MVH WILSON ; 1 SPEC 
INSECT-TIPULIDAE

31054 REF SPEC-F

MEOC,

[DRIFTWOOD CREEK FOSSIL BEDS ]

S SIDE DRIFTWOOD CK,DRIFTWOOD CANYON PROV PARK, E OF SMITHERS,BC

WILSON MVH, 1977,CAN J EARTH SCI, VOL 14, N5,P1145$1146, F I G$ 2G, $2 H$

REMARKS: C,D-MVH WILSON;TWO TIPULIDS ON SLAB

WITHICHNEUMONID WASP ; 1 SLAB

\section{INSECT-TIPULIDAE}

31043 REF SPEC-F

$M E O C$.

[DRIFTWOOD CREEK FOSSIL BEDS

S SIDE DRIFTWOOD CK,DRIFTWOOD CANYON PROV PARK E OF SMITHERS,BC

WILSON MVH, 1977,CAN J EARTH SCI, VOL 14,N5,P1145-

1146. FIG 21,5B

REMARKS:C,D-MVHWILSON ; 1 SPEC

\section{INSECT-TIPULIDAE}

31315 REF SPEC-M

$M$ EOC

[DRIFTWOOD CK FOSSIL BEDS]

S SIDE DRIFTWOOD CK.DRIFTWOOD CANYON PROV PARK, E OF SMITHERS, $8 \mathrm{C}$

WILSON MVH, 1977,CAN J EARTH SCI, VOL 14, N5,P1145-

1146

REMARKS: C,D-MVHWILSON; 1 SPEC

\section{INSECT-TIPULIDAE}

31052 REF SPEC-F

$M$ EOC.

[DRIFTWOOD CREEK FOSSIL BEDS

S SIDE DRIFTWOOD CK,DRIFTWOOD CANYON PROV PARK, E OF SMITHERS, $8 \mathrm{C}$

WILSON MVH, 1977,CAN J EARTH SCI, VOL 14, N5,P1145-

1146. FIG 2F, $5 \mathrm{C}$

REMARKS: C,D-MVHWILSON : 1 SPEC

\section{INSECT-TIPULIDAE}

31034 REF SPEC-F

$M E O C$.

[DRIFTWOOD CREEK FOSSIL BEDS

S SIDE DRIFTWOOD CK.DRIFTWOOD CANYON PROV PARK E OF SMITHERS, BC

WILSON MVH, 1977.CAN J EARTH SCI,VOL 14,N5,P1145-

1146. FIG 5A.6A

REMARKS:C,D-MVHWILSON ; 1 SPEC

\section{INSECT-TRICHOPTERA}

31069 REF SPEC-F

$M$ EOC.

[DRIFTWOOD CREEK FOSSIL BEDS]

S SIDE DRIFTWOOD CK,DRIFTWOOD CANYON PROV PARK, E OF SMITHERS.BC

WILSON MVH, 1977,CAN J EARTH SCI,VOL 14, N5,P1145,FIG $2 E$

REMARKS: C,D-MVHWILSON ; 1 SPEC

\section{INSECT-TRICHOPTERA}

31312 REF SPEC-F

EOC

BELOW MILEPOST 39A ON HORSEFLY-BLACK CK RD,

HORSEFLYR.BC

WILSON MVH, 1977.CAN J EARTH SCI,VOL 14, N5,P1145,FIG 2D,31

REMARKS: C.D-MVH WILSON ; 1 SPEC

\section{INSECT-VELIIDAE}

31044 REF SPEC-M

$M E O C$

[DRIFTWOOD CREEK FOSSIL BEDS]

S SIDE DRIFTWOOD CK, DRIFTWOOD CANYON PROV PARK, E OF SMITHERS.BC

WILSON MVH, 1977,CAN J EARTH SCI, VOL 14,N5,P1144

REMARKS:C.D-MVH WILSON ; 1 SPEC
INSECT-VELIIDAE

31057 REF SPEC.F

$M E O C$,

[DRIFTWOOD CREEK FOSSIL BEDS]

S SIDE DRIFTWOOD CK,DRIFTWOOD CANYON PROV PARK, E OF SMITHERS,BC

WILSON MVH, 1977,CAN J EARTH SCI, VOL 14,N5,P1144,FIG 3D

REMARKS: C,D-MVHWILSON; 1 SPEC

\section{INSECT-VELIIDAE}

31037 REF SPEC-M

$M E O C$,

[DRIFTWOOD CREEK FOSSIL BEDS ]

S SIDE DRIFTWOOD CK,DRIFTWOOD CANYON PROV PARK E OF SMITHERS,BC

WILSON MVH, 1977,CAN J EARTH SCI,VOL 14,N5,P1144

REMARKS: C.D-MVH WILSON; 1 SPEC

\section{INSECT-VELIIDAE}

31059 REF SPEC-M

MEOC,

[DRIFTWOOD CREEK FOSSIL BEDS ]

S SIDE DRIFTWOOD CK,DRIFTWOOD CANYON PROV PARK, E OF SMITHERS,BC

WILSON MVH, 1977,CAN J EARTH SCI,VOL 14, N5,P1144

REMARKS: C,D-MVH WILSON; 1 SPEC

\section{INSECT-VESPIDAE}

31319 REF SPEC-F

$M E O C$,

PRINCETON GP

SITE OF BLAKEBURN NO 3 MINE,S OF COALMONT, W OF PRINCETON,BC

WILSON MVH, 1977,CAN J EARTH SCI,VOL 14,N5,P1152,FIG $7 \mathrm{G}, 8 \mathrm{G}$

REMARKS: C,D-MVHWILSON : 1 SPEC

\section{MEGALEUCTRA NEAVEI RICKER}

7061 P-HOLO

MTERTIARY

[M TERTIARY]

YANTARNY,W KALININGRAD,USSR

RICKER WE, 1935,CAN ENTOMOLOGIST, VOL 67,N12,P259260

REMARKS: P-KRANTZ, 1910 ;LOC GIVEN AS PALMNICKEN, PRUSSIA ; 1 SPEC

SCARABAEIDAE INDET I

30728 REF SPEC-F

PLEIST,

TALARA TAR PITS,PERU

CHURCHER CS, 1966,CAN JOUR ZOOL, VOL 44,P985993,PL2,FIG 14

REMARKS: D-CS CHURCHER, VERT PALAEO;D-1974; DORSAL VIEW OF DAMAGED HEAD; 1 SPEC

\section{SCARABAEIDAE INDET II}

30732 REF SPEC-F

PLEIST,

TALARA TAR PITS,PERU

CHURCHER CS, 1966,CAN JOUR ZOOL, VOL 44,P985993,PL2,FIG 18

REMARKS: D-CS CHURCHER, VERT PALAEO;D-1974; DORSAL VIEW OF HEAD : 1 SPEC

\section{SCARABAEIDAE INDET}

30571 REF SPEC-F

PLEIST,

TALARA TAR PITS.PERL

CHURCHER CS, 1966,CAN JOUR ZOOL, VOL 44,P985993,PL 1,FIG 8

REMARKS:D-CS CHURCHER VERT PALAEO:D- 1974:TOP LEFT OF 9 SPECS MOUNTED ON SAME SLIDE;DORSAL VIEW OF PROFEMUR \& PROTIBIA OF 1 ST LEG $(Q) ; 1$ SLIDE 


\section{SPHINGIDAE INDET}

30729 REF SPEC-F

PLEIST.

TALARA TAR PITS,PERU

CHURCHER CS, 1966,CAN JOUR ZOOL, VOL 44,P985993,PL2,FIG 15

REMARKS: D-CS CHURCHER, VERT PALAEO:D-1974;RIGHT LATERAL VIEW OF PROBOSCIS ; 1 SLIDE

\section{TENEBRIONIDAE INDET}

30574 REF SPEC-F

PLEIST,

TALARA TAR PITS,PERU

CHURCHER CS, 1966,CAN JOUR ZOOL, VOL 44,P985993, PL1,FIG 11

REMARKS: D-CS CHURCHER,VERT PALAEO;D-1974;2ND FROM RIGHT OF 6 SPECS ON SAME SLIDE;DORSAL VIEW OF RIGHT ELYTRON ; 1 SLIDE

\section{TROPISTERNUS SP}

30570 REF SPEC-F

PLEIST,

TALARA TAR PITS,PERU

CHURCHER CS, 1966,CAN JOUR ZOOL, VOL 44,P985993.PL 1,FIG 7

REMARKS: D-CS CHURCHER,VERT PALAEO;D-1974; DORSAL VIEW OF RIGHT ELYTRON ; 1 SLIDE

\section{ARTHROPODA,MISCELLANEOUS}

\section{BRANCHIOCARIS PRETIOSA (RESSER)}

34308 REF SPEC-M

MCA.

STEPHEN FM,BURGESS MBR

RIDGE BETWEEN MT WAPTA\& MT FIELD , 3 MIN OF FIELD,BC BRIGGS DEG, 1976,GSC BULL 264,P6 SPEC COLLECTED BY PE RAYMOND, 1930,MCZ NO 5985: 1 SPEC

\section{PERSPICARIS RECONDITA BRIGGS}

34305 REF SPEC-M

$M C A$.

STEPHEN FM,BURGESS MBR

RIDGE BTWN MT FIELD + WAPTA MT, AT APPROX $2286 \mathrm{M}$, $4.8 \mathrm{KM} \mathrm{N}$ OF FIELD,BC

BRIGGS DEG, 1977,PALAEONTOLOGY,VOL 20,PT3,P606

REMARKS: C-ROM STAFF PARTY, $1975 ; 1$ SPEC
REMARKS: C-ROM STAFF PARTY, 1975 ;COUNTERPART TO

CALCEOCRINUS PUSTULOSUS BROWER

$7395[628 \mathrm{CL}]$ P.HOLO

S,MED

CATARACT GP.MANITOULIN FM

[MANITOULIN DOL,CATARACT]

STONY CK,ONT

Z2 13;BROWER JC,J PALEO,VOL 40,N3, 1966,P627-628,FIG 2C.PL75,FIG 13

REMARKS: C-H JOHNSON, 1930 :SPEC ORIGINALLY

DESCRIBED BY JOHNSON, UNPUB'D PHD THESIS, U OF T,

1934;PUBL'D BY BROWER AS JOHNSON'S SPECIES-

AUTHOR SHOULD BE BROWER; 1 SPEC

CALLOCYSTITES CANADENSIS (BILLINGS)

21186 [992N ] REF SPEC-F

MS.NIAG

CLINTON GP,ROCHESTER FM

[ROCHESTER]

GRIMSBY,ONT

S 199:SCHUCHERT C,SMITH MISC

COLL,VOL47,PT2, 1904,P245-246,PL34,FIG 3

REMARKS: D-BE WALKER ; 1 SPEC

CALLOCYSTITES JEWETTI HALL

21185 [991N] REF SPEC-F

$M S, N I A G$

CLINTON GP,ROCHESTER FM

[ROCHESTER]

GRIMSBY,ONT

S199:SCHUCHERT C.SMITH MISC

COLL,VOL 47,PT2, 1904,P243-245,PL34,FIG 1,2

REMARK S: D-BE WALKER, $1905 ; 1$ SPEC

CHEIROCRINUS GRANDULOSUS PARKS

24117 [920T] P-SYN

MO.CHAMP,MOH,TRENT

TRENTON GP,L COBOURG FM

[L COBOURG]

E OF COLLINGWOOD,ONT

Z213:PARKS WA,TRANS ROY SOC CAN,VOL 22,SECT

4,1928,P73-74,PL1,FIG 11-16

REMARKS: C-WA PARKS, 1927 :SPECS 'A' 'B' 'C' 'D' 'E'

' $F$ ' $=$ FIGS $11,13,15,12,14,16$ RESPECTIVELY'; 6 PCES

COMAROCYSTITES PUNCTATUS BILLINGS

18869 REF SPEC-F

MOCHAMP MOH,TRENT

TRENTON GP

[TRENTON]

OTTAWA ONT

S282;FOERSTE AF,OTT NATUR,VOL30,NO 7, 1916,P73-

79, PL2, FIG 1

REMARKS: P-JE NARRAWAY COLL. 1938; 1 SPEC

CRINOIDEA INCERTAE SEDIS

17489 [1250HR] REF SPEC-F

U O.CINCI,MAYSVILL

DUNDASFM

DON VALLEY QUARRY,TORONTO,ONT

...;FRITZ MA, ONT DEPT MINES,VOL

32.PT7, 1923,P18,PL2,FIG 11-14

REMARKS: C-WA PARKS, 1922 ;COLUMNALS ; 3 PCES

MS NIAG

CLINTON GP,ROCHESTER FM

[ROCHESTER SHALE]

GRIMSBY,ONT

S206:SCHUCHERT C,SMITH MISC COLL,VOL

47,PT2, 1904,P2 12,PL34,FIG 4,5

REMARKS: D-BE WALKER, 1905; 1 SPEC

BROCKOCYSTIS CLINTONENSIS (PARKS)

$7166[372 \mathrm{CL}] \mathrm{P}-\mathrm{HOLO}$

LEPADOCYSTIS CLINTONENSIS PARKS

MS,NIAG

CLINTON GP

[CLINTON]

FORKS OF THE CREDIT, CREDIT R.ONT

S206:PARKS WA, 1910,AMER JOUR SCI, VOL 29,P404.

406, FIG 1,2; FOERSTE AF, 1914,BULL DENISON UNIV SCI

LAB. VOL 17, P 473

REMARKS: C-J TOWNSEND, 1898;D-1909; 1 SPEC
DENDROCRINUS CF DYERI (MEEK)

16975 [7 15HR] S-HYPO

UO.CINCI,MAYSVILL

DUNDAS FM

[DUNDAS ]

W END GARRISON COMMON , TORONTO,ONT

Z2 15; FRITZ MA,ONT DEPT MINES,VOL 32,PT7, 1925,P16-

17.PL2,FIG 7

REMARKS: D-BE WALKER, $1905 ; 1$ SPEC

DEVONOBLASTUS WHITEAVESI REIMANN

20847 P-HOLO

$M D, E R I A N$

HAMILTON GP

[HAMILTON]

BOSANQUET TP.LAMBTON CO,ONT

S438:REIMANN IG BULL BUFF ACAD 
SCI,VOL 17,N 1, 1935,P32,PL1,FIG 8

REMARKS: D-BE WALKER :LOCIN PUBL GIVEN AS

THEDFORD AND VICINITY; 1 SPEC

EUTAXOCRINUS WHITEAVESI SPRINGER

$15737[1432 \mathrm{H}]$ REF SPEC-F

M D.ERIAN

HAMILTON GP

[HAMILTON]

BOS. :NQUET TP,LAMBTON CO.ONT

Z205:SPRINGER F, 1920,CRINOIDEA FLEXIBILIA.P366-

367,PL49,FIG 5A,B

REMARKS: D-BE WALKER, 1905 :LARGER OF 2 SPECS FIG'D 2 SPEC

GLAUCOCRINUS FALCONERI PARKS\&ALCOCK

23822 [610T] P-HOLO

MO.CHAMP,MOH,TRENT

TRENTONGP

[TRENTON]

KIRKFIELD.ONT

Z213:PARKS \& ALCOCK.OTT NATUR,VOL

26,N3\&4.1912,P41-43,PL4,FIG 1,4

REMARKS: C-J TOWNSEND, $1907 ; 1$ SPEC

GLYPTOCRINUS CIRCUMCARINATUS PARKS\&ALCOCK

23876 [668T] P-SYN

MO.CHAMP.MOH,TRENT

TRENTONGP

[TRENTON]

KIRKFIELD,ONT

Z160:PARKS + ALCOCK, OTT NATUR, VOL

26, N3 + 4, 19 12, P43-45, PL4,FIG 2,3

REMARK S: C-J TOWNSEND ;COMPOSITE FIGS DRAWN FROM 2 SPECS;SECOND SPEC NOT LOCATED; 1 SPEC

GLYPTOCRINUS DECADACTYLUS HALL

$17848[1248$ HR] REF SPEC-F

U O,CINCI,MAYSVILL

DUNDAS FM

DON VALLEY QUARRY,TORONTO,ONT

Z160; FRITZ MA, ONT DEPT MINES,VOL 32,PL7, 1925,P15-

16,PL2,FIG 2-5

REMARKS: C-J TOWNSEND :SEVERAL STEMS ON SLAB ; 1 SPEC

GLYPTOCYSTITES MULTIPORUS BILLINGS

18872 REF SPEC?

MO.CHAMP.MOH,TRENT

TRENTONGP

[TRENTON]

OTTAWA, ONT

REMARK S: P-JE NARRAWAY COLL, 1938:SPEC

LABELLED 'PLESIOTYPE';UNKNOWN WHERE

PUBLISHED ; 1 SPEC

HALLICYSTIS IMAGO (HALL)

$21014(A)[705 N(A)]$ REF SPEC-M

M S,NIAG

NIAGARA GP

[NIAGARAN]

BRIDGEPORT,ILL, USA

S209;SCHUCHERT C,SMITHSONIAN MISC COLL,VOL 47,PT

I, 1904,P216,FIG 24

REMARK S: D-BE WALKER, $1905 ; 1$ SPEC

HALLICYSTIS IMAGO (HALL)

$21014(A)[705 N(A)]$ REF SPEC-M

MS.NIAG

NIAGARA GP

[NIAGARAN]

BRIDGEPORT,ILL, USA

S209:SCHUCHERT C.SMITHSONIAN MISC COLL,VOL 47,PT I, 1904,P216,FIG 24

REMARKS: D-BE WALKER, $1905 ; 1$ SPEC
HETEROCRINUS JUVENIS HALL

17486 [ 1247HR] REF SPEC-F

U O.CINCI.MAYSVILL

DUNDAS FM

DON VALLEY QUARRY, TORONTO,ONT

Z212; FRITZ MA, ONT DEPT MINES, VOL 32,PT7, 1925,P 10 12,PL1,FIG 7

REMARKS: C-WA PARKS, 1922 ;SMALL SEPARATE CALYX FIG'D;2SPEC

HETEROCRINUS JUVENIS HALL

17490 [1251HR] REF SPEC-F

U O.CINCI,MAYSVILL

DUNDAS FM

[DUNDAS ]

DON VALLEY QUARRY,TORONTO. ONT

Z212; FRITZ MA, ONT DEPT MINES, VOL 32,PT7, 1925,P 10 12,PL1,FIG 11,12

REMARKS: C-WA PARKS, 1922 ;SINGLE COLUMNAL = FIG $11 ;$ SECT OF STEM $=$ FIG $12 ; 2$ SPEC

HYBOCYSTIS ELDONENSIS PARKS

23779 [566T] P.HOLO

M O.CHAMP,MOH,TRENT

TRENTON GP

[TRENTON]

TRENT VALLEY CANAL, NR KIRKFIELD, ONT

Z211:PARKS WA, OTT NATUR, VOL 21,N 12, 1908,P234.

235,PL2,FIG 4

REMARKS: C-J TOWNSEND, $1907 ; 1$ SPEC

ICHTHYOCRINUS LAEVIS CONRAD

$21306[1123 N$ ] REF SPEC-F

MS,NIAG

CLINTON GP,ROCHESTER FM

[NIAGARA]

GRIMSBY.ONT

Z240:SPRINGER F. 1920,CRINOIDEA FLEXIBILIA,P277.

281,PL33,FIG 13A

REMARKS: D-BE WALKER, $1905 ; 1$ SPEC

IOCRINUS SUBCRASSUS VAR TORONTONENSIS F.

16586 [245HR] P-HOLO

U O.CINCI,MAYSVILL

DUNDAS FM

[DUNDAS]

DON VALLEY QUARAY,TORONTO,ONT

Z212; FRITZ MA,ONT DEPT MINES, VOL

32,PT7, 1925, P13,PL1,FIG 5

REMARKS: C-STUDENTS, $1902 ; 1$ SPEC

LECANOCRINUS MACROPETALUS HALL

$21303[1120 \mathrm{~N}]$ REF SPEC-F

M S,NIAG

CLINTON GP.ROCHESTER FM

[NIAGARAN ]

GRIMSBY,ONT?

Z203:SPRINGER F, 1920,CRINOIDEA FLEXIBILIA,P 129. 130.PL3,FIG 7

REMARKS: D-BE WALKER, 1905 ;SMALLEST OF 3 SPECS IS POSSIBLY THE ONE FIG'D BY SPRINGER ; 3 SPEC

LICHENOCRINUS SP

17488 [1249HR] REF SPEC-F

U O,CINCI,MAYSVILL

DUNDAS FM

[DUNDAS ]

HUMBERVALE, TORONTO,ONT

Z160;FRITZ MA, ONT DEPT MINES, VOL

32,PT7, 1925,P18,PL2,FIG 8,9

REMARKS: C-WA PARKS, 1922 ;FIG 9 INCORRECTLY LABELLED 6 ON PLATE ; 2 SPEC

MESOBLASTUS HAYNESI (CLARKE)

6875 REF SPEC-F

CM.

MT COLEMAN,ALTA

S424:FRITZ \& CLINE, TRANS ROY CAN

INST,VOL21,N46, 1937,P309-312.PL17,FIG 1-9.13

REMARKS: D-AP COLEMAN :SPECS LABELLED $1-6 ; 1^{\prime} 4^{\prime}=F I G$ 
$1-6 ;^{\prime} 2^{\prime}=$ FIG $7:^{\prime} 3^{\prime}=$ = FIG 8; ' 1 ' SECTIONED (SLIDE);OTHERS NOT FIG'D:STRAT UNCERTAIN-BANFF SH OR RUNDLE LS ; 6 SPEC,SLIDE 2230

\section{PENTREMITIDEA CUMMINGSI REIMANN}

902 P-HOLO

M D.ERIAN

HAMILTONGP

THEDFORD.ONT

S4 13:REIMANN IG,BULL BUFF ACAD SCI,VOL 17,N1,P28. 29.PL2,FIG 19,PL4,FIG 6

REMARKS: C-WA PARKS ; 1 SPEC

PERIGLYPTOCRINUS PRISCUS (BILLINGS)

23857 [649T] REF SPEC-M

MO.CHAMP MOH,TRENT

TRENTONGP

[TRENTON]

BALSAM L.ONT

Z190:PARKS WA, 1909, OTTAWA NATURALIST, VOL 23, N9,P153-155

REMARKS: C-J TOWNSEND :SPEC FIG'D BY PARKS IS

MISSING ; 1 SPEC

PLEUROCYSTITES SQUAMOSUS BILLINGS

27211 REF SPEC-M

PLEUROCYSTITES FILITEXTUS BILLINGS

LISKEARD FM

ABANDONED QUARRY ON HILL W OF SPLINT FACTORY,

HAILEYBURY,ONT

S194;BOLTON TE, GSC BULL 187,1970,P61

REMARKS: C-M FRITZ,J MONTEITH, 1949; 1 SPEC

\section{PLEUROCYSTITES SQUAMOSUS BILLINGS}

27214 REF SPEC-M

PLEUROCYSTITES FILITEXTUS BILLINGS

O

LISKEARD FM

ABANDONED QUARRY ON HILL W OF SPLINT FACTORY,

HAILEYBURY, ONT

S194:BOLTON TE, GSC BULL 187,1970,P61

REMARKS: C-M FRITZ,J MONTEITH, 1949;SPEC LABELLED

' 1 ' ON SLAB; 1 SLAB

PLEUROCYSTITES SQUAMOSUS BILLINGS

27214 REF SPEC-M

O.

LISKEARD FM

ABANDONED QUARRY ON HILL W OF SPLINT FACTORY,

HAILEYBURY, ONT

S194;BOLTON TE,GSC BULL 187,1970,P61

REMARKS: C-M FRITZ,J MONTEITH, 1949;SPEC LABELLED

'2' ON SLAB; 1 SLAB

PLEUROCYSTITES SP

27213 REF SPEC-M

O

LISKEARD FM

ABANDONED QUARRY ON HILL W OF SPLINT FACTORY,

HAILEYBURY,ONT

S194:BOLTON TE, GSC BULL 187,1970,P6

REMARKS: C-M FRITZ,J MONTEITH, 1949; 1 SPEC

\section{ECHINODERMATA,ASTEROZOA}

PROMOPALEASTER SOLITARIUS FRITZ

23305 P-SYN

U O,CINCI,MAYSVILL

DUNDAS FM

[DUNDAS ]

HUMBER R TORONTO.ONT

U53;FRITZ MA,ONT DEPT MINES, VOL 32,PT7, 1925,P 19 21.FIG 5,PL2,FIG 19

REMARKS: C-J TOWNSEND :LABELLED COTYPE IN FRITZ'S TYPE CAT;PUBL 'D AS NO 204GS-NO SUCH NO IN ROM RECORDS : 1 SPEC
PROMOPALEASTER SOLITARIUS FRITZ

193 P-SYN

U O,CINCI,MAYSVILL

DUNDASFM

[DUNDAS ]

HUMBER R, TORONTO,ONT

U53;FRITZ MA,ONT DEPT MINES,VOL 32,PT7, 1925,P1921,FIG 4

REMARKS: P- ;PUBL'D AS NO 193HR;SHOULD BE $193 ; 1$ SPEC

PROTASTER WHITEAVESIANUS PARKS

23846 [638T] P-SYN

M O,CHAMP,MOH,TRENT

TRENTON GP

[TRENTON]

KIRKFIELD,ONT

U87:PARKS WA, TRANS ROY CAN INST, VOL $8,1908, P 368$

372,FIG 1-6

REMARKS: C-J TOWNSEND ; 5 SPEC,SLIDE 402

PROTOPALEASTER NARRAWAYI HUDSON

$18881 \mathrm{P}-\mathrm{HOLO}$

M O,CHAMP,MOH,BLKRIV

BLACK RIVER GP

[LR PART BLACK RIVER LS ]

TOP OF SMALL HILL,FEW RODS W OF CITY VIEW PO ,

OTTAWA ONT

U50;HUDSON GH,OTT NATUR, VOL 26,N2, 1912,P 1-13,PL 1-3:

ALSO VOL 26,N3\&4,P45-52

REMARKS: C,P-JE NARRAWAY;P-1938; 1SPEC

SCHUCHERTIA STELLATA (BILLINGS)

18857 REF SPEC-M

MO,CHAMP,MOH,TRENT

TRENTON GP

[TRENTON]

QUEBEC SIDE , CHAUDIERE FALLS, QUE

U65;BRANSTRATOR JW, J PALEO, VOL 46, 1972,P69

REMARKS: P-JE NARRAWAY COLLN, $1938 ; 11$ SPEC

\section{ECHINODERMATA,ECHINOZOA}

BELOCHTHUS ORTHOKOLUS BELL

18874 P-HOLO

MO,CHAMP,MOH,TRENT

TRENTONGP

[TRENTON]

STEAMBOAT LANDING.OTTAWA,ONT

BELL BM, 1976,NY STATE MUS + SCI SERVICE,MEM

21,P77-82,TEXT FIG 8E,PL7,FIG 1-3

REMARKS: P-JE NARRAWAY, 1938; 1SMALL SLAB

BELOCHTHUS ORTHOKOLUS BELL

28179 P.PARA

MO,CHAMP,MOH,TRENT

TRENTON GP

[TRENTON]

PETERBOROUGH,ONT

BELL BM, 1976,NY STATE MUS + SCI SERVICE,MEM 21,P77-82,PL7,FIG 4,5

REMARK S: C-J TOWNSEND; ISOROPHUSELLA INCONDITA

LABELLED 160T-A ON SLAB ; 1 SPEC (SMALL SLAB)

BELOCHTHUS ORTHOKOLUS BELL

23481-C [160T-C] P-PARA

MO.CHAMP,MOH,TRENT

TRENTON GP

[TRENTON]

PETERBOROUGH,ONT

BELL BM, 1976,NY STATE MUS + SCI SERVICE,MEM

21,P77-82,TEXT FIG 8B,C,PL8,FIG 2-5

REMARKS: C-J TOWNSEND : MANY JUVENILE

ISOROPHUSELLA INCONDITA ON SLAB ALSO FIG'D;

FIG'D SPECS ARE NUMBERED ON SLAB AS IN PUBL' $N$; 1

SMALL SLAB 
CRYPTOGOLEUS CHAPMANI (RAYMOND)

28178 REF SPEC-F

MO.CHAMP,MOH,TRENT

TRENTON GP

[TRENTON]

FOOT OF PARLIAMENT HILL, OTTAWA,ONT

BELL BM, 1976,NY STATE MUS + SCI SERVICE,MEM

21.P 131-138.PL 19,FIG 9

REMARKS: P-JE NARRAWAY, 1938; 1SPEC

CRYPTOGOLEUS CHAPMANI (AAYMOND)

23481-B [160T-B] REF SPEC-F

MO.CHAMP,MOH,TRENT

TRENTON GP

[TRENTON]

PETERBOROUGH,ONT

BELL BM, 1976,NY STATE MUS + SCI SERVICE,MEM

21.P 131-138, TEXT FIG 18C.PL20.FIG 2.3

REMARK S: C-J TOWNSEND ;SPEC STORED UNDER

ISOROPHUSELLA INCONDITA (BASSLER); 1 SMALL

SLAB

CRYPTOGOLEUS CHAPMANI (RAYMOND)

28177 REF SPEC-F

M O.CHAMP.MOH,TRENT

TRENTON GP

[TRENTON]

PETERBOROUGH.ONT

BELL BM, 1976, NY STATE MUS + SCI SERVICE,MEM

21,P 131-138, TEXT FIG 17A,PL20,FIG 6-9

REMARK S: C-J TOWNSEND ; 1 SMALL SLAB

CRYPTOGOLEUS RETICULATUS BELL

28176 P-HOLO

MO.CHAMP,MOH,TRENT

TRENTON GP

[TRENTON]

FOOT OF PARLIAMENT HILL, OTTAWA, ONT

BELL BM, 1976,NY STATE MUS + SCI SERVICE,MEM

21,P138-140,TEXT FIG 19A,PL21,FIG 1,2

REMARKS: P-JE NARRAWAY,1938; 1 SPEC

CYCLOCYSTOIDES MILLERI BILLINGS

REMARKS: SEE NARRAWAYELLA RAYMONDI FOERSTE

FOERSTEDISCUS GRANDIS BASSLER

23757 [543T] REF SPEC-F

MO.CHAMP,MOH,TRENT

TRENTON GP

[TRENTON]

TRENT VALLEY CANAL, NR KIRKFIELD, ONT

BELL BM, 1976, NY STATE MUS + SCISERVICE,MEM

21,P66-71, TEXT FIG 5A,B,PL3,FIG 3-5

REMARKS: C-J TOWNSEND; 1 SPEC

ISOROPHUSELLA INCONDITA (RAYMOND)

23481[160T] REF SPEC-F

MO.CHAMP MOH,TRENT

TRENTON GP

[TRENTON]

PETERBOROUGH ONT

-.-;BELL BM, 1976, NY STATE MUS + SCI SERVICE,MEM

21,P 176-190, TEXT FIG 30D-F,31A-C,E,F,32A,B,PL32,FIG 6-7.PL33,FIG 1,3-14

REMARKS: C-J TOWNSEND ;BIBLIO-PL34,FIG 1-8,PL35,FIG 1-4:SPECS ARE NUMBERED ON SLABS AS PUBL'D SPECS 160T-A + 160T-C STORED UNDER BELOCHTHUS ORTHOKOLUS : 5 SMALL SLABS

ISOROPHUSELLA INCONDITA (RAYMOND)

18873

MO.CHAMP.MOH,TRENT

TRENTON GP

[TRENTON]

FOOT OF PARLIAMENT HILL, OTTAWA,ONT

-..-BELL BM, 1976, NY STATEMUS + SCI SERVICE,MEM

21. P176-190 TEXT FIG 29C PL32. FIG 8.9

REMARKS: P-JE NARRAWAY, 1938 ;SPEC A FIG 'D ; 9 SPEC
LEBETODISCUS DICKSONI (BILLINGS)

18848 REF SPEC-F

MO.CHAMP,MOH,TRENT

TRENTON GP

[TRENTON]

OTTAWA,ONT

BELL BM, 1976,NY STATE MUS + SCI SERVICE,MEM

21,P55-65.PL1,FIG 12-13

REMARKS: P-J NARRAWAY, $1938 ; 1$ SPEC

LEBETODISCUS DICK SONI (BILLINGS)

23482 [161T] REF SPEC-F

$M O, C H A M P, M O H, T R E N T$

TRENTON GP

[TRENTON]

PETERBOROUGH,ONT

BELL BM, 1976, NY STATE MUS + SCI SERVICE,MEM

21,P55-65, TEXT FIG 4A,PL2,FIG 7-9

REMARKS: C-J TOWNSEND ; THIS SPEC CONSIDERED BY BELL TO BE THE GSC TYPE $1415 ; 1$ SPEC

LEBETODISCUS DICKSONI (BILLINGS)

18855 REF SPEC-F

MO CHAMP MOH, TRENT

TRENTON GP

[TRENTON]

HULL, QUE

BELL BM, 1976,NY STATE MUS + SCI SERVICE,MEM

21,P55-65,PL1,FIG 3-6

REMARKS: P-JE NARRAWAY, 1938 ;SPECS A + B FIG'D ; 3 SPEC

MACRASTER PSEUDOELEGANS ADKINS

11173 [5799CT] REF SPEC?

$\mathrm{K}, \mathrm{COM}, \mathrm{WASH}$

[WASHITA COMANCHEAN]

7 MIS OF CLEBURNE, JOHNSON CO,TEX,USA

REMARKS: P-JB LITSEY, 1934 L LABELLED PLESIOTYPE,BUT NOT KNOWN WHERE PUBL'D; 1 SPEC

NARRAWAYELLA RAYMONDI FOERSTE

18868 P-HOLO

CYCLOCYSTOIDES HALLIBILLINGS

M O,CHAMP,MOH,TRENT

TRENTON GP

[TRENTON]

AXE FACTORY,HULL,QUE

U206:RAYMOND PE, 1913,GSC MUS BULL 1,P28,FIG

3,PL3,FIG 4;FOERSTE AF,OHIO JOUR SCI,VOL $21, N 2,1920, P 60$

REMARKS: P-JE NARRAWAY COLLN, 1938 :LOC GIVEN AS WALTER'S FACTORY IN ROM RECORDS, AXE FACTORY IN PUBL' $N$; 1 SPEC

\section{ANNELIDA}

ARABELLITES? CLAYTONAE ELLER

26128 P-PARA

MO.CHAMP,MOH,TAENT

TRENTON GP, ROCKLAND FM

LAKEFIELD,ONT

W149;ELLER ER,ANN CARNEGIE MUS, VOL 30,ART

$12,1945, P 140-141$

REMARKS: C-ER ELLER;D-1944 :SLIDE NO 243;NOT FIG'D 1 SPEC

EUNICITES SWEADNERI ELLER

26137 P-PARA

MO,CHAMP,MOH,TRENT

TRENTON GP, COBOUAG FM

COLLINGWOOD.ONT

W149;ELLER ER,ANN CARNEGIE MUS, VOL 30,ART 12,P 180 REMARKS: C-ER ELLER:D-1944 :SLIDE NO 243:NOT FIG'D 1 SPEC 
EUNICITES TODDI ELLER

26118 P-PARA

MO,CHAMP, MOH,TRENT

TRENTON GP, ROCKLAND FM

LINDSAY,ONT

W149;ELLER ER,ANN CARNEGIE MUS,VOL 30,ART $12,1945, P 180$

REM ARKS: C-ER ELLER;D-1944;SLIDE NO 224;NOT FIG'D ; 1 SPEC

\section{EUNICITES TOLMACHOFFI ELLER}

26138 P.PARA

MO.CHAMP.MOH,TRENT

TRENTON GP, COBOURG FM

COLLINGWOOD,ONT

W149:ELLER ER, ANN CARNEGIE MUS, VOL 30,ART

$12,1945, P 177$

REMARK S: C-ER ELLER;D-1944 ;SLIDE NO 244;NOT FIG'D ; 1SPEC

\section{EUNICITES TRENTONENSIS CALEY}

24356 [1198T] P-HOLO

MO.CHAMP.MOH,TRENT

TRENTONGP

[TRENTON]

RLWY CUT, LITTLE CURRENT, MANITOULIN IS, ONT W149;CALEY JF,GSC MEM 202, 1936,P68-69,PL 1,FIG 1 REMARKS: C-JF CALEY, $1933 ; 1$ SPEC

ILDRAITES CAMURUS ELLER

26139 P.PARA

MO,CHAMP,MOH,TRENT

TRENTON GP, COBOURG FM

COLLINGWOOD,ONT

W154:ELLER ER,ANN CARNEGIE MUS,VOL 30,ART 12,P142 REMARKS: C-ER ELLER;D-1944 ;SLIDE NO 245;NOT FIG'D; 1 SPEC

ILDRAITES RARUS ELLER

26119 P-PARA

$M O, C H A M P, M O H, T R E N T$

TRENTON GP, ROCKLAND FM

LINDSAY,ONT

W154;ELLER ER, ANN CARNEGIE MUS,VOL 30,ART

$12,1945, P_{143}$

REMARKS: C-ER ELLER;D-1944 ;SLIDE NO 224;NOT FIG'D ; 1SPEC

LEODICITES ARTUS ELLER

26129 P.PARA

MOCHAMP MOH TRENT

TRENTON GP,ROCKLAND FM

LAKEFIELD,ONT

W149.ELLER ER ANN CARNEGIE MUS, VOL 30 ART $12,1945, P 145$

REMARKS: C-ER ELLER;D-1944 ;SLIDE NO 235;NOT FIG'D ; 1SPEC

LEODICITES BROOKSI ELLER

26130 P.PARA

MO.CHAMP.MOH,TRENT

TRENTON GP, ROCKLAND FM

LAKEFIELD, ONT

W149;ELLER ER, ANN CARNEGIE MUS, VOL 30,ART $12,1945, P 144$

REMARK S: C-ER ELLER:D-1944 :SLIDE NO 236;NOT FIG'D : 1 SPEC

LEODICITES BURIS ELLER

26140 P-PARA

MO CHAMP MOH TRENT

TRENTON GP,COBOURG FM

COLLINGWOOD,ONT

W 149;ELLER ER,ANN CARNEGIE MUS,VOL 30,ART 12, 1945,P $152-153$

REMARKS: C-ER ELLER;D-1944 ;SLIDE NO 246:NOT FIG'D ; 1 SPEC
LUMBRICONEREITES ASSIMILIS ELLER

26120 P.PARA

MO,CHAMP,MOH,TRENT

TRENTON GP,ROCKLAND FM

LINDSAY.ONT

W151;ELLER ER,ANN CARNEGIE MUS, VOL 30,ART 12, 1945,P138-139

REMARKS: C-ER ELLER;D- 1944 ;SLIDE NO 226;NOT FIG'D ; 1SPEC

\section{LUMBRICONEREITES CRASSUS ELLER}

26131 P-PARA

MO,CHAMP, MOH,TRENT

TRENTON GP,ROCKLAND FM

LAKEFIELD,ONT

W151:ELLER ER, ANN CARNEGIE MUS, VOL 30 ,ART $12,1945, P 129$

REMARKS: C-ER ELLER;D-1944 ;SLIDE NO 237;NOT FIG'D ; 1 SPEC

LUMBRICONEREITES JOHNSONI ELLER

26121 P-PARA

MO.CHAMP, MOH, TRENT

TRENTON GP, ROCKLAND FM

LINDSAY,ONT

W151;ELLER ER, ANN CARNEGIE MUS, VOL 30,ART $12,1945, P 132-133$

REMARKS: C-ER ELLER;D-1944 :SLIDE NO 227:NOT FIG'D : 1 SPEC

\section{LUMBRICONEREITES LONGAE ELLER}

26141 P.PARA

MO,CHAMP, MOH, TRENT

TRENTON GP,COBOURG FM

COLLINGWOOD,ONT

W151;ELLER ER, ANN CARNEGIE MUS, VOL 30,ART 12, 1945,P 134-135

REMARKS: C-ER ELLER;D- 1944 ;SLIDE NO 247;NOT FIG'D; 1 SPEC

\section{LUMBRICONEREITES PROJECTUS ELLER}

26132 P-PARA

MO,CHAMP,MOH,TRENT

TRENTON GP,ROCKLAND FM

LAKEFIELD,ONT

W151; ELLER ER,ANN CARNEGIE MUS, VOL 30,ART $12,1945, P 129$

REMARKS: C-ER ELLER;D-1944 ;SLIDE NO 238;NOT FIG'D; 1 SPEC

\section{LUMBRICONEREITES PROJECTUS ELLER}

26122 P-PARA

M O,CHAMP,MOH,TRENT

TRENTON GP.ROCKLAND FM

LINDSAY,ONT

W151; ELLER ER, ANN CARNEGIE MUS, VOL 30,ART $12,1945, P 129$

REMARKS: C-ER ELLER;D- 1944 ;SLIDE NO 228;NOT FIG'D; 1 SPEC

LUMBRICONEREITES WAYICALEY

17754 [1536HR] P-HOLO

U O.CINCI,MAYSVILL

WEKWEMIKONGSING FM

KAGAWONG FALLS, MANITOULIN IS,ONT

W151:CALEY JF GSC MEM 202,1936,P69,PL 1,FIG 3

REMARKS: C-JF CALEY, $1933 ; 1$ SPEC

NEREIDAVUS ALATUS ELLER

26133 P-PARA

MO, CHAMP,MOH,TRENT

TRENTON GP,ROCKLAND FM

LAKEFIELD,ONT

W151; ELLER ER,ANN CARNEGIE MUS, VOL 30,ART 12, 1945,P $189-190$

REMARKS: C-ER ELLER:D- 1944 ;SLIDE NO 230;NOT FIG'D : 1 SPEC 
NEREIDAVUS AVINOFFIELLER

26142 P-PARA

MO.CHAMP,MOH,TRENT

TRENTON GP.COBOURG FM

COLLINGWOOD.ONT

W151:ELLER ER,ANN CARNEGIE MUS,VOL 30, ART $12,1945, \mathrm{P} 188$

REMARKS: C-ER ELLER;D-1944 ;SLIDE NO 248;NOT FIG'D 1 SPEC

PALEOENONITES ACUTUS ELLER

26127 P.PARA

MO.CHAMP MOH,TRENT

TRENTON GP,ROCKLAND FM

KIRKFIELD,ONT

W150;ELLER ER,ANN CARNEGIE MUS,VOL 30,ART 12.1945,P 170

REMARK S: C-ER ELLER;D- 1944 ;SLIDE NO 233;NOT FIG'D 1 SPEC

PALEOENONITES DAVISAE ELLER

26124 P-PARA

MO.CHAMP.MOH,TRENT

TRENTON GP.ROCKLAND FM

LINDSAYONT

W150;ELLER ER,ANN CARNEGIE MUS, VOL 30,ART $12,1945, P 175-176$

REMARKS: C-ER ELLER:D-1944 ;SLIDE NO 230;NOT FIG'D 1 SPEC

PALEOENONITES DILLAE ELLER

26136 P-PARA

MO.CHAMP.MOH,TRENT

TRENTON GP,ROCKLAND FM

LAKEFIELD ONT

W150;ELLER ER,ANN CARNEGIE MUS,VOL 30,ART

$12,1945, P 170-171$

REMARKS: C-ER ELLER;D-1944 ;SLIDE NO 242;NOT FIG'D 1 SPEC

SERPULITES FRITZI PARKS

$17652[1419 \mathrm{HR}]$ P-HOLO

UO.CINCI,MAYSVILL

DUNDAS FM

[DUNDAS ]

LOC 83 (130-135 FT), WORKMAN'S CK ONT

W160;PARKS WA, TRANS ROY SOC CAN, VOL 22,SECT

4. 1928,P72-73,FIG 21,PL1,FIG 18

REMARKS: C-MA FRITZ, $1927 ; 1$ SPEC (2 PCES)

SERPULITES ISOLATUS PARKS

24960 [283U] P-SYN

UO.CINCI,EDEN

COLLINGWOOD FM

CRAIGLEITH ONT

W160:PARKS WA.TRANS ROY SOC CAN, VOL 22,SECT

4.1928,P71-72, FIG 20,PL1,FIG 31

REMARKS: C-WA PARKS, 1927 ;LARGER SPEC FIG'D, FIG 20 2 SPEC

SERPULITES SERRATUS PARKS

24958 [281U] P.SYN

U O.CINCI.EDEN

COLLINGWOOD FM

CRAIGLEITH,ONT

W160:PARKS WA TRANS ROY SOC CAN, VOL 22, SECT

4.1928,P70,FIG 19,PL 1,FIG 29,30

REMARKS: C-WA PARKS, 1927 ;FIG 19 IS SPEC 'B' ; 5 SPEC

STAUROCEPHALITES NETTINGI ELLER

26134 P-PARA

MO.CHAMP.MOH,TRENT

TRENTON GP ROCKLAND FM

LAKEFIELD,ONT

W153:ELLER ER,ANN CARNEGIE MUS,VOL 30,ART 12, 1945,P 162

REMARK S: C-ER ELLER;D- 1944 ;SLIDE NO 240;NOT FIG'D 1 SPEC
UNGULITES SUMNERAE ELLER

26125 P-PARA

MOCHAMP MOH TRENT

TRENTON GP.ROCKLAND FM

LINDSAY,ONT

W152:ELLER ER,ANN CARNEGIE MUS, VOL 30,ART

$12,1945, P 186-187$

REMARKS: C-ER ELLER;D-1944;SLIDE NO 231;NOT FIG'D 1 SPEC

UROTHECAFLAGELLUM MATTHEW

8577 [947CM] P-SYN

$M C A$.

MT STEPHEN,FIELD,BC

W 165:MATTHEW GF, TRANS ROY SOC CAN, VOL 5,SECT 4, 1899, P40-41,PL1,FIG 1A-C

REMARK S: C-GF MATTHEW:D-MACKENZIE ; 1 SPEC

UROTHECA PARVA MATTHEW

$7985[355 \mathrm{CM}] \mathrm{P}-\mathrm{HOLO}$

$M C A$.

STEPHEN FM

MT STEPHEN, FIELD,BC

W165:MATTHEW GF, TRANS ROY SOC CAN, VOL 5,SECT

4,1899,P41,PL7,FIG 2

REMARKS: C-GF MATTHEW;D-MACKENZIE ; 1 SPEC

UROTHECA PERVETUM MATTHEW

8106 [476CM] P-SYN

LCA

ADEYTON GP,BONAVISTAFM

[UETCHEMINIAN]

SMITH SD,NFLD

W165;MATTHEW GF, 1899,NB,P192,PL1,FIG 8;MATTHEW GF TRANS ROY SOC CAN, VOL 5 SECT

4,1899, P 106,PL5, FIG 8

REMARKS: C-GFMATTHEW:D-MACKENZIE :LABELLED COTYPES:STRAT REV AS IN HUTCHINSON, 1962,GSC BULL $88 ; 2$ SPEC

\section{CONODONTOPHORIDA}

GONDOLELLA LAEVIS KOSENDO+KOZITSKAYA

34444 REF SPEC-F

CP,

[SEVILLE LS MBR]

ROCK ISLAND CO,ILL,USA

W60; VON BITTER + MERRILL, 1977, LIFE SCI OCC

PAP, ROM, N29, FIG 2A-E

REMARKS: C-GK MERRILL; 1 SPEC

GONDOLELLA SUBLANCEOLATA GUNNELL

30642 REF SPEC-F

CP,VIRGIL

SHAWNEE GP OREAD FM, HEEBNER MBR

SW NW SEC 7, T75N, R29W, MADISON CO,IOWA, USA W60:VON BITTER PH, 1976, LIFE SCI CONTR,ROM,N 109,P5 10, FIG 3D

REMARKS: C-PH VON BITTER ;SP ELEMENT;LOC 2 , SAMPLE HE-16-3 OF VON BITTER ; 1 SLIDE

GONDOLELLA SUBLANCEOLATA GUNNELL 30643 REF SPEC-F

CP, VIRGIL

SHAWNEE GP, OREAD FM, HEEBNER MBR

SW NW SEC 7. T75N R29W, MADISON CO.IOWA. USA

W60;VON BITTER PH, 1976, LIFE SCI CONTR,ROM, N 109,P510, FIG 3E

REMARKS: C-PH VON BITTER :SP ELEMENT:LOC 2 SAMPLE HE-16-3 OF VON BITTER; 1 SLIDE

GONDOLELLA SUBLANCEOLATA GUNNELL 30644 REF SPEC-F

CP.VIRGIL

SHAWNEE GP.OREAD FM,HEEBNER MBR

SW NW SEC 7,T75N,R29W, MADISON CO,IOWA, USA 
W60; VON BITTER PH, 1976,LIFE SCI CONTR,ROM,N 109,P510, FIG 3F

REMARK S: C-PH VON BITTER ; SP ELEMENT:LOC 2 ,

SAMPLE HE- $16-3$ OF VON BITTER ; 1 SLIDE

GONDOLELLA SUBLANCEOLATA GUNNELL

30645 REF SPEC-F

CP.VIRGIL

SHAWNEE GP, OREAD FM, HEEBNER MBR

SW NW SEC 7,T75N,R29W, MADISON CO,IOWA, USA

W60; VON BITTER PH, 1976,LIFE SCI CONTR,ROM,N109,P510,FIG 3G

REMARKS: C-PH VON BITTER;SP ELEMENT;LOC 2

SAMPLE HE-16-3 OF VON BITTER ; 1 SLIDE

GONDOLELLA SUBLANCEOLATA GUNNELL 30646 REF SPEC-F

CP. VIRGIL

SHAWNEE GP OREAD FM, HEEBNER MBR

SW NW SEC 7,T75N,R29W, MADISON CO,IOWA, USA

W60;VON BITTER PH, 1976, LIFE SCI CONTR,ROM,N109,P510,FIG 3H

REMARK S: C-PH VON BITTER ;SP ELEMENT;LOC 2 ,

SAMPLE HE-16-3 OF VON BITTER : 1 SLIDE

\section{GONDOLELLA SUBLANCEOLATA GUNNELL}

30647 REF SPEC-F

CP. VIRGIL

SHAWNEE GP,OREAD FM, HEEBNER MBR

SE NE SEC 16,T75N,R37W, CASS CO,IOWA,USA

W60; VON BITTER PH, 1976.LIFE SCI CONTR,ROM,N109,P510, FIG 31

REMARK S: C-PH VON BITTER ;SP ELEMENT,LOC 1 ,

SAMPLE HE-15-2 OF VON BITTER ; 1 SLIDE

\section{GONDOLELLA SUBLANCEOLATA GUNNELL}

30648 REF SPEC-F

CP. VIRGIL

SHAWNEE GP OREAD FM, HEEBNER MBR

SE NE SEC 16,T75N, R37W, CASS CO,IOWA, USA

W60;VON BITTER PH, 1976. LIFE SCI CONTR,ROM,N 109,P510,FIG 3J,K

REMARKS: C-PH VON BITTER ;SP ELEMENT,LOC 1 , SAMPLE HE-15-2 OF VON BITTER; 1 SLIDE

\section{GONDOLELLA SUBLANCEOLATA GUNNELL} 30650 REF SPEC-F

CP.VIRGIL

SHAWNEE GP, OREAD FM, HEEBNER MBR

SW NW SEC 7,T75N,R29W, MADISON CO,IOWA, USA W60;VON BITTER PH, 1976, LIFE SCI

CONTR,ROM,N 109,P12,FIG 6A

REMARKS: C-PH VON BITTER :OZ ELEMENT;LOC 2 ,

SAMPLE HE-16-3 OF VON BITTER; 1 SLIDE

GONDOLELLA SUBLANCEOLATA GUNNELL

30651 REF SPEC-F

CP.VIRGIL

SHAWNEE GP,OREAD FM, HEEBNER MBR

SW NW SEC 7,T75N,R29W, MADISON CO,IOWA,USA W60;VON BITTER PH, 1976, LIFE SC

CONTR,ROM,N 109,P12,FIG 6B

REMARKS: C-PH VON BITTER;OZ ELEMENT;LOC 2 ,

SAMPLE HE-16-3 OF VON BITTER ; 1 SLIDE

\section{GONDOLELLA SUBLANCEOLATA GUNNELL}

30652 REF SPEC-F

CP. VIRGIL

SHAWNEE GP OREAD FM , HEEBNER MBR

SW NW SEC 7,T75N, R29W, MADISON CO,IOWA, USA W60;VON BITTER PH, 1976, LIFE SC

CONTR,ROM,N109,P12,FIG 6C

REMARK S: C-PH VON BITTER OZ ELEMENT:LOC 2 SAMPLE HE-16-3 OF VON BITTER ; 1 SLIDE
GONDOLELLA SUBLANCEOLATA GUNNELL 30653 REF SPEC-F

CP.VIRGIL

SHAWNEE GP,OREAD FM, HEEBNER MBR SE NE SEC 16,T75N, R27W, CASS CO,IOWA, USA W60; VON BITTER PH, 1976, LIFE SCI

CONTR, ROM,N 109,P12,FIG 6D,E

REMARKS: C-PH VON BITTER;OZ ELEMENT;LOC 1

SAMPLE HE-15-3 OF VON BITTER ; 1 SLIDE

GONDOLELLA SUBLANCEOLATA GUNNELL

30654 REF SPEC-F

CP.VIRGIL

SHAWNEE GP,OREAD FM, HEEBNER MBR

SE NE SEC 16,T75N,R27W, CASS CO,IOWA, USA

W60:VON BITTER PH, 1976, LIFE SCI

CONTR, ROM, N109,P12,FIG 6E

REMARKS: C-PH VON BITTER;OZ ELEMENT;LOC 1 ,

SAMPLE HE-15-3 OF VON BITTER; 1 SLIDE

GONDOLELLA SUBLANCEOLATA GUNNELL 30655 REF SPEC-F

CP.VIRGIL

SHAWNEE GP OREAD FM HEEBNER MBR

SW NW SEC 7,T75N,R29W, MADISON CO,IOWA,USA W60;VON BITTER PH, 1976, LIFE SCI

CONTR ROM N109,P14.FIG 7A

REMARKS: C-PH VON BITTER ; LO ELEMENT, SINISTRAL LOC 2,SAMPLE HE-16-3 OF VON BITTER; 1 SLIDE

\section{GONDOLELLA SUBLANCEOLATA GUNNELL}

30656 REF SPEC-F

CP, VIRGIL

SHAWNEE GP,OREAD FM, HEEBNER MBR

SW NW SEC 7, T75N,R29W, MADISON CO,IOWA, USA

W60:VON BITTERPH, 1976, LIFE SC

CONTR ROM N109,P14,FIG 6B

REMARKS: C-PH VONBITTER :LO ELEMENT, SINISTRAL: LOC 2,SAMPLE HE-16-3 OF VON BITTER; 1 SLIDE

GONDOLELLA SUBLANCEOLATA GUNNELL 30657 REF SPEC-F

CP. VIRGIL

SHAWNEE GP, OREAD FM, HEEBNER MBR

SW NW SEC 7,T75N,R29W, MADISON CO,IOWA,USA

W60;VON BITTER PH, 1976, LIFE SCI

CONTR, ROM,N 109,P14,FIG 7C

REMARKS: C-PH VON BITTER LO ELEMENT SINISTRAL:

LOC 2,SAMPLE HE-16-3 OF VON BITTER ; 1 SLIDE

GONDOLELLA SUBLANCEOLATA GUNNELL

30658 REF SPEC-F

CP VIRGIL

SHAWNEE GP OREAD FM, HEEBNER MBR

SW NW SEC 7,T75N,R29W, MADISON CO,IOWA, USA

W60:VON BITTER PH, 1976, LIFE SCI

CONTR,ROM,N109,P14,FIG 7D

REMARKS: C-PH VON BITTER; LO ELEMENT,DEXTRAL;LOC 2,SAMPLE HE-16-3 OF VON BITTER ; 1 SLIDE

GONDOLELLA SUBLANCEOLATA GUNNELL

30659 REF SPEC-F

CP, VIRGIL

SHAWNEE GP OREAD FM HEEBNER MBR

SW NW SEC 7,T75N,R29W, MADISON CO,IOWA, USA W60;VON BITTER PH, 1976. LIFE SCI

CONTR, ROM N 109, P 14,FIG 7E,F

REMARKS: C-PH VON BITTER;LO ELEMENT,DEXTRAL;LOC 2.SAMPLE HE-16-3 OF VON BITTER ; 1 SLIDE

\section{GONDOLELLA SUBLANCEOLATA GUNNELL}

30660 REF SPEC-F

CP,VIRGIL

SHAWNEE GP, OREAD FM, HEEBNER MBR

SW NW SEC 7,T75N,R29:V, MADISON CO,IOWA, USA W60;VON BITTER PH, 1976, LIFE SCI

CONTR,ROM.N109,P14,FIG 7G

REMARKS: C-PH VON BITTER :LO ELEMENT,DEXTRAL:LOC 2.SAMPLE HE-16-3 OF VON BITTER; 1 SLIDE 
GONDOLELLA SUBLANCEOLATA GUNNELL 30661 REF SPEC-F

CP.VIRGIL

SHAWNEE GP,OREAD FM, HEEBNER MBR

SW NW SEC 7,T75N,R29W, MADISON CO,IOWA, USA

W60; VON BITTER PH, 1976. LIFE SC

CONTR, ROM,N $109, \mathrm{P} 14, \mathrm{FIG} 7 \mathrm{H}$

REMARK S: C-PH VON BITTER :LO ELEMENT,DEXTRAL;LOC 2,SAMPLE HE-16-3 OF VON BITTER; 1 SLIDE

GONDOLELLA SUBLANCEOLATA GUNNELL

30662 REF SPEC-F

CP.VIRGIL

SHAWNEE GP,OREAD FM, HEEBNER MBR

SE NW SEC $16, T 75 N, R 37 W$, CASS CO, IOWA, USA

W60:VON BITTER PH, 1976, LIFE SCI

CONTR,ROM,N109,P16, FIG 8A,B

REMARKS: C-PH VON BITTER ; HI ELEMENT, SINISTRAL;

LOC 1,SAMPLE HE-15-3 OF VON BITTER; 1 SLIDE

GONDOLELLA SUBLANCEOLATA GUNNELL

30663 REF SPEC-F

CP.VIRGIL

SHAWNEE GP.OREAD FM, HEEBNER MBR

SE NW SEC 16,T75N,R37W, CASS CO,IOWA, USA

W6-; VON BITTER PH, 1976, LIFE SCI

CONTR,ROM,N109,P16,FIG 8C

REMARKS: C-PH VON BITTER;HI ELEMENT, SINISTRAL; LOC 1,SAMPLE HE-15-3 OF VON BITTER; 1 SLIDE

GONDOLELLA SUBLANCEOLATA GUNNELL

30664 REF SPEC-F

CP, VIRGIL

SHAWNEE GP,OREAD FM, HEEBNER MBR

SE NW SEC 16,T75N,R37W, CASS CO,IOWA, USA

W60:VON BITTER PH, 1976, LIFE SCI

CONTR,ROM,N109,P16,FIG 8D

REMARKS: C-PH VON BITTER;HI ELEMENT,DEXTRAL;LOC 1,SAMPLE HE-15-3 OF VON BITTER; 1 SLIDE

GONDOLELLA SUBLANCEOLATA GUNNELL 30665 REF SPEC-F

CP,VIRGIL

SHAWNEE GP, OREAD FM, HEEBNER MBR

SE NW SEC 16,T75N,R37W, CASS CO,IOWA, USA

W60:VON BITTER PH, 1976, CONTR LIFE

SCI,ROM,N 109,P16,FIG 8E

REMARKS: C-PH VON BITTER:HI ELEMENT,DEXTRAL:LOC 1,SAMPLE HE-15-3 OF VON BITTER; 1 SLIDE

GONDOLELLA SUBLANCEOLATA GUNNELL

30666 REF SPEC-F

CP.VIRGIL

SHAWNEE GP,OREAD FM, HEEBNER MBR

SE NW SEC $16, T 75 N, R 37 W$, CASS CO,IOWA, USA

W60:VON BITTER PH, 1976, LIFE SCI

CONTR,ROM,N 109,P 18,FIG 9A

REMARKS: C-PH VON BITTER ; NE ELEMENT SINISTRAL, LOC 1,SAMPLE HE-15-3 OF VON BITTER; 1 SLIDE

GONDOLELLA SUBLANCEOLATA GUNNELL

30667 REF SPEC-F

CP.VIRGIL

SHAWNEE GP OREAD FM, HEEBNER MBR

SE NW SEC 16,T75N,R37W, CASS CO,IOWA,USA

W60:VON BITTER PH, 1976, LIFE SC

CONTR,ROM.N109,P18,FIG 9B

REMARKS: C-PH VON BITTER ;NE ELEMENT, SINISTRAL, LOC 1,SAMPLE HE-15-3 OF VON BITTER ; 1 SLIDE

GONDOLELLA SUBLANCEOLATA GUNNELL

30668 REF SPEC-F

CP.VIRGIL

SHAWNEE GP. OREAD FM. HEEBNER MBR

SE NW SEC 16.T75N,R37W CASS CO,IOWA, USA

W60;VON BITTER PH, 1976, LIFE SCI

CONTR,ROM N109 P18,FIG 9 C

REMARKS: C-PH VON BITTER; NE ELEMENT,SINISTRAL, LOC 1.SAMPLE HE-15-3 OF VON BITTER; 1 SLIDE
GONDOLELLA SUBLANCEOLATA GUNNELL 30669 REF SPEC-F

CP,VIRGIL

SHAWNEE GP,OREAD FM, HEEBNER MBR

SE NW SEC 16,T75N,R37W, CASS CO,IOWA, USA

W60;VON BITTER PH, 1976, LIFE SCI

CONTR, ROM,N 109,P 18,FIG 9D,E

REMARKS: C-PH VON BITTER :NE ELEMENT,DEXTRAL:LOC

1,SAMPLE HE-15-3 OF VON BITTER; 1 SLIDE

GONDOLELLA SUBLANCEOLATA GUNNELL

30670 REF SPEC-F

CP,VIRGIL

SHAWNEE GP,OREAD FM, HEEBNER MBR

SE NW SEC 16,T75N,R37W, CASS CO,IOWA, USA

W60:VON BITTER PH, 1976, LIFE SC

CONTR, ROM,N 109,P 18,FIG 9F,G

REMARKS: C-PH VON BITTER;NE ELEMENT,DEXTRAL;LOC

1,SAMPLE HE-15-3 OF VON BITTER; 1 SLIDE

GONDOLELLA SUBLANCEOLATA GUNNELL

30671 REF SPEC-F

CP.VIRGIL

SHAWNEE GP,OREAD FM, HEEBNER MBR

SE NW SEC 16,T75N,R37W, CASS CO,IOWA, USA

W60;VON BITTER PH, 1976, LIFE SCI

CONTR, ROM,N 109 , P 18. FIG 9H

REMARKS: C-PH VON BITTER ; NE ELEMENT,DEXTRAL;LOC 1,SAMPLE HE-15-3 OF VON BITTER; 1 SLIDE

GONDOLELLA SUBLANCEOLATA GUNNELL 30672 REF SPEC-F

CP,VIRGIL

SHAWNEE GP, OREAD FM, HEEBNER MBR

SW NW SEC 7,T75N,R29W, MADISON CO,IOWA, USA

W60; VON BITTER PH, 1976, LIFE SCI CONTR,ROM, N 109,P2022,FIG $10 \mathrm{~A}$

REMARKS: C-PH VON BITTER ; SYN ELEMENT, SINISTRAL: LOC 2,SAMPLE HE-16-3 OF VON BITTER; 1 SLIDE

GONDOLELLA SUBLANCEOLATA GUNNELL 30673 REF SPEC-F

CP,VIRGIL

SHAWNEE GP, OREAD FM, HEEBNER MBR

SW NW SEC 7 T75N R29W MADISON CO,IOWA USA

W60; VON BITTER PH, 1976, LIFE SCI CONTR,ROM,N 109,P2022, FIG $10 \mathrm{~B}, \mathrm{C}$

REMARKS: C-PH VON BITTER; SYN ELEMENT,SINISTRAL; LOC 2,SAMPLE HE-16-3 OF VON BITTER; 1 SLIDE

GONDOLELLA SUBLANCEOLATA GUNNELL 30674 REF SPEC-F

CP.VIRGIL

SHAWNEE GP,OREAD FM, HEEBNER MBR

SW NW SEC 7,T75N,R29W, MADISON CO,IOWA, USA

W60:VON BITTER PH, 1976. LIFE SCI CONTR, ROM, N 109,P20 22, FIG 10D

REMARKS: C-PH VON BITTER ;SYN ELEMENT, SINISTRAL: LOC 2,SAMPLE HE-16-3 OF VON BITTER ; 1 SLIDE

GONDOLELLA SUBLANCEOLATA GUNNELL

30675 REF SPEC-F

CP. VIRGIL

SHAWNEE GP OREAD FM HEEBNER MBR

SW NW SEC 7,T75N, R29W, MADISON CO,IOWA, USA

W60;VON BITTER PH, 1976. LIFE SCI CONTR,ROM, N 109,P2022,FIG $10 E$

REMARKS: C-PH VON BITTER ;SYN ELEMENT,DEXTRAL; LOC 2.SAMPLE HE-16-3 OF VON BITTER; 1SLIDE

GONDOLELLA SUBLANCEOLATA GUNNELL

30676 REF SPEC-F

CP. VIRGIL

SHAWNEE GP, OREAD FM, HEEBNER MBR

SW NW SEC 7,T75N, R29W, MADISON CO,IOWA, USA

W60;VON BITTER PH, 1976, LIFE SCI CONTR,ROM, N 109, P2022,FIG $10 F$

REMARKS: C-PH VON BITTER:SYN ELEMENT,DEXTRAL; LOC 2,SAMPLE HE-16-3 OF VON BITTER; 1 SLIDE 
GONDOLELLA SUBLANCEOLATA GUNNELL 30677 REF SPEC-F

CP. VIRGIL

SHAWNEE GP,OREAD FM, HEEBNER MBR

SE NE SEC 16,T75N,R37W, CASS CO,IOWA, USA

W60:VON BITTER PH, 1976. LIFE SCI

CONTR, ROM, N 109,P22,FIG 11A-D

REMARKS: C-PH VON BITTER ;TR ELEMENT;LOC 1 ,

SAMPLE HE-15-3 OF VON BITTER ; 1 SLIDE

GONDOLELLA SUBLANCEOLATA GUNNELL

30678 REF SPEC-F

CP.VIRGIL

SHAWNEE GP, OREAD FM, HEEBNER MBR

SE NE SEC 16,T75N,R37W, CASS CO,IOWA, USA

W60;VON BITTER PH, 1976, LIFE SCl

CONTR,ROM,N109,P22,FIG 11E

REMARKS: C-PH VON BITTER ;TR ELEMENT;LOC 1 ,

SAMPLE HE-15-3 OF VON BITTER; 1 SLIDE

GONDOLELLA SUBLANCEOLATA GUNNELL

30626 REF SPEC-F

CP.VIRGIL

SHAWNEE GP,OREAD FM, HEEBNER MBR

SW NW SEC 7,T75N,R29W, MADISON CO,IOWA, USA

W60;VON BITTER PH, 1976, LIFE SCI CONTR,ROM,N 109,P5$10, \mathrm{FIG} 2 \mathrm{~A}$

REMARK S: C-PH VON BITTER ;SP ELEMENT,SINISTRAL; LOC 2,SAMPLE HE-16-3 OF VON BITTER; 1 SLIDE

GONDOLELLA SUBLANCEOLATA GUNNELL

30627 REF SPEC-F

CP.VIRGIL

SHAWNEE GP OREAD FM HEEBNER MBR

SW NW SEC 7,T75N,R29W, MADISON CO,IOWA,USA

W60; VON BITTER PH, 1976, LIFE SCI CONTR,ROM,N 109,P510,FIG 2B

REMARKS: C-PH VON BITTER ;SP ELEMENT,DEXTRAL;LOC 2,SAMPLE HE-16-3 OF VON BITTER;CITED AS ROM 30626 IN FIG CAPTION ; 1 SLIDE

GONDOLELLA SUBLANCEOLATA GUNNELL

30628 REF SPEC-F

CP.VIRGIL

SHAWNEE GP, OREAD FM, HEEBNER MBR

SW NW SEC 7,T75N,R29W, MADISON CO,IOWA,USA

W60;VON BITTER PH, 1976, LIFE SCI

CONTR,ROM,N 109,P12,FIG 2C

REMARKS: C-PH VON BITTER;OZ ELEMENT,SINISTRAL:

LOC 2,SAMPLE HE-16-3 OF VON BITTER; 1 SLIDE

\section{GONDOLELLA SUBLANCEOLATA GUNNELL} 30629 REF SPEC-F

CP,VIRGIL

SHAWNEE GP,OREAD FM, HEEBNER MBR

SW NW SEC 7,T75N, R29W, MADISON CO,IOWA, USA

W60;VON BITTER PH, 1976, LIFE SC

CONTR,ROM,N 109,P12,FIG 2D

REMARKS: C-PH VON BITTER :OZ ELEMENT DEXTRAL:LOC 2,SAMPLE HE-16-3 OF VON BITTER; 1 SLIDE

\section{GONDOLELLA SUBLANCEOLATA GUNNELL}

30630 REF SPEC-F

CP.VIRGIL

SHAWNEE GP OREAD FM, HEEBNER MBR

SW NW SEC 7,T75N,R29W, MADISON CO,IOWA,USA W60;VON BITTER PH, 1976, LIFE SCI CONTR,ROM,N109,P14,FIG 2E

REMARKS: C-PH VON BITTER :LO ELEMENT, SINISTRAL; LOC 2,SAMPLE HE-16-3 OF VON BITTER; 1 SLIDE

\section{GONDOLELLA SUBLANCEOLATA GUNNELL}

30631 REF SPEC-F

CP, VIRGIL

SHAWNEE GP,OREAD FM,HEEBNER MBR

SW NW SEC 7 T75N,R29W MADISON CO,IOWA, USA

W60; VON BITTER PH, 1976, LIFE SC

CONTR,ROM, N 109,P14,FIG 2F

REMARKS: C-PH VON BITTER ; LO ELEMENT,DEXTRAL;LOC 2,SAMPLE HE-16-3 OF VON BITTER; 1 SLIDE
GONDOLELLA SUBLANCEOLATA GUNNELL 30632 REF SPEC-F

CP. VIRGIL

SHAWNEE GP,OREAD FM, HEEBNER MBR

SW NW SEC 7,T75N,R29W, MADISON CO,IOWA, USA

W60; VON BITTER PH, 1976, LIFE SCI

CONTR,ROM,N109,P16,FIG 2G

REMARKS: C-PH VON BITTER ; HI ELEMENT,SINISTRAL:

LOC 2,SAMPLE HE-16-3 OF VON BITTER; 1 SLIDE

GONDOLELLA SUBLANCEOLATA GUNNELL

30641 REF SPEC-F

CP.VIRGIL

SHAWNEE GP,OREAD FM, HEEBNER MBR

SW NW SEC 7,T75N,R29W, MADISON CO,IOWA, USA

W60:VON BITTER PH, 1976, LIFE SCI CONTR,ROM,N109,FIG $3 \mathrm{C}, 4 \mathrm{~A}, 4 \mathrm{~B}$

REMARKS: C-PH VON BITTER :SP ELEMENT;LOC 2 ,

SAMPLE HE-16-3 OF VON BITTER; 1 SLIDE

GONDOLELLA SUBLANCEOLATA GUNNELL 30633 REF SPEC-F

CP. VIRGIL

SHAWNEE GP,OREAD FM, HEEBNER MBR

SW NW SEC 7,T75N,R29W, MADISON CO,IOWA,USA

W60;VON BITTER,PH, 1976, LIFE SCI

CONTR,ROM,N 109,P16,FIG $2 \mathrm{H}$

REMARKS: C-PH VON BITTER:HI ELEMENT,DEXTRAL:LOC 2,SAMPLE HE-16-3 OF VON BITTER; 1 SLIDE

GONDOLELLA SUBLANCEOLATA GUNNELL 30634 REF SPEC-F

CP.VIRGIL

SHAWNEE GP,OREAD FM, HEEBNER MBR

SW NW SEC 7,T75N,R29W, MADISON CO,IOWA, USA

W60:VON BITTER PH, 1976,LIFE SCI CONTR,ROM,P 18,FIG 2

REMARKS: C-PH VON BITTER ;NE ELEMENT,SINISTRAL; LOC 2,SAMPLE HE-16-3 OF VON BITTER ; 1 SLIDE

GONDOLELLA SUBLANCEOLATA GUNNELL 30635 REF SPEC-F

CP.VIRGIL

SHAWNEE GP,OREAD FM, HEEBNER MBR

SE NE SEC 16,T75N,R37W, CASS CO,IOWA, USA

W60;VON BITTER PH, 1976, LIFE SCI

CONTR,ROM,N 109,P18,FIG $2 \mathrm{~J}$

REMARKS: C-PH VON BITTER ;NE ELEMENT,DEXTRAL;LOC 1,SAMPLE HE-15-2 OF VON BITTER; 1 SLIDE

GONDOLELLA SUBLANCEOLATA GUNNELL 30636 REF SPEC-F

CP.VIRGIL

SHAWNEE GP,OREAD FM, HEEBNER MBR

SE NE SEC 16,T75N,R37W, CASS CO,IOWA, USA

W60;VON BITTER PH, 1976, LIFE SCI CONTR,ROM,N 109,P2022, FIG $2 K$

REMARKS: C-PH VON BITTER ; SYN ELEMENT, SINISTRAL; LOC 1,SAMPLE HE-15-3 OF VON BITTER; 1 SLIDE

GONDOLELLA SUBLANCEOLATA GUNNELL 30637 REF SPEC-F

CP.VIRGIL

SHAWNEE GP OREAD FM, HEEBNER MBR

SW NW SEC 7,T75N,R29W, MADISON CO,IOWA USA

W60; VON BITTER PH, 1976, LIFE SCICONTR,ROM,N 109,P2022,FIG 2 L

REMARKS: C-PH VON BITTER ;SYN ELEMENT,DEXTRAL; LOC 1,SAMPLE HE-16-3 OF VON BITTER; 1 SLIDE

GONDOLELLA SUBLANCEOLATA GUNNELL 30638 REF SPEC-F

CP.VIRGIL

SHAWNEE GP,OREAD FM, HEEBNER MBR

SW NW SEC 7,T75N,R29W, MADISON CO,IOWA,USA W60;VON BITTER PH, 1976, LIFE SCI CONTR,ROM,N109,P22,FIG 2M

REMARKS: C-PH VON BITTER :TR ELEMENT:LOC 2 SAMPLE HE-16-3 OF VON BITTER; 1 SLIDE 
GONDOLELLA SUBLANCEOLATA GUNNELL

30639 REF SPEC-F

CP,VIRGIL

SHAWNEE GP,OREAD FM, HEEBNER MBR

SENE SEC $16, T 75 N$, R37W, CASS CO,IOWA, USA

W60;VON BITTER PH, 1976, LIFE SCI CONTR,ROM,N109,P510, FIG $3 A$

REMARKS: G-PH VON BITTER ;SP ELEMENT;LOC 1

SAMPLE HE-15-2 OF VON BITTER; 1 SLIDE

GONDOLELLA SUBLANCEOLATA GUNNELL

30640 REF SPEC-F

CP, VIRGIL

SHAWNEE GP, OREAD FM, HEEBNER MBR

SW NW SEC 7,T75N,R29W, MADISON CO,IOWA,USA

W60;VON BITTER PH, 1976, LIFE SCI CONTR, ROM,N 109,P5$10, F I G$ 3B, 4 C, 4D

REMARKS: C-PH VON BITTER ;SP ELEMENT;LOC 2,

SAMPLE HE-16-3 OF VON BITTER; 1 SLIDE

IDIOGNATHODUS SP,HI ELEMENT

35058 REF SPEC-F

CP.

NE $1 / 4$, SW $1 / 4$, SW $1 / 4$, SEC $26, T 12 N, R 3 E$, COPLEY

TP, KNOX CO,ILL,USA

W62;MERRILL + VON BITTER, 1977,LIFE SCI

CONTR,N112,FIG 9D

REMARKS: C,D-GK MERRILL; 1 SPEC

\section{IDIOGNATHODUS SP,OZ ELEMENT}

35057 REF SPEC-F

$\mathrm{CP}$,

SW 1/4,SW 1/4,SW 1/4,SEC 4,T11N,R9E, CHILLICOTHE

TP,ILL,USA

W62;MERRILL + VON BITTER, 1977,LIFE SCI

CONTR,ROM,N112,FIG 9B

REMARKS: C,D-GK MERRILL ;FIG IS A COMPOSITE

DRAWING ; 3 SPEC (1 SLIDE)

\section{IDIOPRIONIODUS SP}

30679 REF SPEC-F

CP,MISSOURI

KANSAS CITY GP,DENNIS FM,STARK MBR

SARPY CO,NEBR,USA

W50;VON BITTER PH, 1976,LIFE SCI CONTR,ROM,N 109,FIG 17

REMARKS: C-PH VON BITTER ;NATURAL ASSEMBLAGE ; 1 SLIDE

NEOGONDOLELLA CLARKI (KOIKE)

30681 REF SPEC-F

CP.MORROW

MARBLE FALLS FM

SAN SABA CO,TEX,USA

VON BITTER + MERRILL, 1977, LIFE SCI OCC

PAP,ROM, N29,FIG 1D

REMARKS: C-GK MERRILL ; 1 SPEC

NEOGONDOLELLA CLARKI (KOIKE)

30680 REF SPEC-F

CP.MORROW

MARBLE FALLS FM

SAN SABA CO,TEX,USA

VON BITTER + MERRILL, 1977. LIFE SCI OCC

PAP,ROM,N29,FIG 1A-C, 1E-F

REMARKS: C-GK MERRILL; 1 SPEC

\section{NEOGONDOLELLA SP}

30649 REF SPEC-F

$P$,

OGILVIE MTS,YT

VON BITTER PH, 1976,LIFE SCI CONTR,ROM,N109,P10,FIG 5A-D

REMARKS: C-PH VON BITTER ; 1 SPEC
SCOLOPODUS CORNUTIFORMIS BRANSON +MEHL 30576 REF SPEC-F

LO.

ST GEORGE FM

E SHORE STRAIT OF BELLE ISLE , 2-3 MI SW OF CAPE NORMAN,NFLD

W44;BARNES SLACK, 1975, LIFE SCI CONTR,ROM,N 106,FIG $1 A$

REMARKS: C-CR BARNES ;SPEC MOUNTED ON SEM STUB ; 1 SPEC

SCOLOPODUS CORNUTIFORMIS BRANSON + MEHL 30577 REF SPEC-F

LO,

ST GEORGE FM

E SHORE STRAIT OF BELLE ISLE , 2-3 MI SW OF CAPE NORMAN,NFLD

W44;BARNES SLACK, 1975,LIFE SCI CONTR,ROM,N106,FIG $1 B$

REMARKS: C-CR BARNES ;SPEC MOUNTED ON SEM STUB : 1 SPEC

SCOLOPODUS CORNUTIFORMIS BRANSON + MEHL 30578 REF SPEC-F

LO,

ST GEORGE FM

E SHORE STRAIT OF BELLE ISLE , 2-3 MI SW OF CAPE NORMAN,NFLD

W44;BARNES SLACK, 1975,LIFE SCI CONTR,ROM,N 106,FIG $1 C, D$

REMARKS: C-CR BARNES :SPEC SECTIONED,MOUNTED

ON SEM STUB ; 1 SPEC

SCOLOPODUS CORNUTIFORMIS BRANSON + MEHL

30579 REF SPEC-F

L O,

ST GEORGE FM

E SHORE STRAIT OF BELLE ISLE , 2-3 MI SW OF CAPE

NORMAN,NFLD

W44;BARNES SLACK, 1975, LIFE SCI CONTR,ROM,N 106, FIG $1 E, F$

REMARKS: C,D-CR BARNES ;SPEC SECTIONED,MOUNTED ON SEM STUB ; 1 SPEC

SCOLOPODUS EMARGINATUS BARNES + TUKE

30583 REF SPEC-F

LO,

ST GEORGE FM

E SHORE STRAIT OF BELLE ISLE , 2-3 MI SW OF CAPE NORMAN,NFLD

W44;BARNES SLACK, 1975, LIFE SCI CONTR,ROM,N 106,FIG 2C,D

REMARKS: C,D-CR BARNES ;SPEC SECTIONED,MOUNTED ON SEM STUB : 1 SPEC

SCOLOPODUS EMARGINATUS BARNES + TUKE

30584 REF SPEC-F

LO,

ST GEORGE FM

E SHORE STRAIT OF BELLE ISLE , 2-3 MI SW OF CAPE NORMAN,NFLD

W44;BARNES SLACK, 1975,LIFE SCI CONTR,ROM,N 106,FIG 2E,F

REMARKS: C,D-CR BARNES ;SPEC SECTIONED,MOUNTED ON SEM STUB ; 1 SPEC

SCOLOPODUS EMARGINATUS BARNES + TUKE

30585 REF SPEC-F

LO,

ST GEORGE FM

E SHORE STRAIT OF BELLE ISLE , 2-3 MI SW OF CAPE NORMAN,NFLD

W44;BARNES SLACK, 1975,LIFE SCI CONTR,ROM,N 106,FIG $2 A$

REMARKS: D-CR BARNES : PHOTO ONLY:SPEC DESTROYED BY SECTIONING ; 1 PHOTO 
SCOLOPODUS GRACILIS ETHINGTON + CLARK

30587 REF SPEC-F

LO.

BAUMANN FIORD FM, MBR B

CENTRAL ELLESMERE IS,NWT

W44;BARNES SLACK, 1975,LIFE SCI CONTR,ROM,N 106,FIG $3 A$

REMARKS: C.D-CR BARNES ;SPEC MOUNTED ON SEM

STUB ; 1 SPEC

SCOLOPODUS GRACILIS ETHINGTON+CLARK

30588 REF SPEC-F

LO,

BAUMANN FIORD FM, MBR B

CENTRAL ELLESMERE IS,NWT

W44;BARNES SLACK, 1975, LIFE SCI CONTR, ROM,N 106,FIG 3B-D

REMARKS: D-CR BARNES ;PHOTOS ONLY;SPEC

DESTROYED BY SECTIONING ; PHOTOS

SCOLOPODUS GRACILIS ETHINGTON + CLARK

30589 REF SPEC-F

LO,

ST GEORGE FM

E SHORE STRAIT OF BELLE ISLE , 2-3 MI SW OF CAPE

NORMAN,NFLD

W44;BARNES SLACK, 1975, LIFE SCI CONTR,ROM,N106,FIG $4 A, B$

REMARKS: C,D-CR BARNES ;SPEC SECTIONED,MOUNTED ON SEM STUB ; 1 SPEC

SCOLOPODUS MULTICOSTATUS BARNES + TUKE 30591 REF SPEC-F

LO,

ST GEORGE FM

E SHORE STRAIT OF BELLE ISLE , 2-3 MI SW OF CAPE NORMAN,NFLD

W44;BARNES SLACK, 1975,LIFE SCI CONTR,ROM,N106,FIG $2 A$

REMARKS: C,D-CR BARNES ;SPEC MOUNTED ON SEM STUB; 1 SPEC

SCOLOPODUS QUADRAPLICATUS BRANSON + MEHL 30597 REF SPEC-F

$\mathrm{O}$,

ST GEORGE FM

E SHORE STRAIT OF BELLE ISLE , 2-3 MI SW OF CAPE NORMAN,NFLD

W44;BARNES SLACK, 1975,LIFE SCI CONTR,ROM,N106,FIG $5 C-E$

REMARK S: C,D-CR BARNES ;SPEC SECTIONED,MOUNTED ON SEM STUB ; 1 SPEC

SCOLOPODUS QUADRAPLICATUS BRANSON + MEHL 30595 REF SPEC-F

LO,

ST GEORGE FM

E SHORE STRAIT OF BELLE ISLE , 2-3 MI SW OF CAPE

NORMAN NFLD

W44:BARNES SLACK, 1975,LIFE SCI CONTR,ROM,N106,FIG $5 A$

REMARKS: D-CR BARNES : PHOTO ONLY:SPEC

DESTROYED BY SECTIONING ; PHOTO

SCOLOPODUS QUADRAPLICATUS BRANSON+MEHL

30596 REF SPEC-F

LO

ST GEORGE FM

E SHORE STRAIT OF BELLE ISLE , 2-3 MI SW OF CAPE

NORMAN,NFLD

W44:BARNES SLACK, 1975, LIFE SCI CONTR,ROM,N106,FIG $5 B$

REMARKS: D-CR BARNES P PHOTO ONLY·SPEC

DESTROYED BY SECTIONING ; PHOTO

ULRICHODINA PRIMA FURNISH

30608 REF SPEC-F

L O

BAUMANN FIORD FM, MBR B

CENTRAL ELLESMERE IS,NWT
W45;BARNES SLACK, 1975, LIFE SCI CONTR,ROM,N 106,FIG 6C-E

REMARKS: C,D-CR BARNES ; SPEC SECTIONED,MOUNTED ON SEM STUB ; 1 SPEC

ULRICHODINA PRIMA FURNISH

30607 REF SPEC-F

LO

ST GEORGE FM

E SHORE STRAIT OF BELLE ISLE , 2-3 MISW OF CAPE

NORMAN,NFLD

W45;BARNES SLACK, 1975,LIFE SCI CONTR, ROM,N 106,FIG $6 A, B$

REMARKS: D-CR BARNES ;PHOTOS ONLY:SPEC

DESTROYED : PHOTOS

\section{GRAPTOLITHINA}

ACANTHOGRAPTUS GRANTI SPENCER

$21632[1457 N]$ REF SPEC-F

MS.NIAG

LOCKPORT GP

HAMILTON,ONT

V(R)42;BASSLER RS,USNM BULL 65,P56-57,PL2,FIG 1

REMARKS: P-SPENCER COLL, 1919:1 SPEC

BRYOGRAPTUS PATENS MATTHEW

7998 [368CM] REF SPEC-F

$\mathrm{MCA}$.

ST JOHN GP

[ST JOHN,DIV $3 C$ ]

NAVY IS,ST JOHN,NB

V(R)39;MATTHEW GF,TRANS NY ACAD SCI, VOL 14,1895,P268-269,PL48,FIG4A-C

REMARKS: D-W MACKENZIE;ROM RECORDS STATE SPECS MARKED 'G' FIG'D :21 PCES

BRYOGRAPTUS PATENS MATTHEW

$8407[777 \mathrm{CM}(\mathrm{B})]$ P-SYN?

LCA,

ST JOHN GP

[ST JOHN,DIV 3B]

NAVY IS,ST JOHN,NB

V(R)39:MATTHEW GF,TRANS ROY SOC CAN,VOL 10, 1893,PT4,P95-96.PL7,FIG 1A-D

REMARKS: D-W MACKENZIE ;SPEC MARKED 'B' ON SLAB: NO TYPES DESIGNATED IN PUBL' $N$; LABELLED COTYPE: UNCERTAIN IF FIG'D; 1 SPEC

BRYOGRAPTUS SPINOSUS (MATTHEW)

8024 [394CM] REF SPEC-F

$M C A$,

ST JOHN GP

[ST JOHN,DIV 3C]

NAVY IS,ST JOHN,NB

V(R)39:MATTHEW GF TRANS NY ACAD SCI, VOL 14,P269.

270,PL48,FIG 3A-B

REMARKS: D-W MACKENZIE ; 1 SPEC

CALLOGRAPTUS MINUTUS VAR ALTUS GURLEY

$21625(\mathrm{~A})[1450 \mathrm{~N}] \mathrm{P}-\mathrm{HOLO}$

MS,NIAG

LOCKPORT GP

HAMILTON ONT

V(R)38;BASSLER RS,USNM BULL 65, 1909,P 15,FIG 16

REMARKS: P-SPENCER COLL, 1919 ;SPEC MARKED 'A'

FIG'D + DESIGNATED HOLOTYPE IN FIGURE CAPTION : 1 SPEC

CALLOGRAPTUS MINUTUS VAR ALTUS GURLEY

21625 [ $1450 N$ ] P-PARA

MS, NIAG

LOCKPORT GP

HAMILTON,ONT

V(R)38:BASSLER RS.USNM BULL 65, 1909,P 15

REMARK S: P-SPENCER COLL, 1919 ;THIS SPEC NOT FIG'D; OTHER PARATYPES LISTED AS USNM NO $55311 ;$ 1SPEC 
CALYPTOGRAPTUS MICRONEMATODES SPENCER

$21626[1451 \mathrm{~N}]$ REF SPEC-F

MS,NIAG

LOCKPORT GP

HAMILTON,ONT

V(R)43;BASSLER RS, USNM BULL 65, 1909,P39-40,FIG 50

REMARKS: P-SPENCER COLL, 1919 ;LISTED AS NEOTYPE IN FRITZ'S ROM TYPE CAT BUT NOT SO DESIGNATED IN PUBL'N : 1 SPEC (2 PCES)

CLIMACOGRAPTUS AEQUALIS PARKS

24936 [259U] P-HOLO

U O. CINCI,EDEN

U COLLINGWOOD FM

CRAIGLEITH,ONT

V(R) 125:PARKS WA,TRANS ROY SOC CAN,VOL

22, 1928,P65,PL1,FIG 2

REMARKS: C-WA PARKS ; 1 SPEC

\section{CLIMACOGRAPTUS MANITOULINENSIS CALEY}

$17755[1537 \mathrm{HR}]$ P-HOLO

U O.CINCI,EDEN

SHEGUIANDAHFM

LOT 12, CON 12, HOWLAND TP, MANITOULIN IS,ONT

V(R) 125; CALEY JF, GSC MEM 202, 1936,P65-66,FIG 1

REMARKS: C-JF CALEY, 1931; 1SPEC

\section{CLIMACOGRAPTUS MANITOULINENSIS CALEY}

$17756[1538 \mathrm{HR}]$ P-PARA

U O, $\mathrm{CINCI}, \mathrm{EDEN}$

SHEGUIANDAH FM

LOT 12, CON 12, HOWLAND TP, MANITOULIN IS, ONT

V(R) 125;CALEY JF, GSC MEM 202,1936,P65-66,FIG 1

REMARKS: C-JF CALEY, $1931 ; 2$ SPEC

CLIMACOGRAPTUS PROLIFICUS PARKS

24941 [264U]P-SYN

UO, CINCI,EDEN

COLLINGWOODFM

CAMPERDOWN.ONT

V(R)125;PARKS WA, TRANS ROY SOC CAN, VOL

22, 1928,P61-62,FIG 4,5

REMARKS: C-WA PARKS, 1927 ;3 SPECS-A,B,C;A-FIG 5,BFIG $4 ; 3$ SPEC

CLIMACOGRAPTUS ROUGENSIS PARKS

24945 [268U] P-SYN

$\mathrm{O}, \mathrm{CINCl}$,

GLOUCESTERFM

ROUGE R,ONT

V(R)125:PARKS WA, TRANS ROY SOC CAN, VOL

22, 1928,P63-64,FIG 6-8

REMARKS: C-WA PARKS, 1927 ;SPECS A,B,C;A-FIG 6,C-FIG $7, B-F I G ~ 8: 3$ SPEC

CLIMACOGRAPTUS TYPICALIS HALL

24973 [296U] REF SPEC-F

U O, CINCI,EDEN

U COLLINGWOODFM

CRAIGLEITH,ONT

V(R) 125;PARKS WA,TRANS ROY SOC CAN, VOL

22, 1928,SECT 4,P64,FIG 9

REMARKS: C-J TOWNSEND ; 1 SPEC

\section{CLIMACOGRAPTUS CF TYPICALIS HALL}

$17484[1245 \mathrm{HR}]$ REF SPEC-F

U O, CINCI,MAYSVILL

DUNDASFM

[DUNDAS FM,DANFORTH MBR]

8 FT BELOW UPPERMOST BEDS, DON VALLEY

QUARRY,TORONTO,ONT

V(R) 125;FRITZ MA,ONT DEPT

MINES,N32,PT7, 1925,P7,PL1,FIG 6

REMARKS: C-WA PARKS, $1922 ; 1$ SPEC
CLONOGRAPTUS PROXIMATUS MATTHEW

8513 [883CM] P-SYN

$M C A$

ST JOHN GP

[ST JOHN,DIV 3C]

NAVY IS,ST JOHN,NB

V(R)39;MATTHEW GF, TRANS NY ACAD SCI, VOL

14,1895,P265-268, PL48,FIG 1A-D

REMARKS: D-W MACKENZIE;UNCERTAIN WHICH SPECS FIG'D ; 16 PCES

CLONOGRAPTUS SPINOSUS MATTHEW

8460 [830CM] P-SYN?

LCA.

ST JOHN GP

[ST JOHN,DIV 3B]

NAVY IS,ST JOHN NB

V(R)39;MATTHEW GF, TRANS ROY SOC CAN, VOL 10,PT4, 1893,P97,PL7,FIG 2A,B

REMARKS: D-W MACKENZIE :NO TYPES DESIGNATED IN PUBL'N; LABELLED COTYPES;UNCERTAIN WHICH SPEC, IF ANY,IS FIG'D: 11 PCES

DENDROGRAPSUS ? PRIMORDIALIS MATTHEW

8278 [648CM] P-SYN?

$M C A$.

ST JOHN GP

[ST JOHN,DIV 1D]

PORTER'S BK, ST MARTINS,ST JOHN CO NB

V(R)38;MATTHEW EF, TRANS ROY SOC CAN,VOL 3,SECT 4,1886,P31,PL5,FIG 5,5A,B

REMARKS: D-W MACKENZIE ;UNCERTAIN IF THIS IS FIG'D MATERIAL;NO TYPES DESIGNATED IN PUBL'N ; 4 SPEC

DENDROGRAPTUS RAMOSUS SPENCER

$21629[1454 \mathrm{~N}]$ REF SPEC-F

MS,NIAG

LOCKPORT GP

HAMILTON ONT

V(R)38;BASSLER RS,USNM BULL 65,P8-9,FIG 9,PL 1,FIG 3

REMARKS: P-SPENCER COLL, $1919 ; 1$ SPEC

DENDROGRAPTUS SP

17482 [1243HR] REF SPEC-F

U O, CINCI,MAYSVILL

DUNDAS FM

15 FT LEVEL, DON VALLEY QUARRY,TORONTO,ONT V(R)38:FRITZ MA ONT DEPT MINES, VOL

32, PT7, 1925, P3,PL1,FIG 2

REMARKS: C-WA PARKS, 1922 ;SPEC LABELLED ' 1 ' ON

SLAB;2 SLABS WITH NO 1243HR, ONLY ONE WITH

DENDROGRAPTUS ; 1 SPEC

DICTYONEMA EXPANSUM SPENCER

$21609[1434 \mathrm{~N}]$ REF SPEC-F

MS,NIAG

LOCKPORT GP

HAMILTON ONT

V(R)38;BASSLER RS,USNM BULL 65, 1909,P31-33,FIG 37

REMARKS: P-SPENCER COLL, 1919;1SPEC

DICTYONEMA RETIFORME (HALL)

$21610[1435 \mathrm{~N}]$ REF SPEC-F

MS,NIAG

LOCKPORT GP

HAMILTON,ONT

V(R)38;BASSLER RS,USNM BULL 65, 1909,P 18-19,FIG 19,20

REMARKS: P-SPENCER COLL, $1919 \cdot$ FIG 19 IS SPEC

LABELLED 102,FIG 20 IS OTHER SPEC ; 2 SPEC

DICTYONEMA TENELLUM SPENCER

$21642[1467 \mathrm{~N}]$ REF SPEC-F

MS.NIAG

LOCKPORT GP

HAMILTON, ONT

V(R)38;BASSLER RS,USNM BULL 65, 1909,P28-30,FIG 33

REMARKS: P-SPENCER COLL, $1919 ; 1$ SPEC 
DIPLOGRAPTUS FOLIACEOUS VAR GORRELENSIS CALEY

17745 [1526HR] P-HOLO

U O,CINCI,MAYSVILL

WEKWEMIKONGSING FM

GORREL PT, MANITOULIN IS, ONT

V(R)125:CALEY JF, GSC MEM 202, 1936,P66-68,FIG 2

REMARKS: C-JF CALEY, $1932 ; 1$ SPEC

DIPLOGRAPTUS FOLIACEOUS VESPERTINUS RUEDEMANN 17485 [1246HR] REF SPEC-F

O CINCI,MAYS

DUNDASFM

HUMBER R,END OF BLOOR ST , TORONTO,ONT

V(R)125;FRITZ MA, ONT DEPT MINES, VOL 32,PT7,1925,P89,PL1,FIG 8,13

REMARKS: C-WA PARKS, $1922 ; 2$ SPECS CIRCLED ON

SLAB;SPEC ' 1 ' $=$ FIG $8 ; 2$ SLABS

\section{DIPLOGRAPTUS MONTIS PARKS}

24942[265U] P-SYN

O,CINCI EDEN

BLUE MOUNTAIN FM

NR CAMPERDOWN,ONT

V(R) 125;PARKS WA,TRANS ROY SOC CAN, VOL 22,SECT

4,1928,P66-67,FIG 11-13,PL 1,FIG 4

REMARKS: C-WA PARKS, 1926 ;SPEC A-FIG 11,PL 1,FIG 4;8-

FIG 13;C-FIG 12;D-NOT FIG'D ; 4 SPEC (3 SLABS)

DIPLOGRAPTUS SIMILIS CALEY

17744 [1525HR] P-HOLO

U O.CINCI,MAYSVILL

WEKWEMIKONGSING FM

MANITOULIN IS, ONT

V(R) 125;CALEY JF, GSC MEM 202, 1936,P68,FIG 3

REMARKS: C-JF CALEY, 1931 ; LOC IN PUBL'N GIVEN AS

CLAY CLIFF AND LOT 11, CON 11, HOWLAND TP ; 1 SPEC

DIPLOGRAPTUS THECALIS PARKS

$24935[258 \mathrm{U}] \mathrm{P}-\mathrm{HOLO}$

U O, CINCI,EDEN

BLUE MOUNTAIN FM

LOT 371 , CAMPERDOWN,ONT

V(R) 125;PARKS WA, TRANS ROY SOC CAN,VOL 22,SECT

4, 1928,P67-68,PL1,FIG 3

REMARKS: C-WA PARKS, $1926 ; 1$ SPEC

\section{DIPLOGRAPTUS THECALIS PARKS?}

21193 REF SPEC-F

U O.CINCI,EDEN

BLUE MOUNTAIN FM

LOT 371 , CAMPERDOWN,ONT

$V(R) 125$; PARKS WA, TRANS ROY SOC CAN, VOL 22,SECT 4,1928,P67-68,FIG 14

REMARKS: C-WA PARKS, 1926 ;SPEC LABELLED

PARATYPE; 1 SPEC

\section{DIPLOGRAPTUS SP}

24951[274U] REF SPEC-F

U O,CINCI,EDEN

COLLINGWOOD FM

CAMPERDOWN,ONT

V(R) 125:PARKS WA. TRANS ROY SOC CAN,VOL 22, SECT 4, 1928,P69-70,FIG 17,18

REMARKS: C-WA PARKS, 1927 ;SPEC A-FIG 17,B-FIG $18 ; 2$ SPEC

DIPLOGRAPTUS SP

24939 [262U] REF SPEC-F

$\cup O, \mathrm{CINCI}, \mathrm{EDEN}$

COLLINGWOOD FM

CAMPERDOWN,ONT

V(R) 125 ; PARKS WA, TRANS ROY SOC CAN,VOL 22 ,SECT 4,1928,P69,FIG 15

REMARKS: C-WA PARKS, 1927 ; 1 SPEC
DIPLOGRAPTUS SP

24940 [263U] REF SPEC-F

$\cup O, C I N C I, E D E N$

COLLINGWOOD FM

CAMPERDOWN,ONT

V(R) 125 ; PARKS WA, TRANS ROY SOC CAN, VOL 22,SECT 4,1928,P69,FIG 16

REMARKS: C-WA PARKS, 1926 ;GIVEN IN PUBL' 'N AS NO $363 \mathrm{U} ; 1$ SPEC

GLOSSOGRAPTUS CF QUADRIMUCRONATUS (HALL)

24944 [267U] REF SPEC-F

U O,CINCI,EDEN

BLUE MOUNTAINFM

NR CAMPERDOWN,ONT

V(R)126;PARKS WA, TRANS ROY SOC CAN,VOL 22,SECT 4,1928,P65-66,FIG 10

REMARKS: C-WA PARKS, 1927 ; 1 SPEC

\section{GLOSSOGRAPTUS SP}

17482[1243HR] REF SPEC-F

U O,CINCI,MAYSVILL

DUNDAS FM

15FT LEVEL, DON VALLEY QUARRY,TORONTO, ONT

V(R) 122;FRITZMA,ONT DEPT MINES, VOL

32, PT7, 1925, P9,PL 1,FIG 10

REMARKS: C-WA PARKS, 1922 ;SPEC LABELLED ' 2 ' ON

SLAB WITH DENDROGRAPTUS SP; 2 SLABS WITH NO 1243HR;STORED UNDER DENDROGRAPTUS SP ; 1 SPEC

INOCAULIS YORKENSIS FRITZ

17132 [889HR] P-HOLO

U O,CINCI,MAYSVILL

DUNDAS FM

NR WINCHESTER ST BRIDGE, DON VALLEY

QUARRY,TORONTO,ONT

V(R)43;FRITZ MA, ONT DEPT MINES, VOL 32,PT7, 1925,P35,FIG 1

REMARKS: C-J TOWNSEND, $1908: 3$ SPECS,ALL MARKED HOLOTYPE;LARGEST IS FIG'D AND PROBABLY INTENDED AS HOLOTYPE AS AUTHOR REFERS TO A SINGLE SPEC ; 3 SPEC,BROKEN

INOCAULIS ? STRICTUS GURLEY IN BASSLER, 1909

21621 [1446N] P-HOLO

MS,NIAG

LOCKPORT GP

HAMILTON,ONT

V(R)43:BASSLER RS, USNM BULL 65, 1909,P54,FIG

$71, P L 2, F I G 6$

REMARKS: P-SPENCER COLL, $1919 ; 1$ SPEC

MASTIGOGRAPTUS GRACILLIMUS (LESQUEREUX)

17483 [1244HR] REF SPEC-F

U O,CINCI,MAYSVILL

DUNDAS FM

LOWER 2 FT, DON VALLEY QUARRY, TORONTO,ONT

V(R) 56 ;FRITZ MA, ONT DEPT MINES, VOL

32, PT7, 1925, P6 PL 1, FIG 3,14

REMARKS: C-WA PARKS, 1922 ;MATERIAL MENTIONEDIN PUBL'N AND LABELLED 'PLESIOTYPE' BUT DOES NOT RESEMBLE FIGS ; 1 SPEC, 2 SLIDES

MASTIGOGRAPTUS QUADRIBRACHIATUS FRITZ

17541 [ 1304HR] P-HOLO

U O,CINCI,MAYSVILL

DUNDASFM

DON VALLEY QUARRY, TORONTO, ONT

V(R) 56:FRITZMA ONT DEPT MINES, VOL 32,PT7, 1925,P6-7

REMARKS: D-J SATTERLY, 1925 ;NOT FIG'D ; 1 SPEC

ODONTOCAULIS OBPYRIFORMIS GURLEY IN BASSLER

$21622[1447 \mathrm{~N}]$ P-HOLO

MS,NIAG

LOCKPOAT GP

HAMILTON,ONT

V(R)38;BASSLER RS, USNM BULL 65, 1909,P43-44,FIG 55

REMARKS: P-SPENCER COLL, $1919 ; 1$ SPEC 


\section{MISCELLANEOUS AND PLANTS}

ACER PLEISTOCENICUM PENHALLOW 20234 REF SPEC-F

FOSSIL LEAF

N.PLEIST

INTERGLACIAL

DON VALLEY QUARRY,TORONTO,ONT

COLEMAN AP, 1933,ONT DEPT MINES,VOL 41,PT7,P6

REMARKS: D-AP COLEMAN, $1913 ; 1$ SPEC

ACER TORONTONIENSIS PENHALLOW

20211 REF SPEC-F

FOSSIL LEAF

PLEIST.

INTERGLACIAL

DON VALLEY QUARRY,TORONTO,ONT

COLEMAN AP, 1933,ONT DEPT MINES,VOL 41,PT7,P11

REMARKS: D-AP COLEMAN, 1913; 1SPEC

ARTHRARIA BICLAVATA MILLER

17491 [1252HR] REF SPEC-F

UO,CINCI,MAYSVILL

DUNDAS FM

END OF BLOOR ST, HUMBER R,TORONTO,ONT

REMARKS: C-J TOWNSEND : 1 SLAB

BUTHOTREPHIS SUBNODOSA HALL

17497 [1258HR] REF SPEC-F

UO.CINCI,MAYSVILL

DUNDASFM

DON VALLEY QUARRY, TORONTO,ONT

REMARKS: C-J TOWNSEND; 1 SPEC

\section{CALANTICA? SASKATCHEWANENSIS RUSSELL}

28154 P-PARA

UK,CAMPAN

BEARPAW FM,OXARART MBR (LRPART)

LS 10,S 22, T 6,R 26,W 3RD MERID, CYPRESS L,SASK

R277;RUSSELL LS,J PALEONT,VOL 41,N6, 1967,P $1544-$ $1546, F I G 4$

REMARKS: C-LS RUSSELL, 1948 ;CARINA ; 1 SPEC

\section{CALANTICA ? SASKATCHEWANENSIS RUSSELL}

28155 P-PARA

UK,CAMPAN

BEARPAW FM,OXARART MBR (LR PART)

LS 10,S 22,T 6,R 26, W 3RD MERID, CYPRESS L,SASK

R277; RUSSELL LS,J PALEONT, VOL 41,N6,1967,P15441546 , FIG 4

REMARKS: C-LS RUSSELL, 1948 ;CARINA ; 1 SPEC

\section{CALANTICA ? SASKATCHEWANENSIS RUSSELL}

28156 P-PARA

UK,CAMPAN

BEARPAW FM, OXARART MBR (LR PART)

LS $10, S 22, T 6, R 26$, W 3 RD MERID , CYPRESS L,SASK

R277; RUSSELL LS,J PALEONT, VOL 41,N6, 1967,P 15441546, FIG 4

REMARKS: C-LS RUSSELL, 1948 ;CARINA; 1 SPEC

\section{CALANTICA? SASKATCHEWANENSIS RUSSELL}

28157 P-PARA

UK,CAMPAN

BEARPAW FM,OXARART MBR (LR PART)

LS 10,5 22, T 6,R 26, W 3RD MERID, CYPRESS L,SASK

R277;RUSSELL LS,J PALEONT, VOL 41,N6, 1967,P 15441546,FIG 5

REMARKS: C-LS RUSSELL, 1948 ;CARINAL LATERA ; 1 SPEC
CALANTICA ? SASKATCHEWANENSIS RUSSELL

28158 P-PARA

UK,CAMPAN

BEARPAW FM,OXARART MBR (LR PART)

LS 10,S 22,T 6.A 26,W 3RD MERID, CYPRESS L, SASK

R277; RUSSELL LS,J PALEONT, VOL 41,N6, 1967,P15441546.FIG 5

REMARKS: C-LS RUSSELL, 1948 ;CARINAL LATERA ; 1 SPEC

CALANTICA? SASKATCHEWANENSIS RUSSELL 28159 P-PARA

UK,CAMPAN

BEARPAW FM, OXARART MBR (LR PART)

LS 10,S 22,T 6,A 26,W 3RD MERID , CYPRESS L,SASK R277:RUSSELL LS,J PALEONT, VOL 41,N6, 1967,P 15441546, FIG 5

REMARKS: C-LS RUSSELL, $1948 ; 1$ SPEC

CALANTICA? SASKATCHEWANENSIS RUSSELL

28151 P-HOLO

UK,CAMPAN

BEARPAW FM,OXARART MBR (LR PART)

LS 10,S 22,T 6,R 26,W 3RD MERID, CYPRESS L,SASK

R277:RUSSELL LS,J PALEONT,VOL 41,N6, 1967,P 15441546, FIG 1

REMARKS: C-LS RUSSELL, 1948 ;LEFT \& RIGHT SCUTUM PRESUMABLY FROM SAME ANIMAL ; 1 SPEC (2 PARTS)

CALANTICA ? SASKATCHEWANENSIS RUSSELL

28152 P-PARA

UK,CAMPAN

BEARPAW FM,OXARART MBR (LR PART)

LS 10,S 22,T 6,R 26,W 3RD MERID, CYPRESS L,SASK

R277;RUSSELL LS,J PALEONT, VOL 41,N6, 1967,P 1544-

1546,FIG 2,3

REMARKS: C-LS RUSSELL, 1948 ;LEFT SCUTUM ; 1 SPEC

CALANTICA ? SASKATCHEWANENSIS RUSSELL

28153 P-PARA

UK,CAMPAN

BEARPAW FM,OXARART MBR (LRPART)

LS 10,S 22,T 6,R 26,W 3RD MERID, CYPRESS L,SASK

R277;RUSSELL LS,J PALEONT, VOL 41,N6, 1967,P 1544-

1546,FIG 2,3

REMARKS: C-LS RUSSELL, 1948 ;RIGHT SCUTUM ; 1 SPEC

CERATIOCARIS PUSILLUS MATTHEW

22701 [460S ] P-SYN

MS

[SILURIAN,DIV 2]

CUNNINGHAM BK,NEREPIS,NB

R315;MATTHEW GF,TRANS ROY SOC CAN, VOL 6,SECT 4, 1889,P56,PL4,FIG 9

REMARKS: C-GF MATTHEW;D-1913;LABELLED HOLOTYPE; THIS DOES NOT APPEAR TO BE THE FIG'D SPEC ; 1 SPEC

COLEOLUS TENUISTRIATUM 12958 [2348D ] REF SPEC-F

MD

KWATABOAHEGANR,ONT

W134; PARKS WA,ONT DEPT MINES, VOL 13,PT 1, 1904,PL8,FIG 2

REMARKS: C-WA PARKS, 1903 ;STORED UNDER ORTHOCERAS LUXUM; 1 SLAB

CORNULITES STERLINGENSIS (MEEK\&WORTHEN)

17453 [ 1214 HR ] REF SPEC-F

UO,CINCI,MAYSVILL

DUNDAS FM

[DUNDAS]

WATER LEVEL,HUMBER R, 1/2 MI BELOW WESTON,ONT

W137;PARKS FRITZ,ONT DEPT MINES, VOL

31,PT9, 1923,P37-38,PL2,FIG 5, 6

REMARKS:1 SPEC 
CRESEIS CORRUGATA MATTHEW

7965 [335CM ] P-SYN

LO,

[BRETONIAN,DIV 3D]

SUSPENSION BRIDGE, ST JOHN, NB

W 133;MATTHEW GF, TRANS ROY SOC CAN, VOL 10,SECT

4, 1893,P 105,PL7,FIG 12A,B

REMARKS: C-GF MATTHEW;D-MACKENZIE ;SPEC 'A' FIG'D 4 SPEC

CRESEIS MINUTA MATTHEW

7966 [336CM] P-SYN

LO,

[BRETONIAN,DIV 3D ]

SUSPENSION BRIDGE, ST JOHN,NB

W 133:MATTHEW GF, TRANS ROY SOC CAN, VOL 10,SECT 4,1893,P105,PL7,FIG 11A-C

REMARKS: C-GF MATTHEW;D-MACKENZIE ; 5 SPEC

\section{CUPRESSOXYLON MACROCARPOIDES PENHALLOW}

34468 REF SPEC-M

UK,

BELLYRIVER FM

[JUDITH RIVER (BELLY RIVER) SERIES ]

RED DEER R,ALTA

PENHALLOW DP, 1908,OTTAWA NAT, VOL 22,N4,P82

REMARKS: D-DEPT BOTANY,U OF T, 1975; SLIDES CUT

FROM SPECS NUMBERED $319+330$, GIVEN TO

PENHALLOW BY LM LAMBE;RADIAL, TANGENTIAL +

TRANSVERSE SECT OF EACH SPEC;WOOD ; 6 SLIDES

\section{GONIADICHNITES TRICHIFORMIS MATTHEW}

8253 [623CM] P-SYN

$M C A$,

ST JOHN GP

[ST JOHN,DIV C2A]

CATON'S IS, KINGS CO,NB

W194;MATTHEW GF,TRANS ROY SOC CAN, VOL 8,SECT

4,1891,P 160,PL11,FIG 12

REMARKS: C-GF MATTHEW;D-MACKENZIE ; 6 SPEC

\section{HYDROCYTIUM? SILICULA MATTHEW}

$8244[614 \mathrm{CM}]$ P-SYN

LCA,

ST JÖHN GP

[ST JOHN,DIV 1B]

RADCLIFF'S, ST JOHN CO,NB

MATTHEW GF, TRANS ROY SOC CAN, VOL

7,PT4, 1890,P146,PL6,FIG 2

REMARKS: C-GF MATTHEW;D-MACKENZIE ;POSSIBLY SPORE CASES OF ALGAE ; 1 SPEC

HYOLITHELLUS MICANS BILLINGS

8173 [543CM] REF SPEC-M

LCA,

ST JOHN GP

[ST JOHN, ACADIAN,DIV 1B3]

HANFORD BK, ST MARTINS,ST JOHN CO,NB

W 132;MATTHEW GF, TRANS ROY SOC CAN, VOL 11,SECT 4, 1894,P94-95

REMARKS: C-GF MATTHEW;D-MACKENZIE ;LABELLED

PLESIOTYPE;NOT FIG'D ; 2 SPEC

LICROPHYCUS FLABELLUM MILLER\&DYER

17496 [ 1257 HR ] REF SPEC-F

UO,CINCI,MAYSVILL

DUNDAS FM

[DUNDAS ]

CUT ON W SIDE,HUMBER R, 1 MI BELOW LAMBTON,ONT REMARKS: C-J TOWNSEND; 1 SPEC

MARKING

17504 [1265HR ] REF SPEC-F

U O,CINCI,MAYSVILL

DUNDAS FM

HUMBER R.TORONTO ONT

REMARKS: C-STUDENT, 1923 ;MAY BE INORGANIC ; 1 SPEC
MEDUSICHNITES SP FORM B

8468 [838CM] REF SPEC-F

$\mathrm{UCA}$,

ST JOHN GP

[JOHANNIAN,ST JOHN GP,DIV C2A]

TABOR'S BRIDGE, KINGS CO,NB

W234:MATTHEW GF. TRANS ROY SOC CAN,VOL 8,SECT

4.1891,P 145,PL 12,FIG 2

REMARKS: D-W MACKENZIE ;TRACKS ; 1 SPEC

\section{MESOXYLOIDES PLATYPODIUM MASLEN}

32779 P-SYN

UC

[LOWER COAL MEASURES ]

SHORE COLLIERY, SHORE, LITTLEBOROUGH,LANCS, ENG

MASLEN AJ, 1930,ANN OF BOTANY, VOL 44,N175,P515531,PL26,FIG 8

REMARKS: D-BOTANY DEPT, UOF T, 1975:NODULE FROM ROOF OF COLLIERY;OLD BOTANY SLIDE NO 178-BOYD THOMSON COLL;TRANSVERSE SECT;NOS 32778-32782 CUT FROM SAME SPEC;STEM SHOWING LEAF TRACE BUNDLES ; 1 SLIDE

\section{MESOXYLOIDES PLATYPODIUM MASLEN}

32780 P-SYN

UC,

[LOWER COAL MEASURES]

SHORE COLLIERY, SHORE, LITTLEBOROUGH,LANCS,ENG

MASLEN AJ, 1930,ANN OF BOTANY,VOL 44,N175,P515531,PL26,FIG 7

REMARKS: D-BOTANY DEPT,U OF T, 1975; NODULE FROM ROOF OF COLLIERY,OLD BOTANY SLIDE NO 179-BOYD THOMSON COLL;TRANSVERSE SECT;NOS $32778-32782$ CUT FROM SAME SPEC;STEM SHOWING LEAF TRACE BUNDLES ; 1 SLIDE

\section{MESOXYLOIDES PLATYPODIUM MASLEN}

32778 P-SYN

UC

[LOWER COAL MEASURES]

SHORE COLLIERY, SHORE, LITTLEBOROUGH, LANCS,ENG MASLEN AJ, 1930,ANN OF BOTANY, VOL 44,N175,P515-531 REMARKS: D-BOTANY DEPT,U OF T, 1975; NODULE FROM ROOF OF COLLIERY;OLD BOTANY SLIDE NO 117-BOYD THOMSON COLL;TRANSVERSE SECT:NOS 32778-32782 CUT FROM SAME SPEC;STEM SHOWING LEAF TRACE BUNDLES ; 1 SLIDE

MICRICHNUS PALAEOCENUS RUSSELL

28058 P-HOLO

PALEOC

LPASKAPOOFM

RIVER BANK,SEC 29,TP 20,RGE 28,W 4 TH MERID, HIGHWOOD R,ALTA

W211:RUSSELL LS, TRANS ROY CAN INST, VOL

23,PT 1, 1940,P67-74,PL 1-2

REMARKS: C,P-RL FOWLER;STRAT LOC 600' ABOVE CRET-TERT BOUNDARY ; 1 SPEC

MONOCRATERION MAGNIFICUM MATTHEW

$8266[636 \mathrm{CM}] \mathrm{P}-\mathrm{HOLO}$

UCA,

ST JOHN GP

[ST JOHN,DIV C2C ]

S OF DUNNE'S LEDGES, COURTNEY BAY,SIMONDS,NB W2 18:MATTHEW GF, TRANS ROY SOC CAN,VOL 8,SECT 4, 1891,P 161,PL 12, FIG 1, 1A-B

REMARKS: C-GF MATTHEW:D-MACKENZIE ; 1 SPEC

\section{PALAEOCHORDA SETACEA MATTHEW}

8420 [790CM] P-SYN

$\mathrm{MCA}$

BOURINOT GP.DUGALDFM

[LETCHEMINIAN,DIV 1B]

HANFORD BK, ST MARTINS,ST JOHN CO,NB

MATTHEW GF, TRANS ROY SOC CAN, VOL 7,PT 4, 1890,P 145 146,PL6,FIG 1A-G

REMARKS: C-GF MATTHEW;D-MACKENZIE ;POSSIBLE ALGA;STRAT REV AS IN HUTCHINSON, 1952,GSC MEM $263 ; 3$ SPEC 
PALAEOPHYCUS SP INDET

17495 [1256HR] REF SPEC-F

U O.CINCI,MAYSVILL

DUNDAS FM

[DUNDAS]

DON VALLEY QUARRY, TORONTO,ONT

REMARKS: C-J TOWNSEND ; 1 SPEC

PALAEOPHYCUS VIRGATUS HALL

17494 [1255HR] REF SPEC-F

U O,CINCI,MAYSVILL

DUNDAS FM

[DUNDAS ]

BELOW RLWY BRIDGE, HUMBER R,TORONTO,ONT

REMARKS: C-J TOWNSEND ; 3 SPEC

\section{PHYCOIDELLA STICHIDIFERA MATTHEW}

8312 [682CM] P-SYN

CA,

DIV $1 B$

[LETCHEMINIAN]

HANFORD BK, ST MARTINS,ST JOHN CO,NB

MATTHEW GF,TRANS ROY SOC CAN,VOL 7,PT4, 1890,P 144-

145, PL5,FIG 5A-D

REMARKS: C-GF MATTHEW;D-MACKENZIE ; 2 SPEC

PLUMULITES MANUELENSIS MATTHEW

7849 [219CM] P-HOLO

$\mathrm{MCA}$,

ST JOHN GP

[ST JOHN, ACADIAN,C1D]

MANUELS BK, CONCEPTION BAY,NFLD.

2744:MATTHEW GF.TRANS NY ACAD SCI,VOL 15, 1896,P200,PL14,FIG 7

REMARKS: C-GF MATTHEW;D-MACKENZIE ;GENUS

PLUMULITES NOT IN TREATISE; 1 SPEC

PTERYGOTUS GASPESIENSIS RUSSELL

26805 P-HOLO

LD,

GASPE GP (2300' ABOVE BASE), BATTERY POINT FM

SEA CLIFF, 1360' E OF D'AIGUILLON WHARF , GASPE

SOUTH CO,QUE

P30;RUSSELL LS,NAT MUS ANN REPT,BULL 132, 1954,P8387, PL1,FIG 1-3

REMARKS: C-GE LINDBLAD, 1946 ;OLD RECORDS STATE 2 PCES-POS + NEG;ONLY 1 PCE LOCATED; 1 SPEC

PTERYGOTUS GASPESIENSIS RUSSELL

26806 P-PARA

LD,EMSIAN

GASPE GP (2300' ABOVE BASE), BATTERY POINT FM

SEA CLIFF, 1360' E OF D'AIGUILLON WHARF , GASPE

SOUTHCO,QUE

P30:RUSSELL LS,NAT MUS ANN REPT,BULL 132, 1954,P83$87, P L 2, F I G 1,3$

REMARKS: C-GE LINDBLAD, 1946; 1 SPEC

\section{RILL MARKS}

17498 [1259HR] REF SPEC-F

U O,CINCI,MAYSVILL

DUNDAS FM

[DUNDAS ]

FIRST RAVINE, ROSEDALE,TORONTO,ONT

REMARKS: C-J TOWNSEND, 1923 ;MAY BEINORGANIC ; 1 SPEC

\section{RUSOPHYCUS SPINDET}

17493 [1254HR] REF SPEC-F

U O,CINCI,MAYSVILL

DUNDAS FM

[DUNDAS ]

TORONTO,ONT

REMARKS: C-J TOWNSEND :LOCALITY UNCERTAIN-DON

VALLEY QUARRY IN TEXT, HUMBER R IN PLATE CAPTION + ROM RECORDS ; 1 SPEC
SEQUOIA ALBERTENSIS PENHALLOW

34469 P-HOLO

$\mathrm{UK}$.

EDMONTON FM

[EDMONTON SERIES ]

RED DEER R, 100 MI W OF GLEICHEN,ALTA

PENHALLOW DP, 1908, OTTAWA NAT, VOL 22,N4,P83-84,FIG $1-6$

REMARKS: D-DEPT BOTANY,U OF T, 1975:SLIDES MARKED 'TYPE';PROBABLY CUT FROM UNNUMBERED SPEC GIVEN TO PENHALLOW BY LM LAMBE;C-MR. MILLIKEN; STRAT + LOC GIVEN ON SLIDES AS JUDITH R SER, GLEICHEN,BC;RADIAL,TRANS + TANG SECTIONS; WOOD : 3 SLIDES

SEQUOIA ALBERTENSIS PENHALLOW 34959 P-PARA

UK.

BELLY RIVER FM

[JUDITH RIVER (BELLY RIVER) SERIES ]

RED DEER R,ALTA

PENHALLOW DP, 1908,OTTAWA NAT, VOL 22, N4,P83-84

REMARKS: D-DEPT BOTANY,U OF T,1975;SLIDES CUT FROM SPECS NUMBERED $275+276$, GIVEN TO

PENHALLOW BY LM LAMBE;RADIAL, TANGENTIAL + TRANSVERSE SECT OF EACH SPEC;WOOD; 6 SLIDES

SOLENOPORA COMPACTA VAR OUAREAUENSIS FRITZ

23356 P-SYN

MO.CHAMP MOH, BLKRIV

BLACK RIVER GP

[BLACK RIVER]

OUAREAU R,NR JOLIETTE,QUE

REMARKS: C-WA PARKS, 1930 ;SLIDES 42A-D;A,B FIG'D ; 3 SPEC, 4 SLIDES

STENOTHECA CONCENTRICA MATTHEW

$8281[651 \mathrm{CM}]$ P-SYN

MCA.

ST JOHN GP

[ST JOHN, ACADIAN,DIV 1D]

PORTER'S BK, ST MARTINS,ST JOHN CO,NB

W140:MATTHEW GF, TRANS ROY SOC CAN, VOL 3,SECT 4,1886,P57,PL6,FIG 11

REMARKS: C-GF MATTHEW:D-MACKENZIE :LABELLED COTYPES;UNCERTAIN WHICH SPEC FIG'D ; 6 SPEC

STENOTHECA HICKSIANA MATTHEW

7944 [314CM] P-SYN

MCA,

ST JOHN GP

[ST JOHN,ACADIAN,DIV 1D]

PORTER'S BK, ST MARTINS,ST JOHN CO,NB

W140;MATTHEW GF, TRANS ROY SOC CAN, VOL 3,SECT 4.1886,P56-57,PL6,FIG 14

REMARKS: C-GF MATTHEW:D-MACKENZIE ;LABELLED COTYPES ; 2 SPEC

STENOTHECA NASUTA MATTHEW

7943 [313CM] P-SYN

MCA.

ST JOHN GP,DIV $1 C$

[ST JOHN, ACADIAN]

HANFORD BK, ST MARTINS,ST JOHN CO,NB

W 140;MATTHEW GF, TRANS ROY SOC CAN, VOL 3,SECT 4,1886,P58,PL6,FIG 13

REMARKS: C-GF MATTHEW:D-MACKENZIE ;LABELLED HOLOTYPE : 1 SPEC

STENOTHECA RADIATA MATTHEW

7945 [315CM ] P-SYN

$\mathrm{MCA}$,

ST JOHN GP

[ST JOHN, ACADIAN,DIV 1C]

PORTER'S BK, ST MARTINS, ST JOHN CO,NB

W 140:MATTHEW GF, TRANS ROY SOC CAN, VOL 3,SECT 4,1886,P57-58,PL6,FIG 12

REMARKS: C-GF MATTHEW:D-MACKENZIE ;LABELLED COTYPES : 6 SPEC 
TRICHOPHYCUS VENOSUM MILLER

17492 [1253HR] REF SPEC-F

U O,CINCI,MAYSVILL

DUNDASFM

[DUNDAS]

7 FT LEVEL, CUT ON W BANK, HUMBER R, 1 MI BELOW

LAMBTON,ONT

REMARKS: C-J TOWNSEND ; 3 SPEC 


\section{Literature Cited}

Ht+N, J.A and J.O.G. St.NDERSON

1945 Geology of Red Deer and Rosebud Sheets, Alberta. -

Annual Repurt. Albera Research Council, 13: 1-116.

ARUSTRONG, HS

1945 Stigmatella in the Ordovician of the Central Ontario

Basin. - Journal of Paleontology, 19(2): 149-157.

BARNES, C.R. and D.J. ST.ACK

1975 Conodont ultrastructure: the subfamily Acanthodontinac. - Life Sciences Contributions, Royal Ontario Museum. 106: $1-21$.

BASSLER, R.S

1906 The bryozoan fauna of the Rochester Shale. - Bulletin. United States Geological Survey, 292: 1- 137.

1909 Dendroid Graptolites of the Niagaran dolonites at Hamilton, Ontario. - Bulletin, United States National Museum, 65: 1-76

BELL, B.M

1976 A study of North American Edrioasteroidea. - Memoir. New York Stale Museum and Science Service [1974], 21: $1-47$.

BLACKADAR, R.G.

1972 Guide for the preparation of geological maps and reports - Miscellaneous Repor, Geological Survey of Canada [Revised Edition], 16: 1 - 190.

BOLTON, T.E

1957 Silurian stratigraphy and palaeontology of the Niagara Escarpment in Ontario. - Memoir, Geological Survey of Canada, 289: 1-145.

1970 Echinodermata from the Ordovician (Pleurocystites, Cremacrinus) and Silurian (Hemicystites, Protaxocrinus, Macnamaraty/us) of Lake Timiskaming Region, Ontario and Quebec. - In Contributions to Canadian Paleontology, Bulletin, Geological Survey of Canada, 187: 59-66, 146-152.

BRA.NSTRATOR, J.W

1972 Lanthanaster cruciformis, a new Upper Ordovician sea star from Cincinnati, Ohio. - Journal of Paleontology, $46(1): 66-69$

BRIGGS, D.E.G.

1976 The arthropod Branchiocaris n.gen., Middle Cambrian. Burgess Shale, British Columbia. - Bulletin, Geological Survey of Canada, 264: 1 - 29.

1977 Bivalved arthropods from the Cambrian Burgess Shale of British Columbia. - Palaeontology, 20(3): 595-621.

BROWER, J.C.

1966 Functional morphology of Calceocrinidae with description of some new species. - Journal of Paleontology, 40(3): 613-634

CALEY, J.F.

1936 Contributions to the study of the Ordovician of Ontario and Quebec. Part II. The Ordovician of Manitoulin Island, Ontarıo. - Memoir. Geological Survey of Canada, 202: 21 -91

CHURCHFR, C.S.

1966 The insect fauna from the Talara Tar-secps, Peru. Canadian Journal of Zoology, 44: 985.993
COT E.MAN, A P.

1933 The Plessocene of the Toronto Regrou (including the Toronto Interglacial lormation). - Report of the Ontario Department ol Mucs, 1932, 4l(7): 1-69.

COPPER, P

1977 Zygospira and some related Ordovician and Siluran atrypoid brachiopods. - Palaeontology, 20(2): 295- 335

CRANSWICK, J.S, and M.A. FRIIZ

1958 Coral launa of the Upper Abitibi River Limestone. Proceedings, Geological Association of Canada, 10. $31-67$

DEAN, W. I

1972 The trilobite genus Holasaphus Matthew. 1895, in the Middle Cambrian rocks of Nova Scotia and eastern Turkey, - Canadian Journal of Earth Scicnces, 9(3) $266-279$

DOUGLAS, R.J.W., ed.

1970 Geology and economic minerals of Canada. - Report, Geological Survey of Canada Economic Geology, I $1-838$

DYER, W.S

1925 The stratigraphy and paleontology of Toronto and vicinity. Part V. The paleontology of the Credit River Section. - Report of the Ontario Department of Mines, $1923,32(7): 47-88$

ELLER, E.R.

1945 Scolecodonts from the Trenton series (Ordovician) of Ontario, Quebec, and New York. - Annals of the Carnegie Museum, 30: 119-212

FAGERSTROM, J.A.

1971 Brachiopods of the Detroit River Group (Devonian) from southwestern Ontario and adjacent areas of Michigan and Ohio. - Bulletin, Geological Survey of Canada, 204: $1-113$

FOERSTE, A.F.

1914ia The Rogers Gap fauna of central Kentucky. - Journal of the Cincinnati Society of Natural History, 2I(4) $109-156$

1914b Notes on Agelecrinidae and Lepadocystinae, with description of Thresherodiscus and Brockocystis. Journal of the Scientific Laboratories, Denison Universily Bulletin, 17: 399-486

1916 Comarocystites and Caryocrinites. Cystids with pinnuliferous free arms. - Ottawa Naturalist, 30(7.8): $69 \cdot 79,85 \cdot 93$

1919 Notes on Isotelus, Acrolichas, Calymene, and Encrinurus. - Journal of the Scientific Laboratories, Denison University Bulletin, 19: 65-81.

1920 The generic relations of the American Ordovician Lichadidae. - American Journal of Science, 49(289) $26 \cdot 50$

1921 Notes on Arctic Ordovician and Silurian cephalopeds. Chiefly from Boothia Felix-King William L,and, Bache Peninsula, and Bear lsland. - Journal of the Scientific Laboratories, Denison University Bulletin, 19: 247-306.

1928: Devonian cephalopexds from the Moose River Basun. Report of the Ontario Department of Mines, 1928, 37(6): $70-79$

1928b A restudy of American orthoconic Silurian cephalopeds - Journal of the Scientific Laboratories, Denison University Bulleın, 23: $236-320$ 
FOERSTE, A.F and T E, SAVAGE

1927 Ordovician and Silurian cephalopods of the Hudson Bay area. - Journal of the Scientific Laboratories, Denison University Bulletin, 22: 1 - 107.

FRITZ, M.A.

1925 The stratigraphy and palcontology of Toronto and vicinity. Part IV. Hydrozoa, Echinodermata, Trilobita, and Markings. - Report of the Ontario Department of Mines, 1923, 32(7): 1-46.

1926 The stratigraphy and palaeontology of the Workman's Creek section of the Cincinnatian series of Ontario. Transactions, Royal Society of Canada, 20(4): 77 - 107.

1930 Tho new species of fossils from the Palaeozoic rocks of Ontario. - Transactions, Royal Canadian Institute, 17(2): 223- 225 .

1937 Mulrisolenia, a new genus of Paleozoic corals. - Joumal of Paleontology, 11(3): 231-234.

1940 Devonian Bryozoa from Fortin and Malbay Townships, Gaspé County, Quebec. - Contributions of the Royal Ontario Museum of Palaeontology, 4: I - 10.

1941a Catalogue of types in the Royal Ontario Museum of Palaeontology. - Contributions of the Royal Ontario Museum of Palaeontology, 5(1): 1 - 50.

1941 b Fenestrellina multistriata, a new Devonian bryozoan from Quebec. - Journal of Paleontology, I5(1): 94-96.

I941c On Solenopora compacta (Billings) and the new variety Solenopora compacta ouareauensis. - Transactions, Royal Canadian Institute, 23(2): 157-160.

1941d Baltic Ordovician fauna in Gaspé. - Journal of Paleontology, 15(5): 564 .

1942 Catalogue of types in the Royal Ontario Museum of Palaeontology. - Contributions of the Royal Ontario Museum of Palaeontology, 6(2): $1-30$.

1943 Catalogue of types in the Royal Ontario Museum of Palaeontology. - Contributions of the Royal Ontario Museum of Palacontology, 7(3): I- 36.

1944 Catalogue of types in the Royal Ontario Museum of Palaeontology. - Contributions of the Royal Ontario Museun of Palaeontology, 8(4): 1-46.

1945 Catalogue of types in the Royal Ontario Museum of Palaeontology. - Contributions of the Royal Ontario Museum of Palaeontology, 9(5): 1-43.

1946a Catalogue of types in the Royal Ontario Museum of Palaeontology. - Contributions of the Royal Ontario Museum of Palacontology, 10(6): 1-31.

$1946 \mathrm{~b}$ On the Meraspid period of Isotelus maximus Locke. Transactions, Royal Society of Canada, 40(4): 27-32

1951 The pelecypod genus Vlasta in the Ordovician of North America. - Journal of Paleontology, 25(5): 696-698.

1954 Rhopalonaria lambtonensis, n.sp. - Journal of Paleontology, 28(1): 118.

1959 Meraspid period (degree 3) of Pseudogygites latimarginatus (Hall). - Canadian Journal of Zoology, 37: $1117-1121$

1963 An early Middle Pennsylvanian bryozoan fauna from the Banfi area. Alherta. - Bulletin, Canadian Petroleum Geology, 11(1): 54-58.

1964 ?Scutellum regale sp.nov. Fritz from the Silurian of the Hudson Bay area. - Proceedings, Geological Association of Canada, 15: 91 -97.

1970 Redescription of type specimens of the bryozoan Hallopora from the Upper Ordovician of Toronto region, Ontario. - Procecdings, Geological Association of Canadis, 21: 15-23.

1971 The trepostomatous bryozoan Stigmatella catenulata diversa Parks and Dyer (1922), a synonym for Mesotrypa diversa (Parks and Dyer). - Life Sciences Occasional Papers, Royill Ontario Muscum, 18: 1-6.
1973 Redescription of type specimens of bryozoan Stigmatella from the Upper Ordovician of the Toronto region, Ontario. - Life Sciences Contributions, Royal Ontario Museum, 87: 1-31.

1975 Redescription of type specimens of the bryozoan Heterotrypa from Upper Ordovician rocks of the Credit River Valley, Ontario, Canada. - Life Sciences Contributions, Royal Ontario Museum, 101: 1-30.

1976a A microbioherm. In Churcher, C.S., ed. Athlon; Essays on palaeontology in honour of Loris Shano Russell. -

Life Sciences Miscellaneous Publications, Royal Ontario Museum: 18-25.

$1976 \mathrm{~b}$ Redescription of type specimens of species of the bryozoan genera Monticulipora, Mesotrypa, Peronopora. and Prasopora, from the Upper Ordovician rocks of Toronto and vicinity, Ontario, Canada. - Life Sciences Contributions, Royal Ontario Museum, 107: 1-24.

1977 Redescription of type specimens of species of the bryozoan genera Alactoporella. Homotrypa, and Homotrypella, from the Upper Ordovician rocks of the Credit River Valley, Ontario, Canada. - Life Sciences Contributions, Royal Ontario Museum, 111: 1-24.

FRITZ, M.A. and L.M. CLINE

1937 Mesoblastus haynesi (Clark) from Mount Coleman, Alberta. - Transactions, Royal Canadian Institute. 2I(46): $307-312$.

FRITZ, M.A., R.R.H. LEMON and A.W. NORRIS

1957 Stratigraphy and paleontology of the Williams Island Formation. - Proceedings, Geological Association of Canada, 9: $21-47$

FRITZ, M.A. and R.H. WAINES

1956 Stromatoporoids from the Upper Abitibi River limestone. - Proceedings, Geological Association of Canada, 8: $87-118$.

GALLOWAY, J.J. and J. ST.JEAN

1961 Ordovician Siromatoporoidea of North America. Bulletins of American Paleontology, 43(194): 1 - 102.

GIRTY, G.H.

1910 The fauna of the Phosphate Beds of the Park City Formation in Idaho, Wyoming and Utah. - Bulletin. United States Geological Survey, 436: I-82.

GRABAU, A.W.

1909 Description of Monroe fossils. - Geological Series of the Michigan Geological and Biological Survey, 2(1): 87.214.

HALL, J.

1888 Natural History of New York. Palaeontology. Vol. VII. Charles van Benthuysen \& Sons, Albany. New York. 236 pp.

HUDSON, G.H

1912 A fossil starfish with ambulacral covering plates. Ottawa Naturalist, 26(2.3.4): 21 - 26, 45-52.

IIUTCIINSON, R.D.

1952 The stratigraphy and trilobite faunas of the Cambrian sedimentary rocks of Cape Breton Island, Nova Scotia. Memoir, Geological Survey of Canada, 263: 1-124

1962 Cambrian stratigraphy and trilobite fiunas of southeastern Neufoundland. - Bulletin, Geological Survey of Canada, 88: I - 156. 
INTERNATIONAL COMMISSION ON ZOOLOGICAL NOMENCLATURE.

1964 International coule of zoological nomenclature, adopted by the 15th International Congress of Zoology: - Revised edition [Editorial Committee: N.R. Stoll. et al.]. London, Published for the Commission by the International Trust for Zoulogical Nomenclature. 175 pp.

JULL, R.K.

1976 Septal development during hystero-ontogeny in the

Ordovician Tabulate Coral Foerstephyllum. - Journal of

Paleontology; 50(3): 380-391.

KEROHER, G.C. ET AL., eds.

1966 Lexicon of geological names of the United States. -

Bulletin. United States Geological Survey, $1200(1,2,3)$ : $1-4341$.

KNIGHT, J.B.

1941 Paleozoic gastropod genotypes. - Special Paper,

Geological Society of America, 32: 1 - 510.

LUDVIGSEN, $R$.

1977 The Ordovician trilobite Ceraurinus Barton in North America. - Journal of Paleontology, 51(5): 959-972.

MASLEN, A.J.

1930 The structure of Mesorylon platypodium and

Mesoryloides. - Annals of Botany, 44(175): $503-533$.

MATTHEW, G.F.

1883 Illustrations of the fauna of the St. John Group, No. I

The Paradoxides. - Transactions, Royal Society of

Canada, 1882, 1(4): $87-108$.

1885 Illustrations of the fauna of the St. John Group continued: on the Conocoryphea, with further remarks on Paradoxides. - Transactions, Royal Society of Canada, 1884, 2(4): $99-124$

1886 Illustrations of the fauna of the St. John Group continued. No. 3. - Transactions, Royal Society of Canada, 1885, 3(4): 29-84.

1887 On the Cambrian faunas of Cape Breton and New foundland. - Transactions, Royal Society of Canada, $1886,4(4): 147-157$.

1888 Illustrations of the fauna of the St. John Group. No. 4. Transactions, Royal Society of Canada, 1887, 5(4) $115-166$.

1889a On the classification of the Cambrian rocks in Acadia. No. 2. - Canadian Record of Science, 3(5): 303-315.

$1889 \mathrm{~b}$ On the occurrence of Leproplastus in the Acadian Cambrian rocks. - Canadian Record of Science, 3(8): $485-489$.

1890 On Cambrian organisms in Acadia. - Transactions, Royal Society of Canada, 1889, 7(4): 135-162.

1891 a Illustrations of the fauna of the St. John Group. No. 5. Transactions, Royal Society of Canada, 1890, 8(4): $123-166$.

$1891 \mathrm{~b}$ Note on Leproplasius. - Canadian Record of Science, 4(8): $461-462$

1892a Illustrations of the fauna of the St. John Group. No. 6. Transactions, Royal Society of Canada, 1891, 9(4) $33-65$.

1892b Hyolithes gracilis and related forms from the Lower Cambrian of the St. John Group. - Transactions, Royal Society of Canada, 1891, 9(4): 109-111.

1893a Illusirations of the fauna of the St. John Group. No. 7. Transactions, Royal Society of Canada, 1892, 10(4): 95- 109 .

$1893 \mathrm{~b}$ Notes on Cambrian faunas. - Canadian Record of Science, 1892, 5(3): 247.258.

1893c Trematobolus, an articulate brachioped of the inarticulate order. - Canadian Record of Science, 5(5): 276- 279.
Illustrations of the fauna of the St. John Group. No. 8. Transactions, Royal Socicty of Canada, 1893, 11(4): $85-130$

1895a The Protolenus fauna. - Transactions, New York Academy ol Sciences, 14: 101-153.

1895b Two new Cambrian graptolites with notes on other species of Graptolitidae of that age. - Transactions, New York Academy of Sciences, 14: 262-273.

1896 Faunas of the Puradoxides beds in eastern North America, No. 1. - Transactions, New York Academy of Sciences, 15: 192-247.

1897a Studies on Cambrian faunas. - Transactions, Royal Society of Canada, 2nd series, 3(4): 165-203.

1897 b The oldest Siphonotreta. - Geological Magazine, n.s., decade 4, 4(2): $68-71$.

1899a Studies on Cambrian faunas, no.2. - Transactions, Royal Society of Canada, 1898, 2nd series, 4(4): 123-150.

1899 b Studies on Cambrian faunas, no.3. - Transactions, Royal Society of Canada, 2nd series, 5(4): 39-123.

1899c A new Cambrian trilobite. - Bulletin, Natural History Society of New Brunswick, 4(17): 137-142.

1899d Preliminary notice of the Etcheminian fauna of Newfoundland. - Bulletin, Natural History Society of New Brunswick, 4(18): 189-196.

1899e Preliminary notice of the Etcheminian fauna of Cape Breton. - Bulletin, Natural History Society of New Brunswick, 4(18): 198 - 208.

1901 New species of Cambrian fossils from Cape Breton. Bulletin, Natural History Society of New Brunswick, 4(19): $269-286$

1902a Additional notes on the Cambrian of Cape Breton, with descriptions of new species. - Butletin, Natural History Society of New Brunswick, 4(20): 375-426.

1902b Notes on Cambrian faunas. - Transactions, Royal Society of Canada, 8(4): $93-112$.

1902c Ostracoda of the basal Cambrian rocks in Cape Breton. Canadian Record of Science, 8(7): 437-470.

1903 Report on the Cambrian rocks of Cape Breton. - S.E. Dawson, Ottawa. 246 pp.

MCLEAN, R.A

1976 Middle Devonian cystiphyllid corals from the Hume Formation, northwestern Canada. - Bulletin, Geological Survey of Canada, 274: 1-180.

MERRILL, G.K. and P.H. VON BITTER

1977 Apparatus of the Pennsylvanian conodont genus Neognathodus. - Life Sciences Contributions, Royal Ontario Museum, 112: 1-22

OKULITCH, V.J.

1937 Notes on Fletcheria incerta (Billings) and Flercheria sinclairi n.sp. - Transactions, Royal Canadian Institute, 2l(46): 313-36.

1938 Some Black River corals. - Transactions, Royal Society of Canada, 32(4): 87-111

1939 Lichenaria coboconkensis, a new coral from the Ordovician of Ontario. - Journal of Paleontology, 13(5): 514

1940 Revision of type Pleospongia from eastern Canada. Transactions, Royal Society of Canada, 34(4): 75-88.

1942 New Pterygometopinae from the Ordovician of Ontario and Quebec. - Transactions, Royal Socicty of Canada, series 3, 4(4): 99-106.

1943 North American Pleospongia. - Special Paper, Geological Society of America, 48: $1-112$

$1904 a$ Devonian fauna of the Kwataboahegan River. - Report of the Ontario Bureau of Mincs, 13(1): 180.191. 
Hudson Bay Slope. - American Journal of Science, 168. n.s. 18(17): 135-140

1907 The Stromatoporoids of the Guelph Formation in Ontario. - Geological Series, University of Toronto Studies, 4: 1-40[133-172].

1908a Niagara Stromatoporoids. - Geological Series, University of Toronto Studies, 5: 1 - 68 [173-240].

$1908 \mathrm{~b}$ On an occurrence of Hybocystis in Ontario. - Ottawa Naturalist, $21(12): 232-236$

$1908 \mathrm{c}$ Notes on the ophiurıan genus Protaster, with description of a new species. - Transactions, Royal Canadian Institute, 8(3): 363 - 372

1909a Silurian Stromatoporoids of America (exclusive of Niagara and Guelph). - Geological Series, University of Toronto Studies, 6: 1-52[241-292].

$1909 \mathrm{~b}$ Note on the ornamentation of Periglyptocrimus priscus. Ottawa Naturalist, 23(9): 153 - 155.

1910a A new cystid from the Clinton Formation of Ontario Lepadocystis clintonensis. - American Journal of Science, 4th series, 29: $404-406$.

$1910 \mathrm{~b}$ Ordovician Stromatoporoids of America. - Geological Series, University of Toronto Studies, 7: 1-52[293-344].

1915 Palaeozoic fossils from a region southwest of Hudson Bay. - Transactions, Royal Canadian Institute, 11(1): 3-95.

1921 On Triarthrus canadensis, Triarihrus glaber and Triarthrus spinosus. - Transactions, Royal Society of Canada, 15(4): 47-51.

1928 Faunas and stratigraphy of the Ordovician Black Shales and related rocks in southern Ontario. - Transactions, Royal Society of Canada, 22(4): $39-92$.

1933 New species of stromatoporoids, sponges, and corals from the Silurian strata of Baie des Chaleurs. Geological Series, University of Toronto Studies, 33: I - 40 .

1936 Devonian stromatoporoids of North America. Part 1. Geological Series, University of Toronto Studies, 39 : I - 125 .

PARKS, W.A. and F.J. ALCOCK

1912 On two new crinoids from the Trenton Formation of Ontario. - Ottawa Naturalist, 26(3,4): 41 - 45

PARKS, W.A. and W.S. DYER

1922 The stratigraphy and paleontology of Toronto and vicinity. Part 11 - The Molluscoidea. - Report of the Ontario Department of Mines, 1921, 30(7): 1-59.

PARKS, W.A. and M.A. FRITZ.

1923 The stratigraphy and paleontology of Toronto and vicinity. Part 111 - Gastropoda, Ccphalopoda, and Vermes. - Report of the Ontario Department of Mines, I922, 31(9): 1-45

PENHALLOW, D.P.

1908 Report on a collection of fossil woods from the Cretaceous of Alberta. - Ottawa Naturalist, 22(4): $82-88$.

POJETA, J.,JR, and B. RUNNEGAR

1976 The palcontology of rostroconch mollusks and the early history of the Phylum Mollusca. - Professional Paper, Geological Survey of the United States, 968: I-88.

RAYMOND, P.E.

1910 Notes on Ordovician trilobitcs. I1. Asaphidae from the Beckmantown. - Annals of the Carnegic Museum, 7(1) $35-45$.
1913 Notes on Cyclocystoides. - Museum Bulletin, Geological Survey of Canada, 1: $23-32$

1914 Notes on the ontogeny of Isotelus gigas Dekay. Bulletin. Museum of Comparative Zoology, Harvard. 58(5): $247-263$.

RAYMOND, P.E. and D.C. BARTON

1913 A revision of the American species of Ceraurus. Bulletin. Museum of Comparative Zoology, Harvard, 54(20): $525-543$.

RAYMOND, P.E. and J.E. NARRAWAY

1906 A new American Cybele. - Annals of the Carnegie Museum, 3(4): 599-604.

1908 Notes on Ordovician trilobites: Illaenidae from the Black River Limestone near Ottawa, Canada. - Annals of the Carnegie Museum, 4(4): 242 - 255.

1910 Notes on Ordovician trilobites. III. Asaphidae from the Lowville and Black River. - Annals of the Carnegie Museum, 7(1): 46-59

REIMANN, I.G

1935 New species and some new occurrences of Middle Devonian Blastoids. - Bulletin, Buffalo Academy of Science, 17(1): 23-45

RICKER, W.E.

1935 New Canadian Perlids (Part 11). - Canadian Entomologist, 67(12): 256-264

RIGBY, J.K.

1970 Brachiospongia luberculata James, from the Ordovician of Central Ontario. - Journal of Paleontology, 44(6): $1139-1142$

RUSSELL, L.S

1940 Micrichnus tracks from the Paskapoo Formation of Alberta. - Transactions, Royal Canadian Institute, 23(1) $67-74$.

1941 Prograngerella, a new ancestral land snail from the Upper Cretaceous of Alberta. - Journal of Paleontology, 15(3): $309-311$

1943 Marine fauna of the Eastend Formation of Saskatchewan. - Journal of Paleontology, 17(3): 281-288.

1952 Molluscan fauna of the Kishenehn Formation, southeastern British Columbia. - Bulletin, National Museum of Canada, 126: 120-141.

1954 A new species of Eurypterid from the Devonian of Gaspé. - Bulletin, National Museum of Canada, 132: $83-87$.

1967 A pedunculate cirripede from Upper Crctaccous rocks of Saskatchewan. - Journal of Palcontology, 4l(6): 1544 - 1563

1974 Fauna and correlation of the Ravenscrag Formation (Paleocene) of southwestern Saskatchewan. - Life Scicnces Contributions, Royal Ontario Museum, 102: 1 -53 .

1976 Pelecypods of the Hell Creek Formation (Uppcrmost Cretaceous) of Garfield County, Montana. - Canadian Journal of Earth Sciences, 13(2): 355-388

SCHENK, E.T. and J.H. MiMASTIRS

1956 Procedurc in Taxonomy. 3rd cdition. Enlarged and in part rewritten by A.M. Keen and S.W. Muller. - Stanford University Press, California. $149 \mathrm{pp}$

SCHUCHERT, C.

1904 On Siluric and Devonic Cystidea and Camomocrinus. Sinithsonian Miscellaneous Collections, 47(2): 201 - 272. 
1937 The Guelph and Framesa Formaltons of the Ontaru Peninsula. - Transictions, Royal Canidian Institute, 21(2): $317-362$.

SINCLAIR. G.W

1940 A discuswon of the genus. Metcocomblaria with descriptions of neu species. - Tramsatctions, Royall Society of Canada. 34(4): $101-121$

1942 The Chazy Conularidat and their congeners. - Annals of the Carnegic Museum, 29(10): 219.241

1944 Votes on the genera Archaeoconularia and Eocomularia - Transactions, Royal Society ol Canada. 38(4): 87.95

1949 The Ordovician trilobite Eobronteus. - Journal ol Paleontology, 23(1): $45-56$.

SPRINGER, F.

1920 The Crinoidea Flexibilia - Smithsonian Institution. publication 2501. (1.2): 1-485; 1- 158.

SPROULE, J.C

1936 Contributions to the study of the Ordovician of Ontario and Quebec, Part III. A study ol the Cobourg Formation.

- Memoir. Geological Survey of Canada, 202: 93-117.

STEHART, B.H

1920 The stratigraphy and paleontology of Toronto and vicinity. Part 1 - The Pelecypoda. - Report of the Ontario Department of Mines, 1920, 29(6): 5-58.

TOZER, E. T

1956 Uppermost Cretaceous and Paleocene non-marine molluscan faunas of western Alberta. - Memoir,

Geological Survey of Canada, 280: 1-125.

TYRRELL, J.B.

1913 Hudson Bay exploring expedition 1912. - Repon of the Ontario Bureau of Mines, 1912, 22(1): 161-209

ULRICH, E O and R.S, BASSLER

1931 Cambrian bivalved Crustacea of the order Conchostraca. - Proceedings, United States National Museum, 78(4): $1-130$.

VON BITTER, P.H

1976 The apparatus of Gondolella sublanceolata Gunnell (Conodontophorida, Upper Pennsylvanian) and its relationship to Illinella typica Rhodes. - Life Sciences Contributions, Royal Ontario Museum, 109: 1- +4

VON BITTER, P.H. and G.K. MERRILL

1977 Neogondolelliform conodonts of Early and Middle Pennsylvanian age. - Life Sciences Occasional Papers, Royal Ontario Museum, 29: 1 - 12

WALCOTT, C.D.

1905 Cambrian Brachiopoda with descriptions of new genera and species. - Proceedings, United States National Museum, 28: 227 - 337

1912 Cambrian Brachiopoda. - Monograph, United States Geological Survey, 51(1,2): 1-872; 1-363.

WATERHOL'SE, J.B

1969 Relationship between the living genus Pholadomya Sowerby and Upper Paleozoic pelecypoxts. - Lethaia, 2(2): $99-119$.

WATERHOUSE, J.B. and S. PIYASIN

1970 Mid-Permian brachopads from Khao Phrik. Thaland. Palaeontographica, 135 (A): 83.197
WESTR RMA.NN, G.E.(i.

1971 Form, structure, and function ol shell and suphuncle in colled Mesozoic ammonouds. - Lile Seiences

Contributions, Royal Ontario Museum, 78: 1 - 39.

WHITINGTON, H.H.

1953 North American Bathyuridace and Létessegeidac

(Trulubita). - Journall of Paleontology. 27(5): 6+7-678.

WILSON, A.t

1921 The range of certain Lower Ordovician faunas of the Ottawa Valley. - Museum Bulletin. Geological Survey ol Canada. 33: $19-58$.

WILSON, M.V.H.

1977 New records of insect families from the freshwater Middle Eocene of British Columbia. - Canadian Journal of Earth Sciences, 14(5): 1139-1155

ZITTELL, VON K.A

1927 Text-book of Paleontology. Vol. I [2nd edition] . Macmillan \& Co., London. 839 pp. 


ISBN 0-88854-216-X

ISSN $0082-5093$ 